\title{
cells
}

Fibroblast Growth

Factor Receptor

(FGFR) Signaling

Pathway in Tumor

Edited by

Klaus Holzmann and Brigitte Marian

Printed Edition of the Special Issue Published in Cells 
Fibroblast Growth Factor Receptor (FGFR) Signaling Pathway in Tumor 



\section{Fibroblast Growth Factor Receptor (FGFR) Signaling Pathway in Tumor}

Editors

Klaus Holzmann

Brigitte Marian 


\section{Editors}

Klaus Holzmann

Medical University of Vienna

Austria
Brigitte Marian

Medical University of Vienna

Austria

Editorial Office

MDPI

St. Alban-Anlage 66

4052 Basel, Switzerland

This is a reprint of articles from the Special Issue published online in the open access journal Cells (ISSN 2073-4409) (available at: https://www.mdpi.com/journal/cells/special_issues/FGFR_ tumor).

For citation purposes, cite each article independently as indicated on the article page online and as indicated below:

LastName, A.A.; LastName, B.B.; LastName, C.C. Article Title. Journal Name Year, Article Number, Page Range.

ISBN 978-3-03936-784-9 (Hbk)

ISBN 978-3-03936-785-6 (PDF)

(C) 2020 by the authors. Articles in this book are Open Access and distributed under the Creative Commons Attribution (CC BY) license, which allows users to download, copy and build upon published articles, as long as the author and publisher are properly credited, which ensures maximum dissemination and a wider impact of our publications.

The book as a whole is distributed by MDPI under the terms and conditions of the Creative Commons license CC BY-NC-ND. 


\section{Contents}

About the Editors $\ldots \ldots \ldots \ldots \ldots \ldots \ldots \ldots \ldots \ldots \ldots \ldots \ldots$

Klaus Holzmann and Brigitte Marian

Importance of Translational Research for Targeting Fibroblast Growth Factor Receptor Signaling in Cancer

Reprinted from: Cells 2019, 8, 1191, doi:10.3390/cells8101191 _ . . . . . . . . . . . . . 1

Gerd Jomrich, Xenia Hudec, Felix Harpain, Daniel Winkler, Gerald Timelthaler,

Thomas Mohr, Brigitte Marian and Sebastian F. Schoppmann

Expression of FGF8, FGF18, and FGFR4 in Gastroesophageal Adenocarcinomas

Reprinted from: Cells 2019, 8, 1092, doi:10.3390/cells8091092 . . . . . . . . . . . . . . . .

Gregor Vlacic, Mir A. Hoda, Thomas Klikovits, Katharina Sinn, Elisabeth Gschwandtner, Katja Mohorcic, Karin Schelch, Christine Pirker, Barbara Peter-Vörösmarty, Jelena Brankovic, Balazs Dome, Viktoria Laszlo, Tanja Cufer, Ales Rozman, Walter Klepetko, Bettina Grasl-Kraupp, Balazs Hegedus, Walter Berger, Izidor Kern and Michael Grusch Expression of FGFR1-4 in Malignant Pleural Mesothelioma Tissue and Corresponding Cell Lines and its Relationship to Patient Survival and FGFR Inhibitor Sensitivity

Reprinted from: Cells 2019, 8, 1091, doi:10.3390/cells8091091 . . . . . . . . . . . . . . . .

Burcu Emine Celik-Selvi, Astrid Stütz, Christoph-Erik Mayer, Jihen Salhi, Gerald Siegwart and Hedwig Sutterlüty

Sprouty3 and Sprouty4, Two Members of a Family Known to Inhibit FGF-Mediated Signaling, Exert Opposing Roles on Proliferation and Migration of Glioblastoma-Derived Cells

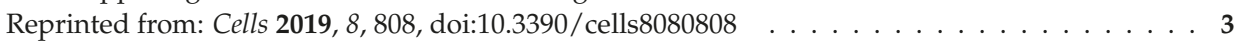

Monica Nanni, Danilo Ranieri, Flavia Persechino, Maria Rosaria Torrisi and Francesca Belleudi

The Aberrant Expression of the Mesenchymal Variant of FGFR2 in the Epithelial Context Inhibits Autophagy

Reprinted from: Cells 2019, 8, 653, doi:10.3390/cells8070653 . . . . . . . . . . . . 5

Patrycja Szybowska, Michal Kostas, Jørgen Wesche, Antoni Wiedlocha

and Ellen Margrethe Haugsten

Cancer Mutations in FGFR2 Prevent a Negative Feedback Loop Mediated by the ERK1/2 Pathway

Reprinted from: Cells 2019, 8, 518, doi:10.3390/cells8060518 _ . . . . . . . . . . . .

Katalin Csanaky, Michael W. Hess and Lars Klimaschewski

Membrane-Associated, Not Cytoplasmic or Nuclear, FGFR1 Induces Neuronal Differentiation Reprinted from: Cells 2019, 8, 243, doi:10.3390/cells8030243 . . . . . . . . . . . . . . . 91

Maria Francesca Santolla, Adele Vivacqua, Rosamaria Lappano,

Damiano Cosimo Rigiracciolo, Francesca Cirillo, Giulia Raffaella Galli, Marianna Talia,

Giuseppe Brunetti, Anna Maria Miglietta, Antonino Belfiore and Marcello Maggiolini

GPER Mediates a Feedforward FGF2/FGFR1 Paracrine Activation Coupling CAFs to Cancer

Cells toward Breast Tumor Progression

Reprinted from: Cells 2019, 8, 223, doi:10.3390/cells8030223 . . . . . . . . . . . . . . 107 
Robert Hanes, Else Munthe, Iwona Grad, Jianhua Han, Ida Karlsen, Emmet McCormack, Leonardo A. Meza-Zepeda, Eva Wessel Stratford and Ola Myklebost

Preclinical Evaluation of the Pan-FGFR Inhibitor LY2874455 in FRS2-Amplified Liposarcoma Reprinted from: Cells 2019, 8, 189, doi:10.3390/cells8020189 _ . . . . . . . . . . . . . . 129

Ana Jimenez-Pascual and Florian A. Siebzehnrubl

Fibroblast Growth Factor Receptor Functions in Glioblastoma

Reprinted from: Cells 2019, 8, 715, doi:10.3390/cells8070715

Jinglin Zhang, Patrick M. K. Tang, Yuhang Zhou, Alfred S. L. Cheng, Jun Yu, Wei Kang and Ka Fai To

Targeting the Oncogenic FGF-FGFR Axis in Gastric Carcinogenesis

Reprinted from: Cells 2019, 8, 637, doi:10.3390/cells8060637 _ . . . . . . . . . . . . . 157

Shuyan Dai, Zhan Zhou, Zhuchu Chen, Guangyu Xu and Yongheng Chen

Fibroblast Growth Factor Receptors (FGFRs): Structures and Small Molecule Inhibitors

Reprinted from: Cells 2019, 8, 614, doi:10.3390/cells8060614 . . . . . . . . . . . . . . . . . . 173

Malgorzata Czyz

Fibroblast Growth Factor Receptor Signaling in Skin Cancers

Reprinted from: Cells 2019, 8, 540, doi:10.3390/cells8060540 . . . . . . . . . . . . . . . . . . 189

Aroosha Raja, Inkeun Park, Farhan Haq and Sung-Min Ahn

FGF19-FGFR4 Signaling in Hepatocellular Carcinoma

Reprinted from: Cells 2019, 8, 536, doi:10.3390/cells8060536 . . . . . . . . . . . . . . . . 209

Marta Latko, Aleksandra Czyrek, Natalia Porębska, Marika Kucińska, Jacek Otlewski, Małgorzata Zakrzewska and Łukasz Opaliński

Cross-Talk between Fibroblast Growth Factor Receptors and Other Cell Surface Proteins

Reprinted from: Cells 2019, 8, 455, doi:10.3390/cells8050455 _ . . . . . . . . . . . . 225

Liwei Lang and Yong Teng

Fibroblast Growth Factor Receptor 4 Targeting in Cancer: New Insights into Mechanisms and Therapeutic Strategies

Reprinted from: Cells 2019, 8, 31, doi:10.3390/cells8010031 


\section{About the Editors}

Klaus Holzmann (Prof. Dr.) is a senior scientist at the Comprehensive Cancer Center of the Medical University Vienna, with extensive experience in basic and translational cancer research, including techniques in molecular and cellular biology, to study fibroblast growth factor signaling and immortality for targeting and marker development. He is an active member of the American Association for Cancer Research (AACR), and has 75 publications in peer-reviewed journals and book chapters, and more than $1400 \mathrm{SCI}$ citations, with an h-score of 24 . He has coordinated, and participated in, national funded projects, and was previously a panel evaluator for the European Research Executive Agency (REA).

Brigitte Marian (Prof. Dr.) is a senior scientist at the Institute of Cancer Research at the Medical University of Vienna. She has long-standing experience in basic and translational cancer research as a principle investigator of peer-reviewed projects. The focus of her work is the investigation of tumor-specific signaling pathways aimed at marker and drug development. She has published more than 100 papers, with 1700 citations, and an h-index of 31. 



\title{
Importance of Translational Research for Targeting Fibroblast Growth Factor Receptor Signaling in Cancer
}

\author{
Klaus Holzmann * and Brigitte Marian * \\ Medical University of Vienna, Comprehensive Cancer Center, Department of Medicine I, Division of Cancer \\ Research, Borschkegasse 8a, 1090 Vienna, Austria \\ * Correspondence: klaus.holzmann@meduniwien.ac.at (K.H.); brigitte.marian@meduniwien.ac.at (B.M.)
}

Received: 25 September 2019; Accepted: 1 October 2019; Published: 2 October 2019

Fibroblast growth factors (FGFs) are a large family of protein ligands that exert a wide range of biological effects in many organs/tissues by activating receptors (FGFRs) of the tyrosine kinase superfamily [1,2]. They are crucial for embryonic development as well as for tissue maintenance and repair in the adult organism [3]. Based on these physiological functions it is not surprising that FGFR signaling is dysregulated in practically every malignancy that has been analyzed in this context [4]. The FGFR activation is common in different tumor types, but only $<10 \%$ of all tumors sequenced carry FGFR aberrations, such as gene amplifications, mutations and rearrangements [5]. Most commonly affected (up to $32 \%$ ) are specific tumor types such as urothelial, breast, endometrial and squamous cell lung cancer. The more frequent mechanism is the upregulation of FGFs to establish autocrine and paracrine loops [6-8]. This adds an additional layer of complexity, because the secreted factors also affect cells of the microenvironment while FGFs produced in the microenvironment may stimulate the cancer cells [9].

Efforts to target FGF signaling in tumors have been going on for about a decade and produced several mostly multi-target compounds that inhibit vascular endothelial growth factor and platelet-derived growth factor in addition to FGFRs. Several such inhibitors are already in clinical trials or used as cancer drugs [10,11]. With regard to the FGFR family, FGFRs1-3 are so closely related that small molecule inhibitors usually affect all 3 in a similar way. Only for FGFR4 with its distinctly different kinase domain, a specific inhibitor has been developed [10,12].

There is still much we do not know: the intricate signaling network underlying the impact of FGFs on the growth, survival and invasiveness of cancer cells and the interaction of FGF-signaling with healthy cells in a paracrine manner driving angiogenesis and metastasis need to be further elucidated to define therapeutic targets and predictive markers for cancer therapy. Since 2017 several excellent articles about general FGFR targeting in cancer have been published, e.g., [10,13]. However, a translational perspective of targeting FGFR signaling for specific cancer subtypes was currently the main topic of only a limited number of review articles, e.g., for squamous cell lung cancer [14], breast cancer [15], endometrial cancer [16], pancreatic cancer [17], prostate cancer [18], and focusing on FGFR4 signaling in hepatocarcinogenesis [19].

This Special Issue of Cells undertakes to cover translational research on FGFR signaling from basic science to clinical studies with strong emphasis on the improvement of knowledge for clinical application. Our call for this special issue entitled "Fibroblast Growth Factor Receptor (FGFR) Signaling Pathway in Tumor" resulted in a total of 15 published articles, including seven reviews.

This specific collection of seven review articles delineate expression and targeting options extending the current knowledge about the aforementioned cancer subtypes for glioblastoma [20], gastric cancer [21] and skin cancer [22] and provides updates about hepatocellular carcinoma and targeting FGFR4 signaling [23,24]. It includes structural information about FGFRs important for development 
of small molecule inhibitors [25] and offers information about the regulation of FGFRs especially by plasma membrane-embedded partner proteins that may act as coreceptors [26]. In hepatocellular carcinomas [23], but also in some other malignancies [24], upregulation of FGFR4 is coupled to secretion of FGF19 to form an autocrine loop and offers a promising therapeutic target- especially as FGFR4-specific targeting compounds have been developed and are already in clinical trials [24]. Dai et al. give a comprehensive overview of the development of FGFR inhibitors and their specificities in relation to their interaction with the FGFR kinase domains [25]. Czys reports in her review on melanomas that alterations in FGF-signaling are not driving the malignant process, but they do increase with tumor progression and contribute to more aggressive phenotypes and therapy resistance [22]. Consequently, targeting FGFRs is suggested for combination therapy [22]. Similar observations have been reported for other malignancies, such as colon cancer [27,28], mesothelioma [29], and lung cancer [30].

Of the reports on original data, two articles by Nanni et al. and Csanaky et al. contribute results on FGFR-dependent signaling and its biological impact on autophagy and differentiation in non-malignant in vitro cell models [31,32]. FGFR variant expression and subcellular localization are essential for the observed biological effects that could impact carcinogenesis. For example, the expression of mesenchymal FGFR variants, such as the IIIc alternative splicing variant in epithelial tumor cells, may increase FGFR signaling via paracrine FGF ligand effects [33]. Szybowska et al. analyzed the impact of FGFR2 mutations on downstream signaling and feed-back loops [34]. Santolla et al. address the issue of tumor cell-microenvironment cross-talk, as they report on interaction with the G-protein estrogen receptor upregulating FGF2 in cancer associated fibroblasts that in turn impacts on the FGFR1 expressing breast cancer cells [35].

More tumor type-specific aspects are taken up in four research articles. Celik-Selvi et al. studied members of the Sprouty protein family that are well-known to inhibit FGFR signaling but some show a tumor-promoting function in brain cancer [36]. Vlacic et al. report about the expression of FGFRs and their prognostic significance in a very rare malignancy—malignant pleural mesothelioma [37]—and Jomrich et al. have analyzed FGFs as prognostic markers in adenocarcinomas of the esophageal-gastric junction [38]. Sarcomas exhibit predominant FGFR1 expression that can be specifically blocked in vitro in human and canine cell models [39]. FGFR expression profiles and blocking capacity were identical and support future comparative research in both species. In this Special Issue, a preclinical study in vivo by Hanes et al. identified amplified FRS2 as the determinant of response to FGFR-inhibitors in high-grade metastatic dedifferentiated liposarcoma, thus paving the way for clinical trials with a pan-FGFR inhibitor that may be more potent to block FGFR signaling in this specific sarcoma subtype [40].

In conclusion, the data presented in this Special Issue extends our knowledge on targeting FGFR signaling for cancer therapy to new compounds/strategies and to new tumor types. They also demonstrate the need for further translational research to decipher the complex role of FGFR signaling for improved targeting in different cancer subtypes.

Author Contributions: Conceptualization, writing and editing: K.H. and B.M.

Acknowledgments: The special issue editors appreciate the timely submission and high quality of the manuscripts offered for this special issue. The support by assistant editors Jena Jin and Billie Jiao is greatly valued.

Conflicts of Interest: The authors declare no conflict of interest.

\section{References}

1. Belov, A.A.; Mohammadi, M. Molecular mechanisms of fibroblast growth factor signaling in physiology and pathology. Cold Spring Harb. Perspect. Biol. 2013, 5. [CrossRef]

2. Ornitz, D.M.; Itoh, N. The fibroblast growth factor signaling pathway. Wiley Interdiscip. Rev. Dev. Biol. 2015, 4, 215-266. [CrossRef] [PubMed] 
3. Turner, N.; Grose, R. Fibroblast growth factor signalling: From development to cancer. Nat. Rev. Cancer 2010, 10, 116. [CrossRef] [PubMed]

4. Tanner, Y.; Grose, R.P. Dysregulated fgf signalling in neoplastic disorders. Semin. Cell Dev. Biol. 2016, 53, 126-135. [CrossRef] [PubMed]

5. Helsten, T.; Elkin, S.; Arthur, E.; Tomson, B.N.; Carter, J.; Kurzrock, R. The fgfr landscape in cancer: Analysis of 4,853 tumors by next-generation sequencing. Clin. Cancer Res. 2016, 22, 259-267. [CrossRef] [PubMed]

6. Gauglhofer, C.; Paur, J.; Schrottmaier, W.C.; Wingelhofer, B.; Huber, D.; Naegelen, I.; Pirker, C.; Mohr, T.; Heinzle, C.; Holzmann, K.; et al. Fibroblast growth factor receptor 4: A putative key driver for the aggressive phenotype of hepatocellular carcinoma. Carcinogenesis 2014, 35, 2331-2338. [CrossRef] [PubMed]

7. Metzner, T.; Bedeir, A.; Held, G.; Peter-Vorosmarty, B.; Ghassemi, S.; Heinzle, C.; Spiegl-Kreinecker, S.; Marian, B.; Holzmann, K.; Grasl-Kraupp, B.; et al. Fibroblast growth factor receptors as therapeutic targets in human melanoma: Synergism with braf inhibition. J Invest Derm. 2011, 131, 2087-2095. [CrossRef]

8. Sonvilla, G.; Allerstorfer, S.; Stattner, S.; Karner, J.; Klimpfinger, M.; Fischer, H.; Grasl-Kraupp, B.; Holzmann, K.; Berger, W.; Wrba, F.; et al. Fgf18 in colorectal tumour cells: Autocrine and paracrine effects. Carcinogenesis 2008, 29, 15-24. [CrossRef]

9. Clayton, N.S.; Wilson, A.S.; Laurent, E.P.; Grose, R.P.; Carter, E.P. Fibroblast growth factor-mediated crosstalk in cancer etiology and treatment. Dev. Dyn. 2017, 246, 493-501. [CrossRef] [PubMed]

10. Babina, I.S.; Turner, N.C. Advances and challenges in targeting fgfr signalling in cancer. Nat. Rev. Cancer 2017, 17, 318-332. [CrossRef]

11. Heinzle, C.; Sutterluty, H.; Grusch, M.; Grasl-Kraupp, B.; Berger, W.; Marian, B. Targeting fibroblast-growth-factor-receptor-dependent signaling for cancer therapy. Expert Opin. Ther.Targets 2011, 15, 829-846. [CrossRef] [PubMed]

12. Katoh, M. Fgfr inhibitors: Effects on cancer cells, tumor microenvironment and whole-body homeostasis (review). Int. J. Mol. Med. 2016, 38, 3-15. [CrossRef] [PubMed]

13. Chae, Y.K.; Ranganath, K.; Hammerman, P.S.; Vaklavas, C.; Mohindra, N.; Kalyan, A.; Matsangou, M.; Costa, R.; Carneiro, B.; Villaflor, V.M.; et al. Inhibition of the fibroblast growth factor receptor (fgfr) pathway: The current landscape and barriers to clinical application. Oncotarget 2017, 8, 16052-16074. [CrossRef]

14. Hashemi-Sadraei, N.; Hanna, N. Targeting fgfr in squamous cell carcinoma of the lung. Target. Oncol. 2017, 12, 741-755. [CrossRef] [PubMed]

15. Perez-Garcia, J.; Munoz-Couselo, E.; Soberino, J.; Racca, F.; Cortes, J. Targeting fgfr pathway in breast cancer. Breast 2018, 37, 126-133. [CrossRef]

16. Winterhoff, B.; Konecny, G.E. Targeting fibroblast growth factor pathways in endometrial cancer. Curr. Probl. Cancer 2017, 41, 37-47. [CrossRef] [PubMed]

17. Kang, X.; Lin, Z.; Xu, M.; Pan, J.; Wang, Z.W. Deciphering role of fgfr signalling pathway in pancreatic cancer. Cell Prolif. 2019, 52, e12605. [CrossRef]

18. Teishima, J.; Hayashi, T.; Nagamatsu, H.; Shoji, K.; Shikuma, H.; Yamanaka, R.; Sekino, Y.; Goto, K.; Inoue, S.; Matsubara, A. Fibroblast growth factor family in the progression of prostate cancer. J. Clin. Med. 2019, 8, 183. [CrossRef]

19. Alvarez-Sola, G.; Uriarte, I.; Latasa, M.U.; Urtasun, R.; Barcena-Varela, M.; Elizalde, M.; Jimenez, M.; Rodriguez-Ortigosa, C.M.; Corrales, F.J.; Fernandez-Barrena, M.G.; et al. Fibroblast growth factor 15/19 in hepatocarcinogenesis. Dig. Dis. 2017, 35, 158-165. [CrossRef]

20. Jimenez-Pascual, A.; A Siebzehnrubl, F. Fibroblast growth factor receptor functions in glioblastoma. Cells 2019, 8, 715. [CrossRef]

21. Zhang, J.; Tang, P.M.K.; Zhou, Y.; Cheng, A.S.L.; Yu, J.; Kang, W.; To, K.F. Targeting the oncogenic fgf-fgfr axis in gastric carcinogenesis. Cells 2019, 8, 637. [CrossRef] [PubMed]

22. Czyz, M. Fibroblast growth factor receptor signaling in skin cancers. Cells 2019, 8, 540. [CrossRef] [PubMed]

23. Raja, A.; Park, I.; Haq, F.; Ahn, S.M. Fgf19-fgfr4 signaling in hepatocellular carcinoma. Cells 2019, 8, 536. [CrossRef] [PubMed]

24. Lang, L.; Teng, Y. Fibroblast growth factor receptor 4 targeting in cancer: New insights into mechanisms and therapeutic strategies. Cells 2019, 8, 31. [CrossRef]

25. Dai, S.; Zhou, Z.; Chen, Z.; Xu, G.; Chen, Y. Fibroblast growth factor receptors (fgfrs): Structures and small molecule inhibitors. Cells 2019, 8, 614. [CrossRef] 
26. Latko, M.; Czyrek, A.; Porebska, N.; Kucinska, M.; Otlewski, J.; Zakrzewska, M.; Opalinski, L. Cross-talk between fibroblast growth factor receptors and other cell surface proteins. Cells 2019, 8, 455. [CrossRef]

27. Ahmed, M.A.; Selzer, E.; Dorr, W.; Jomrich, G.; Harpain, F.; Silberhumer, G.R.; Mullauer, L.; Holzmann, K.; Grasl-Kraupp, B.; Grusch, M.; et al. Fibroblast growth factor receptor 4 induced resistance to radiation therapy in colorectal cancer. Oncotarget 2016, 7, 69976-69990. [CrossRef]

28. Erdem, Z.N.; Schwarz, S.; Drev, D.; Heinzle, C.; Reti, A.; Heffeter, P.; Hudec, X.; Holzmann, K.; Grasl-Kraupp, B.; Berger, W.; et al. Irinotecan upregulates fibroblast growth factor receptor 3 expression in colorectal cancer cells, which mitigates irinotecan-induced apoptosis. Transl. Oncol. 2017, 10, 332-339. [CrossRef]

29. Schelch, K.; Hoda, M.A.; Klikovits, T.; Münzker, J.; Ghanim, B.; Wagner, C.; Garay, T.; Laszlo, V.; Setinek, U.; Dome, B.; et al. Fibroblast growth factor receptor inhibition is active against mesothelioma and synergizes with radio- and chemotherapy. Am. J. Respir. Crit. Care Med. 2014, 190, 763-772. [CrossRef]

30. Fischer, H.; Taylor, N.; Allerstorfer, S.; Grusch, M.; Sonvilla, G.; Holzmann, K.; Setinek, U.; Elbling, L.; Cantonati, H.; Grasl-Kraupp, B.; et al. Fibroblast growth factor receptor-mediated signals contribute to the malignant phenotype of non-small cell lung cancer cells: Therapeutic implications and synergism with epidermal growth factor receptor inhibition. Mol. Cancer Ther. 2008, 7, 3408-3419. [CrossRef]

31. Nanni, M.; Ranieri, D.; Persechino, F.; Torrisi, M.R.; Belleudi, F. The aberrant expression of the mesenchymal variant of fgfr2 in the epithelial context inhibits autophagy. Cells 2019, 8, 653. [CrossRef] [PubMed]

32. Csanaky, K.; Hess, M.W.; Klimaschewski, L. Membrane-associated, not cytoplasmic or nuclear, fgfr1 induces neuronal differentiation. Cells 2019, 8, 243. [CrossRef] [PubMed]

33. Holzmann, K.; Grunt, T.; Heinzle, C.; Sampl, S.; Steinhoff, H.; Reichmann, N.; Kleiter, M.; Hauck, M.; Marian, B. Alternative splicing of fibroblast growth factor receptor igiii loops in cancer. J. Nucleic Acids 2012, 2012, 950508. [CrossRef] [PubMed]

34. Szybowska, P.; Kostas, M.; Wesche, J.; Wiedlocha, A.; Haugsten, E.M. Cancer mutations in fgfr2 prevent a negative feedback loop mediated by the erk1/2 pathway. Cells 2019, 8, 518. [CrossRef] [PubMed]

35. Santolla, M.F.; Vivacqua, A.; Lappano, R.; Rigiracciolo, D.C.; Cirillo, F.; Galli, G.R.; Talia, M.; Brunetti, G.; Miglietta, A.M.; Belfiore, A.; et al. Gper mediates a feedforward fgf2/fgfr1 paracrine activation coupling cafs to cancer cells toward breast tumor progression. Cells 2019, 8, 223. [CrossRef]

36. Celik-Selvi, B.E.; Stutz, A.; Mayer, C.E.; Salhi, J.; Siegwart, G.; Sutterluty, H. Sprouty3 and sprouty4, two members of a family known to inhibit fgf-mediated signaling, exert opposing roles on proliferation and migration of glioblastoma-derived cells. Cells 2019, 8, 808. [CrossRef]

37. Vlacic, G.; Hoda, M.A.; Klikovits, T.; Sinn, K.; Gschwandtner, E.; Mohorcic, K.; Schelch, K.; Pirker, C.; Peter-Vörösmarty, B.; Brankovic, J.; et al. Expression of fgfr1-4 in malignant pleural mesothelioma tissue and corresponding cell lines and its relationship to patient survival and fgfr inhibitor sensitivity. Cells 2019, 8, 1091. [CrossRef]

38. Jomrich, G.; Hudec, X.; Harpain, F.; Winkler, D.; Timelthaler, G.; Mohr, T.; Marian, B.; Schoppmann, S.F. Expression of fgf8, fgf18, and fgfr4 in gastroesophageal adenocarcinomas. Cells 2019, 8, 1092. [CrossRef]

39. Schweiger, N.; Hauck, M.; Steinhoff, H.; Sampl, S.; Reifinger, M.; Walter, I.; Kreilmeier, T.; Marian, B.; Grusch, M.; Berger, W.; et al. Canine and human sarcomas exhibit predominant fgfr1 expression and impaired viability after inhibition of signaling. Mol. Carcinog. 2015, 54, 841-852. [CrossRef]

40. Hanes, R.; Munthe, E.; Grad, I.; Han, J.; Karlsen, I.; McCormack, E.; Meza-Zepeda, L.A.; Stratford, E.W.; Myklebost, O. Preclinical evaluation of the pan-fgfr inhibitor ly2874455 in frs2-amplified liposarcoma. Cells 2019, 8, 189. [CrossRef] 
Article

\title{
Expression of FGF8, FGF18, and FGFR4 in Gastroesophageal Adenocarcinomas
}

\author{
Gerd Jomrich ${ }^{1,2}$, Xenia Hudec ${ }^{2}$, Felix Harpain ${ }^{1,2}$, Daniel Winkler ${ }^{3}$, Gerald Timelthaler ${ }^{2}$, \\ Thomas Mohr ${ }^{2}$, Brigitte Marian ${ }^{2, *}$ and Sebastian F. Schoppmann ${ }^{1}$ \\ 1 Department of Surgery, Medical University of Vienna and Gastroesophageal Tumor Unit, Comprehensive \\ Cancer Center (CCC), Spitalgasse 23, 1090 Vienna, Austria; gerd.jomrich@meduniwien.ac.at (G.J.); \\ felix.harpain@meduniwien.ac.at (F.H.); sebastian.schoppmann@meduniwien.ac.at (S.F.S.) \\ 2 Department of Medicine I, Institute of Cancer Research, Medical University of Vienna, Borschkegasse 8a, \\ 1090 Vienna, Austria; xenia.hudec@meduniwien.ac.at (X.H.); gerald.timelthaler@meduniwien.ac.at (G.T.); \\ thomas.mohr@meduniwien.ac.at (T.M.) \\ 3 Department of Statistics and Operations Research, University of Vienna, Oskar Morgenstern Platz 1, \\ 1090 Vienna, Austria; Daniel.Winkler@wu.ac.at \\ * Correspondence: brigitte.marian@meduniwien.ac.at; Tel.: +43-1-40160-57522
}

Received: 15 June 2019; Accepted: 12 September 2019; Published: 16 September 2019

\begin{abstract}
Even though distinctive advances in the field of esophageal cancer therapy have occurred over the last few years, patients' survival rates remain poor. FGF8, FGF18, and FGFR4 have been identified as promising biomarkers in a number of cancers; however no data exist on expression of FGF8, FGF18, and FGFR4 in adenocarcinomas of the esophago-gastric junction (AEG). A preliminary analysis of the Cancer Genome Atlas (TCGA) database on FGF8, FGF18, and FGFR4 mRNA expression data of patients with AEG was performed. Furthermore, protein levels of FGF8, FGF18, and FGFR4 in diagnostic biopsies and post-operative specimens in neoadjuvantly treated and primarily resected patients using immunohistochemistry were investigated. A total of 242 patients was analyzed in this study: 87 patients were investigated in the TCGA data set analysis and 155 patients in the analysis of protein expression using immunohistochemistry. High protein levels of FGF8, FGF18, and FGFR4 were detected in $94(60.7 \%), 49(31.6 \%)$ and $84(54.2 \%)$ patients, respectively. Multivariable Cox proportional hazard regression models revealed that high expression of FGF8 was an independent prognostic factor for diminished overall survival for all patients and for neoadjuvantly treated patients. By contrast, FGF18 overexpression was significantly associated with longer survival rates in neoadjuvantly treated patients. In addition, FGF8 protein level correlated with Mandard regression due to neoadjuvant therapy, indicating potential as a predictive marker. In summary, FGF8 and FGF18 are promising candidates for prognostic factors in adenocarcinomas of the esophago-gastric junction and new potential targets for new anti-cancer therapies.
\end{abstract}

Keywords: FGF8; FGF18; FGFR4; adenocarcinoma of the esophagogastric junction; neoadjuvant therapy

\section{Introduction}

Esophageal Cancer (EC) is the eighth most common cancer worldwide. Whereas the number of esophageal squamous cell carcinomas (ESCC) is decreasing, the number of adenocarcinomas of the esophago-gastric junction (AEG) is increasing dramatically [1]. Despite improvements in diagnostics and the use of multimodal approaches, combining surgical resection with perioperative chemo-(radio) therapy, overall prognosis of AEG remains poor [2,3]. Survival rates vary considerably among patients with AEG, and an appreciable proportion of patients with advanced stages develop recurrence, even after initially curative resection $[4,5]$. Therapy response is often limited due to a number of inherent mechanisms of resistance [6]. This problem is aggravated by the heterogeneity in malignant tumors, 
containing a small subpopulation of cancer stem-like cells (CSC), characterized by a long lifespan and enhanced survival capacity that supports drug resistance $[7,8]$. Stem cell characteristics of CSCs are governed by the activity of distinct stem cell specific regulatory pathways leading to cancer relapse as well as chemo- and radio-resistance [9]. The role of CD133- and CD44-positive subpopulation in EC has been described recently [10-12] and the wnt-, notch-, hedgehog-, and hippo-pathways have been identified as stem cell specific targets driving therapy resistance and relapse [10-13]. Both CSC-specific signaling pathways and the survival capacity of a larger tumor cell pool might influence the therapy response.

Specifically, FGFs have found their way into anti-cancer therapy as targets to overcome resistance to chemotherapy in a number of different malignancies [14]. FGFs play a major physiological role in embryonic development and tissue repair by mediating strong survival signals via activation of the direct receptor substrate FRS2 $\alpha$, and the RAS- and PI3K-pathways [15-17]. In cancers the pathway might be deregulated by manifold-mechanisms causing either hyperactivation or even constitutively active FGFR-dependent survival signaling [14]. Both expression of specific receptors and up-regulation of autocrine FGF ligands have been found to be associated with resistance to chemo-(radiation) as well as to targeted therapy [18-22]. Previously, our group has studied a CD44-positive stem-like population in colorectal cancer (CRC) and identified a wnt-driven FGF18-dependent autocrine-signaling loop as a strong driver of tumor cell survival $[8,23]$. Furthermore, we demonstrated a progressive up regulation of FGF18 in CRC [24]. The growth factor induces autocrine survival signaling via the FGF receptor FGFR3-IIIc and blocking of this receptor inhibits tumor growth by inducing apoptosis [25]. Alternatively, FGF18 effects may be mediated by FGFR4, a receptor for which a polymorphic variant exists that causes substitution of an arginine for a glycine at position 388 in the transmembrane domain [26-28]. FGF8 is known to play an important role in embryonic development $[29,30]$. In tumors, overexpression of FGF8 is associated with diminished survival based on stimulating anti-apoptotic pathways mediated by the IIIc splice variants of FGFR1, 2, 3 as well as FGFR4 [28,31,32]. Recently, we could show that the expression of FGF8 was strongly associated with the regression grade in neoadjuvantly treated colorectal cancer patients [33].

Until now, little has been known about the role of FGFs and their receptors in AEG, in particular to the best of our knowledge no data was published describing the expression of FGF 8, 18, and FGFR4 in AEG. Therefore, the aim of this study is to investigate the role of FGF 8, 18, and FGFR4 in AEG in order to define predictive markers and possibly identify suitable new targets for multimodal therapies.

\section{Materials and Methods}

\subsection{Preliminary TCGA (The Cancer Genome Atlas) Analysis}

Data (HTSeq counts) for AEG were downloaded from the TCGA-ESCA project, preprocessed, and normalized using the TCGABiolinks package of R [34]. Optimal cutoff values for gene expression were determined by maximizing the log-rank statistics using the survminer package of $R$ [35]. Differentially expressed genes where determined using TCGABiolinks, employing the edgeR algorithm with exact testing [36]. Gene expression of relevant KEGG pathways was visualized using pathview $[37,38]$.

\subsection{Patient Selection}

Patients who underwent a resection of gastroesophageal adenocarcinomas between January 1992 and April 2012 at the Department of Surgery at the Medical University Vienna were identified from a prospectively maintained database. Patients with distant metastasis at time of diagnosis were excluded. The study was approved by the Ethics Committee of the Medical University of Vienna, Austria, according to the declaration of Helsinki (EK 1652/2016). Patients with locally advanced AEG received neoadjuvant chemotherapy according to the recommendation of the interdisciplinary tumor board meeting. Regression grade to neoadjuvant chemotherapy was classified as defined by Mandard 
A.M. et al. [39]. The tumor stage was conducted according to the pathological tumor-node-metastasis (TNM) classification of the Union for International Cancer Control (UICC), 7th edition.

\subsection{Immunohistochemistry}

Immunohistochemistry (IHC) was performed on paraffin-embedded specimens fixed in $4 \%$ buffered formalin, using 3- $\mu$ m-thick histological sections. Furthermore, per case two tissue cylinders with a 2.0 millimeter diameter were punched from representative tissue areas to build a tissue micro array (TMA), as described previously [40]. Expression of FGF8, FGF18, and FGFR4 was detected by using polyclonal rabbit antibodies as follows: FGF8 antibody (Abcam, Cambridge, UK, ab203030) in a dilution of 1:600, FGF18 antibody (Assay Biotech, Fremont, CA, USA, C12364) in a dilution of 1:500, and FGFR4 antibody (Santa Cruz Biotechnology, Dallas, TX, USA, sc-124) in a dilution of 1:400, respectively. Secondary antibody was biotinylated and coupled to an avidin-biotin-HRP complex (Thermo Scientific ${ }^{\mathrm{TM}}$ Lab Vision ${ }^{\mathrm{TM}}$ UltraVision $^{\mathrm{TM}}$ LP, Waltham, MA, USA). 3,30-diaminobenzidine (DAB; Chromogen) was used to visualize the staining and counterstaining was achieved with hematoxylin. Antibodies used in this study were optimized for gastroesophageal adenocarcinomas on colorectal cancer tissue with known expression from previously published studies [33,41]. Two observers (J.G. and H.F.) independently reviewed all slides. For the quantitative evaluation of expression, only epithelial cells were investigated. Immunostaining scores (0-12) of FGF8, FGF18, and FGFR4 were calculated as the products of the staining intensity $(0=$ negative, $1=$ weak, $2=$ moderate or $3=$ strong expression $)$ and points (0-4) were given for the percentages of tumor cells showing positive staining $0(<1 \%), 1(1-10 \%)$, $2(10-50 \%), 3(51-80 \%)$, and $4(>80 \%)$. Tumors were considered to have high expression with final scores exceeding the median score. Tumors showing expression equal or below the median were considered as being low or absent.

\subsection{Statistical Analysis}

Statistical analysis was performed using the R Statistical Software, Vienna, Austria (Version 3.6) with the "survival" package [42,43]. Univariable and multivariable analyses were conducted using the Cox proportional hazard model. The graphical analysis was performed using the Kaplan-Meier estimator. Plotting was performed using the "survminer" package [35]. The significance of differences in survival times were determined with a log-rank test. Correlations between clinicopathological parameters and FGF8, FGF18, and FGFR4 expression levels were analyzed with the $x^{2}$ test. In order to measure statistical dependence between FGF 8 and FGF 18 the non-parametric Kendall's rank correlation was used.

Overall survival (OS) was defined as the time between surgery and the patients' death. Death from causes other than AEG or survival until the end of the observation was considered as censored observations.

\section{Results}

\subsection{Preliminary TCGA (The Cancer Genome Atlas) Analysis}

While investigating mRNA expression data of patients with AEG $(n=87)$ available from the TCGA data base, overexpression of FGF8, FGF18, and FGFR4 was found in 64, 43, 12 cases, respectively. No significant correlation of overexpression of FGF8, FGF18, and FGFR4 and clinicopathological parameters (tumor stage, lymph node status and age) was found. Survival analysis using Kaplan-Meier curves for visualization, found significantly better OS rates for patients with FGF18 overexpressing tumors $(p=0.017)$. No significance could be found for FGF8 and FGFR4 (Figure 1a-c). 

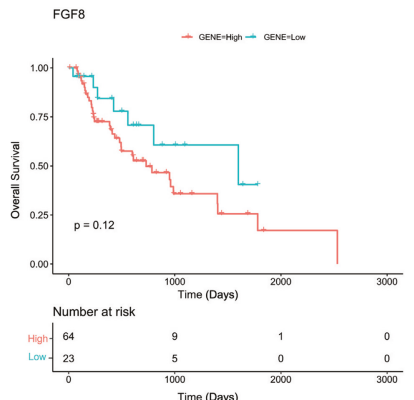

b
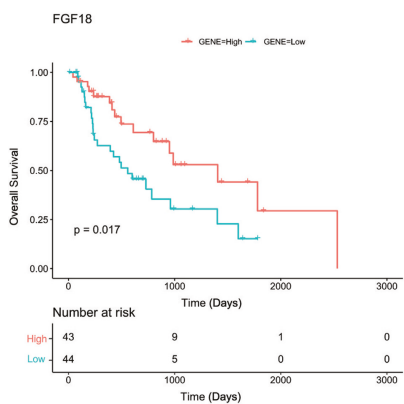

Time (Days)
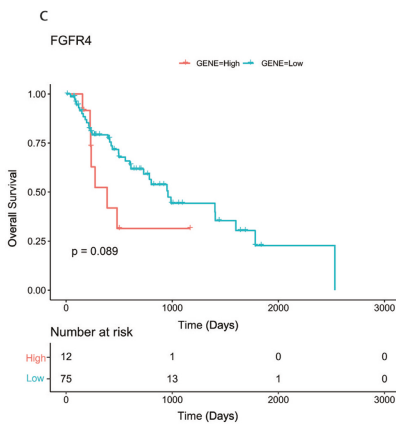

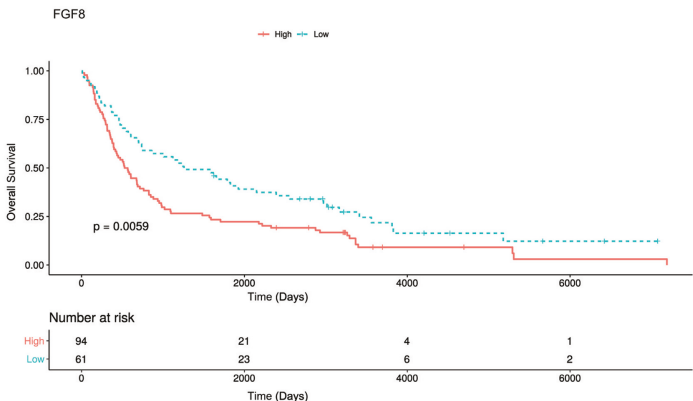

e
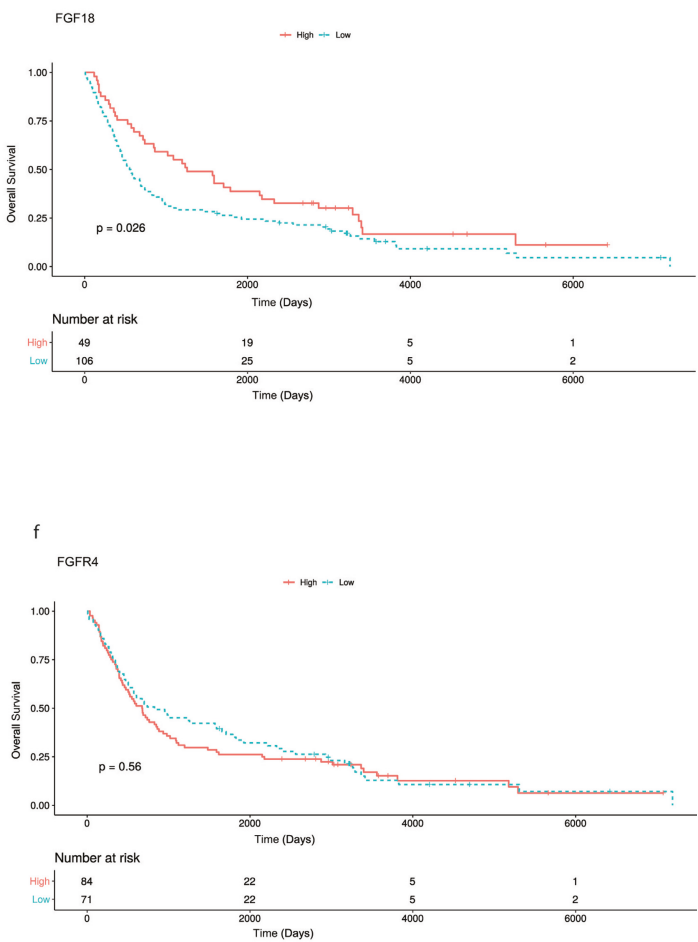

Figure 1. Kaplan-Meier curves of overall survival of patients with adenocarcinomas of the esophago-gastric junction. (a-c) Patients from TCGA data set analysis: high FGF8, FGF18, and FGFR4 expression compared with those with low/absent FGF8, FGF18, and FGFR4 expression. (d-f) Patients from the immunohistological analysis: high FGF8, FGF18, and FGFR4 expression compared with those with low/absent FGF8, FGF18, and FGFR4 expression.

\subsection{Immunohistochemical Analysis of Tumor Tissue Samples}

A total of 155 patients (124 males, $80 \%$ ) with histologically verified AEG were investigated for this study. From 10 patients full section slides were investigated to confirm staining quality for all 
antibodies used in this study. Tissue specimens of the tumors were stained for FGF8, FGF18, FGFR4, cytokeratin 7 (CK7) and the proliferation marker Ki67 (Figure 2). For all 3 markers staining was predominantly seen in the cytoplasm of tumor cells. Weaker staining was also observed in the tumor stroma. For quantification, only tumor cell staining was assessed. High expression of FGF8, FGF18, and FGFR4 was found in $94(60.7 \%), 49(31.6 \%)$ and $84(54.2 \%)$, respectively (Figure 3a-c) as compared to low expressing areas (Figure $3 \mathrm{~d}-\mathrm{f}$ ). Each marker had a distinct expression pattern with no correlation between individual markers. Correlation of clinicopathological parameters and expression of FGF8, FGF18, and FGFR4 in the tumor tissue revealed significant correlations of the FGF8 protein level with tumor size ((y)pT), UICC stage, and Mandard regression grade (Table 1). FGFR4 protein level only correlates with gender and for FGF18 no relationship with any clinical parameter could be observed (compiled in Table 1).
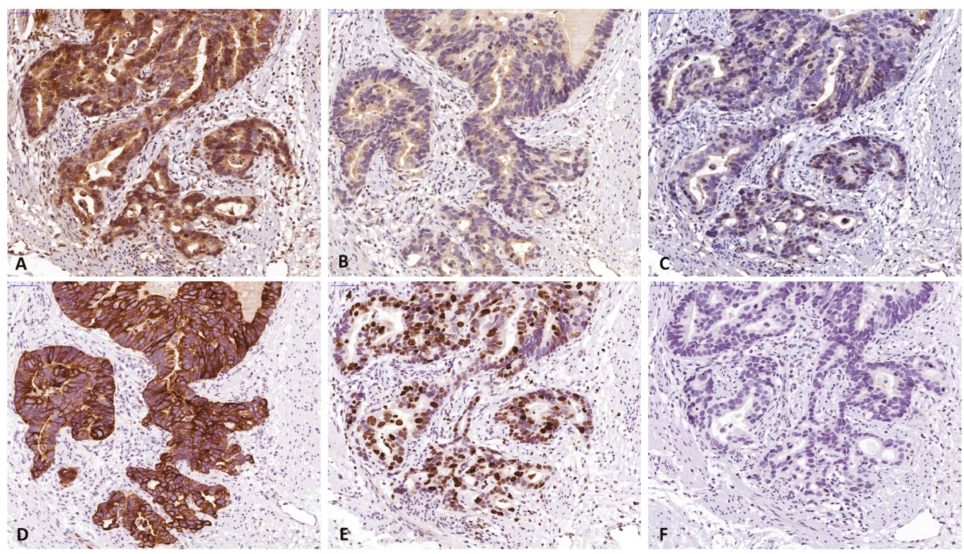

Figure 2. Specimen of adenocarcinomas of the esophago-gastric junction stained for (a) FGF8, (b) FGF18 and (c) FGFR4. Positive staining was found in the tumor cells and to a lesser degree in the microenvironment. FGF8 and FGFR4 expression were primarily found in the nucleus, while FGF18 expression was mainly found in the cytoplasm. For quantitative evaluation, only epithelial cells were investigated. Corresponding sections stained by CK7 (d), Ki67 (e), and negative control (f). (The bar corresponds to $50 \mu \mathrm{m}$.) Original magnification $\times 400$ all).
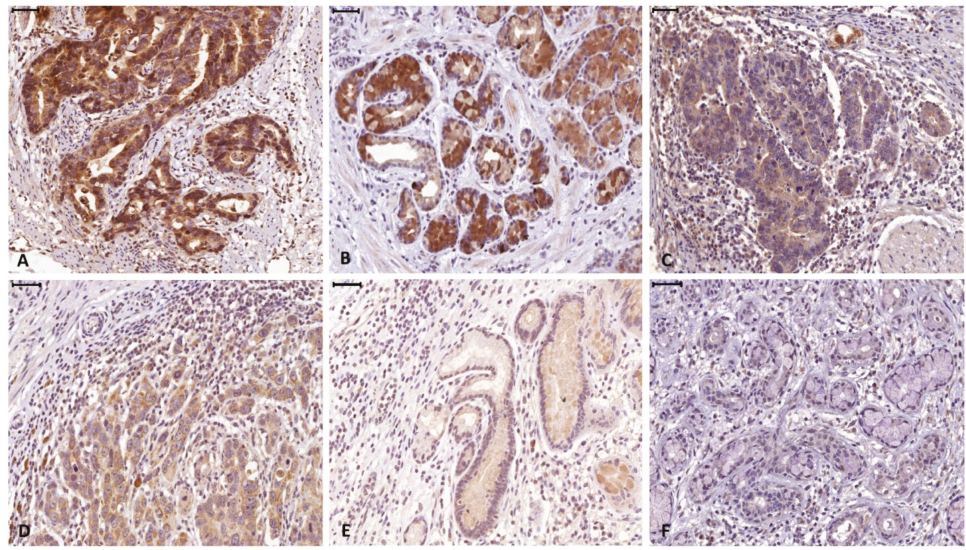

Figure 3. Representative high (a-c) and low (d-f) expressing tumor section of FGF8 (a and d), FGF18 (b and $\mathbf{e})$, and FGFR4 (c and $\mathbf{f}$ ). 


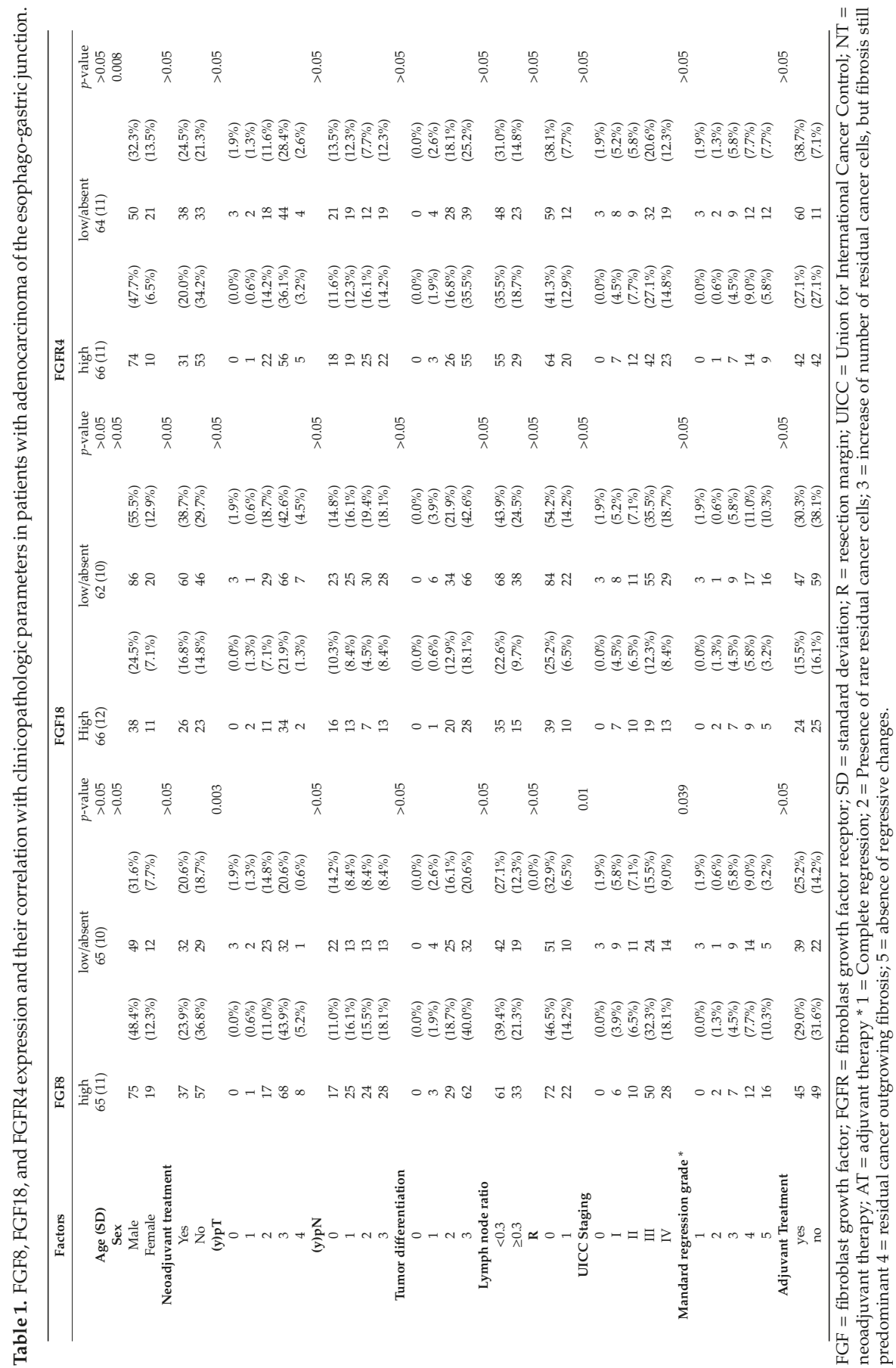


$69(44.5 \%)$ patients received neoadjuvant treatment. Median time of OS was 23 months (range 0.3-236.0 months) and 134 patients died during the time of observation. The rate of 3- and 5-year OS was $38.7 \%$ and $29.7 \%$, respectively. However, Kaplan-Meier analysis shows a significant correlation between high FGF8 expression $(p=0.006)$ and reduced patients' OS, high FGF18 expression $(p=0.026)$ was significantly associated with longer patients' OS. No significance was found for FGFR4 expression and patients' survival (Figure 1d-f).

Univariable Cox proportional hazard regression revealed that high expression of FGF8 (HR 0.61, 95\% CI 0.43-0.87, $p=0.006$ ), advanced tumor stage (HR 2.74, 95\% CI 1.37-5.50, $p=0.005$ ), poor tumor differentiation (HR $0.57,95 \%$ CI $0.39-0.83, p=0.003$ ), high lymph node ratio (HR 1.93, 95\% CI 1.35-2.77, $p<0.001$ ), positive resection margin (HR 2.06, 95\% CI 1.36-3.10, $p<0.001$ ), and receiving adjuvant treatment (HR 1.55, 95\% CI 1.10-2.18, $p=0.013$ ) were significantly associated with impaired patients OS, whereas high expression of FGF18 (HR 0.6, 95\% CI 0.45-0.95, $p=0.027$ ), negative lymph node status (HR 0.23, 95\% CI 0.14-0.39, $p<0.001$ ), and low UICC staging (HR 0.34, 95\% CI 0.19-0.63, $p<0.001$ ), were significantly associated with improved OS (Table 2). Univariable subgroup analysis revealed significant correlation for tumor size, lymph node status and UICC stage, and OS in both neoadjuvantly treated and primarily resected patients. Tumor differentiation and resection margin were found to be significantly associated with OS only in neoadjuvantly treated patients and lymph node ratio only in primarily resected patients (Table 2). Further results of univariable subgroup analysis of neoadjuvantly treated and primarily resected patients can be found in Table 2 as well.

For multivariable Cox proportional hazard regression analysis separate models for FGF8, FGF18, and FGFR4 were used. Besides FGF8, FGF18, and FGFR4 the factors age, gender, tumor differentiation, UICC stage, lymph node ratio, adjuvant treatment, and Mandard regression grade (in neoadjuvantly treated patients only) were included. In multivariable analysis, high FGF8 (HR 0.68, 95\% CI 0.46-0.99, $p=0.04$ ) was identified as the only independent predictor for shorter OS. Subgroup analysis of neoadjuvantly treated and primarily resected patients revealed that high FGF8 (HR 0.43, 95\% CI 0.22-0.82, $p=0.011$ ) and FGF18 (HR 0.44, 95\% CI 0.22-0.86, $p=0.017$ ) in neoadjuvantly treated but not in primarily resected patients remained as independent predictors for OS (Table 3 ).

\section{Discussion}

Despite significant improvements in diagnosis, surgical techniques and multimodal perioperative therapies over the last few years, survival rates of patients suffering from adenocarcinoma of the esophago-gastric junction remain poor. To investigate and understand the pathophysiological mechanisms of tumorigenesis in these cancers might be the key to better therapies and therefore to improved survival rates.

In this study, we show the utility of FGF8 and FGF18 as independent prognostic markers in AEG for the first time.

FGF and FGFR as targets have recently found their way into anti-cancer therapy, especially to overcome chemo- and radio-resistance [14]. Recently, our group investigated the role of FGF8, FGF18, and FGFR4 in colo-(rectal) and hepatocellular cancer [23,24,31,41,44]. However, data of FGF and FGFR expression in adenocarcinomas of the esophago-gastric junction were limited until now. Therefore, a TCGA analysis and immunohistochemistry, including tumor tissue from patients before and after neoadjuvant treatment, was performed to investigate the prognostic role of expression of FGF8, FGF18, and FGFR4 in adenocarcinomas of the esophago-gastric junction. 


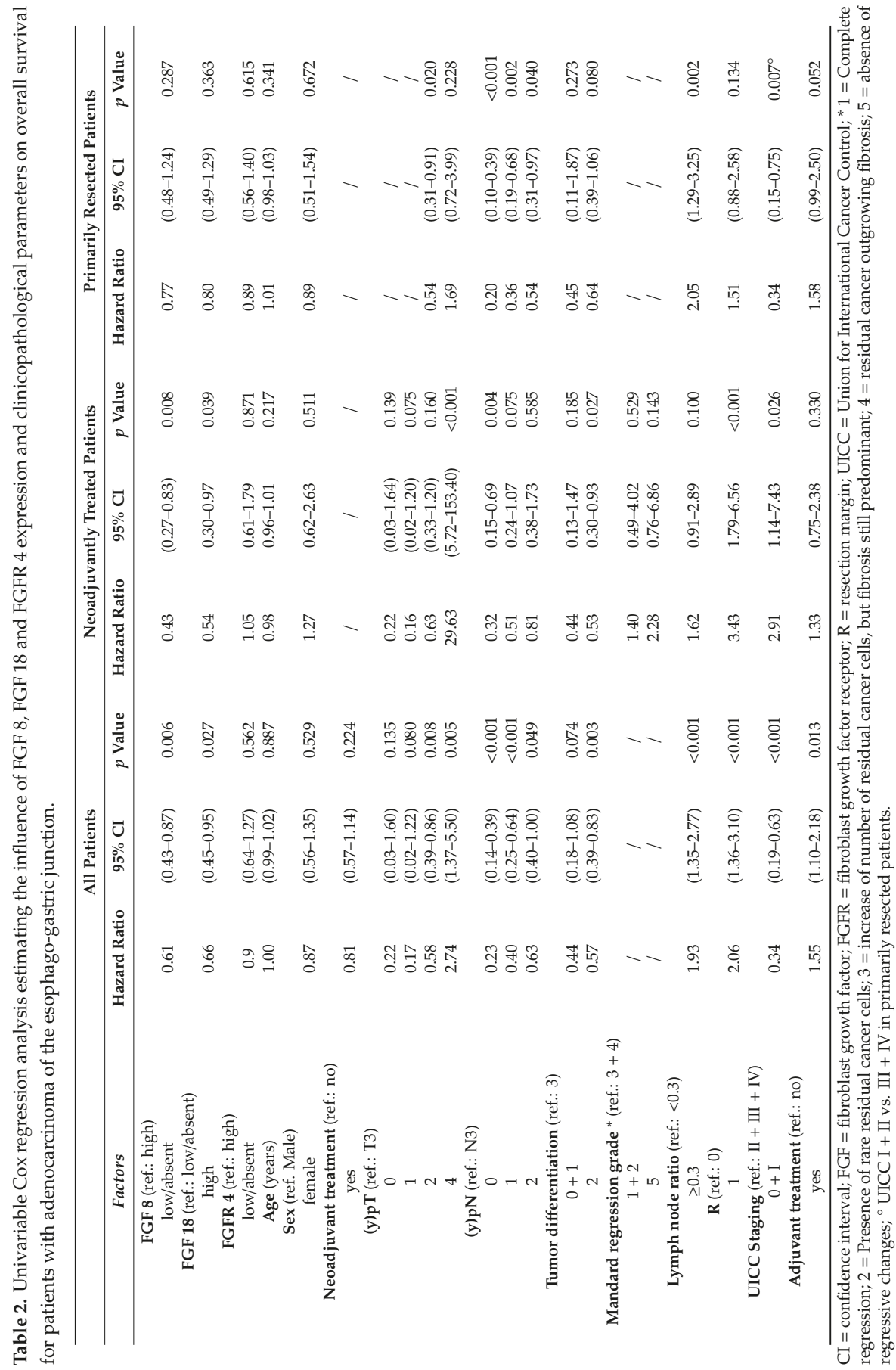


Cells 2019, 8, 1092

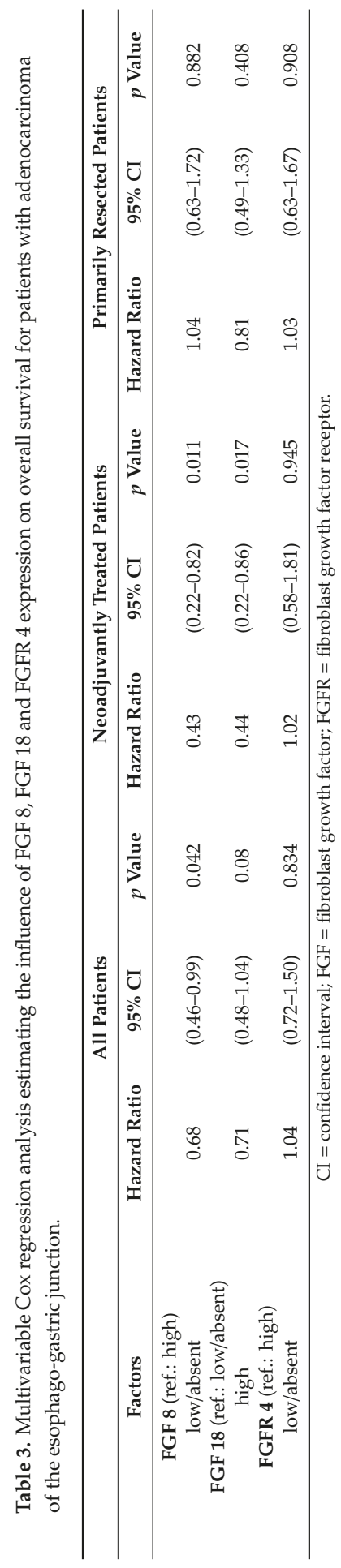


The analysis revealed significantly shorter OS when tumors were highly abundant in FGF8 in the cohort of all patients $(p=0.04)$ and the subgroup of neoadjuvantly treated patients $(p=0.011)$. This goes in good accordance with published data: overexpression of androgen related FGF8 is known to play a crucial role in prostate and colorectal cancer. Furthermore, Harpain et al. recently found that FGF8 induced therapy resistance in neoadjuvantly treated rectal cancer patients $[33,45]$. In this study FGF8 expression was found to correlate with Mandard regression grade, suggesting a role of the growth factor in therapy response. Investigating this correlation in a larger cohort would be of high interest.

With regard to FGF18, our observations in AEG contradict older reports: our previously published findings on FGF18 expression demonstrated increased tumor cell survival and migration caused by FGF18 expression in colorectal cancer [25]. In gastric cancer cell lines, Zhang et al. published data of poor survival when tumors were high in FGF18 and related it this to ERK-MAPK signaling [23,24,46]. In contrast to these results, our data show a significantly improved OS in neoadjuvantly treated patients with adenocarcinomas of the esophago-gastric junction when the tumors are high in FGF18. Analysis of the TCGA data set of AEG patients showed similar results, supporting the conclusion that FGF18 is a positive prognostic factor. Previously, we published data on ETV1 and MK2 expression in adenocarcinomas of the esophago-gastric junction, showing that nuclear overexpression of ETV1 was associated with significantly better patients OS [40]. A potential explanation for the protective role of ETV1 and FGF18 overexpression might be found in the ERK-MAPK signaling pathway as mentioned above. However, our findings remain controversial and need further investigation.

Among all the samples tested, overexpression of FGF8 and FGF18 was found in 31 (20\%) cases. However, analysis of a potential correlation between FGF8 and FGF18 overexpression in tumor tissue found no significant correlation between these two markers (Kendall's rank correlation).

Interestingly, no significant correlation between the overexpression of FGFR4 and OS was found in our analysis. Based on our previously published data on the prognostic role of FGFR4 in colorectal cancer and data on FGFR4 expression on esophageal squamous cell carcinoma, one would anticipate finding alike results in patients with adenocarcinomas of the esophago-gastric junction [41,44,47]. However, our findings are supported by our TCGA analysis of FGFR4 expression in patients with adenocarcinomas of the esophago-gastric junction, showing no significant correlation between expression and OS.

Even though the results of this study demonstrate that FGF8 and FGF18 are independent prognostic factors in patients with adenocarcinomas of the esophago-gastric junction, our study has certain limitations: one is the potential selection bias which was inevitably associated with only partial availability of tumor tissue, especially diagnostic biopsies before neoadjuvantly treated patients. Another limitation might be the retrospective nature of this single center research study. This is balanced by the fact that patient recruitment is ongoing and the patient database is maintained prospectively. Regarding the scoring method used in this study, one potential weak point has to be mentioned. Until now, no data exists on the expression of FGF8, FGF18, and FGFR4 on adenocarcinomas of the esophago-gastric junction investigated by immunohistochemistry and no signifier scoring method is available. Therefore, based on recently published recommendations for IHC scoring, conventional visual scoring based on our previously gained experiences using these antibodies on CRC tissue was used as appropriate [48-50]. However, further investigations on the expression of FGF8, FGF18, and FGFR4, using other methods including digital image analysis are urgently needed.

\section{Conclusions}

In conclusion, this is the first study that investigated the prognostic role of FGF8 and FGF18 including a subgroup analysis of neoadjuvantly treated and primarily resected patients using a preliminary TCGA analysis and immunohistochemistry demonstrating FGF8 and FGF18 as independent prognostic factors in resectable AEG. Furthermore, this is the first study comparing the expression of FGF8, FGF18, and FGFR4 in tumor tissue available before and after neoadjuvant treatment. However, 
due to the unexpected results of FGF18 overexpression and its protective nature, further investigations evaluating FGF8 and FGF18 as potential therapeutic targets are urgently needed.

Author Contributions: Conceptualization, G.J., B.M. and S.F.S.; methodology, G.J., X.H. and F.H.; software, D.W., G.T., T.M.; validation, B.M., S.F.S.; formal analysis, W.D. and T.M.; investigation, J.G., B.M.; resources, B.M., S.F.S.; data curation, G.J., F.H.; writing—original draft preparation, G.J.; writing—review and editing, G.J. and B.M.; visualization D.W., G.T. and T.M.; supervision, B.M., S.F.S.; project administration, B.M., S.F.S.

Funding: This research received no external funding.

Acknowledgments: The authors would like to thank Andrea Beer for her help to acquire diagnostic biopsies.

Conflicts of Interest: The authors declare no conflict of interest.

\section{References}

1. Ferlay, J.; Soerjomataram, I.; Dikshit, R.; Eser, S.; Mathers, C.; Rebelo, M.; Parkin, D.M.; Forman, D.; Bray, F. Cancer incidence and mortality worldwide: Sources, methods and major patterns in globocan 2012. Int. J. Cancer 2015, 136, E359-E386. [CrossRef] [PubMed]

2. Burmeister, B.H.; Smithers, B.M.; Gebski, V.; Fitzgerald, L.; Simes, R.J.; Devitt, P.; Ackland, S.; Gotley, D.C.; Joseph, D.; Millar, J.; et al. Surgery alone versus chemoradiotherapy followed by surgery for resectable cancer of the oesophagus: A randomised controlled phase iii trial. Lancet Oncol. 2005, 6, 659-668. [CrossRef]

3. Reynolds, J.V.; Muldoon, C.; Hollywood, D.; Ravi, N.; Rowley, S.; O’Byrne, K.; Kennedy, J.; Murphy, T.J. Long-term outcomes following neoadjuvant chemoradiotherapy for esophageal cancer. Ann. Surg. 2007, 245, 707-716. [CrossRef] [PubMed]

4. Christein, J.D.; Hollinger, E.F.; Millikan, K.W. Prognostic factors associated with resectable carcinoma of the esophagus. Am. Surg. 2002, 68, 258-262; discussion 262-263. [PubMed]

5. Gertler, R.; Stein, H.J.; Langer, R.; Nettelmann, M.; Schuster, T.; Hoefler, H.; Siewert, J.R.; Feith, M. Long-term outcome of 2920 patients with cancers of the esophagus and esophagogastric junction: Evaluation of the new union internationale contre le cancer/american joint cancer committee staging system. Ann. Surg. 2011, 253, 689-698. [CrossRef] [PubMed]

6. Ku, G.Y.; Ilson, D.H. Esophagogastric cancer: Targeted agents. Cancer Treat. Rev. 2010, 36, 235-248. [CrossRef] [PubMed]

7. Rassouli, F.B.; Matin, M.M.; Saeinasab, M. Cancer stem cells in human digestive tract malignancies. Tumour Biol. 2016, 37, 7-21. [CrossRef]

8. Schulenburg, A.; Ulrich-Pur, H.; Thurnher, D.; Erovic, B.; Florian, S.; Sperr, W.R.; Kalhs, P.; Marian, B.; Wrba, F.; Zielinski, C.C.; et al. Neoplastic stem cells: A novel therapeutic target in clinical oncology. Cancer 2006, 107, 2512-2520. [CrossRef]

9. Borah, A.; Raveendran, S.; Rochani, A.; Maekawa, T.; Kumar, D.S. Targeting self-renewal pathways in cancer stem cells: Clinical implications for cancer therapy. Oncogenesis 2015, 4, e177. [CrossRef]

10. Honing, J.; Pavlov, K.V.; Mul, V.E.; Karrenbeld, A.; Meijer, C.; Faiz, Z.; Smit, J.K.; Hospers, G.A.; Burgerhof, J.G.; Kruyt, F.A.; et al. Cd44, shh and sox2 as novel biomarkers in esophageal cancer patients treated with neoadjuvant chemoradiotherapy. Radiother Oncol. 2015, 117, 152-158. [CrossRef]

11. Sui, Y.P.; Jian, X.P.; Ma, L.I.; Xu, G.Z.; Liao, H.W.; Liu, Y.P.; Wen, H.C. Prognostic value of cancer stem cell marker cd133 expression in esophageal carcinoma: A meta-analysis. Mol. Clin. Oncol. 2016, 4, 77-82. [CrossRef] [PubMed]

12. Hang, D.; Dong, H.C.; Ning, T.; Dong, B.; Hou, D.L.; Xu, W.G. Prognostic value of the stem cell markers cd133 and abcg2 expression in esophageal squamous cell carcinoma. Dis. Esophagus 2012, 25, 638-644. [CrossRef] [PubMed]

13. Qian, X.; Tan, C.; Wang, F.; Yang, B.; Ge, Y.; Guan, Z.; Cai, J. Esophageal cancer stem cells and implications for future therapeutics. Onco. Targets Ther. 2016, 9, 2247-2254. [PubMed]

14. Heinzle, C.; Sutterluty, H.; Grusch, M.; Grasl-Kraupp, B.; Berger, W.; Marian, B. Targeting fibroblast-growth-factor-receptor-dependent signaling for cancer therapy. Expert Opin. Ther. Targets 2011, 15, 829-846. [CrossRef] [PubMed]

15. Beenken, A.; Mohammadi, M. The fgf family: Biology, pathophysiology and therapy. Nat. Rev. Drug Discov. 2009, 8, 235-253. [CrossRef] [PubMed] 
16. Knights, V.; Cook, S.J. De-regulated fgf receptors as therapeutic targets in cancer. Pharmacol. Ther. 2010, 125, 105-117. [CrossRef]

17. Powers, C.J.; McLeskey, S.W.; Wellstein, A. Fibroblast growth factors, their receptors and signaling. Endocr. Relat. Cancer 2000, 7, 165-197. [CrossRef] [PubMed]

18. Kono, S.A.; Marshall, M.E.; Ware, K.E.; Heasley, L.E. The fibroblast growth factor receptor signaling pathway as a mediator of intrinsic resistance to egfr-specific tyrosine kinase inhibitors in non-small cell lung cancer. Drug Resist. Updat. 2009, 12, 95-102. [CrossRef] [PubMed]

19. Motomura, K.; Hagiwara, A.; Komi-Kuramochi, A.; Hanyu, Y.; Honda, E.; Suzuki, M.; Kimura, M.; Oki, J.; Asada, M.; Sakaguchi, N.; et al. An fgf1:Fgf2 chimeric growth factor exhibits universal fgf receptor specificity, enhanced stability and augmented activity useful for epithelial proliferation and radioprotection. Biochim. Biophys. Acta 2008, 1780, 1432-1440. [CrossRef]

20. Pardo, O.E.; Arcaro, A.; Salerno, G.; Raguz, S.; Downward, J.; Seckl, M.J. Fibroblast growth factor-2 induces translational regulation of bcl-xl and bcl-2 via a mek-dependent pathway: Correlation with resistance to etoposide-induced apoptosis. J. Biol. Chem. 2002, 277, 12040-12046. [CrossRef]

21. Pardo, O.E.; Lesay, A.; Arcaro, A.; Lopes, R.; Ng, B.L.; Warne, P.H.; McNeish, I.A.; Tetley, T.D.; Lemoine, N.R.; Mehmet, H.; et al. Fibroblast growth factor 2-mediated translational control of iaps blocks mitochondrial release of smac/diablo and apoptosis in small cell lung cancer cells. Mol. Cell Biol. 2003, 23, 7600-7610. [CrossRef] [PubMed]

22. Roidl, A.; Berger, H.J.; Kumar, S.; Bange, J.; Knyazev, P.; Ullrich, A. Resistance to chemotherapy is associated with fibroblast growth factor receptor 4 up-regulation. Clin. Cancer Res. 2009, 15, 2058-2066. [CrossRef] [PubMed]

23. Koneczny, I.; Schulenburg, A.; Hudec, X.; Knofler, M.; Holzmann, K.; Piazza, G.; Reynolds, R.; Valent, P.; Marian, B. Autocrine fibroblast growth factor 18 signaling mediates wnt-dependent stimulation of cd44-positive human colorectal adenoma cells. Mol. Carcinog. 2015, 54, 789-799. [CrossRef] [PubMed]

24. Sonvilla, G.; Allerstorfer, S.; Stattner, S.; Karner, J.; Klimpfinger, M.; Fischer, H.; Grasl-Kraupp, B.; Holzmann, K.; Berger, W.; Wrba, F.; et al. Fgf18 in colorectal tumour cells: Autocrine and paracrine effects. Carcinogenesis 2008, 29, 15-24. [CrossRef] [PubMed]

25. Sonvilla, G.; Allerstorfer, S.; Heinzle, C.; Stattner, S.; Karner, J.; Klimpfinger, M.; Wrba, F.; Fischer, H.; Gauglhofer, C.; Spiegl-Kreinecker, S.; et al. Fibroblast growth factor receptor 3-iiic mediates colorectal cancer growth and migration. Br. J. Cancer 2010, 102, 1145-1156. [CrossRef] [PubMed]

26. Bange, J.; Prechtl, D.; Cheburkin, Y.; Specht, K.; Harbeck, N.; Schmitt, M.; Knyazeva, T.; Muller, S.; Gartner, S.; Sures, I.; et al. Cancer progression and tumor cell motility are associated with the fgfr $4 \arg (388)$ allele. Cancer Res. 2002, 62, 840-847.

27. Heinzle, C.; Erdem, Z.; Paur, J.; Grasl-Kraupp, B.; Holzmann, K.; Grusch, M.; Berger, W.; Marian, B. Is fibroblast growth factor receptor 4 a suitable target of cancer therapy? Curr. Pharm. Des. 2014, 20, 2881-2898. [CrossRef]

28. Zhang, X.; Ibrahimi, O.A.; Olsen, S.K.; Umemori, H.; Mohammadi, M.; Ornitz, D.M. Receptor specificity of the fibroblast growth factor family. The complete mammalian fgf family. J. Biol. Chem. 2006, 281, 15694-15700. [CrossRef]

29. Brewer, J.R.; Mazot, P.; Soriano, P. Genetic insights into the mechanisms of fgf signaling. Genes Dev. 2016, 30, 751-771. [CrossRef]

30. Tickle, C.; Munsterberg, A. Vertebrate limb development-the early stages in chick and mouse. Curr. Opin. Genet. Dev. 2001, 11, 476-481. [CrossRef]

31. Gauglhofer, C.; Sagmeister, S.; Schrottmaier, W.; Fischer, C.; Rodgarkia-Dara, C.; Mohr, T.; Stattner, S.; Bichler, C.; Kandioler, D.; Wrba, F.; et al. Up-regulation of the fibroblast growth factor 8 subfamily in human hepatocellular carcinoma for cell survival and neoangiogenesis. Hepatology 2011, 53, 854-864. [CrossRef] [PubMed]

32. Mattila, M.M.; Harkonen, P.L. Role of fibroblast growth factor 8 in growth and progression of hormonal cancer. Cytokine Growth Factor Rev. 2007, 18, 257-266. [CrossRef] [PubMed]

33. Harpain, F.; Ahmed, M.A.; Hudec, X.; Timelthaler, G.; Jomrich, G.; Mullauer, L.; Selzer, E.; Dorr, W.; Bergmann, M.; Holzmann, K.; et al. Fgf8 induces therapy resistance in neoadjuvantly radiated rectal cancer. J. Cancer Res. Clin. Oncol. 2019, 145, 77-86. [CrossRef] [PubMed] 
34. Colaprico, A.; Silva, T.C.; Olsen, C.; Garofano, L.; Cava, C.; Garolini, D.; Sabedot, T.S.; Malta, T.M.; Pagnotta, S.M.; Castiglioni, I.; et al. Tcgabiolinks: An r/bioconductor package for integrative analysis of tcga data. Nucleic Acids Res. 2016, 44, e71. [CrossRef] [PubMed]

35. Kassambara, A.; Kosinski, M.; Biecek, P.; Fabian, S. Survminer: Drawing survival curves using "ggplot2". Available online: https://rpkgs.datanovia.com/survminer/index.html (accessed on 10 June 2019).

36. Chen, Y.; Lun, A.; McCarthy, D.; Robinson, M.; Phipson, B.; Hu, Y.; Zhou, X.; Robinson, M.D.; Smyth, G.K. Edger: Empirical analysis of digital gene expression data in r. Available online: https://bioconductor.org/ packages/release/bioc/html/edgeR.html (accessed on 10 June 2019).

37. Kanehisa, M.; Goto, S. Kegg: Kyoto encyclopedia of genes and genomes. Nucleic Acids Res. 2000, 28, 27-30. [CrossRef] [PubMed]

38. Luo, W.; Brouwer, C. Pathview: An r/bioconductor package for pathway-based data integration and visualization. Bioinformatics 2013, 29, 1830-1831. [CrossRef] [PubMed]

39. Mandard, A.M.; Dalibard, F.; Mandard, J.C.; Marnay, J.; Henry-Amar, M.; Petiot, J.F.; Roussel, A.; Jacob, J.H.; Segol, P.; Samama, G.; et al. Pathologic assessment of tumor regression after preoperative chemoradiotherapy of esophageal carcinoma. Clinicopathologic correlations. Cancer 1994, 73, 2680-2686. [CrossRef]

40. Jomrich, G.; Maroske, F.; Stieger, J.; Preusser, M.; Ilhan-Mutlu, A.; Winkler, D.; Kristo, I.; Paireder, M.; Schoppmann, S.F. Mk2 and etv1 are prognostic factors in esophageal adenocarcinomas. J. Cancer 2018, 9 , 460-468. [CrossRef]

41. Ahmed, M.A.; Selzer, E.; Dorr, W.; Jomrich, G.; Harpain, F.; Silberhumer, G.R.; Mullauer, L.; Holzmann, K.; Grasl-Kraupp, B.; Grusch, M.; et al. Fibroblast growth factor receptor 4 induced resistance to radiation therapy in colorectal cancer. Oncotarget 2016, 7, 69976-69990. [CrossRef]

42. R Development Core Team. R: A Language and Environment for Statistical Computing; R Foundation for Statistical Computing: Vienna, Austria, 2018; Available online: https:/www.gbif.org/tool/81287/r-a-languageand-environment-for-statistical-computing (accessed on 10 June 2019).

43. Therneau, T. A Package for Survival Analysis in S. Version 2.38. 2015. Available online: https://cran.r-project. org/web/packages/survival/citation.html (accessed on 10 June 2019).

44. Heinzle, C.; Gsur, A.; Hunjadi, M.; Erdem, Z.; Gauglhofer, C.; Stattner, S.; Karner, J.; Klimpfinger, M.; Wrba, F.; Reti, A.; et al. Differential effects of polymorphic alleles of fgf receptor 4 on colon cancer growth and metastasis. Cancer Res. 2012, 72, 5767-5777. [CrossRef]

45. Dorkin, T.J.; Robinson, M.C.; Marsh, C.; Bjartell, A.; Neal, D.E.; Leung, H.Y. Fgf8 over-expression in prostate cancer is associated with decreased patient survival and persists in androgen independent disease. Oncogene 1999, 18, 2755-2761. [CrossRef] [PubMed]

46. Zhang, J.; Zhou, Y.; Huang, T.; Wu, F.; Pan, Y.; Dong, Y.; Wang, Y.; Chan, A.K.Y.; Liu, L.; Kwan, J.S.H.; et al. Fgf18, a prominent player in fgf signaling, promotes gastric tumorigenesis through autocrine manner and is negatively regulated by mir-590-5p. Oncogene 2019, 38, 33-46. [CrossRef] [PubMed]

47. Shim, H.J.; Shin, M.H.; Kim, H.N.; Kim, J.H.; Hwang, J.E.; Bae, W.K.; Chung, I.J.; Cho, S.H. The prognostic significance of fgfr4 gly388 polymorphism in esophageal squamous cell carcinoma after concurrent chemoradiotherapy. Cancer Res. Treat. 2016, 48, 71-79. [CrossRef] [PubMed]

48. Meyerholz, D.K.; Beck, A.P. Principles and approaches for reproducible scoring of tissue stains in research. Lab. Invest. 2018, 98, 844-855. [CrossRef]

49. Meyerholz, D.K.; Beck, A.P. Fundamental concepts for semiquantitative tissue scoring in translational research. ILAR J. 2018, 59, 13-17. [CrossRef] [PubMed]

50. Lin, F.; Prichard, J. (Eds.) Handbook of Practical Immunohistochemistry: Frequently Asked Questions, 2nd ed.; Springer: New York, NY, USA, 2015; p. xv. 764p.

(C) 2019 by the authors. Licensee MDPI, Basel, Switzerland. This article is an open access article distributed under the terms and conditions of the Creative Commons Attribution (CC BY) license (http://creativecommons.org/licenses/by/4.0/). 

Article

\title{
Expression of FGFR1-4 in Malignant Pleural Mesothelioma Tissue and Corresponding Cell Lines and its Relationship to Patient Survival and FGFR Inhibitor Sensitivity
}

\author{
Gregor Vlacic ${ }^{1,+}$, Mir A. Hoda ${ }^{2,+}$, Thomas Klikovits ${ }^{2}$, Katharina Sinn ${ }^{2}$, \\ Elisabeth Gschwandtner ${ }^{2}$, Katja Mohorcic ${ }^{1}$, Karin Schelch ${ }^{3}$, Christine Pirker ${ }^{3}$, \\ Barbara Peter-Vörösmarty ${ }^{3}$, Jelena Brankovic ${ }^{3}$, Balazs Dome ${ }^{2,4,5}$, Viktoria Laszlo ${ }^{2,4}$, \\ Tanja Cufer ${ }^{1}$, Ales Rozman ${ }^{1}$, Walter Klepetko ${ }^{2}$, Bettina Grasl-Kraupp ${ }^{3}$, Balazs Hegedus ${ }^{6}$, \\ Walter Berger ${ }^{3}$, Izidor Kern ${ }^{1}$ and Michael Grusch ${ }^{3, *}$ \\ 1 University Clinic for Respiratory and Allergic Diseases Golnik, 4204 Golnik, Slovenia; \\ gregor.vlacic@klinika-golnik.si (G.V.); katja.mohorcic@klinika-golnik.si (K.M.); \\ tanja.cufer@klinika-golnik.si (T.C.); ales.rozman@klinika-golnik.si (A.R.); izidor.kern@klinika-golnik.si (I.K.) \\ 2 Translational Thoracic Oncology Laboratory, Division of Thoracic Surgery, Department of Surgery, Medical \\ University of Vienna, 1090 Vienna, Austria; mir.hoda@meduniwien.ac.at (M.A.H.); \\ thomas.klikovits@meduniwien.ac.at (T.K.); katharina.sinn@meduniwien.ac.at (K.S.); \\ elisabeth.gschwandtner@meduniwien.ac.at (E.G.); balazs.dome@meduniwien.ac.at (B.D.); \\ viktoria.laszlo@meduniwien.ac.at (V.L.); walter.klepetko@meduniwien.ac.at (W.K.) \\ 3 Institute of Cancer Research, Department of Medicine I, Medical University of Vienna, 1090 Vienna, Austria; \\ karin.schelch@meduniwien.ac.at (K.S.); christine.pirker@meduniwien.ac.at (C.P.); \\ barbara.peter-voeroesmarty@meduniwien.ac.at (B.P.-V.); jelena.brankovic@meduniwien.ac.at (J.B.); \\ bettina.grasl-kraupp@meduniwien.ac.at (B.G.-K.); walter.berger@meduniwien.ac.at (W.B.) \\ 4 Department of Tumor Biology, National Koranyi Institute of Pulmonology, 1085 Budapest, Hungary \\ 5 Department of Thoracic Surgery, National Institute of Oncology-Semmelweis University, \\ 1085 Budapest, Hungary \\ 6 Department of Thoracic Surgery, University Medicine Essen-Ruhrlandklinik, 45239 Essen, Germany; \\ balazs.hegedues@rlk.uk-essen.de \\ * Correspondence: michael.grusch@meduniwien.ac.at; Tel.: +431-40160-57556 \\ + These authors contributed equally to this work.
}

Received: 31 July 2019; Accepted: 7 September 2019; Published: 16 September 2019

\begin{abstract}
Malignant pleural mesothelioma (MPM) is a devastating malignancy with limited therapeutic options. Fibroblast growth factor receptors (FGFR) and their ligands were shown to contribute to MPM aggressiveness and it was suggested that subgroups of MPM patients could benefit from FGFR-targeted inhibitors. In the current investigation, we determined the expression of all four FGFRs (FGFR1-FGFR4) by immunohistochemistry in tissue samples from 94 MPM patients. From 13 of these patients, we were able to establish stable cell lines, which were subjected to FGFR1-4 staining, transcript analysis by quantitative RT-PCR, and treatment with the FGFR inhibitor infigratinib. While FGFR1 and FGFR2 were widely expressed in MPM tissue and cell lines, FGFR3 and FGFR4 showed more restricted expression. FGFR1 and FGFR2 showed no correlation with clinicopathologic data or patient survival, but presence of FGFR3 in $42 \%$ and of FGFR4 in $7 \%$ of patients correlated with shorter overall survival. Immunostaining in cell lines was more homogenous than in the corresponding tissue samples. Neither transcript nor protein expression of FGFR1-4 correlated with response to infigratinib treatment in MPM cell lines. We conclude that FGFR3 and FGFR4, but not FGFR1 or FGFR2, have prognostic significance in MPM and that FGFR expression is not sufficient to predict FGFR inhibitor response in MPM cell lines.
\end{abstract}

Keywords: malignant pleural mesothelioma; FGFR; overall survival; immunohistochemistry; infigratinib sensitivity 


\section{Introduction}

Malignant pleural mesothelioma (MPM) is a devastating malignancy arising from mesothelial cells lining the chest cavity. Asbestos is the main causative agent for MPM but the latency period between exposure and MPM manifestation can be more than 40 years [1]. While strict regulations on the use of asbestos have been implemented in many countries, there is still widespread use and mining of asbestos in parts of the world leading to an ongoing rise in global incidence [2]. MPM is highly refractory to conventional therapies and the prognosis is generally poor with a median overall survival of little more than one year. Chemotherapy with cisplatin and pemetrexed yields a modest survival benefit, which can be slightly improved only in selected patients by addition of bevacizumab and combination with surgery and/or radiotherapy as additional treatment modalities [3]. Despite multiple clinical studies investigating targeted therapies in MPM, no effective new treatments have been identified in this area, while immunotherapy seems to be moderately effective in a subgroup of patients [3,4]. Genomic analysis of MPM has identified recurrent mutations and structural aberrations mostly in tumor suppressor genes including BAP1, NF2, TP53, SETD2, and CDKN2A, which are difficult to target directly $[5,6]$. However, there is also compelling evidence for hyperactivation of growthand survival-promoting signals in several pathways including the Hippo [7,8], phosphatidylinositol 3-kinase (PI3K) [9], and fibroblast growth factor receptor (FGFR) [10,11] signaling axes, that could provide more 'druggable' targets. Others and we have previously reported the overexpression of FGFR1 and several FGFs in MPM cell models and tissue specimens [10-12]. Moreover, we have described the growth-promoting and EMT-inducing capabilities of FGF2 in MPM cells, identified the miR-16 family members as regulators of FGFR1 and FGFR4 [13] and characterized in preclinical models the potential benefit of combining FGFR inhibition with chemotherapy or radiation $[11,14]$. Recently, a link between FGFR inhibitor sensitivity, FGF9/18 mediated FGFR3 activation, and loss of BAP1 was established [15]. Nevertheless, a comparative analysis of the expression of all four FGFRs in MPM tissues has not been performed so far.

In the current investigation, we therefore focus on expression of the four existing FGFRs (FGFR1-4) in MPM tissue and corresponding patient-derived cell lines as well as their relationship to MPM prognosis and potential prediction of response to FGFR kinase inhibition.

\section{Materials and Methods}

\subsection{Clinical Samples}

Patients: 94 MPM patients were included in the study and full clinical follow_up data was available in 81 patients, 41 from Austria (Medical University of Vienna, Vienna, Austria) and 40 from Slovenia (University Clinic for Respiratory and Allergic Diseases Golnik, Golnik, Slovenia). All patients were referred for diagnosis and treatment to one of the two institutions between 2006 and 2015. MPM diagnosis was histologically confirmed during routine clinical work-up in all patients. Patients were staged clinically and pathologically according to the IMIG staging system [16]. Details on patients' characteristics and treatment modalities are depicted in Table 1. 
Table 1. Patient characteristics

\begin{tabular}{cccc}
\hline \multirow{2}{*}{ All Patients } & & $n$ & $\%$ \\
\hline \multirow{2}{*}{ Sex } & $<60$ & 22 & 27 \\
\cline { 2 - 4 } & $\geq 60$ & 59 & 73 \\
\cline { 2 - 4 } & female & 22 & 27 \\
\hline \multirow{2}{*}{ Histology } & male & 59 & 73 \\
\cline { 2 - 4 } & non-epithelioid & 22 & 27 \\
\hline \multirow{2}{*}{ Stage } & epithelioid & 59 & 73 \\
\cline { 2 - 4 } & early & 28 & 35 \\
\hline \multirow{3}{*}{ Treatment overview } & late & 53 & 65 \\
\cline { 2 - 4 } & BSC & 15 & 19 \\
\cline { 2 - 4 } & CHT & 33 & 41 \\
\cline { 2 - 4 } & CHT + RT & 3 & 4 \\
\cline { 2 - 4 } & CHT + S & 11 & 14 \\
\hline \multirow{2}{*}{ Star } & TMT & 19 & 23
\end{tabular}

BSC: best supportive care; CHT: chemotherapy; RT: radiotherapy; S: surgery; TMT: trimodality therapy.

Tumor samples: All tumor samples were obtained during diagnostic procedures or at the time of surgery (macroscopic complete resection). Histological specimens were fixed in formalin and embedded in paraffin (FFPE). One $3 \mu \mathrm{m}$ section from a representative, tumor-rich FFPE block was stained by hematoxylin/eosin to confirm and locate malignant areas and consecutive sections were used for FGFR1-4 immunohistochemistry. Clinical data and tumor blocks were retrospectively collected for all cases according to the corresponding local ethic committees (Ethical Committee of University of Vienna; Ethical approval number: 904/2009; Date: 9 December 2019).

\subsection{Immunohistochemistry}

Primary antibodies against FGFR1 (sc-121-G), FGFR2 (sc-122), FGFR3 (sc-123), and FGFR4 (sc-124) from Santa Cruz Biotechnology (Dallas, TX, USA) have been extensively used and characterized in multiple tissues and cell models [17-19] and were used here as previously described [20]. In brief, $3 \mu \mathrm{m}$ sections were cut from FFPE blocks. Immunohistochemistry was performed on a Ventana Benchmark XT (Ventana Medical Systems, Tucson, AZ, USA) for antibodies FGFR2-4. Pretreatment with CC1 for 56 min (CC1: Ventana 950-124) was performed, incubation time for primary antibody was $32 \mathrm{~min}$ at $37^{\circ} \mathrm{C}$ (for FGFR4 $60 \mathrm{~min}$ ), dilution 1:50, counterstaining with hematoxylin (Ventana: 760-2021) and bluing reagent (760-2037) was done for 4 min each. For FGFR1, incubation with primary antibody was performed at a 1:50 dilution for $1 \mathrm{~h}$ at ambient temperature and subsequent processing was done with the ImmPRESS HRP Anti goat IgG polymer detection kit (Vector Laboratories, Peterborough, United Kingdom) as per the manufacturer's recommendations. A semi-quantitative three tier scoring system was used to assess the intensity of the cytoplasmic and/or nuclear staining - 0 if there was no staining, 1 if there was faint positivity in at least $50 \%$ of tumor cells, and 2 if there was an unequivocally strong positivity in at least $50 \%$ of tumor cells.

\subsection{Cell Lines}

Establishment of cell lines from clinical samples followed previously described protocols [21]. Cells were cultured in RPMI medium with $10 \%$ fetal bovine serum (FBS) at $37{ }^{\circ} \mathrm{C}$ in a humidified atmosphere with $5 \% \mathrm{CO}_{2}$. Cell line authentication was done by array comparative genomic hybridization and STR analysis [11,22]. All cell lines were regularly checked for mycoplasma contamination. 


\subsection{Generation of Cell Blocks}

Cells were grown to about $80 \%$ confluency in $10 \mathrm{~cm}$ dishes, washed with cold PBS and scraped into $15 \mathrm{~mL}$ tubes in PBS. After centrifugation, the supernatants were discarded and the pellets were

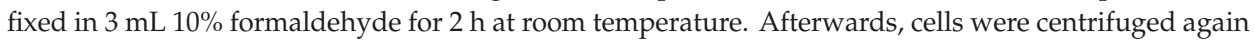
and excess formaldehyde was discarded. HistoGel (HG-4000-12, Thermo Fisher Scientific, Waltham, MA, USA) was liquefied in boiling water for $5 \mathrm{~min}$ and cell pellets were resuspended in equal volumes (ca. $50 \mu \mathrm{L}$ ) of HistoGel. The HistoGel cell mixture was pipetted as a single drop per sample onto a parafilm mounted on a cold plate. After $30 \mathrm{~min}$, the solidified drops were placed into $3 \mathrm{~mL}$ of $70 \%$ ethanol and kept at $4{ }^{\circ} \mathrm{C}$ until paraffin embedding. Further processing and immunostaining were performed using the same antibodies and procedures as for the tissue specimens.

\subsection{Drug Treatment}

For determining FGFR inhibitor sensitivity, 3000 cells per well were seeded into 96-well plates in growth medium containing 10\% FCS. After $24 \mathrm{~h}$ for recovery, cells were treated with concentrations ranging from 0.1 to $10 \mu \mathrm{M}$ of the FGFR1-3 inhibitor infigratinib (BGJ398) or the FGFR4-specific inhibitor BLU9931. Controls received vehicle (DMSO) only. After 72 h, DNA content of cells was analyzed by SYBR green detection as previously published [13]. $\mathrm{IC}_{50}$ values were determined from dose-response curves with GraphPad Prism (GraphPad Software, San Diego, CA, USA).

\subsection{Quantitative Real-Time Reverse Transcription PCR (qRT-PCR)}

Cells were grown in $25 \mathrm{~cm}^{2}$ flasks to about $80 \%$ confluence and total RNA was extracted with the innuPREP RNA mini kit (Analytik Jena, Jena, Germany) according to the manufacturer's instructions. RNA was reverse transcribed with M-MLV reverse transcriptase (Thermo Fisher Scientific) and the resulting cDNAs were used as templates for qRT-PCR analysis with Taqman assays (FGFR1: Hs00913142m1, FGFR2: Hs01552918m1, FGFR3: Hs00179829m1, FGFR4: Hs01106908m1, all from Thermo Fisher Scientific). Relative transcript levels were calculated as $2^{-\Delta C} \mathrm{t} \times 10^{5}$ normalized to the two house-keeping genes GAPDH (Hs99999905m1) and beta-actin (Hs99999903m1).

\subsection{Statistical Analysis}

Categorical data was compared by performing Fishers' exact or chi-square tests. Overall survival (OS) was defined as time between initial MPM diagnosis and date of death or last follow-up. OS was estimated by the Kaplan-Meier method and a log rank test was used to calculate survival differences between two groups. Pearson's correlation coefficients were determined to explore the relationship between two continuous variables. All results were considered statistically significant when $\mathrm{P}<0.05$ two-sided. Analyses were performed using the SPSS Statistics 23.0 package (SPSS Inc., Chicago, IL, USA) and GraphPad Prism.

\section{Results}

\subsection{FGFR1 and FGFR2 Show Strong Staining in MPM Tissue}

Staining for FGFR1-4 was performed in tumor tissue from 94 MPM patients and evaluated using a three-tier semi quantitative scoring system. Representative examples of staining are shown in Figure 1A. Overall, the tumors showed a strong staining for FGFR1 and FGFR2, whereas FGFR3 and FGFR4 showed weaker and more restricted expression (Figure 1B). The staining was mostly granular and evenly distributed for FGFR1 and FGFR2, localized in the cytoplasm and in the nucleus with a higher intensity in the latter. The cases that stained positive for FGFR3 and FGFR4 showed a more scattered distribution of staining with focal areas of more intensive staining and other areas with faint but still convincingly positive staining. A less intense-mostly cytoplasmic-reaction was occasionally seen in some areas that appeared to be composed mostly of non-tumoral cells. 
A

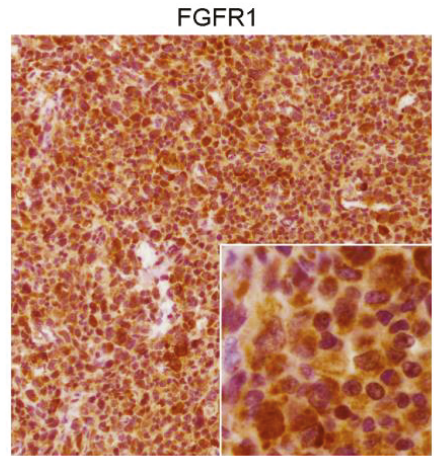

FGFR3

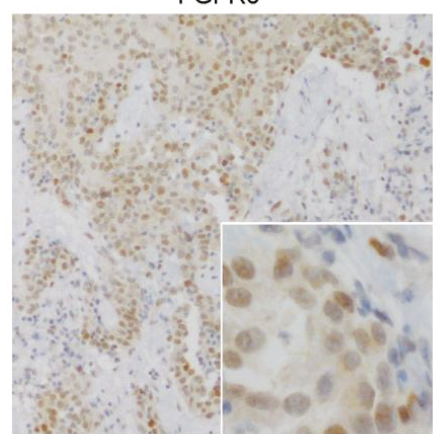

B

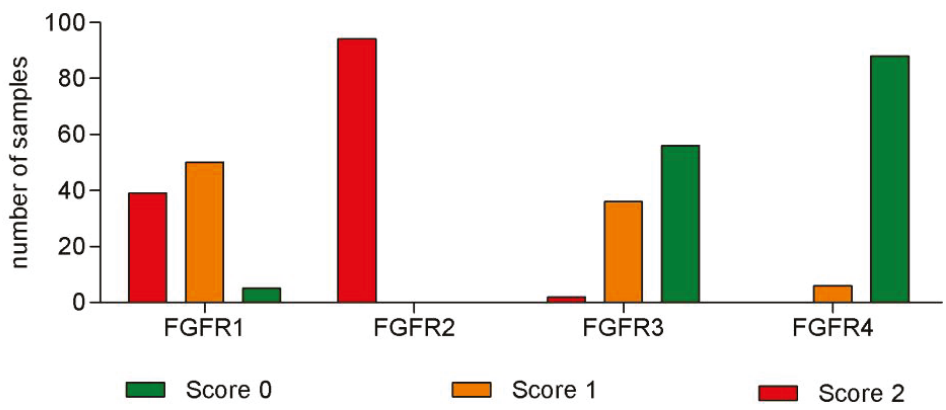

Figure 1. Expression of FGFR1-4 in MPM tissue. (A) Representative images of MPM tissue specimens stained for FGFR1 (score 2, epithelioid), FGFR2 (score 2, epithelioid), FGFR3 (score 1, epithelioid), and FGFR4 (score 1, epithelioid). Scale bar $=25 \mu \mathrm{m}$. (B) Distribution of staining intensities of FGFR1-4 in 94 MPM tissue specimens.

\subsection{Expression of FGFR3 and FGFR4 But Not of Other FGFRs Correlates with Patient Survival}

Clinical follow_up data was available for 81 patients. All patients showed a strong FGFR2 staining and therefore no correlation with clinical parameters was performed in this group. Staining for FGFR1 and FGFR3 was not correlated with age, sex, histology, stage, or type of treatment (Tables S1 and S2), whereas FGFR4 staining was found in 4 of 22 females but only 2 of 59 males (Table S3). Due to the small numbers of biphasic $(\mathrm{N}=13)$ and sarcomatoid $(\mathrm{N}=7)$ tumors, these two categories were combined as non-epithelioid for statistical analyses. Despite the absence of significant correlations 
between staining pattern and histology for any of the FGFRs, an overall trend towards low or absent staining was observed for sarcomatoid tumors (Figure 2A, Tables S1-S3).

A
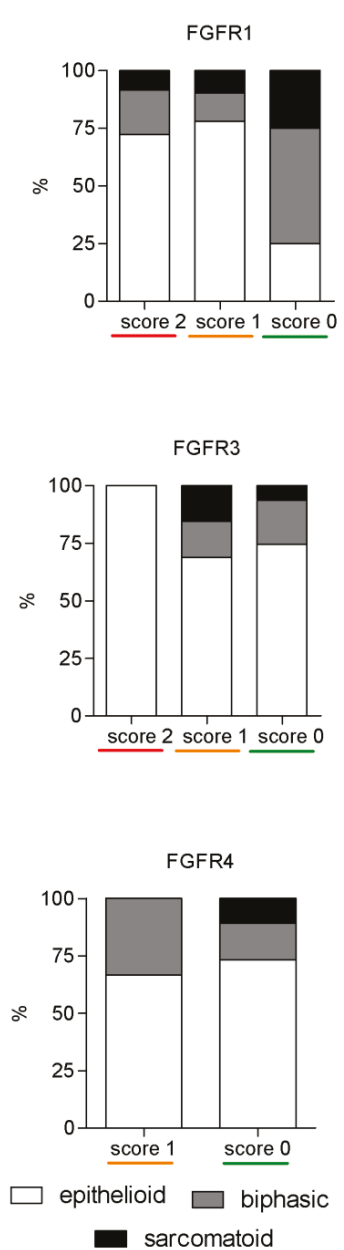

B
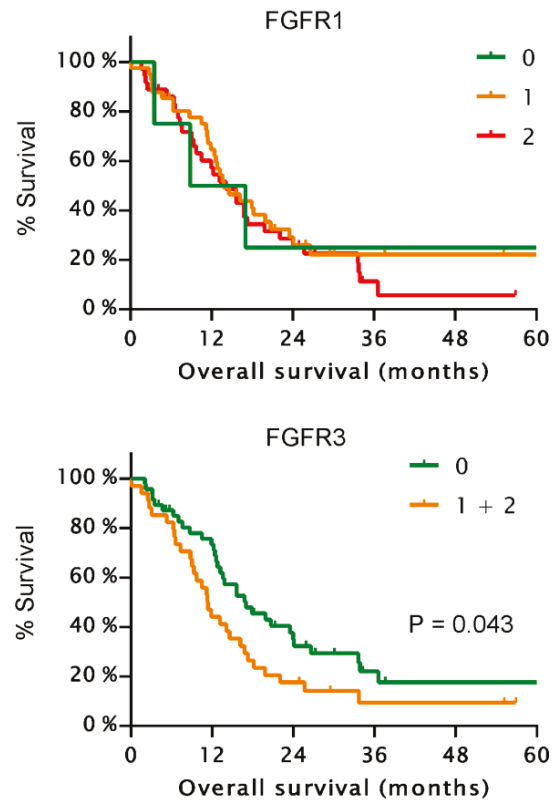

FGFR4

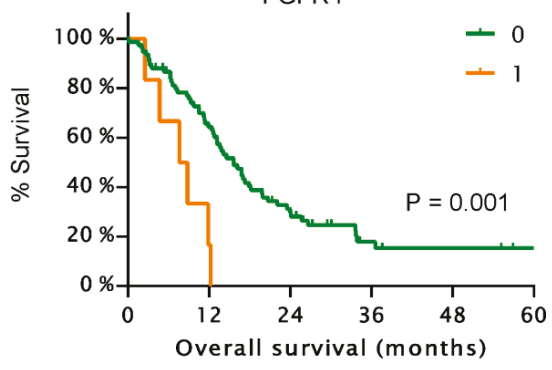

Figure 2. Correlation of FGFR expression with histology and patient prognosis. (A) Percentage of epithelioid, biphasic, and sarcomatoid tumors within the different staining groups of FGFR1 (upper panel), FGFR3 (middle panel), and FGFR4 (lower panel). (B) Kaplan-Meier curves for overall survival of MPM patients with different staining scores of FGFR1 (upper panels), FGFR3 (middle panel), and FGFR4 (lower panel).

FGFR1 staining intensity did not show a significant correlation with patient survival and also the four patients with complete absence of FGFR1 staining had similar OS (Figure 2B). Only 2 patients had a staining score of 2 for FGFR3 and therefore were pooled with the 32 patients with a staining score of 1 . Forty-seven patients showed absence of FGFR3 staining and had a significantly longer OS $(\mathrm{P}=0.043)$. FGFR4 expression, was detectable only in 6 of 81 patients $(7.4 \%)$ and showed a highly significant correlation with a shorter OS $(\mathrm{P}=0.0027)$. 


\subsection{FGFR Inhibitor Sensitivity in Patient-Derived Cell Lines}

From 13 patients analyzed in our study, establishment of stable cell lines was successful. These cell lines were tested for their response to the FGFR inhibitor infigratinib. Cell viability was determined after $72 \mathrm{~h}$ and $\mathrm{IC}_{50}$ values were calculated from dose-response curves (Figure 3A, Table 2). Of the 13 cell lines, 3 had an $\mathrm{IC}_{50}$ value below $0.5 \mu \mathrm{M}$ and were classified as sensitive, 4 cell lines had calculated $\mathrm{IC}_{50}$ levels above $10 \mu \mathrm{M}$ or showed no inhibition even at the highest concentration and were classified as resistant. The remaining six cell lines showed intermediate sensitivity. When sensitive, resistant and intermediate MPM cell lines were compared with respect to FGFR staining scores of the corresponding tissue specimens, there was no correlation between FGFR inhibitor sensitivity and the score of either FGFR1 or FGFR3 (Figure 3B, FGFR2 is not shown due to indiscriminately high staining in all samples). A similar picture also emerged when the sum of the scores for all FGFRs (not shown) or for all FGFRs with the exception of FGFR4 (which is poorly inhibited by infigratinib) was considered.

\section{A}

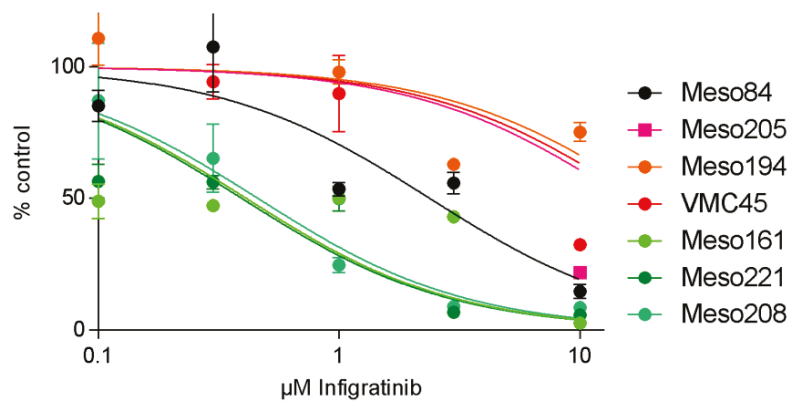

B

FGFR1

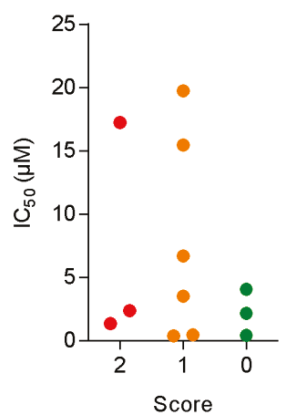

FGFR3

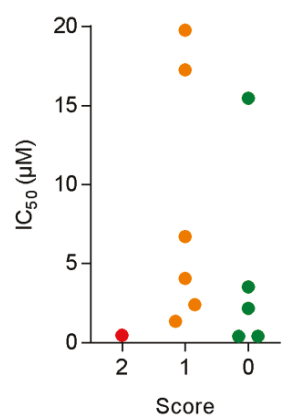

FGFR1-3

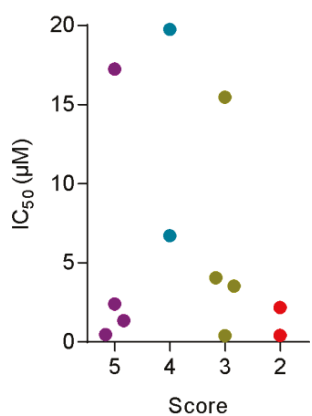

Figure 3. Sensitivity of patient-derived cell lines to the FGFR inhibitor infigratinib. (A) MPM cell lines were incubated with increasing concentrations of infigratinib or vehicle (DMSO) as control and cell number was determined after $72 \mathrm{~h}$. Dose-response curves were calculated with GraphPad Prism. Three sensitive cell lines $\left(\mathrm{IC}_{50}<1 \mu \mathrm{M}\right)$, one intermediate cell line $\left(1 \mu \mathrm{M}<\mathrm{IC}_{50}<10 \mu \mathrm{M}\right)$, and three resistant cell lines $\left(\mathrm{IC}_{50}>10 \mu \mathrm{M}\right)$ are shown in green, black, and red, respectively. (B) Infigratinib $\mathrm{IC}_{50}$ values of the cell lines were plotted against the IHC scores for FGFR1, FGFR3, or the sum of FGFR1-3 of the corresponding tumors from which the cell lines were established. 
Table 2. Cell line characteristics

\begin{tabular}{ccccccc}
\hline \multirow{2}{*}{ Cell Line } & \multirow{2}{*}{ Histology } & $\begin{array}{c}\text { IC }_{\mathbf{5 0}}(\boldsymbol{\mu M}) \\
\text { Infigratinib }\end{array}$ & FGFR1 & FGFR2 & FGFR3 & FGFR4 \\
\hline Meso49 & bi & n.i. & 2 & 2 & 1 & 0 \\
Meso62 & sarc & 2.18 & 2 & 2 & 1 & 0 \\
Meso84 & sarc & 2.39 & 2 & 2 & 1 & 0 \\
Meso92 & bi & 3.53 & 2 & 2 & 1 & 0 \\
Meso161 & bi & 0.41 & 2 & 2 & 1 & 0 \\
Meso189 & epi & 1.34 & 2 & 2 & 1 & 0 \\
Meso194 & epi & 19.76 & 2 & 2 & 1 & 0 \\
Meso205 & epi & 15.47 & 2 & 2 & 1 & 0 \\
Meso208 & epi & 0.46 & 2 & 2 & 1 & 0 \\
Meso221 & epi & 0.39 & 2 & 2 & 1 & 0 \\
VMC28 & epi & 6.71 & 2 & 2 & 1 & 0 \\
VMC40 & bi & 4.06 & 2 & 2 & 1 & 0 \\
VMC45 & epi & 17.24 & 2 & 2 & 1 & 0 \\
\hline
\end{tabular}

epi: epithelioid; bi: biphasic; sarc: sarcomatoid; n.i.: no inhibition.

\subsection{FGFR Expression in Patient-Derived Cell Lines}

Since the staining pattern of the tumors showed considerable heterogeneity and it was unclear whether cell lines derived from a tumor would have the same FGFR expression as the corresponding tumor, we generated cell blocks from all 13 cell lines of our panel and stained them according to the same protocol that had been used for the tumor specimens. Interestingly, the staining patterns of the cell lines were much more homogenous than those of the tumors they had been derived from (Figure 4A and Table 2). All 13 cell lines showed strong staining for FGFR1 and FGFR2, weak staining for FGFR3 and lacked detectable levels of FGFR4 (Table 2, Figure 4A). Due to this very homogenous protein expression pattern, it was obvious that the observed differences in sensitivity to infigratinib cannot be explained by expression differences in FGFR receptor proteins.

Therefore, we determined mRNA levels of FGFR1-4 in the whole cell line panel to be able to compare mRNA and protein levels and see whether FGFR mRNA levels might be better predictors of FGFR inhibitor sensitivity of the cell lines than staining patterns. The highest mRNA levels were found for FGFR1, whereas FGFR2, 3, and 4 were expressed at considerably lower levels (Figure 4B). Indeed, even the lowest mRNA expression level of FGFR1 was several fold higher than the highest level of any other FGFR. Plotting FGFR1 expression levels against infigratinib sensitivity showed no correlation between FGFR1 mRNA level and infigratinib $\mathrm{IC}_{50}$ (Figure $4 \mathrm{C}, \mathrm{R}^{2}=0.041, \mathrm{P}=0.5485$ ). Despite their lower expression levels, we also compared FGFR2-4 mRNA expression to infigratinib sensitivity, but no significant correlations were observed (Figure S1). There was, however, a positive correlation between FGFR3 and FGFR4 mRNA expression and a weak inverse correlation between FGFR1 and FGFR2 mRNA expression (Figure S2).

Finally, since FGFR4 was the only FGFR linked to shorter survival in MPM patients and since FGFR4 is poorly inhibited by infigratinib, we also tested the FGFR4-specific inhibitor BLU9931 in our cell line panel. Overall, $\mathrm{IC}_{50}$ values of BLU9931 were about an order of magnitude higher than those of infigratinib, and three of the cell lines showed no inhibition (Table S4). While the correlation between high FGFR4 mRNA and BLU9931 sensitivity did not reach statistical significance, notably, the two cell lines with the highest FGFR4 mRNA showed the highest sensitivity towards the FGFR4 inhibitor (Figure 4D). 
A FGFR1 FGFR2

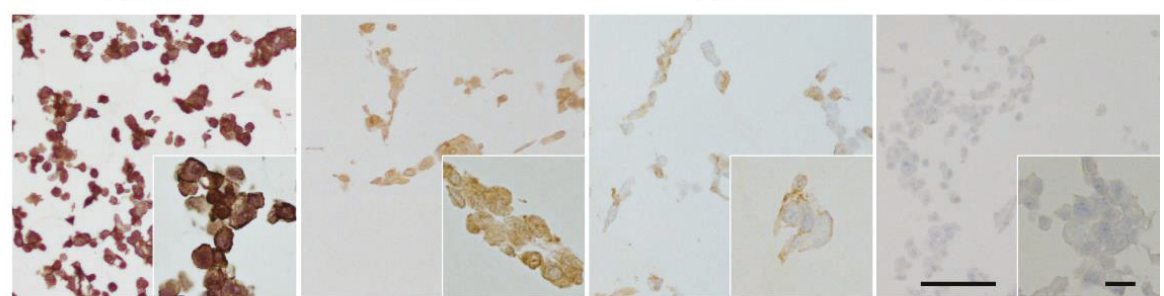

B

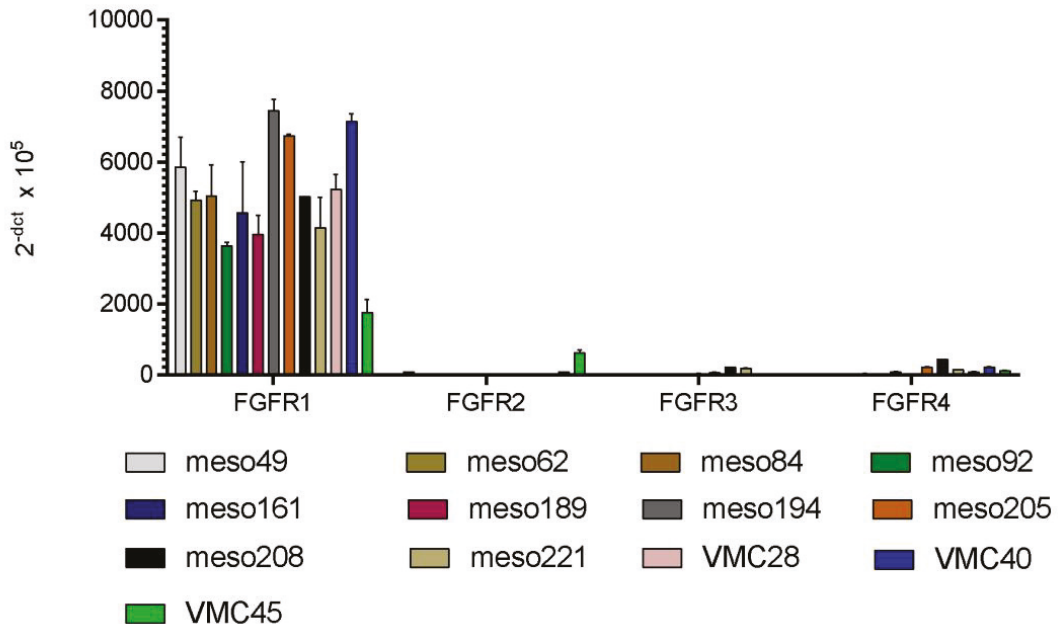

C

D
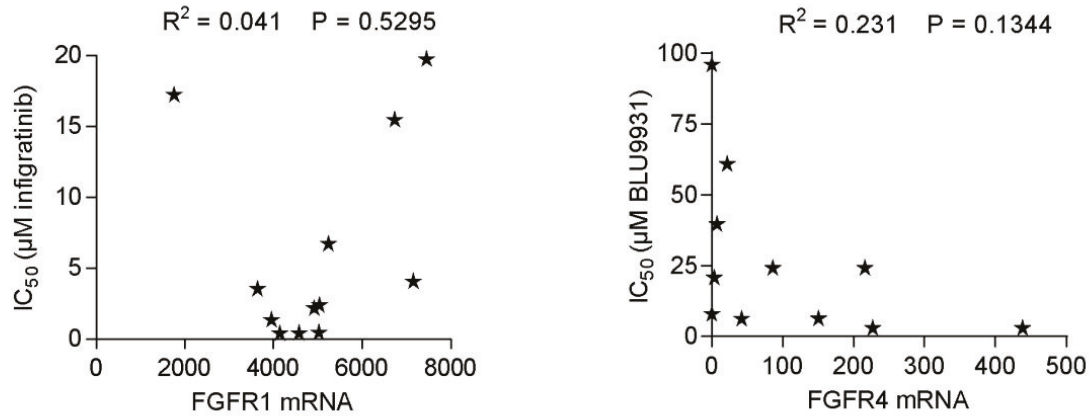

Figure 4. Expression of FGFR1-4 in patient-derived cell lines. (A) Representative images from cell blocks stained for FGFR1 (Meso208), FGFR2 (Meso161), FGFR3 (VMC45), and FGFR4 (Meso205). Scale bar $=100 \mu \mathrm{m}$ in overview images and $10 \mu \mathrm{m}$ in high magnification insets. (B) Total mRNA was isolated from logarithmically growing cell lines and subjected to cDNA synthesis. Quantitative RT-PCR was performed by Taqman assays for FGFR1-4. Expression levels were plotted as $2^{-\mathrm{dCt}} \times$ $10^{5}$ normalized to the house-keeping genes GAPDH and beta-actin. (C) Infigratinib $\mathrm{IC}_{50}$ values were plotted as function of FGFR1 mRNA expression level. (D) BLU9931 IC 50 values were plotted as function of FGFR4 mRNA expression levels. 


\section{Discussion}

Tyrosine kinase inhibitors (TKI) targeting mainly FGFRs (like infigratinib [23] or erdafitinib [24]) or co-targeting FGFRs in addition to VEGFR and other RTKs (like nintedanib [25] or lenvatinib [26,27]) are considered as promising therapeutic agents for a number of different malignancies. Nevertheless, it has remained largely unclear which patients have the highest likelihood of achieving a benefit from these agents. Gene amplifications, mutations, and translocations have been reported for FGFRs in various malignancies. FGFR1 amplification, for instance, occurs in non-small cell lung cancer (NSCLC) and breast cancer patients [28]. Fusion oncogenes containing parts of FGFR1-3 and a number of different partners were found in smaller percentages of patients with glioblastoma, bladder cancer, and a number of additional malignancies [29]. Point mutations of FGFR4 were reported in rhabdomyosarcoma [30]. While some of these genetic FGFR alterations were shown to predict for sensitivity to FGFR inhibitor treatment [23], in other studies (e.g., in lung and breast cancer) the correlation between FGFR1 amplification and FGFR inhibitor sensitivity was less clear [31,32]. In addition to structural alterations in FGFRs, also abberant expression patterns of the four FGFRs are being explored for associations with disease course and response to chemotherapy and kinase inhibitors, for instance in glioblastoma, gastric cancer, and skin cancers [33-35]. Moreover, FGFRs are also expressed on stromal cells and were shown to mediate interaction of cancer cells and cancer-associated fibroblasts [36].

In MPM, several reports have excluded genetic aberrations in specific FGFRs to occur at significant frequencies but have reported sensitivity of subgroups of MPM cells to FGFR inhibition by pharmacologic and genetic approaches $[10,11,15]$. Results from our group show that strongly reduced ERK and AKT phosphorylation in response to treatment with the FGFR inhibitor PD166866 occurs only in sensitive MPM cells [11]. This suggests that repressing FGFR-dependent hyperactivation of both pathways is one prerequisite for FGFR inhibitor sensitivity. In line with this, it was shown that antiproliferative effects of the multikinase inhibitor sorafenib were most pronounced in tumor-initiating mesothelioma cells which expressed high levels of FGF2, leading to autocrine activation of FGFR1 [37]. These cells showed a repression of high basal ERK and MEK phosphorylation levels by sorafenib in the absence of detectable basal AKT phosphorylation. Marek et al. found a link between high FGFR1 protein expression determined by immunoblotting in MPM cell lines and sensitivity to the FGFR inhibitors ponatinib and FP1039/GSK3052230 [10]. The latter inhibitor was recently tested in a phase Ib study in MPM [38]. Quispel-Janssen et al. reported that loss of BAP1 was linked to increased FGFR inhibitor sensitivity and elevated FGFR/FGF expression in MPM cells [15]. The multikinase inhibitor nintedanib targeting VEGFR, FGFR, and PDGFR has shown promising results in preclinical [22] and early clinical trials in MPM [39] but failed to show a benefit in a subsequent phase III study [40]. However, since the clinical study was intended to test nintedanib as an antiangiogenic therapy, no biomarkers were used in this study to select patients on the basis of tumor cell expression of specific nintedanib targets.

Our data confirm that co-expression of several FGFRs is common in MPM tissue. This is in line for instance with hepatocellular carcinoma (HCC) [20] where recent data suggest high expression of FGFR2 and FGFR3 as potential biomarkers for infigratinib response [41]. Our data, however, did not reveal a connection between FGFR expression and infigratinib efficacy. The partly nuclear staining of FGFRs in tumor cells was previously noted by others and us and has been linked to invasive behavior for instance in breast cancer [11,42]. In contrast to the widely expressed FGFR1 and FGFR2, FGFR3 and FGFR4 staining identified subgroups of MPM patients with significantly worse prognosis. FGFR4 was previously reported as a growth driver in conjunction with FGF19 overexpression in HCC [43-45] and has been shown to be of negative prognostic impact in NSCLC [46]. Interestingly, FGF1, FGF2, and FGF18, which are the three most abundantly expressed FGFs in MPM cells [11], all have high affinity for FGFR4 [47]. High FGF2 levels in serum and pleural effusions were previously shown to correlate with poor patient survival in MPM [48], whereas for other FGFs data are largely missing. Strategies for targeting FGFR4 are currently being developed [49,50], and, while further research is 
required, it seems encouraging in this respect that the MPM cell lines with the highest FGFR4 mRNA expression show the strongest inhibition by the FGFR4-specific agent BLU9931.

FGFR3 was previously shown to be relevant for sensitivity to the FGFR inhibitor AZD4547 in MPM cells by transmitting FGF9 signals [15]. Moreover, increased expression of FGFR3 was shown in that study to be associated with mutated BAP1 in gene expression data of MPM patients. In our data, FGFR3 expression was generally weaker and more restricted on protein and mRNA level when compared to FGFR1 and correlated with shorter OS in MPM patients but not with infigratinib sensitivity in cell lines. Of the three sensitive cell lines in our panel, two had a deletion or mutation in BAP1, whereas one showed no obvious alteration. Mutated BAP1, however, was also present in one of the three resistant cell lines [51].

An obvious discrepancy exists with respect to the strong FGFR2 staining and comparatively low mRNA expression data in our cell lines. This could either be explained by cross-reactivity of the antibody with additional antigens or by the presence of FGFR2 splice variants not detected by the Taqman probe used for mRNA analysis. A notable observation of our study is the much more homogenous staining in cell lines compared to the corresponding tumors. This could indicate that culture conditions could favor specific expression patterns either via selection for the survival of cells with, e.g., high FGFR1 expression or via providing a more homogenous microenvironment that leads to a more homogenous expression.

Overall, our study demonstrates that FGFR3 and FGFR4 have prognostic value in MPM, whereas the other FGFRs are often co-expressed but do not correlate with patient survival or clinicopathologic parameters. Further studies in larger patient collectives will be required to clarify the prognostic value of FGFR expression for the different histological subtypes of MPM. Our data confirm the existence of an FGFR inhibitor sensitive subgroup of MPM cell lines, but we have not found a satisfactory predictive capacity to identify sensitive cell lines in our panel by analyzing FGFR1-4 expression. This leads us to postulate that additional, still undiscovered factors play a major part in controlling FGFR inhibitor sensitivity in MPM.

Supplementary Materials: The following are available online at http://www.mdpi.com/2073-4409/8/9/1091/s1, Figure S1: Correlation analysis of infigratinib sensitivity with FGFR mRNA expression levels; Figure S2: Correlation analysis of FGFR mRNA expression levels; Table S1: Correlation of FGFR1 staining pattern with clinical and pathology data; Table S2: Correlation of FGFR3 staining pattern with clinical and pathology data; Table S3: Correlation of FGFR4 staining pattern with clinical and pathology data; Table S4: Sensitivity of the MPM cell lines to BLU9931.

Author Contributions: Conceptualization, M.A.H., I.K., G.V., and M.G.; Methodology, G.V., I.K., B.P.-V., J.B., K.S. (Karin Schelch), and M.A.H.; Software T.K. and G.V.; Validation, M.A.H., T.K., M.G., and I.K.; Formal analysis, B.P.-V. and J.B.; Investigation, C.P., B.P.-V., and K.S. (Karin Schelch); Resources, A.R., T.C., W.K., B.H., and W.B.; Data curation, C.P., K.S. (Katharina Sinn), E.G., K.M., M.A.H., and T.K.; Writing-original draft preparation, M.A.H., T.K., G.V., I.K., and M.G; Writing-review and editing, B.D., V.L., A.R., W.B., B.H., B.G.-K., and T.C.; Visualization G.V., M.G., and T.K.; Supervision, W.B., W.K., B.D., T.C., I.K., and M.G.; Project administration, M.A.H. and M.G.; Funding acquisition, M.A.H., K.S. (Karin Schelch), and M.G.

Funding: This work was funded by the Ingrid Shaker Nessman Cancer Research Association (M.A.H.), the Anniversary Fund (no. 16912, M.G.) of the Austrian National Bank (OeNB) and the Austrian Science Fund (FWF, no. T 1062-B33, K.S., I-2872-B28, B.H., I 3977, B.D.).

Acknowledgments: We are grateful to Barbara Dekan and Jennifer Hsu for help with cell line establishment.

Conflicts of Interest: The authors declare no conflict of interest.

\section{References}

1. Yap, T.A.; Aerts, J.G.; Popat, S.; Fennell, D.A. Novel insights into mesothelioma biology and implications for therapy. Nat. Rev. Cancer 2017, 17, 475-488. [CrossRef] [PubMed]

2. Stayner, L.; Welch, L.S.; Lemen, R. The worldwide pandemic of asbestos-related diseases. Annu. Rev. Public Health 2013, 34, 205-216. [CrossRef] [PubMed] 
3. Mutti, L.; Peikert, T.; Robinson, B.W.S.; Scherpereel, A.; Tsao, A.S.; de Perrot, M.; Woodard, G.A.; Jablons, D.M.; Wiens, J.; Hirsch, F.R.; et al. Scientific Advances and New Frontiers in Mesothelioma Therapeutics. J. Thorac. Oncol. 2018, 13, 1269-1283. [CrossRef] [PubMed]

4. Scherpereel, A.; Mazieres, J.; Greillier, L.; Lantuejoul, S.; Do, P.; Bylicki, O.; Monnet, I.; Corre, R.; Audigier-Valette, C.; Locatelli-Sanchez, M.; et al. Nivolumab or nivolumab plus ipilimumab in patients with relapsed malignant pleural mesothelioma (IFCT-1501 MAPS2): A multicentre, open-label, randomised, non-comparative, phase 2 trial. Lancet Oncol. 2019, 20, 239-253. [CrossRef]

5. Bueno, R.; Stawiski, E.W.; Goldstein, L.D.; Durinck, S.; De Rienzo, A.; Modrusan, Z.; Gnad, F.; Nguyen, T.T.; Jaiswal, B.S.; Chirieac, L.R.; et al. Comprehensive genomic analysis of malignant pleural mesothelioma identifies recurrent mutations, gene fusions and splicing alterations. Nat. Genet. 2016, 48, 407-416. [CrossRef]

6. Hmeljak, J.; Sanchez-Vega, F.; Hoadley, K.A.; Shih, J.; Stewart, C.; Heiman, D.; Tarpey, P.; Danilova, L.; Drill, E.; Gibb, E.A.; et al. Integrative Molecular Characterization of Malignant Pleural Mesothelioma. Cancer Discov. 2018, 8, 1548-1565. [CrossRef] [PubMed]

7. Mizuno, T.; Murakami, H.; Fujii, M.; Ishiguro, F.; Tanaka, I.; Kondo, Y.; Akatsuka, S.; Toyokuni, S.; Yokoi, K.; Osada, H.; et al. YAP induces malignant mesothelioma cell proliferation by upregulating transcription of cell cycle-promoting genes. Oncogene 2012, 31, 5117-5122. [CrossRef]

8. Sekido, Y. Targeting the Hippo Pathway Is a New Potential Therapeutic Modality for Malignant Mesothelioma. Cancers 2018, 10, 90. [CrossRef]

9. Altomare, D.A.; You, H.; Xiao, G.H.; Ramos-Nino, M.E.; Skele, K.L.; De Rienzo, A.; Jhanwar, S.C.; Mossman, B.T.; Kane, A.B.; Testa, J.R. Human and mouse mesotheliomas exhibit elevated AKT/PKB activity, which can be targeted pharmacologically to inhibit tumor cell growth. Oncogene 2005, 24, 6080-6089. [CrossRef]

10. Marek, L.A.; Hinz, T.K.; von Massenhausen, A.; Olszewski, K.A.; Kleczko, E.K.; Boehm, D.; Weiser-Evans, M.C.; Nemenoff, R.A.; Hoffmann, H.; Warth, A.; et al. Nonamplified FGFR1 is a growth driver in malignant pleural mesothelioma. Mol. Cancer Res. 2014, 12, 1460-1469. [CrossRef]

11. Schelch, K.; Hoda, M.A.; Klikovits, T.; Munzker, J.; Ghanim, B.; Wagner, C.; Garay, T.; Laszlo, V.; Setinek, U.; Dome, B.; et al. Fibroblast growth factor receptor inhibition is active against mesothelioma and synergizes with radio- and chemotherapy. Am. J. Respir. Crit. Care Med. 2014, 190, 763-772. [CrossRef] [PubMed]

12. Blackwell, C.; Sherk, C.; Fricko, M.; Ganji, G.; Barnette, M.; Hoang, B.; Tunstead, J.; Skedzielewski, T.; Alsaid, H.; Jucker, B.M.; et al. Inhibition of FGF/FGFR autocrine signaling in mesothelioma with the FGF ligand trap, FP-1039/GSK3052230. Oncotarget 2016, 7, 39861-39871. [CrossRef] [PubMed]

13. Schelch, K.; Kirschner, M.B.; Williams, M.; Cheng, Y.Y.; van Zandwijk, N.; Grusch, M.; Reid, G. A link between the fibroblast growth factor axis and the miR-16 family reveals potential new treatment combinations in mesothelioma. Mol. Oncol. 2018, 12, 58-73. [CrossRef] [PubMed]

14. Schelch, K.; Wagner, C.; Hager, S.; Pirker, C.; Siess, K.; Lang, E.; Lin, R.; Kirschner, M.B.; Mohr, T.; Brcic, L.; et al. FGF2 and EGF induce epithelial-mesenchymal transition in malignant pleural mesothelioma cells via a MAPKinase/MMP1 signal. Carcinogenesis 2018, 39, 534-545. [CrossRef] [PubMed]

15. Quispel-Janssen, J.M.; Badhai, J.; Schunselaar, L.; Price, S.; Brammeld, J.; Iorio, F.; Kolluri, K.; Garnett, M.; Berns, A.; Baas, P.; et al. Comprehensive Pharmacogenomic Profiling of Malignant Pleural Mesothelioma Identifies a Subgroup Sensitive to FGFR Inhibition. Clin. Cancer Res. 2018, 24, 84-94. [CrossRef] [PubMed]

16. Rusch, V.W. A proposed new international TNM staging system for malignant pleural mesothelioma. From the International Mesothelioma Interest Group. Chest 1995, 108, 1122-1128. [CrossRef]

17. Sanna-Cherchi, S.; Sampogna, R.V.; Papeta, N.; Burgess, K.E.; Nees, S.N.; Perry, B.J.; Choi, M.; Bodria, M.; Liu, Y.; Weng, P.L.; et al. Mutations in DSTYK and dominant urinary tract malformations. N. Engl. J. Med. 2013, 369, 621-629. [CrossRef] [PubMed]

18. Saucedo, L.; Buffa, G.N.; Rosso, M.; Guillardoy, T.; Gongora, A.; Munuce, M.J.; Vazquez-Levin, M.H.; Marin-Briggiler, C. Fibroblast Growth Factor Receptors (FGFRs) in Human Sperm: Expression, Functionality and Involvement in Motility Regulation. PLoS ONE 2015, 10, e0127297. [CrossRef] [PubMed]

19. Streit, S.; Mestel, D.S.; Schmidt, M.; Ullrich, A.; Berking, C. FGFR4 Arg388 allele correlates with tumour thickness and FGFR4 protein expression with survival of melanoma patients. Br. J. Cancer 2006, 94, 1879-1886. [CrossRef] 
20. Gauglhofer, C.; Sagmeister, S.; Schrottmaier, W.; Fischer, C.; Rodgarkia-Dara, C.; Mohr, T.; Stattner, S.; Bichler, C.; Kandioler, D.; Wrba, F.; et al. Up-regulation of the fibroblast growth factor 8 subfamily in human hepatocellular carcinoma for cell survival and neoangiogenesis. Hepatology 2011, 53, 854-864. [CrossRef]

21. Sagmeister, S.; Eisenbauer, M.; Pirker, C.; Mohr, T.; Holzmann, K.; Zwickl, H.; Bichler, C.; Kandioler, D.; Wrba, F.; Mikulits, W.; et al. New cellular tools reveal complex epithelial-mesenchymal interactions in hepatocarcinogenesis. Br. J. Cancer 2008, 99, 151-159. [CrossRef] [PubMed]

22. Laszlo, V.; Valko, Z.; Kovacs, I.; Ozsvar, J.; Hoda, M.A.; Klikovits, T.; Lakatos, D.; Czirok, A.; Garay, T.; Stiglbauer, A.; et al. Nintedanib Is Active in Malignant Pleural Mesothelioma Cell Models and Inhibits Angiogenesis and Tumor Growth In Vivo. Clin. Cancer Res. 2018, 24, 3729-3740. [CrossRef] [PubMed]

23. Guagnano, V.; Kauffmann, A.; Wohrle, S.; Stamm, C.; Ito, M.; Barys, L.; Pornon, A.; Yao, Y.; Li, F.; Zhang, Y.; et al. FGFR genetic alterations predict for sensitivity to NVP-BGJ398, a selective pan-FGFR inhibitor. Cancer Discov. 2012, 2, 1118-1133. [CrossRef] [PubMed]

24. Tabernero, J.; Bahleda, R.; Dienstmann, R.; Infante, J.R.; Mita, A.; Italiano, A.; Calvo, E.; Moreno, V.; Adamo, B.; Gazzah, A.; et al. Phase I Dose-Escalation Study of JNJ-42756493, an Oral Pan-Fibroblast Growth Factor Receptor Inhibitor, in Patients with Advanced Solid Tumors. J. Clin. Oncol. 2015, 33, 3401-3408. [CrossRef] [PubMed]

25. Ledermann, J.A.; Hackshaw, A.; Kaye, S.; Jayson, G.; Gabra, H.; McNeish, I.; Earl, H.; Perren, T.; Gore, M.; Persic, M.; et al. Randomized phase II placebo-controlled trial of maintenance therapy using the oral triple angiokinase inhibitor BIBF 1120 after chemotherapy for relapsed ovarian cancer. J. Clin. Oncol. 2011, 29, 3798-3804. [CrossRef] [PubMed]

26. Schlumberger, M.; Jarzab, B.; Cabanillas, M.E.; Robinson, B.; Pacini, F.; Ball, D.W.; McCaffrey, J.; Newbold, K.; Allison, R.; Martins, R.G.; et al. A Phase II Trial of the Multitargeted Tyrosine Kinase Inhibitor Lenvatinib (E7080) in Advanced Medullary Thyroid Cancer. Clin. Cancer Res. 2016, 22, 44-53. [CrossRef] [PubMed]

27. Reck, M.; Kaiser, R.; Mellemgaard, A.; Douillard, J.Y.; Orlov, S.; Krzakowski, M.; von Pawel, J.; Gottfried, M.; Bondarenko, I.; Liao, M.; et al. Docetaxel plus nintedanib versus docetaxel plus placebo in patients with previously treated non-small-cell lung cancer (LUME-Lung 1): A phase 3, double-blind, randomised controlled trial. Lancet Oncol. 2014, 15, 143-155. [CrossRef]

28. Dienstmann, R.; Rodon, J.; Prat, A.; Perez-Garcia, J.; Adamo, B.; Felip, E.; Cortes, J.; Iafrate, A.J.; Nuciforo, P.; Tabernero, J. Genomic aberrations in the FGFR pathway: Opportunities for targeted therapies in solid tumors. Ann. Oncol. 2014, 25, 552-563. [CrossRef]

29. Parker, B.C.; Engels, M.; Annala, M.; Zhang, W. Emergence of FGFR family gene fusions as therapeutic targets in a wide spectrum of solid tumours. J. Pathol. 2014, 232, 4-15. [CrossRef]

30. Taylor, J.G.t.; Cheuk, A.T.; Tsang, P.S.; Chung, J.Y.; Song, Y.K.; Desai, K.; Yu, Y.; Chen, Q.R.; Shah, K.; Youngblood, V; et al. Identification of FGFR4-activating mutations in human rhabdomyosarcomas that promote metastasis in xenotransplanted models. J. Clin. Investig. 2009, 119, 3395-3407. [CrossRef]

31. Kotani, H.; Ebi, H.; Kitai, H.; Nanjo, S.; Kita, K.; Huynh, T.G.; Ooi, A.; Faber, A.C.; Mino-Kenudson, M.; Yano, S. Co-active receptor tyrosine kinases mitigate the effect of FGFR inhibitors in FGFR1-amplified lung cancers with low FGFR1 protein expression. Oncogene 2016, 35, 3587-3597. [CrossRef] [PubMed]

32. Golfmann, K.; Meder, L.; Koker, M.; Volz, C.; Borchmann, S.; Tharun, L.; Dietlein, F.; Malchers, F.; Florin, A.; Buttner, R.; et al. Synergistic anti-angiogenic treatment effects by dual FGFR1 and VEGFR1 inhibition in FGFR1-amplified breast cancer. Oncogene 2018, 37, 5682-5693. [CrossRef] [PubMed]

33. Jimenez-Pascual, A.; Siebzehnrubl, F.A. Fibroblast Growth Factor Receptor Functions in Glioblastoma. Cells 2019, 8, 715. [CrossRef] [PubMed]

34. Zhang, J.; Tang, P.M.K.; Zhou, Y.; Cheng, A.S.L.; Yu, J.; Kang, W.; To, K.F. Targeting the Oncogenic FGF-FGFR Axis in Gastric Carcinogenesis. Cells 2019, 8, 637. [CrossRef] [PubMed]

35. Czyz, M. Fibroblast Growth Factor Receptor Signaling in Skin Cancers. Cells 2019, 8, 540. [CrossRef] [PubMed]

36. Santolla, M.F.; Vivacqua, A.; Lappano, R.; Rigiracciolo, D.C.; Cirillo, F.; Galli, G.R.; Talia, M.; Brunetti, G.; Miglietta, A.M.; Belfiore, A.; et al. GPER Mediates a Feedforward FGF2/FGFR1 Paracrine Activation Coupling CAFs to Cancer Cells toward Breast Tumor Progression. Cells 2019, 8, 223. [CrossRef] [PubMed]

37. Pattarozzi, A.; Carra, E.; Favoni, R.E.; Wurth, R.; Marubbi, D.; Filiberti, R.A.; Mutti, L.; Florio, T.; Barbieri, F.; Daga, A. The inhibition of FGF receptor 1 activity mediates sorafenib antiproliferative effects in human malignant pleural mesothelioma tumor-initiating cells. Stem. Cell Res. Ther. 2017, 8, 119. [CrossRef] 
38. Van Brummelen, E.M.J.; Levchenko, E.; Domine, M.; Fennell, D.A.; Kindler, H.L.; Viteri, S.; Gadgeel, S.; Lopez, P.G.; Kostorov, V.; Morgensztern, D.; et al. A phase Ib study of GSK3052230, an FGF ligand trap in combination with pemetrexed and cisplatin in patients with malignant pleural mesothelioma. Investig. New Drugs 2019. [CrossRef]

39. Grosso, F.; Steele, N.; Novello, S.; Nowak, A.K.; Popat, S.; Greillier, L.; John, T.; Leighl, N.B.; Reck, M.; Taylor, P.; et al. Nintedanib Plus Pemetrexed/Cisplatin in Patients With Malignant Pleural Mesothelioma: Phase II Results From the Randomized, Placebo-Controlled LUME-Meso Trial. J. Clin. Oncol. 2017, 35, 3591-3600. [CrossRef]

40. Scagliotti, G.V.; Gaafar, R.; Nowak, A.K.; Nakano, T.; van Meerbeeck, J.; Popat, S.; Vogelzang, N.J.; Grosso, F.; Aboelhassan, R.; Jakopovic, M.; et al. Nintedanib in combination with pemetrexed and cisplatin for chemotherapy-naive patients with advanced malignant pleural mesothelioma (LUME-Meso): A double-blind, randomised, placebo-controlled phase 3 trial. Lancet Respir. Med. 2019, 7, 569-580. [CrossRef]

41. Huynh, H.; Lee, L.Y.; Goh, K.Y.; Ong, R.; Hao, H.X.; Huang, A.; Wang, Y.; Graus Porta, D.; Chow, P.; Chung, A. Infigratinib Mediates Vascular Normalization, Impairs Metastasis, and Improves Chemotherapy in Hepatocellular Carcinoma. Hepatology 2019, 69, 943-958. [CrossRef] [PubMed]

42. Chioni, A.M.; Grose, R. FGFR1 cleavage and nuclear translocation regulates breast cancer cell behavior. J. Cell Biol. 2012, 197, 801-817. [CrossRef] [PubMed]

43. Gauglhofer, C.; Paur, J.; Schrottmaier, W.C.; Wingelhofer, B.; Huber, D.; Naegelen, I.; Pirker, C.; Mohr, T.; Heinzle, C.; Holzmann, K.; et al. Fibroblast growth factor receptor 4: A putative key driver for the aggressive phenotype of hepatocellular carcinoma. Carcinogenesis 2014, 35, 2331-2338. [CrossRef] [PubMed]

44. Hagel, M.; Miduturu, C.; Sheets, M.; Rubin, N.; Weng, W.; Stransky, N.; Bifulco, N.; Kim, J.L.; Hodous, B.; Brooijmans, N.; et al. First Selective Small Molecule Inhibitor of FGFR4 for the Treatment of Hepatocellular Carcinomas with an Activated FGFR4 Signaling Pathway. Cancer Discov. 2015, 5, 424-437. [CrossRef] [PubMed]

45. Raja, A.; Park, I.; Haq, F.; Ahn, S.M. FGF19-FGFR4 Signaling in Hepatocellular Carcinoma. Cells 2019, 8, 536. [CrossRef] [PubMed]

46. Huang, H.P.; Feng, H.; Qiao, H.B.; Ren, Z.X.; Zhu, G.D. The prognostic significance of fibroblast growth factor receptor 4 in non-small-cell lung cancer. Onco Targets Ther. 2015, 8, 1157-1164. [CrossRef] [PubMed]

47. Heinzle, C.; Erdem, Z.; Paur, J.; Grasl-Kraupp, B.; Holzmann, K.; Grusch, M.; Berger, W.; Marian, B. Is fibroblast growth factor receptor 4 a suitable target of cancer therapy? Curr. Pharm. Des. 2014, 20, 2881-2898. [CrossRef] [PubMed]

48. Strizzi, L.; Vianale, G.; Catalano, A.; Muraro, R.; Mutti, L.; Procopio, A. Basic fibroblast growth factor in mesothelioma pleural effusions: Correlation with patient survival and angiogenesis. Int. J. Oncol. 2001, 18, 1093-1098. [CrossRef] [PubMed]

49. Lang, L.; Teng, Y. Fibroblast Growth Factor Receptor 4 Targeting in Cancer: New Insights into Mechanisms and Therapeutic Strategies. Cells 2019, 8, 31. [CrossRef]

50. Dai, S.; Zhou, Z.; Chen, Z.; Xu, G.; Chen, Y. Fibroblast Growth Factor Receptors (FGFRs): Structures and Small Molecule Inhibitors. Cells 2019, 8, 614. [CrossRef]

51. Pirker, C.; Bilecz, A.; Grusch, M.; Mohr, T.; Laszlo, V.; Stockhammer, P.; Lötsch, D.; Gojo, J.; Gabler, L.; Spiegl-Kreinecker, S.; et al. Activating TERT promoter mutations in malignant pleural mesothelioma: Association with prognosis and altered genomic signature. J. Thorac. Oncol. 2019. under review.

(C) 2019 by the authors. Licensee MDPI, Basel, Switzerland. This article is an open access article distributed under the terms and conditions of the Creative Commons Attribution (CC BY) license (http://creativecommons.org/licenses/by/4.0/). 
Article

\title{
Sprouty3 and Sprouty4, Two Members of a Family Known to Inhibit FGF-Mediated Signaling, Exert Opposing Roles on Proliferation and Migration of Glioblastoma-Derived Cells
}

\author{
Burcu Emine Celik-Selvi, Astrid Stütz, Christoph-Erik Mayer, Jihen Salhi, Gerald Siegwart and \\ Hedwig Sutterlüty * \\ Institute of Cancer Research, Department of Medicine I, Comprehensive Cancer Center, \\ Medica University of Vienna, A-1090 Vienna, Austria \\ * Correspondence: hedwig.sutterluety@meduniwien.ac.at; Tel.: +43-1-40160-57526
}

Received: 26 June 2019; Accepted: 29 July 2019; Published: 1 August 2019

\begin{abstract}
Dysregulation of receptor tyrosine kinase-induced pathways is a critical step driving the oncogenic potential of brain cancer. In this study, we investigated the role of two members of the Sprouty (Spry) family in brain cancer-derived cell lines. Using immunoblot analyses we found essential differences in the pattern of endogenous Spry3 and Spry4 expression. While Spry4 expression was mitogen-dependent and repressed in a number of cells from higher malignant brain cancers, Spry3 levels neither fluctuated in response to serum withdrawal nor were repressed in glioblastoma (GBM)-derived cell lines. In accordance to the well-known inhibitory role of Spry proteins in fibroblast growth factor (FGF)-mediated signaling, both Spry proteins were able to interfere with FGF-induced activation of the MAPK pathway although to a different extent. In response to serum solely, Spry4 exerts its role as a negative regulator of MAPK activation. Ectopic expression of Spry4 inhibited proliferation and migration of GBM-originated cells, positioning it as a tumor suppressor in brain cancer. In contrast, elevated Spry3 levels accelerated both proliferation and migration of these cell lines, while repression of Spry3 levels using shRNA caused a significant diminished growth and migration velocity rate of a GBM-derived cell line. This argues for a tumor-promoting function of Spry3 in GBMs. Based on these data we conclude that Spry3 and Spry4 fulfill different if not opposing roles within the cancerogenesis of brain malignancies.
\end{abstract}

Keywords: Sprouty proteins; brain cancer; FGF-mediated signaling; tumor suppressor; tumor promoter

\section{Introduction}

The term brain cancer summarizes multiple subtypes of tumors originating from different tissues of the central nervous system [1]. The most prevalent type of brain tumors are gliomas which arise from glial or precursor cells. They include, among others, lower graded astrocytoma (AC) and oligodendroglioma (ODG), as well as the WHO Grade IV classified glioblastoma multiforme (GBM) and its variant gliosarcoma (GS). GBM are the most common brain tumors and patients have a poor prognosis with a five-year survival rate of only 5.6\% [2]. A group of neuronal tumors arising in the central but also in the autonomic nervous system are the rare neuroblastoma (NB) which are the second most common tumors in children [3]. Like in all human cancer cells, malignant transformation in gliomas is driven by typical chromosomal changes. The Cancer Genome Atlas project identified alterations in the network regulated by receptor-tyrosine kinases (RTK) as a frequent molecular cause of these cancers. Important molecules responsible for transducing the signals like the epidermal growth 
factor receptor (EGFR), the phosphatidylinositol 3-kinases (PI3K), NRAS and BRAF are frequently altered to a more efficient state, while inhibitors of their activities like neurofibromin (NF1) and the Phosphatase and TENsin homolog (PTEN) are often deleted or less effective [4].

Sprouty (Spry) proteins which represent modulators of RTK-driven signaling pathways were first identified as inhibitors of fibroblast growth factor (FGF)-induced signaling in Drosophila [5]. In humans, four homologues were described [6]. In contrast to the other Spry family members which are ubiquitously expressed in all tissues [6], the Spry3 encoding gene localizes to the pseudoautosomal region 2 and its expression is rarely documented. Only in brain and glia, Spry3 expression is doubtless detected [7]. Spry proteins fulfill important functions in many RTK-mediated signal transduction cascades. Primarily, they are known to interfere specifically with MAPK-ERK activation [8-10], but in other systems they were shown to influence the PI3K pathway as well [11]. Additionally, Spry proteins are able to interfere with phospholipase C-induced pathways [12]. In contrast to their manifold inhibitory function on RTK-mediated pathways, Spry proteins are able to interact with the E3-ubiquitin ligase c-Cbl and thereby constrict the degradation of some RTKs as shown for the EGFR [13]. Considering their functions in fine tuning of the cellular response to RTK-inducing signals, members of the Spry family are good candidates for an important role in the tumorigenesis of different cells. Accordingly, Spry2 and/or Spry4 are shown to act as tumor-suppressors in cancer originated from, e.g., lung [14-16], liver [17], breast [18,19], prostate [20] and bone [21]. In other types of tumors, members of the Spry protein family fulfill a tumor-promoting task as it was demonstrated for Spry2 in colon carcinoma [22,23] and for Spry1 in rhabdomyosarcoma [24]. In brain tumors, repression of Spry2 has been shown to interfere with proliferation of GBM-derived cell lines and tumor formation $[25,26]$. Compatible with the tumor-promoting function of Spry2 in brain, the Spry proteins are important for other neuronal processes. Spry2 as well as Spry4 downregulation is associated with promoted axon outgrowth $[27,28]$, and Spry1, Spry2 and Spry4 inhibit FGF-induced processes in the cerebellum [29]. Data generated in Xenopus document that Spry3 is important in regulating axon branching of motoneurons [30], and the finding that Spry3 is associated with autism susceptibility indicates a further role in the human brain [7].

In the presented study, we investigated the expression of Spry3 and Spry4 in brain cancer-derived cells and analyzed how a modulation of their expression influences the behavior of glioblastoma-derived cell lines.

\section{Material and Methods}

\subsection{Cell Lines}

The astrocytoma-derived cells (SW1088) and both neuroblastoma-derived cell lines (SK-N-DZ and SK-N-FI), as well as the glioblastoma-derived cell lines DBTRG-05MG, T98G and U373 and the oligodendroglioma-derived cell line Hs683 were purchased from the American Type Culture Collection (ATCC). NMC-G1, a cell line established from an astrocytoma, and AM-38, a glioblastoma originated cell line, were obtained from the JCRB cell bank. Cell lines LN40 and LN140 were kindly provided by Dr. Tribolet (Lausanne). Cell lines BTL1529, BTL2177 and BTL53 were established from glioblastoma diagnosed patients and BTL1376 and BTL2175 from gliosarcoma patients at the Neuromed Campus in Linz (NML) as described [31]. The cell line VBT72 was established from a glioblastoma at the Institute for Cancer Research [31]. These cell lines were kindly provided by Walter Berger (Medical University of Vienna). All cells were cultured in the recommended medium containing $10 \%$ fetal calf serum (FCS) and supplemented with penicillin $(100 \mathrm{U} / \mathrm{mL})$ and streptomycin $(100 \mu \mathrm{g} / \mathrm{mL})$ at $37^{\circ} \mathrm{C}$ in $7.5 \% \mathrm{CO}_{2}$.

\subsection{Adenoviral Infection of Cells}

The coding sequence of human Spry3 was amplified by PCR using Pfx Polymerase (Invitrogen) with upstream primer 5-AGCTCTGGATCCATGGATGCTGCGGTGACAGAT-3 (Spry3-s) and downstream primer 5-TAGCGAATTCCTCGAGTCATACAGACTTT-3 (Spry2-as) to add appropriate cloning 
sites. The amplified DNA fragments were subsequently cloned via BamHI/EcoRI into a pADlox plasmid to generate pADlox-Spry3. To construct an adenovirus expressing shRNA directed against Spry3, the CMV promoter of pADlox was exchanged by the human U6 promoter of the pSilencer Vector. Two oligonucleotides harboring an shRNA directed against Spry3 were annealed: sh-Spry3 sense 5'-TCG AGC GCA GCT GTT CAA TAG GCA GAA TTT GTT GAA GCT TGA ACA AAT TCT GCC TAT TGA ACA GCT GCG CTC TTT TTT-3' and shSpry3 as 5'-AAT TAA AAA AGA GCG CAG CTG TTC AAT AGG CAG AAT TTG TTC AAG CTT CAA CAA ATT CTG CCT ATT GAA CAG CTG CGC-3'. The double stranded DNA with overlapping XhoI and EcoRI sites was then inserted in the digested pAdloxU6 vector to obtain pADlox-shSpry3. To obtain a virus directed against Spry4, two oligonucleotides (5'-TCGAGCTCAGCTCGCTACCTCCGCGGCGATGTTGAAGCTTGAACATCGCCGCGGAGGTAGC GAGCTGAGCTGTTTTTT- ${ }^{\prime}$ ' and 5'-AATTAAAAAACAGCTCAGCTCGCTACCTCCGCGGCGATGTT CAAGCTTCAACATCGCCGCGGAGGTAGCGAGCTGAGC-3') were annealed and subcloned the same way to construct pADlox-shSpry4. The correct cloning was confirmed by sequencing analysis. Recombinant viruses were produced as described [32]. Adenoviruses expressing Spry4 or control proteins (luciferase, lacZ or CFP) were already generated [21,33].

The optimal concentrations of the viruses for each cell line was determined by infecting the cells with different dilution of adenoviruses expressing Cyan Fluorescence Protein (CFP). The viral concentration of the adenoviruses expressing different proteins were calculated according to their $\mathrm{OD}_{260}$. For infection, viruses were diluted in serum-free medium.

\subsection{Cell Signaling Assay}

For analyzing ERK phosphorylation, $10^{5}$ cells were seeded into $\varnothing 6 \mathrm{~cm}$ tissue culture plates in DMEM medium containing 10\% FCS. Twenty-four hours later, the cells were washed with and incubated in serum-free medium. Next day cells were infected with adenoviruses and incubated for another 2 days before $20 \%$ FCS or 10ng/mL FGF2 were added.

\subsection{Scratch Assay}

For the scratch assay, $6 \times 10^{5}$ cells were infected with adenoviruses expressing the control proteins, Spry3 or Spry4, respectively. A total of $24 \mathrm{~h}$ post infection, cells were transferred into a 6-well plate. The next day, three straight scratches per well were introduced into the monolayer using a sterile yellow pipette tip. To remove debris, cells were washed twice with $1 \times$ PBS. Finally, $3 \mathrm{~mL}$ of DMEM supplemented with $10 \%$ FCS were added. The closing of the scratch was pictured by the VISITRON Live Cell Imaging System (Visitron, Puchheim, Germany) at 10x magnification using VisiView Software. The running time was set to $40 \mathrm{~h}$ and for monitoring a time interval of $30 \mathrm{~min}$ was chosen. Using ImageJ software, gap width of three scratches were calculated every two hours. Migration velocity was assessed by applying linear regression using GraphPad Prism software. Migration velocity of three independent experiments were compared.

\subsection{Growth Curve}

Growth curves were performed and analyzed as described [21]. Each growth curve was counted in triplicate and after assessing the continuity of the growth by depicting it in a semi-logarithmical graph, the doubling time was calculated by applying an exponential growth equation. The calculated doubling times of at least three independent experiments were compared to each other and differences between two groups were calculated using an unpaired t-test.

\subsection{Immunoblot}

Immunoblotting was carried out as described [34]. The antisera against Spry4 and Spry3 were produced and affinity-purified as described [15]. The Spry3 antibodies were raised against the N-terminal 200 amino acids of the human homolog. As a loading control, antibodies against GAPDH (sc-365062) and ERK 1/2 (sc-514302) were purchased from Santa Cruz. Antibodies against 
phosphorylated extracellular signal-regulated kinase (pERK) (\#9101) were received from Cell Signaling Technology. The horseradisch peroxidase-coupled secondary antibodies were purchased from GE Healthcare.

\section{Results}

3.1. In Brain Cancer-Derived Cell Lines Spry3 Protein is Commonly Expressed Independent of Mitogen Availability

First, we investigated if Spry3 expression is influenced by the grade of malignancy or the histological background of brain cancer-derived cells. In order to analyze Spry3 protein levels, antibodies had to be produced, affinity-purified and their sensitivity as well as their specificity had to be assessed. To analyze their sensitivity, U373 cells were infected with decreasing amounts of adenovirus expressing Spry3 protein. As depicted in Figure 1A, the antibodies detected a single band at $33 \mathrm{kDa}$ and in cells infected with decreasing titers of Spry3-encoding adenoviruses, the intensity of the detected band corresponded to the amount of introduced viruses while the cellular protein content was comparable. To control the specificity, all four Spry proteins were ectopically expressed by using the respective adenoviruses. Two days after infection, sufficient amounts of all Spry proteins were expressed, but the Spry3 antibody only detected Spry3 (Figure 1B). In the subsequent experiment, we determined the endogenous levels of Spry3 in different brain cancer-derived cell lines. To analyze if, like it was shown for Spry2 and Spry4 [35], Spry3 protein expression is dependent on mitogens in the cellular environment, serum was withdrawn from part of the cells $(-)$, and their Spry3 levels were compared to those of cells cultivated in the presence of serum (+). In only 1/17 cell lines Spry3 protein was undetectable. Most of the cell lines express detectable amounts of Spry3 proteins which appear in a distinguishable pattern of bands. Usually the slower migrating bands were more abundant in the presence of serum indicating that a serum-dependent modification is applied to Spry3 (Figure 1C). Concerning the influence of the histopathologic origin, we observed that in the more advanced GBM-derived cell lines the expression of Spry3 was on average higher than in cells originated from the lower graded ODG and AC (Figure 1D,E). The highest expression of Spry3 was detected in the two NB-derived bone morrow metastases. These observations would favor rather an oncogenic than a tumor-suppressing function of Spry3 in brain cancers. Interestingly, the serum had not the expected influence on Spry3 expression, as half of the cell lines failed to adapt their Spry3 expression in response to mitogen availability. In five of the cell lines, its expression even slightly increased (less than 2-fold) if serum was withdrawn. A more pronounced change of Spry3 in response to serum in form of an increase or decrease was only observed in one cell line each (Figure 1D,F). Therefore, it is unlikely that mitogen-induced signals play an important role in regulating the expression of Spry3. 


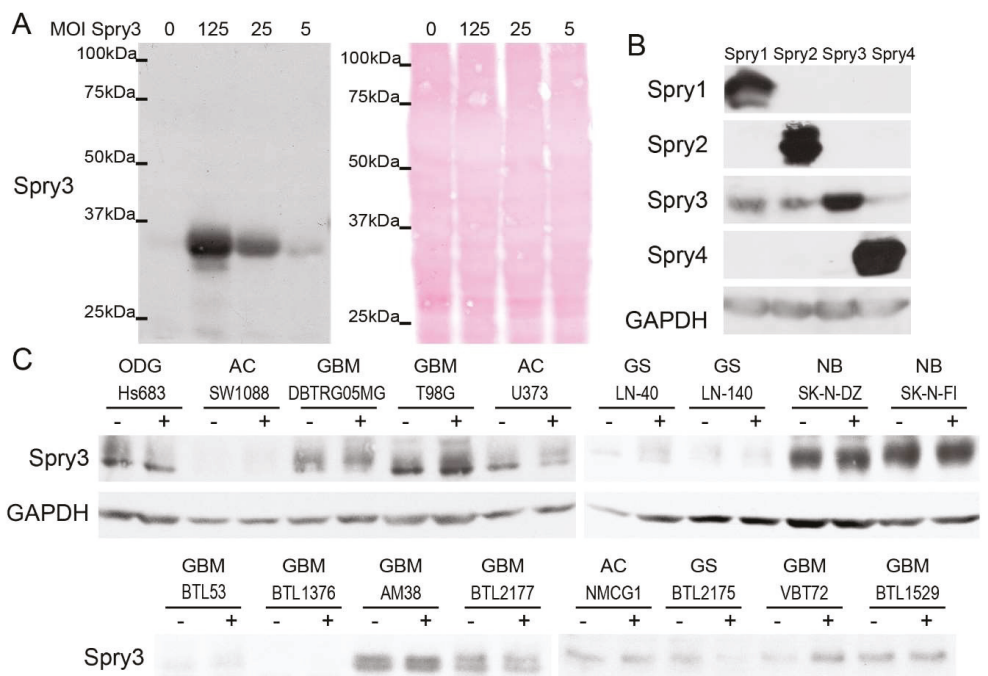

GAPDH

D

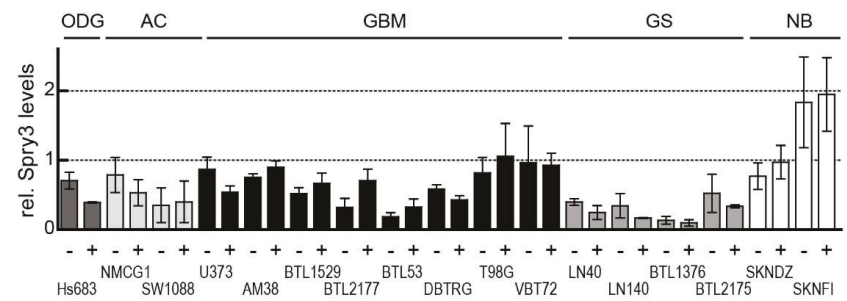

E

$\mathrm{F}$
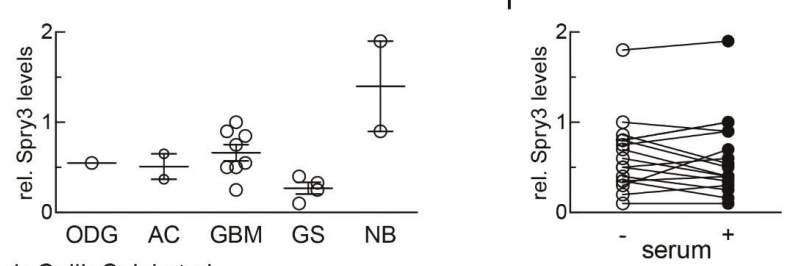

Figure 1; Celik-Selvi et al.

Figure 1. Expression of Spry3 protein in brain cancer-derived cell lines. (A) U373 cells were infected with decreasing amounts of adenoviruses expressing Spry3 protein and an immunoblot using Spry3 antibodies was performed. Equal loading was verified by Ponceau S staining of the immunoblot. (B) Adenoviruses expressing Spry1, Spry2, Spry3 or Spry4 were introduced into U373 cells. A total of $48 \mathrm{~h}$ post-infection cells were harvested and proteins were isolated. An immunoblot sequentially probed with all of the indicated antibodies is depicted. (C) Logarithmically growing cell lines derived from oligodendroglioma (ODG), astrocytoma (AC), glioblastoma (GBM), gliosarcoma (GS) and neuroblastoma (NB) were cultured for $24 \mathrm{~h}$ without (-) and with (+) serum. Using Western blot, endogenous Spry3 and GAPDH proteins were determined. (D) Amounts of Spry3 proteins were measured as ratio to an external control (MG63) by Image Quant software and normalized to GAPDH. Quantification results of 2-3 Western blots depicted as mean \pm SEM are shown in a column graph. Cell lines were sorted according to their histopathological origin. (E) A scatterplot presenting the Spry3 expression across the histopathological subgroups of brain cancer is shown. (F) Calculated Spry3 levels from cells grown in serum-deprived (open circle) and -supplemented (closed circle) mediums are compared. 
3.2. Spry4 Protein Expression is Repressed in Cell Lines Derived from More Malignant Brain Tumors, but Usually Still Serum-Dependent

In order to investigate that growth factor-induced signaling in the analyzed cell lines is able to sufficiently influence the negative feedback loop responsible for controlling Spry protein expression, Spry4 protein levels were determined in comparison. In some cell lines, Spry4 expression was very prominent, but in five of them, we were not able to detect Spry4 proteins. Like in the case of Spry3, Spry4 frequently appeared in more than one migrating form (Figure 2A). Compared to the levels detected in cells derived from lower graded patients' tissues, usually Spry4 expression in GBM and GS is strongly repressed, although in few of these cell lines Spry4 protein was definitively abundant (Figure 2B,C). Only in five of the cells lines the expression of Spry4 in serum-free conditions was insignificantly changed when compared to the parallel in serum cultivated cell counterparts. Seven of the brain-derived cell lines displayed a more than twofold decrease of Spry4 protein as a consequence of serum starvation. Moreover, in three of them the detected difference between the serum and non-serum condition exceeded a fivefold dimension (Figure 2B,D). When Spry3 and Spry4 expression in the different brain-derived cell lines were compared (Figure 2E), we found that there was no correlation indicating that Spry3 and Spry4 expression are regulated by independent mechanisms. 
A

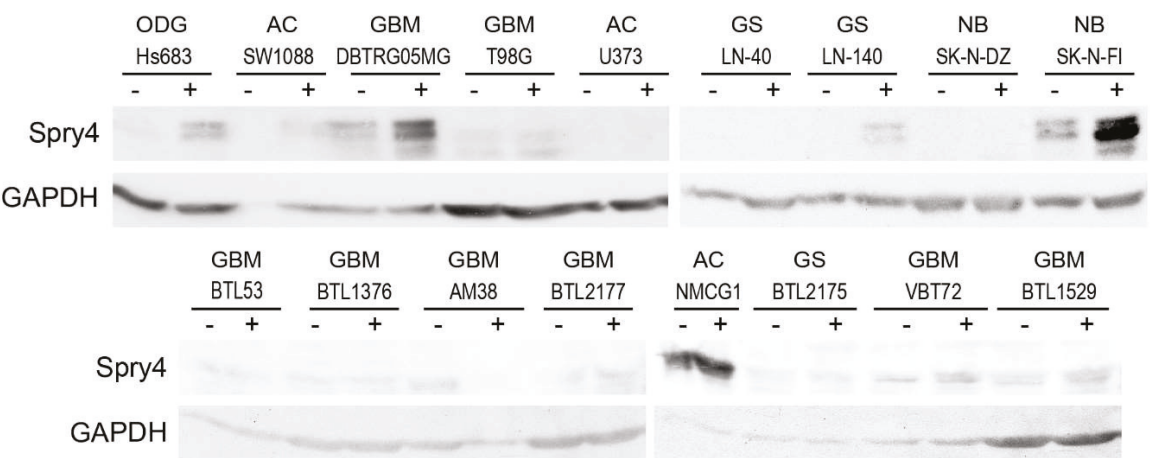

B

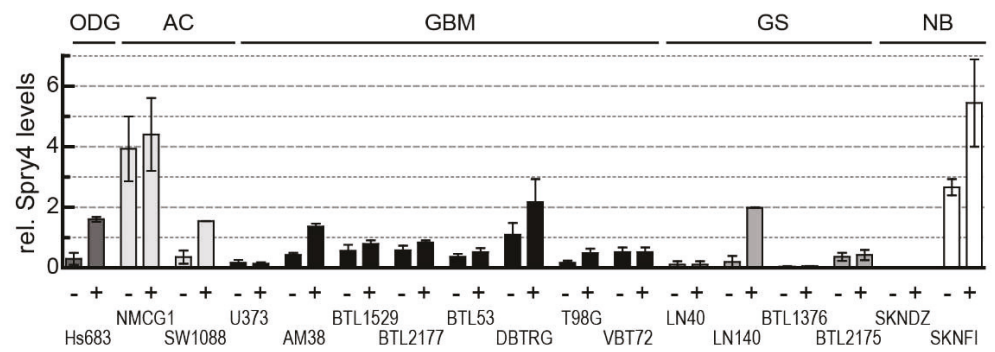

C

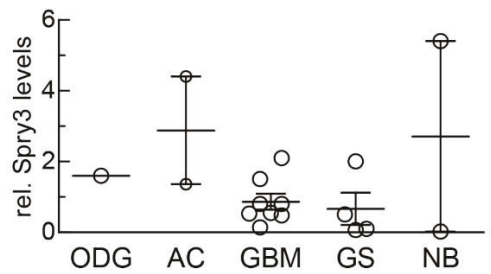

E

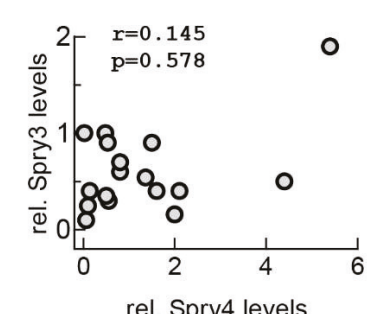

rel. Spry4 levels

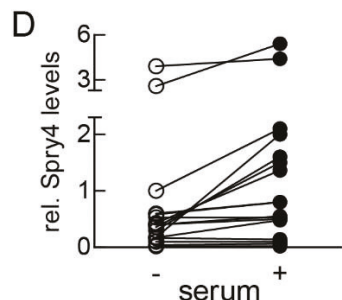

Figure 2. Expression analysis of endogenous Spry4 protein in brain cancer cells. (A) Spry4 protein levels in 17 brain cancer-derived cell lines which were cultured devoid of (-) and with (+) serum. GAPDH served as loading control. (B) Quantification of Spry4 was performed using Image Quant 5.0. An external control was arbitrarily set as 1 and loading differences were adjusted to GAPDH expression. A column graph summarizes the results of 2-3 independent experiments. (C) Spry4 expression in serum-supplemented growth condition was compared. A scattered dot-plot grouping the cells according to the histological origin is depicted. (D) A comparison of Spry4 levels detected in starved (open circle) and stimulated (closed circle) cells is presented. (E) Correlation of Spry3 and Spry4 expression was calculated using GraphPad Prism. 
3.3. MAPK Activation in Response to FGF and Serum is Effectively Inhibited by Spry4 While Spry3 Failed to Fulfill This Function in the Presence of Serum

To analyze if Spry3 and Spry4 are able to interfere with FGF-induced signaling, U373 cells were serum-deprived for 3 days before FGF2 was added for 5, 10 and $20 \mathrm{~min}$. Within the starvation period a portion of cells were infected with viruses expressing Spry3, Spry4 or a control protein. In control treated cells, adding of FGF induced the MAPK pathway after 10 min as measured by determining the fraction of pERK (Figure 3A). In cells expressing excessive amounts of Spry3, like in the control cells, activation of the MAPK pathway was also observed after $10 \mathrm{~min}$, but the extent of phosphorylation was less pronounced (Figure 3A,C). In case of ectopic Spry4 expression we detected that the proportion of activated ERK in serum-starved conditions was clearly less distinct. The addition of FGF caused an augmentation of the pERK levels, which was less intense than in the other two groups (Figure 3A,C). These data evince the inhibitory role of Spry proteins on FGF-mediated signaling, but demonstrated that Spry4 was more potent concerning interference with MAPK activation than Spry3.

In order to asses if the two Spry forms differ concerning their potential to inhibit MAPK activation in response to serum, a respective cell signaling assay was applied. In response to serum, ERK was immediately phosphorylated to a much higher extent (at least 10 times the value observed in starved cells) than in FGF-treated cells (two- to threefold induction). When Spry3 was expressed, the induction was slightly delayed but the amplitude was not significantly diminished. In contrast, Spry4 inhibited ERK phosphorylation significantly. As already observed in case of FGF induction, the basal pERK levels of cells cultivated in the absence of mitogens was clearly diminished, but also the maximal levels of pERK phosphorylation were reduced in comparison to the cells expressing a control or Spry3 protein. These data demonstrate that Spry4 can potently interfere with induction of the MAPK and indicate that Spry4 was more potent concerning interference with MAPK activation than Spry3. 
A

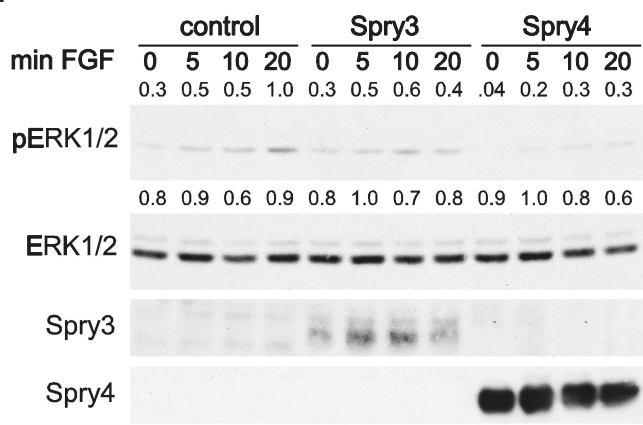

B

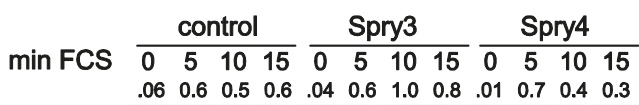

pERK1/2

$\begin{array}{llllllllllllll}0.6 & 0.5 & 0.6 & 1.0 & 0.8 & 01 & 0.7 & 0.4 & 0.3\end{array}$

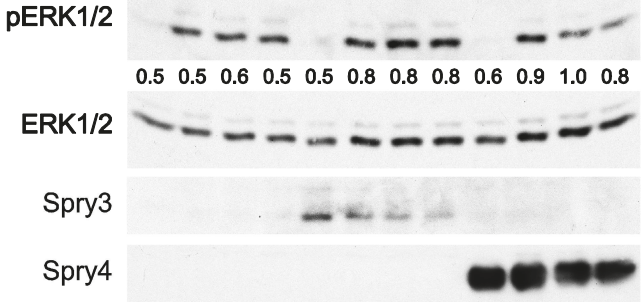

C

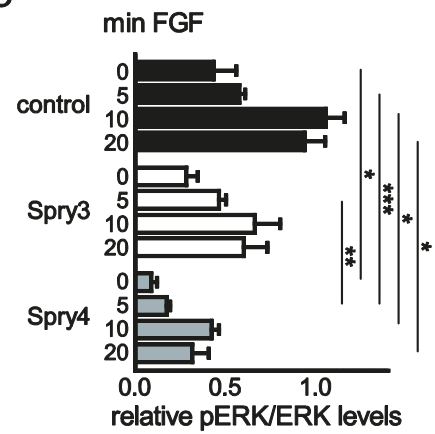

D

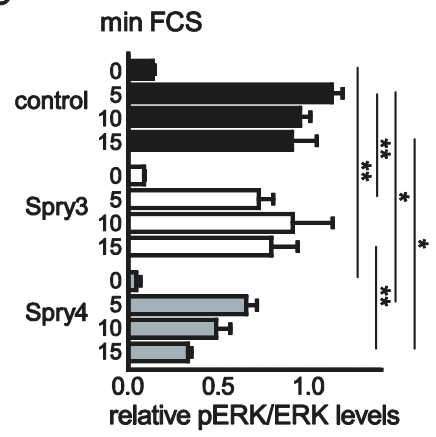

Figure 3. Influence of Spry3 and Spry4 proteins on ERK activation by FGF2 and serum. Glioblastoma (GBM)-derived cells (U373) were serum-starved for $24 \mathrm{~h}$ and then infected with adenoviruses expressing either a control protein (luciferase), Spry3 or Spry4. Two days later, cells were incubated with FGF2 (A) or serum (B) for the indicated times. Representative immunoblots of an experiment using antibodies recognizing pERK1/2 and total ERK1/2 are shown. Expression of Spry3 and Spry4 were verified by the respective antibodies. Using ImageQuant 5.0, the pERK1/2 bands detected were quantified and normalized to the corresponding values obtained for the ERK expression. The highest values were arbitrarily set as 1 . The results of the quantification for the presented blots are depicted. (C) A summary of calculated mean values \pm SEM of the pERK/ERK values from three experiments using FGF2 to stimulate the cells is depicted. Significance between the three groups was calculated by using a one-way ANOVA test in GraphPad prism. (D) The bands of pERK and ERK in response to serum were densitometrically quantified using ImageQuant 5.0, and the highest values of each experiment were set as 1 . The graph summarizes three experiments. Significance was determined by a one-way ANOVA test in using GraphPad prism software. ${ }^{*} p<0.05$; ${ }^{* *} p<0.01 ; * * * p<0.001$.

\subsection{In Brain Cancer-Derived Cell Lines, Spry3 and Spry4 Expressions Have an Opposing Effect on Cell Proliferation}

To investigate if Spry3 and Spry4 interfere with cell proliferation in brain cancer-derived cells, we selected DBTRG-05MG and U373 cell lines to apply ectopic overexpression of the respective Spry proteins. Both of these cell lines were easy to infect by adenoviruses as tested by using CFP expressing adenoviruses (data not shown). Furthermore, in DBTRG-05MG Spry3 appears mainly in its slower migrating form and Spry4 levels are pronounced while in U373 Spry3 mainly appears in its faster migrating form and a shift is only detected after serum addition. Spry4 was not detected in this cell line (Figures 1C and 2A). To measure cell proliferation, growth curve analyses were performed. In DBTRG-05MG, Spry3 expressing cells double significantly faster ( $0.9 \pm 0.01$ doublings per day) than control treated cells $(0.8 \pm 0.02)$ while Spry4 expression decelerate the proliferation process to only 
0.69 doublings per day (Figure 4A,B). Corroborating in U373, Spry3 accelerate cell proliferation from $0.56 \pm 0.01$ to $0.63 \pm 0.01$ doublings per day and Spry 4 expression inhibits cell expansion to $0.51 \pm 0.01$ (Figure 4C,D). In both cell lines, Spry3 and Spry4 proteins are clearly overexpressed if the respective adenoviruses are applied (Figure 4E).

These data demonstrate that cell proliferation is promoted by Spry3 and suppressed by Spry 4 expression arguing for an opposing effect of these Spry members.

A

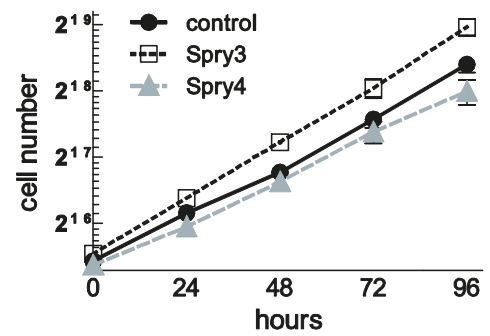

C

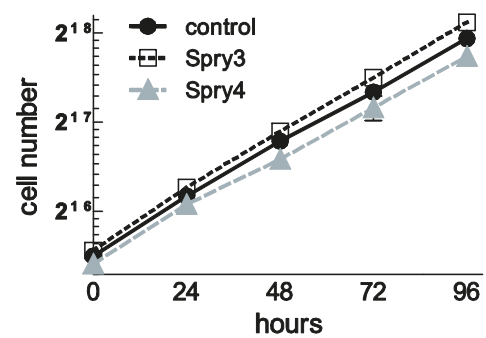

E

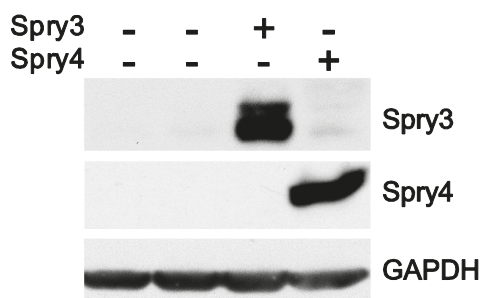

B
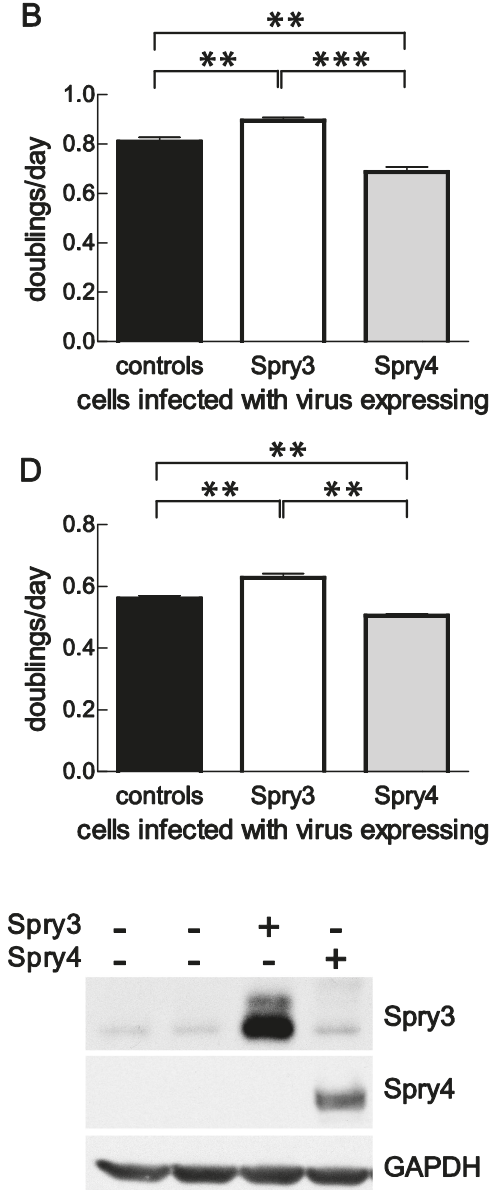

Figure 4. Influence of ectopic Spry3 and Spry4 expression on cell proliferation. Proliferation of cells overexpressing the indicated proteins was assessed by growth curve analysis. (A) The number of DBTRG-05MG cells were counted every $24 \mathrm{~h}$ for 5 days and are depicted as growth curves using a semi-logarithmical scale. A representative growth curve of three replicates is depicted. (B) Using GraphPad Prism, doubling times of at least three independent growth curve analyses performed with DBTRG-05MG cells were calculated and presented as mean doublings per day \pm SEM. (C) A representative growth curve of U373 cell line is shown. (D) Using exponential growth equations, doubling times of U373MG cells were calculated and shown as doublings per day. Significance was assessed using an unpaired t-test in GraphPad Prism and mean \pm SEM are shown. ${ }^{*} p<0.05 ;{ }^{* *} p<0.01$; *** $p<0.001$ (E) Overexpression of Spry3 and Spry4 in the GBM cell lines DBTRG-05MG (left) and U373 (right) were verified by immunoblotting. 


\subsection{Spry3 and Spry4 Exert a Contrary Effect on the Migratory Capabilities of Brain Cells}

Aberrant cell migration is another RTK-mediated process contributing to the malignancy of cancer cells. Therefore, we next investigated if the expression of Spry3 and Spry4 proteins modulate the closure of the gap in a scratch assay. In DBTRG-05MG, ectopic expression of the Spry3 protein has a prominent influence on cell migration by augmenting their velocity from $26.1 \pm 1.4$ to $36.1 \pm 0.5 \mu \mathrm{m} / \mathrm{h}$. In contrast, Spry 4 expression slows down these cells to $21.3 \pm 0.99 \mu \mathrm{m} / \mathrm{h}$ (Figure $5 \mathrm{~A}, \mathrm{~B})$. Both effects were significant.

A

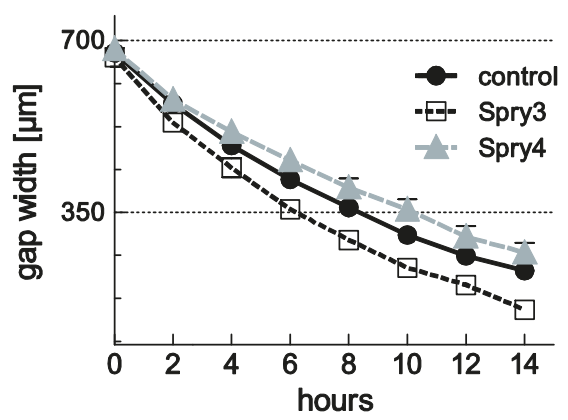

C

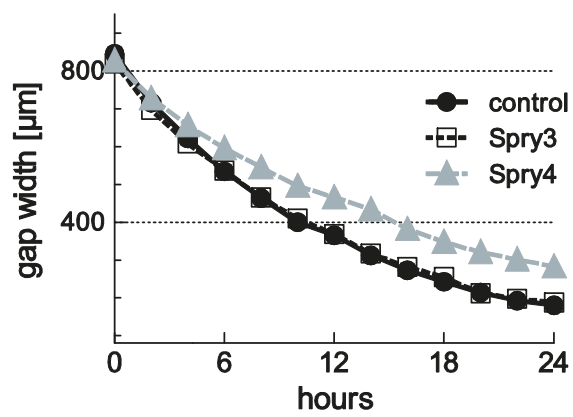

B
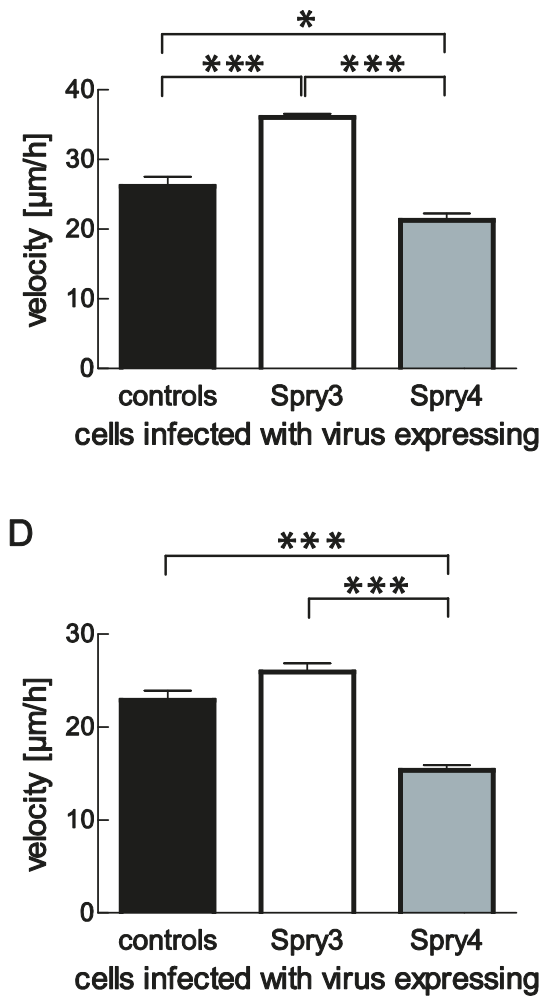

Figure 5. Influence of Spry3 and Spry4 expression on cell migration of GBM-derived cell lines. (A) Scratch assay was performed in DBTRG-05MG cells infected with adenoviruses expressing the indicated proteins. Representative curves of distance coverage were obtained by measuring decreasing gap widths of three replicative scratches at every two-hour time points using ImageJ. (B) Using linear regression, migration velocities were calculated. Means of at least three experimentations \pm SEM are summarized as column bars. (C) Representative measurements of replicative gap closure in a close layer of U-373 MG cell expressing the indicated proteins are shown. (D) Velocities of at least three experiments were calculated using linear regression in GraphPad Prism and summarized in a graph depicting means \pm SEM. An unpaired t-test was used to acquire significance. $p<0.05 ;{ }^{* *} p<0.01$; *** $p<0.001$.

Compared to DBTRG-05MG, U373 cells are slower migrating and the effect of the Spry proteins was less developed. Spry3 has no significant effect on the velocity of gap closure although a slightly faster calculated average velocity points towards a positive effect of its expression on cell migration. 
In accordance with the data obtained in DBTRG-05MG, Spry4 expression in U373 delays the closure of the gap significantly. The control-treated cells move with a speed of $22.9 \pm 1.0 \mu \mathrm{m} / \mathrm{h}$ towards the opposite front, while in the presence of Spry4 as an average speed only $15.4 \pm 0.5 \mu \mathrm{m} / \mathrm{h}$ were calculated (Figure 5C,D).

These observations indicate that in brain cells, Spry3 and Spry4 exert different effects not only on cell proliferation but also on cell migration.

\subsection{Repressed Expression of Spry3 Inhibits Cell Proliferation and Migration}

To further verify our observations, we wanted to investigate if lowered Spry expressions would influence cell proliferation in the opposite way than their overexpression. Therefore, an adenovirus expressing a shRNA directed against Spry3 was introduced into DBTRG-05MG and Spry3 levels were compared to the ones in control-treated and Spry3 overexpressing cells. As depicted in Figure 6A, expression of shSpry3 failed to modulate Spry3 levels, while the overexpression was successfully applied. In contrast, in U373 cells, expression of shRNA targeting Spry3 mRNA resulted in clearly lowered levels of Spry3 protein (Figure 6B). Since Spry4 is not expressed in detectable amounts in U373, it was just useful to express shSpry4 in DBTRG-05MG, where similar to the application of shSpry3, the endogenous expression of the protein was unaffected by expressing a shRNA directed against Spry 4 (data not shown). Next, we performed a growth curve analysis using shSpry3 in U373 cells. Reduced Spry3 expression caused an inhibition of cell proliferation while in parallel overexpression accelerated the doubling of these cells (Figure 6C). Compared to control cells, doubling of shSpry3-treated cells was reduced from 0.58 to 0.50 doublings per day, substantiating an oncogenic effect of Spry3 in brain cancer (Figure 6D). To evaluate if a repression of Spry3 in addition to its interference with proliferation is also influencing cell migration, Spry 3 levels of U373 cells were modulated by treatment with the respective adenoviruses and a scratch assay was performed. The time to close the gap was significantly delayed when Spry3 levels were lowered (Figure 6E). On average, cells expressing a shRNA targeting Spry3 cover a distance of $21 \mu \mathrm{m}$ in an hour, while control treated cells move about 1.2-fold faster, while Spry3 overexpression had no significant influence on the velocity of gap closure (Figure 6F). These data demonstrate that like proliferation, cell migration of GBM-derived cells is hindered if less Spry3 proteins are present confirming the tumor-promoting function of Spry3. 
A

$\begin{array}{llll}\text { Spry3 } & - & - & + \\ \text { shSpry3 } & + & - & - \\ \text { Spry3 } & & & \end{array}$

C

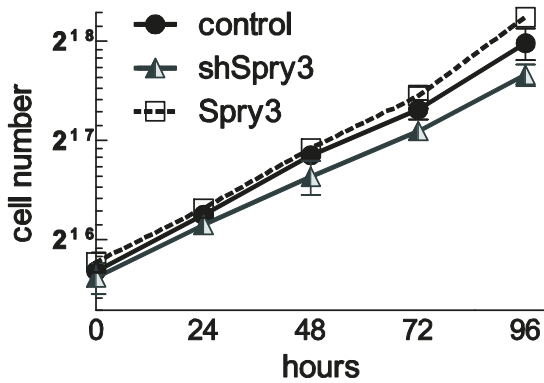

E

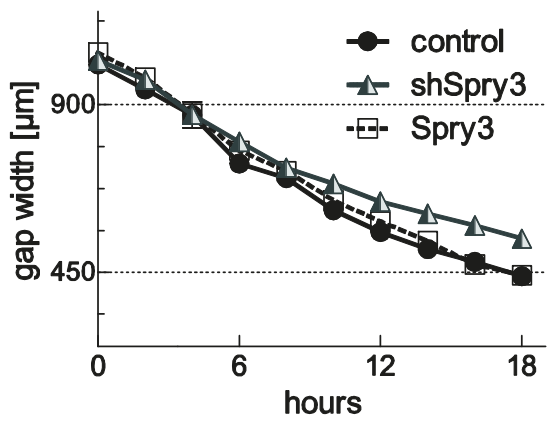

B

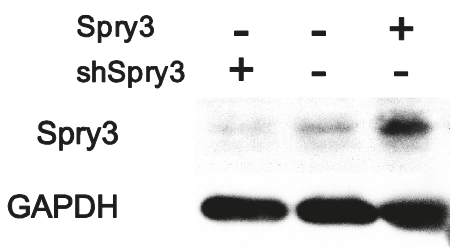

D

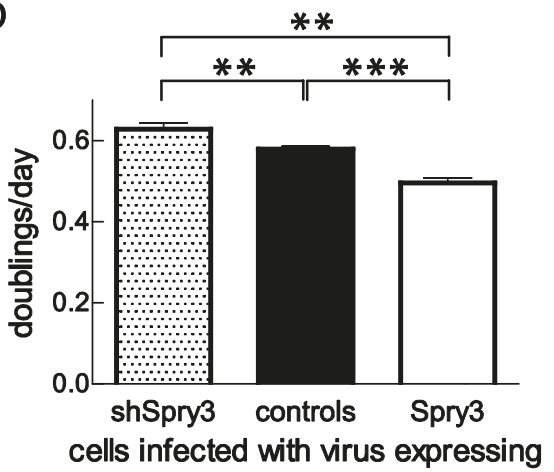

F

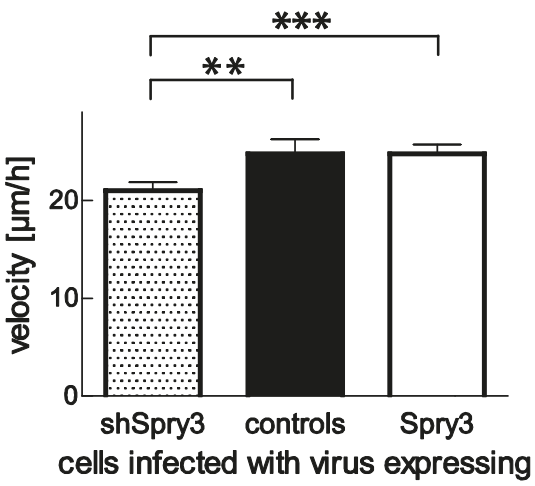

Figure 6. Verification of Spry3 impact on cell proliferation and migration by downregulation of the endogenous protein levels. DBTRG-05MG (A) and U373 (B) cells infected with adenoviruses expressing Spry3, shSpry3 or a control protein were analyzed concerning their Spry3 protein levels. (C) Three days after infection with the indicated viruses a growth curve analysis was performed in U373. (D) The doubling time of three experiments was calculated by performing an exponential growth equation and the mean doublings per day \pm SEM are depicted. (E) U373 cell expressing the indicated proteins were cultured to form a close layer before a scratch assay was performed. Measurements of three replicative gaps were performed every two hours and a representative experiment is shown. (F) Velocities of three experiments were calculated using linear regression in GraphPad Prism and a summary is depicted. Using an unpaired t-test, significance was determined. ${ }^{* *} p<0.01 ;{ }^{* * *} p<0.001$.

\section{Discussion}

Deregulated signal transduction is one of the most frequent alterations contributing to malignancy of brain cancer. In this study we provide data showing that Spry3 and Spry4 expression may be 
altered in brain cancer and affect cell proliferation and migration in opposing ways. Both Spry proteins are expressed in most of the brain cancer-derived cell lines, and two bands with a slightly different migration velocity can be detected. In case of Spry3, the faster migrating band is more frequently detected in the cells cultivated in the absence of serum. Although posttranslational modification of Spry3 is not reported, it is likely that analog to the other family members [36] the protein is phosphorylated at serine and/or tyrosine residues and that one of these potential modification is causing a shift in the gel.

A comparison of the Spry3 and Spry4 protein levels in the different cell lines revealed that the expression of these two Spry family members does not correlate. While Spry3 expression was on average elevated in cell lines originated from higher malignant tumors, Spry4 tended to be repressed in GBM and GS compared to cells derived from lower graded cancers. In accordance with our observation, an earlier report describes that Spry4 is often missing or deleted in gliomas [37]. Indeed, in several of the GBMs analyzed we were unable to detect this Spry isoform. Similarly, in lung $[14,38]$ and breast cancer [39], a repression of Spry4 is coinciding with a postulated tumor-suppressive function. With regard to Spry3, in normal brain tissue its expression is well documented, but due to its low abundance in other tissues, expression data in cancers are rarely available [6,7]. To our knowledge, only one report by Sirivatanauksorn et al. investigated the RNA level of Spry3 as well as Spry4 in hepatocellular cancer, and comparable to our observations in the brain, Spry4 mRNA levels were downregulated in liver cancer-associated tissue, while Spry3 expression was unaltered [40]. With respect to Spry2, data generated on RNA level clearly points towards an upregulation of this Spry member in GBM when compared to non-tumor tissue [25], while a study exploring protein levels in immunohistochemistry suggests downregulation of Spry2 in higher malignant brain cancers when compared to lower graded tumors [41]. Another obvious different variable concerning regulation of Spry3 and Spry4 is their dependency on mitogen availability. Like Spry1 in lung cancer cells [34], Spry3 protein levels fail to fluctuate in response to serum-withdrawal in brain cancer-derived cells. In contrast, Spry4 expression is usually manifold augmented when serum-containing factors are supplied. This is in accordance with observations in lung [35,42], prostate and osteosarcoma [42]. Additionally, it is reported that in neuronal cells derived from the dorsal root ganglion, Spry4 can be induced as a consequence of FGF2 as well as NFG supplementation [28]. Differences in Spry3 and Spry4 expression control are furthermore reported in bovine ovarian granulosa cells where Spry4 was increased in response to FGF1 and FGF4, while in parallel Spry3 levels were lowered [43].

Concerning their impact on the cellular behavior, we observed that ectopic expression of Spry3 is augmenting the growth and migration rate of different GBM-derived cell lines. Corroborating, repression of its protein levels as achieved by introducing a specific shRNA resulted in diminished cell proliferation. This would suggest that this Spry protein member exerts a tumor-promoting role in brain cancers. Accordingly, two different reports suggest that Spry2 is advantageous for the malignancy of GBM $[25,26]$. Knock-down of its expression decelerates cell proliferation of GBM cell lines $[25,26]$ while astrocytes were unaffected by modulated Spry2 levels [25]. Additionally Spry2 was identified as prognostic marker for GBM patients survival [25]. Although in most tissues Spry proteins fulfill the function of tumor-suppressors, individual Spry proteins are promoting tumorigenic potential here and there [44]. Spry2, for example, is above its function in GBM, shown to promote colon cancer malignancy by increasing proliferation, migration, tumor growth [23] and invasion [22] of colon cancer cells. In case of Spry1, an oncogenic function of the protein was demonstrated in the embryonal subtype of rhabdomyosarcoma [24].

In contrast to Spry3 and Spry2, Spry4 expression is inhibiting cell migration and proliferation of GBM-derived cell lines and is able to inhibit ERK phosphorylation in FGF2- and serum-induced as well as in unstimulated GBM-derived cells. An opposing role of Spry4 to other Spry proteins is already signified in colon carcinomas. Zhou et al. [45] demonstrated that Spry4 expression interferes with in vitro and in vivo cell proliferation of colon cancer cells. In contrast, Spry2 and Spry1 are fulfilling oncogenic functions in these tumors [22,23,46]. Additionally, in osteosarcoma [21] and in 
ovarian cancer [47], a tumor-suppressing role for Spry2 but not Spry4 was explicitly highlighted. Nonetheless, cell migration is specifically inhibited by Spry4 expression in prostate [48], pancreatic [49] and endothelial cells [50]. A concordant inhibition of proliferation and migration in case of Spry4 expression is reported for breast [18] and lung cancer cells [14]. Additionally, Spry4 can fulfill a tumor-suppressing role by interfering with angiogenic signals and thereby inhibits neovascularization and tumor growth [51].

\section{Conclusion}

In summary, our study describes that Spry3 and Spry4 exert different roles in brain cancer. Spry3 potentiates the tumorigenic potential of glioblastoma cells and Spry4 functions as tumor-suppressing protein in this entity.

Author Contributions: Conceptualization, B.E.C.-S., C.-E.M. and H.S.; methodology, B.E.C.-S. A.S., C.-E.M., J.S., and G.S.; validation, B.E.C.S., A.S., C.E.M., and J.S.; formal analysis, B.E.C.S. and H.S.; investigation, B.E.C.S., A.S. and J.S.; resources, C.E.M., J.S., and G.S.; data curation, H.S.; writing-original draft preparation, B.E.C.S and H.S.; writing-review and editing, B.E.C.S., A.S. and H.S.; visualization, B.E.C.S. and H.S.; supervision, H.S.; project administration, H.S.; and funding acquisition, H.S.

Funding: This research was funded by Herzfelder'sche Familienstiftung grant number 2015 and Hochschuljubiläumsstiftung grant number H-210512/2015...

Acknowledgments: We thank Walter Berger and Lisa Gabler for providing the cell lines. Daniel Valcanover, Gabriel Kaufmann, Dorra Boumaiza, Gerald Timelthaler and Angelina Doriguzzi for their technical assistance and Alina Bayer for her help with producing a Spry3 antigen.

Conflicts of Interest: The authors declare no conflict of interest.

\section{References}

1. Furnari, F.B.; Fenton, T.; Bachoo, R.M.; Mukasa, A.; Stommel, J.M.; Stegh, A.; Hahn, W.C.; Ligon, K.L.; Louis, D.N.; Brennan, C.; et al. Malignant astrocytic glioma: Genetics, biology, and paths to treatment. Genes Dev. 2007, 21, 2683-2710. [CrossRef] [PubMed]

2. Kruchko, C.; Ostrom, Q.T.; Boscia, A.; Truitt, G.; Gittleman, H.; Barnholtz-Sloan, J.S. CBTRUS Statistical Report: Primary Brain and Other Central Nervous System Tumors Diagnosed in the United States in 2011-2015. Neuro-Oncol. 2018, 20, iv1-iv86. [CrossRef]

3. Schilling, F.H.; Spix, C.; Berthold, F.; Erttmann, R.; Fehse, N.; Hero, B.; Klein, G.; Sander, J.; Schwarz, K.; Treuner, J.; et al. Neuroblastoma screening at one year of age. N. Engl. J. Med. 2002, 346, 1047-1053. [CrossRef] [PubMed]

4. Cancer Genome Atlas Research, N. Comprehensive genomic characterization defines human glioblastoma genes and core pathways. Nature 2008, 455, 1061-1068. [CrossRef] [PubMed]

5. Hacohen, N.; Kramer, S.; Sutherland, D.; Hiromi, Y.; Krasnow, M.A. Sprouty encodes a novel antagonist of FGF signaling that patterns apical branching of the Drosophila airways. Cell 1998, 92, 253-263. [CrossRef]

6. Minowada, G.; Jarvis, L.A.; Chi, C.L.; Neubuser, A.; Sun, X.; Hacohen, N.; Krasnow, M.A.; Martin, G.R. Vertebrate Sprouty genes are induced by FGF signaling and can cause chondrodysplasia when overexpressed. Development 1999, 126, 4465-4475. [PubMed]

7. Ning, Z.; McLellan, A.S.; Ball, M.; Wynne, F.; O’Neill, C.; Mills, W.; Quinn, J.P.; Kleinjan, D.A.; Anney, R.J.; Carmody, R.J.; et al. Regulation of SPRY3 by X chromosome and PAR2-linked promoters in an autism susceptibility region. Hum. Mol. Genet. 2015, 24, 7450. [CrossRef]

8. Sasaki, A.; Taketomi, T.; Kato, R.; Saeki, K.; Nonami, A.; Sasaki, M.; Kuriyama, M.; Saito, N.; Shibuya, M.; Yoshimura, A. Mammalian Sprouty4 suppresses Ras-independent ERK activation by binding to Raf1. Nat. Cell Biol. 2003, 5, 427-432. [CrossRef]

9. Hanafusa, H.; Torii, S.; Yasunaga, T.; Nishida, E. Sprouty1 and Sprouty2 provide a control mechanism for the Ras/MAPK signalling pathway. Nat. Cell Biol. 2002, 4, 850-858. [CrossRef]

10. Gross, I.; Morrison, D.J.; Hyink, D.P.; Georgas, K.; English, M.A.; Mericskay, M.; Hosono, S.; Sassoon, D.; Wilson, P.D.; Little, M.; et al. The receptor tyrosine kinase regulator Sprouty1 is a target of the tumor suppressor WT1 and important for kidney development. J. Biol. Chem. 2003, 278, 41420-41430. [CrossRef] 
11. Edwin, F.; Singh, R.; Endersby, R.; Baker, S.J.; Patel, T.B. The tumor suppressor PTEN is necessary for human Sprouty 2-mediated inhibition of cell proliferation. J. Biol. Chem. 2006, 281, 4816-4822. [CrossRef] [PubMed]

12. Akbulut, S.; Reddi, A.L.; Aggarwal, P.; Ambardekar, C.; Canciani, B.; Kim, M.K.; Hix, L.; Vilimas, T.; Mason, J.; Basson, M.A.; et al. Sprouty proteins inhibit receptor-mediated activation of phosphatidylinositol-specific phospholipase C. Mol. Biol. Cell 2010, 21, 3487-3496. [CrossRef] [PubMed]

13. Wong, E.S.; Lim, J.; Low, B.C.; Chen, Q.; Guy, G.R. Evidence for direct interaction between Sprouty and Cbl. J. Biol. Chem. 2001, 276, 5866-5875. [CrossRef] [PubMed]

14. Tennis, M.A.; Van Scoyk, M.M.; Freeman, S.V.; Vandervest, K.M.; Nemenoff, R.A.; Winn, R.A. Sprouty-4 inhibits transformed cell growth, migration and invasion, and epithelial-mesenchymal transition, and is regulated by Wnt7A through PPARgamma in non-small cell lung cancer. Mol. Cancer Res. 2010, 8, 833-843. [CrossRef] [PubMed]

15. Sutterluty, H.; Mayer, C.E.; Setinek, U.; Attems, J.; Ovtcharov, S.; Mikula, M.; Mikulits, W.; Micksche, M.; Berger, W. Down-regulation of Sprouty2 in non-small cell lung cancer contributes to tumor malignancy via extracellular signal-regulated kinase pathway-dependent and -independent mechanisms. Mol. Cancer Res. 2007, 5, 509-520. [CrossRef]

16. Shaw, A.T.; Meissner, A.; Dowdle, J.A.; Crowley, D.; Magendantz, M.; Ouyang, C.; Parisi, T.; Rajagopal, J.; Blank, L.J.; Bronson, R.T.; et al. Sprouty-2 regulates oncogenic K-ras in lung development and tumorigenesis. Genes Dev. 2007, 21, 694-707. [CrossRef]

17. Fong, C.W.; Chua, M.S.; McKie, A.B.; Ling, S.H.; Mason, V.; Li, R.; Yusoff, P.; Lo, T.L.; Leung, H.Y.; So, S.K.; et al. Sprouty 2, an inhibitor of mitogen-activated protein kinase signaling, is down-regulated in hepatocellular carcinoma. Cancer Res. 2006, 66, 2048-2058. [CrossRef]

18. Vanas, V.; Muhlbacher, E.; Kral, R.; Sutterluty-Fall, H. Sprouty4 interferes with cell proliferation and migration of breast cancer-derived cell lines. Tumour Biol. 2014, 35, 4447-4456. [CrossRef]

19. Lo, T.L.; Yusoff, P.; Fong, C.W.; Guo, K.; McCaw, B.J.; Phillips, W.A.; Yang, H.; Wong, E.S.; Leong, H.F.; Zeng, Q.; et al. The ras/mitogen-activated protein kinase pathway inhibitor and likely tumor suppressor proteins, sprouty 1 and sprouty 2 are deregulated in breast cancer. Cancer Res. 2004, 64, 6127-6136. [CrossRef]

20. Kwabi-Addo, B.; Wang, J.; Erdem, H.; Vaid, A.; Castro, P.; Ayala, G.; Ittmann, M. The expression of Sprouty1, an inhibitor of fibroblast growth factor signal transduction, is decreased in human prostate cancer. Cancer Res. 2004, 64, 4728-4735. [CrossRef]

21. Rathmanner, N.; Haigl, B.; Vanas, V.; Doriguzzi, A.; Gsur, A.; Sutterluty-Fall, H. Sprouty2 but not Sprouty4 is a potent inhibitor of cell proliferation and migration of osteosarcoma cells. FEBS Lett. 2013, 587, 2597-2605. [CrossRef]

22. Barbachano, A.; Ordonez-Moran, P.; Garcia, J.M.; Sanchez, A.; Pereira, F.; Larriba, M.J.; Martinez, N.; Hernandez, J.; Landolfi, S.; Bonilla, F.; et al. SPROUTY-2 and E-cadherin regulate reciprocally and dictate colon cancer cell tumourigenicity. Oncogene 2010, 29, 4800-4813. [CrossRef]

23. Holgren, C.; Dougherty, U.; Edwin, F.; Cerasi, D.; Taylor, I.; Fichera, A.; Joseph, L.; Bissonnette, M.; Khare, S. Sprouty-2 controls c-Met expression and metastatic potential of colon cancer cells: Sprouty/c-Met upregulation in human colonic adenocarcinomas. Oncogene 2010, 29, 5241-5253. [CrossRef]

24. Schaaf, G.; Hamdi, M.; Zwijnenburg, D.; Lakeman, A.; Geerts, D.; Versteeg, R.; Kool, M. Silencing of SPRY1 triggers complete regression of rhabdomyosarcoma tumors carrying a mutated RAS gene. Cancer Res. 2010, 70, 762-771. [CrossRef]

25. Park, J.W.; Wollmann, G.; Urbiola, C.; Fogli, B.; Florio, T.; Geley, S.; Klimaschewski, L. Sprouty2 enhances the tumorigenic potential of glioblastoma cells. Neuro-Oncol. 2018, 20, 1044-1054. [CrossRef]

26. Walsh, A.M.; Kapoor, G.S.; Buonato, J.M.; Mathew, L.K.; Bi, Y.; Davuluri, R.V.; Martinez-Lage, M.; Simon, M.C.; O'Rourke, D.M.; Lazzara, M.J. Sprouty2 Drives Drug Resistance and Proliferation in Glioblastoma. Mol. Cancer Res. 2015, 13, 1227-1237. [CrossRef]

27. Hausott, B.; Vallant, N.; Schlick, B.; Auer, M.; Nimmervoll, B.; Obermair, G.J.; Schwarzer, C.; Dai, F.; Brand-Saberi, B.; Klimaschewski, L. Sprouty2 and -4 regulate axon outgrowth by hippocampal neurons. Hippocampus 2012, 22, 434-441. [CrossRef]

28. Hausott, B.; Vallant, N.; Auer, M.; Yang, L.; Dai, F.; Brand-Saberi, B.; Klimaschewski, L. Sprouty2 down-regulation promotes axon growth by adult sensory neurons. Mol. Cell. Neurosci. 2009, 42, 328-340. [CrossRef] 
29. Yu, T.; Yaguchi, Y.; Echevarria, D.; Martinez, S.; Basson, M.A. Sprouty genes prevent excessive FGF signalling in multiple cell types throughout development of the cerebellum. Development 2011, 138, 2957-2968. [CrossRef]

30. Panagiotaki, N.; Dajas-Bailador, F.; Amaya, E.; Papalopulu, N.; Dorey, K. Characterisation of a new regulator of BDNF signalling, Sprouty3, involved in axonal morphogenesis in vivo. Development 2010, 137, 4005-4015. [CrossRef]

31. Benke, S.; Agerer, B.; Haas, L.; Stoger, M.; Lercher, A.; Gabler, L.; Kiss, I.; Scinicariello, S.; Berger, W.; Bergthaler, A.; et al. Human tripartite motif protein 52 is required for cell context-dependent proliferation. Oncotarget 2018, 9, 13565-13581. [CrossRef]

32. Sutterluty, H.; Chatelain, E.; Marti, A.; Wirbelauer, C.; Senften, M.; Muller, U.; Krek, W. p45SKP2 promotes p27Kip1 degradation and induces S phase in quiescent cells. Nat. Cell Biol. 1999, 1, 207-214. [CrossRef]

33. Vanas, V.; Haigl, B.; Stockhammer, V.; Sutterluty-Fall, H. MicroRNA-21 Increases Proliferation and Cisplatin Sensitivity of Osteosarcoma-Derived Cells. PLoS ONE 2016, 11, e0161023. [CrossRef]

34. Kral, R.M.; Mayer, C.E.; Vanas, V.; Gsur, A.; Sutterluty-Fall, H. In non-small cell lung cancer mitogenic signaling leaves Sprouty1 protein levels unaffected. Cell. Biochem. Funct. 2014, 32, 96-100. [CrossRef]

35. Mayer, C.E.; Haigl, B.; Jantscher, F.; Siegwart, G.; Grusch, M.; Berger, W.; Sutterluty, H. Bimodal expression of Sprouty2 during the cell cycle is mediated by phase-specific Ras/MAPK and c-Cbl activities. Cell. Mol. Life Sci. 2010, 67, 3299-3311. [CrossRef]

36. Impagnatiello, M.A.; Weitzer, S.; Gannon, G.; Compagni, A.; Cotten, M.; Christofori, G. Mammalian sprouty-1 and -2 are membrane-anchored phosphoprotein inhibitors of growth factor signaling in endothelial cells. J. Cell Biol. 2001, 152, 1087-1098. [CrossRef]

37. Zhang, W.; Lv, Y.; Xue, Y.; Wu, C.; Yao, K.; Zhang, C.; Jin, Q.; Huang, R.; Li, J.; Sun, Y.; et al. Co-expression modules of NF1, PTEN and sprouty enable distinction of adult diffuse gliomas according to pathway activities of receptor tyrosine kinases. Oncotarget 2016, 7, 59098-59114. [CrossRef]

38. Doriguzzi, A.; Salhi, J.; Sutterluty-Fall, H. Sprouty4 mRNA variants and protein expressions in breast and lung-derived cells. Oncol. Lett. 2016, 12, 4161-4166. [CrossRef]

39. Faratian, D.; Sims, A.H.; Mullen, P.; Kay, C.; Um, I.; Langdon, S.P.; Harrison, D.J. Sprouty 2 is an independent prognostic factor in breast cancer and may be useful in stratifying patients for trastuzumab therapy. PLOS ONE 2011, 6, e23772. [CrossRef]

40. Sirivatanauksorn, Y.; Sirivatanauksorn, V.; Srisawat, C.; Khongmanee, A.; Tongkham, C. Differential expression of sprouty genes in hepatocellular carcinoma. J. Surg. Oncol. 2012, 105, 273-276. [CrossRef]

41. Kwak, H.J.; Kim, Y.J.; Chun, K.R.; Woo, Y.M.; Park, S.J.; Jeong, J.A.; Jo, S.H.; Kim, T.H.; Min, H.S.; Chae, J.S.; et al. Downregulation of Spry2 by miR-21 triggers malignancy in human gliomas. Oncogene 2011, 30, 2433-2442. [CrossRef]

42. Doriguzzi, A.; Haigl, B.; Gsur, A.; Sutterluty-Fall, H. The increased Sprouty4 expression in response to serum is transcriptionally controlled by Specific protein 1. Int. J. Biochem. Cell Biol. 2015, 64, 220-228. [CrossRef]

43. Jiang, Z.; Price, C.A. Differential actions of fibroblast growth factors on intracellular pathways and target gene expression in bovine ovarian granulosa cells. Reproduction 2012, 144, 625-632. [CrossRef]

44. Masoumi-Moghaddam, S.; Amini, A.; Morris, D.L. The developing story of Sprouty and cancer. Cancer Metastasis Rev. 2014, 33, 695-720. [CrossRef]

45. Zhou, X.; Xie, S.; Yuan, C.; Jiang, L.; Huang, X.; Li, L.; Chen, Y.; Luo, L.; Zhang, J.; Wang, D.; et al. Lower Expression of SPRY4 Predicts a Poor Prognosis and Regulates Cell Proliferation in Colorectal Cancer. Cell. Physiol. Biochem. 2016, 40, 1433-1442. [CrossRef]

46. Zhang, Q.; Wei, T.; Shim, K.; Wright, K.; Xu, K.; Palka-Hamblin, H.L.; Jurkevich, A.; Khare, S. Atypical role of sprouty in colorectal cancer: Sprouty repression inhibits epithelial-mesenchymal transition. Oncogene 2016, 35, 3151-3162. [CrossRef]

47. Masoumi-Moghaddam, S.; Amini, A.; Wei, A.Q.; Robertson, G.; Morris, D.L. Sprouty 2 protein, but not Sprouty 4 , is an independent prognostic biomarker for human epithelial ovarian cancer. Int. J. Cancer 2015, 137, 560-570. [CrossRef]

48. Wang, J.; Thompson, B.; Ren, C.; Ittmann, M.; Kwabi-Addo, B. Sprouty4, a suppressor of tumor cell motility, is down regulated by DNA methylation in human prostate cancer. Prostate 2006, 66, 613-624. [CrossRef]

49. Jaggi, F.; Cabrita, M.A.; Perl, A.K.; Christofori, G. Modulation of endocrine pancreas development but not beta-cell carcinogenesis by Sprouty4. Mol. Cancer Res. 2008, 6, 468-482. [CrossRef] 
50. Gong, Y.; Yang, X.; He, Q.; Gower, L.; Prudovsky, I.; Vary, C.P.; Brooks, P.C.; Friesel, R.E. Sprouty4 regulates endothelial cell migration via modulating integrin beta3 stability through c-Src. Angiogenesis 2013, 16, 861-875. [CrossRef]

51. Taniguchi, K.; Ishizaki, T.; Ayada, T.; Sugiyama, Y.; Wakabayashi, Y.; Sekiya, T.; Nakagawa, R.; Yoshimura, A. Sprouty4 deficiency potentiates Ras-independent angiogenic signals and tumor growth. Cancer Sci. 2009, 100, 1648-1654. [CrossRef] [PubMed]

(C) 2019 by the authors. Licensee MDPI, Basel, Switzerland. This article is an open access article distributed under the terms and conditions of the Creative Commons Attribution (CC BY) license (http://creativecommons.org/licenses/by/4.0/). 


\title{
The Aberrant Expression of the Mesenchymal Variant of FGFR2 in the Epithelial Context Inhibits Autophagy
}

\author{
Monica Nanni ${ }^{1,+}$, Danilo Ranieri ${ }^{1,+}$, Flavia Persechino ${ }^{1}$, Maria Rosaria Torrisi ${ }^{1,2,+}$ and \\ Francesca Belleudi ${ }^{1, *, \dagger}$ \\ 1 Laboratory affiliated to Istituto Pasteur Italia-Fondazione Cenci Bolognetti, Department of Clinical and \\ Molecular Medicine, Sapienza University of Rome, 00185 Rome, Italy \\ 2 S. Andrea University Hospital, 00189 Rome, Italy \\ * Correspondence: francesca.belleudi@uniroma1.it; Tel.: +39-06-33775995 \\ + These authors contributed equally.
}

Received: 29 May 2019; Accepted: 28 June 2019; Published: 29 June 2019

\begin{abstract}
Signaling of the epithelial splice variant of fibroblast growth factor receptor 2 (FGFR2b) triggers both differentiation and autophagy, while the aberrant expression of the mesenchymal FGFR2c isoform in epithelial cells induces impaired differentiation, epithelial mesenchymal transition (EMT) and tumorigenic features. Here we analyzed in the human keratinocyte cell line, as well as in primary cultured cells, the possible impact of FGFR2c forced expression on the autophagic process. Biochemical and quantitative immunofluorescence analysis, coupled to the use of autophagic flux sensors, specific substrate inhibitors or silencing approaches, showed that ectopic expression and the activation of FGFR2c inhibit the autophagosome formation and that AKT/MTOR is the downstream signaling mainly involved. Interestingly, the selective inhibition of AKT or MTOR substrates caused a reversion of the effects of FGFR2c on autophagy, which could also arise from the imbalance of the interplay between AKT/MTOR pathway and JNK1 signaling in favor of JNK1 activation, BCL-2 phosphorylation and possibly phagophore nucleation. Finally, silencing experiments of depletion of ESRP1, responsible for FGFR2 splicing and consequent FGFR2b expression, indicated that the switching from FGFR2b to FGFR2c isoform could represent the key event underlying the inhibition of the autophagic process in the epithelial context. Our results provide the first evidence of a negative impact of the out-of-context expression of FGFR2c on autophagy, suggesting a possible role of this receptor in the modulation of the recently proposed negative loop between autophagy and EMT during carcinogenesis.
\end{abstract}

Keywords: FGFR2c; autophagy; keratinocyte; MTOR; JNK1

\section{Introduction}

The fibroblast growth factor receptors (FGFR1-4) are four receptor tyrosine kinases regulating key processes, such as cell proliferation, differentiation, migration and survival $[1,2]$. The alternative splicing of the IgIII loop in FGFR1-3, generates the FGFRIIIb or the FGFRIIIc isoforms, which are mainly expressed in epithelial and mesenchymal tissues, respectively [3]. Deregulation of FGF/FGFR signaling can play either oncogenic or tumor suppressive roles [2,4]. In this regard, the epithelial isoform of FGFR2 (FGFR2b) is a well-recognized regulator of epidermal differentiation and skin homeostasis [5-7] exerting a tumor suppressive role in vitro and in vivo $[8,9]$. According to these studies, our group has demonstrated that FGFR2b controls the entire program of human keratinocyte differentiation [10-12] and that PKC $\delta$ and PKC $\alpha$ signaling downstream FGFR2b are involved in different steps of this process [12]. However, we also found that, in the same epidermal tissue context, the altered FGFR2 
splicing and the aberrant expression of the mesenchymal FGFR2c isoform induces changes in the specificity for FGFs, leading to impairment of differentiation [13], epithelial mesenchymal transition (EMT) and early tumorigenic features [14]. The observation that FGFR2b/FGFR2c switching is also induced in keratinocytes by the E5 oncoprotein of human papillomavirus 16 (HPV16E5) [15], which is expressed in the early stages of virus infection, further supports the hypothesis that FGFR2c aberrant expression might be a precocious event in epithelial tumorigenesis.

At the light of growing evidences showing that FGFs would control cell differentiation by regulating autophagy in several tissues [16-18], we have also demonstrated that FGFR2b signaling triggers the autophagic process in human keratinocytes, showing that FGFR2b-induced autophagy and receptor-mediated early differentiation are interplaying events [19-21] and that JNK1 is the downstream signaling pathway at the crossroad between them [21,22].

However, autophagy not only regulates several biological functions, such as cell differentiation, but can also play either onco-suppressive or oncogenic roles in cancer, depending on its stage. In particular, during the initial steps of tumorigenesis autophagy, it appears to be linked to a negative loop to EMT, in established tumors this process has a pro-survival effect and its possible interplay with EMT remains still debated [23-25]. Therefore, keeping in mind the role of FGFR2c in driving EMT, here we pointed on to establish if and how the aberrant expression of FGFR2c could impact on autophagy. The results obtained showed that FGFR2c expression and signaling in epithelial context negatively interfere with the autophagic process, suggesting that this interference could significantly contribute to cancerogenesis.

\section{Results}

\subsection{FGFR2c Expression and Signaling Inhibit Autophagy in Human Keratinocytes}

To analyze the effect of FGFR2c expression and signaling on autophagy and to compare it to that previously described by us for FGFR2b [19-22], we took advantage of the human keratinocyte $\mathrm{HaCaT}$ clones stably transduced with $\mathrm{pBp}$-FGFR2b or pBp-FGFR2c retroviral constructs or with empty $\mathrm{pBp}$ vector, as negative control [14]. Cells were left untreated or stimulated with FGF7, the specific ligand of FGFR2b, or with FGF2, which does not bind to FGFR2b, but is able to activate other FGFRs including FGFR2c. Western blot analysis showed that, while FGF7 stimulation increased the levels of the band corresponding to the lipidated form of the microtubule associated protein 1 light chain 3 (MAP1LC3/LC3-II) in all clones (Figure 1A), as expected [19-22], FGF2 treatment significantly decreased them only in cells ectopically expressing FGFR2c (Figure 1A). Both the FGFR2b-dependent induction and FGFR2c-mediated inhibition of autophagy were abolished by the specific FGFR2 tyrosine kinase inhibitor SU5402 (Figure 1A), demonstrating that, in both cases, the signaling of FGFR2 isoforms was required. Since it is widely accepted that autophagy is not only a post-translationally regulated, but also a transcriptionally controlled process [26] and we have previously shown that FGFR2b signaling plays a role in this transcriptional control [20,21], we wondered whether FGFR2c might impact on autophagy also by affecting the expression of LC3 gene. To this aim, the mRNA transcript levels of LC3, which we have previously demonstrated to be increased in response to FGF7 in keratinocytes [20,21], were estimated by real-time relative RT-PCR. The results showed that, while FGF7 stimulation increased the expression of this gene in all clones (Figure 1B), FGF2 treatment does not affect them, even in HaCaT-pBp FGFR2c (Figure 1B). The impact of FGFR2c aberrant expression and signaling on autophagy was also investigated by immunofluorescence approach. Quantitative immunofluorescence analysis showed that, while LC3 signal intensity, as well as the number of LC3 positive dots per cell, were increased by FGF7 stimulation in all clones (Figure 1C), they appeared reduced in response to FGF2 only in HaCaT pBp-FGFR2c cells (Figure 1C). Again, all the observed effects were abolished by SU5402 (Figure 1C), confirming the requirement of FGFR2 isoform activation. Thus, the ectopic expression of FGFR2c and its signaling, which is known to exert an oncogenic outcome in human keratinocytes, appear also to negatively impact on autophagy. 
A
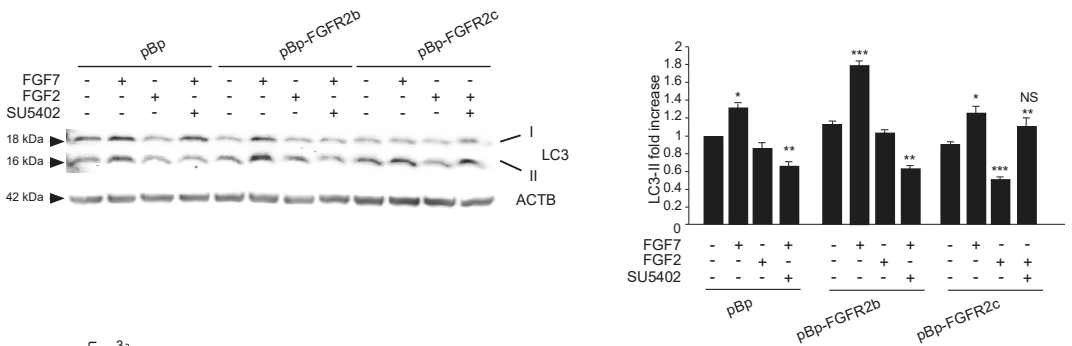

B

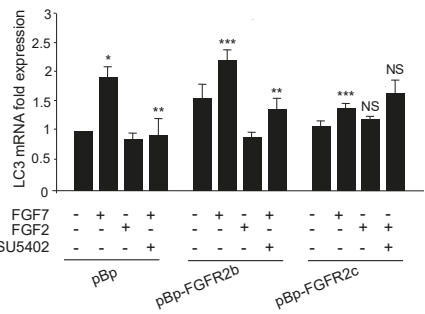

C
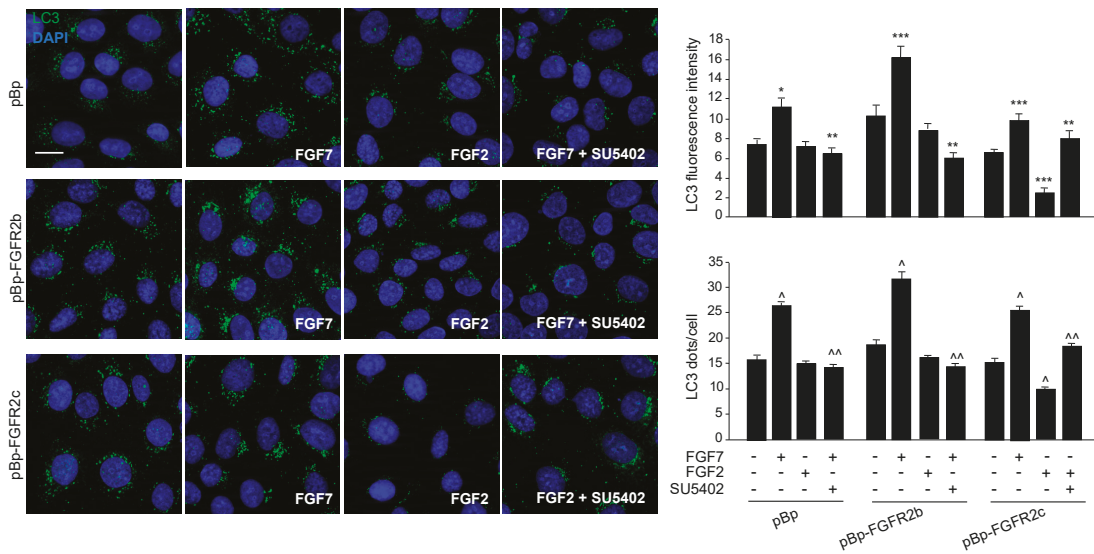

Figure 1. The ectopic expression of FGFR2c and its signaling inhibit autophagy in human keratinocytes. $\mathrm{HaCaT}$ cells, stably transduced with pBp-FGFR2b or pBp-FGFR2c constructs or with the empty pBp retroviral vector as control, were left untreated or stimulated with FGF7 or FGF2 in presence or absence of the FGFR2 tyrosine kinase inhibitor SU5402 as described in Material and Methods. (A) Western blot analysis showed that, while FGF7 stimulation increases the levels of LC3-II in all clones, FGF2 stimulation significantly decreased them only in $\mathrm{HaCaT}$ pBp-FGFR2c cells. All the observed effects were abolished by the presence of SU5402. Equal loading was assessed with the anti-ACTB antibody. For the densitometric analysis the values from three independent experiments were normalized and expressed as fold increases and are reported as mean values \pm standard deviations (SD). Student's $t$ test was performed, and significance levels are defined as $P<0.05 .{ }^{*} p<0.05$ and ${ }^{* * *} p<0.001$ vs the corresponding FGF-unstimulated cells; ** $p<0.05$ vs the corresponding SU5402-untreated cells; not significant (NS) vs the corresponding FGF-unstimulated, SU5402-untreated cells. (B) Real-time Reverse Transcriptase-Polymerase Chain Reaction (RT-PCR) analysis shows that while FGF7 stimulation induces 
the increases of LC3 mRNA transcripts in all clones, FGF2 treatment does not affect them. The results observed in HaCaT pBp and pBp-FGFR2b upon FGF7 stimulation were abolished by SU5402. Results are expressed as mean values \pm SE. Student's $t$ test was performed, and significance levels were defined as $P<0.05$. ${ }^{*} p<0.01,{ }^{* * * *} p<0.05$ and NS vs the corresponding FGF-unstimulated cells; ${ }^{* *} p<0.05$ and NS vs the corresponding SU5402-untreated-cells. (C) Quantitative immunofluorescence analysis shows that LC3 signal intensity was increased by FGF7 stimulation in all clones, but it appears strongly reduced upon FGF2 treatment only in HaCaT pBp-FGFR2c cells. The observed effects were abolished by SU5402 treatment. Quantitative analysis of the fluorescence intensity and LC3 positive dots per cell were performed as described in Materials and Methods, and the results are expressed as mean values \pm standard errors (SE). The student's $t$ test was performed, and significance levels were defined as $P<0.05$. ${ }^{*} p<0.01,{ }^{* * *} p<0.001$ and ${ }^{\wedge} p<0.0001$, vs the corresponding FGF-unstimulated cells; ${ }^{* *} p<0.001$ and ${ }^{\wedge} p<0.0001$ vs the corresponding SU5402-untreated cells.

\subsection{The Autophagosome Formation is the Autophagic Step Impaired by FGFR2c Expression and Signaling}

The amount of intracellular autophagosomes usually depends on the balance between their formation and their lysosomal-mediated degradation. Therefore, in order to assess how the ectopic FGFR2c could impact on the autophagic flux, the levels of the well-known autophagy substrate SQSTM1/p62 (sequestosome 1) was estimated by Western blot analysis. The evident decrease of the $62 \mathrm{kDa}$ band corresponding to SQSTM1, observed in all clones upon FGF7 stimulation (Figure 2A), confirmed the ability of FGFR2b signaling to trigger mainly the autophagosome assembly. In contrast, the significant increase of the SQSTM1 band, observed exclusively in HaCaT pBp-FGFR2c clones and only in response to FGF2 (Figure 2A), indicated that FGFR2c signaling might act via the inhibition of new autophagosome formation, rather than by accelerating their turnover. The observed effects were abolished by SU5402 (Figure 2A), confirming the requirement of receptor isoform activation. Since it is well known that SQSTM1 can be also transcriptionally regulated under conditions that modulate autophagy, we also investigated its mRNA expression levels in $\mathrm{HaCaT}$ clones stimulated as above. The results showed that FGF7 stimulation induced an evident decrease of SQSTM1 mRNA transcripts in all clones (Figure 2B), while FGF2 treatment did not significantly impact on them (Figure 2B). The ability of FGFR2c to negatively interfere with the phagosome formation, rather than their turnover, was also investigated using fluorescence approaches, transfecting $\mathrm{HaCaT}$ clones with a pDest-mCherry-EGFP-LC3 tandem construct [27]. In fact, mCherry-EGFP-LC3 is an autophagic flux sensor, since EGFP fluorescence (green) is quenched in acidic environments, whereas mCherry (red) is an acidic-stable fluorescent tag: The nascent autophagosomes are both red and green (yellow) labeled, whereas the acidic autolysosomes appear red, as a consequence of the EGFP quenching. Quantitative fluorescence analysis, performed on transfected cells left untreated or stimulated with FGFR2 ligands as above, showed that, while FGF7 stimulation increased both yellow and red dots (corresponding to autophagosomes and autophagolysosomes, respectively) (Figure 2C), FGF2 treatment significantly decreased them in HaCaT pBp-FGFR2c cells (Figure 2C). These results further confirmed that FGFR2c activation appear to inhibit new autophagosome assembly. 
A

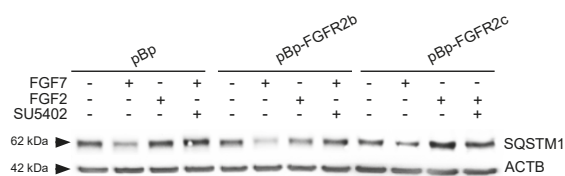

B
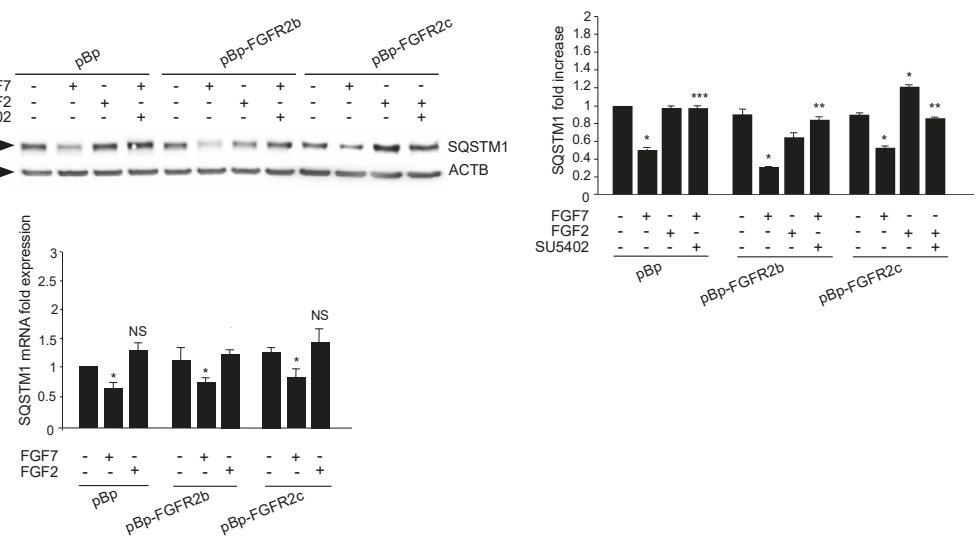

C
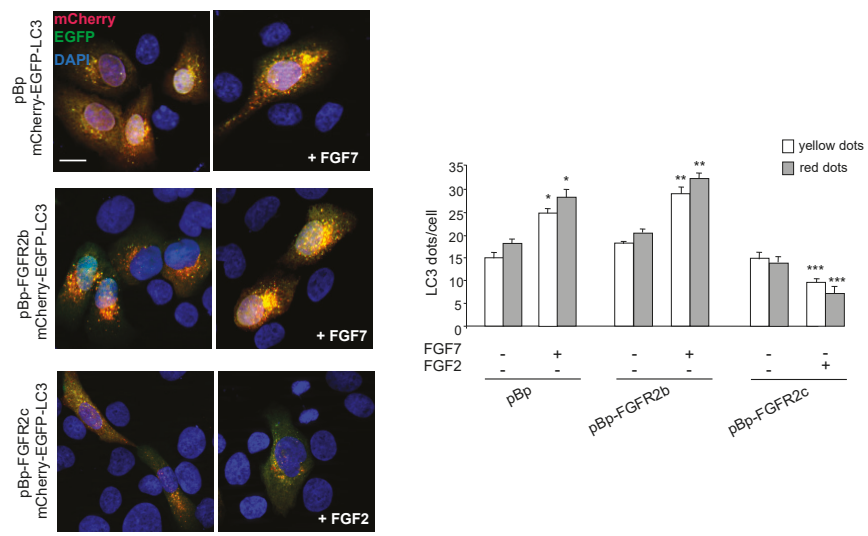

D
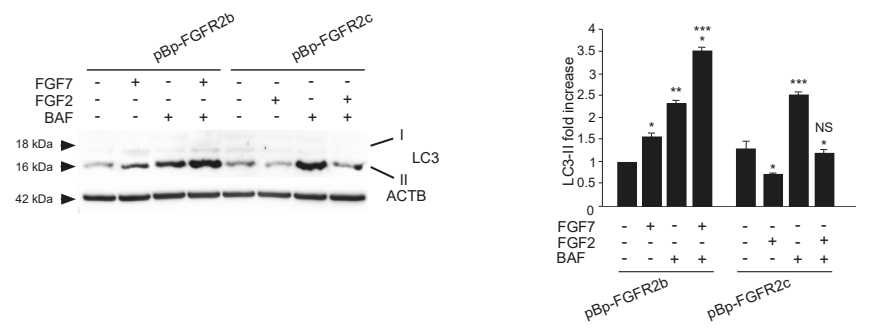

Figure 2. FGFR2c expression and signaling inhibits the autophagosome formation. (A) HaCaT pBp, pBp-FGFR2b or pBp-FGFR2c cells were left untreated or stimulated with FGF7 or FGF2 in presence or absence of the FGFR2 tyrosine kinase inhibitor SU5402 as above. Western blot analysis shows that, the $62 \mathrm{kDa}$ band corresponding to SQSTM1 was significantly increased only in HaCaT pBp-FGFR2c clones after FGF2 stimulation, while it decreases in all clones upon FGF7 stimulation. All the observed effects were abolished by SU5402. Equal loading was assessed with anti-ACTB antibody. Densitometric analysis and the student's $t$ test were performed as reported above. ${ }^{*} p<0.05$ vs the corresponding FGF-unstimulated cells; ** $p<0.05$ and ${ }^{* * *} p<0.001$ vs the corresponding SU5402-untreated cells. (B) $\mathrm{HaCaT}$ clones were stimulated with FGFR2 ligands as above. Real-time RT-PCR analysis shows that 
FGF7 stimulation decreased SQSTM1 mRNA transcripts in all clones, while FGF2 treatment did not affect them. Results are expressed as mean values \pm SE. The student's $t$ test was performed, and significance levels were defined as $P<0.05$. ${ }^{*} p<0.01$ vs the corresponding FGF7-unstimulated cells; NS vs the corresponding FGF7-unstimulated cells. (C) HaCaT clones were transiently transfected with mCherry-EGFP-LC3 construct. Cells were then left untreated or stimulated with FGF7 or FGF2 as above. Quantitative fluorescence analysis showed that, while FGF7 stimulation increased both yellow and red dots in $\mathrm{HaCaT}$ pBp and $\mathrm{HaCaT}$ pBp-FGFR2b cells, FGF2 stimulation decreased them in $\mathrm{HaCaT}$ pBp-FGFR2c cells. Quantitative analysis was performed as described in Materials and Methods, and results were expressed as mean values \pm SE. The student's $t$ test was performed as reported in the legend to Figure 1B. ${ }^{*} p<0.05$ and ${ }^{* *} p<0.001$ vs the corresponding FGF7-unstimulated cells; ${ }^{* * *} p<0.01$ vs the corresponding FGF2-unstimulated cells. (D) HaCaT pBp-FGFR2b and pBp-FGFR2c clones were left untreated or stimulated with FGF7 or FGF2 in the presence or absence of bafilomycin A1 for the last $3 \mathrm{~h}$. Western blot analysis showed that the increase in the levels of LC3-II upon FGF7 stimulation observed in HaCaT pBp-FGFR2b was further enhanced by bafilomycin while the decrease of LC3-II observed in HaCaT pBp-FGFR2c cells upon FGF2 stimulation was not recovered in the presence of the drug. Equal loading was assessed with anti-ACTB antibody. Densitometric analysis and the student's $t$ test were performed as reported above. ${ }^{*} p<0.05$ vs the corresponding FGF-unstimulated cells; ${ }^{* *} p<0.05,{ }^{* * *} p<0.01$ and NS vs the corresponding bafilomycin-untreated cells.

Finally, we monitored the LC3-II levels in the presence or absence of the well-known inhibitor of the autophagosome-lysosome fusion bafilomycin A1. Western blot analysis showed that the effect of increase of LC3-II band generally induced by this drug (Figure 2D) was not found in HaCaT pBp-FGFR2c upon FGF2 stimulation (Figure 2D). These findings demonstrated that the observed decrease of LC3-II induced by FGF2 in HaCaT pBp-FGFR2c cannot be ascribed to an acceleration of the autophagic flux.

Since we have previously shown that FGFR2b signaling plays a role in the transcriptional control of several ATG genes, other than LC3 [20,21], we also assessed whether FGFR2c might impact on autophagy by affecting some of these genes To this aim, the mRNA transcript levels of the BECN1 and ATG5 were estimated by real-time RT-PCR. The results showed that FGF2 treatment did not affect them, even in HaCaT-pBp FGFR2c (Figure S1). Therefore, we feel confident to conclude that the interference exerted by FGFR2c on autophagy does not occur at the transcriptional level.

\subsection{AKT/MTOR is the Downstream Pathway Responsible for FGFR2c-Mediated Inhibition of Autophagy}

To search for the possible downstream signaling pathways responsible for FGFR2c-mediated autophagic repression, MAPK/ERK1/2 and AKT/MTOR signaling were first considered, since they represent the main pathways involved in FGFR-mediated inhibition of autophagy [16,28-30]. Western blot analysis demonstrated that, only in cells ectopically expressing FGFR2c, FGF2 stimulation triggered ERK1/2, as well as AKT and MTOR phosphorylation (Figure 3); these effects were abolished by the FGFR2 kinase inhibitor SU5402 (Figure 3), confirming their dependence from FGFR2c activation. Then, in order to assess the possible involvement of these two pathways in FGFR2c-mediated repression of autophagy, we took advantage of specific substrate inhibitors: The ERK1/2 upstream substrates MAP2K/MEK1/2 inhibitor PD0325901 [31], the AKT inhibitor AKT-I-1/2 [32] and the widely used MTOR inhibitor rapamycin. The efficiency of each inhibitor was first assayed by Western blot analysis (Figure S2A). Then, we analyzed their effects on LC3-II expression in HaCaT pBp controls and HaCaT pBp-FGFR2c left untreated or stimulated with FGF2, as above. Surprisingly, Western blot analysis showed that, in the presence of either AKT inhibitor or rapamycin, but not of MEK1/2 inhibitor, the decrease of LC3-II levels induced by FGF2 stimulation in pBp-FGFR2c cells was not only recovered, but significantly increased, compared to the corresponding unstimulated cells (Figure 4A). These results indicated that the selective block of AKT/MTOR pathway was able to revert the effects on autophagy. No effects of all the inhibitors were detectable in cells not stimulated with FGF2 or in pBp control cells (Figure 4A), suggesting that, at least in our keratinocyte model, the ERK1/2 or AKT/MTOR shut-off 
did not significantly interfere with basal autophagy. The crucial role of AKT/MTOR pathway in FGFR2c-mediated inhibition of autophagy was also confirmed by immunofluorescence approaches, demonstrating that FGF2 stimulation dampened LC3 signal intensity, as well as reduced the LC3 positive dots per cell, only in HaCaT pBp-FGFR2c clones (Figure 4B); however, in the presence of the AKT inhibitor or rapamycin, this effect was completely reversed, resulting in a visible increase of the staining and number of LC3 dots (Figure 4B). Thus, AKT/MTOR is the FGFR2c downstream pathway responsible for autophagy inhibition; nevertheless, if this pathway is selectively switched-off, the negative effect of FGFR2c signaling cascade on autophagic process is not only recovered, but even reversed in autophagy induction. In order to further assess the interesting outcome of AKT/MTOR shut-off on FGFR2c-mediated autophagic effects, we carried out specific protein depletion by siRNA. $\mathrm{HaCaT}$ pBp and HaCaT pBp-FGFR2c clones were transfected with MTOR siRNA or with an unrelated siRNA, as negative control, and the efficiency of MTOR depletion was checked by Western blot analysis (Figure S2B). After siRNA transfection, cells were left untreated or stimulated with FGF2 as above. Western blot analysis showed that the decrease of LC3-II, evident in HaCaT pBp-FGFR2c control siRNA cells upon FGF2 stimulation (Figure 4C), turned into a clear increase upon MTOR depletion (Figure 4C).
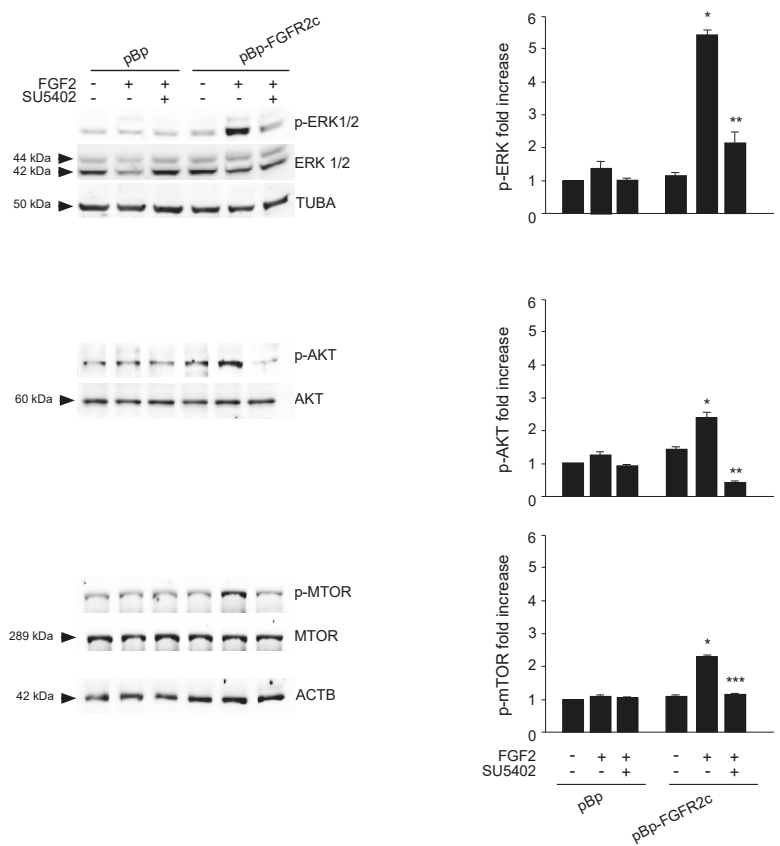

Figure 3. FGF2 stimulation activates ERK1/2 and AKT/MTOR signaling pathways in HaCaT pBp-FGFR2c. HaCaT pBp and HaCaT pBp-FGFR2c clones were left untreated or stimulated with FGF2 in the presence or absence of the FGFR2 tyrosine kinase inhibitor SU5402, as above. Western blot analysis performed using an antibody directed against the phosphorylated form of ERK1/2, AKT and MTOR demonstrates the activation of each substrates only in HaCaT pBp-FGFR2c upon FGF2 stimulation. These effects were abolished by SU5402 treatment. Equal loading was assessed with anti-ERK1/2, anti-AKT, and anti-MTOR antibodies. Densitometric analysis and Student's $t$ test were performed as reported above. ${ }^{*} p<0.05$ vs the corresponding FGF2-unstimulated cells; ${ }^{* *} p<0.05$ and **** $p<0.01$ vs the corresponding SU5402-untreated cells. 
A
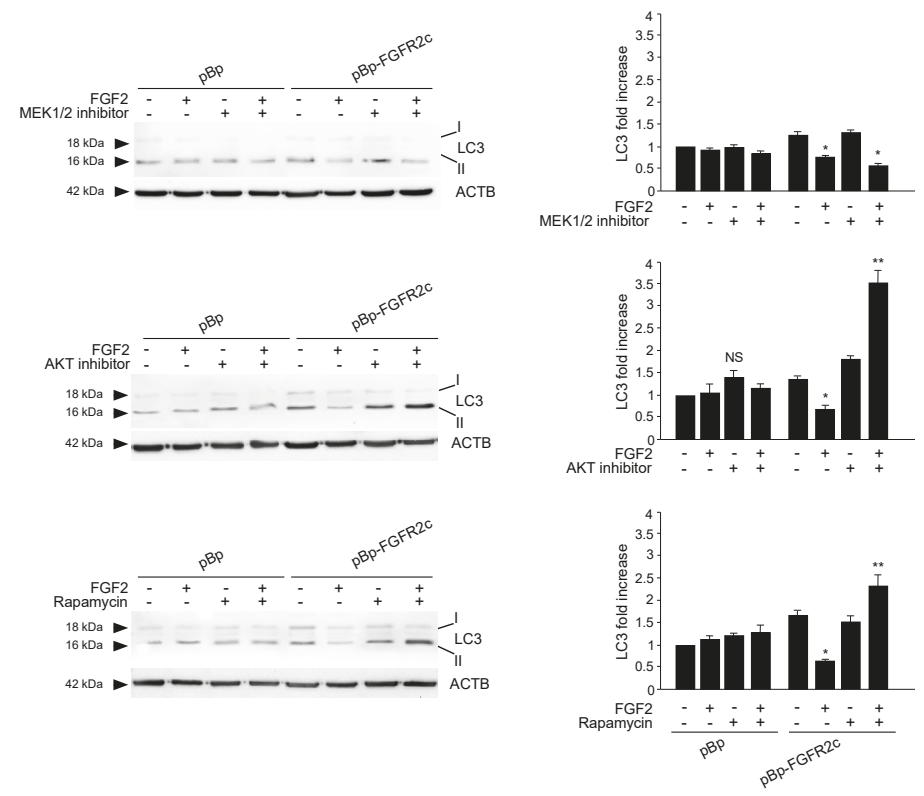

B
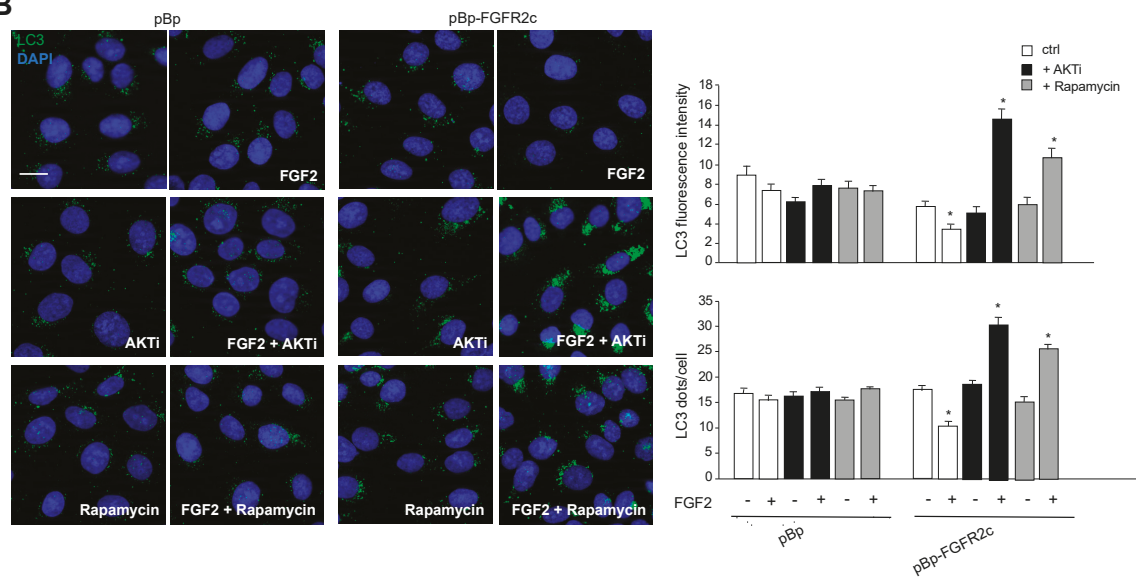

C
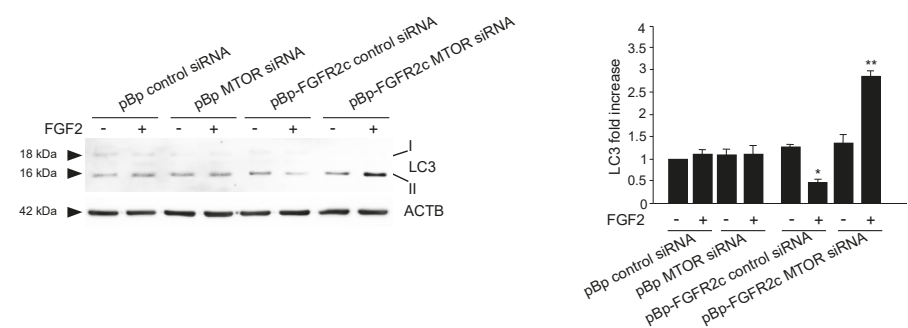

Figure 4. AKT/MTOR is the signaling pathway downstream FGFR2c responsible for FGFR2c-mediated inhibition of autophagy. (A) HaCaT pBp and HaCaT pBp-FGFR2c clones were left untreated or stimulated 
with FGF2 in the presence or absence of the indicated substrate inhibitors as reported in Materials and Methods. Western blot analysis shows that both AKT inhibitor and rapamycin increased the level of LC3-II upon FGF2 stimulation in pBp-FGFR2c cells compared to the corresponding unstimulated cells, while MEK1/2 inhibitor shows no effects. No effects of all the inhibitors are detectable in pBp-FGFR2c cells not stimulated with FGF2 or in pBp control cells. Equal loading was assessed with anti-ACTB antibody. Densitometric analysis and the student's $t$ test were performed as reported above. ${ }^{*} p<0.05$ vs the corresponding FGF2-unstimulated cells; ${ }^{* *} p<0.05$ and NS vs the corresponding substrate inhibitor-untreated cells. (B) HaCaT pBp and HaCaT pBp-FGFR2c clones were left untreated or stimulated with FGF2 in the presence or absence of AKT inhibitor or rapamycin as reported in Materials and Methods. Quantitative immunofluorescence analysis showed that both AKT inhibitor and rapamicyn reversed the inhibitory effect of FGF2 on LC3 signal intensity and on LC3 positive dots formation only in HaCaT pBp-FGFR2c clones. Quantitative analysis was performed as described in Materials and Methods, and results are expressed as mean values \pm SE. The student's $t$ test was performed as reported above. ${ }^{*} p<0.001$ vs the corresponding FGF2-unstimulated cells. (C) HaCaT pBp and $\mathrm{HaCaT}$ pBp-FGFR2c clones were transiently transfected with MTOR siRNA or an unrelated siRNA as a control. Cells were then left untreated or stimulated with FGF2 as described in Materials and Methods. Western blot analysis shows that the decrease of LC3-II, observed in HaCaT pBp-FGFR2c control siRNA cells upon FGF2 stimulation, was reversed upon MTOR depletion. Equal loading was assessed with anti-ACTB antibody. Densitometric analysis and the student's $t$ test were performed as reported in the legend to Figure 1A. ${ }^{*} p<0.05$ and ${ }^{* *} p<0.01$ vs the corresponding FGF2-unstimulated cells.

2.4. The Reversion of the Effects of FGFR2c on Autophagy Upon the Selective Block of AKT/MTOR Pathway is Accompanied by JNK1 Activation

Searching for the signaling events possibly involved in the reversion of autophagic response to FGFR2c, we focused our attention on MAPK8/JNK1 pathway. In fact, it has been recently proposed that AKT/MTOR signaling exerts an inhibitory function on JNK [33], which is the pathway involved in the induction of autophagy mediated by various FGFRs [17], including FGFR2b [21,22]. In fact, PI3K/AKT/MTOR and JNK signaling are not independent pathways, but a complex network playing important biological roles in cancer [33] and cooperating in the control of different events, including autophagy [34]. Therefore, it is reasonable to suppose that, in our cellular model of keratinocytes ectopically expressing FGFR2c, the induction of autophagy upon AKT/MTOR shut down could be the consequence of the imbalance of the interplay between AKT/MTOR and JNK pathways in favor of JNK1 activation. In order to ascertain it, we checked the phosphorylation levels of JNK1 in the presence of AKT inhibitor: Western blot analysis showed that, in cells expressing FGFR2c, the basal phosphorylation of JNK1 was significantly decreased by FGF2 stimulation (Figure 5), but this effect was reversed by the presence of the AKT inhibitor (Figure 5). Overall, these results suggested that FGFR2c-induced repression of autophagy involves the AKT/MTOR pathway, which also inhibits JNK1 signaling. In fact, upon the selective block of AKT/MTOR pathway, JNK1 phosphorylation/activation increases and contributes to autophagy stimulation. 

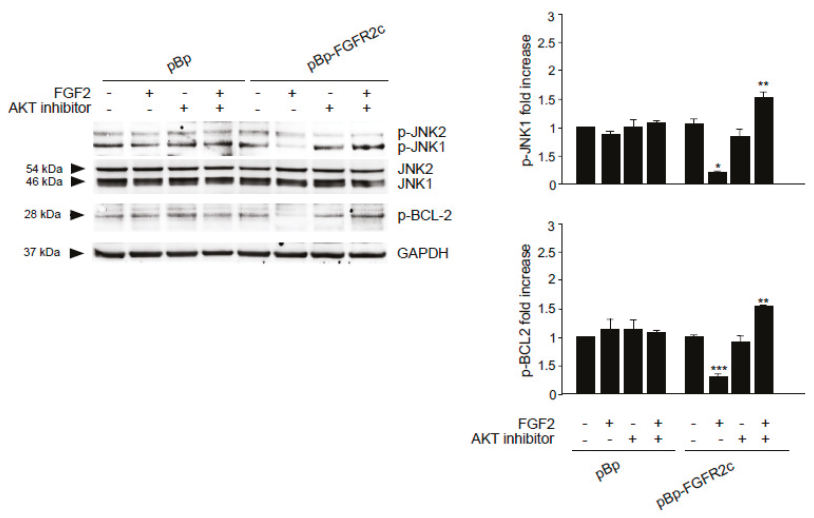

Figure 5. AKT signaling is required for FGFR2c-mediated inhibition of JNK e BCL-2 phosphorylation. $\mathrm{HaCaT}$ pBp and HaCaT pBp-FGFR2c clones were left untreated or stimulated with FGF2 in the presence or absence of AKT inhibitor as reported in Materials and Methods. Western blot analysis performed using antibody directed against the phosphorylated form of JNK and BCL-2, demonstrated that FGF2 stimulation decreases the basal phosphorylation of both substrates, while AKT inhibitor reverses this effect. Densitometric analysis and the student's $t$ test were performed as reported in the legend to Figure $1 \mathrm{~A} .{ }^{*} p<0.05$ and ${ }^{* * *} p<0.01$ vs the corresponding FGF2-unstimulated cells; ${ }^{* *} p<0.05$ vs the corresponding AKT inhibitor-untreated cells.

It is well known that JNK1 signaling triggers the autophagic step of phagophore nucleation via BCL-2 phosphorylation and consequent BECN1 release from the BCL-2/BECN1 inhibitory complex [35-37]. This mechanism has been described for the autophagy triggered by FGFR4 [17] and FGFR2b [21]. Therefore, we wondered if FGFR2c activation, which inhibits JNK1 downstream signaling, could also negatively impact on BCL-2 phosphorylation. Western blot analysis showed that the basal phosphorylation of BCL-2 in HaCaT pBp-FGFR2c clones appeared decrease by FGF2 stimulation (Figure 5), but the presence of AKT inhibitor reversed this effect (Figure 5). Thus, while FGFR2c signaling appears to repress autophagy via AKT/MTOR phosphorylation/activation, which also negatively impact on JNK1-mediated phosphorylation of BCL-2, the selective block of AKT/MTOR pathway appears to redirect it toward JNK1 activation, BCL-2 phosphorylation and possibly phagophore nucleation.

\subsection{Switching From the Epithelial FGFR2b to the Mesenchymal FGFR2c Isoform Underlies the FGF2-Mediated Inhibition of Autophagy in Epithelial Context}

The epithelial splicing regulatory proteins (ESRPs), and in particular, the ESRP1 isoform are responsible for the FGFR2 splicing and consequent expression of the epithelial FGFR2b isoform $[15,38]$. Therefore, in order to assess if the FGFR2b versus FGFR2c isoform switching could represent a key event responsible for FGF2-induced inhibition of the autophagic process in epithelial context, we forced this event in keratinocytes, performing ESRP1 depletion by siRNA approach. HaCaT cells where transfected with ESRP1 siRNA (HaCaT ESRP1 siRNA) or with an unrelated siRNA (HaCaT control siRNA), as control, and then stimulated with FGF7 or FGF2 in the presence or not of the FGFR2 kinase inhibitor SU5402, as reported above. The efficiency of ESRP1 depletion was verified through either molecular (Figure 6A, left panel) and biochemical approaches (Figure 6A, right panel), while its effect on FGFR2 isoform expression was quantitated by real-time relative RT-PCR, using human fibroblasts (HFs) as positive control for FGFR2c expression. The results showed that ESRP1 depletion led to a significant decrease of FGFR2b expression (Figure 6B, left panel) and to the appearance of FGFR2c (Figure 6B, right panel), indicating that the correct splicing of the FGFR2 gene, occurring in epithelial context, had been impaired. Then, we focused our attention on the autophagic events. Western blot 
analysis showed that, the increase of LC3-II in response to FGF7, visible in HaCaT control siRNA cells (Figure 6C) and attributable to FGFR2b activation and signaling, was abolished by ESRP1 depletion (Figure 6C). In contrast, in response to FGF2 stimulation, these HaCaT ESRP1 siRNA cells showed a rate of LC3-II decrease (Figure 6C), as well as of MTOR phosphorylation/activation (Figure 6D) comparable to that observed in HaCaT pBp-FGFR2c cells (see Figures 1A and 3). These results were also confirmed in parallel experiments, using skin-derived primary human keratinocytes (HKs) transfected with ESRP1 siRNA or control siRNA and stimulated with FGF7 or FGF2 as above. Molecular and biochemical approaches were used to verify the efficiency of ESRP1 depletion (Figure 6E), as well as its ability to lead FGFR2b down-regulation and ex-novo expression of FGFR2c (Figure 6F). Finally, Western blot analysis confirmed that ESRP1 depletion was able to abolish the autophagic response of HKs to FGF7 and to sensitize them to FGF2, inducing an LC3-II decrease (Figure 6G) and a MTOR phosphorylation/activation (Figure 6H) comparable to that observed in HaCaT ESRP1 siRNA cells. These results suggested that an altered FGFR2 splicing and the consequent switch from FGFR2b to FGFR2c in epithelial context might drive to autophagy inhibition.

A
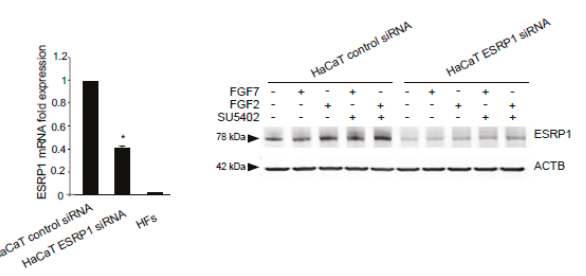

B

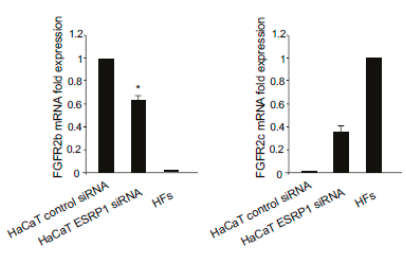

C
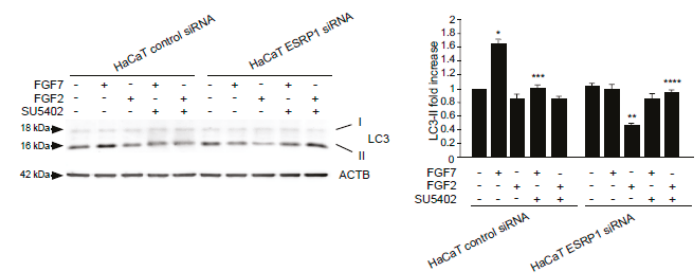

D
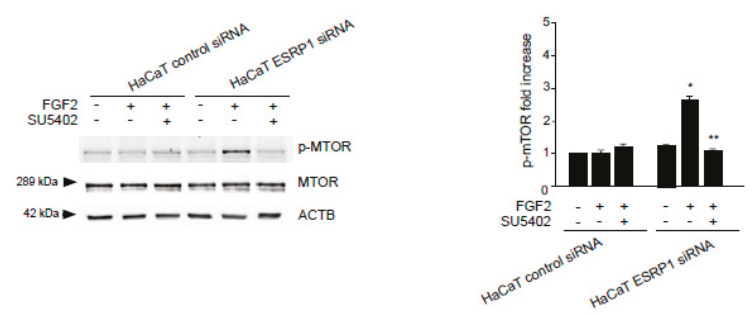

Figure 6. Cont. 
E

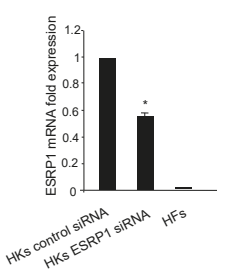

$\mathbf{F}$

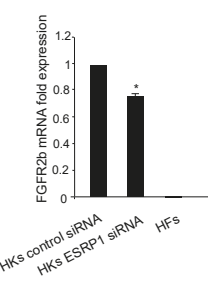

G
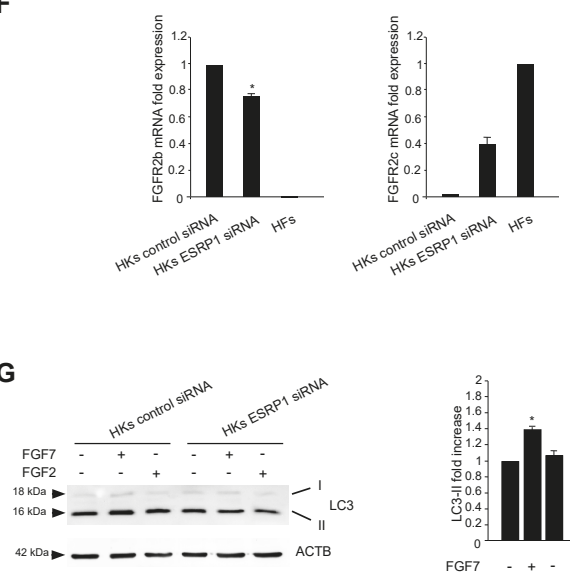

H

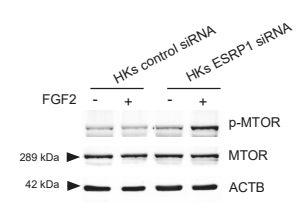

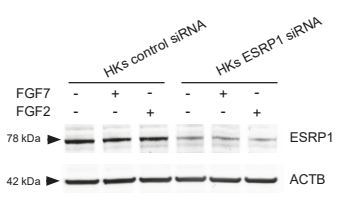
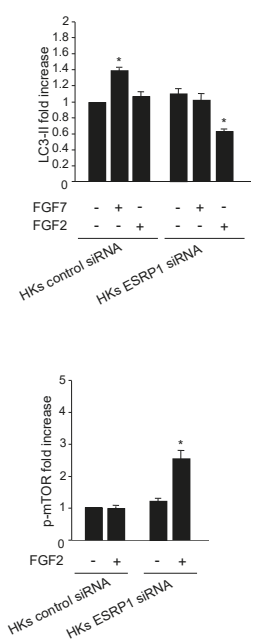

Figure 6. ESRP1 depletion by siRNA in haCaT cells results in aberrant FGFR2 splicing and FGF2-mediated inhibition of autophagy. (A) HaCaT cells were transiently transfected with ESRP1 siRNA or an unrelated siRNA as a control. Cells were then left in complete medium (left panel). Alternatively, cells were left untreated or stimulated with FGF7 or FGF2 in the presence or absence of the FGFR2 tyrosine kinase inhibitor SU5402 as above (right panel). Real-time RT-PCR analysis (left panel) and Western blot analysis (right panel) show the efficiency of ESRP1 depletion. Results are expressed as mean values \pm SE. The student's $t$ test was performed, and significance levels are defined as $P$ values of $\pm 0.05 .{ }^{*} p<0.01$ vs the corresponding control siRNA cells. For the Western blot analysis, equal loading was assessed with anti-ACTB antibody. (B) HaCaT cells were transiently transfected with ESRP1 siRNA or an unrelated siRNA as a control and then left in complete medium. Real-time RT-PCR analysis shows that ESRP1 depletion leads to a significant decrease of FGFR2b expression (left panel) and to the appearance of FGFR2c (right panel). Results are expressed as mean values \pm SE. The student's $t$ test was performed, and significance levels are defined as $P$ values of $0.05 .{ }^{*} p<0.05$ vs 
the corresponding control siRNA cells. (C) HaCaT cells were transiently transfected with ESRP1 siRNA or an unrelated siRNA as a control. Cells were left untreated or stimulated with FGF7 or FGF2 in the presence or absence of the FGFR2 tyrosine kinase inhibitor SU5402 as above. Western blot analysis shows that ESRP1 depletion abolishes the increase of LC3 induced by FGF7 while it induces a decrease of LC3 upon FGF2 stimulation. Equal loading was assessed with anti-ACTB antibody. Densitometric analysis and the student's $t$ test were performed as reported in the legend to Figure $1 \mathrm{~A} .{ }^{*} p<0.05$ and ${ }^{* *} p<0.01$ vs the corresponding FGF-unstimulated cells; ${ }^{* * *} p<0.01$ and ${ }^{* * *} p<0.05$ vs the corresponding SU5402-untreated cells. (D) HaCaT cells were transiently transfected with ESRP1 siRNA or an unrelated siRNA as a control and then left untreated or stimulated with FGF2 in presence or absence of SU5402 as above. Western blot analysis performed using antibody directed against the phosphorylated form of MTOR shows the phosphorylation of this substrates upon FGF2 stimulation in HaCaT ESRP1 siRNA cells. Equal loading was assessed with anti-ACTB antibody. Densitometric analysis and the student's $t$ test were performed as reported in the legend to Figure $1 \mathrm{~A} .{ }^{*} p<0.05$ vs the corresponding FGF-unstimulated cells; ${ }^{* *} p<0$. 001 vs the corresponding SU5402-untreated cells. (E) HKs cells were transiently transfected with ESRP1 siRNA or an unrelated siRNA as a control and left in complete medium (left panel) or stimulated with FGF7 or FGF2 as above (right panel). Real-time RT-PCR analysis (left panel) and Western blot analysis (right panel) showed the efficiency of ESRP1 depletion. Results are expressed as mean values \pm SE. The student's $t$ test was performed, and significance levels are defined as P values of \pm 0.05 . ${ }^{*} p<0.05$ vs the corresponding control siRNA cells. For the Western blot analysis, equal loading was assessed with anti-ACTB antibody. (F) HKs cells were transiently transfected with ESRP1 siRNA or an unrelated siRNA as a control and then left in complete medium. Real-time RT-PCR analysis showed that ESRP1 depletion lead to a significant decrease of FGFR2b expression (left panel) and to the appearance of FGFR2c (right panel). Results are expressed as mean values \pm SE. The student's $t$ test was performed, and significance levels are defined as $P$ values of $\pm 0.05 .{ }^{*} p<0.01$ vs the corresponding control siRNA cells. (G) HKs cells were transiently transfected with ESRP1 siRNA or an unrelated siRNA as a control. Cells were left untreated or stimulated with FGF7 or FGF2 as above. Western blot analysis shows that ESRP1 depletion abolished the increase of LC3 induced by FGF7 while it induced a decrease of LC3 upon FGF2 stimulation. Equal loading was assessed with anti-ACTB antibody. Densitometric analysis and the student's $t$ test were performed as reported in the legend to Figure $1 \mathrm{~A} .{ }^{*} p<0.05$ vs the corresponding FGF-unstimulated cells. (H) HKs cells were transiently transfected with ESRP1 siRNA or an unrelated siRNA as a control and then left untreated or stimulated with FGF2. Western blot analysis performed using antibody directed against the phosphorylated form of MTOR shows that ESRP1 depletion induces the phosphorylation of this substrates upon FGF2 stimulation. Equal loading was assessed with anti-ACTB antibody. Densitometric analysis and the student's $t$ test were performed as reported in the legend to Figure $1 \mathrm{~A} .{ }^{*} p<0.05$ vs the corresponding control siRNA cells.

\section{Discussion}

The human genome consists of 20,000-25,000 genes, 95\% of which undergo alternative splicing, ensuring the higher proteome diversity [39]. Isoforms arising from the splicing events show distinct and often opposing functions and several studies have suggested that the aberrant splicing represents a crucial event in cancer [39]. The high frequency of splicing aberrations in neoplastic diseases has made necessary new strategies for therapeutic approaches pointed on targeting the aberrant variants and their signaling.

In agreement with the proposed central role of the aberrant splicing in tumorigenesis, the altered splicing of FGFR2 and the consequent appearance of the mesenchymal FGFR2c isoform was observed in several carcinomas [40-44]. Consistent with this clinical evidence, recent studies from our group have demonstrated that the FGFR2 isoform switching and aberrant expression of the mesenchymal FGFR2c isoform in human keratinocytes induces impaired differentiation [13] and EMT [14,15]. The initiation of a pathological type III EMT is also accompanied by the appearance of tumorigenic features [14] indicating that FGFR2 aberrant splicing might represent the precocious event driving the early step of carcinogenesis. 
Very recently it is emerging that EMT and autophagy are tumor cell responses to microenvironment stresses occurring in different stages of cancer progression [24,25]. These responses appear to be mutually exclusive and they regulate each other in a complex negative loop [24,25]. However, still many open questions remain to be answered to clarify how tumor cells decide whether to enter one or the other cell stress response. To address tumor cell heterogeneity, the identification of hub molecules able to regulate this intricate interplay appears a promising goal for cancer therapy.Starting from our recent results dealing with the ability of the epithelial FGFR2b isoform in promoting autophagy [19-22], we speculated that the ectopic FGFR2c might play a central role in the regulation of the negative crosstalk between EMT and the autophagic process in epithelial context. Consistent with this hypothesis, using biochemical, molecular and immunofluorescence approaches, we demonstrated here that the ectopic expression of FGFR2c in normal human keratinocytes efficiently counteracts autophagy. Moreover, forcing the aberrant splicing of FGFR2 in keratinocytes via ESRP1 depletion by siRNA approach, we found that the switching from the epithelial FGFR2b to the mesenchymal FGFR2c isoforms could be the specific event underlying the negative impact on autophagy in epithelial context. This is consistent with the fact that dysregulations in RNA alternative splicing is linked to EMT induction and tumor development [39]. In this regard, RNA splicing regulators, such as ESRPs, are emerging as key proteins playing both oncogenic and tumor suppressive roles via the modulation of RNA isoforms involved in oncogenic signaling pathways [45]. Therefore, we might speculate that, at least in the epidermal context, the down-regulation of ESPRs proteins, responsible for FGFR2 isoform switching, would be the molecular event crucial not only for EMT induction [15] but also for autophagy repression.

Since our results also provide elements to assume that FGFR2c-induced inhibition of the autophagic process is not transcriptionally regulated, we further progressed on the identification of the molecular mechanisms underlying FGFR2c-mediated inhibition of autophagy, identifying AKT/MTOR as the crucial pathway involved (Figure 7). We also observed that the selective inhibition of AKT or MTOR, but not that of MEK1/2, was able not just to dampen, but even to reverse the effects of FGFR2c signaling on autophagy (Figure 7). Interestingly, this unexpected event was accompanied by JNK1 activation. JNK1 signaling is known to be involved in the induction of autophagy mediated by various FGFRs [17], including FGFR2b $[21,22]$ and proceeds via BCL-2 phosphorylation, which in turn allows phagophore nucleation [35-37]. Indee, our results showed that both JNK1 and BCL-2 phosphorylation appeared repressed by FGFR2c activation, but strongly activated upon AKT/MTOR shut-off. These findings are in agreement with previous observations of a cooperative crosstalk between PI3K/AKT/MTOR and JNK pathways in the regulation of autophagy also in different cellular contexts, such as PC12 cells and various cancer cell lines [34,46]. In addition, increasing evidences revealed that AKT and JNK pathways interact with each other and that AKT signaling inhibits JNK and different mechanisms have been proposed [33]: In fact, AKT would counteract JNK activation antagonizing the formation of the MAPK8IP1/JIP1-JNK complex [47,48], as well as interfering with the activation of JNK upstream kinases, such as MAP3K5/ASK1, MAP2K4/7/MKK4/7 and MLK [33]. However, since the PI3K/AKT pathway plays essential roles in cancer [33], understanding the molecular mechanisms regulating its crosstalk with JNK may essentially contribute to clarify the specific involvement of each of these pathways in tumor development. Moreover, since autophagy and EMT negatively regulate each other in tumor cells, it is reasonable to suppose that AKT/MTOR inhibition would be able not only to restore autophagy, but also to reverse FGFR2c-induced EMT and tumorigenic features: Further future investigations will be performed in order to address this topic.

Overall, since FGFR2c is simultaneously responsible for the unbalance of the negative crosstalk between autophagy and EMT in favor of this latter, as well as for impairment of differentiation and induction of tumorigenic features, we can conclude that this receptor, expressed as a consequence of an altered splicing event, could be one of the crucial molecular drivers of epithelial deregulation during tumorigenesis. On the other hand, the selective inhibition of specific pathways downstream FGFR2c, 
such as AKT/MTOR, could represent an effective tool to interfere with the oncogenic autophagy/EMT crosstalk and consequently to counteract the early steps of carcinogenesis.

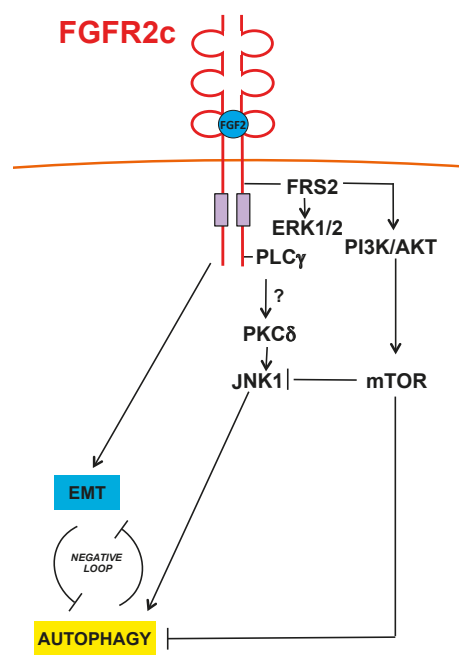

Figure 7. Schematic drawing of the proposed role of FGFR2c and its signaling in the regulation of autophagy and EMT interplay. FGFR2c inhibits autophagy via AKT/MTOR pathway, which also negatively interferes with JNK1 signaling.

\section{Materials and Methods}

\subsection{Cells and Treatments}

The human keratinocyte cell line HaCaT, stably expressing FGFR2c (pBp-FGFR2c), overexpressing FGFR2b (pBp-FGFR2b) or the empty vector ( $\mathrm{pBp}$ ) were cultured in Dulbecco's modified eagle's medium (DMEM), supplemented with $10 \%$ fetal bovine serum (FBS) plus antibiotics. Primary cultures of human keratinocytes and human fibroblasts derived from healthy skin (HKs and HFs, respectively) were obtained from patients attending the Dermatology Unit of the Sant'Andrea Hospital of Rome; all patients were extensively informed and their consent for the investigation was given and collected in written form in accordance with guidelines approved by the management of the Sant'Andrea Hospital. The research was done in agreement with the guidelines of the Helsinki declaration, according to a protocol study approved by the Ethical Committee of Sant'Andrea University Hospital (Prot. CE n. 1591/2013). Primary cells were isolated and cultured as previously described $[49,50]$.

$\mathrm{HaCaT}$ clones were transiently transfected with the pDest-mCherry-EGFP tandem expression vector containing LC3 (HaCaT mCherry-EGFP-LC3) [27].

For RNA interference and MTOR or ESRP1 silencing, cells were transfected with MTOR small interfering RNA (MTOR siRNA) (Santa Cruz Biotechnology, Inc., Santa Cruz, CA, USA; SC35409), ESRP1 siRNA (Santa Cruz Biotechnology, SC77526), or an unrelated siRNA as a control, using Lipofectamine 2000 transfection reagent (Life Technologies, Carlsbad, CA, USA; 11668-019) or Fugene HD (Promega, Madison, WI, E2311) according to the manufacturer's protocol.

For growth factors stimulation, cells were left untreated or incubated with FGF7 (Upstate Biotechnology, Lake Placid, NY, 01-118) or with FGF2 (PeproTech, London, BFGF 100-188) 100 ng/mL for $24 \mathrm{~h}$ at $37^{\circ} \mathrm{C}$. To induce activation and signaling of FGFR2 isoforms, cells were serum starved and incubated with FGF7 or FGF2 $100 \mathrm{ng} / \mathrm{mL}$ for $10 \mathrm{~min}$ at $37^{\circ} \mathrm{C}$. For inhibition of FGFR2b and FGFR2c tyrosine kinase activity, cells were pre-incubated with a specific FGFR2 tyrosine kinase inhibitor, SU5402 $25 \mu \mathrm{M}$ (Calbiochem, Nottingham, UK; 572630) for $1 \mathrm{~h}$ before treatments with growth factors (GFs). 
To inhibit ERK, AKT, or MTOR cells were incubated with the MEK1/2-specific inhibitor PD0325901

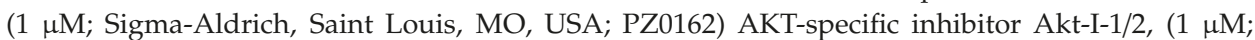
Calbiochem, 124005), or with the specific MTOR inhibitor rapamycin (100 nM; Cell Signaling Technology, Beverly, MA, USA; 9904) respectively, for $1 \mathrm{~h}$ at $37^{\circ} \mathrm{C}$ before being treated with FGF2 in the presence of each inhibitor.

To irreversibly block the fusion between autophagosomes and lysosomes, cells were incubated with bafilomycin A1 (20 nM; Sigma-Aldrich, B1793) for $3 \mathrm{~h}$ at $37^{\circ} \mathrm{C}$ after treatment with GFs in the presence of the inhibitor.

\subsection{Immunoflurescence}

$\mathrm{HaCaT}$ clones, grown on coverslips, were fixed with $4 \%$ paraformaldehyde in phosphate-buffered saline (PBS) for $30 \mathrm{~min}$ at $25^{\circ} \mathrm{C}$, followed by treatment with $0.1 \mathrm{M}$ glycine for $20 \mathrm{~min}$ at $25^{\circ} \mathrm{C}$ and with $0.1 \%$ Triton $\mathrm{X}-100$ for an additional $5 \mathrm{~min}$ at $25^{\circ} \mathrm{C}$ to allow permeabilization. Cells were then incubated for $1 \mathrm{~h}$ at $25^{\circ} \mathrm{C}$ with the following primary antibodies: Mouse monoclonal anti-LC3 (1:100 in PBS, 5F10 Nanotools, Teningen, Germany, 0231). The primary antibodies were visualized using goat anti-mouse IgG-Alexa Fluor 488 (1:200 in PBS, Life Technologies, Carlsbad, CA, A11001) for $30 \mathrm{~min}$ at $25^{\circ} \mathrm{C}$. Nuclei were stained with DAPI (1:1000 in PBS; Sigma-Aldrich, D9542). Coverslips were finally mounted with Mowiol (Sigma) for observation. Fluorescence signals were analyzed by scanning cells in a series of sequential sections with an ApoTome System (Zeiss) connected with an Axiovert 200 inverted microscope (Zeiss); image analysis was performed by the Axiovision software (Zeiss) and images were obtained by 3D reconstruction of the total number of the serial optical sections. Quantitative analysis of the fluorescence intensity was performed by the Axiovision software (Zeiss), analyzing 10 different fields randomly taken from 3 independent experiments. Quantitative analysis of LC3-positive dots per cell was performed analyzing 100 cells for each sample in 5 different microscopy fields from 3 different experiments. Results are shown as means \pm standard error (SE). The student's $t$ test was performed and significance levels have been defined as $p<0.05$.

\subsection{Western Blot Analysis}

Cells were lysed in a buffer containing $50 \mathrm{mM}$ HEPES, pH 7.5, $150 \mathrm{mM} \mathrm{NaCl}, 1 \%$ glycerol, 1\% Triton X-100, $1.5 \mathrm{mM} \mathrm{MgCl}$, $5 \mathrm{mM}$ EGTA, supplemented with protease inhibitors ( $10 \mathrm{~g} / \mathrm{mL}$ aprotinin, $1 \mathrm{mM}$ phenylmethylsulfonyl fluoride [PMSF], $10 \mu \mathrm{g} / \mathrm{mL}$ leupeptin) and phosphatase inhibitors (1 $\mathrm{mM}$ sodium orthovanadate, $20 \mathrm{mM}$ sodium pyrophosphate, $0.5 \mathrm{M} \mathrm{NaF}$ ). A range of 20 to $50 \mu \mathrm{g}$ of total protein was resolved under reducing conditions by 8 or $12 \%$ SDS-PAGE and transferred to reinforced nitrocellulose (BA-S 83; Schleicher \& Schuell, Keene, NH, USA; BA-S83). The membranes were blocked with 5\% nonfat dry milk (Bio-Rad Laboratories, Hercules, CA, USA, 170-6404) in PBS 0.1\% Tween 20 (Bio-Rad, 170-6531) and incubated with anti-SQSTM1 (BD Bioscience, San Josè, CA, USA, 610833), anti-phospho-JNK (anti-p-JNK) (Thr183/Tyr185, Cell Signaling, 9255S), anti-p-MTOR (Ser 2448, Cell Signaling, 5536S), monoclonal antibodies or with anti-LC3 (MBL, Woburn, MA, PD014), anti ESRP1 (Sigma-Aldrich, HPA023719), anti-p-p44/42 mitogen-activated protein kinase (MAPK) (p-ERK1/2) (Thr202/Tyr204; Cell Signaling, 9101S), anti-p-AKT (Ser 473; Cell Signaling, 9271), anti-p-BCL2 (Ser 70; Cell Signaling, 2827) polyclonal antibodies, followed by enhanced chemiluminescence (ECL) detection (Thermo Scientific, Rockford, IL, USA; 34580).

The membranes were rehydrated by washing in PBS/Tween-20, stripped with $100 \mathrm{mM}$ mercaptoethanol and $2 \%$ SDS for $30 \mathrm{~min}$ at $55^{\circ} \mathrm{C}$ and probed again with, anti-AKT (H-136; Santa Cruz Biotechnology, sc-8312), anti-p44/42 MAPK (ERK1/2) (137F5, Cell Signaling, 4695S), anti-JNK (Cell Signaling, 9252S), anti- $\alpha$-TUBA (Cell Signaling, 2148S) polyclonal antibodies or with anti-MTOR (7C10, Cell Signaling, 2983S), anti-ACTB (Sigma-Aldrich, A5441) monoclonal antibody to estimate the protein equal loading.

Densitometric analysis was performed using Quantity One Program version 4.6.8 (Bio-Rad). The resulting values from three different experiments were normalized, expressed as fold increase 
respect to the control value and reported in graph as mean values \pm standard deviation (SD). The student's $t$ test was performed and significance levels have been defined as $p<0.05$.

\subsection{Primers}

Oligonucleotide primers necessary for target genes and the housekeeping gene were chosen by using the online tool Primer-BLAST [51] and purchased from Invitrogen (Invitrogen, Carlsbad, CA, USA). The following primers were used: For the MAP1LC3B target gene: $5^{\prime}$-CGCACCTTCGAACA AAGAG-3' (sense) and 5'-CTCACCCTTGTATCGTTCTATTATCA-3' (antisense); for the BECN1 target gene: 5'-GGATGGTGTCTCTCGCAGAT-3' (sense) and 5'-TTGGCACTTTCTGTGGACAT-3' (antisense); for the ATG5 target gene: $5^{\prime}$-CAACTTGTTTCACGCTATATCAGG-3' (sense) and 5'-CAC TTTGTCAGTTACCAACGTCA-3' (antisense); for ESRP1 target gene: $5^{\prime}$-GGCTCGGATGAGAAGGA GTT-3' (sense), 5'-GCACTTCGTGCAACTGTCC-3' (antisense), for FGFR2b target gene: 5'-CGTGGA AAAGAACGGCAGTAAATA-3' (sense), 5'-GAACTATTTATCCCCGAGTGCTTG-3' (antisense); for FGFR2c target gene: 5' - TGAGGACGCTGGGGAATATACG-3 (sense), 5'-TAGTCTGGGGAAGCTGT AATCTCCT 3' (antisense); for the SQSTM1 target gene: 5'-AGCTGCCTTGTACCCACATC-3 (sense), 5'- CAGAGAAGCCCATGGACAG-3' (antisense); for the 18S rRNA housekeeping gene: $5^{\prime}$-CGAGCCGCCTGGATACC-3' (sense) and 5'-CATGGCCTCAGTTCCGAAAA-3' (antisense). For each primer pair, we performed no-template control and no-reverse-transcriptase control (reverse transcription [RT]-negative) assays, which produced negligible signals

\subsection{RNA Extraction and cDNA Synthesis}

RNA was extracted using the TRIzol method (Invitrogen, Carlsbad, CA, USA; 15596018) according to the manufacturer's instructions and eluted with $0.1 \%$ diethylpyrocarbonate (DEPC)-treated water. Each sample was treated with DNase I (Invitrogen, 18068-015). The total RNA concentration was quantitated by spectrophotometry; $1 \mu \mathrm{g}$ of total RNA was used for reverse transcription using the iScriptTM cDNA synthesis kit (Bio-Rad, 170-8891) according to the manufacturer's instructions.

\subsection{PCR Amplification and Real-Time Quantitation}

Real-time RT-PCR was performed using the iCycler real-time detection system (iQ5 Bio-Rad) with optimized PCR conditions. The reactions were carried out in a 96-well plate using $\mathrm{QQ}$ SYBR green supermix (Bio-Rad, 1708882), adding forward and reverse primers for each gene and $1 \mu l$ of diluted template cDNA to a final reaction mixture volume of $15 \mu$ l. All assays included a negative control and were replicated three times. The thermal cycling program was performed as described previously [52]. Real-time quantitation was performed with the help of the iCycler IQ optical system software, version 3.0a (Bio-Rad), according to the manufacturer's manual. Results are reported as mean values $\pm \mathrm{SE}$ from three different experiments in triplicate. The student's $t$ test was performed, with significance levels defined as $P$ values $<0.05$.

Supplementary Materials: The following are available online at http://www.mdpi.com/2073-4409/8/7/653/s1, Figure S1: FGFR2c-induced inhibition of autophagy is not transcriptionally regulated, Figure S2: Biochemical evaluation of the efficiency of specific signaling pathway substrate inhibitors and siRNAs.

Author Contributions: M.R.T and F.B. have made substantial contribution to conception and design of the project, M.N., D.R., M.R.T and F.B. have been involved in drafting the manuscript and has given final approval of the version to be published. M.N., D.R. and F.P. have made substantial contributions to acquisition and analysis of the data and have given final approval of the version to be published.

Funding: This work was partially supported by grants from MIUR- Ministero dell'Istruzione dell'Università e della Ricerca and from AIRC-Associazione Italiana per la Ricerca sul Cancro, Italy (Grant number: IG 15858).

Conflicts of Interest: The authors declare that they have no competing interests. 


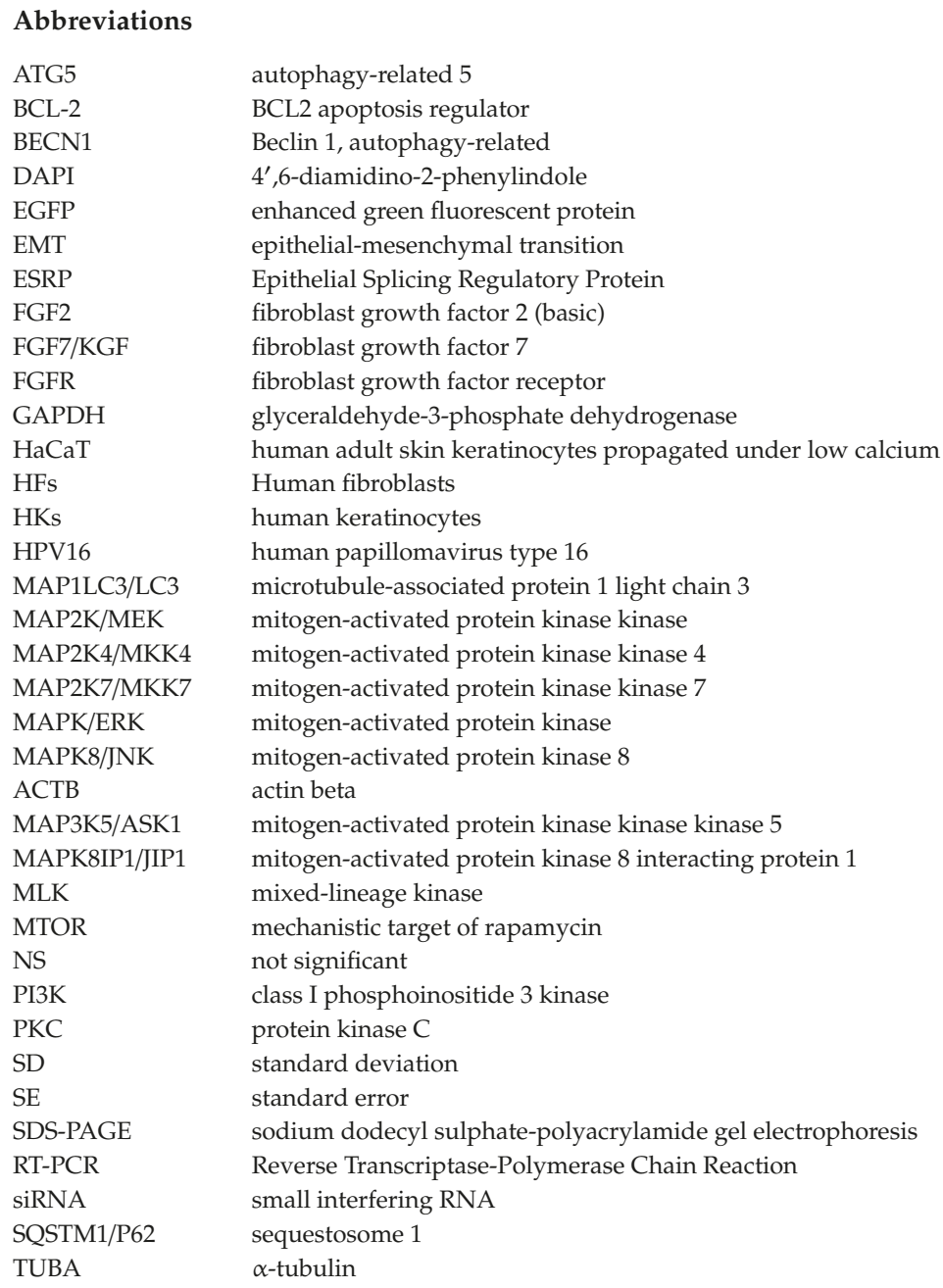

\section{References}

1. Brewer, J.R.; Mazot, P.; Soriano, P. Genetic insights into the mechanisms of FGF signaling. Genes Dev. 2016, 30, 751-771. [CrossRef] [PubMed]

2. Tanner, Y.; Grose, R.P. Dysregulated FGF signalling in neoplastic disorders. Semin. Cell Dev. Biol. 2016, 53, 126-135. [CrossRef] [PubMed]

3. Turner, N.; Grose, R. Fibroblast growth factor signalling: From development to cancer. Nat. Rev. Cancer 2010, 10, 116-129. [CrossRef]

4. Brooks, A.N.; Kilgour, E.; Smith, P.D. Molecular pathways: Fibroblast growth factor signaling: A new therapeutic opportunity in cancer. Clin. Cancer Res. 2012, 18, 1855-1862. [CrossRef] [PubMed]

5. Petiot, A.; Conti, F.J.; Grose, R.; Revest, J.M.; Hodivala-Dilke, K.M.; Dickson, C. A crucial role for FGFR-IIIb signalling in epidermal development and hair follicle patterning. Development 2003, 130, 5493-5501. [CrossRef] [PubMed]

6. Grose, R.; Fantl, V.; Werner, S.; Chioni, A.M.; Jarosz, M.; Rudling, R.; Cross, B.; Hart, I.R.; Dickson, C. The role of fibroblast growth factor receptor $2 \mathrm{~b}$ in skin homeostasis and cancer development. EMBO J. 2007, 26, 1268-1278. [CrossRef] 
7. Yang, J.; Meyer, M.; Müller, A.K.; Böhm, F.; Grose, R.; Dauwalder, T.; Verrey, F.; Kopf, M.; Partanen, J.; Bloch, W. Fibroblast growth factor receptors 1 and 2 in keratinocytes control the epidermal barrier and cutaneous homeostasis. J. Cell Biol. 2010, 188, 935-952. [CrossRef]

8. Feng, S.; Wang, F.; Matsubara, A.; Kan, M.; McKeehan, W.L. Fibroblast growth factor receptor 2 limits and receptor 1 accelerates tumorigenicity of prostate epithelial cells. Cancer Res. 1997, 58, 1509-1514.

9. Zhang, Y.; Wang, H.; Toratani, S.; Sato, J.D.; Kan, M.; McKeehan, W.L.; Okamoto, T. Growth inhibition by keratinocyte growth factor receptor of human salivary adenocarcinoma cells through induction of differentiation and apoptosis. Proc. Natl. Acad. Sci. USA. 2001, 98, 11336-11340. [CrossRef]

10. Belleudi, F.; Purpura, V.; Torrisi, M.R. The receptor tyrosine kinase FGFR2b/KGFR controls early differentiation of human keratinocytes. PLoS ONE 2011, 6, e24194. [CrossRef]

11. Purpura, V.; Belleudi, F.; Caputo, S.; Torrisi, M.R. HPV16 E5 and KGFR/ FGFR2b interplay in differentiating epithelial cells. Oncotarget 2013, 4, 192-205. [CrossRef] [PubMed]

12. Rosato, B.; Ranieri, D.; Nanni, M.; Torrisi, M.R.; Belleudi, F. Role of FGFR2b expression and signaling in keratinocyte differentiation: Sequential involvement of PKC $\delta$ and PKC $\alpha$. Cell Death Dis. 2018, 9, 565. [CrossRef] [PubMed]

13. Ranieri, D.; Rosato, B.; Nanni, M.; Belleudi, F.; Torrisi, M.R. Expression of the FGFR2c mesenchymal splicing variant in human keratinocytes inhibits differentiation and promotes invasion. Mol. Carcinog. 2018, 57, 272-283. [CrossRef] [PubMed]

14. Ranieri, D.; Rosato, B.; Nanni, M.; Magenta, A.; Belleudi, F.; Torrisi, M.R. Expression of the FGFR2 mesenchymal splicing variant in epithelial cells drives epithelial-mesenchymal transition. Oncotarget 2016, 7, 5440-5460. [CrossRef] [PubMed]

15. Ranieri, D.; Belleudi, F.; Magenta, A.; Torrisi, M.R. HPV16 E5 expression induces switching from FGFR2b to FGFR2c and epithelial-mesenchymal transition. Int. J. Cancer 2015, 137, 61-72. [CrossRef] [PubMed]

16. Zhang, J.; Liu, J.; Huang, Y.; Chang, J.Y.F.; Liu, L.; McKeehan, W.L.; Martin, J.F.; Wang, F. FRS2 $\alpha$-mediated FGF signals suppress premature differentiation of cardiac stem cells through regulating autophagy activity. Circ. Res. 2012, 110, e29-e39. [CrossRef] [PubMed]

17. Cinque, L.; Forrester, A.; Bartolomeo, R.; Svelto, M.; Venditti, R.; Montefusco, S.; Polishchuk, E.; Nusco, E.; Rossi, A.; Medina, D. FGF signalling regulates bone growth through autophagy. Nature 2015, 528, 272-275. [CrossRef]

18. Wang, X.; Qi, H.; Wang, Q.; Zhu, Y.; Wang, X.; Jin, M.; Tan, Q.; Huang, Q.; Xu, W.; Li, X. FGFR3/fibroblast growth factor receptor 3 inhibits autophagy through decreasing the ATG12-ATG5 conjugate, leading to the delay of cartilage development in achondroplasia. Autophagy 2015, 11, 1998-2013. [CrossRef]

19. Belleudi, F.; Purpura, V.; Caputo, S.; Torrisi, M.R. FGF7/KGF regulates autophagy in keratinocytes: A novel dual role in the induction of both assembly and turnover of autophagosomes. Autophagy 2014, 10, 803-821. [CrossRef]

20. Belleudi, F.; Nanni, M.; Raffa, S.; Torrisi, M.R. HPV16 E5 deregulates the autophagic process in human keratinocytes. Oncotarget 2015, 6, 9370-9386. [CrossRef]

21. Nanni, M.; Ranieri, D.; Rosato, B.; Torrisi, M.R.; Belleudi, F. Role of fibroblast growth factor receptor $2 b$ in the cross talk between autophagy and differentiation: Involvement of Jun $\mathrm{N}$-terminal protein kinase signaling. Mol. Cell. Biol. 2018, 38, e00119-18.

22. Nanni, M.; Ranieri, D.; Raffa, S.; Torrisi, M.R.; Belleudi, F. Interplay between FGFR2b-induced autophagy and phagocytosis: Role of PLC $\gamma$-mediated signaling. J. Cell Mol. Med. 2018, 22, 668-683. [CrossRef] [PubMed]

23. Mowers, E.E.; Sharifi, M.N.; Macleod, K.F. Autophagy in cancer metastasis. Oncogene 2017, 36, 1619-1630. [CrossRef] [PubMed]

24. Marcucci, F.; Ghezzi, P.; Rumio, C. The role of autophagy in the cross-talk between epithelial-mesenchymal transitioned tumor cells and cancer stem-like cells. Mol. Cancer 2017, 16, 3. [CrossRef] [PubMed]

25. Marcucci, F.; Rumio, C. How tumor cells choose between epithelial-mesenchymal transition and autophagy to resist stress-therapeutic implications. Front. Pharmacol. 2018, 9, 714. [CrossRef] [PubMed]

26. Füllgrabe, J.; Ghislat, G.; Cho, D.H.; Rubinszteinet, D.C. Transcriptional regulation of mammalian autophagy at a glance. J. Cell Sci. 2016, 129, 3059-3066. [CrossRef] [PubMed]

27. Pankiv, S.; Clausen, T.H.; Lamark, T.; Brech, A.; Bruun, J.A.; Outzen, H.; Øvervatn, A.; Bjørkøy, G.; Johansen, T. p62/ SQSTM1 binds directly to Atg8/LC3 to facilitate degradation of ubiquitinated protein aggregates by autophagy. J. Biol. Chem. 2007, 282, 24131-24145. [CrossRef] [PubMed] 
28. Lin, X.; Zhang, Y.; Liu, L.; McKeehan, W.L.; Shen, Y.; Song, S.; Wang, F. FRS2 $\alpha$ is essential for the fibroblast growth factor to regulate the MTOR pathway and autophagy in mouse embryonic fibroblasts. Int. J. Biol. Sci. 2011, 7, 1114-1121. [CrossRef]

29. Chen, Y.; Xie, X.; Li, X.; Wang, P.; Jing, Q.; Yue, J.; Liu, Y.; Cheng, Z.; Li, J.; Song, H. FGFR antagonist induces protective autophagy in FGFR1-amplified breast cancer cell. Biochem. Biophys. Res. Commun. 2016, 474, 1-7. [CrossRef]

30. Yuan, H.; Li, Z.M.; Shao, J.; Ji, W.X.; Xia, W.; Lu, S. FGF2/FGFR1 regulates autophagy in FGFR1-amplified non-small cell lung cancer cells. J. Exp. Clin. Cancer Res. 2017, 36, 72. [CrossRef]

31. Nakanishi, Y.; Mizuno, H.; Sase, H.; Fujii, T.; Sakata, K.; Akiyama, N.; Aoki, Y.; Aoki, M.; Ishii, N. ERK signal suppression and sensitivity to CH5183284/Debio 1347, a selective FGFR inhibitor. Mol. Cancer Ther. 2015, 14, 2831-2839. [CrossRef] [PubMed]

32. Bain, J.; Plater, L.; Elliott, M.; Shpiro, N.; Hastie, C.J.; McLauchlan, H.; Klevernic, I.; Arthur, J.S.; Alessi, D.R.; Cohen, P. The selectivity of protein kinase inhibitors: A further update. Biochem. J. 2007, 408, 297-315. [CrossRef] [PubMed]

33. Zhao, H.F.; Wang, J.; Tony To, S.S. The phosphatidylinositol 3-kinase/Akt and c-Jun N-terminal kinase signaling in cancer: Alliance or contradiction? Int. J. Oncol. 2015, 47, 429-436. [CrossRef] [PubMed]

34. Rodríguez-Blanco, J.; Martín, V.; García-Santos, G.; Herrera, F.; Casado-Zapico, S.; Antolín, I.; Rodriguez, C. Cooperative action of JNK and AKT/MTOR in 1-methyl-4-phenylpyridinium-induced autophagy of neuronal PC12 cells. J. Neurosci. Res. 2012, 90, 1850-1860. [CrossRef] [PubMed]

35. Zhou, Y.Y.; Li, Y.; Jiang, W.Q.; Zhou, L.F. MAPK/JNK signalling: A potential autophagy regulation pathway. Biosci. Rep. 2015, 35, e00199.

36. Russell, R.C.; Yuan, H.X.; Guan, K.L. Autophagy regulation by nutrient signaling. Cell Res. 2014, $24,42-57$. [CrossRef]

37. Wei, Y.; Pattingre, S.; Sinha, S.; Bassik, M.; Levine, B. JNK1-mediated phosphorylation of Bcl-2 regulates starvation-induced autophagy. Mol. Cell. 2008, 30, 678-688. [CrossRef]

38. Warzecha, C.C.; Sato, T.K.; Nabet, B.; Hogenesch, J.B.; Carstens, R.P. ESRP1 and ESRP2 are epithelial cell-type-specific regulators of FGFR2 splicing. Mol. Cell. 2009, 33, 591-601. [CrossRef]

39. Wang, B.D.; Lee, N.H. Aberrant RNA Splicing in Cancer and Drug Resistance. Cancers 2018, $10,458$. [CrossRef]

40. Kawase, R.; Ishiwata, T.; Matsuda, Y.; Onda, M.; Kudo, M.; Takeshita, T.; Naito, Z. Expression of fibroblast growth factor receptor 2 IIIc in human uterine cervical intraepithelial neoplasia and cervical cancer. Int. J. Oncol. 2010, 36, 331-340.

41. Matsuda, Y.; Hagio, M.; Seya, T.; Ishiwata, T. Fibroblast growth factor receptor 2 IIIc as a therapeutic target for colorectal cancer cells. Mol. Cancer Ther. 2012, 11, 2010-2020. [CrossRef] [PubMed]

42. Ishiwata, T.; Matsuda, Y.; Yamamoto, T.; Uchida, E.; Korc, M.; Naito, Z. Enhanced expression of fibroblast growth factor receptor 2 IIIc promotes human pancreatic cancer cell proliferation. Am. J. Pathol. 2012, 180, 1928-1941. [CrossRef] [PubMed]

43. Zhao, Q.; Caballero, O.L.; Davis, I.D.; Jonasch, E.; Tamboli, P.; Yung, W.K.; Weinstein, J.N.; Kenna Shaw for TCGA research network; Strausberg, R.L.; Yao, J. Tumor-specific isoform switch of the fibroblast growth factor receptor 2 underlies the mesenchymal and malignant phenotypes of clear cell renal cell carcinomas. Clin. Cancer Res. 2013, 19, 2460-2472. [CrossRef] [PubMed]

44. Peng, W.X.; Kudo, M.; Fujii, T.; Teduka, K.; Naito, Z. Altered expression of fibroblast growth factor receptor 2 isoform IIIc: Relevance to endometrioid adenocarcinoma carcinogenesis and histological differentiation. Int. J. Clin. Exp. Pathol. 2014, 7, 1069-1076. [PubMed]

45. Urbanski, L.M.; Leclair, N.; Anczuków, O. Alternative-splicing defects in cancer: Splicing regulators and their downstream targets, guiding the way to novel cancer therapeutics. Wiley Interdiscip. Rev. RNA 2018, 9, e1476. [CrossRef]

46. Liu, J.; Zheng, L.; Zhong, J.; Wu, N.; Liu, G.; Lin, X. Oleanolic acid induces protective autophagy in cancer cells through the JNK and MTOR pathways. Oncol. Rep. 2014, 32, 567-572. [CrossRef] [PubMed]

47. Kim, A.H.; Yano, H.; Cho, H.; Meyer, D.; Monks, B.; Margolis, B.; Birnbaum, M.J.; Chao, M.V. Akt1 regulates a JNK scaffold during excitotoxic apoptosis. Neuron 2002, 35, 697-709. [CrossRef]

48. Pan, J.; Pei, D.S.; Yin, X.H.; Hui, L.; Zhang, G.Y. Involvement of oxidative stress in the rapid Akt1 regulating a JNK scaffold during ischemia in rat hippocampus. Neurosci. Lett. 2006, 392, 47-51. [CrossRef] 
49. Cardinali, G.; Ceccarelli, S.; Kovacs, D.; Aspite, N.; Lotti, L.V.; Torrisi, M.R.; Picardo, M. Keratinocyte growth factor promotes melanosome transfer to keratinocytes. J. Investig. Dermatol. 2005, 125, 1190-1199. [CrossRef]

50. Raffa, S.; Leone, L.; Scrofani, C.; Monini, S.; Torrisi, M.R.; Barbara, M. Cholesteatoma-associated fibroblasts modulate epithelial growth and differentiation through KGF/FGF7 secretion. Histochem. Cell Biol. 2012, 138, 251-269. [CrossRef]

51. Ye, J.; Coulouris, G.; Zaretskaya, I.; Cutcutache, I.; Rozen, S.; Madden, T.L. Primer-BLAST: A tool to design target-specific primers for polymerase chain reaction. BMC Bioinform. 2012, 13, 134. [CrossRef] [PubMed]

52. Avitabile, D.; Genovese, L.; Ponti, D.; Ranieri, D.; Raffa, S.; Calogero, A.; Torrisi, M.R. Nucleolar localization and circadian regulation of Per2S, a novel splicing variant of the Period 2gene. Cell Mol. Life Sci. 2014, 71, 2547-2559. [CrossRef] [PubMed]

(C) 2019 by the authors. Licensee MDPI, Basel, Switzerland. This article is an open access article distributed under the terms and conditions of the Creative Commons Attribution (CC BY) license (http://creativecommons.org/licenses/by/4.0/). 

Article

\title{
Cancer Mutations in FGFR2 Prevent a Negative Feedback Loop Mediated by the ERK1/2 Pathway
}

\author{
Patrycja Szybowska ${ }^{1,2}$, Michal Kostas ${ }^{2,3}$, Jørgen Wesche ${ }^{2,3}$, Antoni Wiedlocha ${ }^{1,2,4}$ \\ and Ellen Margrethe Haugsten $2,3, *$ \\ 1 Department of Molecular Cell Biology, Institute for Cancer Research, The Norwegian Radium Hospital, Oslo \\ University Hospital, Montebello, 0379 Oslo, Norway; Patrycja.Szybowska@rr-research.no (P.S.); \\ Antoni.Wiedlocha@rr-research.no (A.W.) \\ 2 Centre for Cancer Cell Reprogramming, Institute of Clinical Medicine, Faculty of Medicine, University of \\ Oslo, Montebello, 0379 Oslo, Norway; Michal.Janusz.Kostas@rr-research.no (M.K.); \\ Jorgen.Wesche@rr-research.no (J.W.) \\ 3 Department of Tumor Biology, Institute for Cancer Research, The Norwegian Radium Hospital, \\ Oslo University Hospital, Montebello, 0379 Oslo, Norway \\ 4 Military Institute of Hygiene and Epidemiology, 01-163 Warsaw, Poland \\ * Correspondence: Ellen.M.Haugsten@rr-research.no; Tel.: +47-2278-1785
}

Received: 17 April 2019; Accepted: 28 May 2019; Published: 29 May 2019

\begin{abstract}
Tight regulation of signaling from receptor tyrosine kinases is required for normal cellular functions and uncontrolled signaling can lead to cancer. Fibroblast growth factor receptor 2 (FGFR2) is a receptor tyrosine kinase that induces proliferation and migration. Deregulation of FGFR2 contributes to tumor progression and activating mutations in FGFR2 are found in several types of cancer. Here, we identified a negative feedback loop regulating FGFR2 signaling. FGFR2 stimulates the Ras/MAPK signaling pathway consisting of Ras-Raf-MEK1/2-ERK1/2. Inhibition of this pathway using a MEK1/2 inhibitor increased FGFR2 signaling. The putative ERK1/2 phosphorylation site at serine 780 (S780) in FGFR2 corresponds to serine 777 in FGFR1 which is directly phosphorylated by ERK1/2. Substitution of S780 in FGFR2 to an alanine also increased signaling. Truncated forms of FGFR2 lacking the C-terminal tail, including S780, have been identified in cancer and S780 has been found mutated to leucine in bladder cancer. Substituting S780 in FGFR2 with leucine increased FGFR2 signaling. Importantly, cells expressing these mutated versions of S780 migrated faster than cells expressing wild-type FGFR2. Thus, ERK1/2-mediated phosphorylation of S780 in FGFR2 constitutes a negative feedback loop and inactivation of this feedback loop in cancer cells causes hyperactivation of FGFR2 signaling, which may result in increased invasive properties.
\end{abstract}

Keywords: FGFR2; ERK1/2; phosphorylation; serine; negative feedback loop; cancer

\section{Introduction}

Tight regulation of receptor tyrosine kinase signaling is required for specific cellular responses, such as cell growth, differentiation, migration, and apoptosis. Inadequate regulation of signaling is a common event in cancer development and enhanced receptor signaling promotes tumor growth [1]. The receptor tyrosine kinase, FGFR2 (fibroblast growth factor receptor 2) is a transmembrane, cell-surface localized receptor that belongs to a family of four related receptors [2]. FGFR2 is activated by FGF ligands and induces various downstream signaling molecules. Deregulation of FGFR2 contributes to tumor progression and activating mutations in FGFR2 have been found in different types of cancer, like gastric cancer, breast cancer, and endometrial carcinoma [3,4]. In addition, activating mutations have been found in skeletal disorders, like Apert syndrome and Crouzon syndrome [5]. Clearly, precise regulation of FGFR2 signaling is important to prevent diseases. 
Upon ligand binding, FGFRs dimerize. This, in turn, activates the tyrosine kinase domain of the receptor by trans-autophosphorylation [2]. FGFRs mediate signaling by recruiting specific molecules that bind to phosphorylated tyrosines, triggering a number of signaling pathways. The docking protein FRS2 (FGFR substrate 2) is phosphorylated by the activated receptor, creating phosphotyrosine docking sites for proteins containing SH2-domains. By binding to FRS2, the adaptor protein Grb2 (growth factor receptor-bound protein 2) activates the Ras/ mitogen-activated protein kinase (MAPK) pathway and the phosphoinositide 3-kinase (PI3K)/Akt pathway [2]. Ras activates the kinase activity of Raf, which phosphorylates MEK1/2. MEK then phosphorylates ERK1/2 (extracellular signal-regulated kinase) which activates 90 kDa Ribosomal S6 Kinase 2 (RSK2), among other downstream targets. Activated FGFRs also recruit and phosphorylate phospholipase $\mathrm{C} \gamma(\mathrm{PLC} \gamma)$, which culminates in the activation of protein kinase C (PKC) [2].

In comparison to the well-studied activation of FGFRs, the mechanisms leading to deactivation of the receptor are not fully understood. It is known that the signal from the activated receptor can be attenuated by internalization and degradation in lysosomes [6,7]. After internalization, FGFR ubiquitination marks the receptor for degradation [6,8]. Depending on the receptor type, the bound ligand, and possibly also the cell type and the context, FGFRs might also be recycled back to the cell surface instead of being transported to lysosomes, which may result in prolonged signaling [7].

FGFR signaling is also regulated by phosphatases. Recently, we have shown that a phosphatase, PTPRG, directly dephosphorylates activated FGFRs [9]. Proteins that regulate FGFR signaling, such as MAPK phosphatase 3 (MKP3) and Sprouty 1/2, are negative regulators that are induced or activated by FGF signaling and act on downstream signaling molecules [10]. In addition, FGFR signaling can be regulated by inhibitory phosphorylation, forming negative feedback loops that attenuate the signals. It has been shown that active ERK1/2 can phosphorylate FRS2 on threonine residues. This leads to reduced tyrosine phosphorylation of FRS2 and therefore reduced downstream signaling [11]. On the receptor level, two such negative feedback loops have been identified for FGFR1 [12,13]. It has been shown that upon FGFR1 activation/tyrosine phosphorylation, the receptor is also phosphorylated at serine 777 (S777) directly by activated ERK1/2. S777 phosphorylation reduces the tyrosine phosphorylation in the kinase domain of the receptor and thus also reduces signaling [12]. In addition, the serine/threonine kinase RSK2, which is activated through the Ras-MAPK pathway, can also bind to FGFR1 and phosphorylate FGFR1 at serine 789 [13]. This phosphorylation seems to be required for proper endocytosis and ubiquitination of FGFR1. Preventing RSK2 activation or mutation of S789 leads to increased signaling [13]. It is not clear if the other FGFRs are also regulated by such negative feedback loops.

Here, we have investigated whether a similar negative feedback loop mediated by ERK1/2 also exists for FGFR2. Inhibition of the ERK1/2 signaling pathway, using a MEK1/2 inhibitor (U0126), led to sustained FGFR2 phosphorylation. Moreover, substitution of serine 780 (S780) in FGFR2 for alanine also resulted in sustained FGFR2 activation. S780 in FGFR2 is equivalent to the ERK1/2 substrate S777 in FGFR1. Several truncated forms of FGFR2 lacking the C-terminal tail, including S780, have been identified in cancer. In addition, $\mathrm{S} 780$ has been found mutated to leucine in a patient with bladder cancer. Substituting S780 in FGFR2 with leucine also increased FGFR2 signaling. More importantly, cells expressing the mutated versions of S780 were migrating faster than cells expressing wild-type FGFR2. Possibly, the lack of MAPK-dependent negative feedback gives FGFR2-expressing cancer cells an advantage. These results also indicate that care should be taken when the MAPK-pathway is inhibited in cancer.

\section{Materials and Methods}

\subsection{Materials, Antibodies, and Compounds}

The following antibodies were used: Mouse anti-phospho-ERK1/2 (Thr202/Tyr204) (\#9106), rabbit anti-ERK1/2 (\#9102), mouse anti-phospho-FGFR (Tyr653/654) (\#3476), rabbit anti-FGFR2 (\#11835), rabbit anti-FGFR2 (N-terminal) (\#23328), rabbit anti-FGFR1 (\#9749), rabbit anti-FGFR3 (\#4574), rabbit anti-FGFR4 (\#8562), rabbit anti-phospho-PLC $\gamma$ (Tyr783) (\#14008), and rabbit anti-phospho-RSK2 
(Ser 227) (\#3556) from Cell Signaling Technology (Leiden, The Netherlands) and mouse anti- $\gamma$-tubulin (T6557) from Sigma-Aldrich (St. Louis, MO, USA). Fluorescently labelled secondary antibodies were from Jackson ImmunoResearch Laboratories (Cambridgeshire, UK). HRP-conjugated secondary antibodies were from Jackson ImmunoResearch Laboratories and Agilent (Santa Clara, CA, USA).

U0126 (1144) was from Tocris Bioscience (Bristol, UK). PD173074 was from Calbiochem (San Diego, CA, USA). Cycloheximide, recombinant EGF, mowiol, heparin, and protein-G-sepharose were from Sigma Aldrich. Restriction enzymes were from New England Biolabs (Ipswich, MA, USA). Adenosine triphosphate $\left[\gamma^{-}{ }^{32} \mathrm{P}\right] 3000 \mathrm{Ci} / \mathrm{mmol}$ EasyTides was purchased from PerkinElmer (Norwalk, CT, USA). PhosSTOP phosphatase inhibitor cocktail and cOmplete EDTA-free protease inhibitor cocktail were from Roche (Basel, Switzerland). Hoechst 33342, DyLight 550 NHS Ester, and recombinant active ERK1 with glutathione S-transferase (GST) tag (\#PV3311) were from Thermo Fisher Scientific (Waltham, MA, USA). Recombinant FGF1 was prepared as previously described [14]. FGF1 was labelled with DyLight 550 (DL550-FGF1) following the manufacturer's procedures.

\subsection{Plamids and siRNAs}

cDNA encoding full-length human FGFR2 (IIIc) (NCBI: NM_000141) was cut out from the pCMV6-XL4 cDNA clone (Origene Technologies, Rockville, MD, USA) as an EcoRI-XbaI fragment and ligated into pcDNA3 (Thermo Fisher Scientific, Waltham, MA, USA). The resulting plasmid was further cut with KpnI to remove the upstream untranslated region. To remove the untranslated region downstream of the gene, the plasmid was partially cut with Tth111I, followed by cutting with XbaI. The plasmid was furthermore treated with T4 DNA polymerase (New England Biolabs, Ipswich, MA, USA) to make blunt ends and then ligated. Note that the XbaI and the Tth111I sites were destroyed. After sequencing, a point mutation in the N-terminal region was discovered (G183V). This point mutation was mutated back (generating a glycine at the 138 position) using site-directed mutagenesis with the following primer: 5-CGCTGCCCAGCCGGGGGGAACCCAATGCCAACC-3. pcDNA3 hFGFR2 was used as a template to generate pcDNA3 hFGFR2 S780A, S780D, and S780L. The following primers were used: S780A; 5-CCTCTCGAACAGTATGCACCTAGTTACCCTGAC-3, S780D; 5-CCTCTCGAACAGTATGACCCTAGTTACCCTGAC-3, S780L; and 5-CCTCTCGAACAGTATCTACC TAGTTACCCTGAC-3. All constructs were verified by sequencing (Eurofins Genomics, Ebersberg, Germany). pcDNA3 hFGFR1 and pcDNA3 hFGFR4 have been described previously $[7,15]$ and pcDNA3 hFGFR3 was a generous gift from Dr. A. Yayon (ProChon Biotech, Ness Ziona, Israel).

\subsection{Cell Lines and Transfection}

To generate U2OS cells stably expressing FGFR2, FGFR2 S780A, FGFR2 S780D, and FGFR2 S780L, Fugene 6 transfection reagent (Promega, Madison, WI, USA) was used according to the manufacturer's protocol. Clones were selected with $1 \mathrm{mg} / \mathrm{mL}$ geneticin and then the clones were chosen based on their receptor expression levels analyzed by immunofluorescence and Western blotting. Throughout the paper, clone \#1 of the particular stable cell line is used if nothing else is stated.

The cells were propagated in Dulbecco's Modified Eagle Medium (DMEM) supplemented with 10\% fetal bovine serum, $100 \mathrm{U} / \mathrm{mL}$ penicillin, and $100 \mu \mathrm{g} / \mathrm{mL}$ streptomycin in a $5 \% \mathrm{CO}_{2}$ atmosphere at $37^{\circ} \mathrm{C}$.

Transient transfection was performed using Fugene 6 transfection reagent according to the manufacturer's protocol. Cells were analyzed 16-24 h after transfection.

\subsection{Western Blotting}

Cells were treated as indicated and then lysed in Laemmli sample buffer (Bio-Rad, Oxford, UK). Proteins in the cell lysates were separated on a gradient (4-20\%) sodium dodecyl sulfate-polyacrylamide gel electrophoresis (SDS-PAGE) and then blotted onto a membrane using the TransBlot ${ }^{\circledR}$ Turbo Transfer system (Bio-Rad). Membranes were then incubated with indicated primary antibodies followed by corresponding secondary antibody coupled to HRP. Bands were visualized by chemiluminscence using SuperSignal ${ }^{\mathrm{TM}}$ West Dura Extended Duration Substrate (Thermo Fisher Scientific, Waltham, 
MA, USA) or SuperSignal ${ }^{\mathrm{TM}}$ West Femto Maximum Sensitivity Substrate (Thermo Fisher Scientific). In some cases, antibodies were stripped from the membranes using Pierce Stripping buffer and the membranes were reprobed. The images were prepared using ImageLab Software (Bio-Rad) and Adobe Illustrator CS4 14.0.0 (San Jose, CA, USA). Quantification of bands of interest was performed in Fiji ImageJ software [16]. Lane normalization factor (LNF) was determined by dividing the intensity of the $\gamma$-tubulin bands on its highest signal in each blot.

\subsection{Microscopy}

Cells, seeded onto coverslips, were treated as indicated and fixed in $4 \%$ formaldehyde. The cells were then permeabilized with $0.1 \%$ triton X-100, stained with indicated antibodies and Hoechst 33342 and mounted in mowiol. Confocal images were acquired with a $63 \mathrm{X}$ objective on a Zeiss confocal Laser Scanning Miscroscope (LSM) 780 (Jena, Germany). Images were prepared in Fiji Image J software and Adobe Illustrator CS4 14.0.0. Images for quantification of p-FGFR and DL550-FGF1 signal intensities were taken with identical settings and the quantification was performed with Fiji Image J software. The same threshold was used for all images in the same experiment. Due to background staining in the nuclei, p-FGFR intensities in the nuclei were subtracted from the total intensities in the corresponding cell.

\subsection{In Vitro Phosphorylation Assay}

The cells were starved for $2 \mathrm{~h}$ in serum-free media and lysed in lysis buffer $(20 \mathrm{mM}$ phosphate-Na pH 7.4, $150 \mathrm{mM} \mathrm{NaCl}, 1 \mathrm{mM}$ Ethylenediaminetetraacetic acid (EDTA), 1\% Triton X-100, protease inhibitors). The receptors were immunoprecipitated for $1 \mathrm{~h}$ using anti-N-terminal-FGFR2 antibodies pre-bound to protein-G-sepharose, washed 3 times with $1 \mathrm{M} \mathrm{NaCl}$ and treated with $1 \mu \mathrm{M}$ PD173074 for $30 \mathrm{~min}$. The kinase reaction was performed on beads using $50 \mathrm{ng}$ recombinant active ERK1 and $50 \mu \mathrm{Ci}$ ATP- $\gamma_{-}{ }^{32} \mathrm{P}$ (per $100 \mu \mathrm{L}$ reaction) in $50 \mathrm{mM}$ HEPES-Na pH 7.5, $20 \mathrm{mM} \mathrm{MgCl} 2,5 \mathrm{mM}$ Ethylene Glycol Tetraacetic Acid (EGTA), and phosphatase inhibitors for $30 \mathrm{~min}$ at $30^{\circ} \mathrm{C}$. The reaction was quenched with $20 \mathrm{mM}$ EDTA. Then, the immunoprecipitated receptors were washed 3 times $(25 \mathrm{mM}$ HEPES-Na pH 7.5, $1 \mathrm{mM}$ EDTA) and released from the beads in SDS-loading buffer by $15 \mathrm{~min}$ at $95^{\circ} \mathrm{C}$ and subjected to SDS-PAGE before analysis with autoradiography and immunoblotting.

\subsection{Cell Migration}

Cells sparsely seeded in IncuCyte Image Lock 96-well plates (Essen BioSciences, Hertfordshire, UK) were imaged every $10 \mathrm{~min}$ for $21 \mathrm{~h}$ by IncuCyte ${ }^{\circledR}$ S3 Live Cell Analysis System with IncuCyte ${ }^{\circledR}$ S3 Software (V2018B) (Essen BioSciences). In all experiments, cells were either left untreated or treated with FGF1 $(100 \mathrm{ng} / \mathrm{mL})$ and heparin $(20 \mathrm{U} / \mathrm{mL})$. Images were analyzed with IncuCyte ${ }^{\circledR}$ S3 Software (V2018B) and Fiji ImageJ software with Manual Tracking and Chemotaxis and Migration Tool (ibidi GmbH, Planegg, Germany).

\section{Results}

\subsection{Inhibition of MEK1/2 Increases FGFR2 Signaling}

Signaling from FGFRs is regulated by mechanisms such as endocytic trafficking $[6,7]$ and dephosphorylation by phosphatases (PTPRG) [9]. Recently, we identified a negative feedback loop that involves direct phosphorylation of serine 777 (S777) in the C-terminal tail of FGFR1 by active ERK1/2 [12]. Phosphorylation of S777 in FGFR1 is necessary for proper attenuation of FGFR1 signaling and treatment of cells with U0126, a MEK1/2 inhibitor, leads to increased activation of FGFR1. To investigate if a similar ERK1/2-mediated negative feedback loop also exists for FGFR2, we treated cells with U0126 MEK1/2 inhibitor and investigated tyrosine phosphorylation status of FGFR2 at different time-points after addition of FGF1. Since FGFR levels are low in many cells and endogenous FGFRs can be difficult to detect, we generated U2OS cells stably expressing FGFR2 IIIc (U2OS-R2). In contrast to parental U2OS cells, our U2OS-R2 cells endocytose detectable amounts of DL550-FGF1 (FGF1 labelled with 
DyLight550) and are strongly stained with anti-FGFR2 antibodies (Figure 1a). U2OS cells do not express detectable levels of any of the four FGFRs (Figure S1a and [17]) and, although the antibody that we use against phosphorylated FGFR (p-FGFR antibody) recognizes all four receptors, only the ectopic FGFR in the stably transfected U2OS cells is detected.

First, we investigated which doses of the MEK1/2 inhibitor (U0126) efficiently inhibit ERK1/2 activation upon FGF1 stimulation in U2OS-R2 cells (Figure 1b). MEK1/2 is upstream of ERK1/2 in the Ras/MAPK signaling pathway. Incubating the cells with increasing concentrations of U0126 demonstrated that $20 \mu \mathrm{M}$ U0126 efficiently blocked ERK1/2 activation. Next, we treated the cells with $20 \mu \mathrm{M}$ U0126 and compared the receptor activation in treated cells versus untreated cells. The levels of tyrosine-phosphorylated FGFR2 were increased in U0126 treated cells compared to untreated cells (Figure 1c). Similar effects were observed in two additional clones of U2OS-R2 (Figure S1b). To investigate this effect further, we also stained cells with antibodies against tyrosine phosphorylated FGFR (p-FGFR) and compared the intensity of p-FGFR staining between indicated treatments (Figure 1d). When resting cells or cells treated with FGF1 together with FGFR inhibitor (PD173074) were stained with p-FGFR antibodies, we could detect a bright signal in the nucleus. We considered this as unspecific staining by the antibody. Thus, upon quantification, the intensity of the nuclear p-FGFR antibody staining was subtracted from that of the total cell. Interestingly, in cells treated with FGF1, we observed a clear increase in p-FGFR antibody intensity in the cytosol compared to resting cells or PD173074 treated cells. As expected, we could also observe a high degree of co-localization between DL550-FGF1 and p-FGFR antibody staining (Figure 1d, second panel). When cells were treated with FGF1 and U0126 (to prevent ERK1/2 signaling), we detected an increase in p-FGFR antibody staining compared to FGF1 treatment alone. Taken together, our data indicates that a similar feedback mechanism as to that found for FGFR1 might also exist in the case of FGFR2. We conclude that ERK1/2 signaling is required for attenuation of FGFR2 signaling.

Since ERK1/2 signaling can be activated by other receptor tyrosine kinases as well, we investigated if activation of ERK1/2 prior to FGFR2 activation would influence the response to FGF1. To test this, we treated cells with EGF 30 min prior to stimulation with FGF1 and compared the levels of FGFR2 tyrosine phosphorylation to that in cells not pretreated with EGF. First, we investigated whether EGF activates ERK1/2 signaling in U2OS-R2 cells. We observed a peak of ERK1/2 phosphorylation 10-20 min after addition of EGF. Indeed, ERK1/2 is active in U2OS-R2 cells during this 30 min period of stimulation with EGF (Figure 2a).

Next, we stimulated cells for 30 min with EGF before activation of FGFR by addition of FGF1. Interestingly, reduced levels of tyrosine-phosphorylated FGFR2 was observed in cells pretreated with EGF (Figure 2b). These data indicate a dual role for the ERK1/2 signaling-mediated feedback loop in FGFR2 signaling. Not only does it function to ensure proper attenuation of FGFR2 signaling, it also ensures accurate responses to FGF1 stimulation. In an environment where the ERK1/2 pathway is activated by other receptor tyrosine kinases, the response to FGF1 is less pronounced than in resting cells. In this way, different receptors may cross-talk to prevent excess signaling. 


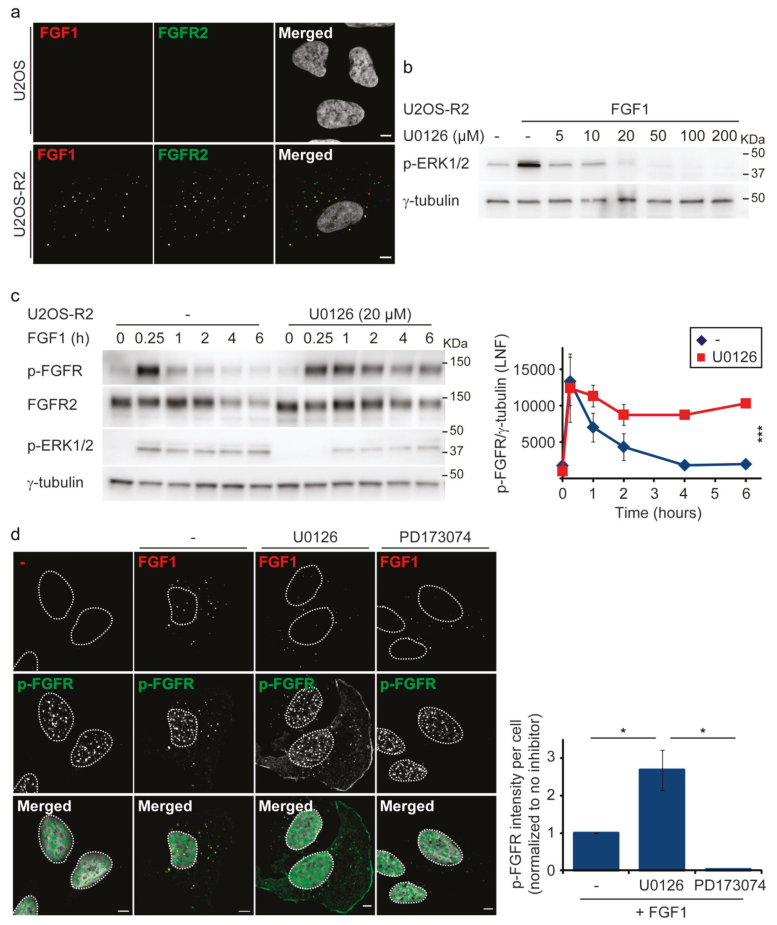

Figure 1. Inhibition of the ERK1/2 pathway prolongs FGFR2 signaling. (a) U2OS cells or U2OS cells stably transfected with FGFR2IIIc (U2OS-R2) were treated with $200 \mathrm{ng} / \mathrm{mL}$ DL550-FGF1 in the presence of heparin (50 U/mL) for $30 \mathrm{~min}$. The cells were then fixed, stained with anti-FGFR2 antibodies and Hoechst, and analyzed by confocal microscopy. The images were taken at fixed intensity settings, and brightness/contrast was adjusted in the same way for all images. Representative images are shown. Scale bar: $5 \mu \mathrm{M}$. (b) U2OS-R2 cells were kept in serum-free media for $2 \mathrm{~h}$ prior to stimulation for $30 \mathrm{~min}$ with $200 \mathrm{ng} / \mathrm{mL}$ FGF1 in the presence of heparin $(20 \mathrm{U} / \mathrm{mL})$ and increasing concentrations of U0126. Cells were then lysed and the lysates were analyzed by immunoblotting using the indicated antibodies. A p in front of the name of the antibody indicates that it recognizes the phosphorylated form of the protein. One representative experiment is shown. (c) U2OS-R2 cells were kept in serum-free media for $2 \mathrm{~h}$ before addition of $100 \mathrm{ng} / \mathrm{mL}$ FGF1 and heparin $(20 \mathrm{U} / \mathrm{mL})$ in the presence or absence of U0126 $(20 \mu \mathrm{M})$ for indicated periods of time. Cycloheximide $(10 \mu \mathrm{g} / \mathrm{mL})$ was added at the beginning of the starvation period and kept throughout the experiment. After lysis, the cellular material was analyzed with immunoblotting using the indicated antibodies. A p in front of the name of the antibody indicates that it recognizes the phosphorylated form of the protein. Quantifications of three independent experiments are presented in the graph. The bands corresponding to phosphorylated receptor were normalized to Lane normalization factor (LNF) ( $\gamma$-tubulin). Error bars denote the standard deviation. The difference between U0126 treated cells versus untreated cells was significant $(p \leq 0.001,3$-way ANOVA, Holm-Sidak test, $\mathrm{n}=3$ ). (d) U2OS-R2 cells were kept in serum-free media for $2 \mathrm{~h}$ before addition of $200 \mathrm{ng} / \mathrm{mL}$ DL550-FGF1 and heparin $(50 \mathrm{U} / \mathrm{mL})$ for $30 \mathrm{~min}$. The cells were pretreated with U0126 $(20 \mu \mathrm{M})$ or PD173074 (50 nM) 30 min before addition of FGF1 as indicated. The cells were then fixed, stained with anti-p-FGFR antibodies and Hoechst, and analyzed by confocal microscopy. Scale bar: $5 \mu$ M. Quantifications of two independent experiments were performed as described in materials and methods and are presented in the graph. In total, 60 cells treated with FGF1 alone, 60 cells treated with FGF1 and U0126, and 38 cells treated with FGF1 and PD173074 were quantified. Outliers were removed according to the $1.5^{*} \mathrm{IQR}$ outlier rule. Error bars denote the standard error of the mean $(\mathrm{SEM}), \mathrm{n}=2$. Due to a general variation in the intensity between the two experiments, the means in each experiment were normalized to the mean of cells treated with FGF1 alone (no inhibitor) in the corresponding experiment. ( $p \leq 0.05$, two-sided $\mathrm{t}$ test on normalized data, $\mathrm{n}=2$ ). 


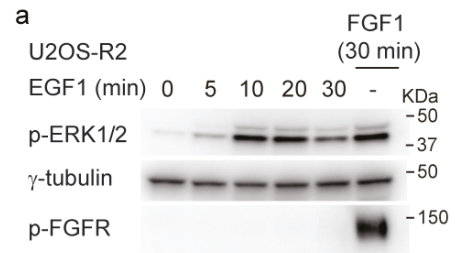

b
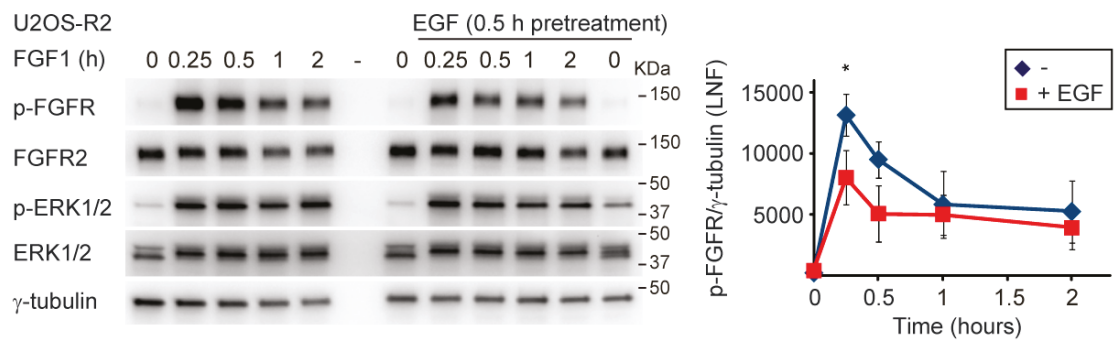

Figure 2. Pretreatment with EGF reduces the response to FGF1. (a) U2OS-R2 cells were kept in serum-free media for $2 \mathrm{~h}$ and then $100 \mathrm{ng} / \mathrm{mL}$ EGF or $100 \mathrm{ng} / \mathrm{mL}$ FGF1 was added to the cells. The cells were lysed after the indicated periods of time. A p in front of the name of the antibody indicates that it recognizes the phosphorylated form of the protein. (b) U2OS-R2 cells were kept in serum-free media for $2 \mathrm{~h}$. Then, EGF (20-100 ng/mL) was added to the samples as indicated. After $30 \mathrm{~min}$, the cells were stimulated with $20 \mathrm{ng} / \mathrm{mL}$ FGF1 and heparin $(10 \mathrm{U} / \mathrm{mL})$ and lysed after the indicated periods of time. Cycloheximide $(10 \mu \mathrm{g} / \mathrm{mL})$ was added at the beginning of the starvation period and kept throughout the experiment. After lysis, the cellular material was analyzed by immunoblotting using the indicated antibodies. A p in front of the name of the antibody indicates that it recognizes the phosphorylated form of the protein. Quantifications of three independent experiments are presented in the graph. The time-point of $30 \mathrm{~min}$ is only from two experiments. The bands corresponding to phosphorylated receptor were normalized to LNF ( $\gamma$-tubulin). Error bars denote the standard deviation. The difference between EGF-pretreated cells versus cells not treated with EGF was significant at the time point of 15 $\min \left({ }^{*} p \leq 0.05,1\right.$-way ANOVA, Tukey test, $\left.\mathrm{n}=3\right)$.

\subsection{Mutation of Serine 780 in FGFR2 Leads to Increased FGFR2 Activity}

Since the phosphorylation site of ERK1/2 in FGFR1 (S777) is already identified, we wanted to investigate if the corresponding serine in FGFR2 is important for proper downregulation of FGFR2 signaling. By sequence alignment, we identified S780 in FGFR2 to correspond to FGFR1 S777 (Figure 3a). Interestingly, in both receptors, the particular serine is followed by a proline and thus forms an ERK1/2 phosphorylation motif (pS/T-P) [18]. We therefore decided to substitute serine 780 in FGFR2 with alanine. Alanine represents a site that cannot be phosphorylated. Next, we prepared U2OS cells stably expressing FGFR2 S780A (U2OS-R2 S780A).

We then investigated whether FGFR2 S780A is expressed to similar levels as the wild-type receptor and if it maintained normal FGFR2 properties. We therefore stimulated cells with FGF1 and analyzed the lysates using Western blotting. First of all, the levels of FGFR2 wild-type and FGFR2 S780A seem comparable in the two clones (Figure 3b). Secondly, we noticed that FGFR2 S780A is able to activate the main downstream signaling pathways similarly to wild-type FGFR2 (Figure 3b). In addition, the mutated receptor was able to bind FGF1 at the cell surface and internalize FGF1 into early endosomes similarly to wild-type FGFR2 (Figure 3c). Comparable results were confirmed in two additional clones of U2OS-R2 wild-type and U2OS-R2 S780A (Figure S2a,b). Moreover, FGFR2 S780A co-localizes with DL550-FGF1, similarly to FGFR2 wild-type (Figure 3d).

We then analyzed the level of FGFR tyrosine phosphorylation over time in FGFR2 S780A-expressing cells. Compared to wild-type expressing cells, FGFR2 activation was sustained in U2OS-R2 S780A 
(Figure 4a and Figure S3). This effect was similar to the effect observed upon U0126 treatment. It is therefore likely that this serine, also in the case of FGFR2, is phosphorylated by ERK1/2.

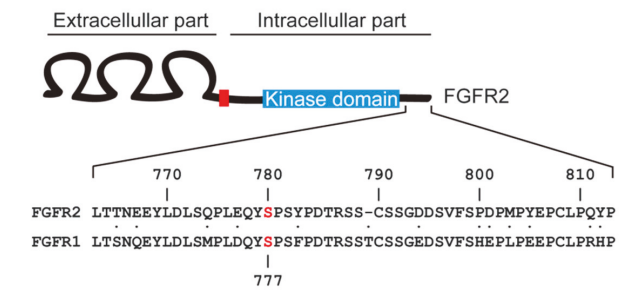

C

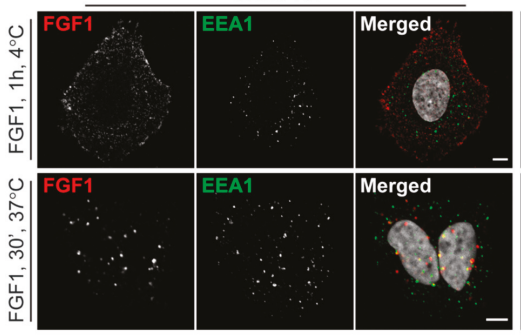

U2OS-R2

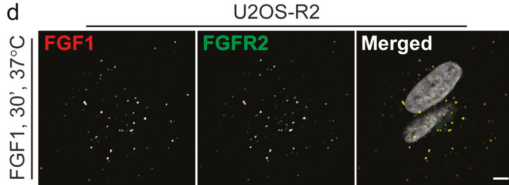

b

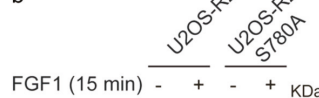

p-FGFR

FGFR2

p-PLC $\gamma$

p-RSK2

p-ERK1/2

$\gamma$-tubulin

U2OS-R2 S780A

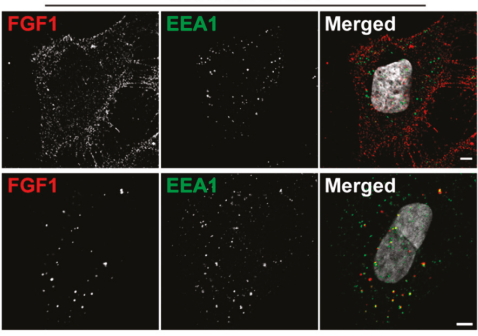

U2OS-R2 S780A

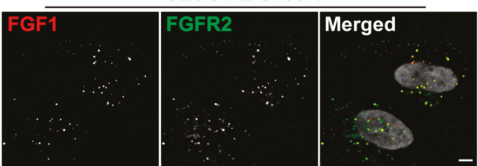

Figure 3. Characterization of cell lines stably expressing FGFR2 S780A mutant. (a) A pairwise sequence alignment tool from EMBL-EBI was used to align the C-terminal tails of FGFR2 and FGFR1. S780 in FGFR2 corresponds to S777 in FGFR1 (labelled in red in the figure). Numbers refer to the amino acid numbering used for human FGFR2 (NCBI: NM_000141). (b) U2OS-R2 cells or U2OS-R2 S780A cells were kept in serum-free media for two hours and then treated or not with $100 \mathrm{ng} / \mathrm{mL}$ FGF1 for $15 \mathrm{~min}$ in the presence of heparin $(20 \mathrm{U} / \mathrm{mL})$. After lysis, the cellular material was analyzed by immunoblotting using the indicated antibodies. A $p$ in front of the name of the antibody indicates that it recognizes the phosphorylated form of the protein. One representative experiment is shown. (c) U2OS-R2 or U2OS-R2 S780A cells were kept at $4{ }^{\circ} \mathrm{C}$ with DL550-FGF1 for one hour in the presence of heparin $(50 \mathrm{U} / \mathrm{mL})$. Next, the cells were either fixed directly (upper panel) or incubated for $30 \mathrm{~min}$ at $37^{\circ} \mathrm{C}$ before fixation (lower panel). The cells were then stained with anti-EEA1 antibodies and Hoechst and analyzed by confocal microscopy. Representative images are shown. Scale bar: $5 \mu \mathrm{M}$. (d) U2OS-R2 or U2OS-R2 S780A cells were treated with $200 \mathrm{ng} / \mathrm{mL}$ DL550-FGF1 in the presence of heparin $(50 \mathrm{U} / \mathrm{mL})$ for $30 \mathrm{~min}$. The cells were then fixed, stained with anti-FGFR2 antibodies and Hoechst, and analyzed by confocal microscopy. Representative images are shown. Scale bar: $5 \mu \mathrm{M}$. 


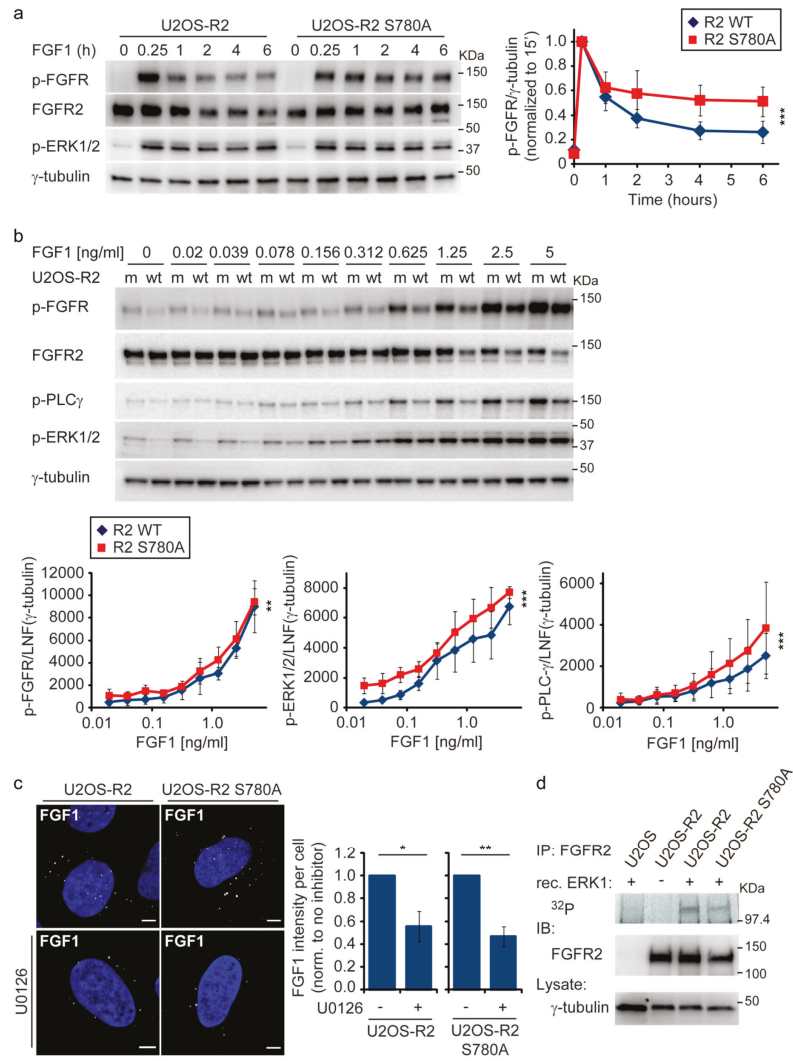

Figure 4. Signaling from FGFR2 S780A is prolonged compared to wild-type FGFR2. (a) U2OS-R2 wild-type or U2OS-R2 S780A cells were kept for two hours in serum-free media before addition of 100 $\mathrm{ng} / \mathrm{mL}$ FGF1 and heparin $(20 \mathrm{U} / \mathrm{mL})$ for the indicated periods of time. Cycloheximide $(10 \mu \mathrm{g} / \mathrm{mL}) \mathrm{was}$ added at the beginning of the starvation period and kept throughout the experiment. After lysis, the cellular material was analyzed by immunoblotting using the indicated antibodies. A p in front of the name of the antibody indicates that it recognizes the phosphorylated form of the protein. Quantifications of five independent experiments are presented in the graph. The bands corresponding to phosphorylated receptor were normalized to $\gamma$-tubulin and within each experiment to the time point of $15 \mathrm{~min}$. Error bars denote the standard deviation. The difference between FGFR2 wild-type and the S780A mutant was significant ( $p \leq 0.001$, 3-way ANOVA, Holm-Sidak test, $\mathrm{n}=5$ ). (b) U2OS-R2 wild-type (wt) or U2OS-R2 S780A (m) cells were kept for two hours in serum-free media before addition of indicated concentrations of FGF1 in the presence of heparin $(20 \mathrm{U} / \mathrm{mL})$ for $15 \mathrm{~min}$. After lysis, the cellular material was analyzed by immunoblotting using the indicated antibodies. A p in front of the name of the antibody indicates that it recognizes the phosphorylated form of the protein. Quantifications of three independent experiments are presented in the graph. The bands corresponding to the phosphorylated receptor/ERK1/2/PLC $\gamma$ were normalized to LNF ( $\gamma$-tubulin). Error bars denote the standard deviation. The difference between FGFR2 wild-type and S780A mutant was significant ${ }^{* *} p \leq 0.01{ }^{* * *} p \leq 0.001$, 3-way ANOVA, Holm-Sidak test, $\mathrm{n}$ =3). (c) Internalization of DL550-FGF1 in FGFR2 and FGFR2 S780A cells is reduced upon U0126 treatment. U2OS-R2 and U2OS-R2 S780A cells were incubated with $200 \mathrm{ng} / \mathrm{mL}$ DL550-FGF1 and heparin (50 U/mL) for $30 \mathrm{~min}$. The cells were pretreated as indicated with U0126 $(20 \mu \mathrm{M})$ for $30 \mathrm{~min}$ before addition of FGF1. 
The cells were then fixed, stained with Hoechst, and analyzed by confocal microscopy. The images were taken at fixed intensity settings, and brightness/contrast was adjusted in the same way for all images. Scale bar: $5 \mu \mathrm{M}$. Quantifications of four independent experiments were performed as described in materials and methods and are presented in the graph. In total, 269 U2OS-R2 cells, 262 U2OS-R2 cells treated with U0126, 247 U2OS-R2 S780A cells, and 213 U2OS-R2 S780A cells treated with U0126 were quantified. Outliers were removed according to the $1.5^{*} \mathrm{IQR}$ outlier rule. Error bars denote the SEM $(n=4)$. Due to a general variation in the intensity between experiments, the means of U0126 treated cells for each cell line in each experiment were normalized to the mean of the corresponding cell line in the same experiment $\left({ }^{* *} p \leq 0.05,{ }^{*} p \leq 0.05\right.$, two-sided $\mathrm{t}$ test, $\left.\mathrm{n}=4\right)$. (d) In vitro phosphorylation of FGFR2 by active recombinant ERK1. Lysates from U2OS, U2OS-R2, and U2OS-R2 S780A cells were subjected to FGFR2 immunoprecipitation (IP). The immunoprecipitated materials were next incubated with $\left[\gamma^{-}{ }^{32} \mathrm{P}\right]$-labelled adenosine triphosphate and recombinant active ERK1 (rec. ERK1) in the presence of PD173074. After washing, the samples were subjected to SDS-PAGE and analyzed by autoradiography and immunoblotting (IB). One representative experiment is shown.

In the previous experiments, higher concentrations of FGF1 were used to activate the receptor. We wanted to test if increased FGFR activity also occurred at lower concentrations of FGF1. We treated U2OS-R2 and U2OS-R2 S780A with different concentrations of FGF1 starting at $0.02 \mathrm{ng} / \mathrm{mL}$ (Figure 4b). Tyrosine phosphorylation of the receptor and its main downstream signaling pathways were then analyzed with Western blotting. We observed a slight increase in the levels of tyrosine-phosphorylated FGFR2 as well as in the levels of phosphorylated ERK1/2 and PLC- $\gamma$ in S780A-expressing cells compared to wild-type cells with low concentrations of FGF1. This experiment was performed after $15 \mathrm{~min}$ of FGF1 treatment where the effect is not at its highest. However, although the increase in signaling in FGFR2 S780A cells was modest, it was consistent at all concentrations tested. We therefore conclude that the negative feedback loop is operational at both lower and higher concentrations of ligand and at early time points.

Increased signaling can be a result of reduced receptor endocytosis. In Figure 1d, we detected more surface staining and less uptake of DL550-FGF1 in cells treated with U0126 (MEK1/2 inhibitor). This indicates a decrease in endocytosis when MEK1/2-ERK1/2 signaling is inhibited. However, from our previous work on FGFR1, despite a decrease in endocytosis upon MEK1/2-ERK1/2 inhibition, this effect was not due to lack of ERK1/2 phosphorylation of the receptor but rather a lack of a second serine phosphorylation event in FGFR1 mediated by RSK2 [13]. To investigate this, we compared the uptake of DL550-FGF1 in U2OS-R2 wild-type and U2OS-R2 S780A mutant cells in the presence of U0126 (Figure 4c). Upon U0126 treatment, the uptake of DL550-FGF1 was reduced similarly in both cell lines. Thus, the lack of phosphorylation on S780 is probably not the reason for the reduced endocytosis upon MEK1/2 inhibition. Other phosphorylation events mediated by components of the MAPK signaling pathways might be important for proper FGFR2 endocytosis.

Next, we wanted to test if ERK1/2 directly phosphorylates FGFR2. We therefore immunoprecipitated FGFR2 from cell lysates and incubated the immunoprecipitated receptor with recombinant active ERK1 and radioactive $\left[\gamma_{-}{ }^{32} \mathrm{P}\right]$-labelled adenosine triphosphate (ATP). The experiment was performed in the presence of PD173074 (FGFR inhibitor) to prevent autophosphorylation of the receptor. Using autoradiography, we could observe a band representing phosphorylated FGFR2 in the presence of active ERK1 (Figure 4d). This band was somewhat reduced in the sample from FGFR2 S780A cells. Thus, it seems that ERK1 directly phosphorylates FGFR2 on S780A. Since the phosphorylation of FGFR2 S780A is only partially reduced, we cannot exclude that other sites in FGFR2 might be phosphorylated by ERK1.

In order to study the role of S780 in FGFR2 further, we also prepared cell lines stably expressing FGFR2 S780D. The negatively charged aspartic acid might mimic constitutive phosphorylation of the residue. First, we verified that U2OS-R2 S780D cells were able to activate the main signaling pathways as wild-type FGFR2 (Figure S4a). We then investigated the tyrosine phosphorylation levels of FGFR2 upon FGF1 stimulation in U2OS-R2 S780D cells. Unfortunately, we observed the 
same effect of serine 780 mutated to an aspartic acid as we observed for FGFR2 S780A (Figure S4b). This is not surprising, as the mimicry of a phosphorylated serine by an aspartic acid often fails to reproduce the function of the phosphorylated serine [19]. We think that, instead of mimicking a constitutively phosphorylated serine, FGFR2 S780D rather displays a site that has lost its ability to become phosphorylated. Thus, FGFR2 S780D acts similarly to FGFR2 S780A and shows increased FGFR2 tyrosine phosphorylation.

\subsection{Possible Role of Serine 780 in FGFR2 in Cancer Progression}

We next investigated if the ERK1/2-mediated negative feedback loop possibly could play a role in cancer progression. By exploring databases reporting known alterations in cancer (cBioPortal; http://www.cbioportal.org and COSMIC (Catalogue of Somatic Mutations in Cancer); http://cancer. sanger.ac.uk) [20-22], we found several alterations that might influence the negative feedback loop in FGFR2 (Figure 5a). First, several truncated versions of FGFR2 lacking the C-terminal tail, including S780, have been identified in thyroid, skin, endometrial, and gastric cancers. In these cases, the negative feedback loop will not be operational and the receptor signaling may not be properly attenuated. This could potentially contribute to cancer progression. Secondly, several mutations in the close proximity of $\mathrm{S} 780$ have also been identified, including the glutamic acid at position 777 to a lysine and tyrosine 779 to a cysteine (Figure 5a). It is possible that these mutations influence S780 phosphorylation and receptor activity. Especially, the exchange of a negatively charged glutamic acid to a positively charged lysine might affect the properties of this region. Interestingly, serine 780 in FGFR2 has been found mutated to leucine in a patient with bladder cancer (Figure 5a). We decided to investigate the effect of this mutation further.

First of all, we generated U2OS cells stably expressing FGFR2 S780L (U2OS-R2 S780L) and confirmed that FGFR2 S780L cells were able to activate signaling pathways similarly to wild-type FGFR2 (Figure 5b and Figure S5a). Moreover, the levels of FGFR2 wild-type and FGFR2 S780L seem comparable and the mutated receptor is able to bind FGF1 at the cell surface and internalize FGF1 into early endosomes similarly to wild-type FGFR2 (Figure $5 \mathrm{c}$ and Figure S5b). In addition, the internalized DL550-FGF1 co-localizes well with anti-FGFR2 staining (Figure 5d).

We then analyzed the levels of FGFR2 tyrosine phosphorylation over time in FGFR2 S780L-expressing cells stimulated with FGF1. Compared to wild-type expressing cells, FGFR2 activation was prolonged in U2OS-R2 S780L (Figure 6a). Similar results were observed in two additional clones of U2OS-R2 S780L (Figure S6).

Clearly, the mutation of serine 780 to leucine leads to increased receptor signaling, which may be an advantage for cancer cells. Most cancer deaths $(\sim 90 \%)$ are caused by metastasis [23]. In order to metastasize and spread to distant organs, cancer cells need to be mobile and able to migrate. We therefore tested the mobility of U2OS cells stably expressing wild-type or S780 mutants. Since clonal variations might occur, we tested three different clones of each. Cells were seeded sparsely to allow for random migration and then imaged every $10 \mathrm{~min}$ for $21 \mathrm{~h}$. We observed that stimulation of cells with FGF1 increased the migration velocities of all cell lines (Figure 6b and Videos S1 and S2). Moreover, U2OS cells expressing either of the mutant forms of S780 (A/L) migrated significantly faster than wild-type expressing cells in the presence of FGF1 (Figure 6b and Videos S2-S4). Preventing the negative feedback loop in FGFR2 by mutation of S780 causes increased signaling and, as a consequence, increased cell migration. In a cancer setting, this might contribute to disease progression. 
a

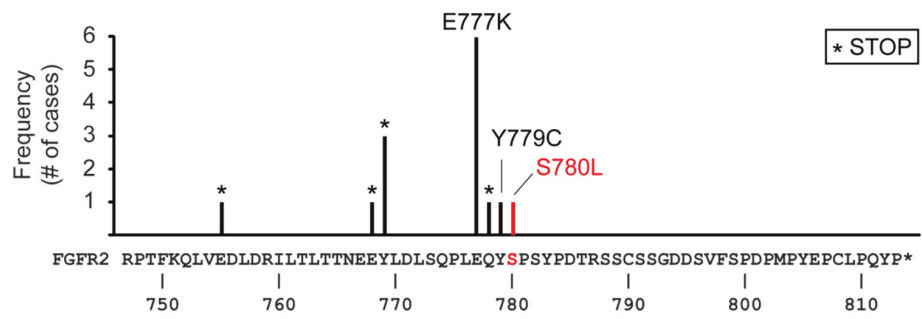

b

C

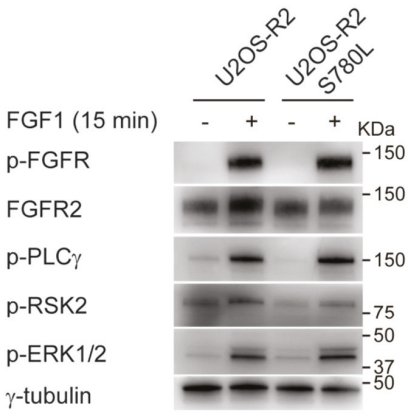

U2OS-R2 S780L

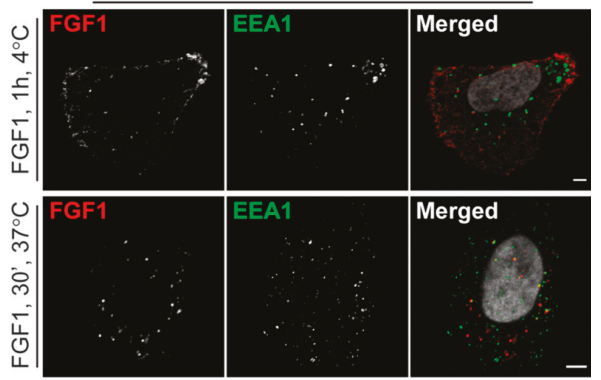

d U2OS-R2 S780L

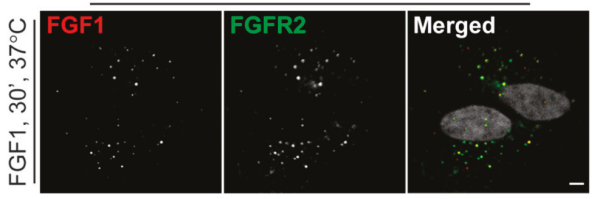

Figure 5. FGFR2 S780 is mutated in cancer. (a) The sequence of the C-terminal tail of FGFR2. Variations that might influence S780 phosphorylation and have been identified in cancer patients are indicated. An asterisk indicates a stop codon. Numbers refer to the amino acid numbering used for human FGFR2 (NCBI: NM_000141). The variations are reported in cBioPortal and COSMIC. (b) U2OS-R2 cells or U2OS-R2 S780L cells were kept in serum-free media for two hours and then treated or not with $100 \mathrm{ng} / \mathrm{mL}$ FGF1 for $15 \mathrm{~min}$ in the presence of heparin $(20 \mathrm{U} / \mathrm{mL})$. After lysis, the cellular material was analyzed by immunoblotting using the indicated antibodies. A p in front of the name of the antibody indicates that it recognizes the phosphorylated form of the protein. One representative experiment is shown. (c) U2OS-R2 S780L cells were kept at $4{ }^{\circ} \mathrm{C}$ with DL550-FGF1 for one hour. Next, the cells were either fixed directly (upper panel) or incubated for $30 \mathrm{~min}$ at $37^{\circ} \mathrm{C}$ before fixation (lower panel). The cells were then stained with anti-EEA1 antibodies and Hoechst and analyzed by confocal microscopy. Representative images are shown. Scale bar: $5 \mu \mathrm{M}$. (d) U2OS-R2 S780L cells were treated with $200 \mathrm{ng} / \mathrm{mL}$ DL550-FGF1 in the presence of heparin $(50 \mathrm{U} / \mathrm{mL})$ for $30 \mathrm{~min}$. The cells were then fixed, stained with anti-FGFR2 antibodies and Hoechst, and analyzed by confocal microscopy. Representative images are shown. Scale bar: $5 \mu \mathrm{M}$. 
a
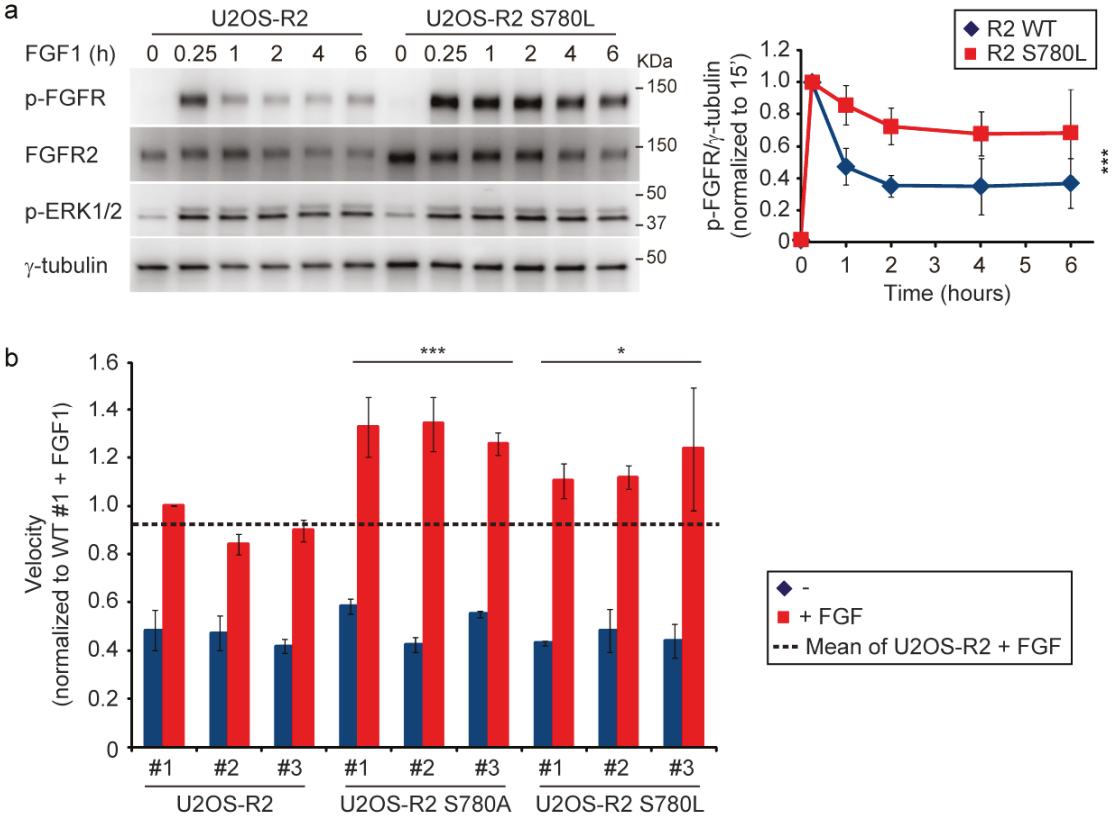

Figure 6. Lack of S780 phosphorylation increases the biological response to FGF1. (a) U2OS-R2 wild-type or U2OS-R2 S780L cells were kept in serum-free media for two hours before addition of $100 \mathrm{ng} / \mathrm{mL}$ FGF1 and heparin $(20 \mathrm{U} / \mathrm{mL})$ for indicated periods of time. Cycloheximide $(10 \mu \mathrm{g} / \mathrm{mL})$ was added at the beginning of the starvation period and kept throughout the experiment. After lysis, the cellular material was analyzed by immunoblotting using the indicated antibodies. A p in front of the name of the antibody indicates that it recognizes the phosphorylated form of the protein. Quantifications of three independent experiments are presented in the graph. The bands corresponding to phosphorylated receptor were normalized to $\gamma$-tubulin and within each experiment to the time point of $15 \mathrm{~min}$. Error bars denote the standard deviation. The difference between FGFR2 wild-type and S780L mutant was significant ( $p \leq 0.001$, 3-way ANOVA, Holm-Sidak test, $\mathrm{n}=3$ ). (b) Three different clones of U2OS-R2 wild-type, U2OS-R2 S780A, and U2OS-S780L cells were seeded into Image Lock 96-well plates. The cells were left untreated or stimulated with FGF1 $(100 \mathrm{ng} / \mathrm{mL})$ in the presence of heparin $(20 \mathrm{U} / \mathrm{mL})$ and imaged every $10 \mathrm{~min}$ over a period of $21 \mathrm{~h}$ by IncuCyte ${ }^{\circledR}$ S3 Live Cell Analysis System. The graph represents the mean velocities normalized to U2OS-R2 clone \#1 with FGF1 of three independent experiments. The total number of cells tracked: U2OS-R2 \#1 (-/+): 138/140, \#2 (-/+): 111/138, \#3 (-/+): 106/141, U2OS-R2 S780A \#1 (-/+): 139/167, \#2 (-/+): 135/142, \#3 (-/+): 128/142, U2OS-R2 S780L \#1 (-/+): 97/174, \#2 (-/+): 110/153, \#3 (-/+): 121/139. Error bars denote the SEM $(\mathrm{n}=3)$. The difference between the wild-type and S780 mutants were significant ${ }^{* * *} p \leq 0.001,{ }^{*} p \leq 0.05$, student $\mathrm{t}$ test, $\mathrm{n}=3$ ).

\section{Discussion}

We have identified a negative feedback loop, mediated by the ERK1/2 pathway that regulates FGFR2 signaling. First, we found that inhibition of the ERK1/2-pathway leads to sustained FGFR2 signaling. Next, we found that substituting serine 780 in FGFR2 with alanine or leucine results in increased signaling. Serine 780 in FGFR2 is followed by a proline and thus forms an ERK1/2 phosphorylation motif (pS/T-P). In addition, S780 in FGFR2 corresponds to S777 in FGFR1. S777 in FGFR1 has previously been shown to be phosphorylated directly by ERK1/2. Taken together, we propose that ERK1/2-mediated phosphorylation of S780 in FGFR2 acts as a negative feedback loop to prevent excess signaling. This was evident when cells were pretreated with EGF to activate ERK1/2 prior to FGF1 stimulation. In activated cells, the response to FGF1 was lower than in resting cells. The 
feedback loop may function to fine-tune FGFR2 signaling in an environment where signaling is already on, preventing a further increase in signaling. We observed that cells lacking S780 (mutated to alanine or leucine) migrate faster than wild-type expressing cells. Since migration is important for spreading of cancer cells and metastasis, clearly the lack of this negative feedback loop gives cancer cells an advantage. Indeed, S780L has been identified in a patient with bladder cancer. In addition, several truncated forms of FGFR2 lacking S780 have also been identified in cancer. Maintaining the negative feedback loop ensures accurate signaling and preventing the feedback loop (either by mutation of S780 in FGFR2 or by inhibition of ERK1/2 signaling) could cause cancer progression.

Aberrant signaling through the Ras-Raf-MEK1/2-ERK1/2 pathway has been implicated in many types of cancer and is a promising therapeutic target. Although BRaf- and MEK-inhibitor monoor combination-therapy have shown promising effects in cancer patients, many patients develop resistance and experience disease progression [24]. These resistance mechanisms include reactivation of the MAPK and/or the PI3K/Akt pathway. Examples of common resistance mechanisms include NRas mutation, BRaf v600 amplification, loss of PTEN, PI3KCA mutation, and RTK activation [24]. Since ERK $1 / 2$ is the only activator in the pathway with the ability to stimulate a wide variety of downstream substrates, it has emerged as an attractive therapeutic target. Despite the discovery of ERK1/2 many decades ago, ERK1/2 inhibitors have so far not been successfully implemented in the clinic [25]. One possible reason is that activated ERK1/2 stimulates inhibitory phosphorylation of many upstream factors and kinases, such as MEK, Raf, and different RTKs (including FGFR2), which prevent extensive signaling $[25,26]$. It is therefore worth considering that sole inhibition of the ERK1/2 signaling pathway in cancer could give rise to increased FGFR signaling through other signaling pathways (for example PI3K/Akt). Indeed, a recent study showed an increase in FGFR signaling upon MEK inhibition in KRas-driven lung cancer [27]. Therefore, caution should be taken when considering the use of MEK/ERK pathway inhibitors in cancer patients with FGFR2 expression.

Interestingly, a similar negative feedback loop involving ERK1/2-mediated phosphorylation has been identified for EGFR. In this case, ERK1/2-mediated signaling phosphorylates EGFR at threonine 669 (T669) [28,29]. Phosphorylation of this residue, which is localized in the juxtamembrane region of the receptor, was shown to reduce the tyrosine phosphorylation levels of EGFR. It seems that the T669-phosphorylated juxtamembrane region in EGFR has a reduced ability to cross-activate the other receptor of the dimer [30]. Although S780 is located in the C-terminal tail of FGFR2, it is possible that local conformational changes introduced by the phosphorylation at $\mathrm{S} 780$ reduce its cross-activation. It is also possible that a local conformational change in the receptor, caused by phosphorylation of S780, makes FGFR2 a better substrate for tyrosine dephosphorylation. Another attractive possibility is that phosphorylated S780 directly recruits a negative regulator such as a phosphatase or a scaffolding protein. Interestingly, serine 779 (S782 according to our numbering) in FGFR2 is phosphorylated by active $\mathrm{PKC} \varepsilon$ and provides a docking site for the adaptor protein 14-3-3 [31]. However, in this case, phosphorylation of S779 (S782) seems to be required for sustained ERK1/2 activation and thus does not function as a negative feedback loop. It will be interesting to understand how these two phosphorylation events at S779 (S782) and S780 in FGFR2 work in partnership. It is also possible that S780 phosphorylation plays a role in receptor endocytosis and degradation. Previously, we found that S789 in FGFR1 is phosphorylated by RSK2 and seems to be required for proper internalization [13]. Interestingly, when we treated FGF1 stimulated cells with U0126 and stained for p-FGFR, we observed increased p-FGFR staining close to the cell surface (Figure 1d). We also observed less FGF1 internalized. However, when U2OS-R2 S780A cells were treated with U0126, the uptake of DL550-FGF1 was reduced to a similar extent as in U2OS-R2 wild-type cells (Figure 4c). It is possible that an RSK2-mediated feedback loop similar to that observed for FGFR1 exists also for FGFR2. U0126 inhibits both ERK1/2 and its downstream target, RSK2. There are also other examples of receptors that are serine-phosphorylated similarly to FGFR2, FGFR1, and EGFR. The Met receptor is phosphorylated by active PKC $\delta / \varepsilon$ at serine 985 in the juxtamembrane region [32]. Substitution of serine 985 by alanine resulted in increased tyrosine phosphorylation of Met. Similarly to FGFR2 and FGFR1, it is not clear what causes the reduced 
tyrosine phosphorylation in this case. Taken together, serine and threonine phosphorylation of receptor tyrosine kinases might be a common event that regulates receptor activity. A better understanding of these events will provide useful information when targeting receptor tyrosine kinases in cancer.

Although the mutation of serine 780 in FGFR2 to leucine clearly increases FGFR2 tyrosine phosphorylation levels and FGF1-stimulated cell migration, the role of this mutation in cancer is not clear. The mutation was found in a patient with bladder cancer. Although increased signaling and increased migration are traits that normally would benefit cancer cells, the role of FGFR2 signaling in bladder cancer is not fully understood and the FGFR2b isoform has been suggested to act as a tumor suppressor in the urothelium. It has been reported that reduction of FGFR2b levels in urothelial cancer samples correlate with decreased survival [33] and the chromosomal arm 10q, where the FGFR2 gene is located, is often lost in advanced bladder cancer [34]. It should be noted that $10 \mathrm{q}$ also contains the tumor suppressor PTEN [35]. Moreover, expression of FGFR2b in urothelial cells lacking endogenous FGFR2 led to reduced proliferation and reduced tumorigenicity in nude mice [36]. On the other hand, increased FGFR2c expression has been reported in a model of epithelial-to-mesechymal transition (EMT) in bladder cancer cells [37] and recently, the U.S. Food and Drug Administration (FDA) approved Balversa (Erdafitinib), a pan FGFR-inhibitor for clinical use in patients with locally advanced or metastatic urothelial carcinoma with FGFR2 and FGFR3 aberrations [38]. This is the first targeted-FGFR therapy approved for clinical use and the first targeted therapy in advanced urothelial carcinoma. Alongside the approval of the drug, the FDA approved an RT-PCR-based diagnostic test to identify patients with FGFR3 mutations or FGFR2 fusions. It is possible that FGFR2 plays a tumor-suppressing role in earlier stages of bladder cancer, but could have tumor-promoting effects in certain patients with advanced bladder cancer.

The S780L mutation is only reported once in the COSMIC database and at such low frequency that the significance is questionable. On the other hand, truncated versions of FGFR2 lacking S780 have been identified in cancer patients (Figure 5a). In addition, an alternatively spliced form of FGFR2, FGFR2IIIb-C3, is also lacking S780 [39]. In contrast to full-length FGFR2IIIb, FGFR2IIIb-C3 is only identified in human cancer samples. Aberrant expression of FGFR2IIIb-C3 in SUM-52 breast cancer cells resulted in sustained signaling leading to transformation [40]. A tyrosine phosphorylation site (corresponding to Y766 in FGFR1) is also lacking in FGFR2IIIb-C3 and could explain the increased signaling and transformation capabilities of FGFR2IIIb-C3. However, mutation of only this tyrosine in full-length FGFR2 did not lead to increased signaling [41]. Thus, loss of other mechanisms maintained by the C-terminal tail of FGFR2 might cause the increased signaling and transforming potential of FGFR2IIIb-C3. We propose that lack of S780 in FGFR2IIIb-C3 could promote its transforming capabilities. Interestingly, a patient with endometrial cancer was identified with the activating mutation N549H in FGFR2 and a truncated C-terminal tail (cBioPortal; http://www.cbioportal.org) [20,21]. This combination of alterations in FGFR2 will clearly impact signaling output and could be even more cancer-promoting than versions with either mutation alone.

In summary, we have identified an ERK1/2-mediated negative feedback-loop in FGFR2. We propose that lack of this feedback loop could give cancer cells an advantage and, indeed, variants of FGFR2 lacking the feedback loop have been identified in several human cancers. We conclude therefore that, in addition to the previously reported activating mutations in the kinase domain of FGFRs [4], mutations in the C-terminal tail of FGFR2 may also cause hyperactivation of the receptors.

Supplementary Materials: The following are available online at http://www.mdpi.com/2073-4409/8/6/518/s1, Figure S1: Inhibition of ERK1/2 prolongs FGFR2 signaling in several U2OS-R2 clones. Figure S2: Characterization of U2OS-R2 S780A clones. Figure S3: Signaling from FGFR2 S780A is prolonged compared to wild-type FGFR2. Figure S4: FGFR2 S780D mutant is not phosphomimetic. Figure S5: Characterization of U2OS-R2 S780L clones. Figure S6: Signaling from FGFR2 S780L is prolonged compared to wild-type FGFR2. Video S1: Migration of U2OS-R2 cells in the absence of FGF1. Video S2: Migration of U2OS-R2 cells stimulated with FGF1. Video S3: Migration of U2OS-R2 S780A cells stimulated with FGF1. Video S4: Migration of U2OS-R2 S780L cells stimulated with FGF1. 
Author Contributions: Conceptualization, P.S., E.M.H., A.W., and J.W.; methodology, P.S. and E.M.H.; validation, P.S., and E.M.H.; formal analysis, E.M.H., P.S., and M.K.; investigation, P.S., J.W., A.W., and M.K.; writing-original draft preparation, P.S. and E.M.H.; writing—review and editing, P.S., E.M.H., M.K., A.W., J.W.; visualization, P.S., and E.M.H.; supervision, E.M.H., and A.W.; project administration, E.M.H.; funding acquisition, E.M.H., A.W., and J.W. All authors read and approved the manuscript.

Funding: This work was supported by the Norwegian Cancer Society. E.M.H. holds a Career fellowship (project 6842225). This work was partially supported by The Research Council of Norway through its Centers of Excellence funding scheme, project number 262652

Acknowledgments: The results shown here are in part based upon data generated by the TCGA Research Network: https://www.cancer.gov/tcga.

Conflicts of Interest: The authors declare no conflict of interest.

\section{References}

1. Sangwan, V.; Park, M. Receptor tyrosine kinases: Role in cancer progression. Curr. Oncol. 2006, 13, 191-193.

2. Ornitz, D.M.; Itoh, N. The Fibroblast Growth Factor signaling pathway. Wiley Interdiscip. Rev. Dev. Biol. 2015, 4, 215-266. [CrossRef] [PubMed]

3. Wesche, J.; Haglund, K.; Haugsten, E.M. Fibroblast growth factors and their receptors in cancer. Biochem. J. 2011, 437, 199-213. [CrossRef] [PubMed]

4. Babina, I.S.; Turner, N.C. Advances and challenges in targeting FGFR signalling in cancer. Nat. Rev. Cancer 2017, 17, 318-332. [CrossRef]

5. Ornitz, D.M.; Marie, P.J. Fibroblast growth factor signaling in skeletal development and disease. Genes Dev. 2015, 29, 1463-1486. [CrossRef]

6. Haugsten, E.M.; Malecki, J.; Bjorklund, S.M.; Olsnes, S.; Wesche, J. Ubiquitination of fibroblast growth factor receptor 1 is required for its intracellular sorting but not for its endocytosis. Mol. Biol. Cell 2008, 19, 3390-3403. [CrossRef] [PubMed]

7. Haugsten, E.M.; Sorensen, V.; Brech, A.; Olsnes, S.; Wesche, J. Different intracellular trafficking of FGF1 endocytosed by the four homologous FGF receptors. J. Cell Sci. 2005, 118, 3869-3881. [CrossRef] [PubMed]

8. Belleudi, F.; Leone, L.; Maggio, M.; Torrisi, M.R. Hrs regulates the endocytic sorting of the fibroblast growth factor receptor 2b. Exp. Cell Res. 2009, 315, 2181-2191. [CrossRef] [PubMed]

9. Kostas, M.; Haugsten, E.M.; Zhen, Y.; Sorensen, V.; Szybowska, P.; Fiorito, E.; Lorenz, S.; Jones, N.; de Souza, G.A.; Wiedlocha, A.; et al. Protein Tyrosine Phosphatase Receptor Type G (PTPRG) Controls Fibroblast Growth Factor Receptor (FGFR) 1 Activity and Influences Sensitivity to FGFR Kinase Inhibitors. Mol. Cell Proteom. 2018, 17, 850-870. [CrossRef]

10. Goetz, R.; Mohammadi, M. Exploring mechanisms of FGF signalling through the lens of structural biology. Nat. Rev. Mol. Cell Biol. 2013, 14, 166-180. [CrossRef]

11. Lax, I.; Wong, A.; Lamothe, B.; Lee, A.; Frost, A.; Hawes, J.; Schlessinger, J. The docking protein FRS2alpha controls a MAP kinase-mediated negative feedback mechanism for signaling by FGF receptors. Mol. Cell 2002, 10, 709-719. [CrossRef]

12. Zakrzewska, M.; Haugsten, E.M.; Nadratowska-Wesolowska, B.; Oppelt, A.; Hausott, B.; Jin, Y.; Otlewski, J.; Wesche, J.; Wiedlocha, A. ERK-mediated phosphorylation of fibroblast growth factor receptor 1 on Ser777 inhibits signaling. Sci. Signal. 2013, 6, ra11. [CrossRef]

13. Nadratowska-Wesolowska, B.; Haugsten, E.M.; Zakrzewska, M.; Jakimowicz, P.; Zhen, Y.; Pajdzik, D.; Wesche, J.; Wiedlocha, A. RSK2 regulates endocytosis of FGF receptor 1 by phosphorylation on serine 789. Oncogene 2014, 33, 4823-4836. [CrossRef] [PubMed]

14. Wesche, J.; Malecki, J.; Wiedlocha, A.; Ehsani, M.; Marcinkowska, E.; Nilsen, T.; Olsnes, S. Two nuclear localization signals required for transport from the cytosol to the nucleus of externally added FGF-1 translocated into cells. Biochemistry 2005, 44, 6071-6080. [CrossRef]

15. Klingenberg, O.; Wiedlocha, A.; Rapak, A.; Khnykin, D.; Citores, L.; Olsnes, S. Requirement for C-terminal end of fibroblast growth factor receptor 4 in translocation of acidic fibroblast growth factor to cytosol and nucleus. J. Cell Sci. 2000, 113, 1827-1838. [PubMed]

16. Schindelin, J.; Arganda-Carreras, I.; Frise, E.; Kaynig, V.; Longair, M.; Pietzsch, T.; Preibisch, S.; Rueden, C.; Saalfeld, S.; Schmid, B.; et al. Fiji: An open-source platform for biological-image analysis. Nat. Methods 2012, 9, 676-682. [CrossRef] 
17. Haugsten, E.M.; Zakrzewska, M.; Brech, A.; Pust, S.; Olsnes, S.; Sandvig, K.; Wesche, J. Clathrin- and dynamin-independent endocytosis of FGFR3-implications for signalling. PLOS ONE 2011, 6, e21708. [CrossRef]

18. Unal, E.B.; Uhlitz, F.; Bluthgen, N. A compendium of ERK targets. FEBS Lett. 2017, 591, 2607-2615. [CrossRef] [PubMed]

19. Chen, Z.; Cole, P.A. Synthetic approaches to protein phosphorylation. Curr. Opin. Chem. Biol. 2015, 28, 115-122. [CrossRef]

20. Gao, J.; Aksoy, B.A.; Dogrusoz, U.; Dresdner, G.; Gross, B.; Sumer, S.O.; Sun, Y.; Jacobsen, A.; Sinha, R.; Larsson, E.; et al. Integrative analysis of complex cancer genomics and clinical profiles using the cBioPortal. Sci. Signal. 2013, 6, pl1. [CrossRef] [PubMed]

21. Cerami, E.; Gao, J.; Dogrusoz, U.; Gross, B.E.; Sumer, S.O.; Aksoy, B.A.; Jacobsen, A.; Byrne, C.J.; Heuer, M.L.; Larsson, E.; et al. The cBio cancer genomics portal: An open platform for exploring multidimensional cancer genomics data. Cancer Discov. 2012, 2, 401-404. [CrossRef] [PubMed]

22. Forbes, S.A.; Beare, D.; Boutselakis, H.; Bamford, S.; Bindal, N.; Tate, J.; Cole, C.G.; Ward, S.; Dawson, E.; Ponting, L.; et al. COSMIC: Somatic cancer genetics at high-resolution. Nucleic Acids Res. 2017, 45, D777-D783. [CrossRef] [PubMed]

23. Chaffer, C.L.; Weinberg, R.A. A perspective on cancer cell metastasis. Science 2011, 331, 1559-1564. [CrossRef] [PubMed]

24. Kakadia, S.; Yarlagadda, N.; Awad, R.; Kundranda, M.; Niu, J.; Naraev, B.; Mina, L.; Dragovich, T.; Gimbel, M.; Mahmoud, F. Mechanisms of resistance to BRAF and MEK inhibitors and clinical update of US Food and Drug Administration-approved targeted therapy in advanced melanoma. Oncotargets Ther. 2018, 11, 7095-7107. [CrossRef]

25. Liu, F.; Yang, X.; Geng, M.; Huang, M. Targeting ERK, an Achilles' Heel of the MAPK pathway, in cancer therapy. Acta Pharm. Sin. B 2018, 8, 552-562. [CrossRef] [PubMed]

26. Lake, D.; Correa, S.A.; Muller, J. Negative feedback regulation of the ERK1/2 MAPK pathway. Cell. Mol. Life Sci. 2016, 73, 4397-4413. [CrossRef] [PubMed]

27. Manchado, E.; Weissmueller, S.; Morris, J.P.; Chen, C.C.; Wullenkord, R.; Lujambio, A.; de Stanchina, E.; Poirier, J.T.; Gainor, J.F.; Corcoran, R.B.; et al. A combinatorial strategy for treating KRAS-mutant lung cancer. Nature 2016, 534, 647-651. [CrossRef]

28. Northwood, I.C.; Gonzalez, F.A.; Wartmann, M.; Raden, D.L.; Davis, R.J. Isolation and characterization of two growth factor-stimulated protein kinases that phosphorylate the epidermal growth factor receptor at threonine 669. J. Biol. Chem. 1991, 266, 15266-15276.

29. Takishima, K.; Griswold-Prenner, I.; Ingebritsen, T.; Rosner, M.R. Epidermal growth factor (EGF) receptor T669 peptide kinase from 3T3-L1 cells is an EGF-stimulated "MAP" kinase. Proc. Natl. Acad. Sci. USA 1991, 88, 2520-2524. [CrossRef]

30. Sato, K.; Shin, M.S.; Sakimura, A.; Zhou, Y.; Tanaka, T.; Kawanishi, M.; Kawasaki, Y.; Yokoyama, S.; Koizumi, K.; Saiki, I.; et al. Inverse correlation between Thr-669 and constitutive tyrosine phosphorylation in the asymmetric epidermal growth factor receptor dimer conformation. Cancer Sci. 2013, 104, 1315-1322. [CrossRef] [PubMed]

31. Lonic, A.; Powell, J.A.; Kong, Y.; Thomas, D.; Holien, J.K.; Truong, N.; Parker, M.W.; Guthridge, M.A. Phosphorylation of serine 779 in fibroblast growth factor receptor 1 and 2 by protein kinase C(epsilon) regulates Ras/mitogen-activated protein kinase signaling and neuronal differentiation. J. Biol. Chem. 2013, 288, 14874-14885. [CrossRef]

32. Gandino, L.; Longati, P.; Medico, E.; Prat, M.; Comoglio, P.M. Phosphorylation of serine 985 negatively regulates the hepatocyte growth factor receptor kinase. J. Biol. Chem. 1994, 269, 1815-1820.

33. Diez de Medina, S.G.; Chopin, D.; El, M.A.; Delouvee, A.; LaRochelle, W.J.; Hoznek, A.; Abbou, C.; Aaronson, S.A.; Thiery, J.P.; Radvanyi, F. Decreased expression of keratinocyte growth factor receptor in a subset of human transitional cell bladder carcinomas. Oncogene 1997, 14, 323-330. [CrossRef] [PubMed]

34. Cappellen, D.; Gil Diez de Medina, S.; Chopin, D.; Thiery, J.P.; Radvanyi, F. Frequent loss of heterozygosity on chromosome 10q in muscle-invasive transitional cell carcinomas of the bladder. Oncogene 1997, 14, 3059-3066. [CrossRef] [PubMed] 
35. Hurst, C.D.; Platt, F.M.; Taylor, C.F.; Knowles, M.A. Novel tumor subgroups of urothelial carcinoma of the bladder defined by integrated genomic analysis. Clin. Cancer Res. Off. J. Am. Assoc. Cancer Res. 2012, 18, 5865-5877. [CrossRef]

36. Ricol, D.; Cappellen, D.; El, M.A.; Gil-Diez-de-Medina, S.; Girault, J.M.; Yoshida, T.; Ferry, G.; Tucker, G.; Poupon, M.F.; Chopin, D.; et al. Tumour suppressive properties of fibroblast growth factor receptor 2-IIIb in human bladder cancer. Oncogene 1999, 18, 7234-7243. [CrossRef] [PubMed]

37. Chaffer, C.L.; Brennan, J.P.; Slavin, J.L.; Blick, T.; Thompson, E.W.; Williams, E.D. Mesenchymal-to-epithelial transition facilitates bladder cancer metastasis: Role of fibroblast growth factor receptor-2. Cancer Res. 2006, 66, 11271-11278. [CrossRef]

38. Nadal, R.; Bellmunt, J. Management of metastatic bladder cancer. Cancer Treat. Rev. 2019, 76, 10-21. [CrossRef]

39. Cha, J.Y.; Maddileti, S.; Mitin, N.; Harden, T.K.; Der, C.J. Aberrant receptor internalization and enhanced FRS2-dependent signaling contribute to the transforming activity of the fibroblast growth factor receptor 2 IIIb C3 isoform. J. Biol. Chem. 2009, 284, 6227-6240. [CrossRef]

40. Moffa, A.B.; Tannheimer, S.L.; Ethier, S.P. Transforming potential of alternatively spliced variants of fibroblast growth factor receptor 2 in human mammary epithelial cells. Mol. Cancer Res. 2004, 2, 643-652.

41. Moffa, A.B.; Ethier, S.P. Differential signal transduction of alternatively spliced FGFR2 variants expressed in human mammary epithelial cells. J. Cell Physiol. 2007, 210, 720-731. [CrossRef] [PubMed]

(C) 2019 by the authors. Licensee MDPI, Basel, Switzerland. This article is an open access article distributed under the terms and conditions of the Creative Commons Attribution (CC BY) license (http://creativecommons.org/licenses/by/4.0/). 


\title{
Membrane-Associated, Not Cytoplasmic or Nuclear, FGFR1 Induces Neuronal Differentiation
}

\author{
Katalin Csanaky ${ }^{1}$, Michael W. Hess ${ }^{2}$ and Lars Klimaschewski ${ }^{1, *}$ \\ 1 Division of Neuroanatomy, Medical University of Innsbruck, 6020 Innsbruck, Austria; \\ katalin.csanaky@i-med.ac.at \\ 2 Division of Histology and Embryology, Medical University of Innsbruck, 6020 Innsbruck, Austria; \\ michael.hess@i-med.ac.at \\ * Correspondence: lars.klimaschewski@i-med.ac.at; Tel.: +43-512-9003-71160
}

Received: 31 January 2019; Accepted: 8 March 2019; Published: 14 March 2019

\begin{abstract}
The intracellular transport of receptor tyrosine kinases results in the differential activation of various signaling pathways. In this study, optogenetic stimulation of fibroblast growth factor receptor type 1 (FGFR1) was performed to study the effects of subcellular targeting of receptor kinases on signaling and neurite outgrowth. The catalytic domain of FGFR1 fused to the algal light-oxygen-voltage-sensing (LOV) domain was directed to different cellular compartments (plasma membrane, cytoplasm and nucleus) in human embryonic kidney (HEK293) and pheochromocytoma (PC12) cells. Blue light stimulation elevated the pERK and pPLC $\gamma 1$ levels in membrane-opto-FGFR1-transfected cells similarly to ligand-induced receptor activation; however, no changes in pAKT levels were observed. PC12 cells transfected with membrane-opto-FGFR1 exhibited significantly longer neurites after light stimulation than after growth factor treatment, and significantly more neurites extended from their cell bodies. The activation of cytoplasmic FGFR1 kinase enhanced ERK signaling in HEK293 cells but not in PC12 cells and did not induce neuronal differentiation. The stimulation of FGFR1 kinase in the nucleus also did not result in signaling changes or neurite outgrowth. We conclude that FGFR1 kinase needs to be associated with membranes to induce the differentiation of PC12 cells mainly via ERK activation.
\end{abstract}

Keywords: optogenetics; FGF2; ERK; AKT; receptor kinase; neurite outgrowth; HEK293; PC12

\section{Introduction}

The fibroblast growth factor receptor (FGFR) family comprises four closely related receptors (FGFR1-4) consisting of a signal peptide, three extracellular immunoglobulin (Ig)-like domains, an acidic box, a single transmembrane helix, and an intracellular split tyrosine kinase domain [1]. The binding of FGF ligands results in receptor dimerization and autophosphorylation of the cytoplasmic kinase domains. Following the recruitment of various adapter molecules, several intracellular signaling pathways are activated, of which, the Ras/extracellular signal-regulated kinase (ERK) and phosphatidylinositol-3 kinase (PI3K)/AKT signaling cascades play a key role in neuronal differentiation and axon growth [2]. All FGFR subtypes drive the same signaling cascades but with different strengths [3].

Activated FGFR complexes are endocytosed and partly recycled to the plasma membrane or transported to late endosomes/multivesicular bodies followed by degradation in lysosomes [4]. The internalization of activated FGFR1 does not implicate the inactivation of the receptor or attenuation of signaling, but allows ongoing and even stronger signaling activities particularly of the ERK pathway. In neurons, ERK1/2 activation requires receptor internalization, while AKT activation does not [5]. We and others have demonstrated that the extent of the stimulation of the major signaling pathways involved in neuronal differentiation and axon outgrowth is crucially dependent on the amount and 
localization of activated kinase domains. For example, leupeptin, a tripeptide inhibitor of cysteine and aspartic acid cleaving proteases, prevents the lysosomal degradation of FGFR1 and promotes basic fibroblast growth factor (FGF2)-induced axon regeneration by enhanced receptor recycling [6].

In addition to its membrane localization, FGFR1 is constitutively found in the cytoplasm and nucleus [7]. The nuclear translocation of FGFR1 is importin-dependent [8] and results in the binding of the receptor to transcriptionally active chromatin which drives the gene expression of FGF2 and tyrosine hydroxylase [9]. Furthermore, interaction with RSK1 promotes FGFR1 release from the pre-Golgi compartments to the cytoplasm, increases the mobile population of FGFR1 and facilitates the nuclear accumulation of FGFR1 [10,11]. Nerve growth factor (NGF) has also been suggested to utilize integrative nuclear FGFR1 signaling (INFS) for its gene-activating functions [12]. In addition, INFS is apparently involved in the dendritic outgrowth of sympathetic neurons treated with bone morphogenetic protein [13].

The plethora of FGFR-dependent biological effects is not only explained by differences in subcellular receptor targeting but also by the differential expression of adapter proteins and other signaling components. Since the duration and kinetics of receptor activation play a decisive role in determining the functional and morphological outcome [14], FGFR1 represents an ideal object for optogenetic manipulation.

Recently, light-activatable FGF receptors that are incapable of ligand binding were developed [15]. Chimeric opto-fibroblast growth factor receptors (opto-FGFRs) exhibiting the catalytic domain were fused to the algal light-oxygen-voltage-sensing (LOV) domain for light-induced dimerization. These constructs lack the extracellular receptor domain and are, therefore, insensitive to endogenous or exogenous ligands. Short pulses of blue light induce LOV domain dimerization which results in the transphosphorylation of two receptor kinase molecules. Receptor phosphorylation diminishes rapidly after light stimulation and is followed by the inactivation of the main signaling pathways. Thus, intracellular FGFR activity can be externally controlled by repeated light stimulation. In this study, plasma membrane-targeted (mem-opto-FGFR1), cytoplasmic (cyto-opto-FGFR1) and nuclear FGFR1 (nucl-opto-FGFR1) were produced and studied with regard to signal pathway activation and neurite outgrowth.

\section{Materials and Methods}

\subsection{Plasmid Construction}

Mem-opto-FGFR1, which is in the pcDNA3.1(-) plasmid backbone, was described previously [15]. To clone cyto-opto-FGFR1, the myristoylation signal (MYR) of mem-opto-FGFR1 was replaced by the Kozak sequence using reverse PCR. Three nuclear localization sequences (NLSs) were inserted into cyto-opto-FGFR1 to obtain nucl-opto-FGFR1. Fluorescently labeled versions of opto-FGFR1 were produced by inserting mVenus $(\mathrm{mV})$ (Figure 1). All plasmids that were generated were verified by sequencing. 

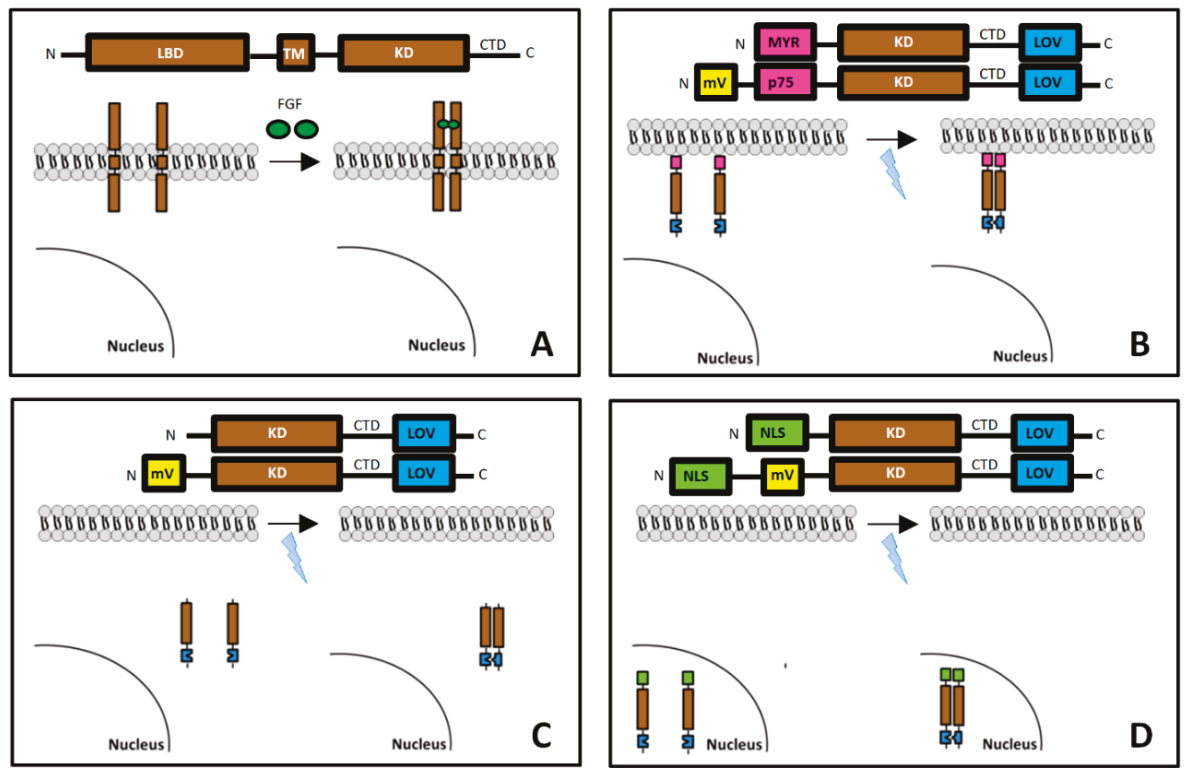

Figure 1. Design of light-controlled opto-fibroblast growth factor receptors (FGFR1s) and their molecular architecture: sequence of the different FGFR1 coding genes, spatial relation of FGFR1s to the surface membrane and nucleus, and their activation/dimerization. (A) Naturally occurring full-length FGFR1 consists of the extracellular ligand-binding (LBD), transmembrane (TM), kinase (KD) and C-terminal tail (CTD) domains. (B) Artificial mem-opto-FGFR1 is anchored to the plasma membrane with an N-terminal myristoylation signal (MYR) followed by the KD, CTD and LOV domain ( $\mathrm{mV}$-mem-opto-FGFR1 is inserted into the plasma membrane by incorporation of the transmembrane domain of p75). Fluorescent mVenus and the light-oxygen-voltage-sensing (LOV) protein are separated to avoid non-specific activation of the LOV domain by mVenus (by Förster resonance energy transfer). mVenus can be detected by excitation with green light $(514 \mathrm{~nm})$ that does not activate the LOV domain. (C) Cyto-opto-FGFR1 consists of only the KD and the LOV domain. (D) Three nuclear localization sequence (NLS) signals are inserted into nucl-opto-FGFR1 with or without mVenus.

\subsection{Cell Culture and Transfection}

Human embryonic kidney cells (HEK293, ATCC, Manassas, VA, USA) were grown in Dulbecco's Modified Eagle's Medium (DMEM, Sigma, St. Louis, MI, USA) supplemented with $10 \%$ fetal bovine serum (FBS; Thermo Fisher Scientific, Waltham, MA, USA, TFS) and 1\% antibiotic-antimycotic solution (100 U/mL penicillin, $100 \mu \mathrm{g} / \mathrm{mL}$ streptomycin, $0.25 \mu \mathrm{g} / \mathrm{mL}$ amphotericin, TFS). Rat adrenal pheochromocytoma (PC12) cells (Sigma) were grown in RPMI (Roswell Park Memorial Institute) 1640 (TFS) supplemented with $10 \%$ horse serum, $5 \%$ FBS, and $1 \%$ antibiotic-antimycotic solution on dishes coated with $50 \mu \mathrm{g} / \mathrm{mL}$ collagen type I (Merck, Kenilworth, NJ, USA). Human glioblastoma cells (U251) were cultivated in RPMI 1640 cell culture medium supplemented with 5\% FBS and 1\% antibiotic-antimycotic solution. All cell lines were grown under standard conditions in humidified atmosphere at $37^{\circ} \mathrm{C}$, with $5 \% \mathrm{CO}_{2}$. Transient transfections were performed with Lipofectamine 2000 (Invitrogen, Carlsbad, CA, USA) according to the manufacturer's protocol.

\subsection{Confocal Laser Scanning Microscopy}

For the localization of mV-opto-FGFR1s in HEK293 cells, $4 \times 10^{5}$ cells were seeded on \#1.5 coverslips and co-transfected with $1 \mu \mathrm{g}$ mV-opto-FGFR1 and $1 \mu \mathrm{g}$ LifeAct-mCherry (Addgene \#40908). Six hours after transfection, $25 \mu \mathrm{M}$ importazole (Sigma) was added to the cells overnight to prevent nuclear 
import of the mV-nucl-opto-FGFR1 construct. Cells were fixed in $4 \%$ formaldehyde solution (made from paraformaldehyde; PFA), stained with Hoechst 33258 dye, mounted in ProLong Diamond Antifade Mountant (TFS) and imaged using SP8 confocal laser scanning microscopy (Leica, Wetzlar, Germany).

\subsection{Immunogold Electron Microscopy}

For immunogold electron microscopy, Tokuyasu cryosection and cryo-based pre-embedding immunogold labelling were applied [16-18]. HEK293 cells were transfected with $1 \mu \mathrm{g}$ $\mathrm{mV}$-mem-opto-FGFR1 in 10-cm diameter dishes and kept overnight in DMEM supplemented with 10\% FBS and $1 \%$ antibiotic-antimycotic solution. Cells were fixed with $4 \%(w / v)$ buffered formaldehyde solution for $>3$ days at room temperature (RT). Ultra-thin cryosections were labelled with goat anti-GFP (\#600-101-215, 1:500, Rockland, Limerick, PA, USA), followed by rabbit anti-goat IgG 15-nm colloidal gold conjugates (\#EM.RAG15, 1:50, British Biocell Intl., Cardiff, UK). U251 cells were cultured on sapphire coverslips (Ø $3 \mathrm{~mm}$ ), transfected with $0.2 \mu \mathrm{g} \mathrm{mV}$-mem-opto-FGFR1 and kept overnight in RPMI 1640 supplemented with 5\% FBS and 1\% antibiotic-antimycotic solution. Samples were then subjected to high-pressure freezing, freeze-substitution, and rehydration, followed by pre-embedding labelling. Primary antibodies were goat anti-GFP (\#600-101-215, 1:2000, Rockland), secondary antibodies were Nanogold ${ }^{\circledR}-$ Fab' $^{\prime}$ rabbit anti-goat IgG $(\mathrm{H}+\mathrm{L})$ (\#2006; 1:700), followed by silver enhancement (SE) with HQ-Silver ${ }^{\circledR}$ (\#2012, all from Nanoprobes) and standard plastic embedding. Sections were analyzed by transmission electron microscopy.

\subsection{Stimulation and Immunoblot}

HEK293 cells were seeded on 6-well dishes $\left(1 \times 10^{6}\right.$ per dish, Corning, NY, USA $)$ and transfected with either $2 \mu \mathrm{g}$ opto-FGFR1 or $2 \mu \mathrm{g}$ FGFR1-eGFP [19]. Six hours after transfection, the medium was replaced with reduced serum medium $(0.5 \%$ FBS) and the cells were incubated overnight. One day after transfection, opto-FGFR1-transfected cells were stimulated for $5 \mathrm{~min}$ by $2.5 \mu \mathrm{W} / \mathrm{mm}^{2}$ blue light in a reptile egg incubator (PT2499, Exo Terra, Hagen, Holm, Germany) equipped with 300 light-emitting diodes. The blue light intensity was measured with a digital power meter connected to the Microscope Power Sensor Head (Thorlabs, Newton, NJ, USA). Cells that were kept in the dark (wrapped in aluminum foil) in the same incubator served as controls. FGFR1-eGFP-transfected cells were stimulated by treating them with $100 \mathrm{ng} / \mathrm{mL}$ FGF2 (Stemcell, Vancouver, BC, Canada) and $1 \mu \mathrm{g} / \mathrm{mL}$ heparin sulfate (HepS, Sigma) for $5 \mathrm{~min}$.

The lysis of light-stimulated cells was performed in the incubator under blue light. The dark samples were lysed under non-stimulating red light. Cells were harvested in $50 \mu \mathrm{L}$ lysis buffer on ice, sonicated and centrifuged (13,000 rpm, $\left.20 \mathrm{~min}, 4^{\circ} \mathrm{C}\right)$. To prevent ERK signaling, $50 \mu \mathrm{M}$ MAPK kinase inhibitor (PD98059; Sigma) was added to the cells $2 \mathrm{~h}$ prior to light activation or FGF2 stimulation. For the measurement of ERK kinetics, the ligand was washed out two times with PBS and lysates made after 5, 15 and $30 \mathrm{~min}$. In the case of opto-FGFR1-transfected cells, the lysates were obtained after 5,15 and $30 \mathrm{~min}$ of dark period. A total of $30 \mu \mathrm{g}$ protein per lane was separated by SDS-PAGE and transferred to Immobilon-FL PVDF membranes (Merck, Kenilworth, NJ, USA). Blots were incubated with primary antibodies in Odyssey Blocking Buffer (LI-COR) overnight at $4{ }^{\circ} \mathrm{C}$ (pFGFR1, \#3476, 1:1000; ERK1/2, \#9107, 1:1000; pERK1/2, \#9101, 1:2000; AKT, \#2920, 1:1000; pAKT, \#4060, 1:2000; pPLC $\gamma 1$, \#2821, 1:2000, all from Cell Signaling Technology/CST, Danvers, MA, USA; PLC $\gamma 1$, \#16955, 1:1000, Abcam; GAPDH, sc-32233, 1:500, Santa Cruz Biotechnology, Dallas, TX, USA). Secondary antibodies (IRDye 800CW goat anti-rabbit IgG, \#926-32231, and IRDye 680RD goat anti-mouse IgG, \#926-68070, both 1:20.000, LI-COR, Lincoln, NE, USA) were applied for $1 \mathrm{~h}$ at RT. Signals were recorded with an infrared fluorescence detection system (Odyssey Fc Imaging System).

Long-term light and ligand stimulation for neurite outgrowth assay and immunocytochemistry pheochromocytoma (PC12) cells was used for neuronal differentiation experiments. Cell lines enriched with mV-mem-opto-FGFR1-, mV-cyto-opto-FGFR1- or mV-nucl-opto-FGFR1-transfected cells were obtained as follows. Cells with a low passage number $(<\mathrm{P} 10)$ were seeded on 6-well plates (BD) and 
transiently transfected with $1 \mu \mathrm{g}$ of plasmids. After $24 \mathrm{~h}$, the culture medium was replaced by a medium containing $300 \mu \mathrm{g} / \mathrm{mL}$ Geneticin (G418, TFS), which was changed every other day. When sufficient numbers of resistant cell colonies were observed, the cells were triturated with fire-polished Pasteur pipettes for $10 \mathrm{~min}$ and then transferred to collagen-coated culture dishes. For microscopic analysis, cells were plated on 35-mm diameter collagen-coated imaging dishes (IBIDI). PC12 cells were also transiently transfected with $1 \mu \mathrm{g}$ FGFR1-eGFP. After $6 \mathrm{~h}$, cells were cultured in RPMI 1640 supplemented with $10 \%$ horse serum, $5 \%$ FBS, and $1 \%$ antibiotic-antimycotic solution overnight followed by a starvation medium supplemented with $2 \mathrm{mM} \mathrm{L-glutamin,} \%$ antibiotic-antimycotic solution, and N2 supplement (100×, TFS) for 2 h. FGFR1-eGFP-transfected cells and naive PC12 cells were then treated for $48 \mathrm{~h}$ with $100 \mathrm{ng} / \mathrm{mL}$ FGF2 $+1 \mu \mathrm{g} / \mathrm{mL} \mathrm{HepS}$ and with $100 \mathrm{ng} / \mathrm{mL}$ nerve growth factor (NGF), respectively. For light stimulation, the dishes were placed in a humidified atmosphere at $37^{\circ} \mathrm{C}$ with $5 \% \mathrm{CO}_{2}$ in an incubator with light-emitting diodes as described above. The blue light intensity was measured with a digital power meter and set to $2.5 \mu \mathrm{W} / \mathrm{mm}^{2}$. The control cells were kept in the dark in the same incubator during stimulation. A repetitive $5 \mathrm{~min}$ ON and $55 \mathrm{~min}$ OFF cycle was used for stimulation over $48 \mathrm{~h}$.

Cells were then fixed with 4\% PFA for $15 \mathrm{~min}$ and their neurites were visualized by immunocytochemistry. Neuron-specific class III beta-tubulin (1:1000, $1 \mathrm{~h}$ at RT; R\&D system) and Alexa 568 goat anti-mouse IgG (1:1000, $2 \mathrm{~h}$ at RT; TFS) were applied as the primary and secondary antibody, respectively. Inverted fluorescence microscopy (AxioVert, Zeiss, Oberkochen, Germany) at $40 \times$ magnification was used for imaging. Neurite outgrowth was defined if cells exhibited at least one process of more than one cell body diameter length.

For immunocytochemistry, PC12 cells enriched with mV-opto-FGFR1-transfected cells were used. Following serum starvation, light stimulation and fixation, cells were incubated overnight with primary antibodies (pERK1 /2, \#9101, 1:400, CST; pAKT, \#4060, 1:400, CST) followed by incubation at RT for $2 \mathrm{~h}$ with Alexa 546 goat anti-rabbit IgG (1:1000, TFS). SP8 confocal laser scanning microscopy (Leica) was used for cell imaging. Controls included the omission of primary antibodies (negative) and stimulation of cells with NGF (positive).

\subsection{Quantification of Results and Statistical Analysis}

The total neurite length (TNL, sum of the length of all neurites extending from the cell body), maximal distance (MD, length of longest neurite) and number of neurites per cell were quantified (Figure S1). Metamorph software version 4.6 (Visitron Systems, Puchheim, Germany) was used for the quantification of neurite outgrowth and immunocytochemistry. Band intensities of the Western blots were densitometrically quantified using Image Studio LiteVer 5.2. GraphPad Prism software was used for statistical analysis (two-way ANOVA with Sidak correction).

\section{Results}

\subsection{Localization of $m V$-opto-FGFR1s}

In HEK293 cells, mV-mem-opto-FGFR1 localized predominantly to the plasma membrane and to endosomes (Figure 2A1-A4), while mV-cyto-opto-FGFR1 diffused freely in the cytoplasm and nucleus (Figure 2B1-B4). The mV-nucl-opto-FGFR1 construct was found exclusively in the nucleus with enrichment of the protein in presumptive nuclear speckle domains (Figure 2C1-C4). Inhibition of the importin $\beta$ transport receptors with $25 \mu \mathrm{M}$ importazole resulted in diffuse cytoplasmic yellow fluorescence of $\mathrm{mV}$-nucl-opto-FGFR1, indicating a partial block of the nuclear import (Figure S2). Immunogold electron microscopy of the thawed cryosections revealed $\mathrm{mV}$-mem-opto-FGFR1 in the plasma membrane, in (late) endosomes/multivesicular bodies (MVBs) and lysosomes of HEK293 cells (Figure 2D1-D3). These data were further confirmed by a complementary immunogold labeling method applied to a different cell line (U251 glioblastoma cells) in which the same subcellular distribution of $\mathrm{mV}$-mem-opto-FGFR1 was found (Figure S3). 

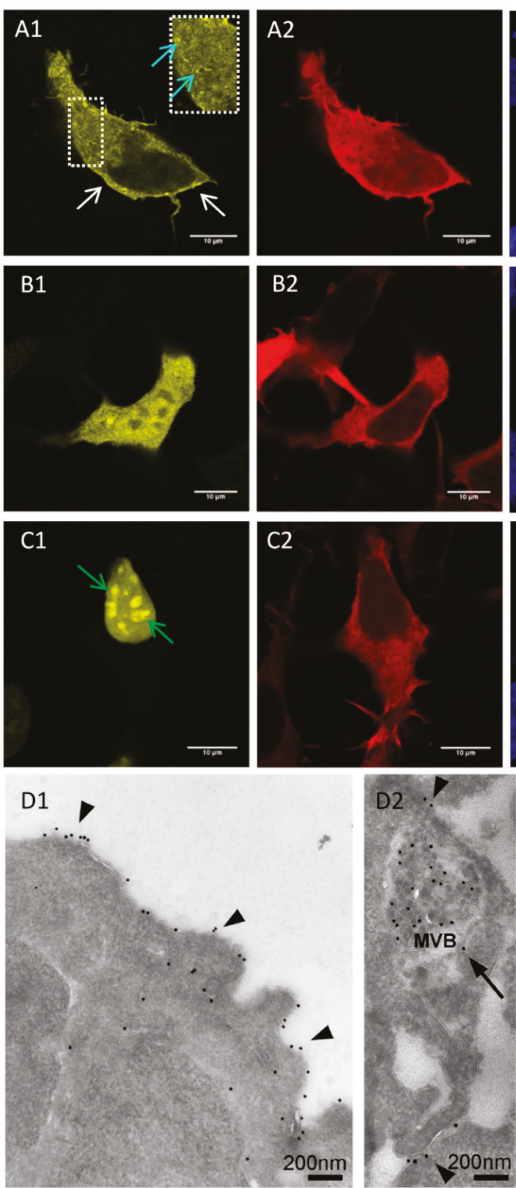
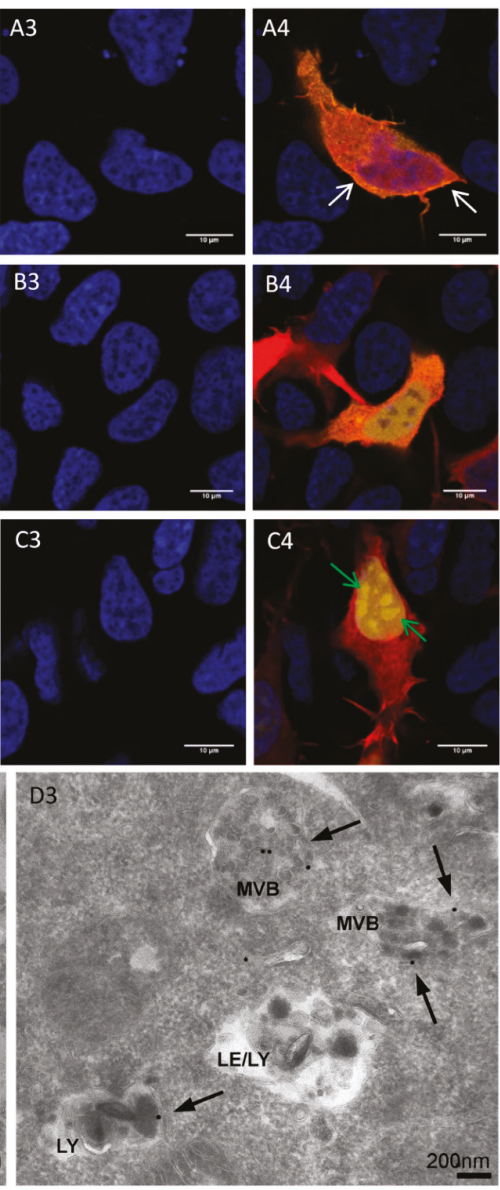

Figure 2. Light and electron microscopic localization of mV-opto-FGFR1s in human embryonic kidney (HEK293) cells. (A-C) Immunofluorescence microscopy of cells co-transfected with mV-opto-FGFR1s and LifeAct-mCherry to visualize cell bodies and all cytoplasmic processes. (A1) mV-mem-opto-FGFR1 is observed in the plasma membrane (white arrows) and in endosomes (inset; cyan arrows) indicating endosomal receptor internalization. (B1) mV-cyto-opto-FGFR1-transfected cells reveal diffuse yellow fluorescence in the cytoplasm and nucleus of transfected cells. (C1) mV-nucl-opto-FGFR1 is only located in the nucleus (nuclear speckle domains are indicated by green arrows). Fixed cell nuclei are stained with Hoechst (blue) and A4-C4 represent overlays. Scale bars for all images are $10 \mu \mathrm{m}$. (D) Immunogold electron microscopy of the thawed cryosections reveals $\mathrm{mV}$-mem-opto-FGFR1 in the plasma membrane (arrowheads in D1 and D2) and in the limiting membrane as well as inside various endocytic compartments (arrows in D2 and D3); MVB = multivesicular body, LE = late endosome, LY = lysosome.

\subsection{Opto-FGFR1-Dependent Signaling Pathway Activation in HEK293 Cells}

For all quantitative signaling assays, the levels of active FGFR1 (pFGFR1) and the control protein (GAPDH) were determined to normalize for different transfection efficiencies (Figure 3A,B). Immunoblot results showed that serum starvation (naive state) significantly reduced the basal level of pERK but not the basal levels of pAKT or pPLC $\gamma 1$ in HEK293 cells. The overexpression of FGFR1-eGFP induced ERK and AKT activation which was further increased by adding FGF2 for 5 min. No marked changes in PPLC $\gamma 1$ levels were observed following FGF2 treatment. 


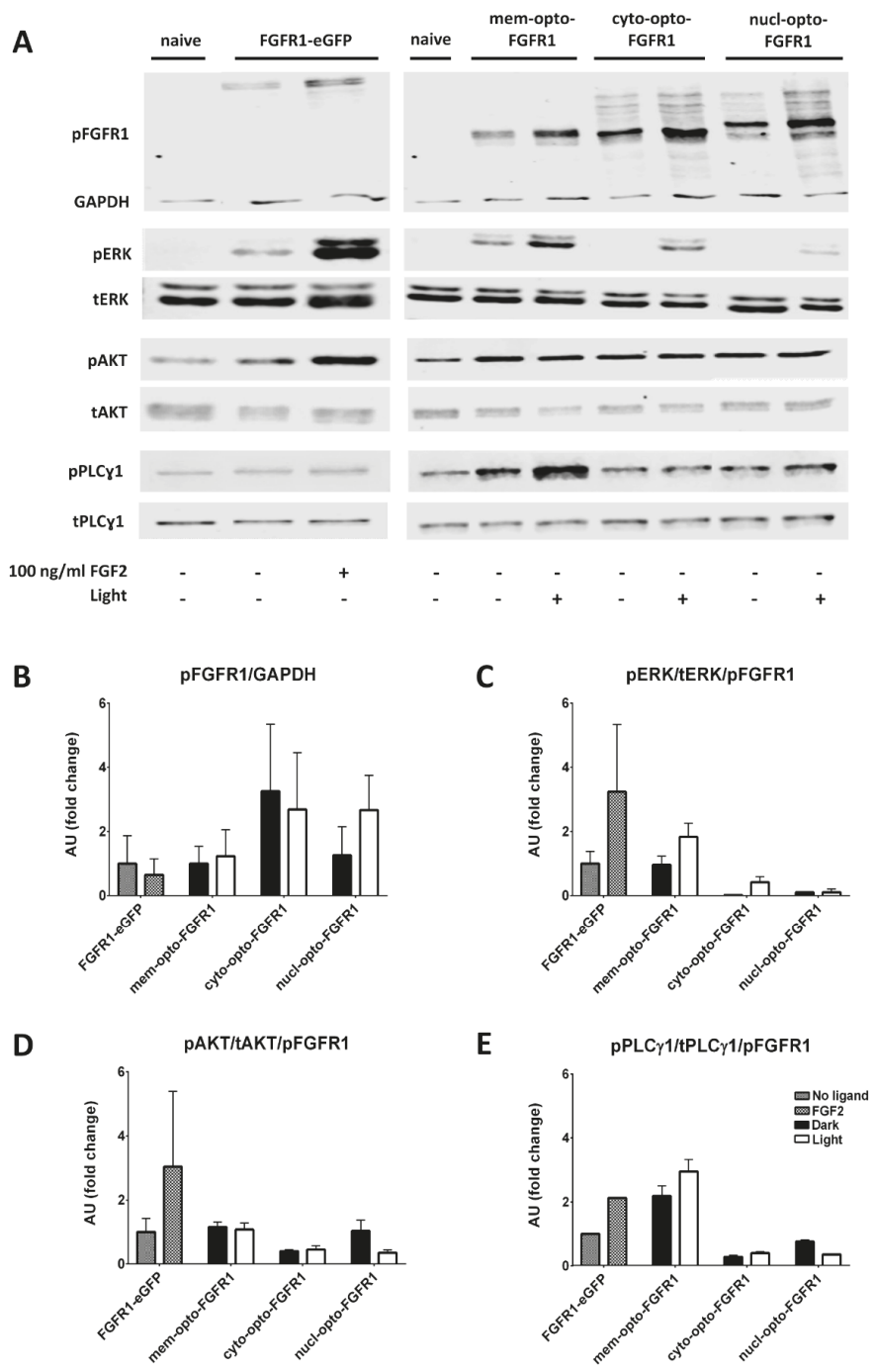

Figure 3. Immunoblot analysis of light- and FGF2-induced activation of key signaling molecules in HEK293 cells. (A) Representative examples of Western blots for pFGFR1/GAPDH, pERK/tERK, pAKT/tAKT and pPLCy1/tPLCy1 are shown. (B-E) Average intensities of ERK, AKT, and PLCy1 phosphorylation were quantified after normalization for pFGFR1/GAPDH to control for differences in plasmid expression levels. All ratios are relative to FGFR1-eGFP $(=1)$ and calculated from three independent experiments (mean \pm SEM).

The overexpression of mem-opto-FGFR1 alone (without light stimulation) resulted in receptor autoactivation, as indicated by increased pERK and pPLC $\gamma 1$ but unchanged pAKT levels (Figure 3A). Autoactivation was not observed after cyto-opto-FGFR1 or nucl-opto-FGFR1 transfection. Blue light stimulation for $5 \mathrm{~min}$ following mem-opto-FGFR1 or cyto-opto-FGFR1 transfection elevated the pERK levels (Figure 3C). Ligand-induced ERK activation lasted longer, whereas light-induced ERK activation was terminated faster (30 min versus 5-15 min, Figure S4). Minimal ERK activation was observed after transfection with nucl-opto-FGFR1. Unexpectedly, the pAKT levels remained unchanged after 
blue light stimulation of HEK293 cells following transfection with any of the opto-FGFR1 constructs (Figure 3D). On the other hand, the pPLC $\gamma 1 / \mathrm{tPLC} \gamma 1$ ratio slightly increased after blue light stimulation of mem-opto-FGFR1- but not of cyto- or nucl-opto-FGFR1-transfected cells (Figure 3E).

\subsection{Immunocytochemistry of $m V$-opto-FGFR1-Transfected PC12 Cells}

Immunocytochemistry was performed instead of protein blotting to visualize signaling pathway activation in PC12 cells (due to their lower transfection rate). Similarly to HEK293 cells, mem-opto-FGFR1-transfected pheochromocytoma cells exhibited increased pERK levels, as indicated by intense pERK immunolabeling in the cytoplasm (Figure 4A2/A6,G). As with HEK293 cells, no AKT activation was observed following intermittent blue light stimulation (Figure 4B2/B6,H). Furthermore, the pERK and pAKT levels were unchanged in stimulated mV-cyto-opto-FGFR1 (Figure 4C2/C6,D2/D6) or mV-nucl-opto-FGFR1-expressing PC12 cells (Figure 4E2/E6,F2/F6).
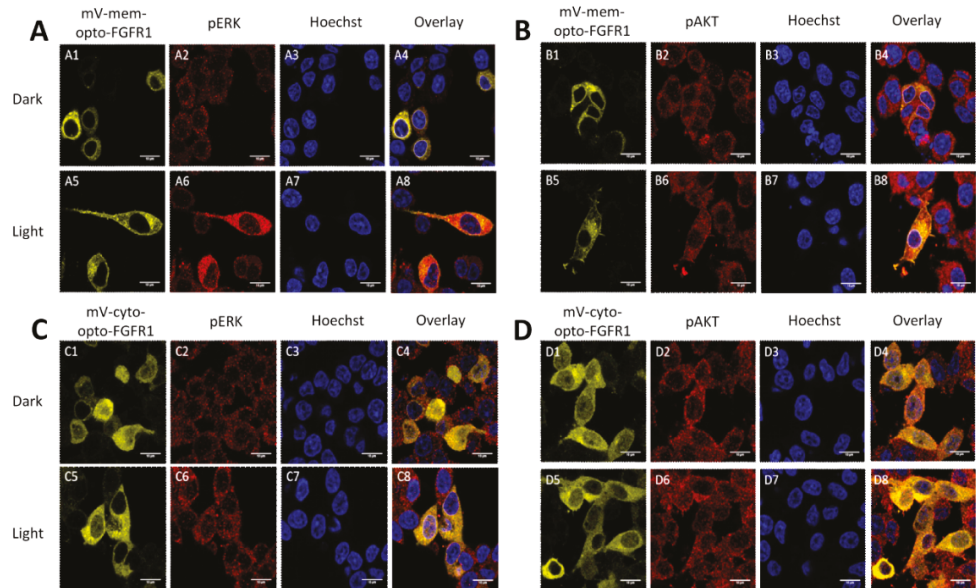

PERK

Hoechst
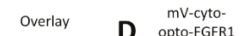

PAKT

Hoechst

Overlay
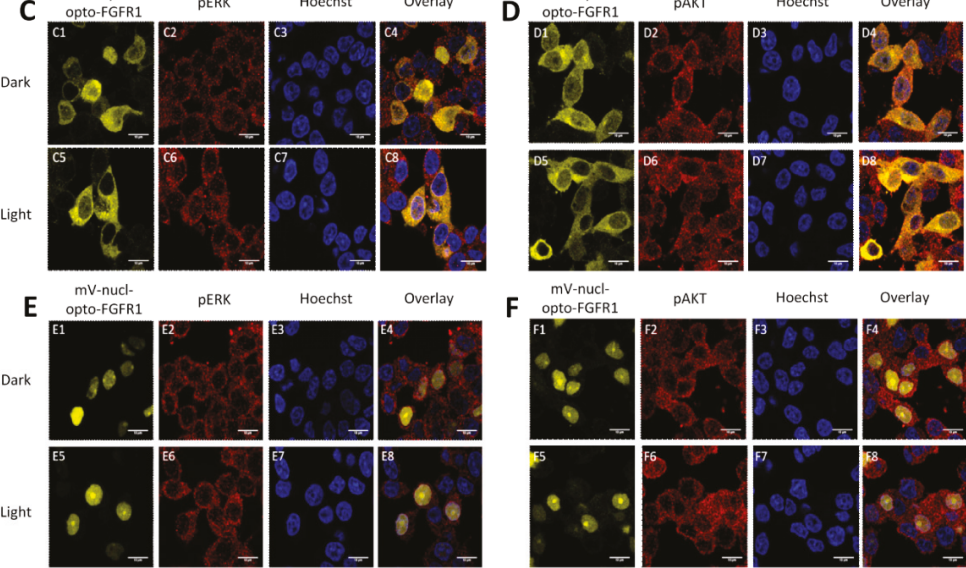

Overlay

mV-nucl-

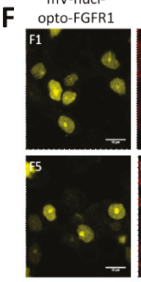

PAKT

Hoechst
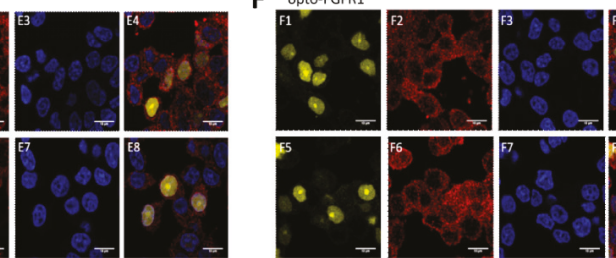

Overlay

G PERK

H

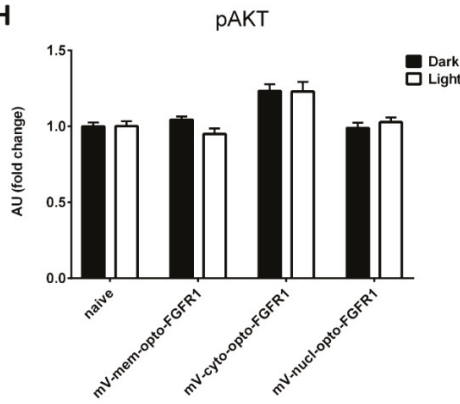

Figure 4. Light-induced ERK and AKT activation in mV-opto-FGFR1-transfected PC12 cells. Although mV-mem-opto-FGFR1-transfected cells exhibit short neurite extensions (sprouts) in the dark 
(A1), blue light induces neuronal differentiation with long, slender neurites in transfected cells (A5). (A) Significantly increased cytoplasmic pERK levels following light stimulation of mV-mem-opto-FGFR1-transfected cells, while non-transfected cells show no changes. (C,E) The pERK level is unchanged after stimulation of $\mathrm{mV}$-cyto- and nucl-opto-FGFR1-transfected cells. (B,D,F) The fluorescence intensities of pAKT signals are similar in mV-opto-FGFR1-transfected and non-transfected cells in the dark and no changes are observed after blue light stimulation. Images are acquired using the same laser intensity for both dark and light in each fluorescent channel and presented without adjusting contrast or subtracting background. $(\mathbf{G}, \mathbf{H})$ Quantification of average fluorescence intensities. Results are calculated from two independent experiments and presented as mean \pm SEM $(30<\mathrm{n}<60)$, $* * * * * 0.0001$. Scale bars $=10 \mu \mathrm{m}$.

\subsection{Neuronal Differentiation of PC12 Cells Induced by Blue Light}

PC12 cells exhibited no spontaneous or FGF2-induced neurite outgrowth, suggesting that the clone used in the present study does not express significant levels of endogenous FGF receptors (Figure 5A and Figure S5). In fact, all four FGFR mRNAs are endogenously expressed but the levels are low, particularly for FGFR1 (Figure S5E). Two days after treatment with NGF, neuronal differentiation was observed (Figure 5B; $120 \pm 11.9 \mu \mathrm{m}$ total neurite length, TNL, Figure 5K; $52.7 \pm 4 \mu \mathrm{m}$ of maximal neurite length, MD, Figure 5L; $2.6 \pm 0.12$ processes extending from the cell body, Figure 5M). Cells transiently transfected with FGFR1-eGFP revealed significantly longer neurites compared to naive cells (Figure 5C) and increased neurite initiation (Figure 5M). FGF2 treatment further enhanced neuronal differentiation with long neurites (Figure 5D). Although the autoactivation of $\mathrm{mV}$-mem-opto-FGFR1 induced mild neurite outgrowth in the dark state (Figure 5E), blue light stimulation resulted in dramatically increased neuronal differentiation (Figure 5F,K) which was significantly inhibited by prior PD98059 treatment (Figure S6). A significant increase in the number of neurites extending from $\mathrm{mV}$-mem-opto-FGFR1-transfected cells after blue light stimulation was observed as well as significantly longer neurites when compared to NGF and FGF2 treatment (Figure 5L,M). Cells expressing either mV-cyto-opto-FGFR1 or mV-nucl-opto-FGFR1 showed flattened, spindle-shaped morphology with short cytoplasmic extensions but failed to grow processes longer than one cell body in diameter (Figure 5G-J). 

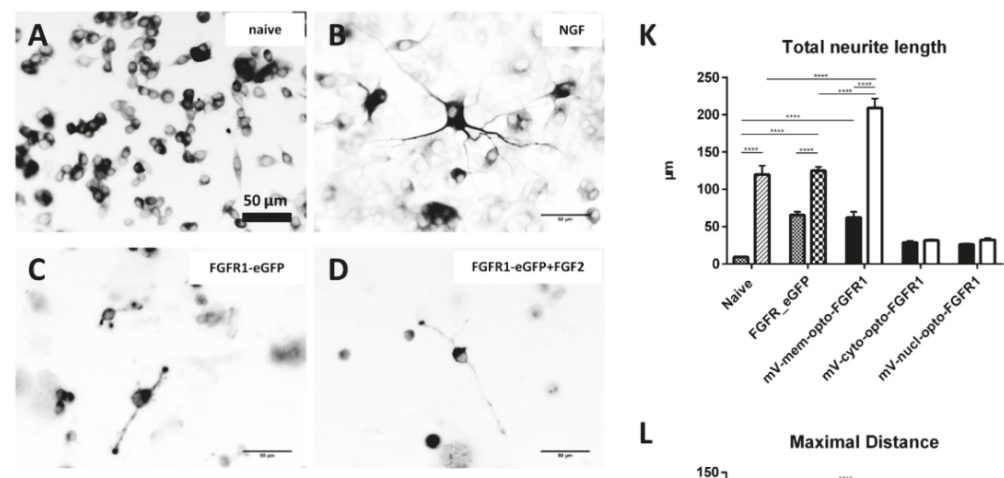

$\mathbf{L}$
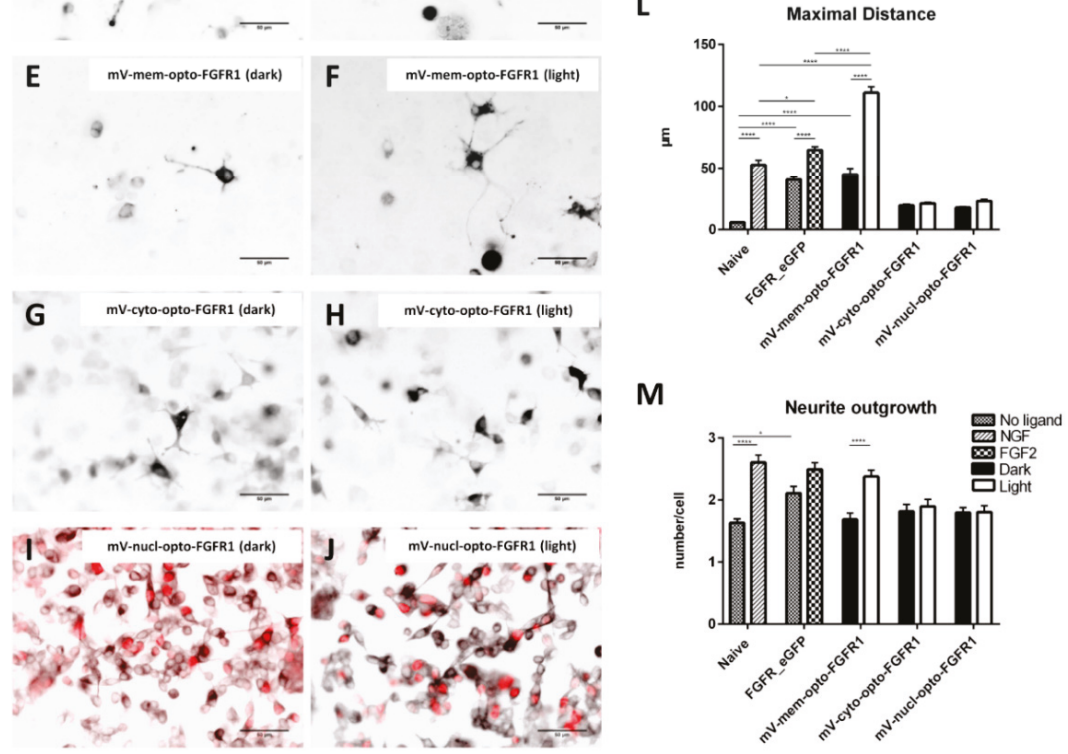

Figure 5. Ligand- and light-induced neurite outgrowth by pheochromocytoma (PC12) cells. (A-J) Inverted immunofluorescence images following neuron-specific class III $\beta$-tubulin staining to identify neurites (red nuclei in nucl-opto-FGFR1 cells allow identification of transfected cells in I/J). (K-M) Quantification of morphological parameters (total neurite outgrowth, longest process and number of processes per cell; see Figure S1 for details). Results are calculated from three independent experiments and presented as mean $\pm \operatorname{SEM}(50<\mathrm{n}<100),{ }^{*} p<0.05,{ }^{* * * *} p<0.0001$. Scale bars $=50 \mu \mathrm{m}$.

\section{Discussion}

Light-sensitive G-protein-coupled receptors (e.g., rhodopsin) occur naturally, whereas light-sensitive receptor tyrosine kinases (RTKs) need to be artificially produced. Recent studies have been aimed at subcellular targeting of opto-TrkA and light-gated adenylate cyclase [20,21]. In addition, various membrane-associated opto-RTK constructs were synthesized, such as opto-TrkB [22] and three different opto-FGFR1 constructs $[15,23,24]$. One of the light-activated FGFR1 proteins (through the homointeraction of cryptochrome 2) induced cell polarization and directed cell migration through changes in the actin-tubulin cytoskeleton [23]. Furthermore, opto-FGFR1 was applied for light-induced sprouting of human bronchial epithelial cells [15].

The opto-FGFR1 constructs used here were designed for specific targeting of the kinase domain to only the plasma membrane, cytoplasm, and nucleus, respectively, to investigate the possible effects of subcellular FGFR kinase activation on signal pathway induction and neurite 
outgrowth as a biological read-out. Similarly to full-length FGFR1, immunoelectron microscopy revealed that $\mathrm{mV}$-mem-opto-FGFR1s were anchored to the plasma membrane, internalized and transported to multivesicular bodies (MVBs)/late endosomes and lysosomes [25,26]. Although our construct was expected to only attach to membranes (plasma membrane, endosomal/lysosomal), $\mathrm{mV}$-mem-opto-FGFR1 was also occasionally observed in the cytoplasm and nucleus. It is known that internalized full-length FGFR1 may be released from endosomes and travels to the nucleus through importin $\beta$-mediated translocation and that newly synthetized FGFR1 may enter the nucleus directly as well [27-30].

Intranuclear FGFR1 is localized within nuclear matrix-attached speckle domains in the form of large discrete spots [31-33]. In this study, such fluorescence patterns were also observed in mV-nucl-opto-FGFR1-transfected cells exhibiting the split kinase domain of FGFR1 coupled to three NLSs. Biologically active, soluble kinase fragments are also created by cleavage at the transmembrane domain [34,35]. Similarly to these natural cytoplasmic FGFR1 fragments, mV-cyto-opto-FGFR1 constructs lacking specific targeting signals diffuse freely in the cytoplasm.

In this study, the activation of ERK but not of AKT was observed in cells expressing membrane-associated or, to a lesser extent, cytoplasmic opto-FGFR1 after blue light stimulation. The light-induced activation of both pathways has been described in connection with membrane-localized opto-TrkA and -TrkB receptors and with cytoplasmic opto-TrkA [20,22]. Moreover, significant ERK phosphorylation was observed after targeting active kinase domains of FGFR1, FGFR3 and FGFR4 to the plasma membrane [36]. Our findings are consistent with these and earlier studies from our own group demonstrating that FGFR1, in contrast to TrkA, exerts a significantly stronger influence on the ERK than on the AKT pathway [37-39].

Opto-constructs provide additional advantages such as that activation ceases shortly after switching off the light stimulus. In contrast to ligand-dependent RTK signaling, light-induced stimulation is terminated faster. In other studies, ERK nuclear translocation and immunoblot assays indicated similar ERK phosphorylation kinetics following light-induced Raf1 and Ras recruitment, as observed in our experiments [40,41]. However, although PI3K/AKT was expected to be activated after FGFR phosphorylation, we did not observe changes in the pAKT levels after light stimulation of opto-FGFR1s in transfected HEK293 and PC12 cells. These variations in signal pathway activation between different cell lines and different modes of induction may be explained by the differential expression of signaling adapters and possible crosstalk, particularly of the ERK- and AKT-dependent pathways [42,43].

PC12 cells are widely used to study neuronal differentiation. NGF induces biochemical, electrophysiological and morphological changes in these cells, recapitulating many characteristic features of differentiated sympathetic neurons [44,45]. The optogenetic activation of Trk receptors and stimulation of the Raf-MEK-ERK cascade have also been demonstrated to induce neuronal differentiation [41]. Due to the selective activation of ERK in the latter study, the neurites were longer than those of NGF-treated cells which could be explained by multiple downstream pathway activation including PI3K/AKT-mediated process branching. Taken together, we suggest that repetitive ERK stimulation acts as the primary driver of neurite extension, whereas AKT-dependent pathways primarily stimulate the formation of branches [46].

In this study, PC12 cells transfected with mV-mem-opto-FGFR1 exhibited longer neurites than NGF- or FGF2-treated cells and significantly more neurites extended from the cell body. Interestingly, light-induced stimulation of mem-opto-FGFR1 did not increase pAKT levels as compared to dark control cells, suggesting that the effects on neurite outgrowth are mainly, if not exclusively, dependent on the ERK pathway. Our immunocytochemistry results showed intense pERK immunolabeling in the cytoplasm, but only weak fluorescence in the nucleus $55 \mathrm{~min}$ after blue light stimulation. In contrast to other cell lines, the activated ERK2 variant (ERK2-MEK1 fusion protein) diffusely localized to the cytoplasm and to extending cellular processes. This localization pattern suggests that ERK2 is involved in promoting neurite formation, not only through its actions on gene transcription but also through 
effects at the site of neurite extension [47]. Light-induced neuronal differentiation was not observed in $\mathrm{mV}$-cyto- or in nucl-opto-FGFR1-transfected cells, corroborating earlier studies by Donoghue and his coworkers who achieved differentiation from plasma membrane-bound FGFR kinase domains in PC12 cells $[36,48]$. However, the full-length FGFR1 targeted to the nucleus also induced PC12 cell differentiation [12,49]. As discussed above, the binding of nuclear FGFR1 to CREB-binding protein (CBP) or to ribosomal S6 kinase isoform 1 (RSK1) may be involved in nuclear FGFR1 signaling $[49,50]$. Nevertheless, the activated kinase domain alone does not appear to be sufficient to modify gene expression, resulting in neuronal differentiation.

In summary, we demonstrated that only membrane-bound opto-FGFR1 constructs are capable of activating the ERK pathway sufficiently to induce neuronal cell differentiation in PC12 cells. We assume that fully functional signaling platforms only form in association with membranes. Active RTKs apparently recruit different adaptors and scaffold proteins in plasma membranes as compared to endosomal and other membranes which exhibit different curvatures and phosphatidylinositol compositions. Finally, the present study provides further evidence that FGFR1-dependent signaling pathways and neurite outgrowth can be controlled and manipulated optogenetically, which allows the study of subcellular receptor activation with spatial and temporal precision.

Supplementary Materials: Supplementary materials are available on http:/ /www.mdpi.com/2073-4409/8/3/ $243 /$ s1.

Author Contributions: Conceptualization, L.K.; methodology and formal analysis, K.C. and M.W.H.; writing-original draft preparation, K.C.; writing-review and editing, M.W.H. and L.K.; supervision, L.K.; funding acquisition, K.C. and L.K.

Funding: This study was supported by the intramural funding program of the Medical University Innsbruck for young scientist MUI-START, Project 2016-01-003, to K.C.

Acknowledgments: Special thanks go to Markus Reindl for providing HEK293 and U251 cells. K.C. is grateful to Barbara Hausott, Barbara Fogli and Martina Wick for help with several methods used in this study. Maximilian Klein helped in quantification of PC12 neuronal differentiation. Barbara Witting and Karin Gutleben are acknowledged for excellent technical assistance.

Conflicts of Interest: The authors declare no conflict of interest.

\section{References}

1. Lee, P.L.; Johnson, D.E.; Cousens, L.S.; Fried, V.A.; Williams, L.T. Purification and complementary DNA cloning of a receptor for basic fibroblast growth factor. Science 1989, 245, 57-60. [CrossRef]

2. Zhou, F.Q.; Snider, W.D. Intracellular control of developmental and regenerative axon growth. Philos. Trans. R. Soc. Lond. B. Biol. Sci. 2006, 361, 1575-1592. [CrossRef] [PubMed]

3. Eswarakumar, V.P.; Lax, I.; Schlessinger, J. Cellular signaling by fibroblast growth factor receptors. Cytokine Growth Factor Rev. 2005, 16, 139-149. [CrossRef] [PubMed]

4. Platta, H.W.; Stenmark, H. Endocytosis and signaling. Curr. Opin. Cell Biol. 2011, 23, 393-403. [CrossRef]

5. MacInnis, B.L.; Campenot, R.B. Retrograde support of neuronal survival without retrograde transport of nerve growth factor. Science 2002, 295, 1536-1539. [CrossRef] [PubMed]

6. Hausott, B.; Vallant, N.; Hochfilzer, M.; Mangger, S.; Irschick, R.; Haugsten, E.M.; Klimaschewski, L. Leupeptin enhances cell surface localization of fibroblast growth factor receptor 1 in adult sensory neurons by increased recycling. Eur. J. Cell Biol. 2012, 91, 129-138. [CrossRef] [PubMed]

7. Stachowiak, E.K.; Maher, P.A.; Tucholski, J.; Mordechai, E.; Joy, A.; Moffett, J.; Coons, S.; Stachowiak, M.K. Nuclear accumulation of fibroblast growth factor receptors in human glial cells-association with cell proliferation. Oncogene 1997, 14, 2201-2211. [CrossRef] [PubMed]

8. Planque, N. Nuclear trafficking of secreted factors and cell-surface receptors: New pathways to regulate cell proliferation and differentiation, and involvement in cancers. Cell Commun. Signal. 2006, 4, 7. [CrossRef] [PubMed] 
9. Peng, H.; Myers, J.; Fang, X.; Stachowiak, E.K.; Maher, P.A.; Martins, G.G.; Popescu, G.; Berezney, R.; Stachowiak, M.K. Integrative nuclear FGFR1 signaling (INFS) pathway mediates activation of the tyrosine hydroxylase gene by angiotensin II, depolarization and protein kinase C. J. Neurochem. 2002, 81, 506-524. [CrossRef]

10. Dunham-Ems, S.M.; Pudavar, H.E.; Myers, J.M.; Maher, P.A.; Prasad, P.N.; Stachowiak, M.K. Factors controlling fibroblast growth factor receptor-1's cytoplasmic trafficking and its regulation as revealed by FRAP analysis. Mol. Biol. Cell 2006, 17, 2223-2235. [CrossRef]

11. Stachowiak, M.K.; Birkaya, B.; Aletta, J.M.; Narla, S.T.; Benson, C.A.; Decker, B.; Stachowiak, E.K. Nuclear FGF receptor-1 and CREB binding protein: An integrative signaling module. J. Cell. Physiol. 2015, 230, 989-1002. [CrossRef] [PubMed]

12. Lee, Y.W.; Stachowiak, E.K.; Birkaya, B.; Terranova, C.; Capacchietti, M.; Claus, P.; Aletta, J.M.; Stachowiak, M.K. NGF-induced cell differentiation and gene activation is mediated by integrative nuclear FGFR1 signaling (INFS). PLoS ONE 2013, 8, e68931. [CrossRef] [PubMed]

13. Horbinski, C.; Stachowiak, E.K.; Chandrasekaran, V.; Miuzukoshi, E.; Higgins, D.; Stachowiak, M.K. Bone morphogenetic protein-7 stimulates initial dendritic growth in sympathetic neurons through an intracellular fibroblast growth factor signaling pathway. J. Neurochem. 2002, 80, 54-63. [CrossRef]

14. Li, X.; Wang, C.; Xiao, J.; McKeehan, W.L.; Wang, F. Fibroblast growth factors, old kids on the new block. Semin. Cell Dev. Biol. 2016, 53, 155-167. [CrossRef] [PubMed]

15. Grusch, M.; Schelch, K.; Riedler, R.; Reichhart, E.; Differ, C.; Berger, W.; Inglés-Prieto, Á.; Janovjak, H. Spatio-temporally precise activation of engineered receptor tyrosine kinases by light. EMBO J. 2014, 33, 1713-1726. [CrossRef]

16. Tokuyasu, K.T. A technique for ultracryotomy of cell suspensions and tissues. J. Cell Biol. 1973, 57, 551-565. [CrossRef]

17. Liou, W.; Geuze, H.J.; Slot, J.W. Improving structural integrity of cryosections for immunogold labeling. Histochem. Cell Biol. 1996, 106, 41-58. [CrossRef]

18. Hess, M.W.; Vogel, G.F.; Yordanov, T.E.; Witting, B.; Gutleben, K.; Ebner, H.L.; de Araujo, M.E.G.; Filipek, P.A.; Huber, L.A. Combining high-pressure freezing with pre-embedding immunogold electron microscopy and tomography. Traffic 2018, 19, 639-649. [CrossRef] [PubMed]

19. Jin, Y.; Pasumarthi, K.B.; Bock, M.E.; Lytras, A.; Kardami, E.; Cattini, P.A. Cloning and expression of fibroblast growth factor receptor-1 isoforms in the mouse heart: Evidence for isoform switching during heart development. J. Mol. Cell. Cardiol. 1994, 26, 1449-1459. [CrossRef] [PubMed]

20. Duan, L.; Hope, J.M.; Guo, S.; Ong, Q.; François, A.; Kaplan, L.; Scherrer, G.; Cui, B. Optical Activation of TrkA Signaling. ACS Synth. Biol. 2018, 7, 1685-1693. [CrossRef] [PubMed]

21. Tsvetanova, N.G.; von Zastrow, M. Spatial encoding of cyclic AMP signaling specificity by GPCR endocytosis. Nat. Chem. Biol. 2014, 10, 1061-1065. [CrossRef]

22. Chang, K.Y.; Woo, D.; Jung, H.; Lee, S.; Kim, S.; Won, J.; Kyung, T.; Park, H.; Kim, N.; Yang, H.W.; et al. Light-inducible receptor tyrosine kinases that regulate neurotrophin signalling. Nat. Commun. 2014, 5, 4057. [CrossRef] [PubMed]

23. Kim, N.; Kim, J.M.; Lee, M.; Kim, C.Y.; Chang, K.Y.; Heo, W.D. Spatiotemporal control of fibroblast growth factor receptor signals by blue light. Chem. Biol. 2014, 21, 903-912. [CrossRef]

24. Reichhart, E.; Ingles-Prieto, A.; Tichy, A.M.; McKenzie, C.; Janovjak, H. A Phytochrome Sensory Domain Permits Receptor Activation by Red Light. Angew. Chem. Int. Ed. Engl. 2016, 55, 6339-6342. [CrossRef] [PubMed]

25. Haugsten, E.M.; Sørensen, V.; Brech, A.; Olsnes, S.; Wesche, J. Different intracellular trafficking of FGF1 endocytosed by the four homologous FGF receptors. J. Cell Sci. 2005, 118, 3869-3881. [CrossRef]

26. Irschick, R.; Trost, T.; Karp, G.; Hausott, B.; Auer, M.; Claus, P.; Klimaschewski, L. Sorting of the FGF receptor 1 in a human glioma cell line. Histochem. Cell Biol. 2013, 139, 135-148. [CrossRef] [PubMed]

27. Maher, P.A. Nuclear Translocation of fibroblast growth factor (FGF) receptors in response to FGF-2. J. Cell Biol. 1996, 134, 529-536. [CrossRef]

28. Małecki, J.; Wiedłocha, A.; Wesche, J.; Olsnes, S. Vesicle transmembrane potential is required for translocation to the cytosol of externally added FGF-1. EMBO J. 2002, 21, 4480-4490. [CrossRef]

29. Reilly, J.F.; Maher, P.A. Importin beta-mediated nuclear import of fibroblast growth factor receptor: Role in cell proliferation. J. Cell Biol. 2001, 152, 1307-1312. [CrossRef] 
30. Stachowiak, M.K.; Fang, X.; Myers, J.M.; Dunham, S.M.; Berezney, R.; Maher, P.A.; Stachowiak, E.K. Integrative nuclear FGFR1 signaling (INFS) as a part of a universal "feed-forward-and-gate" signaling module that controls cell growth and differentiation. J. Cell. Biochem. 2003, 90, 662-691. [CrossRef]

31. Somanathan, S.; Stachowiak, E.K.; Siegel, A.J.; Stachowiak, M.K.; Berezney, R. Nuclear matrix bound fibroblast growth factor receptor is associated with splicing factor rich and transcriptionally active nuclear speckles. J. Cell. Biochem. 2003, 90, 856-869. [CrossRef] [PubMed]

32. Stachowiak, M.K.; Maher, P.A.; Joy, A.; Mordechai, E.; Stachowiak, E.K. Nuclear localization of functional FGF receptor 1 in human astrocytes suggests a novel mechanism for growth factor action. Brain. Res. Mol. Brain. Res. 1996, 38, 161-165. [CrossRef]

33. Stachowiak, M.K.; Maher, P.A.; Joy, A.; Mordechai, E.; Stachowiak, E.K. Nuclear accumulation of fibroblast growth factor receptors is regulated by multiple signals in adrenal medullary cells. Mol. Biol. Cell 1996, 7, 1299-1317. [CrossRef] [PubMed]

34. Chioni, A.M.; Grose, R. FGFR1 cleavage and nuclear translocation regulates breast cancer cell behavior. J. Cell. Biol. 2012, 197, 801-817. [CrossRef] [PubMed]

35. Coleman, S.J.; Bruce, C.; Chioni, A.M.; Kocher, H.M.; Grose, R.P. The ins and outs of fibroblast growth factor receptor signalling. Clin. Sci. (Lond) 2014, 127, 217-231. [CrossRef] [PubMed]

36. Hart, K.C.; Robertson, S.C.; Kanemitsu, M.Y.; Meyer, A.N.; Tynan, J.A.; Donoghue, D.J. Transformation and Stat activation by derivatives of FGFR1, FGFR3, and FGFR4. Oncogene 2000, 19, 3309-3320. [CrossRef]

37. Klimaschewski, L.; Nindl, W.; Feurle, J.; Kavakebi, P.; Kostron, H. Basic fibroblast growth factor isoforms promote axonal elongation and branching of adult sensory neurons in vitro. Neuroscience 2004, 126, 347-353. [CrossRef]

38. Hausott, B.; Schlick, B.; Vallant, N.; Dorn, R.; Klimaschewski, L. Promotion of neurite outgrowth by fibroblast growth factor receptor 1 overexpression and lysosomal inhibition of receptor degradation in pheochromocytoma cells and adult sensory neurons. Neuroscience 2008, 153, 461-473. [CrossRef]

39. Hausott, B.; Rietzler, A.; Vallant, N.; Auer, M.; Haller, I.; Perkhofer, S.; Klimaschewski, L. Inhibition of fibroblast growth factor receptor 1 endocytosis promotes axonal branching of adult sensory neurons. Neuroscience 2011, 188, 13-22. [CrossRef]

40. Toettcher, J.E.; Weiner, O.D.; Lim, W.A. Using optogenetics to interrogate the dynamic control of signal transmission by the Ras/Erk module. Cell 2013, 155, 1422-1434. [CrossRef]

41. Zhang, K.; Duan, L.; Ong, Q.; Lin, Z.; Varman, P.M.; Sung, K.; Cui, B. Light-mediated kinetic control reveals the temporal effect of the Raf/MEK/ERK pathway in PC12 cell neurite outgrowth. PLoS ONE 2014, 9, e92917. [CrossRef]

42. Hayashi, H.; Tsuchiya, Y.; Nakayama, K.; Satoh, T.; Nishida, E. Down-regulation of the PI3-kinase/Akt pathway by ERK MAP kinase in growth factor signaling. Genes Cells 2008, 13, 941-947. [CrossRef] [PubMed]

43. Hensel, N.; Baskal, S.; Walter, L.M.; Brinkmann, H.; Gernert, M.; Claus, P. ERK and ROCK functionally interact in a signaling network that is compensationally upregulated in Spinal Muscular Atrophy. Neurobiol. Dis. 2017, 108, 352-361. [CrossRef] [PubMed]

44. Angelastro, J.M.; Klimaschewski, L.; Tang, S.; Vitolo, O.V.; Weissman, T.A.; Donlin, L.T.; Shelanski, M.L.; Greene, L.A. Identification of diverse nerve growth factor-regulated genes by serial analysis of gene expression (SAGE) profiling. Proc. Natl. Acad. Sci. USA 2000, 97, 10424-10429. [CrossRef]

45. Greene, L.A.; Tischler, A.S. Establishment of a noradrenergic clonal line of rat adrenal pheochromocytoma cells which respond to nerve growth factor. Proc. Natl. Acad. Sci. USA 1976, 73, 2424-2428. [CrossRef] [PubMed]

46. Hausott, B.; Klimaschewski, L. Membrane turnover and receptor trafficking in regenerating axons. Eur. J. Neurosci. 2016, 43, 309-317. [CrossRef]

47. Robinson, M.J.; Stippec, S.A.; Goldsmith, E.; White, M.A.; Cobb, M.H. A constitutively active and nuclear form of the MAP kinase ERK2 is sufficient for neurite outgrowth and cell transformation. Curr. Biol. 1998, 8, 1141-1150. [CrossRef]

48. Webster, M.K.; Donoghue, D.J. Enhanced signaling and morphological transformation by a membrane-localized derivative of the fibroblast growth factor receptor 3 kinase domain. Mol. Cell. Biol. 1997, 17, 5739-5747. [CrossRef] 
49. Peng, H.; Moffett, J.; Myers, J.; Fang, X.; Stachowiak, E.K.; Maher, P.; Kratz, E.; Hines, J.; Fluharty, S.J.; Mizukoshi, E.; et al. Novel nuclear signaling pathway mediates activation of fibroblast growth factor-2 gene by type 1 and type 2 angiotensin II receptors. Mol. Biol. Cell 2001, 12, 449-462. [CrossRef]

50. Hu, Y.; Fang, X.; Dunham, S.M.; Prada, C.; Stachowiak, E.K.; Stachowiak, M.K. 90-kDa ribosomal S6 kinase is a direct target for the nuclear fibroblast growth factor receptor 1 (FGFR1): Role in FGFR1 signaling. J. Biol. Chem. 2004, 279, 29325-29335. [CrossRef]

(C) 2019 by the authors. Licensee MDPI, Basel, Switzerland. This article is an open access article distributed under the terms and conditions of the Creative Commons Attribution (CC BY) license (http:/ / creativecommons.org/licenses/by/4.0/). 



\title{
GPER Mediates a Feedforward FGF2/FGFR1 Paracrine Activation Coupling CAFs to Cancer Cells toward Breast Tumor Progression
}

\author{
Maria Francesca Santolla ${ }^{1}$, Adele Vivacqua ${ }^{1}$, Rosamaria Lappano ${ }^{1}$, \\ Damiano Cosimo Rigiracciolo ${ }^{1}$, Francesca Cirillo ${ }^{1}$, Giulia Raffaella Galli ${ }^{1}$, Marianna Talia ${ }^{1}$, \\ Giuseppe Brunetti ${ }^{2}$, Anna Maria Miglietta ${ }^{3}$, Antonino Belfiore ${ }^{4}$ and Marcello Maggiolini ${ }^{1, *}$ \\ 1 Department of Pharmacy, Health and Nutritional Sciences, University of Calabria, 87036 Rende, Italy; \\ mariafrancesca.santolla@unical.it (M.F.S.); adele.vivacqua@unical.it (A.V.); \\ rosamaria.lappano@unical.it (R.L.); damianorigiracciolo@yahoo.it (D.C.R.); francesca.cirillo@unical.it (F.C.); \\ giulia.r.galli@gmail.com (G.R.G.); mariannatalia11@gmail.com (M.T.) \\ 2 University of Natural Resources and Life Sciences, 1180 Vienna, Austria; giusep.bru@gmail.com \\ 3 Regional Hospital, 87100 Cosenza, Italy; annamariamiglietta@virgilio.it \\ 4 Endocrinology, Department of Clinical and Experimental Medicine, University of Catania, Garibaldi-Nesima \\ Hospital, 95122 Catania, Italy; antonino.belfiore@unict.it \\ * Correspondence: marcellomaggiolini@yahoo.it or marcello.maggiolini@unical.it
}

Received: 18 February 2019; Accepted: 4 March 2019; Published: 7 March 2019

\begin{abstract}
The FGF2/FGFR1 paracrine loop is involved in the cross-talk between breast cancer cells and components of the tumor stroma as cancer-associated fibroblasts (CAFs). By quantitative PCR (qPCR), western blot, immunofluorescence analysis, ELISA and ChIP assays, we demonstrated that $17 \beta$-estradiol (E2) and the $G$ protein estrogen receptor (GPER) agonist G-1 induce the up-regulation and secretion of FGF2 via GPER together with the EGFR/ERK/c-fos/AP-1 signaling cascade in (ER)-negative primary CAFs. Evaluating the genetic alterations from METABRIC and TCGA datasets, we then assessed that FGFR1 is the most frequently amplified FGFRs family member and its amplification/expression associates with shorter survival rates in breast cancer patients. Therefore, in order to assess the functional FGF2/FGFR1 interplay between CAFs and breast cancer cells, we generated the FGFR1-knockout MDA-MB-231 cells using CRISPR/Cas9 genome editing strategy. Using conditioned medium from estrogen-stimulated CAFs, we established that the activation of FGF2/FGFR1 paracrine signaling triggers the expression of the connective tissue growth factor (CTGF), leading to the migration and invasion of MDA-MB-231 cells. Our findings shed new light on the role elicited by estrogens through GPER in the activation of the FGF2/FGFR1 signaling. Moreover, our findings may identify further biological targets that could be considered in innovative combination strategies halting breast cancer progression.
\end{abstract}

Keywords: cancer-associated fibroblasts; GPER; breast cancer; estrogen; FGFR1; FGF2

\section{Introduction}

Cross-talk between stromal and epithelial cells plays an important role in diverse pathophysiological conditions, including malignant diseases [1-3]. In this regard, it has been largely reported that the acquisition of an aggressive phenotype does not depend exclusively on the intrinsic cancer cell properties, but also on stromal features [4]. For instance, cancer-associated fibroblasts (CAFs), one of the most abundant cell types within the tumor microenvironment, coordinate a multifaceted biochemical program that promotes cancer cell proliferation, migration, invasion, epithelial-mesenchymal transition (EMT), and angiogenesis [5]. Indeed, it has been shown that paracrine 
mediators secreted by CAFs, such as cytokines and growth factors, exert an important role in the acquisition of malignant features [6,7].

The fibroblast growth factor (FGF)-FGF receptor (FGFR) axis is one of the major signal transduction pathways mediating the interaction between tumor stroma and cancer cells $[8,9]$. FGFRs family includes four highly conserved transmembrane receptor tyrosine kinases (FGFR1-4) and one receptor that binds to FGF ligands, although it lacks the intracellular kinase domain (FGFR5, also known as FGFRL1) [10]. FGFRs can be activated either in an autocrine fashion by FGFs produced by the tumor cells or in a paracrine manner by FGFs secreted by the stromal components [11]. The activation of FGFRs triggers the phosphorylation of extracellular signal-regulated kinase (ERK), phosphatidylinositol 3-kinase (PI3K), and other transduction pathways, regulating many physiological processes including embryogenesis, tissue homeostasis, and angiogenesis [12,13]. Abnormal activation of the FGFR1-mediated signaling pathway can be caused by translocation, point mutation and amplification of the FGFR1 gene, hence leading to malignant transformation and cancer progression $[9,14,15]$. Likewise, increased FGF2 levels have been observed in plasma samples of patients affected by diverse malignancies, such as leukemia and lung and breast cancers, especially when metastases are present $[16,17]$. Among diverse stimuli, FGF2 expression and secretion can be regulated by estrogens $[18,19]$, which act mainly through the classical estrogen receptors (ER) $\alpha$ and ER $\beta$ leading to the proliferation, migration and survival of breast cancer cells [20]. The $G$ protein estrogen receptor (GPER, also called GPR30) has been identified as a further receptor able to mediate the action of estrogens in numerous pathophysiological conditions [21,22]. GPER activation induces a network of signal transduction pathways including activation of the epidermal growth factor receptor (EGFR), intracellular cyclic adenosine monophosphate (AMP) accumulation, calcium mobilization, activation of ERK1/2 and PI3K [23]. Moreover, GPER triggers the expression of various genes involved in the growth and migration of diverse estrogen-responsive tumors [24-27]. Of note, the stimulatory action mediated by estrogenic GPER signaling has been also evidenced in breast primary CAFs revealing the existence of a functional cooperation between these important components of the tumor stroma and cancer cells [28-31]. Recent studies have shown that a functional interaction between ER and FGFR-mediated pathways may occur toward breast cancer progression, indicating that the simultaneous inhibition of both receptors could be considered in more comprehensive treatments $[11,32,33]$. GPER was also involved in the estrogen-induced regulation of FGF2 toward the autocrine stimulation of the cognate receptor FGFR1 in astroglial cells [19].

Here, we provided novel insights into the ability of estrogens to regulate a feedforward FGF2/FGFR1 activation between the ER-negative CAFs and breast cancer cells. On the basis of our findings, GPER may be included among the factors facilitating the estrogen-activated cross-talk within the tumor microenvironment toward breast tumor progression.

\section{Materials and Methods}

\subsection{Reagents}

We purchased (1-[4-(-6-bromobenzol [1,3] diodo-5-yl)-3a,4,5,9b-tetrahidro3H5cyclopenta[c] quinolin-8yl]-ethanone) (G-1), (3aS,4R,9bR)-4-(6-Bromo-1, 3-benzodioxol-5-yl)-3a,4,5,9b-3H-cyclopenta [c]quinolone (G15) from Tocris Bioscience (Space, Milan, Italy); 17 $\beta$-Estradiol (E2), Wortmannin (WM) from Sigma-Aldrich (Milan, Italy); PD173074 from Selleckchem (DBA, Milan, Italy); PD98059 from Calbiochem (DBA, Milan, Italy); tyrphostin AG1478 from Biomol Research Laboratories (Milan, Italy) and recombinant human Fibroblast Growth Factor (FGF2) 100-18B, from PEPROTECH (SIAL, Rome, Italy). All compounds were solubilized in dimethyl sulfoxide (DMSO), except for FGF2, which was dissolved in aqueous buffer $(0.1 \%$ BSA). 


\subsection{Cell Cultures}

MDA-MB-231, MCF-7, and SkBr3 breast cancer cells were obtained from the ATCC (Manassas, USA). MDA-MB-231 and MCF-7 cells were maintained in DMEM/F12 (Life Technologies, Milan, Italy), $10 \%$ fetal bovine serum (FBS) and 1\% of penicillin/streptomycin, while SkBr3 cells were maintained in RPMI-1640 (Life Technologies, Milan, Italy), 10\% fetal bovine serum (FBS), and $1 \%$ of penicillin/streptomycin (Life Technologies, Milan, Italy). Cells were used less than six months after resuscitation and mycoplasma negativity was tested monthly. CAFs were extracted from invasive mammary ductal carcinomas obtained from mastectomies, while normal fibroblasts (NFs) were isolated from a non-cancerous breast tissue at least $2 \mathrm{~cm}$ from the outer tumor margin, as previously described $[25,28,34]$. Primary cells cultures of breast fibroblasts were characterized by immunofluorescence. Cells were incubated with anti-vimentin (V9, sc-6260), anti-cytokeratin 14 (LL001 sc-53,253) and anti-fibroblast activated protein $\alpha(\mathrm{FAP} \alpha)(\mathrm{H}-56)$ antibodies that were obtained from Santa Cruz Biotechnology (DBA, Milan, Italy) (Supplementary Figure S1). All cells were grown in a $37^{\circ} \mathrm{C}$ incubator with $5 \% \mathrm{CO}_{2}$ and switched to medium without serum and phenol red the day before treatments to be processed for immunoblot and quantitative PCR (qPCR) assays.

\subsection{Gene Expression Studies}

Total RNA was extracted and complementary DNA (cDNA) was synthesized by reverse transcription as described in our previous work [35]. Quantitative PCR (qPCR) assays were performed using platform Quant Studio7 Flex Real-Time PCR System (Life Technologies, Milan, Italy). Gene-specific primers were designed using Primer Express version 2.0 software (Applied Biosystems). For FGF2, FGFR1, c-fos, CTGF and the ribosomal protein 18S, which was used as a control gene to obtain normalized values, the primers were: $5^{\prime}$-AGTGTGTGCTAACCGTTACCT-3' (FGF2 forward) and 5'-ACTGCCCAGTTCGTTTCAGTG-3' (FGF2 reverse); 5' - CCCGTAGCTCCATATTGGACA-3' (FGFR1 forward) and 5' - TTTGCCATTTTTCAACCAGCG-3' (FGFR1 reverse); $5^{\prime}$-CGAGCCCTTTGATGACT TCCT-3' (c-fos forward) and 5'-GGAGCGGGCTGTCTCAGA-3' (c-fos reverse); $5^{\prime}$-ACCTGTGGGATG GGCATCT-3' (CTGF forward) and 5'-CAGGCGGCTCTGCTTCTCTA-3' (CTGF reverse); $5^{\prime}$ - GGCGT CCCCCAACTTCTTA-3' (18S forward) and 5'-GGGCATCACAGACCTGTTATT-3' (18S reverse). Assays were performed in triplicate and the results were normalized with control mRNA levels of 18S. Relative mRNA levels were calculated using the ${ }^{\Delta \Delta} \mathrm{Ct}$ method comparing to control group.

\subsection{Gene silencing Experiments and Plasmids}

Cells were transfected by X-treme GENE 9 DNA Transfection Reagent (Roche Diagnostics, Sigma-Aldrich, Milan, Italy) for $24 \mathrm{~h}$ before treatments with a control vector and a specific shRNA sequence for each target gene. The short hairpin (sh)RNA constructs to knock down the expression of GPER and CTGF, and the unrelated shRNA control constructs have been described previously $[24,30,36]$. The plasmid DN/c-fos, which encodes for c-fos mutant that heterodimerizes with c-fos dimerization partners but does not allow DNA binding, was a kind gift from Dr C. Vinson (NIH, Bethesda, MD, USA).

\subsection{CRISPR/Cas9-Mediated FGFR1 Knockout}

Short guide RNA (sgRNA) sequence targeting human FGFR1 was designed using the E-CRISP sgRNA Designer (http:/ / www.e-crisp.org/E-CRISP/) and cloned into the pSpCas9 (BB)-2A-Puro (PX459) vector (kindly provided by Dr. W.T. Khaled, University of Cambridge, UK) according to the protocol described in Ran et al. [37]. The FGFR1 sgRNA sequence is as follows: sgFGFR1: 5'-CGGCCTAGCGGTGCAGAGTG-3'. Then, the plasmid with sgRNA was transiently transfected into MDA-MB-231 cells using Lipofectamine LTX (Life Technologies, Milan Italy). Two days after transfection the cells were selected via growth in a medium contained $1 \mu \mathrm{g} / \mathrm{mL}$ puromycin dihydrochloride (Sigma-Aldrich, Milan, Italy). After puromycin selection, the puromycin-resistant 
colonies were picked and expanded in regular medium. Then, immunoblots for FGFR1 protein were performed to evaluate the efficiency of the FGFR1 knockout.

\subsection{Immunofluorescence Microscopy}

$50 \%$ percent confluent cultured grown on coverslips were serum deprived for $24 \mathrm{~h}$ and then treated for $6 \mathrm{~h}$ with E2 and G-1 alone and in combination with G15, as indicated. Where required, cells were previously transfected for $24 \mathrm{~h}$ with shRNA or shGPER (as described above) and then treated for $6 \mathrm{~h}$ with E2 and G-1. Next, cells were fixed in 4\% paraformaldehyde in PBS, permeabilized with $0.2 \%$ Triton X-100, washed 3 times with PBS and incubated at $4{ }^{\circ} \mathrm{C}$ overnight with a mouse primary antibody against FGF2 (C-2) (Santa Cruz Biotechnology, DBA, Milan, Italy). After incubation, the slides were extensively washed with PBS, probed with donkey anti-mouse IgG-FITC (1:300; purchased from Alexa Fluor, Life Technologies, Milan Italy) and 4, 6-diamidino-2-phenylindole dihydrochloride (DAPI) (1:1000; Sigma-Aldrich, Milan, Italy). Then, the images were obtained using the Cytation 3 Cell Imaging Multimode reader (BioTek, AHSI, Milan Italy) and analyzed by the Gen5 software (BioTek, AHSI, Milan Italy).

\subsection{Conditioned Medium Derived from CAFs}

CAFs were cultured in regular growth medium, then washed twice with PBS and transferred in medium without serum for $24 \mathrm{~h}$. Next, CAFs were treated for $6 \mathrm{~h}$ with E2 and G-1 alone and in combination with G15, as indicated; then, cells were washed twice with PBS and cultured for additional $12 \mathrm{~h}$ with fresh serum-free medium. Thereafter, the supernatants were collected, centrifuged at $3500 \mathrm{rpm}$ for $5 \mathrm{~min}$ to remove cell debris and used as conditioned medium in the appropriate experiments.

\subsection{Western Blot Analysis}

CAFs and MDA-MB-231 cells were processed to obtain protein lysates for western blot analysis as previously described [29]. Primary antibodies were as follows: GPER (AB137479) (Abcam, Euroclone Milan, Italy); CTGF (TA806803) (OriGene Technologies, DBA, Milan, Italy); FGFR1 (\#9740) and p-FGFR1 (\#3476) (CST, Euroclone Milan, Italy); c-fos (E8), phosphorylated extracellular signal-regulated kinase (ERK) (E-4), ERK2 (C-14), p-AKT1/2/3 (Ser 473)-R, AKT/1/2/3 (H-136) and $\beta$-actin (AC-15) (Santa Cruz Biotechnology, DBA, Milan, Italy). Proteins were detected by horseradish peroxidase-linked secondary antibodies (Biorad, Milan, Italy) and revealed using the chemiluminescent substrate for western blotting Westar Nova 2.0 (Cyanagen, Biogenerica, Catania, Italy).

\subsection{Enzyme-Linked Immunosorbent Assay}

The concentrations of FGF2 in conditioned medium collected from CAFs were measured using human FGF2 ELISA Kit (Thermo Fisher Scientific, Monza Italy), according to the manufacturer's instructions. Briefly, after incubation with conditioned media for $2 \mathrm{~h}$ at room temperature (RT), the plates were washed four times using $1 \mathrm{X}$ wash buffer, then $100 \mu \mathrm{L}$ Hu FGF2 Biotin conjugate solution were added into each well, except the chromogen blanks, for $1 \mathrm{~h}$ at RT. Next, the plates were washed four times using $1 \mathrm{X}$ wash buffer and then $100 \mu \mathrm{L}$ 1X Streptavidin-HPR solution were added into each well for $30 \mathrm{~min}$ at RT. Following incubation, plates were washed four times using $1 \mathrm{X}$ wash buffer and a colour-substrate solution was added to each well. After incubation in the dark for $30 \mathrm{~min}$ at RT, $100 \mu \mathrm{L}$ of stop solution was used to stop reaction. Then, the plates were read at $450 \mathrm{~nm}$ on a Microplate Spectrophotometer Epoch ${ }^{\mathrm{TM}}$ (BioTek, AHSI, Milan Italy). 


\subsection{Chromatin Immunoprecipitation (ChIP) Assay}

Chip experiments were performed as previously described [29]. The primers used to amplify a region containing an AP-1 site located into the FGF2 promoter sequence were: $5^{\prime}$-GTTTCTA CAAGGAGGCACGTC-3' (Fw) and 5'-GAGATGCCAAATCTGATGCCA-3' (Rv). qPCR data were normalized respect to unprocessed lysates (Input DNA) and the results were reported as fold changes respect to nonspecific $\operatorname{IgG}$.

\subsection{Analysis of Public Datasets from METABRIC and TCGA and Kaplan-Meier Plotter}

Images of genomic alterations in Molecular Taxonomy of Breast Cancer International Consortium (METABRIC) and The Cancer Genome Atlas (TCGA) databases were captured from cBioPortal (http: / / www.cbioportal.org) [38,39]. Prognostic values of mRNA expression or copy-number (CN) gains of FGFR1 from METABRIC [40,41] breast cancer samples were analyzed by Kaplan-Meier survival curves. Long-rank test was used for statistical analysis. The mRNA expression z-Scores of FGFR1 and CTGF were retrieved from METABRIC [40,41] breast cancer samples analyzed for gene expression using Illumina Human v3 microarray. Data were processed using the Python programming language to identify correlation patterns among different genes. In particular, pairwise linear regressions of mRNA levels between FGFR1 and CTGF were calculated. The Pearson correlation coefficients measured the magnitude of the linear relationship between genes.

\subsection{Polarization Assay}

FGFR1 (WT) and FGFR1 (KO) MDA-MB-231 cells were serum deprived for $24 \mathrm{~h}$ and then exposed for $8 \mathrm{~h}$ to conditioned media collected from CAFs or to FGF2, as indicated. Then cells were processed as previously described $[28,42]$.

\subsection{Scratch Assay}

FGFR1 (WT) and FGFR1 (KO) MDA-MB-231 cells were seeded into 12-well plates and they were allowed to grow in regular growth medium until they were $70-80 \%$ confluent. Next, cells were switched in medium without serum and after $24 \mathrm{~h}$ a p200 pipette tip was used to create a scratch of the cell monolayer. Cells were washed twice with PBS and then incubated at $37^{\circ} \mathrm{C}$ with conditioned media collected from CAFs or with FGF2 for $24 \mathrm{~h}$, as indicated. Pictures were photographed at $0 \mathrm{~h}$ and $24 \mathrm{~h}$ after scratching using inverted phase contrast microscope $(5 \times$ magnification). The rate of cell migration was calculated by quantifying the \% of wound closure area using the WCIF ImageJ software, according to the formula:

$$
\% \text { of wound closure }=[(\mathrm{At}=0 \mathrm{~h})-(\mathrm{At}=\Delta \mathrm{h}) /(\mathrm{At}=0 \mathrm{~h})] \times 100 \%
$$

\subsection{Transwell Migration and Invasion Assays}

Transwell $8 \mu \mathrm{m}$ polycarbonate membrane (Costar, Sigma-Aldrich, Milan, Italy) was used to evaluate in vitro migration and invasion of FGFR1 (WT) and FGFR1 (KO) MDA-MB-231 cells. $5 \times 10^{4}$ cells in $300 \mu \mathrm{L}$ serum-free medium were seeded in the upper chamber, coated with (invasion assay) or without (migration assay) Corning ${ }^{\circledR}$ Matrigel ${ }^{\circledR}$ Growth Factor Reduced (GFR) Basement Membrane Matrix (Biogenerica, Catania, Italy) at a 1:3 dilution. Medium containing 10\% FBS was then added into the lower chamber as a chemoattractant. $4 \mathrm{~h}$ after seeding, cells on the upper surface of the membrane were removed by wiping with Q-tip, and invaded or migrated cells were fixed with $100 \%$ methanol, stained with Giemsa (Sigma-Aldrich, Milan, Italy), photographed using Cytation 3 Cell Imaging Multimode Reader (BioTek, AHSI, Milan Italy) and counted using the WCIF ImageJ software. 


\subsection{Statistical Analysis}

Data were analyzed by one-way ANOVA with Dunnett's multiple comparisons, where applicable, using GraphPad Prism, 6.01 (GraphPad Software, Inc., San Diego, CA, USA). $\left({ }^{*}\right) p<0.05$ and $\left({ }^{* *}\right) p<0.01$ were considered statistically significant.

\subsection{Ethics Approval and Consent to Participate}

All procedures are conformed to the Helsinki Declaration for the research on humans. Signed informed consent was obtained from all patients and the experimental research has been performed with the ethical approval provided by the "Comitato Etico Regione Calabria, Cosenza, Italy" (approval code: 166, 2 December 2016).

\section{Results}

\subsection{GPER Mediates the Induction of FGF2 Expression by E2 and G-1 in Breast Cancer-Associated} Fibroblasts (CAFs)

Previous studies have shown that estrogens acting either through ER or GPER up-regulate FGF2 expression and secretion in both normal and cancer cells [19,32,43]. In order to provide novel insights into the FGF2 regulation by estrogens within the tumor microenvironment, we sought to address whether estrogens may regulate FGF2 levels in ER-negative/ GPER-positive CAFs isolated from breast tumor patients (see material and methods section). Worthy of note, both E2 and G-1 induced the expression of FGF2 at the mRNA (Figure 1a,b) and protein levels (Figure 1c) in CAFs. However, the response to E2 and G-1 was no longer observed after GPER silencing (Figure 1d, Supplementary Figure S2) or using the GPER antagonist G15 (Figure 2a,b). In contrast, E2 and G-1 were not able to elicit FGF2 up-regulation in fibroblasts derived from noncancerous breast tissue (data not shown). By performing ELISA experiments, we then observed that the secretion of FGF2 in CAFs medium upon treatments with E2 and G-1 is abrogated treating cells with the GPER antagonist G15 (Figure 2c). As GPER activation induces the stimulation of diverse transduction pathways [23], we also found that FGF2 up-regulation prompted by E2 and G-1 was prevented either by the EGFR tyrosine kinase inhibitor AG1478 (AG) or the MEK inhibitor PD98059 (PD), but not by the PI3K inhibitor Wortmannin (WM) (Supplementary Figure S3a,b). Taken together, these findings indicate that, in CAFs, both E2 and G-1 induce FGF2 expression through the GPER-EGFR-ERK1/2 signaling cascade. 


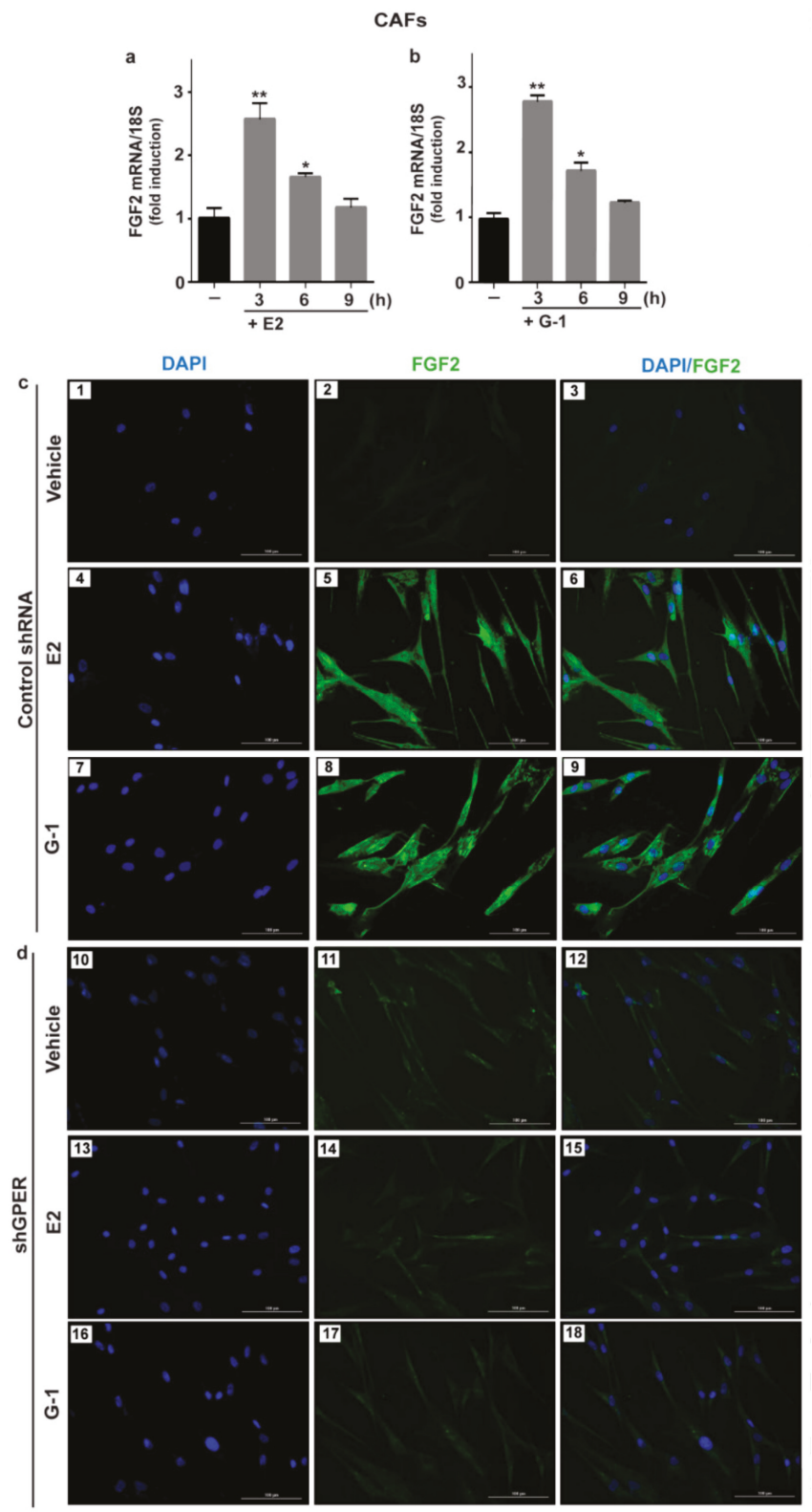

CAFs

Figure 1. E2 and G-1 induce FGF2 expression through GPER in CAFs. 10 nM E2 (a) and 100 nM G-1 (b) induced FGF2 mRNA expression, as evaluated by quantitative PCR (qPCR). Values were normalized to $18 \mathrm{~S}$ expression and shown as fold changes of FGF2 mRNA expression upon E2 and G-1 treatments respect to cells exposed to vehicle (). Each column represents the mean \pm standard deviation (SD) of three independent experiments performed in triplicate. $\left(^{* *}\right)$ indicates $p<0.01$ and $\left(^{*}\right)$ indicates $p<0.05$. (c,d) FGF2 protein expression by immunofluorescence in CAFs transfected for $24 \mathrm{~h}$ with control shRNA (panels 1-9) or sh G protein estrogen receptor (shGPER) (panels 10-18) and then treated for $6 \mathrm{~h}$ with vehicle, $10 \mathrm{nM}$ E2 and $100 \mathrm{nM} \mathrm{G-1}$, as indicated. FGF2 accumulation is shown by the green signal, nuclei are stained by 4, 6-diamidino-2-phenylindole dihydrochloride (DAPI) (blue signal), scale bar $=100 \mu \mathrm{m}$. Images shown are representative of two independent experiments. 

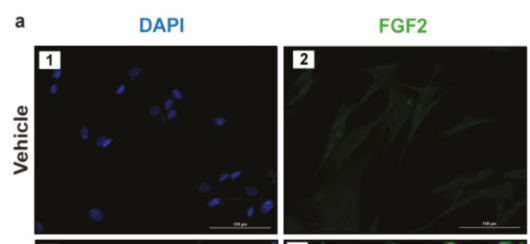

CAFs
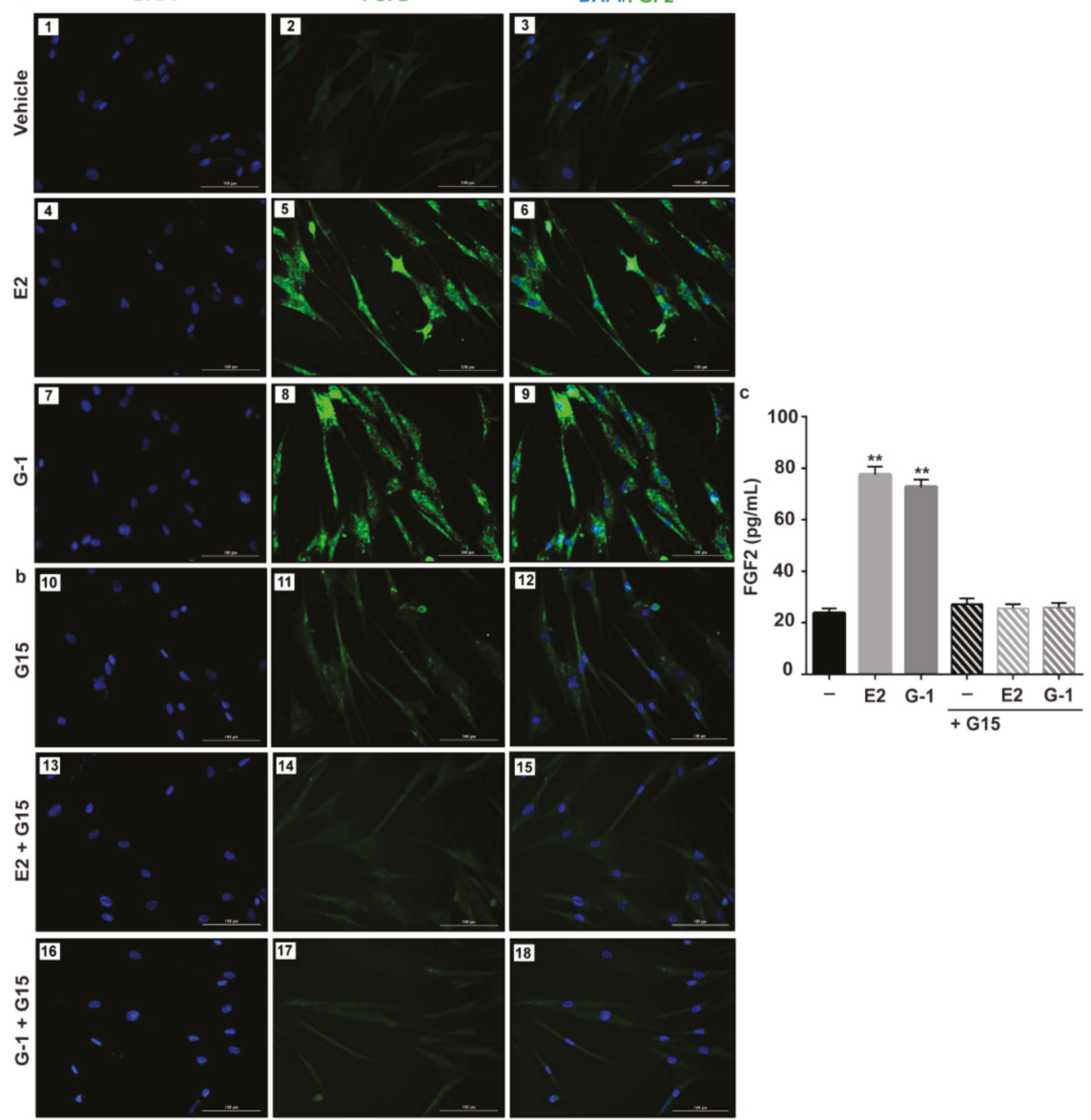

Figure 2. GPER mediates the up-regulation and the secretion of FGF2 by E2 and G-1 in CAFs. FGF2 protein expression by immunofluorescence in CAFs treated for $6 \mathrm{~h}$ with vehicle, $10 \mathrm{nM}$ E2 and $100 \mathrm{nM}$ G-1, alone (panels 1-9) (a) and in combination with 100 nM GPER antagonist G15 (panels 10-18) (b). FGF2 accumulation is shown by the green signal, nuclei are stained by DAPI (blue signal), scale bar $=100 \mu \mathrm{m}$. Images shown are representative of two independent experiments. (c) ELISA of FGF2 levels in supernatants collected from CAFs treated for $18 \mathrm{~h}$ with vehicle (-), $10 \mathrm{nM}$ E2 and $100 \mathrm{nM} \mathrm{G-1}$ alone and in combination with $100 \mathrm{nM}$ GPER antagonist G15. Each column represents the mean \pm SD of three independent experiments performed in triplicate. $\left.{ }^{* *}\right)$ indicates $p<0.01$.

\section{2. c-fos is Involved in the FGF2 up-Regulation Induced by Estrogenic GPER Signaling in CAFs}

As the activation of GPER-EGFR-ERK1/2 transduction pathway leads to c-fos expression [22,29], we determined c-fos response at both mRNA and protein levels upon E2 and G-1 exposure in CAFs (Figure 3a-c). Then, we established that both ligands trigger the recruitment of $c-f o s$ to the AP-1 site located within the FGF2 promoter region (Figure 3d,e). Further supporting these results, the up-regulation of FGF2 protein expression induced by E2 and G-1 was prevented transfecting CAFs with a dominant negative form of c-fos (DN/c-fos) (Figure 3f,g). Collectively, the abovementioned findings suggest that, in CAFs, GPER along with the EGFR-ERK1/2-c-fos-AP-1 signaling pathway mediates FGF2 expression in response to E2 or G-1. 

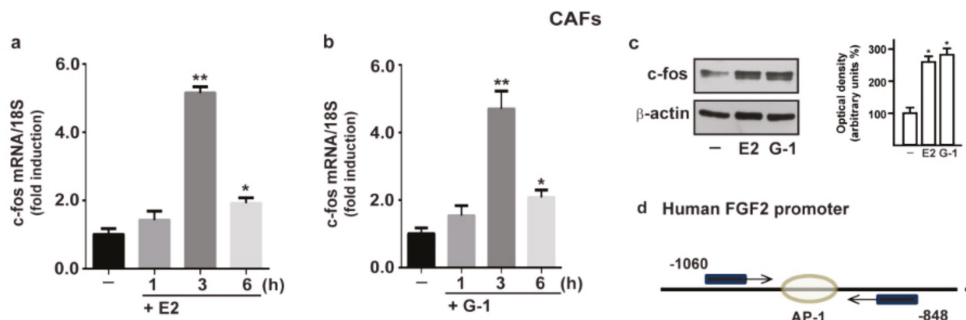

d Human FGF2 promoter
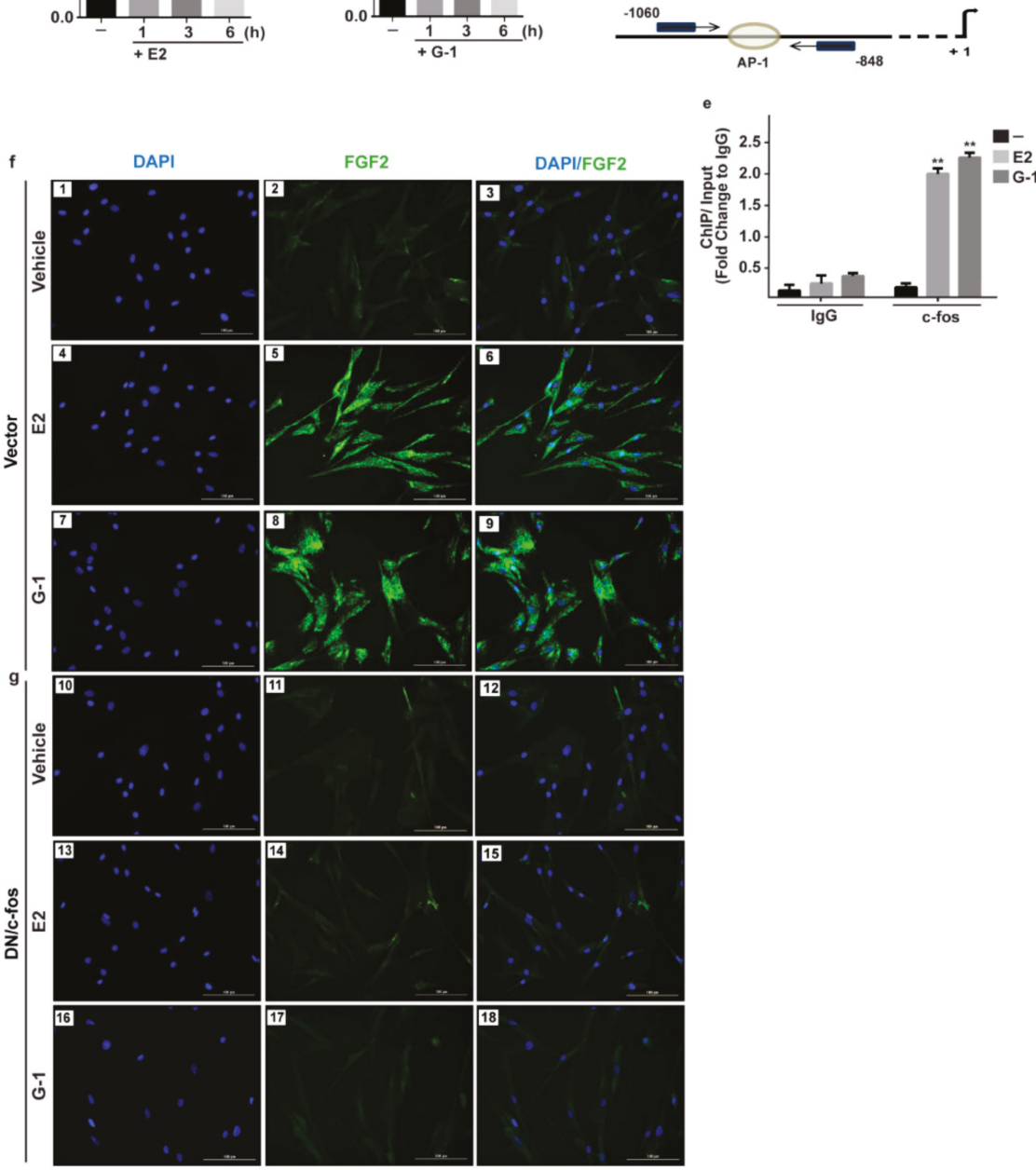

Figure 3. The oncogene fos (c-fos) is involved in the up-regulation of FGF2 by E2 and G-1 in CAFs. 10 nM E2 (a) and 100 nM G-1 (b) induced c-fos mRNA expression, as evaluated by qPCR. Values were normalized to $18 \mathrm{~S}$ expression and shown as fold changes of c-fos mRNA expression upon E2 and G-1 treatments respect to cells exposed to vehicle (-). (c) The treatment for $3 \mathrm{~h}$ with $10 \mathrm{nM}$ E2 and $100 \mathrm{nM} \mathrm{G-1}$ up-regulated c-fos protein, which is recruited to the AP-1 site located within the FGF2 promoter region (-1060/-848; the transcriptional start site is indicated as + 1), as ascertained by Chromatin Immunoprecipitation (ChIP)-qPCR assay (d,e). Data were normalized to the input and reported as fold changes respect to Immunoblobulin $\mathrm{G}(\mathrm{IgG})$. Each column represents the mean $\pm \mathrm{SD}$ of three independent experiments performed in triplicate. In immunoblot experiments $\beta$-actin served 
as a loading control, side panels show densitometric analysis of the blot normalized to the loading control. $\left({ }^{*}\right)$ indicates $p<0.05$ and $\left({ }^{* *}\right)$ indicates $p<0.01$. (f) FGF2 protein expression by immunofluorescence in CAFs transfected for $18 \mathrm{~h}$ with a vector (panels 1-9), or (g) with a construct encoding for a dominant negative form of c-fos (DN/c-fos) (panels 10-18) and then treated for $6 \mathrm{~h}$ with vehicle, $10 \mathrm{nM}$ E2 and $100 \mathrm{nM} \mathrm{G}-1$, as indicated. FGF2 accumulation is evidenced by the green signal, nuclei are stained by DAPI (blue signal), scale bar $=100 \mu \mathrm{m}$. Images shown are representative of two independent experiments.

\subsection{Conditioned Medium (CM) from Estrogens-Stimulated CAFs Activates the FGFR1-ERK1/2-AKT Transduction Pathway in MDA-MB-231 Cells}

Previous studies have shown that the activation of the FGF2-FGFR1 autocrine and/or paracrine loop plays an important role toward the migration and invasion of cancer cells [44-46]. Moreover, FGFR1 has been found highly amplified in breast cancer patients and associated with endocrine resistance [11,14]. In accordance with these observations, a large-scale genomic analysis of METABRIC and TCGA databases allowed us to assess not only that FGFR1 represents the most amplified receptor of the FGFRs family members but also that FGFR1 amplification occurs in nearly $14 \%$ of breast cancer patients (Figure 4a,b) [38,39]. Of note, breast cancer patients with either higher expression or copy number (CN) gains of FGFR1 are associated with shorter overall survival rates respect to the rest of the cohort (Figure 4c,d). Taken into account the aforementioned results, we focused on FGF2-FGFR1 signaling in the context of paracrine communication between CAFs and breast cancer cells. In this vein, we used MDA-MB-231 cells as model system because these cells did not express FGF2 [47], but rather displayed high expression levels of FGFR1 (Supplementary Figure S4). In order to better investigate the role of FGFR1 in our experimental model, we used CRISPR/Cas9 genome editing technology to generate FGFR1 knockout (KO) MDA-MB-231 cell line (Figure 4e,f). Given that both E2 and G-1 stimulate the expression and the secretion of FGF2 in CAFs (see Figure 1; Figure 2), we then ascertained that CM from E2 and G-1 treated-CAFs induces FGFR1 phosphorylation in MDA-MB-231 cells, as well as the stimulation of the two main pathways downstream FGFR1 activation, such as ERK1/2 and AKT [11,48] (Figure 5a-c). Next, in parallel experiments, the FGFR1 inhibitor PD173074 was found to abolish both ERK1/2 and AKT phosphorylation (Figure 5a-c). Accordingly, CM obtained from E2 and G-1 treated-CAFs did not induce ERK1/2 and AKT activation in FGFR1 (KO) MDA-MB-231 cells (Figure 5d-g). 

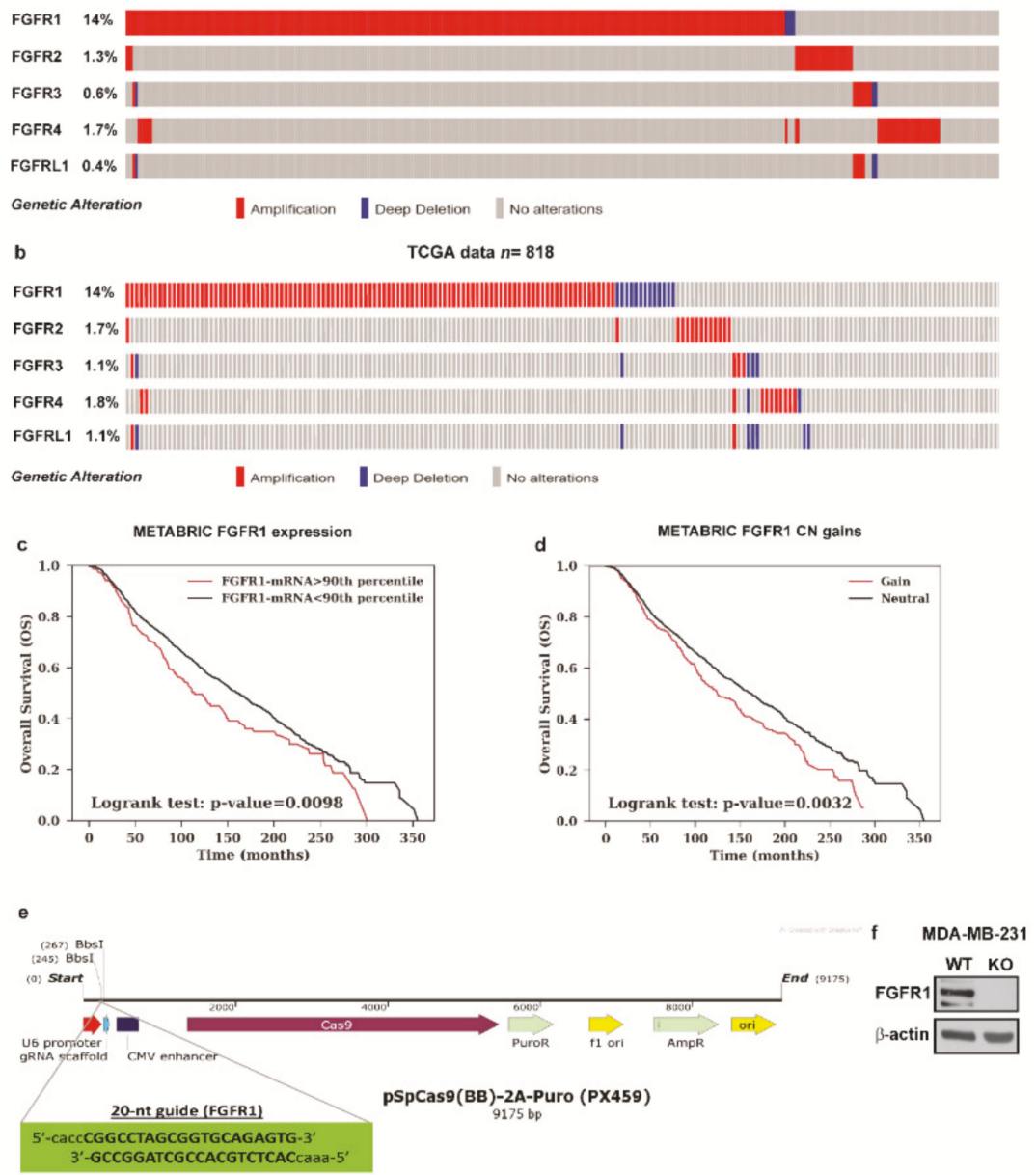

Figure 4. Analysis of METABRIC and TCGA datasets and CRISPR/Cas9-mediated FGFR1 knockout $(\mathrm{KO})$ in MDA-MB-231 cells. (a,b) The OncoPrint of genomic alteration of FGFRs members showed that FGFR1 is the most amplified receptor of the family in human breast cancer patients. Each row represents a gene and each column represents a tumor sample. Red bars indicate gene amplifications, blue bars deep deletions and grey bars no alterations. (c,d) Kaplan-Meir plots show the overall survival (OS) from METABRIC dataset between patients with normal or high FGFR1 mRNA expression or between patients with copy number $(\mathrm{CN})$ gains or without (neutral). Statistical analysis was performed using the long-rank test. (e) Schematic representation of the pX459 plasmid and the sgRNA sequence used to generate FGFR1 (KO) MDA-MB-231 cells. (f) Immunoblots of lysates generated from FGFR1 (WT) and FGFR1 (KO) MDA-MB-231 cells. $\beta$-actin served as loading control. Immunoblots shown are representative of three independent experiments. 


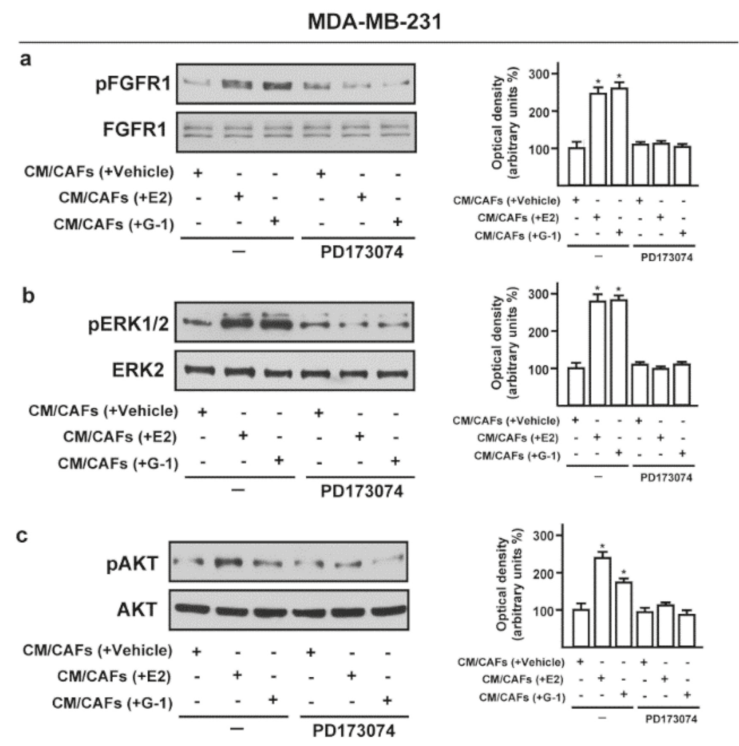

MDA-MB-231
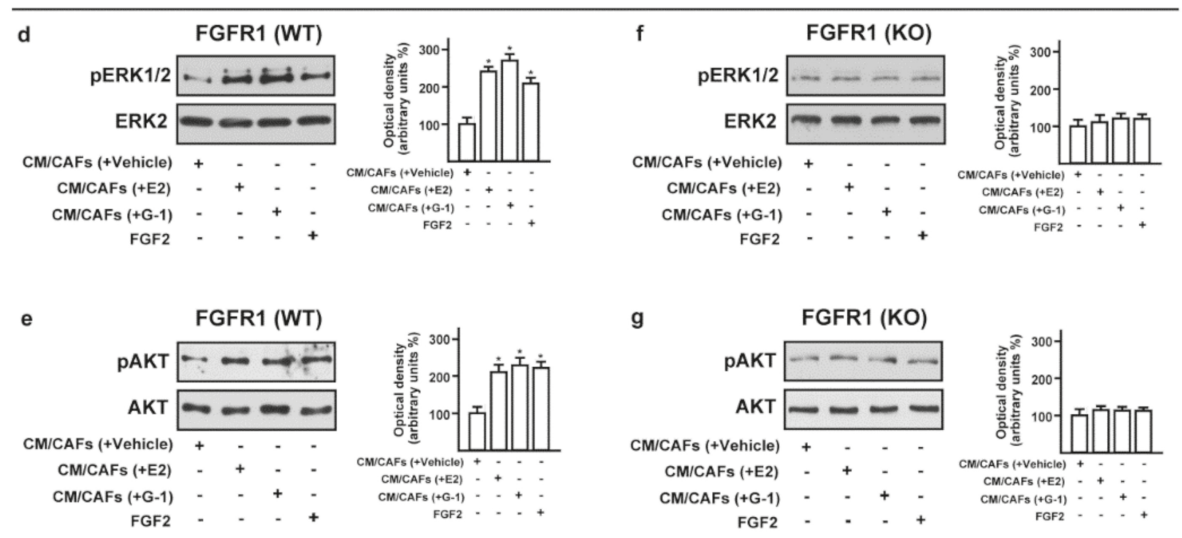

Figure 5. Conditioned medium (CM) from estrogen-stimulated CAFs induces the activation of FGFR1- signaling pathway in MDA-MB-231 cells. (a-c) Phosphorylation of FGFR1, ERK1/2, AKT in MDA-MB-231 cells exposed for $1 \mathrm{~h}$ to CM from CAFs treated for $18 \mathrm{~h}$ with vehicle [CM/CAFs (+vehicle)], $10 \mathrm{nM}$ E2 [CM/CAFs (+E2)] or $100 \mathrm{nM} \mathrm{G-1} \mathrm{[CM/CAFs} \mathrm{(+G-1)],} \mathrm{alone} \mathrm{and} \mathrm{in} \mathrm{the} \mathrm{presence}$ of $1 \mu \mathrm{M}$ FGFR1 inhibitor PD173074. (d,e) Activation of ERK1/2 and AKT in FGFR1 (WT) MDA-MB-231 cells upon exposure for $1 \mathrm{~h}$ to $\mathrm{CM}$ from CAFs treated for $18 \mathrm{~h}$ with $10 \mathrm{nM}$ E2 [CM/CAFs (+E2)], $100 \mathrm{nM}$ G-1 [CM/CAFs (+G-1)]; (f,g) In FGFR1 (KO) MDA-MB-231 cells cultured in the same conditions as described above, the activation of ERK1/2 and AKT was no longer observed. FGF2 at $25 \mathrm{nM}$ was used as positive control. FGFR1, ERK2, AKT and $\beta$-actin served as loading control, as indicated. Side panels show densitometric analysis of the blots normalized to the loading controls. Immunoblots shown are representative of three independent experiments. $\left({ }^{*}\right)$ indicates $p<0.05$.

\subsection{FGF2/FGFR1 Paracrine Activation Up-Regulates CTGF Expression in MDA-MB-231 Cells}

A synergic action between FGF2 and connective tissue growth factor (CTGF) may occur in diverse pathophysiological conditions [49-51]. Hence, we analyzed the mRNA levels of FGFR1 and CTGF 
in METABRIC breast cancer patients database $[40,41]$ and we found a positive correlation between FGFR1 and CTGF expression (Figure 6a). Therefore, we investigated the involvement of FGF2/FGFR1 paracrine activation by estrogen-stimulated CAFs on CTGF expression in MDA-MB-231 cells. Worthy of note, CM collected from E2 and G-1 treated-CAFs triggered CTGF expression at both mRNA (Figure 6b) and protein levels (Figure 6c,d) in FGFR1 (WT) MDA-MB-231 cells but not in FGFR1 (KO) MDA-MB-231 cells. Next, the up-regulation of CTGF protein levels observed in the aforementioned conditions was also abrogated in the presence of the FGFR1 inhibitor PD173074, the MEK inhibitor PD98059 as well as the PI3K inhibitor Wortmannin (WM) (Figure 6e-g). Altogether, these findings suggest that FGF2/FGFR1 paracrine activation induced by estrogen-stimulated CAFs prompts CTGF expression through the involvement of ERK1/2 and AKT signaling cascades in MDA-MB-231 cells.

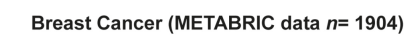

a
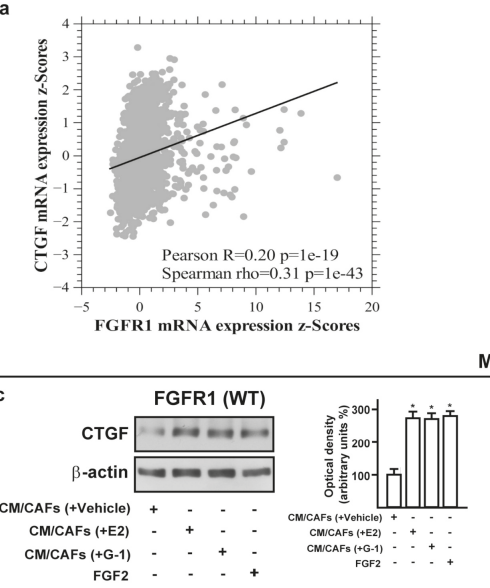

MDA-MB-231

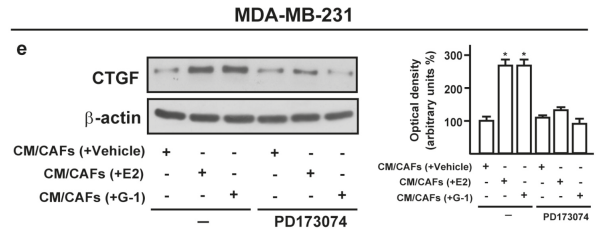

f

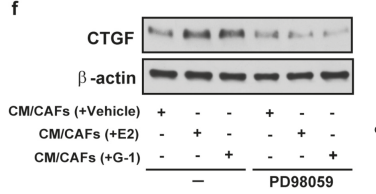

g
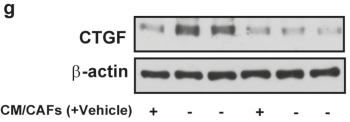

CM/CAFs (+E2)

CM/CAFs (+G-1)

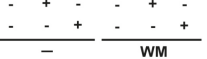

MDA-MB-231

b

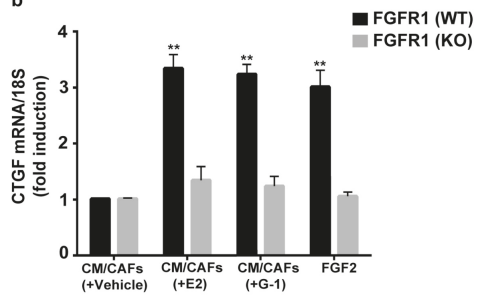

MDA-MB-231

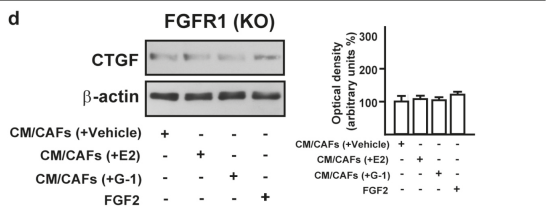

Figure 6. Conditioned medium (CM) from estrogen-stimulated CAFs up-regulates CTGF levels through FGFR1 signaling pathway in MDA-MB-231 cells. (a) Pairwise linear regressions of FGFR1 versus CTGF mRNA levels were performed on METABRIC dataset of 1904 breast tumor samples. Scatter plot shows 
positive correlation between FGFR1 and CTGF expression. (b-d) CTGF mRNA and protein levels in FGFR1 (WT) and FGFR1 (KO) MDA-MB-231 cells exposed for $3 \mathrm{~h}$ to CM from CAFs treated for $18 \mathrm{~h}$ with vehicle [CM/CAFs (+vehicle)], $10 \mathrm{nM}$ E2 [CM/CAFs (+E2)] or $100 \mathrm{nM} \mathrm{G-1}$ [CM/CAFs (+G-1)], or exposed to $25 \mathrm{nM}$ FGF2, as positive control, evaluated by qPCR and western blot. In RNA experiments, values were normalized to the expression of $18 \mathrm{~S}$ and shown as fold changes of CTGF mRNA expression upon CM from CAFs treated with E2 and G-1 respect to cells exposed to CM from $\mathrm{CAFs}$ treated with vehicle. Each column represents the mean $\pm \mathrm{SD}$ of three independent experiments performed in triplicate. (e-g) Up-regulation of CTGF protein expression in MDA-MB-231 cells exposed for $3 \mathrm{~h}$ to $\mathrm{CM}$ from CAFs treated for $18 \mathrm{~h}$ with vehicle [CM/CAFs (+vehicle)], $10 \mathrm{nM}$ E2 [CM/CAFs (+E2)], or $100 \mathrm{nM} \mathrm{G-1}$ [CM/CAFs (+G-1)] was no longer observed in the presence of $1 \mu \mathrm{M}$ FGFR1 inhibitor PD173074, $10 \mu \mathrm{M}$ MEK inhibitor PD98059 or $100 \mathrm{nM}$ PI3K inhibitor Wortmannin (WM). $\beta$-actin served as loading control. Side panels show densitometric analysis of the blots normalized to the loading controls. Immunoblots shown are representative of three independent experiments.

$(* *)$ indicates $p<0.01$ and $(*)$ indicates $p<0.05$.

\subsection{FGF2/FGFR1 Paracrine Activation Induces Cell Migration and Invasion Through CTGF in} MDA-MB-231 Cells

Upon FGF2/FGFR1 activation, breast cancer cells may acquire invasive phenotype features modulating the expression of cell junction proteins, promoting a spindle-like morphology and increasing cell motility [52-54]. Recapitulating the abovementioned results, CM collected from E2 and G-1 treated-CAFs increased spindle-like morphology in FGFR1 (WT) MDA-MB-231 cells, but not in FGFR1 (KO) MDA-MB-231 cells as evaluated by the polarity index (Figure 7a,b). Performing scratch (Supplementary Figure S5a,b) and transwell assays (Figure 7c,d), we then observed that the migration and invasion of FGFR1 (WT) MDA-MB-231 cells promoted by CM from E2 and G-1 treated-CAFs were no longer evident in FGFR1 (KO) MDA-MB-231 cells as well as using the FGFR1 inhibitor PD173074 (data not shown). Next, we found that, in MDA-MB-231 cells, CTGF silencing prevents the migratory and invasive effects triggered by CM obtained from E2 and G-1 treated-CAFs (Figure 8a,b). Taken together, these results suggest that CTGF is involved by FGF2/FGFR1 paracrine activation toward important biological features elicited in MDA-MB-231 cells. 
MDA-MB-231
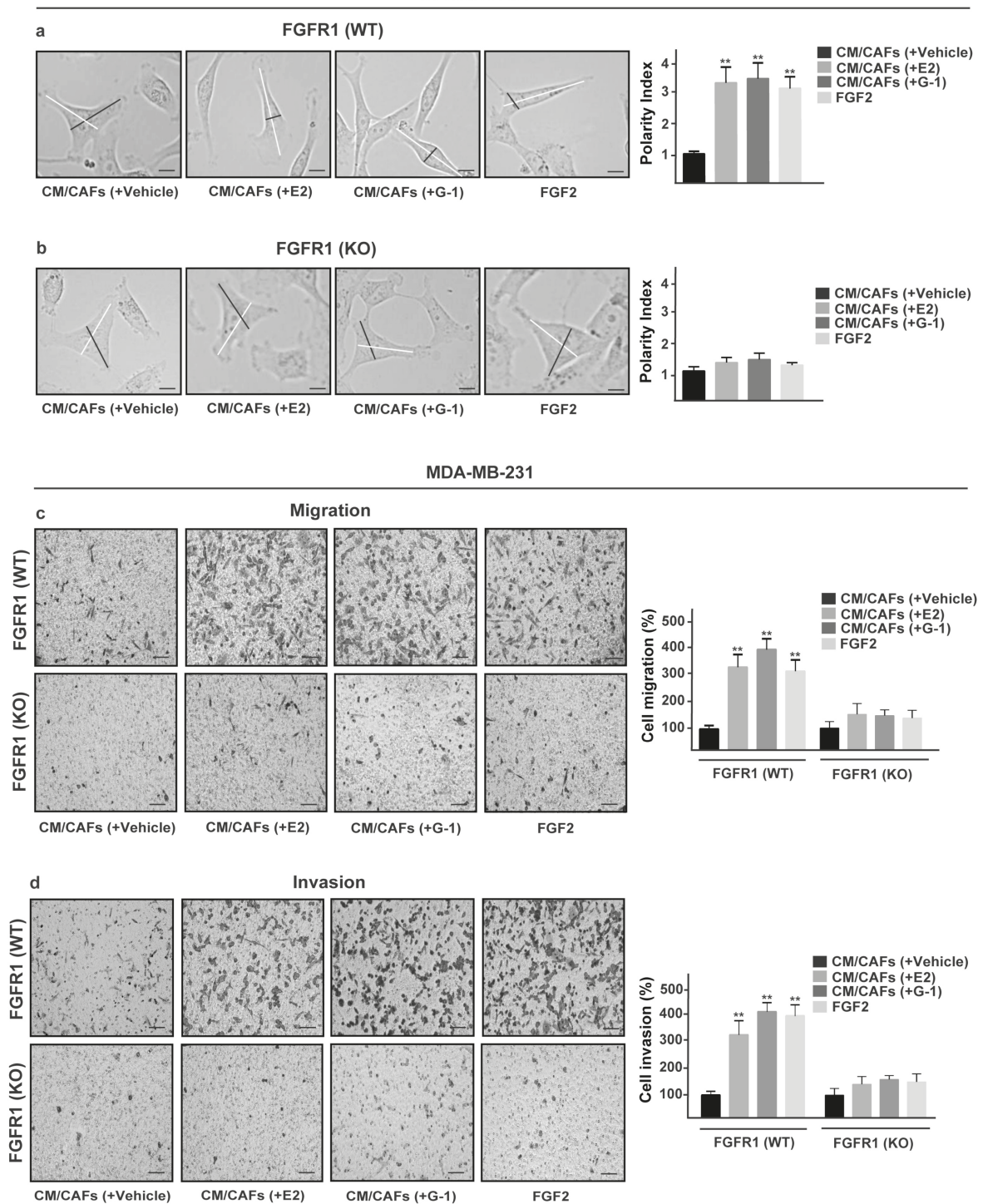

Figure 7. FGFR1 paracrine activation promotes migration and invasion in MDA-MB-231 cells. (a) FGFR1 (WT) and (b) FGFR1 (KO) MDA-MB-231 cells were cultured for $8 \mathrm{~h}$ in CM from CAFs treated for $18 \mathrm{~h}$ with vehicle [CM/CAFs (+vehicle)], $10 \mathrm{nM}$ E2 [CM/CAFs (+E2)] or $100 \mathrm{nM} \mathrm{G}-1$ [CM/CAFs (+G-1)], or exposed to 25 nM FGF2, as positive control. Lines traced on cells were used to calculate the Polarity Index (PI). White lines define the migratory axis and black lines the transversal axis. $\mathrm{PI}=1.0$ indicates a polygonal shape, whereas a value $>1.0$ defines ranges of migratory shapes. Scale bar $=30 \mu \mathrm{m}$. Images shown are representative of 30 random fields acquired in three independent experiments. Transwell assays were used to assess cell migration (c) and invasion (d) in FGFR1 (WT) and FGFR1 (KO) MDA-MB-231 cells cultured for $8 \mathrm{~h}$ in CM from CAFs treated for $18 \mathrm{~h}$ with vehicle [CM/CAFs (+vehicle)], $10 \mathrm{nM}$ E2 [CM/CAFs (+E2)] or $100 \mathrm{nM} \mathrm{G-1} \mathrm{[CM/CAFs} \mathrm{(+G-1)],} \mathrm{or} \mathrm{exposed} \mathrm{to}$ $25 \mathrm{nM}$ FGF2, as positive control. Cells were counted in at least 10 random fields at $10 \times$ magnification, in three independent experiments performed in triplicate. Scale bar $\left.=200 \mu \mathrm{m},{ }^{* *}\right)$ indicates $p<0.01$. 


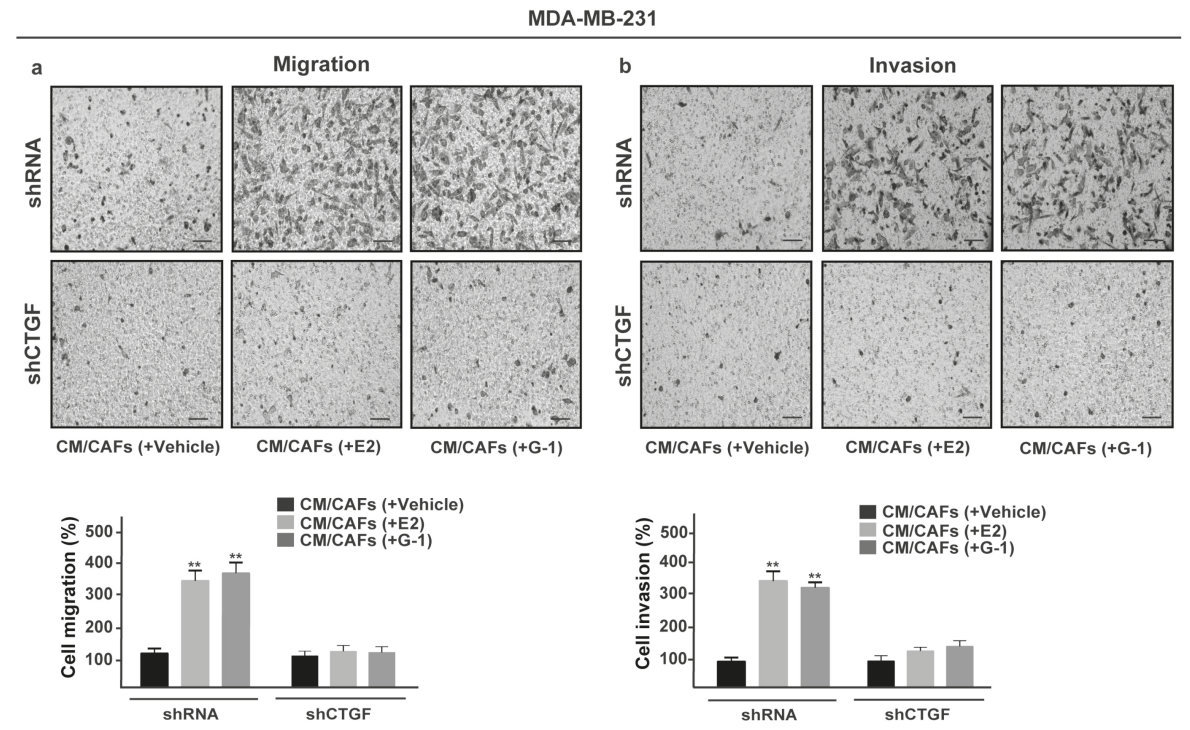

Figure 8. CTGF is required for migration and invasion induced by FGFR1 paracrine activation in MDA-MB-231 cells. Transwell assays were used to assess cell migration (a) and invasion (b) in MDA-MB-231 cells transfected for $24 \mathrm{~h}$ with control shRNA or shCTGF and then cultured for $8 \mathrm{~h}$ in $\mathrm{CM}$ from CAFs treated for $18 \mathrm{~h}$ with vehicle [CM/CAFs (+vehicle)], $10 \mathrm{nM}$ E2 [CM/CAFs (+E2)] or $100 \mathrm{nM} \mathrm{G}-1$ [CM/CAFs (+G-1)]. Cells were counted in at least 10 random fields at $10 \times$ magnification, in three independent experiments performed in triplicate. Scale bar $\left.=200 \mu \mathrm{m},{ }^{* *}\right)$ indicates $p<0.01$.

\section{Discussion}

In the current study, we provide novel evidence regarding the role of GPER in the regulation of FGF2 expression triggered by estrogens within the tumor microenvironment. In particular, using primary patient-derived breast CAFs, we ascertained that both E2 and the selective GPER agonist G-1 induce the expression and secretion of FGF2 activating the GPER/EGFR/ERK/c-fos/AP-1 signaling cascade. Analyzing publicly available databases, we then showed that FGFR1 is the most frequently amplified receptor of the FGFRs family along with its association with shorter survival rates in breast cancer patients [38-41]. In addition, focusing on the FGF2/FGFR1 functional interaction that occurs between CAFs and breast cancer cells, we determined that FGF2 secretion by estrogens-treated CAFs prompts the up-regulation of CTGF expression through the FGFR1-ERK1/2-AKT signaling cascade in MDA-MB-231 cells. As biological counterpart, we found that cell motility and invasiveness triggered by the FGF2/FGFR1 paracrine activation are abrogated by CTGF silencing.

In recent years, considerable attention has been deserved to the involvement of the tumor stroma toward cancer development [55]. In this regard, it has been shown that the interactions between tumor cells and the associated stroma represent a solid relationship that impacts disease initiation, progression, and patient prognosis [56]. For instance, CAFs acting as main players within the tumor stroma, provide a supportive microenvironment for aggressive features of cancer cells [57-60]. Indeed, CAFs are able to sustain cancer cell growth together with the invasion and metastasis via paracrine actions elicited by cytokines and growth factors released in the tumor microenvironment [6,61]. To date, in breast malignancies $\sim 80 \%$ of stromal fibroblasts acquire the activated landscapes of CAFs that boost the proliferation of cancer cells at both the primary and the metastatic sites [62]. Additionally, CAFs may increase the in situ estrogen production, which contributes to the development of breast carcinomas through a multifaceted interactions among different transduction pathways $[18,63]$. In this vein, several lines of evidence have shown that cancer cells may acquire aberrant growth and invasion 
properties through the dysregulation of the FGF/FGFR signaling [8,64], as highlighted in large-scale analyses of human cancer genomes [65-68]. Moreover, the up-regulation and secretion of FGF2 toward the stimulation of FGFR1 signaling in breast cancers was reported to occur upon estrogen stimulation through the classical ER [32,43]. GPER has been also involved in the stimulatory effects exerted by estrogens and its expression was associated with the tumor size, the distant metastasis, and the recurrence of breast malignancies [21-23]. Likewise, the role of GPER has been ascertained in CAFs toward the proliferation, migration, and spreading of breast tumor cells [22]. In accordance with these findings, our current results provide new data showing that GPER mediates the expression and secretion of FGF2 in breast CAFs leading to the paracrine activation of the FGFR1-ERK1/2-AKT transduction signaling along with important biological responses in MDA-MB-231 cells.

Metastasis, the leading cause of mortality for breast cancer patients, is a complex and multi-stage process that comprise cellular transformation and tumor growth, angiogenesis, and invasion of target organs $[69,70]$. In this context, it has been reported that EMT may prompt diverse processes of the metastatic cascade [71,72]. Accordingly, recent studies have shown that FGFR1 activation promotes EMT and metastasis through different signaling pathways in various tumors as prostate, breast, and lung cancers $[17,46,73]$. High FGF2 expression and secretion have been found in triple-negative breast cancer cell lines, in particular in those showing a mesenchymal phenotype [47,74]. In addition, several factors including vascular endothelial growth factor (VEGF), PC-cell-derived growth factor (PCDGF), epidermal growth factor (EGF), and CTGF were demonstrated to confer migratory and metastatic properties to breast cancer cells $[75,76]$. As CTGF is concerned, mechanical stresses, cytokines, and growth factors stimulations have been reported to be able to alter its expression levels toward the cytoskeletal reorganization and migratory features in breast cancer cells $[24,75,77,78]$. Previous studies have also suggested a correlation between FGF2 levels and CTGF-activated signaling in different pathophysiological conditions [49-51]. Further corroborating these findings, in the present study a positive correlation between FGFR1 and CTGF expression was assessed by a bioinformatic analysis on 1904 breast tumor samples retrieved from METABRIC dataset. Next, we demonstrated that the paracrine activation of the FGF2/FGFR1 transduction pathway prompts the expression of CTGF, which was involved in the migratory and invasive responses observed in MDA-MB-231 cells.

\section{Conclusions}

Our findings indicate that GPER mediates a feed-forward FGF2/FGFR1 engagement within the tumor microenvironment linking CAFs to breast cancer cells toward tumor progression. Moreover, on the basis of the present data GPER may be included among the transduction mechanisms involved in the FGF2/FGFR1 paracrine activation that may contribute to breast cancer development.

Supplementary Materials: The following are available online at http:/ /www.mdpi.com/2073-4409/8/3/223/s1, Figure S1. Characterization of primary cultured CAFs and NFs; Figure S2. Efficacy of GPER silencing; Figure S3. Up-regulation of FGF2 expression by E2 and G-1 is mediated by the GPER-EGFR-ERK1/2 transduction pathway in CAFs; Figure S4. mRNA expression of FGF2 and FGFR1 in MDA-MB-231, SkBr3 and MCF-7 cells; Figure S5. Scratch assay upon exposure to CM from estrogen-stimulated CAFs in MDA-MB-231 cells.

Author Contributions: M.F.S., A.B., and M.M. conceived the study and carried out its design. M.F.S., A.V., R.L., D.C.R., F.C., G.R.G., and M.T. performed the experiments. M.F.S., A.V., and R.L. analyzed the data. G.B. conducted the bioinformatics analyses. A.M.M. provided breast tumor samples. M.F.S., A.B., and M.M. wrote the paper. All authors read and approved the final manuscript.

Funding: This study was supported by the Italian Association for Cancer Research (AIRC, IG 21322). AB was supported by AIRC-IG 19242. MFS was supported by the Fondazione Umberto Veronesi (Post-Doctoral Fellowship 2019).

Acknowledgments: We are grateful to Walid T. Khaled for his helpful support and the plasmids provided.

Conflicts of Interest: The authors declare no conflict of interest. 


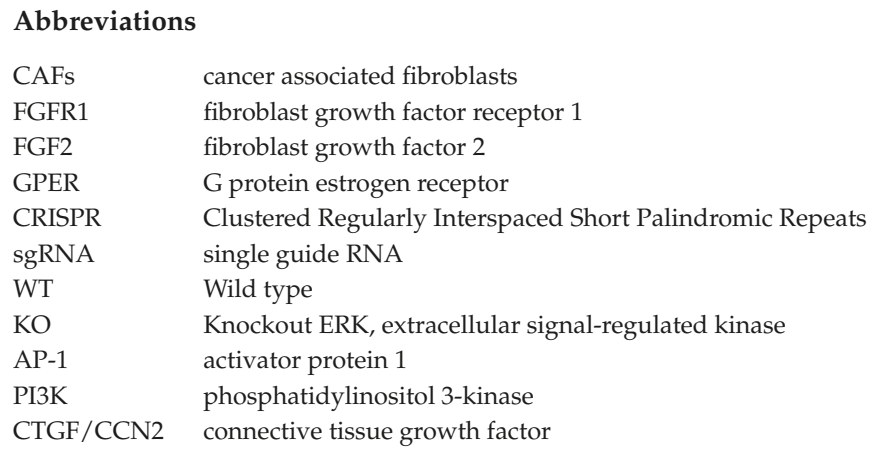

\section{References}

1. Hanahan, D.; Coussens, L.M. Accessories to the crime: Functions of cells recruited to the tumor microenvironment. Cancer Cell 2012, 21, 309-322. [CrossRef] [PubMed]

2. Hayward, S.W.; Wang, Y.; Cao, M.; Hom, Y.K.; Zhang, B.; Grossfeld, G.D.; Sudilovsky, D.; Cunha, G.R. Malignant transformation in a nontumorigenic human prostatic epithelial cell line. Cancer Res. 2001, 61, 8135-8142. [PubMed]

3. Procacci, P.; Moscheni, C.; Sartori, P.; Sommariva, M.; Gagliano, N. Tumor-stroma cross-talk in human pancreatic ductal adenocarcinoma: A focus on the effect of the extracellular matrix on tumor cell phenotype and invasive potential. Cells 2018, 7, 158. [CrossRef] [PubMed]

4. Marsh, T.; Pietras, K.; McAllister, S.S. Fibroblasts as architects of cancer pathogenesis. Biochim. Biophys. Acta Mol. Basis Dis. 2013, 1832, 1070-1078. [CrossRef] [PubMed]

5. Kalluri, R. The biology and function of fibroblasts in cancer. Nat. Rev. Cancer 2016, 16, 582-598. [CrossRef] [PubMed]

6. Han, Y.; Zhang, Y.; Jia, T.; Sun, Y. Molecular mechanism underlying the tumor-promoting functions of carcinoma-associated fibroblasts. Tumor Biol. 2015, 36, 1385-1394. [CrossRef] [PubMed]

7. Augsten, M. Cancer-associated fibroblasts as another polarized cell type of the tumor microenvironment. Front. Oncol. 2014, 4, 62. [CrossRef] [PubMed]

8. Carter, E.P.; Fearon, A.E.; Grose, R.P. Careless talk costs lives: Fibroblast growth factor receptor signalling and the consequences of pathway malfunction. Trends Cell Biol. 2015, 25, 221-233. [CrossRef] [PubMed]

9. Katoh, M. FGFR inhibitors: Effects on cancer cells, tumor microenvironment and whole-body homeostasis (Review). Int. J. Mol. Med. 2016, 38, 3-15. [CrossRef] [PubMed]

10. Sleeman, M.; Fraser, J.; McDonald, M.; Yuan, S.; White, D.; Grandison, P.; Kumble, K.; Watson, J.D.; Murison, J.G. Identification of a new fibroblast growth factor receptor, FGFR5. Gene 2001, 271, 171-182. [CrossRef]

11. Babina, I.S.; Turner, N.C. Advances and challenges in targeting FGFR signalling in cancer. Nat. Rev. Cancer 2017, 17, 318-332. [CrossRef] [PubMed]

12. Dieci, M.V.; Arnedos, M.; Andre, F.; Soria, J.C. Fibroblast growth factor receptor inhibitors as a cancer treatment: From a biologic rationale to medical perspectives. Cancer Discov. 2013, 3, 264-279. [CrossRef] [PubMed]

13. Englinger, B.; Kallus, S.; Senkiv, J.; Laemmerer, A.; Moser, P.; Gabler, L.; Groza, D.; Kowol, C.; Heffeter, P.; Grusch, M.; et al. Lysosomal sequestration impairs the activity of the preclinical FGFR inhibitor PD173074. Cells 2018, 7, 259. [CrossRef] [PubMed]

14. Formisano, L.; Young, C.D.; Bhola, N.; Giltnane, J.M.; Estrada, M.V.; Arteaga, C.L. FGFR1 is associated with resistance to interaction with estrogen receptor (ER) $\alpha$ endocrine therapy in ER+/FGFR1-amplified breast cancer. Cancer Res. 2015, 75, 2435. [CrossRef]

15. Wynes, M.W.; Hinz, T.K.; Gao, D.; Martini, M.; Marek, L.A.; Ware, K.E.; Edwards, M.G.; Böhm, D.; Perner, S.; Helfrich, B.A.; et al. FGFR1 mRNA and protein expression, not gene copy number, predict FGFR TKI sensitivity across all lung cancer histologies. Clin. Cancer Res. 2014, 20, 3299-3309. [CrossRef] [PubMed] 
16. Ruotsalainen, T.; Joensuu, H.; Mattson, K.; Salven, P. High pretreatment serum concentration of basic fibroblast growth factor is a predictor of poor prognosis in small cell lung cancer. Cancer Epidemiol. Biomarkers Prev. 2002, 11, 1492-1495. [PubMed]

17. Brown, W.S.; Tan, L.; Smith, A.; Gray, N.S.; Wendt, M.K.; Wendt, M.; Taylor, M.; Schiemann, B.; Sossey-Alaoui, K.; Schiemann, W.; et al. Covalent targeting of fibroblast growth factor receptor inhibits metastatic breast cancer. Mol. Cancer Ther. 2016, 15, 2096-2106. [CrossRef] [PubMed]

18. Ren, Y.; Jia, H.H.; Xu, Y.Q.; Zhou, X.; Zhao, X.H.; Wang, Y.F.; Song, X.; Zhu, Z.Y.; Sun, T.; Dou, Y.; et al. Paracrine and epigenetic control of CAF-induced metastasis: The role of HOTAIR stimulated by TGF- $\beta 1$ secretion. Mol. Cancer 2018, 17, 5. [CrossRef] [PubMed]

19. Huang, C.; Yuan, P.; Wu, J.; Huang, J. Estrogen regulates excitatory amino acid carrier 1 (EAAC1) expression through sphingosine kinase 1 (SphK1) transacting FGFR-mediated ERK signaling in rat C6 astroglial cells. Neuroscience 2016, 319, 9-22. [CrossRef] [PubMed]

20. Jia, M.; Dahlman-Wright, K.; Gustafsson, J.A. Estrogen receptor alpha and beta in health and disease. Best Pract. Res. Clin. Endocrinol. Metab. 2015, 29, 557-568. [CrossRef] [PubMed]

21. Prossnitz, E.R.; Maggiolini, M. Mechanisms of estrogen signaling and gene expression via GPR30. Mol. Cell. Endocrinol. 2009, 308, 32-38. [CrossRef] [PubMed]

22. Lappano, R.; Maggiolini, M. G protein-coupled receptors: Novel targets for drug discovery in cancer. Nat. Rev. Drug Discov. 2011, 10, 47-60. [CrossRef] [PubMed]

23. Maggiolini, M.; Picard, D. The unfolding stories of GPR30, a new membrane-bound estrogen receptor. J. Endocrinol. 2010, 204, 105-114. [CrossRef] [PubMed]

24. Pandey, D.P.; Lappano, R.; Albanito, L.; Madeo, A.; Maggiolini, M.; Picard, D. Estrogenic GPR30 signalling induces proliferation and migration of breast cancer cells through CTGF. EMBO J. 2009, 28, 523-532. [CrossRef] [PubMed]

25. De Francesco, E.M.; Pellegrino, M.; Santolla, M.F.; Lappano, R.; Ricchio, E.; Abonante, S.; Maggiolini, M. GPER mediates activation of HIF1 $\alpha$ /VEGF signaling by estrogens. Cancer Res. 2014, 74, 4053-4064. [CrossRef] [PubMed]

26. Lappano, R.; Santolla, M.F.; Pupo, M.; Sinicropi, M.S.; Caruso, A.; Rosano, C.; Maggiolini, M. MIBE acts as antagonist ligand of both estrogen receptor $\alpha$ and GPER in breast cancer cells. Breast Cancer Res. 2012, 14, R12. [CrossRef] [PubMed]

27. Lappano, R.; Rosano, C.; Santolla, M.F.; Pupo, M.; de Francesco, E.M.; de Marco, P.; Ponassi, M.; Spallarossa, A.; Ranise, A.; Maggiolini, M. Two novel GPER agonists induce gene expression changes and growth effects in cancer cells. Curr. Cancer Drug Targets 2012, 12, 531-542. [CrossRef] [PubMed]

28. De Marco, P.; Lappano, R.; De Francesco, E.M.; Cirillo, F.; Pupo, M.; Avino, S.; Vivacqua, A.; Abonante, S.; Picard, D.; Maggiolini, M. GPER signalling in both cancer-associated fibroblasts and breast cancer cells mediates a feedforward IL1 $\beta /$ IL1R1 response. Sci. Rep. 2016, 6, 24354. [CrossRef] [PubMed]

29. Santolla, M.F.; Avino, S.; Pellegrino, M.; De Francesco, E.M.; De Marco, P.; Lappano, R.; Vivacqua, A.; Cirillo, F.; Rigiracciolo, D.C.; Scarpelli, A.; et al. SIRT1 is involved in oncogenic signaling mediated by GPER in breast cancer. Cell Death Dis. 2015, 6, e1834. [CrossRef] [PubMed]

30. De Marco, P.; Bartella, V.; Vivacqua, A.; Lappano, R.; Santolla, M.F.; Morcavallo, A.; Pezzi, V.; Belfiore, A.; Maggiolini, M. Insulin-like growth factor-I regulates GPER expression and function in cancer cells. Oncogene 2012, 32, 678-688. [CrossRef] [PubMed]

31. Vivacqua, A.; Sebastiani, A.; Miglietta, A.; Rigiracciolo, D.; Cirillo, F.; Galli, G.; Talia, M.; Santolla, M.; Lappano, R.; Giordano, F.; et al. miR-338-3p is regulated by estrogens through GPER in breast cancer cells and cancer-associated fibroblasts (CAFs). Cells 2018, 7, 203. [CrossRef] [PubMed]

32. Fillmore, C.M.; Gupta, P.B.; Rudnick, J.A.; Caballero, S.; Keller, P.J.; Lander, E.S.; Kuperwasser, C. Estrogen expands breast cancer stem-like cells through paracrine FGF/Tbx3 signaling. Proc. Natl. Acad. Sci. USA 2010, 107, 21737-21742. [CrossRef] [PubMed]

33. Balko, J.M.; Mayer, I.A.; Sanders, M.E.; Miller, T.W.; Kuba, M.G.; Meszoely, I.M.; Wagle, N.; Garraway, L.A.; Arteaga, C.L. Discordant cellular response to presurgical letrozole in bilateral synchronous ER+ breast cancers with a KRAS mutation or FGFR1 gene amplification. Mol. Cancer Ther. 2012, 11, 2301-2305. [CrossRef] [PubMed] 
34. De Francesco, E.M.; Lappano, R.; Santolla, M.F.; Marsico, S.; Caruso, A.; Maggiolini, M. HIF-1alpha/GPER signaling mediates the expression of VEGF induced by hypoxia in breast cancer associated fibroblasts (CAFs). Breast Cancer Res. 2013, 15, R64. [CrossRef] [PubMed]

35. Santolla, M.F.; De Francesco, E.M.; Lappano, R.; Rosano, C.; Abonante, S.; Maggiolini, M. Niacin activates the $G$ protein estrogen receptor (GPER)-mediated signalling. Cell. Signal. 2014, 26, 1466-1475. [CrossRef] [PubMed]

36. Pisano, A.; Santolla, M.F.; De Francesco, E.M.; De Marco, P.; Rigiracciolo, D.C.; Perri, M.G.; Vivacqua, A.; Abonante, S.; Cappello, A.R.; Dolce, V.; et al. GPER, IGF-IR, and EGFR transduction signaling are involved in stimulatory effects of zinc in breast cancer cells and cancer-associated fibroblasts. Mol. Carcinog. 2017, 56, 580-593. [CrossRef] [PubMed]

37. Ran, F.A.; Hsu, P.D.P.; Wright, J.; Agarwala, V.; Scott, D.A.; Zhang, F. Genome engineering using the CRISPR-Cas9 system. Nat. Protoc. 2013, 8, 2281-2308. [CrossRef] [PubMed]

38. Cerami, E.; Gao, J.; Dogrusoz, U.; Gross, B.E.; Sumer, S.O.S.; Aksoy, B.A.B.; Jacobsen, A.; Byrne, C.J.; Heuer, M.L.; Larsson, E.; et al. The cBio cancer genomics portal: An open platform for exploring multidimensional cancer genomics data. Cancer Discov. 2012, 2, 401-404. [CrossRef] [PubMed]

39. Gao, J.; Aksoy, B.A.; Dogrusoz, U.; Dresdner, G.; Gross, B.; Sumer, S.O.; Sun, Y.; Jacobsen, A.; Sinha, R.; Larsson, E.; et al. Integrative analysis of complex cancer genomics and clinical profiles using the cBioPortal. Sci.Signal. 2013, 6, 1-19. [CrossRef] [PubMed]

40. Curtis, C.; Shah, S.P.; Chin, S.F.; Turashvili, G.; Rueda, O.M.; Dunning, M.J.; Speed, D.; Lynch, A.G.; Samarajiwa, S.; Yuan, Y.; et al. The genomic and transcriptomic architecture of 2000 breast tumours reveals novel subgroups. Nature 2012, 486, 346-352. [CrossRef] [PubMed]

41. Pereira, B.; Chin, S.F.; Rueda, O.M.; Vollan, H.K.M.; Provenzano, E.; Bardwell, H.A.; Pugh, M.; Jones, L.; Russell, R.; Sammut, S.J.; et al. The somatic mutation profiles of 2433 breast cancers refines their genomic and transcriptomic landscapes. Nat. Commun. 2016, 7, 11479. [CrossRef] [PubMed]

42. Vicente-Manzanares, M.; Koach, M.A.; Whitmore, L.; Lamers, M.L.; Horwitz, A.F. Segregation and activation of myosin IIB creates a rear in migrating cells. J. Cell Biol. 2008, 183, 543-554. [CrossRef] [PubMed]

43. Siegfried, J.M.; Farooqui, M.; Rothenberger, N.J.; Dacic, S.; Stabile, L.P. Interaction between the estrogen receptor and fibroblast growth factor receptor pathways in non-small cell lung cancer. Oncotarget 2017, 8, 24063-24076. [CrossRef] [PubMed]

44. Coleman, S.J.; Chioni, A.-M.; Ghallab, M.; Anderson, R.K.; Lemoine, N.R.; Kocher, H.M.; Grose, R.P. Nuclear translocation of FGFR1 and FGF2 in pancreatic stellate cells facilitates pancreatic cancer cell invasion. EMBO Mol. Med. 2014, 6, 467-481. [CrossRef] [PubMed]

45. Tanner, Y.; Grose, R.P. Dysregulated FGF signalling in neoplastic disorders. Semin. Cell Dev. Biol. 2016, 53, 126-135. [CrossRef] [PubMed]

46. Wang, K.; Ji, W.; Yu, Y.; Li, Z.; Niu, X.; Xia, W.; Lu, S. FGFR1-ERK1/2-SOX2 axis promotes cell proliferation, epithelial-mesenchymal transition, and metastasis in FGFR1-amplified lung cancer. Oncogene 2018, 37, 5340-5354. [CrossRef] [PubMed]

47. Sharpe, R.; Pearson, A.; Herrera-Abreu, M.T.; Johnson, D.; Mackay, A.; Welti, J.C.; Natrajan, R.; Reynolds, A.R.; Reis-Filho, J.S.; Ashworth, A.; et al. FGFR signaling promotes the growth of triple-negative and basal-like breast cancer cell lines both in vitro and in vivo. Clin. Cancer Res. 2011, 17, 5275-5286. [CrossRef] [PubMed]

48. Lundin, L.; Rönnstrand, L.; Cross, M.; Hellberg, C.; Lindahl, U.; Claesson-Welsh, L. Differential tyrosine phosphorylation of fibroblast growth factor (FGF) receptor-1 and receptor proximal signal transduction in response to FGF-2 and heparin. Exp. Cell Res. 2003, 287, 190-198. [CrossRef]

49. Xu, R.; Zhao, H.; Muhammad, H.; Dong, M.; Besenbacher, F.; Chen, M. Dual-delivery of FGF-2/CTGF from silk fibroin/PLCL-PEO coaxial fibers enhances MSC proliferation and fibrogenesis. Sci. Rep. 2017, 7, 8509. [CrossRef] [PubMed]

50. Aoyama, E.; Kubota, S.; Takigawa, M. CCN2/CTGF binds to fibroblast growth factor receptor 2 and modulates its signaling. FEBS Lett. 2012, 586, 4270-4275. [CrossRef] [PubMed]

51. Chujo, S.; Shirasaki, F.; Kondo-Miyazaki, M.; Ikawa, Y.; Takehara, K. Role of connective tissue growth factor and its interaction with basic fibroblast growth factor and macrophage chemoattractant protein-1 in skin fibrosis. J. Cell. Physiol. 2009, 220, 189-195. [CrossRef] [PubMed] 
52. Yang, M.; Yu, X.; Li, X.; Luo, B.; Yang, W.; Lin, Y.; Li, D.; Gan, Z. TNFAIP3 is required for FGFR1 activation-promoted proliferation and tumorigenesis of premalignant DCIS. COM human mammary epithelial cells. Breast Cancer Res. 2018, 20, 1-14. [CrossRef] [PubMed]

53. Compagni, A.; Wilgenbus, P.; Impagnatiello, M.A.; Cotten, M.; Christofori, G. Fibroblast growth factors are required for efficient tumor angiogenesis. Cancer Res. 2000, 60, 7163-7169. [PubMed]

54. Ronca, R.; Giacomini, A.; Rusnati, M.; Presta, M. The potential of fibroblast growth factor/fibroblast growth factor receptor signaling as a therapeutic target in tumor angiogenesis. Expert Opin. Ther. Targets 2015, 19, 1361-1377. [CrossRef] [PubMed]

55. Liotta, L.A.; Kohn, E.C. The microenvironment of the tumour-host interface. Nature 2001, 411, 375-379. [CrossRef] [PubMed]

56. Quail, D.F.; Joyce, J.A. Microenvironmental regulation of tumor progression and metastasis. Nat. Med. 2013, 19, 1423-1437. [CrossRef] [PubMed]

57. Bhowmick, N.A.; Neilson, E.G.; Moses, H.L. Stromal fibroblasts in cancer initiation and progression. Nature 2004, 432, 332-337. [CrossRef] [PubMed]

58. De Wever, O.; Mareel, M. Role of tissue stroma in cancer cell invasion. J. Pathol. 2003, 200, 429-447. [CrossRef] [PubMed]

59. De Wever, O.; Demetter, P.; Mareel, M.; Bracke, M. Stromal myofibroblasts are drivers of invasive cancer growth. Int. J. Cancer 2008, 123, 2229-2238. [CrossRef] [PubMed]

60. Van der Horst, G.; Bos, L.; van der Pluijm, G. Epithelial plasticity, cancer stem cells, and the tumor-supportive stroma in bladder carcinoma. Mol. Cancer Res. 2012, 10, 995-1009. [CrossRef] [PubMed]

61. Karagiannis, G.S.; Poutahidis, T.; Erdman, S.E.; Kirsch, R.; Riddell, R.H.; Diamandis, E.P. Cancer-associated fibroblasts drive the progression of metastasis through both paracrine and mechanical pressure on cancer tissue. Mol. Cancer Res. 2012, 10, 1403-1418. [CrossRef] [PubMed]

62. Orimo, A.; Gupta, P.B.; Sgroi, D.C.; Arenzana-Seisdedos, F.; Delaunay, T.; Naeem, R.; Carey, V.J.; Richardson, A.L.; Weinberg, R.A. Stromal fibroblasts present in invasive human breast carcinomas promote tumor growth and angiogenesis through elevated SDF-1/CXCL12 secretion. Cell 2005, 121, 335-348. [CrossRef] [PubMed]

63. Yamaguchi, Y.; Hayashi, S. Estrogen-related cancer microenvironment of breast carcinoma. Endocr. J. 2009, 56, 1-7. [CrossRef] [PubMed]

64. Sobhani, N.; Ianza, A.; D'Angelo, A.; Roviello, G.; Giudici, F.; Bortul, M.; Zanconati, F.; Bottin, C.; Generali, D. Current status of fibroblast growth factor receptor-targeted therapies in breast cancer. Cells 2018, 7, 76. [CrossRef] [PubMed]

65. Campbell, J.; Ryan, C.J.; Brough, R.; Bajrami, I.; Pemberton, H.N.; Chong, I.Y.; Costa-Cabral, S.; Frankum, J.; Gulati, A.; Holme, H.; et al. Large-scale profiling of kinase dependencies in cancer cell lines. Cell Rep. 2016, 14, 2490-2501. [CrossRef] [PubMed]

66. Touat, M.; Ileana, E.; Postel-Vinay, S.; André, F.; Soria, J.C. Targeting FGFR signaling in cancer. Clin. Cancer Res. 2015, 21, 2684-2694. [CrossRef] [PubMed]

67. Mohammadi, M.; Froum, S.; Hamby, J.M.; Schroeder, M.C.; Panek, R.L.; Lu, G.H.; Eliseenkova, V.A.; Green, D.; Schlessinger, J.; Hubbard, S.R. Crystal structure of an angiogenesis inhibitor bound to the FGF receptor tyrosine kinase domain. EMBO J. 1998, 17, 5896-5904. [CrossRef] [PubMed]

68. Dutt, A.; Salvesen, H.B.; Chen, T.-H.; Ramos, A.H.; Onofrio, R.C.; Hatton, C.; Nicoletti, R.; Winckler, W.; Grewal, R.; Hanna, M.; et al. Drug-sensitive FGFR2 mutations in endometrial carcinoma. Proc. Natl. Acad. Sci. USA 2008, 105, 8713-8717. [CrossRef] [PubMed]

69. Van't Veer, L.J.; Weigelt, B. Road map to metastasis. Nat. Med. 2003, 9, 999-1000. [CrossRef] [PubMed]

70. Kraljevic, S.P.; Sedic, M.; Bosnjak, H.; Spaventi, S.; Pavelic, K. Metastasis: New perspectives on an old problem. Mol. Cancer 2011, 11, 22. [CrossRef] [PubMed]

71. Lamouille, S.; Xu, J.; Derynck, R. Molecular mechanisms of epithelial-mesenchymal transition. Nat. Rev. Mol. Cell Biol. 2014, 15, 178. [CrossRef] [PubMed]

72. De Craene, B.; Berx, G. Regulatory networks defining EMT during cancer initiation and progression. Nat. Rev. Cancer 2013, 13, 97. [CrossRef] [PubMed]

73. Acevedo, V.D.; Gangula, R.D.; Freeman, K.W.; Li, R.; Zhang, Y.; Wang, F.; Ayala, G.E.; Peterson, L.E.; Ittmann, M.; Spencer, D.M. Inducible FGFR-1 activation leads to irreversible prostate adenocarcinoma and an epithelial-to-mesenchymal transition. Cancer Cell 2007, 12, 559-571. [CrossRef] [PubMed] 
74. Abramson, V.G.; Lehmann, B.D.; Ballinger, T.J.; Pietenpol, J.A. Subtyping of triple-negative breast cancer: Implications for therapy. Cancer 2015, 121, 8-16. [CrossRef] [PubMed]

75. Chen, P.-S.; Wang, M.-Y.; Wu, S.-N.; Su, J.-L.; Hong, C.-C.; Chuang, S.-E.; Chen, M.-W.; Hua, K.-T.; Wu, Y.-L.; Cha, S.-T; et al. CTGF enhances the motility of breast cancer cells via an integrin- $\alpha \mathrm{v} \beta 3$-ERK1/2-dependent S100A4-upregulated pathway. J. Cell Sci. 2007, 120, 2053-2065. [CrossRef] [PubMed]

76. Goltsov, A.; Deeni, Y.; Khalil, H.; Soininen, T.; Kyriakidis, S.; Hu, H.; Langdon, S.; Harrison, D.; Bown, J. Systems analysis of drug-induced receptor tyrosine kinase reprogramming following targeted mono- and combination anti-cancer therapy. Cells 2014, 3, 563-591. [CrossRef] [PubMed]

77. Cicha, I.; Goppelt-Struebe, M. Connective tissue growth factor: Context-dependent functions and mechanisms of regulation. BioFactors 2009, 35, 200-208. [CrossRef] [PubMed]

78. Kang, Y.; Siegel, P.M.; Shu, W.; Drobnjak, M.; Kakonen, S.M.; Cordón-Cardo, C.; Guise, T.A.; Massagué, J. A multigenic program mediating breast cancer metastasis to bone. Cancer Cell 2003, 3, 537-549. [CrossRef]

(C) 2019 by the authors. Licensee MDPI, Basel, Switzerland. This article is an open access article distributed under the terms and conditions of the Creative Commons Attribution (CC BY) license (http:/ / creativecommons.org/licenses/by/4.0/). 
Article

\title{
Preclinical Evaluation of the Pan-FGFR Inhibitor LY2874455 in FRS2-Amplified Liposarcoma
}

\author{
Robert Hanes ${ }^{1,2}$, Else Munthe ${ }^{1}$, Iwona Grad ${ }^{1}$, Jianhua Han ${ }^{3}$, Ida Karlsen ${ }^{3,4}$, \\ Emmet McCormack ${ }^{3,5}$, Leonardo A. Meza-Zepeda ${ }^{1,2,6}$, Eva Wessel Stratford ${ }^{1}$ \\ and Ola Myklebost ${ }^{1,2,7, *}$ \\ 1 Department of Tumor Biology, Institute of Cancer Research, the Norwegian Radium Hospital, \\ Oslo University Hospital, 0379 Oslo, Norway; Robert.Hanes@rr-research.no (R.H.); \\ Else.Munthe@rr-research.no (E.M.); Iwona.Grad@rr-research.no (I.G.); \\ Leonardo.A.Meza-Zepeda@rr-research.no (L.A.M.-Z.); eva.wessel.stratford@rr-research.no (E.W.S.) \\ 2 Norwegian Cancer Genomics Consortium, 0379 Oslo, Norway \\ 3 Centre for Cancer Biomarkers (CCBIO), Department of Clinical Sciences, University of Bergen, 5021 Bergen, \\ Norway; Jianhua.Han@uib.no (J.H.); idakarlsenemail@gmail.com (I.K.); \\ emmet.mc.cormack@med.uib.no (E.M.) \\ 4 KinN Therapeutics AS, 5021 Bergen, Norway \\ 5 Department of Internal Medicine, Hematology Section, Haukeland University Hospital, \\ 5021 Bergen, Norway \\ 6 Genomics Core Facility, Department of Core Facilities, Institute of Cancer Research, the Norwegian Radium \\ Hospital, Oslo University Hospital, 0379 Oslo, Norway \\ 7 Department of Clinical Science, University of Bergen, 5020 Bergen, Norway \\ * Correspondence: ola.myklebost@uib.no; Tel.: +47-5558-4565
}

Received: 16 January 2019; Accepted: 18 February 2019; Published: 21 February 2019

\begin{abstract}
Background: FGFR inhibition has been proposed as treatment for dedifferentiated liposarcoma (DDLPS) with amplified FRS2, but we previously only demonstrated transient cytostatic effects when treating FRS2-amplified DDLPS cells with NVP-BGJ398. Methods: Effects of the more potent FGFR inhibitor LY2874455 were investigated in three DDLPS cell lines by measuring effects on cell growth and apoptosis in vitro and also testing efficacy in vivo. Genome, transcriptome and protein analyses were performed to characterize the signaling components in the FGFR pathway. Results: LY2874455 induced a stronger, longer-lasting growth inhibitory effect and moderate level of apoptosis for two cell lines. The third cell line, did not respond to FGFR inhibition, suggesting that FRS2 amplification alone is not sufficient to predict response. Importantly, efficacy of LY2874455 was confirmed in vivo, using an independent FRS2-amplified DDLPS xenograft model. Expression of FRS2 was similar in the responding and non-responding cell lines and we could not find any major difference in downstream FGFR signaling. The only FGF expressed by unstimulated non-responding cells was the intracellular ligand FGF11, whereas the responding cell lines expressed extracellular ligand FGF2. Conclusion: Our study supports LY2874455 as a better therapy than NVP-BGJ398 for FRS2-amplified liposarcoma, and a clinical trial is warranted.
\end{abstract}

Keywords: FRS2; FGFR; NVP-BGJ398; LY2874455; sarcoma

\section{Introduction}

Sarcomas are rare cancers of mesenchymal origin, accounting for approximately $1 \%$ of all solid cancers, and can be classified into more than 50 distinct histological subtypes [1]. Liposarcomas (LPS), which resemble adipose tissue, are further classified into three main subtypes, well-differentiated/dedifferentiated liposarcoma (WD/DDLPS), myxoid/round cell liposarcoma, and pleomorphic liposarcoma [2]. The heterogeneity makes clinical research and trials challenging. 
However, all together rare cancers comprise one of the largest patient groups, which is in great need of new therapeutic approaches. Therefore, a deeper mechanistic understanding is needed to be able to identify and validate new potential targets. In a previous study, we identified amplifications of multiple genes in the 12q14.1-q15 region in the DDLPS cell line NRH-LS1 and investigated several of these as therapeutic targets [3]. One of these, FRS2, is generally co-amplified with MDM2 in WD/DDLPS [4]. FRS2 codes for an important component of the FGF receptor (FGFR) signaling pathway, which plays crucial roles in multiple biological processes, such as cell growth, survival and differentiation, as well as tumor development and progression [5,6]. FRS2-dependent FGFR signaling is induced through FGFR activation by FGF ligands, and consecutive phosphorylation of FRS2 triggers an intracellular signaling cascade involving RAS/MAPK/ERK and PI3K/AKT [7], leading to oncogenic pro-survival and anti-apoptotic properties and increased proliferation and migration. To date, there are no drugs available that can target FRS2 directly, but attenuating the signal from FGFR, upstream of FRS2, with FGFR inhibitors has been shown to be growth inhibitory in such cells [3,8].

NVP-BGJ398, which is in phase II clinical trials, has been shown to be a potent and selective FGFR inhibitor in a wide panel of cancer cell lines [9]. NVP-BGJ398 has been reported to selectively inhibit FGFR1, -2 and -3 with IC50s of $0.9 \mathrm{nM}, 1.4 \mathrm{nM}$ and $1.0 \mathrm{nM}$, respectively, whereas the IC50 for FGFR4 is $60 \mathrm{nM}$ [10]. Another pan-FGFR inhibitor, LY2874455, recently completed a phase I clinical trial [11], and has been reported to selectively inhibit FGFR1, $-2,-3$, and -4 with IC50s of $2.8 \mathrm{nM}, 2.6 \mathrm{nM}, 6.4 \mathrm{nM}$ and $6 \mathrm{nM}$, respectively [12].

In this study, we have investigated the therapeutic potential of LY2874455 with the aim to improve efficacy for FRS2-amplified DDLPS.

\section{Materials and Methods}

\subsection{Cell Line and Culture Conditions}

The DDLPS cell lines NRH-LS1, established from a patient-derived xenograft as previously described [3] and LPS510 and LPS853, kindly provided by Dr. Jonathan Fletcher, were cultured in RPMI-1640 medium (Sigma-Aldrich, St. Louis, MO, USA) supplemented with $10 \%$ FBS (Sigma-Aldrich), 1\% L-alanyl-L-glutamine (Sigma-Aldrich) and 1\% penicillin-streptomycin (Sigma-Aldrich) and grown at $37^{\circ} \mathrm{C}, 5 \% \mathrm{CO}_{2}$. Short tandem repeat DNA profiling was performed on all cell lines to confirm identity. Cells were negative for mycoplasma using the VenorGeM Mycoplasma Detection Kit (Minerva Biolabs, Berlin, Germany).

\subsection{Drugs}

LY2874455 (\#S7057) (Selleck Chemicals, Munich, Germany) was dissolved in DMSO (Sigma-Aldrich) according to the manufacturer's recommendation. For each experiment the appropriate control (referred to as untreated) was used, with a DMSO concentration corresponding to that used with the highest drug concentration. The concentration of DMSO in the control for $100 \mathrm{nM}$ LY2874455 and NVP-BGJ398 was $0.01 \%$ and for $1 \mu \mathrm{M}$ LY2874455 and NVP-BGJ398 $0.1 \%$.

\subsection{Drug Treatment and Cell Proliferation Assay}

The cellular proliferation rate was measured using a live-cell imaging system, IncuCyte ZOOM (Essen Bioscience, Birmingham, UK) with the corresponding software application (version 2013BRev1), (Essen Bioscience, Birmingham, UK). Cells were seeded into 96-well plates, and drug treatment initiated after 16h, in triplicates. Each drug treatment was performed over a period of 1-2 weeks and done at least three times. Proliferation rate was measured as cell confluence over time every third hour.

\subsection{Apoptosis}

Apoptosis assays were done with the IncuCyte ZOOM. To measure apoptosis, the CellPlayer 96-Well Kinetic Caspase-3/7 reagent containing DEVD-NucViewTM488 (Essen Bioscience) at a 
concentration of $2 \mu \mathrm{M}$ was added at the same time as the drug. Total numbers of apoptotic cells were counted in the green channel $(488 \mathrm{~nm})$. After $96 \mathrm{~h}$, cells were incubated for 30 minutes with $4 \mu \mathrm{M}$ of Nuclear-ID Red DNA stain (Enzo Life Sciences, Farmingdale, NY, USA), and total cell count was measured in the red channel $(566 \mathrm{~nm})$. The percentage of apoptotic cells per well was calculated as the number of apoptotic cells relative to the total number of nuclei.

\subsection{Viability Assay for Dose-Response Curve and IC50 Calculations}

Cell viability was measured using the CellTiter-Glo Luminescent Cell Viability Assay (Promega, Madison, WI, USA). $5 \times 10^{3}$ cells per well were seeded onto a 96 -well flat and clear bottom polystyrene treated microplate (Corning, Corning, NY, USA). The drug treatment was initiated $16 \mathrm{~h}$ after seeding and was applied at concentrations ranging from $0.1 \mathrm{nM}$ to $1000 \mathrm{nM}$. After 72, 96 and $120 \mathrm{~h}$, ATP levels were used as a measure of viability. Relative IC50 for $120 \mathrm{~h}$ was calculated using a four-parameter logistic function [13] based on non-linear regression analysis using SigmaPlot (Systat Software Inc, San Jose, CA, USA) version 12.5.0.38.

\subsection{In Vivo Assay}

Animal experiments were performed according to protocols approved by the National Animal Research Authority (Mattilsynet) in compliance with the European Convention of the Protection of Vertebrates Used for Scientific Purposes (ID 10175). The LS70x xenograft (established directly from a DDLPS tumor) was implanted into the flank of immunodeficient NOD-scid IL2rynull (NSG) mice [14]. When tumors reached $150 \mathrm{~mm}^{3}$, animals were randomized into two groups, each of six mice, and treated twice per day with either $3 \mathrm{mg} / \mathrm{kg}$ LY2874455 or vehicle only ( $2 \%(v / v)$ DMSO, $30 \%(v / v)$ PEG 300, 5\% (v/v) Tween 80 in sterile water), administered by oral gavage. Treatment was performed for 28 days, or until the tumor reached a size of $1 \mathrm{~cm}^{3}$. Tumor growth was measured by caliper measurements twice per week for the duration of treatment. Unpaired two-tailed $t$-test was performed to detect significant differences in tumor volumes ( $p \leq 0.05$ was considered significant).

\subsection{Western Blots}

Cells were treated for $24 \mathrm{~h}$ with either $100 \mathrm{nM}$ LY2874455, $100 \mathrm{nM}$ NVP-BGJ398 or control-treated with the corresponding concentration of DMSO and the last $15 \mathrm{~min}$ with or without $15 \mathrm{ng} / \mathrm{ml}$ of recombinant human FGF1 [15] and $10 \mathrm{U} / \mathrm{mL}$ of Heparin. In vitro cells were washed with PBS and dissolved in SDS lysis buffer. Xenografts were cut into smaller pieces and snap frozen. Proteins were extracted with T-Per lysis buffer (Thermo Fisher Scientific, Waltham, MA, USA), supplemented with protease and phosphatase inhibitors (both from Thermo Fischer Scientific), using the TissueLyser LT (QIAGEN, Venlo, Netherlands). DTT was added to the lysates before boiling. Proteins were separated in a 4-12\% Novex PAGE gel in MOPS running buffer, and transferred to PVDF membranes (Thermo Fisher Scientific). The following antibodies were used: pFRS2-TYR436 (\#3861), AKT (\#9272), pAKT-SER473 (\#9271), ERK (\#9102), pERK-T202/Y204 (\#4370), PLC $\gamma 1$ (\#5690), pPLC $\gamma 1-T Y R 783$ (\#2821) (all from Cell Signaling Technology, Danvers, MA, USA), FRS2 (\#SC8318) (Santa Cruz Biotechnology, Dallas, TX, USA) and $\alpha$-Tubulin (\#CP06) (Merck KGaA, Darmstadt, Germany). All antibodies were diluted 1:1000, except FRS2 (1:500) and $\alpha$-Tubulin (1:2000). Secondary antibodies were rabbit anti-mouse immunoglobulins/HRP (\#P0260) and goat anti-rabbit immunoglobulins/HRP (\#P0448) (Dako, Glostrup, Denmark) at a concentration of $1.3 \mathrm{~g} / \mathrm{L}$ and $0.25 \mathrm{~g} / \mathrm{L}$ respectively. The Western blots were developed using the Supersignal Western Dura substrate (Thermo Fisher Scientific), and detected and quantified on a Syngene G-Box (Synoptics Group, Cambridge, UK) with the GeneSnap (version 7.12, Synoptics Group) and the GeneTools (version 4.3.7.0, Synoptics Group) programs, respectively.

\subsection{Quantitative Real-Time PCR-Based Copy Number Assay}

DNA was isolated from cells using the AllPrep DNA/RNA Mini Kit (QIAGEN) according to the manufacturer's protocol. Quantitative real-time PCR was performed based on absolute quantitation 
using the Applied Biosystems 7900HT fast real-time PCR system (Applied Biosystems, Foster By, CA, USA). The copy numbers of FRS2 (Hs02860563_cn), ALB (Hs05929625_cn) and LSAMP (Hs05902664_cn) were determined using TaqMan copy number assays from Applied Biosystems, ALB and LSAMP were used as endogenous controls, as these have low level of DNA copy number changes in a large panel of liposarcoma samples [16]. The copy numbers were determined using the CopyCaller Software v2.1 program (Applied Biosystems) as described by the manufacturer, and the FRS2 data were normalized to $L S A M P$. The copy numbers were validated using $A L B$ as another endogenous reference gene (data not shown).

\subsection{Quantitative Real-Time PCR Based Expression Assay}

RNA was isolated from cells using the AllPrep DNA/RNA Mini Kit (QIAGEN) according to the manufacturers protocol. cDNA was prepared using $1 \mu \mathrm{g}$ of RNA and the SuperScript VILO Master Mix (Invitrogen, Carlsbad, CA, USA). Quantitative real-time PCR was performed based on $\Delta \triangle \mathrm{Ct}$ relative quantitation using the Applied Biosystems 7900HT fast real-time PCR system (Applied Biosystems). The expression levels of FRS2 were determined using TaqMan gene expression assays (Hs00183614_m1) with human B2M (VIC ${ }^{\circledR} /$ MGB probe) (Applied Biosystems) as internal control for normalization. The relative expression levels were determined using the comparative $\Delta \Delta \mathrm{Ct}$ method as described by the manufacturer. Human Adipose Tissue Total RNA was used as reference (Clontech, Mountain View, CA, USA).

\subsection{RNA Sequencing}

RNA was isolated from the cell lines using the AllPrep DNA/RNA Mini Kit (QIAGEN). mRNA sequencing libraries were prepared using $100 \mathrm{ng}$ of total RNA and the Illumina TruSeq Stranded mRNA Library Prep kit for NeoPrep following the supplier's instructions. The libraries were sequenced on a NextSeq 500 Illumina sequencer (Illumina, San Diego, CA, USA) using a High Output v2 kit chemistry, generating $2 \times 75$ bp paired-end sequence reads. RNA-Seq reads were aligned using STAR aligner (v.2.5.0b) against the human reference genome (UCSC hg19, RefSeq and Gencode gene annotations), and FPKM estimation was generated by Cufflinks 2 using the RNA-seq alignment app at Illumina BaseSpace.

\section{Results}

\subsection{Improved Efficacy Using LY2874455}

When treating NRH-LS1 cells at equivalent concentrations, LY2874455 inhibited growth of the cells more efficiently than did NVP-BGJ398 (Figure 1A). NRH-LS1 cells exposed to $100 \mathrm{nM}$ of LY2874455 were completely growth inhibited after $72 \mathrm{~h}$ (Figure 1A), while treatment with $100 \mathrm{nM}$ NVP-BGJ398 gave only partial growth inhibition at that time point. In contrast to NVP-BGJ398, we found that LY2874455 induced apoptosis in a subpopulation of NRH-LS1 cells (Figure 1B). Furthermore, $100 \mathrm{nM}$ LY2874455 induced, on average, four times higher levels of apoptosis after $96 \mathrm{~h}$, as compared to cells treated with 100 nM NVP-BGJ398 (Figure 1C). As shown in Figure 2, LY2874455 inhibited cell growth in a dose-dependent manner (Figure 2A), with the full effect at approximately $100 \mathrm{nM}$. The IC50 for LY2874455 in NRH-LS1 cells was estimated to $7 \mathrm{nM}$ compared to $47 \mathrm{nM}$ for NVP-BGJ398 (Figure S2), based on viability after $120 \mathrm{~h}$ of treatment (Figure 2B). 
A

B
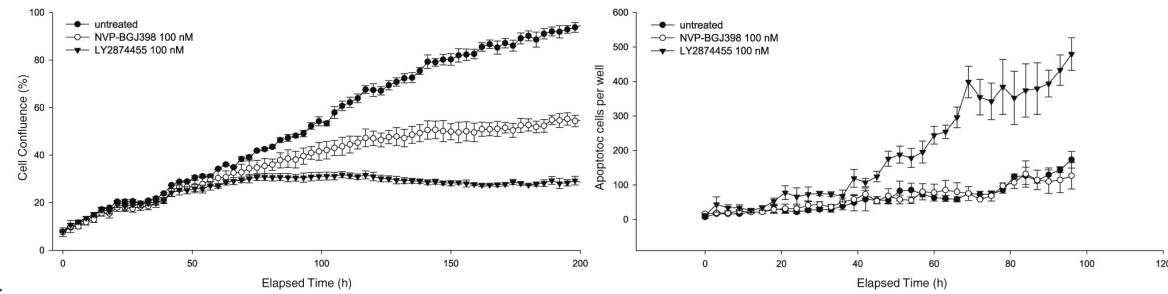

C
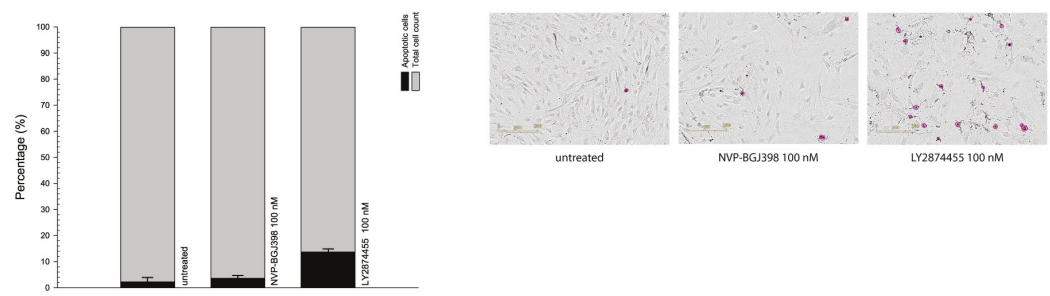

Figure 1. Comparison of the effect of NVP-BGJ398 and LY2874455 on proliferation and apoptosis of NRH-LS1 cells. (A) Proliferation of NRH-LS1 cells after inhibition of FGFR with either NVP-BGJ398 or LY2874455; one representative experiment is shown $(n=3)$, error bars represent the standard error of the mean (SEM) of technical replicates; (B) The number of cells with active caspase $3 / 7$ during $96 \mathrm{~h}$ of treatment with either $100 \mathrm{nM}$ of NVP-BGJ398 or $100 \mathrm{nM}$ LY2874455, one representative experiment is shown $(n=3)$; (C) The percentage of apoptotic cells after $96 \mathrm{~h}$ of treatment with NVP-BGJ398 or LY2874455; the mean of experiments is shown $(n=3)$, error bars represent the standard deviation (SD) of the experiments. Representative images show apoptotic cells outlined in purple based on measured apoptotic signal. For all experiments untreated is with DMSO concentration corresponding to that of the highest drug concentration. 
A

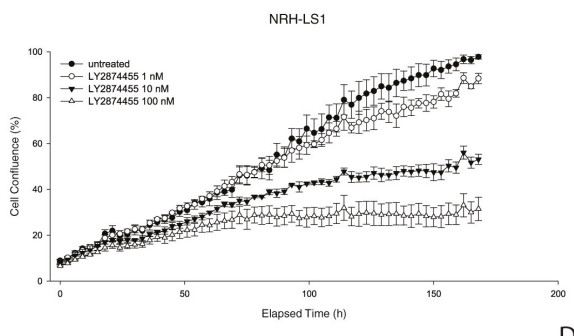

C

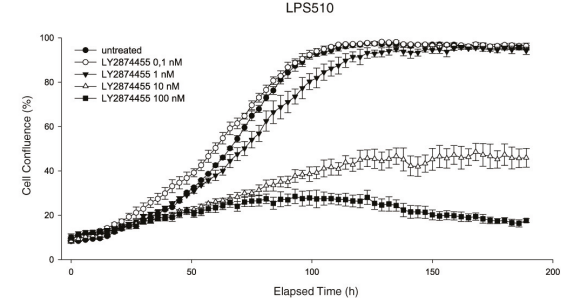

E

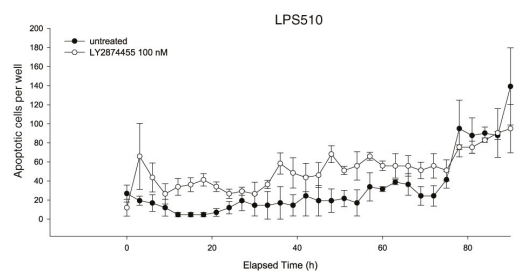

B

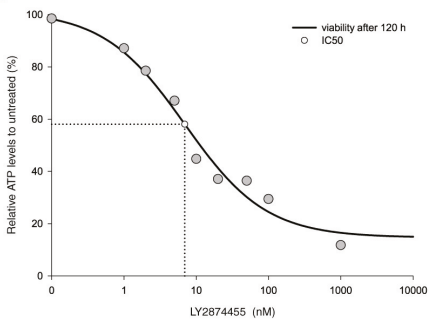

D

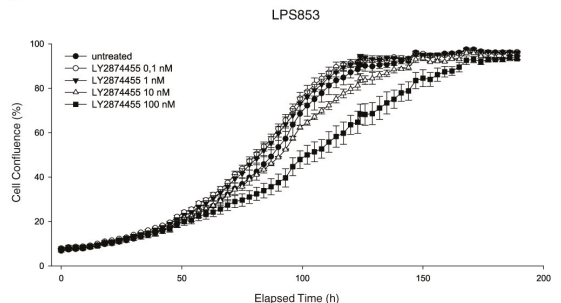

$\mathrm{F}$

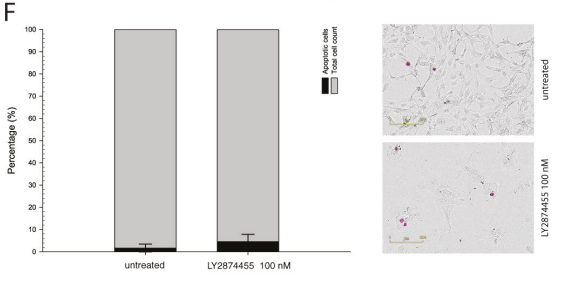

Figure 2. The effect of LY2874455 on the proliferation and viability of NRH-LS1, LPS510 and LPS853 cells. (A) Proliferation of NRH-LS1 cells at different concentrations of LY2874455; one representative experiment is shown $(n=3)$, error bars represent the standard error of the mean (SEM) of technical replicates; (B) The IC50 was estimated at $7 \mathrm{nM}$ based on NRH-LS1 cell viability after $120 \mathrm{~h}$ of treatment with LY2874455; Proliferation of LPS510 (C) and LPS853 (D) cells in the presence of LY2874455. (E) The number of LPS510 cells with active caspase 3/7 during $96 \mathrm{~h}$ of treatment with $100 \mathrm{nM}$ of LY2874455; (C-E) One representative experiment is shown $(n=3)$, error bars represent the standard error of the mean (SEM) of technical replicates; (F) The percentage of apoptotic LPS510 cells after $96 \mathrm{~h}$ of treatment with $100 \mathrm{nM}$ of LY2874455. The mean of experiments is shown $(n=4)$, error bars represent the standard deviation (SD) of the experiments. Representative images show apoptotic cells outlined in purple based on measured apoptotic signal. For all experiments untreated is with DMSO concentration corresponding to that of the highest drug concentration.

\subsection{The Response to FGFR Inhibition in FRS2-Amplified Cell Lines Is Variable}

We next investigated the effect of LY2874455 in two additional FRS2-amplified DDLPS cell lines, LPS510 and LPS853, which have FRS2 copy number and gene expression levels comparable to NRH-LS1 (Figure S1). The expression levels of FRS2 were 10-20 fold higher in all three cell lines compared to both human adipocyte tissue and an undifferentiated immortalized mesenchymal progenitor cell line (iMSC\#3), [17] (Figure S1). LY2874455 inhibited the growth of LPS510 similar to NRH-LS1 (Figure 2C), with IC50 values of 5.5 and 6.9 respectively (Figure S2). In contrast, LPS853 was only modestly inhibited at $100 \mathrm{nM}$ (Figure 2D). Similar levels of apoptosis were observed for both LPS510 and NRH-LS1 after treatment with $100 \mathrm{nM}$ LY2874455, with 9\% for LPS510 cells (Figure 2E,F) and 13\% for NRH-LS1 (Figure 1C) at $96 \mathrm{~h}$. 


\subsection{LY2874455 Induces Long-Lasting Growth Inhibition}

In order to assess duration of the growth inhibitory effects of LY2874455 and NVP-BGJ398, we discontinued drug treatment after $96 \mathrm{~h}$ or $264 \mathrm{~h}$. In contrast to NVP-BGJ398, the growth inhibition of NRH-LS1 and LPS510 was strong after withdrawal of LY2874455, although it was quite significant with LPS510 cells, especially at the highest doses (Figure 3A-D). Both LPS510 and NRH-LS1 maintained growth arrest within the time-frame of the experiment after $264 \mathrm{~h}$ of treatment with LY2874455.

A

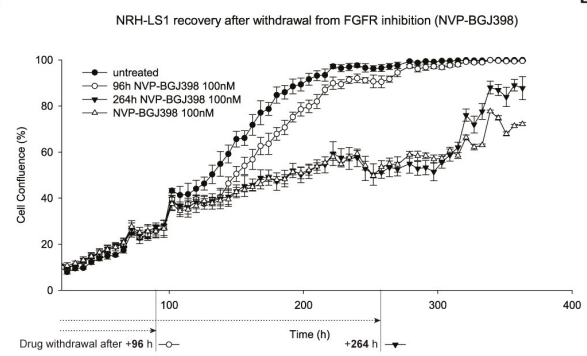

C

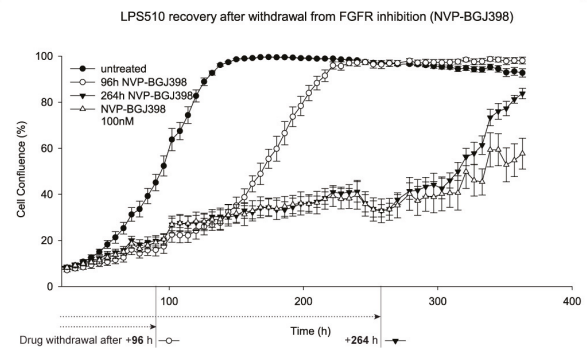

B

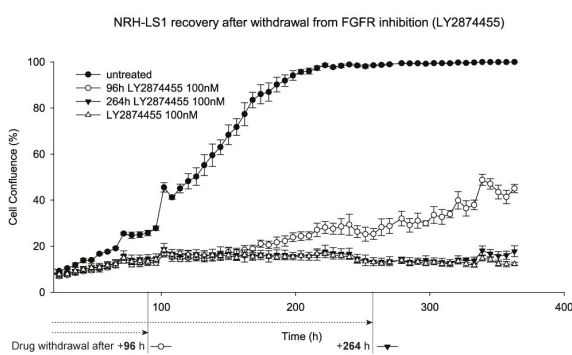

D

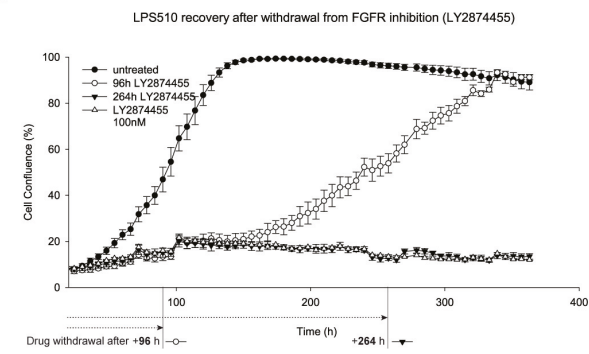

Figure 3. NRH-LS1 and LPS510 cells resume proliferation after withdrawal of treatment with NVP-BGJ398, but not LY2874455. Proliferation of NRH-LS1 (A,B) or LPS510 (C,D) cells treated with $100 \mathrm{nM}$ of NVP-BGJ398 (A,C) or LY2874455 (B,D) continuously or upon withdrawal of the drug after $96 \mathrm{~h}$ or $264 \mathrm{~h}$ of treatment. One representative experiment is shown $(n=3)$, error bars represent the standard error of the mean (SEM) of technical replicates. For all experiments untreated with DMSO concentration corresponding to that of the highest drug concentration.

\subsection{Both FGFR Inhibitors Inactivate MEK/ERK-Dependent Signaling in FRS2-Amplified Cells}

To determine whether the different effects of these drugs could be attributed to the activation or inhibition of different signaling components in the FGFR pathway, we investigated the status of several signaling proteins downstream of FGFR and FRS2 in NRH-LS1 cells upon stimulation with FGF and treatment with NVP-BGJ398 or LY2874455 (Figure 4, quantified in Figure S4A). Although phosphorylation of FRS2 was expected to drive growth in these cells, we only detected phosphorylated FRS2 upon stimulation with exogeneous FGF, probably because pFRS2 levels in unstimulated cells were below the detection limit of the pFRS2 antibody used in this western blot assay. An increased phosphorylation of the downstream signaling protein ERK was also found upon stimulation with FGF1. When NRH-LS1 cells were treated for $24 \mathrm{~h}$ with either $100 \mathrm{nM}$ LY2874455 or 100 nM NVP-BGJ398, FGF1-induced phosphorylation of FRS2 was completely abolished (Figure 4A), supporting the expected drug action, and also endogenous pERK was reduced. Stimulation with FGF1 also induced phosphorylation of PLC $\gamma 1$, an FRS2-independent component of the FGFR pathway, which was completely abolished by the treatment with either FGFR inhibitor. The expression and phosphorylation of AKT was unaffected by FGF1 stimulation or FGFR inhibition (Figure 4A). Thus, 
no clear differences in FGF signaling could be seen that explained the difference in growth inhibition of the two inhibitors in NRH-LS1.

A

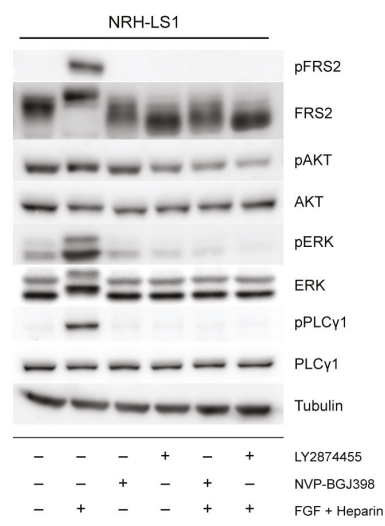

B

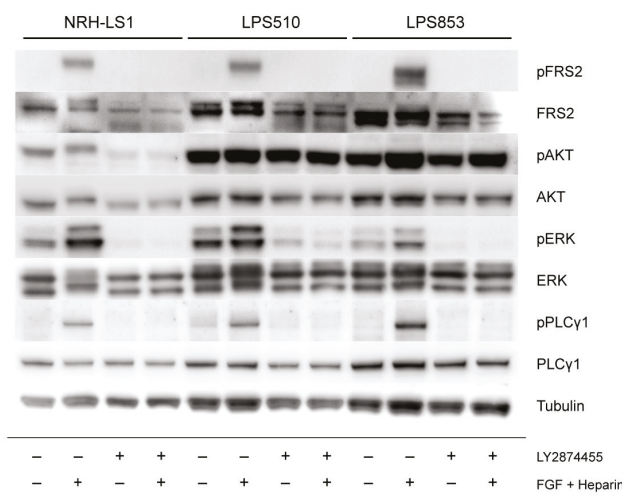

Figure 4. Signaling pathway analysis after FGFR stimulation and inhibition in NRH-LS1, LPS510 and LPS853. Western blots showing (A) The level of phosphorylated and total protein for the indicated proteins in NRH-LS1 cells treated for $24 \mathrm{~h}$ with $100 \mathrm{nM}$ of LY2874455 or $100 \mathrm{nM}$ NVP-BGJ398, with or without FGF stimulation as indicated; (B) The level of phosphorylated and total protein for the indicated proteins in NRH-LS1, LPS510 and LPS853 cells treated for $24 \mathrm{~h}$ with $100 \mathrm{nM}$ of LY2874455, with or without FGF as indicated. In all Western blot experiments $\alpha$-tubulin was used as a loading control.

We further compared the effect of LY2874455 on the same proteins on the three cell lines to understand their difference in sensitivity to FGFR inhibition (Figure 4B, quantified in Figure S4B). The western blots confirmed that FRS2 protein was expressed in all three cell lines, while pFRS2 was below detection in unstimulated cells. Phosphorylation of FRS2, PLC $\gamma 1$, and ERK was induced by stimulation with FGF1 and was inhibited upon treatment with LY2874455 in all three cell lines, showing the ability of these cell lines to respond to FGF stimulation and FGFR inhibition. The phosphorylation levels of AKT were higher in both LPS510 and LPS853 compared to NRH-LS1 but remained unaffected by stimulation or inhibition of FGFR signaling (Figure 4B).

\subsection{The Expression of FGF Receptors Varies in FRS2-Amplified LPS Lines}

In order to investigate why only two out of three LPS cell lines responded to the treatment with FGFR inhibitors and whether this could be explained due to a differential expression of some components of the FGFR pathways, we analyzed transcriptome sequencing data of NRH-LS1, LPS510, and LPS853 for expression of FGFR signaling components upstream of FRS2. Similar relative expression (FPKM) values of 52.0, 51.8 and 44.3 for FRS2 were found in NRH-LS1, LPS510 and LPS853, respectively. All three cell lines also had similar expression levels for FGFR1 with FPKMs of 41.5, 51.5 and 36.1, while FGFR4 was comparably higher expressed in LPS853 with an FPKM of 77.5, compared to 1.2 and 0 in NRH-LS1 and LPS510, respectively. All the cell lines had very low expression of FGFR2 and FGFR3. FGFRL1 is another member of the fibroblast growth factor receptor family, however it lacks the cytoplasmic tyrosine kinase domain and can act as a negative regulator of FGFR signaling [18]. Interestingly, FGFRL1 was higher expressed in LPS853 than in LPS510 and NRH-LS1, with FPKM values of 50.0, 25.8 and 11.1, respectively.

We also found FGF2 to be higher expressed in NRH-LS1 and LPS510 with FPKM values of 19.6 and 21.0 respectively, compared to LPS853 with FPKM of 0.5. In turn, FGF11 expression was higher in LPS853 with FPKM of 24.5, compared to NRH-LS1 and LPS510 with values of 0.4 and 1.2 respectively. 
A heat map that shows the expression of FGF receptors and ligands, as well as adapter proteins, is provided in Figure S3.

\subsection{LY2874455 Inhibits Tumor Growth In Vivo}

Having observed promising therapeutic potential in vitro for two out of three FRS2-amplified LPS cell lines treated with LY2874455, we investigated the effect in vivo. Patient-derived xenograft (PDX) models derived directly from patient material are more representative for drug responses, but the NRH-LS1 PDX model, from which the cell line is derived, grows slowly and was not suitable for preclinical testing. Rather than making a less representative cell line-derived PDX from LPS510, we used the DDLPS patient-derived LS70x PDX [14], which also provided an additional independent model. LS70x has amplification and increased expression of FRS2 comparable to the three cell lines (Figure S1), and grows reasonably well in NSG mice. When tumors reached $150 \mathrm{~mm}^{3}$, mice were treated with $3 \mathrm{mg} / \mathrm{kg}$ twice per day. Already from day 4 of treatment we observed significant inhibition of tumor growth compared to control-treated mice (Figure 5A). To confirm that LY2874455 reduced FGFR signaling in vivo, we performed a kinetic study of FGFR signaling proteins. Tumors were harvested at 3, 24 and $48 \mathrm{~h}$ after last treatment (end of study) and protein lysates were subjected to Western blotting to analyze the phosphorylation levels of FRS2 and ERK. The endogenous level of phosphorylated FRS2 in the LS70x tumors was, as in the cell lines, below detection (data not shown). However, ERK phosphorylation was clearly reduced $3 \mathrm{~h}$ after treatment and remained reduced for at least 24 h (Figure 5B, quantified in Figure S4C).

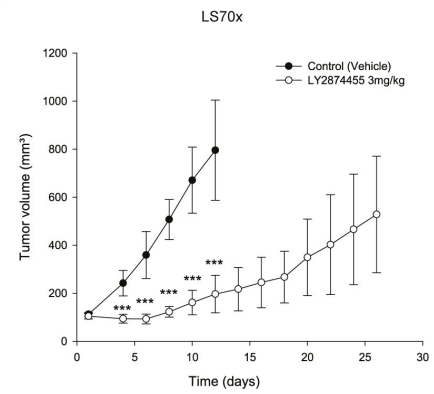

B

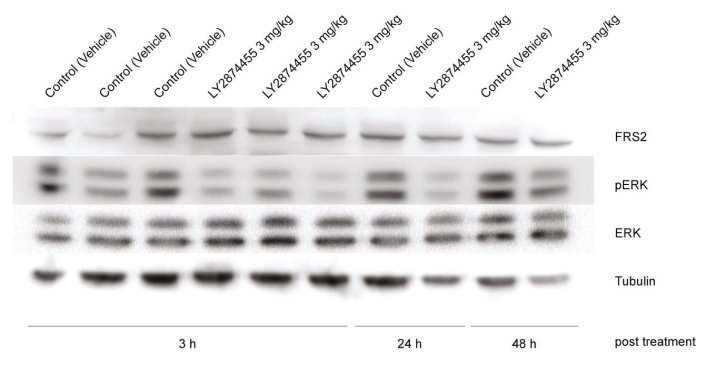

Figure 5. Growth inhibitory effect of LY2874455 in vivo on LS70x, a FRS2-amplified xenograft. (A) in vivo study with FRS2-amplified tumors of LS70x xenografts $(n=6)$ treated twice per day (BID) with LY2874455 $3 \mathrm{mg} / \mathrm{kg}$ or control vehicle for up to 28 days until tumor size reaches a limit of $1 \mathrm{~cm}^{3}$. Data shown as means $\pm \mathrm{SEM}^{* * *} p \leq 0.001$; unpaired two-tailed $t$-test treated versus vehicle treated; (B) Western blots showing the level of phosphorylated and total ERK in lysates extracted from LS70x tumors treated in vivo with vehicle or LY2874455 until endpoint. The tumors were harvested at the indicated time after last treatment. $\alpha$-tubulin is used as loading control.

\section{Discussion}

WD/DDLPS tumors almost invariably have FRS2 amplified [8] and are in great need of new therapies. We cannot expect many new drugs specific for rare cancers, therefore the possibility to repurpose existing drugs for these patients deserves thorough consideration. FGF receptor inhibitors have shown tolerable toxicities in rodent and patients $[10,11]$, thus showing efficacy in preclinical models could pave the way to clinical trials on sarcoma patients.

Our previous in vitro study demonstrated limitations of NVP-BGJ398, since the drug was only transiently cytostatic and the cells quickly regained growth capacity when drug was removed [3]. In this study we further investigated the potential of FGFR inhibition as treatment for FRS2-amplified DDLPS. 
Results were more promising using the FGFR inhibitor LY2874455, which gave improved efficacy in vitro. LY2874455 is reported to have a similar potency against all four FGFRs in biochemical assays and has shown potent activity against FGFR signaling in preclinical studies of several cancer types such as lung, gastric, bladder and multiple myeloma [12]. This drug had a stronger effect on the growth of NRH-LS1 cells, and induced apoptosis more efficiently than NVP-BGJ398 did. Interestingly, LY2874455 had a long-lasting effect on the responding cell lines, with cell growth inhibited several days after the drug treatment was discontinued. The observed effect and higher potency of LY2874455 compared to NVP-BGJ398 could potentially be due to off-target inhibition, since LY2874455 was shown to act as a multi-kinase inhibitor and consequently inhibits a wide range of different kinases [19]. However, the similarity of the response of FGFR signaling by the two inhibitors indicates a similar mode of action.

We hypothesized that amplified FRS2 would potentiate FGFR signaling and drive growth of FRS2-amplified DDLPS. Thus, FRS2 amplification could be a biomarker predicting sensitivity to FGFR inhibitors. Although the three FRS2 amplified cell lines had similar levels of FRS2, LPS853 was resistant, suggesting that $F R S 2$ amplification alone is not sufficient as predictive biomarker. The lack of response of one cell line out of four independent models might not be representative of the patient population, but more refined biomarkers detecting such tumors would be valuable. To identify possible differences in FGFR signaling that could explain the different responses to FGFR inhibition, we analyzed the status of FGFR signaling pathway proteins. The basal level of phosphorylated FRS2 in unstimulated cells was undetectable but was drastically increased upon addition of FGF1 for all the three cell lines, confirming functional FGFR signaling. Both AKT and ERK are downstream of FRS2 and the strong inhibition of ERK phosphorylation when cells were treated with FGFR inhibitor indicated that the FGFR pathway is the predominant activator of ERK in these cells. This is consistent with a study in human bladder cancer, which showed dephosphorylation of FRS2 and ERK by NVP-BGJ398 [10]. We could not detect significant changes in levels of AKT or phosphorylated AKT, which is also consistent with previous studies [20].

We did not observe any FGFR mutations or translocations that could explain the different response to FGFR inhibition (data not shown). Although we observed equal expression of FGFR1 among the cell lines, the non-responding LPS853 cells had considerably higher expression of FGFR4. Furthermore, LPS853 also had a high expression of FGFRL1, which lacks the kinase domain and has been suggested to be a negative regulator of FGFR1 signaling [21]. Although LPS853 cells do not respond to FGF inhibitors with reduced proliferation, FGFR inhibition still prevents endogenous and FGF1-induced ERK phosphorylation in these cells. This implies that FGFR signaling is maintained in LPS853 cells, but that these cells are not dependent on phosphorylated ERK for proliferation. This is known for other cell types as well. Only a subset of KRAS mutated colon, pancreatic and lung cancer depends on MEK/ERK signaling for proliferation, although the cells responds to MEK inhibition with reduced pERK [22]. Often this is due to other rescuing mechanisms. This could explain why LPS853 cells grow independent of this pathway, and also why they respond to higher doses of LY2874455, but not to high doses of NVP-BGJ398 (data not shown) [3], since LY2874455, unlike NVP-BGJ398, has a similar potency against all four FGF receptors in biochemical assays $[12,23]$.

We expect the levels of FGFs in fetal calf serum to be low, but the responding cell lines may produce autocrine FGFs, as was indeed indicated by the RNA-seq data. NRH-LS1 and LPS510 had high expression of FGF2, a ligand for several of the FGF receptors, while LPS853 had high expression of the intracellular FGF11. While the function of FGF11 is not fully known, FGF2 is known to be secreted by adipocytes and to stimulate proliferation upon binding to FGFR1. We hypothesize that the lack of expression of any extracellular FGF ligands in LPS853 cells indicate that they grow independently of FGFR in vitro, but we cannot exclude that they depended on exogeneous FGF in vivo and have adapted to conditions without FGF in vitro. Further functional investigations including knock-down of the different ligands and receptors might solve these issues. 
In summary, our results support LY2874455 as a better drug candidate than NVP-BGJ398 for treatment of FRS2-amplified liposarcoma. LY2874455 also showed significant efficacy in vivo, which is an important finding, since the FGF-regulatory landscape in tissues is different from cell cultures. Whether efficacy of LY2874455 can also be translated to other sarcomas with aberrations in the FGFR pathway, such as amplified, fused or mutated FGFR genes [24], needs to be investigated. We hope these studies could result in a clinical trial for DDLPS patients in great need of new treatments now that phase I clinical trials have shown tolerable toxicities [11].

Supplementary Materials: The following are available online at http://www.mdpi.com/2073-4409/8/2/189/s1, Figures S1-S4.

Author Contributions: R.H.: conceptualization, drug studies, data curation and analysis, project administration, visualization, writing - original draft, review, and editing. I.G.: drug studies, data curation and analysis, writing - review and editing. E.M. (Else Munthe): protein studies, data curation and analysis, writing-review and editing. J.H.: animal studies, data curation and analysis, manuscript review. I.K.: animal studies, data curation and analysis, manuscript review. E.M. (Emmet McCormack): animal studies, data curation and analysis, manuscript review. L.A.M.-Z.: supervision, genomics, data curation and analysis, writing-review and editing. E.W.S.: supervision, design of animal studies, writing - review and editing. O.M.: conceptualization, funding acquisition, project administration, resources, supervision, writing-review and editing.

Funding: This research was funded by the REGIONAL HEALTH AUTHORITY FOR SOUTH-EASTERN NORWAY, grant number 2014047, the NORWEGIAN RESEARCH COUNCIL, grant number 221580, the NORWEGIAN CANCER SOCIETY, grant number PR-2007-0163, and by the LIDDY SHRIVER SARCOMA INITIATIVE for Translational Research in Well- and De-differentiated Liposarcoma.

Acknowledgments: We are grateful to Mona Mari Lindeberg for DNA and RNA isolation and Susanne Lorenz from the Genomics Core Facility at Oslo University Hospital for the sequencing of the cell lines. We would also like to thank Mihaela Popa for contributing to the design and planning of the in vivo experiment. This work was supported by grants from the Norwegian Research Council, The Regional Health Authority of South-Eastern Norway, and the Norwegian Cancer Society.

Conflicts of Interest: The authors declare no conflict of interest.

\section{References}

1. Burningham, Z.; Hashibe, M.; Spector, L.; Schiffman, J.D. The epidemiology of sarcoma. Clin. Sarcoma Res. 2012, 2, 14. [CrossRef] [PubMed]

2. $\quad$ Barretina, J.; Taylor, B.S.; Banerji, S.; Ramos, A.H.; Lagos-Quintana, M.; Decarolis, P.L.; Shah, K.; Socci, N.D.; Weir, B.A.; Ho, A.; et al. Subtype-specific genomic alterations define new targets for soft-tissue sarcoma therapy. Nat. Genet. 2010, 42, 715-721. [CrossRef] [PubMed]

3. Hanes, R.; Grad, I.; Lorenz, S.; Stratford, E.W.; Munthe, E.; Reddy, C.C.S.; Meza-Zepeda, L.A.; Myklebost, O. Preclinical evaluation of potential therapeutic targets in dedifferentiated liposarcoma. Oncotarget 2016, 7, 54583-54595. [CrossRef] [PubMed]

4. Wang, X.; Asmann, Y.W.; Erickson-Johnson, M.R.; Oliveira, J.L.; Zhang, H.; Moura, R.D.; Lazar, A.J.; Lev, D.; Bill, K.; Lloyd, R.V.; et al. High-resolution genomic mapping reveals consistent amplification of the fibroblast growth factor receptor substrate 2 gene in well-differentiated and dedifferentiated liposarcoma. Genes Chromosomes Cancer 2011, 50, 849-858. [CrossRef] [PubMed]

5. Wesche, J.; Haglund, K.; Haugsten, E.M. Fibroblast growth factors and their receptors in cancer. Biochem. J. 2011, 437, 199-213. [CrossRef] [PubMed]

6. Korc, M.; Friesel, R.E. The role of fibroblast growth factors in tumor growth. Curr. Cancer Drug Targets 2009, 9, 639-651. [CrossRef] [PubMed]

7. Eswarakumar, V.P.; Lax, I.; Schlessinger, J. Cellular signaling by fibroblast growth factor receptors. Cytokine Growth Factor Rev. 2005, 16, 139-149. [CrossRef] [PubMed]

8. Zhang, K.; Chu, K.; Wu, X.; Gao, H.; Wang, J.; Yuan, Y.-C.; Loera, S.; Ho, K.; Wang, Y.; Chow, W.; et al. Amplification of FRS2 and activation of FGFR/FRS2 signaling pathway in high-grade liposarcoma. Cancer Res. 2013, 73, 1298-1307. [CrossRef] [PubMed]

9. Guagnano, V.; Kauffmann, A.; Wöhrle, S.; Stamm, C.; Ito, M.; Barys, L.; Pornon, A.; Yao, Y.; Li, F.; Zhang, Y.; et al. FGFR genetic alterations predict for sensitivity to NVP-BGJ398, a selective pan-FGFR inhibitor. Cancer Discov. 2012, 2, 1118-1133. [CrossRef] [PubMed] 
10. Guagnano, V.; Furet, P.; Spanka, C.; Bordas, V.; Le Douget, M.; Stamm, C.; Brueggen, J.; Jensen, M.R.; Schnell, C.; Schmid, H.; et al. Discovery of 3-(2,6-dichloro-3,5-dimethoxy-phenyl)-1\{6-[4-(4-ethyl-piperazin-1-yl)-phenylamino]-pyrimidin-4-yl\}-1-methyl-urea (NVP-BGJ398), a potent and selective inhibitor of the fibroblast growth factor receptor family of receptor tyrosine kinase. J. Med. Chem. 2011, 54, 7066-7083. [CrossRef]

11. Michael, M.; Bang, Y.-J.; Park, Y.S.; Kang, Y.-K.; Kim, T.M.; Hamid, O.; Thornton, D.; Tate, S.C.; Raddad, E.; Tie, J. A Phase 1 Study of LY2874455, an Oral Selective pan-FGFR Inhibitor, in Patients with Advanced Cancer. Target Oncol. 2017, 12, 463-474. [CrossRef] [PubMed]

12. Zhao, G.; Li, W.-Y.; Chen, D.; Henry, J.R.; Li, H.-Y.; Chen, Z.; Zia-Ebrahimi, M.; Bloem, L.; Zhai, Y.; Huss, K.; et al. A novel, selective inhibitor of fibroblast growth factor receptors that shows a potent broad spectrum of antitumor activity in several tumor xenograft models. Mol. Cancer Ther. 2011, 10, 2200-2210. [CrossRef] [PubMed]

13. Sebaugh, J.L. Guidelines for accurate EC50/IC50 estimation. Pharm. Stat. 2011, 10, 128-134. [CrossRef] [PubMed]

14. Kresse, S.H.; Meza-Zepeda, L.A.; Machado, I.; Llombart-Bosch, A.; Myklebost, O. Preclinical xenograft models of human sarcoma show nonrandom loss of aberrations. Cancer 2012, 118, 558-570. [CrossRef] [PubMed]

15. Wesche, J.; Wiedlocha, A.; Falnes, P.O.; Choe, S.; Olsnes, S. Externally added aFGF mutants do not require extensive unfolding for transport to the cytosol and the nucleus in NIH/3T3 cells. Biochemistry 2000, 39, 15091-15100. [CrossRef] [PubMed]

16. Kanojia, D.; Nagata, Y.; Garg, M.; Lee, D.H.; Sato, A.; Yoshida, K.; Sato, Y.; Sanada, M.; Mayakonda, A.; Bartenhagen, C.; et al. Genomic landscape of liposarcoma. Oncotarget 2015, 6, 42429-42444. [CrossRef] [PubMed]

17. Skårn, M.; Noordhuis, P.; Wang, M.-Y.; Veuger, M.; Kresse, S.H.; Egeland, E.V.; Micci, F.; Namløs, H.M.; Håkelien, A.-M.; Olafsrud, S.M.; et al. Generation and characterization of an immortalized human mesenchymal stromal cell line. Stem Cells Dev. 2014, 23, 2377-2389. [CrossRef] [PubMed]

18. Sleeman, M.; Fraser, J.; McDonald, M.; Yuan, S.; White, D.; Grandison, P.; Kumble, K.; Watson, J.D.; Murison, J.G. Identification of a new fibroblast growth factor receptor, FGFR5. Gene 2001, 271, 171-182. [CrossRef]

19. Hagel, M.; Miduturu, C.; Sheets, M.; Rubin, N.; Weng, W.; Stransky, N.; Bifulco, N.; Kim, J.L.; Hodous, B.; Brooijmans, N.; et al. First Selective Small Molecule Inhibitor of FGFR4 for the Treatment of Hepatocellular Carcinomas with an Activated FGFR4 Signaling Pathway. Cancer Discov. 2015, 5, 424-437. [CrossRef]

20. Luo, L.Y.; Kim, E.; Cheung, H.W.; Weir, B.A.; Dunn, G.P.; Shen, R.R.; Hahn, W.C. The tyrosine kinase adaptor protein FRS2 is oncogenic and amplified in high-grade serous ovarian cancer. Mol. Cancer Res. 2014, 13, 502-509. [CrossRef]

21. Trueb, B. Biology of FGFRL1, the fifth fibroblast growth factor receptor. Cell. Mol. Life Sci. 2011, 68, 951-964. [CrossRef] [PubMed]

22. Halilovic, E.; She, Q.-B.; Ye, Q.; Pagliarini, R.; Sellers, W.R.; Solit, D.B.; Rosen, N. PIK3CA mutation uncouples tumor growth and cyclin D1 regulation from MEK/ERK and mutant KRAS signaling. Cancer Res. 2010, 70, 6804-6814. [CrossRef] [PubMed]

23. Wu, D.; Guo, M.; Philips, M.A.; Qu, L.; Jiang, L.; Li, J.; Chen, X.; Chen, Z.; Chen, L. Crystal Structure of the FGFR4/LY2874455 Complex Reveals Insights into the Pan-FGFR Selectivity of LY2874455. PLoS ONE 2016, 11, e0162491. [CrossRef]

24. Asano, N.; Yoshida, A.; Mitani, S.; Kobayashi, E.; Shiotani, B.; Komiyama, M.; Fujimoto, H.; Chuman, H.; Morioka, H.; Matsumoto, M.; et al. Frequent amplification of receptor tyrosine kinase genes in welldifferentiated/dedifferentiated liposarcoma. Oncotarget 2017, 8, 12941. [CrossRef] [PubMed] 


\title{
Fibroblast Growth Factor Receptor Functions in Glioblastoma
}

\author{
Ana Jimenez-Pascual and Florian A. Siebzehnrubl * \\ European Cancer Stem Cell Research Institute, Cardiff University School of Biosciences, Cardiff CF24 4HQ, UK \\ * Correspondence: fas@cardiff.ac.uk; Tel.: +44-29-206-88500
}

Received: 14 May 2019; Accepted: 11 July 2019; Published: 13 July 2019

\begin{abstract}
Glioblastoma is the most lethal brain cancer in adults, with no known cure. This cancer is characterized by a pronounced genetic heterogeneity, but aberrant activation of receptor tyrosine kinase signaling is among the most frequent molecular alterations in glioblastoma. Somatic mutations of fibroblast growth factor receptors (FGFRs) are rare in these cancers, but many studies have documented that signaling through FGFRs impacts glioblastoma progression and patient survival. Small-molecule inhibitors of FGFR tyrosine kinases are currently being trialed, underlining the therapeutic potential of blocking this signaling pathway. Nevertheless, a comprehensive overview of the state of the art of the literature on FGFRs in glioblastoma is lacking. Here, we review the evidence for the biological functions of FGFRs in glioblastoma, as well as pharmacological approaches to targeting these receptors.
\end{abstract}

Keywords: FGFR; review; malignant glioma; brain cancer; astrocytoma; fibroblast growth factor

\section{Introduction}

Fibroblast growth factors (FGFs) were first isolated from bovine brain extracts in 1939 and characterized by their ability to induce proliferation of fibroblasts [1]. It took another 50 years to discover and clone the first of their cognate receptors [2]. FGFRs control many biological functions, including cell proliferation, survival, and cytoskeletal regulation (for review, see [3]). FGFR signaling is important during embryonal development of the CNS, and as a survival mechanism for adult neurons and astrocytes [4-6]. Furthermore, FGFR signaling was found to promote self-renewal and fate specification of neural stem cells [7].

In many cancers, FGFR aberrations have been implicated in tumor development and progression [8,9], and include $F G F R$ overexpression, amplification, mutations, splicing isoform variations, and FGFR translocations [10,11]. While FGFR genomic alterations have been identified in many solid tissue cancers, such events remain rare in glioblastoma (GBM) (Table 1) [12]. Nonetheless, FGFR expression changes in astrocytes can lead to malignant transformation and GBM progression due to the activation of mitogenic, migratory, and antiapoptotic responses [13-15]. Of note, fusions between FGFR and TACC (transforming acidic coiled-coil containing proteins) genes were shown to be oncogenic in GBM [16], and occur in about $3 \%$ of GBM patients [12]. Whole-genome analyses of patient samples have revealed that the number of FGFR mutations and amplifications are generally very low in GBM (FGFR1: 51/3068 samples, FGFR2: 12/2662; FGFR3: 16/2887; FGFR4: 9/2456; cancer.sanger.ac.uk; [17]). Not only are oncogenic mutations in FGFRs rare in GBM, the lack of FGFR passenger mutations (i.e., mutations not providing a survival benefit) suggests that these are selected against, and that the maintenance of dynamic FGFR signaling is important for the development and/or progression of GBM. 
Table 1. Common FGFR genomic aberration in solid tumors. FGF signaling deregulation is involved in the development of many different human cancers. Four FGFR genomic alterations are represented in this table: gene amplification, point mutations, chromosomal translocations, and FGFR splicing isoforms. Each FGFR alteration is linked with the most significant cancers that contain those alterations. The role of the majority of the discovered point mutations in FGFR is unknown in cancer. Adapted from $[10,18]$.

\begin{tabular}{|c|c|c|c|c|}
\hline Gene & $\begin{array}{c}\text { Gene } \\
\text { Amplifications }\end{array}$ & Point Mutations & $\begin{array}{l}\text { Chromosomal } \\
\text { Translocations }\end{array}$ & Splice Variants \\
\hline FGFR1 & $\begin{array}{c}\text { Breast, ovarian, } \\
\text { bladder, and lung } \\
\text { cancer }\end{array}$ & $\begin{array}{l}\text { Majority of cancers. } \\
\text { Example: Melanoma }\end{array}$ & $\begin{array}{c}\text { Stem cell } \\
\text { leukemia/lymphoma } \\
\text { (SCLL), GBM }\end{array}$ & $\begin{array}{c}\text { IIIc: small cell lung } \\
\text { carcinoma } \\
\text { I } \beta \text { : breast cancer and GBM }\end{array}$ \\
\hline FGFR2 & $\begin{array}{l}\text { Breast, gastric, lung } \\
\text { cancer }\end{array}$ & $\begin{array}{c}\text { Majority of cancers. } \\
\text { Example: Endometrial } \\
\text { carcinoma }\end{array}$ & & $\begin{array}{l}\text { IIIb: breast, endometrial, } \\
\text { cervical, lung, pancreatic } \\
\text { and colorectal cancer } \\
\text { IIIc: prostate cancers }\end{array}$ \\
\hline FGFR3 & Bladder cancer & $\begin{array}{c}\text { Majority of cancers. } \\
\text { Example: bladder cancer }\end{array}$ & $\begin{array}{l}\text { GBM, T-cell lymphoma } \\
\text { and bladder }\end{array}$ & IIIc: bladder cancer \\
\hline FGFR4 & Colorectal cancer & $\begin{array}{l}\text { Majority of cancers. } \\
\text { Example: metastatic } \\
\text { breast cancer and } \\
\text { rhabdomyosarcoma }\end{array}$ & & \\
\hline
\end{tabular}

We hypothesize that the neurodevelopmental and cell survival functions of this signaling pathway are at least partly conserved in GBM. Thus, dynamic FGFR signaling needs to be maintained for the survival of GBM cells, and therefore evolutionary pressure selects against both activating and inactivating mutations. Here, we review the current literature on FGFRs in GBM, and the evidence for differential functions of individual FGFRs in brain tumor progression.

\section{FGFR Structure}

There are four known FGFRs, FGFR1-4, which are membrane-bound receptor tyrosine kinases (RTKs). A fifth member of the FGFR family, FGFRL1, is lacking a transmembrane domain and is therefore soluble. FGFRL1 acts as an antagonist to FGFR signaling [19,20]. Structurally, FGFR1-4 consist of three different domains: an extracellular ligand binding domain, a transmembrane domain, and an intracellular domain that interacts with cytoplasmic molecules and transduces FGFR signaling $[8,21,22]$ (Figure 1).

The extracellular domain can bind FGF ligands, heparan sulfate (HS), and extracellular matrix molecules, which can act as a scaffold to enable receptor binding of specific FGFs. It is divided into three immunoglobulin-like (Ig) loops: Ig-I, Ig-II, and Ig-III (also called D1, D2, and D3) [23]. Ig-I is linked to Ig-II by a stretch of 30 acidic residues called the acid box, a unique region of FGFRs [10,24]. Ig-I and the acid box have receptor auto-inhibitory functions [25-27] while the Ig-II and Ig-III subdomains form the ligand binding site of the receptor $[3,15,18,28,29]$. Ig-II contains the heparin/HS binding region and FGF binding activity site, while the junction between Ig-II and Ig-III controls heparin and FGF affinity [21,30-33] (Figure 1).

Multiple FGFR isoforms are generated by alternative splicing of the region encoding for the extracellular domain. This modifies the affinity and sensitivity of the receptors for different FGF ligands [34,35]. Thus, an array of FGFR isoforms is created that can fine-tune the response of cells to the large number of potential FGF ligands available in their specific environment. FGF sensitivity is further modified by co-receptors, such as Klotho family members, which are required for binding of endocrine FGFs [36-38]. 


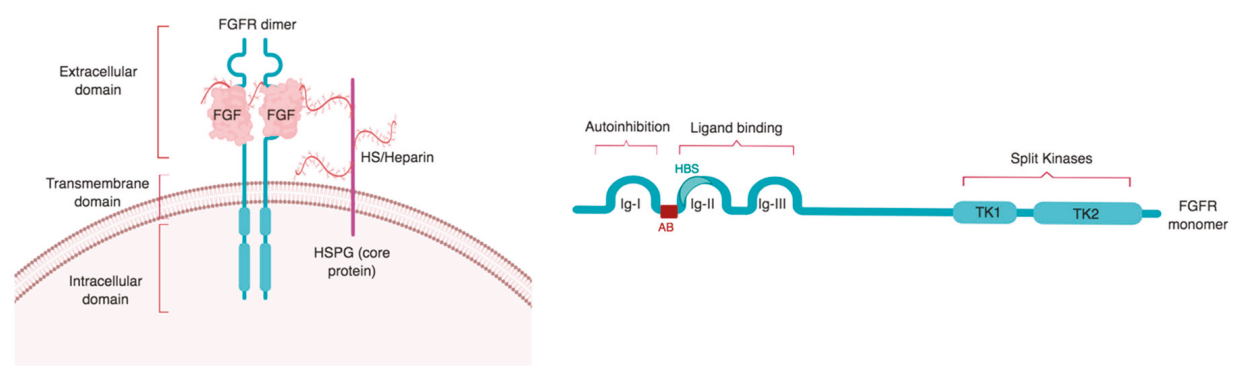

Figure 1. Domain structure of FGFRs: an extracellular domain containing ligand binding site is followed by a single transmembrane domain, and an intracellular domain containing split tyrosine kinases. Left panel: organization of the FGF-FGFR complex at the cell surface. The FGF-FGFR complex is stabilized by a heparin/HS chain of the HS proteoglycan (HSPG). Right panel: The extracellular domain of the receptor is composed of three Ig-like domains: Ig-I, Ig-II, and Ig-III. Ig-I has autoinhibitory capacity while Ig-II and Ig-III form the ligand binding domain. Ig-II contains the heparin/HS binding site (HBS) and is separated from Ig-I by an acid box (AB). The cytoplasmic domain is formed by two tyrosine kinases: tyrosine kinase 1 (TK1) and tyrosine kinase 2 (TK2). Image created with biorender.com.

Alternatively-spliced $\beta$ isoforms of FGFR1 or FGFR2 are produced by the exclusion of the Ig-I domain, which is encoded by exon 3. Due to the auto-inhibitory function of the Ig-I domain, the $\beta$ isoform has considerably higher affinity for FGFs, and is oncogenic $[39,40]$. Retention of the Ig-I domain creates FGFR $\alpha$ isoforms [28].

Additionally, alternative splicing generates two isoforms of the Ig-III domain, known as Ig-IIIb and Ig-IIIc, in FGFR1-3, but not FGFR4 [10,41-43]. Ig-IIIb and Ig-IIIc are generated by exon skipping, and are encoded by exons 8 and 9, respectively (Figure 2). By contrast, exon 7, encoding Ig-IIIa, is present in all splice variants. Different splice-regulatory proteins have been identified that control the splicing of Ig-IIIb, such as regulatory RNA-binding protein (RBP), and the epithelial splicing regulatory proteins (ESRP1/2) [32,40,44].

The expression of FGFR splice variants is tissue-dependent. For example, the Ig-IIIb isoform is more prevalent in epithelial tissues, while Ig-IIIc is preferentially expressed in mesenchymal ones [22,24,32]. Switching of epithelial and mesenchymal isoforms occurs during epithelial-mesenchymal transition, which is known as the IIIb/IIIc switch. Hence, FGFR isoform expression is also related to tissue plasticity, and changes during tissue growth, proliferation, and remodeling [45].

The FGFR transmembrane domain is crucial for transferring the signal from the extracellular to the intracellular domain, the latter consisting of a juxtamembrane domain, two tyrosine kinase domains, and the C-terminal tail $[10,32,46,47]$. FGFR domains are highly conserved among receptors, and the tyrosine kinase domain shares the highest homology. The Ig-III domain is also highly conserved, especially between FGFR1 and FGFR2 [48].

The binding of FGF ligands to HSPGs causes FGFR dimerization and activation in the - $\mathrm{COOH}$ receptor tail of the cytoplasmic tyrosine residues by phosphorylation $[49,50]$. For instance, autophosphorylation of FGFR1 tyrosine $(Y)$ residues occurs in three steps. Firstly, phosphorylation of Y653 leads to a 50-100-fold increase of the catalytic core activation of the intracellular domain. Secondly, Y583, Y463, Y766, and Y585 sites are consequently phosphorylated, and finally, the second tyrosine kinase domain phosphorylation increases the tyrosine kinase activity 10 -fold. This sequence of autophosphorylation follows a specific and controlled order that, if deregulated, can induce malfunction of the pathway [49]. 


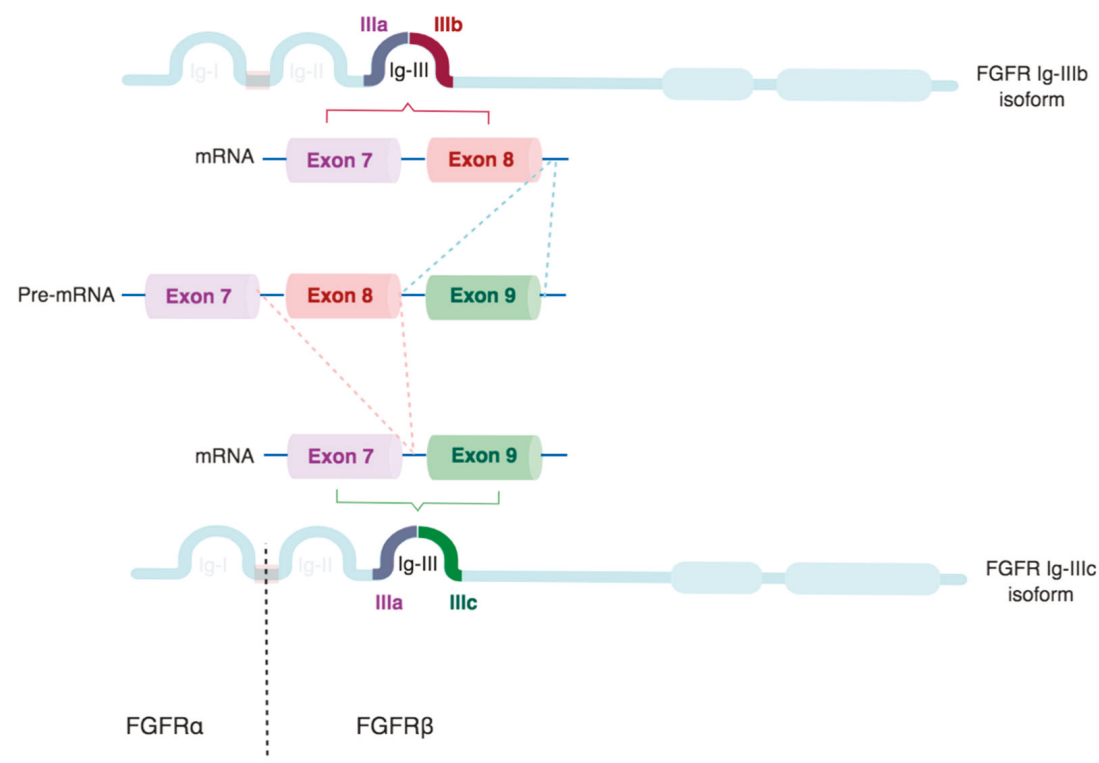

Figure 2. Schematic representation of FGFR splice isoforms. The Ig-III domain of FGFR1-3 is encoded by exons 7-9. Exon 7 encodes Ig-IIIa, which consists of the N-terminal half of the Ig-III loop. The C-terminal half is formed by the IIIb or IIIc sequence, which is generated by the selective inclusion of exons 8 or 9 , respectively. Truncation of the Ig-I loop creates FGFR $\beta$ isoforms (dotted line), while the full-length receptor is termed FGFR $\alpha$. Image created with biorender.com.

Different models have been proposed for FGFR dimerization depending on the ligand-heparinreceptor complex, specificity between the ligand and the receptor, and the heparin length required for the binding $[21,24]$. In the first model, HS increases the association between the receptor and the high affinity binding site of the ligand, forming a ternary complex (1:1:1 FGF-HS-FGFR) that then interacts with a second receptor, inducing FGFR dimerization through the FGF low affinity binding site (2:1:1 FGFR-HS-FGF). On the other hand, the symmetrical model suggests that two individual ternary complexes are formed. The FGFR dimerization will then occur by FGFR-FGFR direct interaction, FGF ligand interaction, or by HS-HS link (2:2:2 FGFR-HS-FGF). In this model, HS enhances FGFR dimerization, but it is not crucial. Finally, according to the asymmetric model, HS attaches to two FGF-FGFR complexes, binding both FGFs but only one of the receptors (2:1:2 FGFR-HS-FGF) [21]. Therefore, although different models have been suggested, more research is needed to clarify the stoichiometry of FGFR dimerization [21,24,51].

\section{FGFR Signaling Cascade}

FGF-FGFR stimulates cell signaling pathways related to cell proliferation, survival, cytoskeletal regulation, and FGFR degradation [10]. Cell proliferation is mainly induced by RAC/JNK and RAS-MAPK signaling pathways [3]. RAC kinases can be activated by the transient phosphorylation of CRK, which simultaneously stimulates RAC phosphorylation trough DOCK1 or SOS/RAS [10]. RAC kinases promote proliferation by the activation of JNK and p38. Alternatively, the RAS/RAF/MEK/ERK signaling pathway can be activated by the FRS2-GRB2-SOS-SHP2 complex assembly or byPKC activation through PLC phosphorylation [18,22].

Cell survival is mainly promoted by phosphorylation of PI3K/AKT signaling through the FRS2-GRB2-GAB1 complex. Finally, FGFRs are also implicated in cytoskeletal regulation, as PLC phosphorylation leads to the hydrolysis of PIP $_{2}$ into IP3, inducing calcium release [18] (Figure 3). 


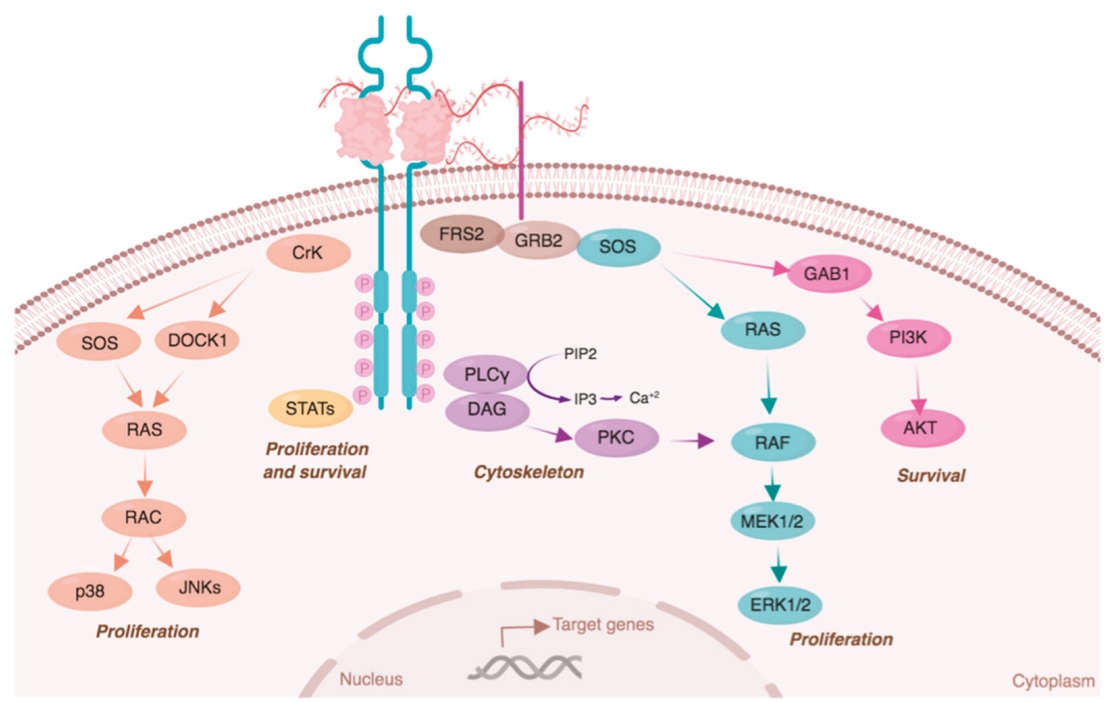

Figure 3. FGFR signaling pathway. After ligand binding, FGFRs dimerize and activate multiple signal transduction pathways. Each pathway induces the expression of specific target genes related to cell proliferation (STATs, RAS/p38/JNKs, and RAS/MAPK/ERK), survival (STATs and PI3K/AKT), and cytoskeleton regulation ( $\mathrm{PLC} / \mathrm{Ca}^{2+}$ ). Kinases are color-coded according to their specific signaling pathway. Image created with biorender.com.

Because FGFR signaling acts upon many biological functions, a regulatory system that controls its timing, spread, and balances its activation is required. This is important as the activation of the signaling cascade depends on FGFR expression and localization to the cell membrane. Therefore, receptor availability depends on the balance between its recycling and degradation rate, which differ among receptors. One of these regulatory systems is FGFR internalization or constitutive endocytosis. FGFR synthesis occurs at a higher level than its internalization. However, after ligand-binding, FGFR internalization from the plasma membrane accelerates [52]. FGFR internalization is primarily mediated by clathrin-dependent endocytosis and requires the SRC-FRS2 complex [53]. The internalization rate depends on the receptor type-FGFR1 has the highest internalization rate and FGFR3 the lowest. Endocytosis of activated FGFRs involves detachment from the SRC complex [54]. FGFRs can then re-translocate to the cytosol, mitochondria, nucleus (to directly regulate gene expression), or to the endosomal compartment for receptor degradation [52]. The latter requires interaction between the FRS2-GRB complex and CBL, and is receptor-independent [22] (Figure 4). Indeed, FGFR1 has more ubiquitination sites than FGFR4, so its degradation rate is likely higher [10].

Other regulatory systems of FGFR signaling are the negative regulators SEF, SPRY1/SPRY4, and MKP1/MKP3. The activation of cell proliferation is counterbalanced by SEF, which negatively regulates ERK and AKT activation [18]. Similarly, SPRY1/SPRY4 reduce proliferation by directly interacting with RAS/RAF kinases or by blocking the FRS2-GRB2-SOS-SHP2 complex. MKP1 and MKP3 also attenuate FGFR signaling by dephosphorylating MAPK and ERK [10] (Figure 4).

FGF signaling is also negatively regulated by the autoinhibitory (Ig-I) domain of the receptors. This is controlled by the electrostatic interactions between the negatively charged acid box with the highly basic heparin binding site in Ig-II [25]. This complex blocks the heparin-FGF binding, minimizing FGFR activation. The auto-inhibitory capacity is crucial for the modulation of the pathway, as the high amount of HSPGs from the cell surface and the extracellular matrix increases the probabilities of FGF-heparin binding and activation of the RTK cascade [24]. 


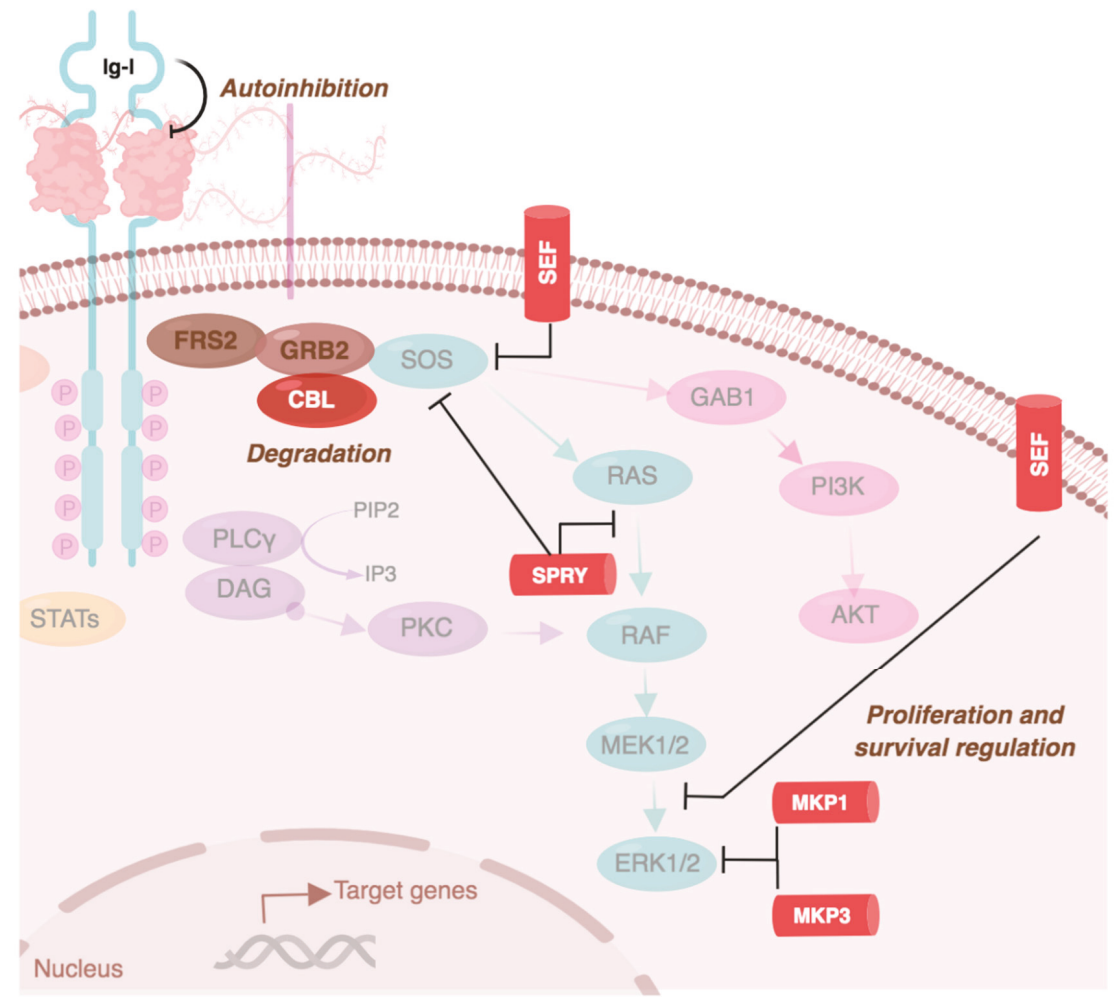

Figure 4. FGFR signaling pathway regulation. FGFR signaling is negatively regulated, partly by CBL (inducing FGFR degradation after receptor internalization), by SEF, SPRY, MKP1, and MPK3 (which negatively regulate proliferation and survival related pathways). FGFRs can also regulate their own activation due to the autoinhibitory function of Ig-I. Image created with biorender.com.

Other factors involved in FGFR pathway regulation are the ligand affinity for the receptor and the ligand amount and availability. Extracellular FGFs are protected and stored by HS proteoglycans. Heparanases are directly involved in FGF signaling regulation, as they cleave the HS chain, thus releasing FGFs in the vicinity of cells. Depending on the cell type and the growth factor released, heparanases are therefore involved in cell growth, differentiation, or stemness maintenance [55]. Likewise, sufficient amounts of ligand and heparin/HSPGs are necessary for stabilizing FGFR dimerization. The necessary ligand concentration is dependent on the ligand-binding affinity of the FGFRs, which depends on the FGFR splice isoforms $[3,8,42]$. For example, FGF2 activates both FGFR1 IIIb and IIIc isoforms, while it has a higher affinity for the isoform IIIc in FGFR2 and FGFR3 [42].

\section{Crosstalk between FGFRs and Other Cell Surface Molecules}

FGFRs can be modulated independently of their ligands by integral cell membrane proteins, such as G-protein-coupled receptors (GPCRs), cell adhesion molecules (CAMs), and other RTKs, which play a crucial role in the induction of specific cell responses and fate during development and cancer [56]. GPCRs can transactivate FGFRs by promoting the activation of matrix metalloproteinases, resulting in cleavage of FGFs, or by directly interacting with FGFRs [57]. GPCR-mediated FGFR1 transactivation is associated with neuronal differentiation, neurite growth, and synaptic plasticity [56]. FGFR1 modulation by GPCRs (e.g., CB1A, 5-HT1A, and mAChR) involves the activation of the SRC-ERK1/2 pathway [57-59]. In C6 glioma cells, crosstalk between the mu-Opioid receptor and 
FGFR1 was shown to activate this signaling cascade, but the specific mechanism is not yet completely understood [60].

FGFR activity is also modulated by CAMs, cell surface proteins that regulate cell-cell interactions and motility. Importantly, the FGFR acid box region is required for the CAM/FGFR interaction [61], hence the FGFR $\beta$ isoforms cannot be transactivated by CAMs. CAMs of the integrin, cadherin, and immunoglobulin (e.g., NCAM and L1-CAM) superfamilies signal through FGFRs to induce neurite outgrowth, cell survival, and oncogenesis [61-66]. Integrins signaling can activate cell proliferation, survival, and invasion [67], and integrin $\alpha 6$ [68] and $\alpha 7$ [69] have been linked to GBM cancer stem cells (GSCs). A recent study suggested that integrin $\alpha 6$ regulates the expression of FGFR1 through ZEB1 and YAP transcription factors [70]. N-cadherin stabilizes FGFR1 and decreases its internalization, thus promoting invasion in breast cancer cells, and N-Cadherin/FGFR crosstalk promotes neurite outgrowth [71]. Of note, the stem cell transcription factor ZEB1 regulates $\mathrm{N}$-Cadherin expression, which is associated with EMT and invasion. It is tempting to speculate that N-Cadherin/FGFR1 interactions could constitute a positive feedback loop in GSCs through the activation of ZEB1 and subsequent induction of N-Cadherin and FGFR1 expression [70,72] (see also Section 5.1). NCAMs physically associate with FGFRs and inhibit the high-affinity binding between these receptors and their canonical ligands [56,73]. Furthermore, polysialic acid-NCAM (PSA-NCAM) has been described as a marker of GBM patient prognosis [74]. This study showed that a targeted expression of PSA-NCAM in C6 glioma cells resulted in increased levels of Olig2, a transcription factor associated with GSCs [75]. While it remains unclear whether this was the result of FGFR transactivation by PSA-NCAM, we have recently shown that OLIG2 can be induced by FGFR1 signaling [72]. Furthermore, the L1-CAM/FGFR1/Anosmin-1 complex regulates neurite branching [76-78] and L1-CAM-mediated FGFR1 transactivation induces glioma cell proliferation and motility [79].

Crosstalk between FGFRs and other RTKs, such as EPHs and PDGFRs, has been identified in $\mathrm{Y} 2 \mathrm{H}$ screens and endothelial cells [80,81]. FGFR/RTKs form a heterocomplex in which the tyrosine kinase domain of the FGFR is phosphorylated by the other receptor [56]. EPHA4 transactivated FGFR1 in the U251 glioma cell line, promoting cell growth and migration, and EPHA4 expression is increased in glioma [82]. Less is known about potential crosstalk between other RTKs and FGFRs in GBM. In summary, FGFR activity can be modulated non-canonically by other cell surface proteins, resulting in the activation of intracellular signaling pathways and cell responses associated with FGFR signaling.

\section{Expression and Functions of FGFRs in Glioblastoma}

Gene expression analysis of TCGA data (GBM 540) revealed profound heterogeneity of FGFR1-4 expression across GBM patients [72]. Below, we discuss the evidence for the functions of individual FGFRs in glioma.

\subsection{FGFR1}

Yamaguchi et al. found that expression of FGFR1 increases with WHO grade in astrocytomas [39], and increased FGFR1 levels in GBM are not due to amplification of the FGFR1 gene [83].

In addition to the increased expression of FGFR1 in malignant gliomas, the ratio of alternatively spliced FGFR1 $\alpha / \beta$ isoforms changes with progression to more aggressive brain cancers. While FGFR1 $\alpha$ is the predominant isoform in normal brain and low-grade gliomas, high-grade gliomas show a shift towards the expression of FGFR1 $\beta[13,39]$. Loss of the FGFR1 $\alpha$ exon increases the receptor-ligand affinity [28], thus, changes in alternative splicing may contribute to GBM malignancy by increasing the sensitivity of tumor cells to FGFs present in their environment.

Functionally, FGFR1 expression in malignant glioma has been associated with increased migration of cancer cells [82]. In this study, a high expression of EPHA4 in glioma cells was found to potentiate FGF2-FGFR1 signaling and promoted cell growth and migration through the AKT/MAPK and RAC1/CDC42 pathways, respectively. Data from our lab support that FGFR1 loss results in reduced tumor invasion in vivo (Jimenez-Pascual and Siebzehnrubl, unpublished observation). 
Loilome and colleagues identified FGFR1 as a potential transducer of FGF2 effects on glioma cell proliferation [84], but whether other FGFRs also contribute was not directly tested. Nevertheless, the pharmacological inhibition of FGFR signaling significantly reduced tumor cell growth in a range of established and patient-derived glioma lines.

The malignancy-promoting effects of FGFR1 were further demonstrated in a study that found FGFR1 signaling promoting radioresistance in glioma cell lines through PLC1 $\gamma$ and HIF1 $\alpha$ [85]. FGFR1 expression is regulated by the stem-cell associated transcription factor ZEB1 [70], suggesting that FGFR1 may be associated with GBM cancer stem cells. We recently performed a comprehensive analysis of the functions of FGFR1-3 in GBM and found that FGFR1 indeed is preferentially expressed on GSCs, where it regulates the expression of the critical stem cell transcription factors SOX2, OLIG2, and ZEB1, thereby promoting tumorigenicity in vivo [72]. In summary, FGFR1 is a key regulator of tumor growth, invasion, therapy resistance, and cancer stemness in malignant glioma.

\subsection{FGFR2}

While FGFR1 is mainly expressed on neurons [6], FGFR2 is the primary FGFR on astrocytes [5]. In contrast to FGFR1, FGFR2 expression decreases with glioma grade [43]. Reduced expression of FGFR2, as well as its IIIb and IIIC isoforms, is associated with a higher tumor grade and poorer survival in glioma patients [43]. Tumors with a higher expression of FGFR2 showed significantly less proliferation, as identified by Ki-67 staining, but whether there is a direct link between FGFR2 signaling and slowing or exiting the cell cycle remains unclear. By contrast, experimental tumors derived from in vivo implantation of $\mathrm{C} 6$ glioma cells exhibited decreased tumor growth after inhibition of FGFR2 signaling by a dominant negative construct [86].

Our recent analysis of cell-surface FGFR expression patterns in GBM stem cell lines indicates that FGFR2 is nevertheless highly prevalent on GBM cells in vitro [72], but it remains to be tested whether FGFR2 loss results in increased proliferation and/or tumorigenicity. Loss of FGFR2 is associated with a loss of Chr. 10q, which in and of itself carries an unfavorable prognosis [87]. It is therefore conceivable that FGFR2 loss is not causally linked to reduced patient survival, and further work is needed to clarify the functional relevance of FGFR2 signaling in GBM.

\subsection{FGFR3}

In a small subset of GBM patients, fusion of the FGFR3 and TACC3 genes generates an oncogenic FGFR3 form [16]. In rare cases, fusion between FGFR1 and TACC1 can occur as well [88]. In FGFR3-TACC3, the FGFR tyrosine kinase domain is fused to the TACC coiled-coil domain, resulting in constitutive activation of the fused receptor. Small-molecule FGFR inhibitors were effective at blocking tumor growth where FGFR-TACC fusion occurred, indicating that the fused receptor is causally linked to tumor development. Overall, FGFR-TACC fusions are found in $\sim 3 \%$ of gliomas and are mutually exclusive with EGFR amplifications. Recently, it has been shown that FGFR-TACC fusions affect cell metabolism, activating oxidative phosphorylation and mitochondrial activity [89]. Of note, we have recently found that GSCs preferentially utilize oxidative phosphorylation and mitochondrial respiration [90], and therefore it would be interesting to investigate whether FGFR-TACC fusions also affect stemness pathways in GBM.

Whether FGFR3 has specific functions that differ from FGFR1 or -2 in GBM, and/or whether signaling through FGFR3 activates specific downstream signaling pathways remains unclear. Of note, global transcriptomic analysis of TCGA and CGGA datasets found increased expression of FGFR3 in the classical and neural subtypes of GBM [91]. Gene ontology analysis showed an association of FGFR3 expression with biological processes of cell differentiation in this study.

A recent study investigating gene expression using single-cell RNA-Seq in GBM patients found that FGFR3 expression is five-fold higher in invasive GBM cells compared to the tumor core [92]. Indeed, FGFR3 was the second highest differentially expressed gene between invasive and tumor core GBM cells. While this suggests that FGFR3 may be functionally associated with tumor invasion, whether FGFR3 signaling is driving GBM invasion remains to be shown. 


\subsection{FGFR4}

Very little evidence exists of the expression of FGFR4 in GBM. An early study found increased expression of the FGFR4 protein, but not mRNA, with increasing grade in astrocytoma [93]. Another study demonstrated the expression of FGFR4 across different GBM cell lines [84]. We recently investigated FGFR protein expression in GBM cells [72]. In our study, FGFR4 was not detectable by western blot in primary patient-derived GBM cells, and analysis of GBM patient data in the TCGA dataset showed heterogeneous, but overall low expression of FGFR4. Moreover, we could not find differences in survival when stratifying patients for FGFR4 high or low expression. More research is needed to fully characterize whether FGFR4 is expressed on subsets of GBM cells, and whether it is functional in these cancers.

\section{FGFRs as Therapeutic Targets in GBM}

Oncogenic FGFR signaling promotes malignancy in many cancers, including CNS malignancies. Thus, pharmacological targeting of FGFRs may be therapeutically beneficial. A number of RTK inhibitors have been developed that show selectivity of FGFRs over other RTKs [18]. While some small-molecule inhibitors also target non-FGFR RTKs (e.g., dovitinib, levatinib, brivanib), others are selective for FGFR1-3 (e.g., PD173074, BGJ398, AZ4547, JNJ-493). To date, no small-molecule inhibitors exist with good selectivity for individual FGFR subtypes or isoforms [18].

Recently, a study identified FGFR signaling as a potential therapeutic target in pediatric glioma using a large-scale shRNA screen [94]. In this study, FGFR inhibitors (AZ4547, dovatinib, PD173074, ponatinib) were more effective in reducing the growth of pediatric glioma cells in vitro than the first-line chemotherapeutic agent Temozolomide.

The selective FGFR inhibitors AZ4547 and BGJ398 have been tested in clinical phase I/II (NCT028224133) and phase II trials (NCT01975701), respectively. AZ4547 was trialed in patients with recurrent IDH wild-type gliomas with FGFR1-TACC1 or FGFR3-TACC3 fusions, but this trial was suspended after analysis of the data from the first 12 patients. A trial of BGJ398 in malignant glioma patients with FGFR1-TACC1 or FGFR3-TACC3 fusion, and/or activating mutation in FGFR1, -2, or -3, was completed, but so far, no results have been published.

A phase I/II trial of the irreversible FGFR inhibitor TAS-120 (NCT02052778) is currently recruiting patients with advanced solid tumors, including brain tumors. As with the AZ4547 and BGJ398 trials, the focus of this trial is on patients with FGFR gene fusions or activating mutations.

Due to the prevalence of FGFRs on many CNS cells, and the importance of FGFR signaling for CNS cell survival, as well as the apparent intratumoral and intertumoral heterogeneity of FGFR expression in GBM, it will be interesting to see whether FGFR inhibitors are successful as monotherapy. Based on the evidence implicating FGFR1 as a GSC regulator, it is further tempting to speculate whether the targeted inhibition of FGFR1 in combination with conventional chemo/radiotherapy could prevent or delay recurrence in GBM.

\section{Conclusions}

Gene expression profiling and whole-genome sequencing data indicate that all four FGFRs are expressed to varying degrees in GBM, underlining the heterogeneity of this disease. Several studies have documented that high-grade gliomas show an increased expression of FGFR1, and decreased expression of FGFR2 [13,39,43,72,83], but in almost all cases, FGFR expression was detected at a global level, and limited or no effort was made to further identify FGFR splice isoforms. A recent report investigating gene expression at the single-cell level [92] found that FGFR3 was expressed the second-highest in invasive GBM cells. This illustrates that much more research is needed to unravel the functions of individual FGFRs and their splice isoforms in brain cancers in general, and GBM in particular. 
To what extent signaling through individual FGFRs contributes to disease progression, and whether individual FGFRs and/or isoforms activate specific pathways linked to different pathobiological aspects of these cancers (e.g., invasion, tumor initiation, therapy resistance) remains largely unknown. Yet, the fact that FGFR subtypes are differentially expressed on cellular subpopulations within the same tumor suggests that individual FGFRs may have divergent functions in GBM [72,92].

The strongest evidence by far indicates that FGFR1 is an important contributor to poor outcome in GBM, and FGFR1 signaling is linked to cancer stemness, invasion, and radioresistance [70,72,85]. However, recent evidence from TCGA datasets highlights that FGFR1-4 are expressed to varying degrees and in different combinations in patient samples [72]. This calls for a more detailed analysis of FGFR distribution across GBM patients and within individual tumors. It is conceivable that different combinations of FGFR subtypes and splice isoforms mediate and/or modulate different aspects of FGF signaling in GBM cells. Only a comprehensive analysis of the cell-surface expression of FGFRs at the single-cell level will dissect the intratumoral heterogeneity of these receptors, and thus provide the foundation for new, targeted approaches to blocking FGFR signaling for glioma therapy.

Author Contributions: Conceptualization, A.J.-P. and F.A.S.; writing-original draft preparation, A.J.-P. and F.A.S.; writing-review and editing, A.J.-P. and F.A.S.; visualization, A.J.-P.; supervision, F.A.S.; project administration, F.A.S.; funding acquisition, F.A.S.

Funding: This research was funded by MRC, grant number MR/S007709/1.

Conflicts of Interest: The authors declare no conflict of interest.

\section{References}

1. Trowell, O.A.; Willmer, E.N. Studies on the growth of tissues in vitro VI. The effects of some tissue extracts on the growth of periosteal fibroblasts. J. Exp. Biol. 1939, 16, 60-70.

2. Lee, P.L.; Johnson, D.E.; Cousens, L.S.; Fried, V.A.; Williams, L.T. Purification and complementary DNA cloning of a receptor for basic fibroblast growth factor. Science 1989, 245, 57-60. [CrossRef] [PubMed]

3. Beenken, A.; Mohammadi, M. The FGF family: Biology, pathophysiology and therapy. Nat. Rev. Drug. Discov. 2009, 8, 235-253. [CrossRef]

4. Guillemot, F.; Zimmer, C. From cradle to grave: The multiple roles of fibroblast growth factors in neural development. Neuron 2011, 71, 574-588. [CrossRef] [PubMed]

5. Miyake, A.; Hattori, Y.; Ohta, M.; Itoh, N. Rat oligodendrocytes and astrocytes preferentially express fibroblast growth factor receptor-2 and -3 mRNAs. J. Neurosci. Res. 1996, 45, 534-541. [CrossRef]

6. Gonzalez, A.M.; Berry, M.; Maher, P.A.; Logan, A.; Baird, A. A comprehensive analysis of the distribution of FGF-2 and FGFR1 in the rat brain. Brain Res. 1995, 701, 201-226. [CrossRef]

7. Frinchi, M.; Bonomo, A.; Trovato-Salinaro, A.; Condorelli, D.F.; Fuxe, K.; Spampinato, M.G.; Mudo, G. Fibroblast growth factor-2 and its receptor expression in proliferating precursor cells of the subventricular zone in the adult rat brain. Neurosci. Lett. 2008, 447, 20-25. [CrossRef] [PubMed]

8. Haugsten, E.M.; Wiedlocha, A.; Olsnes, S.; Wesche, J. Roles of fibroblast growth factor receptors in carcinogenesis. Mol. Cancer Res. 2010, 8, 1439-1452. [CrossRef]

9. Greulich, H.; Pollock, P.M. Targeting mutant fibroblast growth factor receptors in cancer. Trends Mol. Med. 2011, 17, 283-292. [CrossRef]

10. Tiong, K.H.; Mah, L.Y.; Leong, C.O. Functional roles of fibroblast growth factor receptors (FGFRs) signaling in human cancers. Apoptosis 2013, 18, 1447-1468. [CrossRef]

11. Costa, R.; Carneiro, B.A.; Taxter, T.; Tavora, F.A.; Kalyan, A.; Pai, S.A.; Chae, Y.K.; Giles, F.J. FGFR3-TACC3 fusion in solid tumors: Mini review. Oncotarget 2016, 7, 55924-55938. [CrossRef] [PubMed]

12. Lasorella, A.; Sanson, M.; Iavarone, A. FGFR-TACC gene fusions in human glioma. Neuro Oncol. 2017, 19, 475-483. [CrossRef] [PubMed]

13. Morrison, R.S.; Yamaguchi, F.; Saya, H.; Bruner, J.M.; Yahanda, A.M.; Donehower, L.A.; Berger, M. Basic fibroblast growth factor and fibroblast growth factor receptor I are implicated in the growth of human astrocytomas. J. Neuro Oncol. 1994, 18, 207-216. [CrossRef] 
14. Yamada, S.M.; Yamaguchi, F.; Brown, R.; Berger, M.S.; Morrison, R.S. Suppression of glioblastoma cell growth following antisense oligonucleotide-mediated inhibition of fibroblast growth factor receptor expression. Glia 1999, 28, 66-76. [CrossRef]

15. Dienstmann, R.; Rodon, J.; Prat, A.; Perez-Garcia, J.; Adamo, B.; Felip, E.; Cortes, J.; Iafrate, A.J.; Nuciforo, P.; Tabernero, J. Genomic aberrations in the FGFR pathway: Opportunities for targeted therapies in solid tumors. Ann. Oncol. 2014, 25, 552-563. [CrossRef] [PubMed]

16. Singh, D.; Chan, J.M.; Zoppoli, P.; Niola, F.; Sullivan, R.; Castano, A.; Liu, E.M.; Reichel, J.; Porrati, P.; Pellegatta, S.; et al. Transforming fusions of FGFR and TACC genes in human glioblastoma. Science 2012, 337, 1231-1235. [CrossRef]

17. Forbes, S.A.; Beare, D.; Boutselakis, H.; Bamford, S.; Bindal, N.; Tate, J.; Cole, C.G.; Ward, S.; Dawson, E.; Ponting, L.; et al. COSMIC: Somatic cancer genetics at high-resolution. Nucleic Acids Res. 2017, 45, D777-D783. [CrossRef]

18. Dieci, M.V.; Arnedos, M.; Andre, F.; Soria, J.C. Fibroblast growth factor receptor inhibitors as a cancer treatment: From a biologic rationale to medical perspectives. Cancer Discov. 2013, 3, 264-279. [CrossRef]

19. Wiedemann, M.; Trueb, B. Characterization of a novel protein (FGFRL1) from human cartilage related to FGF receptors. Genomics 2000, 69, 275-279. [CrossRef]

20. Steinberg, F.; Zhuang, L.; Beyeler, M.; Kalin, R.E.; Mullis, P.E.; Brandli, A.W.; Trueb, B. The FGFRL1 receptor is shed from cell membranes, binds fibroblast growth factors (FGFs), and antagonizes FGF signaling in Xenopus embryos. J. Biol. Chem. 2010, 285, 2193-2202. [CrossRef]

21. Harmer, N.J.; Ilag, L.L.; Mulloy, B.; Pellegrini, L.; Robinson, C.V.; Blundell, T.L. Towards a resolution of the stoichiometry of the fibroblast growth factor (FGF)-FGF receptor-heparin complex. J. Mol. Biol. 2004, 339, 821-834. [CrossRef] [PubMed]

22. Ahmad, I.; Iwata, T.; Leung, H.Y. Mechanisms of FGFR-mediated carcinogenesis. Biochim. Biophys. Acta 2012, 1823, 850-860. [CrossRef] [PubMed]

23. Ornitz, D.M.; Marie, P.J. Fibroblast growth factor signaling in skeletal development and disease. Genes Dev. 2015, 29, 1463-1486. [CrossRef] [PubMed]

24. Mohammadi, M.; Olsen, S.K.; Ibrahimi, O.A. Structural basis for fibroblast growth factor receptor activation. Cytokine Growth Factor Rev. 2005, 16, 107-137. [CrossRef] [PubMed]

25. Olsen, S.K.; Ibrahimi, O.A.; Raucci, A.; Zhang, F.; Eliseenkova, A.V.; Yayon, A.; Basilico, C.; Linhardt, R.J.; Schlessinger, J.; Mohammadi, M. Insights into the molecular basis for fibroblast growth factor receptor autoinhibition and ligand-binding promiscuity. Proc. Natl. Acad. Sci. USA 2004, 101, 935-940. [CrossRef] [PubMed]

26. Kiselyov, V.V.; Kochoyan, A.; Poulsen, F.M.; Bock, E.; Berezin, V. Elucidation of the mechanism of the regulatory function of the Ig1 module of the fibroblast growth factor receptor 1. Protein Sci. 2006, 15, 2318-2322. [CrossRef]

27. Kalinina, J.; Dutta, K.; Ilghari, D.; Beenken, A.; Goetz, R.; Eliseenkova, A.V.; Cowburn, D.; Mohammadi, M. The alternatively spliced acid box region plays a key role in FGF receptor autoinhibition. Structure 2012, 20, 77-88. [CrossRef] [PubMed]

28. Wang, F.; Kan, M.; Yan, G.; Xu, J.; McKeehan, W.L. Alternately spliced NH2-terminal immunoglobulin-like Loop I in the ectodomain of the fibroblast growth factor (FGF) receptor 1 lowers affinity for both heparin and FGF-1. J. Biol. Chem. 1995, 270, 10231-10235. [CrossRef] [PubMed]

29. Yeh, B.K.; Igarashi, M.; Eliseenkova, A.V.; Plotnikov, A.N.; Sher, I.; Ron, D.; Aaronson, S.A.; Mohammadi, M. Structural basis by which alternative splicing confers specificity in fibroblast growth factor receptors. Proc. Natl. Acad. Sci. USA 2003, 100, 2266-2271. [CrossRef]

30. Rapraeger, A.C.; Krufka, A.; Olwin, B.B. Requirement of heparan sulfate for bFGF-mediated fibroblast growth and myoblast differentiation. Science 1991, 252, 1705-1708. [CrossRef]

31. Yayon, A.; Klagsbrun, M.; Esko, J.D.; Leder, P.; Ornitz, D.M. Cell surface, heparin-like molecules are required for binding of basic fibroblast growth factor to its high affinity receptor. Cell 1991, 64, 841-848. [CrossRef]

32. Gong, S.G. Isoforms of receptors of fibroblast growth factors. J. Cell Physiol. 2014, 229, 1887-1895. [CrossRef]

33. Schlessinger, J.; Plotnikov, A.N.; Ibrahimi, O.A.; Eliseenkova, A.V.; Yeh, B.K.; Yayon, A.; Linhardt, R.J.; Mohammadi, M. Crystal structure of a ternary FGF-FGFR-heparin complex reveals a dual role for heparin in FGFR binding and dimerization. Mol. Cell 2000, 6, 743-750. [CrossRef] 
34. Miki, T.; Bottaro, D.P.; Fleming, T.P.; Smith, C.L.; Burgess, W.H.; Chan, A.M.; Aaronson, S.A. Determination of ligand-binding specificity by alternative splicing: Two distinct growth factor receptors encoded by a single gene. Proc. Natl. Acad. Sci. USA 1992, 89, 246-250. [CrossRef] [PubMed]

35. Chellaiah, A.T.; McEwen, D.G.; Werner, S.; Xu, J.; Ornitz, D.M. Fibroblast growth factor receptor (FGFR) 3. Alternative splicing in immunoglobulin-like domain III creates a receptor highly specific for acidic FGF/FGF-1. J. Biol. Chem. 1994, 269, 11620-11627. [PubMed]

36. Kurosu, H.; Ogawa, Y.; Miyoshi, M.; Yamamoto, M.; Nandi, A.; Rosenblatt, K.P.; Baum, M.G.; Schiavi, S.; Hu, M.C.; Moe, O.W.; et al. Regulation of fibroblast growth factor-23 signaling by klotho. J. Biol. Chem. 2006, 281, 6120-6123. [CrossRef] [PubMed]

37. Urakawa, I.; Yamazaki, Y.; Shimada, T.; Iijima, K.; Hasegawa, H.; Okawa, K.; Fujita, T.; Fukumoto, S.; Yamashita, T. Klotho converts canonical FGF receptor into a specific receptor for FGF23. Nature 2006, 444, 770-774. [CrossRef] [PubMed]

38. Adams, A.C.; Cheng, C.C.; Coskun, T.; Kharitonenkov, A. FGF21 requires betaklotho to act in vivo. PLoS ONE 2012, 7, e49977. [CrossRef] [PubMed]

39. Yamaguchi, F.; Saya, H.; Bruner, J.M.; Morrison, R.S. Differential expression of two fibroblast growth factor-receptor genes is associated with malignant progression in human astrocytomas. Proc. Natl. Acad. Sci. USA 1994, 91, 484-488. [CrossRef]

40. Tomlinson, D.C.; Knowles, M.A. Altered splicing of FGFR1 is associated with high tumor grade and stage and leads to increased sensitivity to FGF1 in bladder cancer. Am. J. Pathol. 2010, 177, 2379-2386. [CrossRef]

41. Eswarakumar, V.P.; Lax, I.; Schlessinger, J. Cellular signaling by fibroblast growth factor receptors. Cytokine Growth Factor Rev. 2005, 16, 139-149. [CrossRef] [PubMed]

42. Holzmann, K.; Grunt, T.; Heinzle, C.; Sampl, S.; Steinhoff, H.; Reichmann, N.; Kleiter, M.; Hauck, M.; Marian, B. Alternative Splicing of Fibroblast Growth Factor Receptor IgIII Loops in Cancer. J. Nucleic Acids 2012, 2012, 950508. [CrossRef] [PubMed]

43. Ohashi, R.; Matsuda, Y.; Ishiwata, T.; Naito, Z. Downregulation of fibroblast growth factor receptor 2 and its isoforms correlates with a high proliferation rate and poor prognosis in high-grade glioma. Oncol. Rep. 2014, 32, 1163-1169. [CrossRef] [PubMed]

44. Matlin, A.J.; Clark, F.; Smith, C.W. Understanding alternative splicing: Towards a cellular code. Nat. Rev. Mol. Cell Biol. 2005, 6, 386-398. [CrossRef] [PubMed]

45. Yamaguchi, A.; Ishii, H.; Morita, I.; Oota, I.; Takeda, H. mRNA expression of fibroblast growth factors and hepatocyte growth factor in rat plantaris muscle following denervation and compensatory overload. Pflugers Arch. 2004, 448, 539-546. [CrossRef]

46. Burgar, H.R.; Burns, H.D.; Elsden, J.L.; Lalioti, M.D.; Heath, J.K. Association of the signaling adaptor FRS2 with fibroblast growth factor receptor 1 (Fgfr1) is mediated by alternative splicing of the juxtamembrane domain. J. Biol. Chem. 2002, 277, 4018-4023. [CrossRef] [PubMed]

47. Sarabipour, S.; Hristova, K. FGFR3 unliganded dimer stabilization by the juxtamembrane domain. J. Mol. Biol. 2015, 427, 1705-1714. [CrossRef] [PubMed]

48. Zhang, Y.; Gorry, M.C.; Post, J.C.; Ehrlich, G.D. Genomic organization of the human fibroblast growth factor receptor 2 (FGFR2) gene and comparative analysis of the human FGFR gene family. Gene 1999, 230, 69-79. [CrossRef]

49. Lew, E.D.; Furdui, C.M.; Anderson, K.S.; Schlessinger, J. The precise sequence of FGF receptor autophosphorylation is kinetically driven and is disrupted by oncogenic mutations. Sci. Signal. 2009, 2, ra6. [CrossRef]

50. Touat, M.; Ileana, E.; Postel-Vinay, S.; Andre, F.; Soria, J.C. Targeting FGFR Signaling in Cancer. Clin. Cancer Res. 2015, 21, 2684-2694. [CrossRef]

51. Ori, A.; Wilkinson, M.C.; Fernig, D.G. The heparanome and regulation of cell function: Structures, functions and challenges. Front. Biosci. 2008, 13, 4309-4338. [CrossRef] [PubMed]

52. Opalinski, L.; Sokolowska-Wedzina, A.; Szczepara, M.; Zakrzewska, M.; Otlewski, J. Antibody-induced dimerization of FGFR1 promotes receptor endocytosis independently of its kinase activity. Sci. Rep. 2017, 7, 7121. [CrossRef] [PubMed]

53. Auciello, G.; Cunningham, D.L.; Tatar, T.; Heath, J.K.; Rappoport, J.Z. Regulation of fibroblast growth factor receptor signalling and trafficking by Src and Eps8. J. Cell Sci. 2013, 126, 613-624. [CrossRef] [PubMed] 
54. Sandilands, E.; Akbarzadeh, S.; Vecchione, A.; McEwan, D.G.; Frame, M.C.; Heath, J.K. Src kinase modulates the activation, transport and signalling dynamics of fibroblast growth factor receptors. EMBO Rep. 2007, 8, 1162-1169. [CrossRef] [PubMed]

55. Kundu, S.; Xiong, A.; Spyrou, A.; Wicher, G.; Marinescu, V.D.; Edqvist, P.D.; Zhang, L.; Essand, M.; Dimberg, A.; Smits, A.; et al. Heparanase promotes glioma progression and is inversely correlated with patient survival. Mol. Cancer Res. 2016. [CrossRef] [PubMed]

56. Latko, M.; Czyrek, A.; Porebska, N.; Kucinska, M.; Otlewski, J.; Zakrzewska, M.; Opalinski, L. Cross-talk between fibroblast growth factor receptors and other cell surface proteins. Cells 2019, 8, 455. [CrossRef] [PubMed]

57. Di Liberto, V.; Mudo, G.; Belluardo, N. Crosstalk between receptor tyrosine kinases (RTKs) and G protein-coupled receptors (GPCR) in the brain: Focus on heteroreceptor complexes and related functional neurotrophic effects. Neuropharmacology 2019, 152, 67-77. [CrossRef]

58. Asimaki, O.; Leondaritis, G.; Lois, G.; Sakellaridis, N.; Mangoura, D. Cannabinoid 1 receptor-dependent transactivation of fibroblast growth factor receptor 1 emanates from lipid rafts and amplifies extracellular signal-regulated kinase 1/2 activation in embryonic cortical neurons. J. Neurochem. 2011, 116, 866-873. [CrossRef]

59. Borroto-Escuela, D.O.; Carlsson, J.; Ambrogini, P.; Narvaez, M.; Wydra, K.; Tarakanov, A.O.; Li, X.; Millon, C.; Ferraro, L.; Cuppini, R.; et al. Understanding the role of GPCR heteroreceptor complexes in modulating the brain networks in health and disease. Front. Cell Neurosci. 2017, 11, 37. [CrossRef]

60. Belcheva, M.M.; Haas, P.D.; Tan, Y.; Heaton, V.M.; Coscia, C.J. The fibroblast growth factor receptor is at the site of convergence between mu-opioid receptor and growth factor signaling pathways in rat C6 glioma cells. J. Pharmacol. Exp. Ther. 2002, 303, 909-918. [CrossRef]

61. Sanchez-Heras, E.; Howell, F.V.; Williams, G.; Doherty, P. The fibroblast growth factor receptor acid box is essential for interactions with $\mathrm{N}$-cadherin and all of the major isoforms of neural cell adhesion molecule. J. Biol. Chem. 2006, 281, 35208-35216. [CrossRef] [PubMed]

62. Williams, E.J.; Furness, J.; Walsh, F.S.; Doherty, P. Activation of the FGF receptor underlies neurite outgrowth stimulated by L1, N-CAM, and N-cadherin. Neuron 1994, 13, 583-594. [CrossRef]

63. Condic, M.L.; Letourneau, P.C. Ligand-induced changes in integrin expression regulate neuronal adhesion and neurite outgrowth. Nature 1997, 389, 852-856. [CrossRef] [PubMed]

64. Boscher, C.; Mege, R.M. Cadherin-11 interacts with the FGF receptor and induces neurite outgrowth through associated downstream signalling. Cell Signal. 2008, 20, 1061-1072. [CrossRef] [PubMed]

65. Meiri, K.F.; Saffell, J.L.; Walsh, F.S.; Doherty, P. Neurite outgrowth stimulated by neural cell adhesion molecules requires growth-associated protein-43 (GAP-43) function and is associated with GAP-43 phosphorylation in growth cones. J. Neurosci. 1998, 18, 10429-10437. [CrossRef] [PubMed]

66. Doherty, P.; Cohen, J.; Walsh, F.S. Neurite outgrowth in response to transfected N-CAM changes during development and is modulated by polysialic acid. Neuron 1990, 5, 209-219. [CrossRef]

67. Bianconi, D.; Unseld, M.; Prager, G.W. Integrins in the spotlight of cancer. Int. J. Mol. Sci. 2016, $17,2037$. [CrossRef] [PubMed]

68. Lathia, J.D.; Gallagher, J.; Heddleston, J.M.; Wang, J.; Eyler, C.E.; Macswords, J.; Wu, Q.; Vasanji, A.; McLendon, R.E.; Hjelmeland, A.B.; et al. Integrin alpha 6 regulates glioblastoma stem cells. Cell Stem Cell 2010, 6, 421-432. [CrossRef]

69. Haas, T.L.; Sciuto, M.R.; Brunetto, L.; Valvo, C.; Signore, M.; Fiori, M.E.; di Martino, S.; Giannetti, S.; Morgante, L.; Boe, A.; et al. Integrin alpha7 is a functional marker and potential therapeutic target in glioblastoma. Cell Stem Cell 2017, 21, 35-50. [CrossRef]

70. Kowalski-Chauvel, A.; Gouaze-Andersson, V.; Baricault, L.; Martin, E.; Delmas, C.; Toulas, C.; Cohen-Jonathan-Moyal, E.; Seva, C. Alpha6-integrin regulates FGFR1 expression through the ZEB1/YAP1 transcription complex in glioblastoma stem cells resulting in enhanced proliferation and stemness. Cancers 2019, 11, 406. [CrossRef]

71. Nguyen, T.; Mege, R.M. N-cadherin and fibroblast growth factor receptors crosstalk in the control of developmental and cancer cell migrations. Eur. J. Cell Biol. 2016, 95, 415-426. [CrossRef] [PubMed]

72. Hale, J.S.; Jimenez-Pascual, A.; Kordowski, A.; Pugh, J.; Rao, S.; Silver, D.J.; Alban, T.; Watson, D.B.; Chen, R.; McIntyre, T.M.; et al. ADAMDEC1 maintains a novel growth factor signaling loop in cancer stem cells. bioRxiv 2019. [CrossRef] 
73. Francavilla, C.; Loeffler, S.; Piccini, D.; Kren, A.; Christofori, G.; Cavallaro, U. Neural cell adhesion molecule regulates the cellular response to fibroblast growth factor. J. Cell Sci. 2007, 120, 4388-4394. [CrossRef] [PubMed]

74. Amoureux, M.C.; Coulibaly, B.; Chinot, O.; Loundou, A.; Metellus, P.; Rougon, G.; Figarella-Branger, D. Polysialic acid neural cell adhesion molecule (PSA-NCAM) is an adverse prognosis factor in glioblastoma, and regulates olig2 expression in glioma cell lines. BMC Cancer 2010, 10, 91. [CrossRef] [PubMed]

75. Ligon, K.L.; Huillard, E.; Mehta, S.; Kesari, S.; Liu, H.; Alberta, J.A.; Bachoo, R.M.; Kane, M.; Louis, D.N.; Depinho, R.A.; et al. Olig2-regulated lineage-restricted pathway controls replication competence in neural stem cells and malignant glioma. Neuron 2007, 53, 503-517. [CrossRef] [PubMed]

76. Bribian, A.; Barallobre, M.J.; Soussi-Yanicostas, N.; de Castro, F. Anosmin-1 modulates the FGF-2-dependent migration of oligodendrocyte precursors in the developing optic nerve. Mol. Cell Neurosci. 2006, 33, 2-14. [CrossRef]

77. Garcia-Gonzalez, D.; Clemente, D.; Coelho, M.; Esteban, P.F.; Soussi-Yanicostas, N.; de Castro, F. Dynamic roles of FGF-2 and Anosmin-1 in the migration of neuronal precursors from the subventricular zone during pre- and postnatal development. Exp. Neurol. 2010, 222, 285-295. [CrossRef]

78. Murcia-Belmonte, V.; Esteban, P.F.; Garcia-Gonzalez, D.; De Castro, F. Biochemical dissection of Anosmin-1 interaction with FGFR1 and components of the extracellular matrix. J. Neurochem. 2010, 115, 1256-1265. [CrossRef]

79. Mohanan, V.; Temburni, M.K.; Kappes, J.C.; Galileo, D.S. L1CAM stimulates glioma cell motility and proliferation through the fibroblast growth factor receptor. Clin. Exp. Metastasis 2013, 30, 507-520. [CrossRef]

80. Chen, P.Y.; Simons, M.; Friesel, R. FRS2 via fibroblast growth factor receptor 1 is required for platelet-derived growth factor receptor beta-mediated regulation of vascular smooth muscle marker gene expression. J. Biol. Chem. 2009, 284, 15980-15992. [CrossRef]

81. Yokote, H.; Fujita, K.; Jing, X.; Sawada, T.; Liang, S.; Yao, L.; Yan, X.; Zhang, Y.; Schlessinger, J.; Sakaguchi, K. Trans-activation of EphA4 and FGF receptors mediated by direct interactions between their cytoplasmic domains. Proc. Natl. Acad. Sci. USA 2005, 102, 18866-18871. [CrossRef] [PubMed]

82. Fukai, J.; Yokote, H.; Yamanaka, R.; Arao, T.; Nishio, K.; Itakura, T. EphA4 promotes cell proliferation and migration through a novel EphA4-FGFR1 signaling pathway in the human glioma U251 cell line. Mol. Cancer Ther. 2008, 7, 2768-2778. [CrossRef] [PubMed]

83. Morrison, R.S.; Yamaguchi, F.; Bruner, J.M.; Tang, M.; McKeehan, W.; Berger, M.S. Fibroblast growth factor receptor gene expression and immunoreactivity are elevated in human glioblastoma multiforme. Cancer Res. 1994, 54, 2794-2799. [PubMed]

84. Loilome, W.; Joshi, A.D.; ap Rhys, C.M.; Piccirillo, S.; Vescovi, A.L.; Gallia, G.L.; Riggins, G.J. Glioblastoma cell growth is suppressed by disruption of fibroblast growth factor pathway signaling. J. Neuro Oncol. 2009, 94, 359-366. [CrossRef] [PubMed]

85. Gouaze-Andersson, V.; Delmas, C.; Taurand, M.; Martinez-Gala, J.; Evrard, S.; Mazoyer, S.; Toulas, C.; Cohen-Jonathan-Moyal, E. FGFR1 induces glioblastoma radioresistance through the PLCgamma/Hif1alpha pathway. Cancer Res. 2016, 76, 3036-3044. [CrossRef] [PubMed]

86. Miraux, S.; Lemiere, S.; Pineau, R.; Pluderi, M.; Canioni, P.; Franconi, J.M.; Thiaudiere, E.; Bello, L.; Bikfalvi, A.; Auguste, P. Inhibition of FGF receptor activity in glioma implanted into the mouse brain using the tetracyclin-regulated expression system. Angiogenesis 2004, 7, 105-113. [CrossRef]

87. Daido, S.; Takao, S.; Tamiya, T.; Ono, Y.; Terada, K.; Ito, S.; Ouchida, M.; Date, I.; Ohmoto, T.; Shimizu, K. Loss of heterozygosity on chromosome $10 \mathrm{q}$ associated with malignancy and prognosis in astrocytic tumors, and discovery of novel loss regions. Oncol. Rep. 2004, 12, 789-795. [CrossRef]

88. Di Stefano, A.L.; Fucci, A.; Frattini, V.; Labussiere, M.; Mokhtari, K.; Zoppoli, P.; Marie, Y.; Bruno, A.; Boisselier, B.; Giry, M.; et al. Detection, characterization, and inhibition of FGFR-TACC fusions in IDH wild-type glioma. Clin. Cancer Res. 2015, 21, 3307-3317. [CrossRef]

89. Frattini, V.; Pagnotta, S.M.; Fan, J.J.; Russo, M.V.; Lee, S.B.; Garofano, L.; Zhang, J.; Shi, P.; Lewis, G.; Zhang, J.; et al. A metabolic function of FGFR3-TACC3 gene fusions in cancer. Nature 2018, 553, 222-227. [CrossRef]

90. Hoang-Minh, L.B.; Siebzehnrubl, F.A.; Yang, C.; Suzuki-Hatano, S.; Dajac, K.; Loche, T.; Andrews, N.; Schmoll Massari, M.; Patel, J.; Amin, K.; et al. Infiltrative and drug-resistant slow-cycling cells support metabolic heterogeneity in glioblastoma. EMBO J. 2018, 37, e98772. [CrossRef] 
91. Wang, Z.; Zhang, C.; Sun, L.; Liang, J.; Liu, X.; Li, G.; Yao, K.; Zhang, W.; Jiang, T. FGFR3, as a receptor tyrosine kinase, is associated with differentiated biological functions and improved survival of glioma patients. Oncotarget 2016, 7, 84587-84593. [CrossRef] [PubMed]

92. Darmanis, S.; Sloan, S.A.; Croote, D.; Mignardi, M.; Chernikova, S.; Samghababi, P.; Zhang, Y.; Neff, N.; Kowarsky, M.; Caneda, C.; et al. Single-cell RNA-Seq analysis of infiltrating neoplastic cells at the migrating front of human glioblastoma. Cell Rep. 2017, 21, 1399-1410. [CrossRef] [PubMed]

93. Yamada, S.M.; Yamada, S.; Hayashi, Y.; Takahashi, H.; Teramoto, A.; Matsumoto, K. Fibroblast growth factor receptor (FGFR) 4 correlated with the malignancy of human astrocytomas. Neurol. Res. 2002, 24, $244-248$. [CrossRef] [PubMed]

94. Schramm, K.; Iskar, M.; Statz, B.; Jager, N.; Haag, D.; Slabicki, M.; Pfister, S.M.; Zapatka, M.; Gronych, J.; Jones, D.T.W.; et al. DECIPHER pooled shRNA library screen identifies PP2A and FGFR signaling as potential therapeutic targets for DIPGs. Neuro Oncol. 2019. [CrossRef] [PubMed]

(C) 2019 by the authors. Licensee MDPI, Basel, Switzerland. This article is an open access article distributed under the terms and conditions of the Creative Commons Attribution (CC BY) license (http://creativecommons.org/licenses/by/4.0/). 



\title{
Targeting the Oncogenic FGF-FGFR Axis in Gastric Carcinogenesis
}

\author{
Jinglin Zhang ${ }^{1,2,3}$, Patrick M. K. Tang ${ }^{1}$, Yuhang Zhou ${ }^{1,2,3}$, Alfred S. L. Cheng ${ }^{4}$, Jun Yu ${ }^{2,5}$, \\ Wei Kang ${ }^{1,2,3, *}$ and Ka Fai To ${ }^{1,2,3, *}$ \\ 1 Department of Anatomical and Cellular Pathology, State Key Laboratory of Translational Oncology, \\ Prince of Wales Hospital, The Chinese University of Hong Kong, Hong Kong, China; \\ jinglinzhang@cuhk.edu.hk (J.Z.); patrick.tang@cuhk.edu.hk (P.M.K.T.); zyhjoe@gmail.com (Y.Z.) \\ 2 Institute of Digestive Disease, State Key Laboratory of Digestive Disease, The Chinese University of Hong \\ Kong, Hong Kong, China; junyu@cuhk.edu.hk \\ 3 Li Ka Shing Institute of Health Science, Sir Y.K. Pao Cancer Center, The Chinese University of Hong Kong, \\ Hong Kong, China \\ 4 School of Biomedical Sciences, The Chinese University of Hong Kong, Hong Kong, China; \\ alfredcheng@cuhk.edu.hk \\ 5 Department of Medicine and Therapeutics, The Chinese University of Hong Kong, Hong Kong, China \\ * Correspondence: weikang@cuhk.edu.hk (W.K.); kfto@cuhk.edu.hk (K.F.T.); \\ Tel.: +(852)-3505-1505 (W.K.); Fax: +(852)-2649-7286 (W.K.)
}

Received: 23 May 2019; Accepted: 24 June 2019; Published: 25 June 2019

\begin{abstract}
Gastric cancer (GC) is one of the most wide-spread malignancies in the world. The oncogenic role of signaling of fibroblast growing factors (FGFs) and their receptors (FGFRs) in gastric tumorigenesis has been gradually elucidated by recent studies. The expression pattern and clinical correlations of FGF and FGFR family members have been comprehensively delineated. Among them, FGF18 and FGFR2 demonstrate the most prominent driving role in gastric tumorigenesis with gene amplification or somatic mutations and serve as prognostic biomarkers. FGF-FGFR promotes tumor progression by crosstalking with multiple oncogenic pathways and this provides a rational therapeutic strategy by co-targeting the crosstalks to achieve synergistic effects. In this review, we comprehensively summarize the pathogenic mechanisms of FGF-FGFR signaling in gastric adenocarcinoma together with the current targeted strategies in aberrant FGF-FGFR activated GC cases.
\end{abstract}

Keywords: FGF; FGFR; gastric cancer; monoclonal antibody; small molecule

\section{Introduction}

Gastric cancer (GC), the third leading cause of cancer death globally, is considered a heterogeneous disease. Although the prevalence has declined over the past decades, more than half of newly diagnosed cases are found to possess local advancement or metastasis [1,2]. Late diagnosis and lack of effective therapeutics still make GC a challenge globally. For decades, researchers have been dedicated to uncover the mysteries behind GC, not only the medication strategies to alleviate or cure the disease, but the key factors for detecting the challenging disease at its early stage. It has been proven that environmental, etiological, and genetic factors largely contribute to GC development, for example, high salt diets, $H$. pylori infections [3], and CDH1 mutations [4,5]. Systematically, in-depth and comprehensive mechanistic studies revealed the crosstalk of oncogenic signaling pathways during GC progression as well as pre-cancerous gastric lesion development [6-9]. Of note, inactivation of the Hippo pathway has been substantially demonstrated in the pathogenesis of GC, via the accumulation of nuclear YAP1 in an uncontrollable manner [10-12]. Moreover, recent studies have further uncovered 
the emerging roles of fibroblast growing factors (FGFs) and their receptors (FGFRs) in the carcinogenesis of some GC subtypes, owing to their molecular characteristics [13]. It has been well documented that the FGF and FGFR families are important regulators for biological development $[14,15]$. Aberration of FGF-FGFR signaling substantially results in skeletal disorders as well as cancer development, including GC [16]. Since genetic aberrations of FGFR2 have been recently defined, it serves as a diagnostic marker and clinical drug target for GC [17-19]. However, development of FGFR2-targeted therapy has been largely decelerated due to recently reported disadvantages. Thus, further investigation of the FGF-FGFR must be continued in order to identify drug targets for GC therapy. This review aims to summarize the updated discoveries and discuss the further prospects of FGF-FGFR signaling in GC pathogenesis and therapy development.

\section{Emerging Role of FGF-FGFR in Solid Tumors}

\subsection{FGF Family Induces Tumor Growth}

FGFRs belong to the receptor tyrosine kinases (RTKs) superfamily. Most of the RTKs are membrane receptors with high affinity to multiple growth factors, cytokines, and hormones, and they contain intracellular domains with tyrosine kinase activity. Canonically, FGFRs are monomers in their inactivation state. Dimerization of the intracellular part occurs after binding with their ligand FGFs. Functional binding of FGF and FGFR leads to cross-phosphorylation and activation of the receptor. Activated FGFRs then transduce biochemical signals into cytosolic activities [20]. Indeed, the FGF family comprises 22 secreted factors that are generally divided into seven subgroups in terms of their phylogenetic relation, homology, and biochemical function [21]. As reported, five FGF subfamilies are released in paracrine and autocrine manners, including FGF1 (FGF1, FGF2), FGF4 (FGF4, FGF5, FGF6), FGF7 (FGF3, FGF7, FGF10, FGF22), FGF8 (FGF8, FGF17, FGF18), and FGF9 (FGF9, FGF16, FGF20). In contrast, the FGF15 (FGF15, FGF19, FGF21, FGF23) subfamily is secreted through endocrine glands as a hormone for metabolic modulation with $\alpha$ - and $\beta$-Klotho family proteins. Nevertheless, there are intracellular FGFs (FGF11, FGF12, FGF13, FGF14) that lack secretory N-terminal peptides, which execute their functions independent of FGFRs [22].

FGFs not only show regulatory roles in cell fate and survival, but also exerts biological functions in tissue regeneration and repair [23,24]. In the last few decades, clinical reports have highlighted the importance of FGFs in tumorigenesis, including excessive cell growth and angiogenesis. For example, basic fibroblast growth factor (bFGF) promotes angiogenesis for hepatoma progression [25], and a follow-up study suggested serum bFGF as a biological indicator for invasive and recurrent hepatocellular carcinoma (HCC) [26]. The clinical significance of bFGF was first recognized in patients who received surgical removal of colorectal cancer (CRC) at serological and pathological levels, where expression of bFGF indicated the independency in lymphatic invasion [27]. In addition, FGF amplification rated $10 \%$ in human malignancies, as overproduction of FGFs enables the communication between epithelial cells and stromal cells in the tumor microenvironment for tumorigenesis [28,29].

\subsection{FGFR Family Drives Oncogenesis}

\subsubsection{Functional Structures of FGFR}

Interestingly, FGF ligands interact with only four FGFRs (FGFR1-4), which are highly conserved in mammals, although FGFs harbor many family members. In general, FGFRs can be classified into three major domains based on their location relative to the cell membrane: (1) a ternary extracellular immunoglobulin (Ig) (domain I, II, III) that is in charge of binding with ligands; (2) a signal-pass transmembrane helix that acts as a connection; and (3) an intracellular tyrosine kinase (TK) that conveys the signals $[30,31]$. Generally speaking, the extracellular part of the FGFR provides binding sites for ligand binding, while the intracellular part is responsible for potentiating the relevant signaling pathways. Between the extracellular domains I and II, there is an acidic box region for the FGFR to 
interact with some molecules other than FGFs, while domains II and III possess the heparin binding site and FGF binding site [32]. The Ig domain III in FGFR1-3 has alternative splicing sites. The domain IIIa remains invariant while the other half varies according to the encoded exon IIIb or IIIc, which are based on tissue-dependent expression $[33,34]$. This means that the FGFRs only differ between certain parts of the Ig that governs the affinity and specificity of their ligands. There is a single-pass transmembrane domain connecting the Ig domains and the intracellular FGFR domains. The intracellular part of the FGFR includes a juxtamembrane domain for phosphotyrosine binding of adaptors, and two tyrosine kinase (TK) domains. As soon as the TK domains are phosphorylated, the downstream cascades are activated to further expand the signal [35]. Special FGFRs devoid of TK activity, namely FGFR5 or FGFRL1, have been identified and proposed as decoys, interfering with downstream signaling pathways [36]. Due to the diversity of receptor structure and transcript sequence, there are a number of FGFR variants that have been identified. For example, the FGFR2 IIIb isoform has high binding affinity to FGF3, FGF7, and FGF10, while its IIIc form is much preferable to FGF2, FGF4, and FGF20 [20,29]. Further investigation may lead to the discovery of a potential FGFR variant for GC management.

\subsubsection{Mechanisms of FGFR in Driving Cancer}

Recently, the oncogenic roles of FGFRs have been extensively demonstrated, and somatic alterations and differential expression patterns of FGFR have been seen in different human cancers. Helsten et al. recently depicted a landscape of FGFR aberrations from a large-cohort high-throughput sequencing of cancer patients. In total, FGFR aberrations were detected in $7.1 \%$ of the malignancies, including gene amplification $(66 \%)$, mutations $(26 \%)$, and rearrangements $(8 \%)$, suggesting the occurrence of FGFR aberration in most cancer types [37]. Mechanistically, FGFR disorder drives oncogenesis mainly via the following mechanisms: (1) FGFR gene amplification: It makes up the majority of the genetic alterations and results in abundant membrane FGFRs, which further augment the activation of its downstream signaling. Gene amplification is common in FGFR1, followed by FGFR2, but rare in FGFR3 and FGFR4. (2) Activating mutations: Most of the mutations exist in the extracellular receptor domains and cause constitutive activation of FGFRs automatically, without the participation of ligands. Activating mutations are frequently found in FGFR2 and FGFR3. (3) FGFRs fusion protein via chromosomal translocation: In this mechanism, the final exon at the C-terminus of the FGFR is replaced by another gene, which results in increasing dimerization and constitutive kinase activity, while ligands are also not required in this manner. (4) Hyperactivation of FGFRs under FGF overproduction from cancer and stromal cells: Additionally, the alternative splicing reconstitutes FGFRs from IIIb to IIIc isoforms, the binding specificity and affinity between FGF and FGFR is altered accordingly. (5) Apart from the genetic alterations of FGF and FGFR, more and more evidence supports that the differential expression of their downstream partners also evidently contributes to the oncogenic progression in multiple cancers [35].

\subsection{Partner Proteins Mediate FGF-FGFR Signal Transduction}

Signal transduction of FGF-FGFR cannot proceed without the participation of partner proteins. Cell adhesion molecules (CAMs), other types of RTKs, and G-Protein-Coupled Receptors (GPCRs) have been found to interact with FGFR family members and regulate a broad range of cell behaviors [38]. Intrinsically, FGFs can be anchored to the extracellular matrix by heparan sulfate proteoglycans (HPSGs) and thus avoid degradation by proteases. FGFs then bind to certain cell-surface FGFRs to form a ternary complex FGF-FGFR-HPSG [39,40]. Otherwise, a deficiency of HPSG results in the enhanced FGF ligand diffusion and failure of the FGF-FGFR signal transduction, which imposes a restriction on cell polarity and motility [41]. As the complex is formed, intracellular tyrosine kinases of FGFR dimerize and cross-phosphorylate on their tyrosine residues at the activation chain. The main intracellular substrates of FGFR are known as phospholipase C (PLC $\gamma$ ) (FRS1), FGFR substrate 2 $($ FRS $2 \alpha$ ), and FGFR substrate 3 (FRS2 $\beta$ ) $[42,43]$. These proteins function as adaptors and are directly phosphorylated by the activating FGFRs $[44,45]$. FRS2 is a lipid-anchored protein and is located on the 
juxtamembrane domain to recruit signaling components toward the receptor in response to stimulation by ligands [46]. The functional domain of the FRS2 recruits growth factor receptor-bound 2 (GRB2) by four main phosphorylation sites (Tyr196, Tyr306, Tyr349, Tyr392) [47]. GRB2 then enrolls either the guanine nucleotide exchange factor son of sevenless (SOS) or the GRB2-associated binding protein 1 (GAB1) [42]. These proteins form a scaffold for initiating downstream signaling and compose a significant part for signal transduction of the FGF-FGFR signaling. It is noted that some negative regulators exist on the cell surface to counteract the effect of FGFR. One such family is called similar expression to FGF (SEF), members of this family interact with the intracellular domain of FGFRs and inhibit downstream responses. In tumors, the expression of SEF is significantly decreased $[48,49]$.

\subsection{Signaling Pathways Respond to FGF-FGFR Activation}

Upon the recruitment and activation of the FGF-FGFR complex, extracellular signals are turned into intracellular events. Cytosolic signaling pathways aroused by the FGF-FGFR complex are recognized as downstream of FGF-FGFR. It has been well-defined that the Ras-dependent mitogen-activated protein kinase (RAS-MAPK), Ras-independent phosphoinositide 3-kinase (PI3K-Akt), $\mathrm{PLC} \gamma-\mathrm{Ca}^{2+}-\mathrm{PKC}$, and Janus kinase-signal transducers and activators of transcription (JAK-STAT) act as canonical downstream signaling pathways of FGF-FGFR [50-53]. On one hand, phosphorylation of FRS2 and GRB2 further initiates the RAS-MAPK and PI3K-AKT signaling pathways by recruiting SOS and GAB1 to the protein complex, respectively. RAS phosphorylates a series of MAPKs such as extracellular signal-regulated kinase 1 (ERK1) and ERK2, which potentiate E26 transformation-specific (ETS) transcription factors to interact and regulate their target genes related to cell proliferation, survival, and transformation $[50,54]$. As a feedback, inhibitory factors can also be induced by FGF signals. Sprouty (SPRY) interrupts the activation of GRB2, and MAPK phosphatase 3 (MKP3) dephosphorylates ERK1/2 [15]. The PI3K-AKT signaling pathway works differently. After FGF stimulation, GRB2 phosphorylates PI3K-AKT and then inhibits nuclear localization of a pro-apoptotic effector, promoting expression of genes associating with cell survival [55]. In contrast, inhibiting FGFR impairs the function of this pathway and leads to retardation of tumor growth and metastasis [51]. On the other hand, phosphorylation of PLC $\gamma$ by the FGFR kinase domain hydrolyzes phosphatidylinositol 4,5-bisphosphate to produce inositol triphosphate (IP3) and diacylglycerol (DAG), which support intracellular calcium release and activate protein kinase C (PKC), respectively [56]. Moreover, it has been suggested that amplification of FGFR is required for the signal transducers and activators of transcription-3 (STAT3) activation in cancers. The interaction of FGFR and STAT3 depends on the involvement of JAK [57]. It should be noted that FGF-FGFR signaling cascades also cooperate with other signaling pathways, including Notch [58], Wnt [59], Hedgehog [60], and BMP signaling [61]. Fine-tuning of the cascades ensures homeostasis among normal cells, but their dysfunction may induce multiple diseases and even cancers.

\section{Deregulation of the FGF-FGFR Signaling in Gastric Carcinogenesis}

\subsection{Significance of FGFR2 in Gastric Tissues}

FGF-FGFR signaling exerts multiple biological functions and effects. FGFR2 isoforms IIIb and IIIc are predominantly expressed in the epithelial and mesenchymal tissues [62-64]. Along with the understanding of FGFR2, their FGF ligands have been gradually identified. Structurally, FGFR2-IIIb bonds to FGF1, FGF3, FGF7, FGF10, and FGF22 in epithelial tissues; while FGFR2-IIIc responds to a number of FGFs (i.e., FGF1, FGF2, FGF4, FGF5, FGF6, FGF8, FGF9, FGF16, FGF17, FGF18, FGF19, and FGF20) in mesenchymal cells [65,66]. Interestingly, different FGFs will result in various downstream effects via FGFR activation. In gastric tissue, FGFR2 is involved in early epithelial growth before differentiation, and FGF10 and FGFR2-IIIb promote proliferation and patterning of the forestomach. In contrast, silence of both FGF10 and FGFR2 severely induces abnormal lining of gastric epithelium [67]. 


\subsection{Aberrant FGF-FGFRs Advance Gastric Tumorigenesis}

FGFR2 not only has physiological roles in normal gastric tissue, but also contributes to the development and progression of GC. Indeed, FGFR2 amplification was detected in GC cells three decades ago [68,69]. The understanding of FGFR2 is extensive, especially in terms of its abnormal genetic alterations that are rare in other FGFR members. FGFR amplification is the main genetic alteration in GC, accounting for up to $9 \%$ in western populations and $1.2-4.9 \%$ in Asian cohorts $[13,70]$. Nevertheless, mutation and fusion genes are rare in GC patients. From tissue-based studies, incidence of FGFR2 amplification is equivalent to that of ERBB2 and KRAS, ranging from $2 \%$ to $9 \%$ according to different methodologies and geographies. Clinical data also manifest that the frequency of FGFR2 amplification basically contributes to diffuse-type GC [71-74]. In addition, amplification of FGFR2 in GC is mutually exclusive with HER2 and KRAS amplification by FISH assay [18,75], suggesting they are independent prognostic biomarkers. Gene amplification is a common cause for mRNA overexpression. In fact, a recent in situ analysis showed that FGFR2 mRNA is highly correlated with FGFR2 amplification in primary cases clinically, where a high expression level of FGFR2 is associated with poor survival rate of GC patients [76]. Recently, FGFR2 overexpression has been detected in a great portion of GC cases by immunohistochemistry staining, the high level FGFR2-IIIb isoform predicts poor overall survival in patients [19]. A retrospective study revealed that FGFR2 expression was negatively associated with relapse-free survival in a Japanese diffuse-type GC cohort. In that study, although association between FGFR2 expression and survival outcomes in patients with stage II/III GC after surgery and S-1 chemotherapy was insignificant, patients with recurrence after five years of treatment made up a relatively large proportion of the high FGFR2 levels, implying the FGFR2 overexpression may be relevant to GC development [77]. FGFR2 may also contribute to drug resistance of GC. A GC model with FGFR2 amplification was sensitive to a FGFR inhibitor AZD4547. However, another study questions the efficacy and safety of AZD4547 in GC patients since their progression-free survival rate did not significantly improve with AZD4547 monotherapy compared with paclitaxel, which may due to the intratumor heterogeneity of the FGFR2 copy-number aberration [78]. Based on these studies, aberrant FGFR2 is largely involved in gastric tumorigenesis and is a candidate to be a diagnostic marker and has the potential to be a therapeutic target for GC treatment. However, challenges will exist until the complexity of the FGFR2 signaling network is resolved.

Autocrine and paracrine FGFs constitute an important functional role in the FGFR2 signaling cascade. In the last two decades, FGF ligands have been reported in multiple cancers, but only a few FGFs were investigated in GC. For example, gastric fibroblast-derived FGF7 increases scirrhous GC cell proliferation in a paracrine manner. Although intrinsic levels of FGF7 are low in GC cells, its corresponding receptor FGFR2 is highly expressed. Subsequently, FGF7 was reported to interact with FGFR2 to promote cell migration and invasion in GC $[79,80]$. On the other hand, a study found that FGF9 triggers proliferation and inhibits apoptosis of GC cells in an autocrine manner in a Chinese GC cohort [81]. At the genetic level, amplification of FGF genes may lead to their overproduction in GC, specifically, FGF10 amplification has been reported in 3\% of GC and in 5.7\% of gastric adenocarcinomas [82,83]. FGF10 is correlated to GC cell invasion and has been suggested as a prognostic biomarker and potential drug target in gastric adenocarcinoma [84]. In our recent study, we explored the FGF mRNA profiling in 10 GC cell lines by microarray analysis, where FGF18 showed the highest expression among all the FGF members. This study also identified clinical correlation of FGF18 and highlighted FGF18 as a potent diagnostic indicator in GC. Upon FGF18 stimulation, cell growth is facilitated by activation of SMAD2/3 and suppression of ATM signaling [85]. Nevertheless, the molecular network of FGF-FGFRs responsible for GC progression remains to be revealed.

\subsection{FGFR2 Crosstalk in GC}

It is believed that the FGFR2 aberration fundamentally contributes to GC development, but how FGFR2 coordinates with other regulatory signaling remains unclear. Investigations of FGFR2 and other oncogenic signaling have been conducted to decipher the comprehensive network. 
The amplification of FGFR2 has been implied to facilitate cell growth in GC through crosstalking with other RTKs. It is reported that activated epidermal growth factor receptor (EGFR), human epidermal growth factor receptor 3 (HER3), and MET correlate with drug hyposensitivity of GC cells with FGFR2 amplification. Interestingly, a combination of an FGFR2 inhibitor and EGFR neutralizing antibody partially enhanced drug sensitivity of GC in vitro and in vivo, suggesting these RTKs may cause drug resistance in cancer cells under FGFR2 inhibition. Eventually, a novel mechanism was identified whereby RTKs can coexpress with FGFR2 and synergistically promote the growth of GC [86]. In contrast, another study reported that HER2, MET, and FGFR2 are mutually exclusive oncogenic drivers, where a large number of HER2-negative patients were highly sensitive to the MET- and FGFR2-targeted therapies [87]. However, these contradictory conclusions may be attributable to the differences of the GC cohorts and the experimental models applied in the studies. One possible reason is that the former study focused on FGFR2 amplification cases where patients were hyposensitive to AZD4547, while the later one concerned both gene amplification and overexpression. Nevertheless, these results examined the potential relationship between FGFR2 and other RTKs, though the underlying molecular mechanisms are not fully understood. Combined therapy for targeting both FGFR2 and RTKs may be a new strategy for clinically treating GC.

In addition, several signaling pathways have been highlighted as downstream of FGFR2 that may also be involved in GC development (Figure 1). Lau et al. revealed a survival mechanism for developing acquired-resistance under FGFR inhibition. They established drug resistance on both primary and patient derived xenograft (PDX) models of various GCs with different FGFR2 amplification levels by applying FGFR2 inhibitors. Interestingly, they observed that MAPK and AKT signaling pathways were dispensable for drug resistance, but the constitutive inhibition of GSK3 $\beta$, which depends on activation of PKC, was required for cell survival [88]. Therefore, the FGFR2-PKC-GSK3 $\beta$ axis is considered as the main mechanism causing resistance in GC during anti-FGFR2 therapy. Additionally, PI3K-Akt-mTOR signaling contributes to the oncogenic activity of FGFR signaling in GC. Huang et al. recently suggested that FGFR2 signaling promotes GC by regulating the expression of Thrombospondin 1 (THBS1) and THBS4 via the PI3K-Akt-mTOR pathway. They indicated that FGF7-FGFR2 signaling upregulates THBS1, while THBS4 is decreased by the FGFR2-Akt cascade [80,89]. These studies established that PI3K-Akt signaling partially contributes to the tumor-promoting function of FGFR2 in GC, although the contribution of the THBS family to GC is still not fully understood. Therefore, further studies are required to reveal the detailed mechanisms. Moreover, epithelial mesenchymal transition (EMT) is a well-known mechanism that facilitates tumor cell transformation and distant metastasis during oncogenic progression. FGF-FGFR signaling has been shown to potentiate EMT [20]. The basic components of EMT, WNT signaling, and Twist-related protein 1 (Twist1) have been found to upregulate FGFR2 in GC cell lines. In turn, FGFR2 further amplifies Twist1 mediated EMT and cell invasion, implying dual inhibition of these pathways is needed for GC therapy [90]. Of note, under the FGFR2 signaling cascade, nuclear accumulation of $\beta$-catenin and EMT transcription factors, such as SNAIL, have also been proposed [91].

Current findings have uncovered the complicated interactions between FGFR2 signaling and other RTKs and oncogenic signaling pathways in GC. These signaling networks trigger primary and secondary resistance of GC cells under treatment and eventually lead to the advanced stage of disease. Fortunately, a better understanding of the FGFR signaling network will gradually help in the development of novel therapeutic options for GC. 


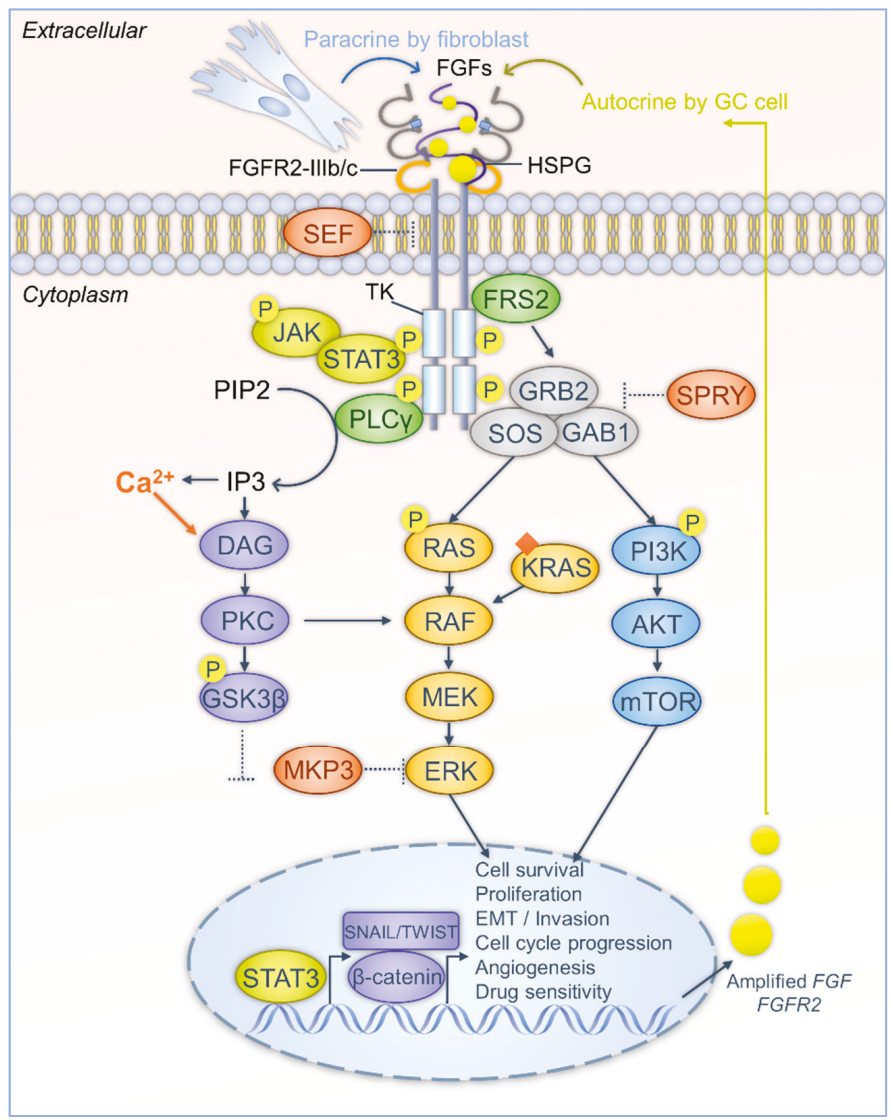

Figure 1. The FGF-FGFR cascade interplays with the downstream signaling network in GC progression. Firstly, FGFR is aberrantly activated in GC cells. FGFs can be released mainly in two ways, in a paracrine manner by gastric fibroblasts, and in an autocrine manner from cancer cells. Gene amplification of FGFs and FGFRs leads to overproduction of FGFs and FGFRs. FGFs are stabilized and bind to FGFR via HSPG. Alternative splicing of FGFR induces two isoforms that highly are expressed in GC. The isoforms show different affinity to FGFs and contribute to diverse cellular processes. The intracellular region of the FGFR has tyrosine kinase (TK) activity. FGF stimulation leads to dimerization, phosphorylation, and activation of FGFR. The inhibitory effect of SEF is attenuated in GC cells. Secondly, after FGFR activation, adaptor proteins are recruited and also activated by phosphorylation. FRS2 further recruits GRB2, GAB1, and SOS to form a complex. The complex activates RAS-MAPK and PI3K-Akt-mTOR signaling pathways and transduces FGF stimulation into transcriptional regulation to forward tumorigenesis. The inhibitory effects of SPRY and MKP3 are abrogated in GC cells. PLC $\gamma$ hydrolyzes PIP2 to IP3, increases $\mathrm{Ca}^{2+}$ levels, triggers DAG-PKC signaling, and phosphorylates GSK3 $\beta$. Then, GSK3 $\beta$ is decreased and $\beta$-catenin is released to the nuclei. $\beta$-catenin and other EMT transcription factors, SNAIL and TWIST, initiate expression of oncogenes that are required for GC progression. Besides, JAK-STAT3 is activated by FGFR and contributes to transcriptional regulation of GC progression. (Arrows represent the activation or release routes; dash dots indicate the weakening of inhibitory effects).

\section{Targeting Aberrant FGF-FGFR Activation in GC by Specific Antibodies or Small Molecules}

As the FGF-FGFR singling plays an oncogenic role in tumorigenesis by crosstalking with or regulating multiple crucial other pathways, targeting of FGF-FGFR by specifically designed therapeutic 
agents has shed light on the precision of medicine [92]. These agents include specific anti-FGFR monoclonal antibodies, FGF traps [93], non-selective RTK inhibitors, and selective RTK inhibitors.

\subsection{Specific Antibodies and FGF Traps}

In the aberrant FGF-FGFR-activation GC cases, anti-FGF (FGF traps) or anti-FGFR monoclonal antibodies might exert anti-cancer effects for the treatment (Table 1). Compared with tyrosine kinase inhibitors, the specific antibodies targeting FGFs or FGFRs have more specificity and less toxicity because they can avoid the off-target effects.

Table 1. A list of anti-FGFR monoclonal antibodies and FGF traps potentially employed in GC.

\begin{tabular}{clc}
\hline Monoclonal Antibodies & Targets & References \\
\hline GAL-FR21 and GAL-FR22 & FGFR2 & {$[94]$} \\
FPA144 (Bemarituzumab) & FGFR2 amplification or overexpression & {$[98]$} \\
BAY 1179470 & FGFR2 amplification or overexpression & {$[95]$} \\
\hline FGF traps & & \\
\hline GSK3052230 & FGFs & {$[93,96]$} \\
NSC12 & FGFs & {$[97]$} \\
\hline
\end{tabular}

The specific monoclonal antibodies generated and effectively employed for targeting FGFRs in GC research are quite limited [22]. They include GAL-FR21 and GAL-FR22 antibodies. GAL-FR21 binds only the FGFR2IIIb isoform, whereas GAL-FR22 and GAL-FR23 can directly bind to both the FGFR2IIIb and FGFR2IIIc isoforms, with binding regions respectively in the D3, D2-D3, and D1 domains of FGFR2. GAL-FR21 and GAL-FR22 block the binding of FGF2, FGF7, and FGF10 to FGFR2IIIb. GAL-FR21 inhibits FGF2- and FGF7-induced phosphorylation of FGFR2, and both antibodies dramatically down-modulate the activation of FGFR2 in SNU16 cells (with FGFR2 amplification). These monoclonal antibodies also effectively inhibit the tumor growth of established SNU16 and OCUM-2M xenografts in mice [94]. Another FGFR2b-specific antibody, FPA144, can not only treat GC patients with FGFR2 amplification, but also patients with FGFR2b overexpression who lack FGFR2 gene amplification. FPA144 is still being evaluated in a phase III clinical trial of GC. Another novel antibody-drug conjugate (ADC), namely BAY 1179470, provides preclinical efficacy. It consists of a fully human FGFR2 monoclonal antibody, which binds to the FGFR2 isoforms FGFR2-IIIb and FGFR2-IIIc, conjugated through a noncleavable linker to a novel derivative of the microtubule-disrupting cytotoxic drug auristatin (FGFR2-ADC). Functional studies demonstrated that FGFR2-ADC administration leads to a significant tumor growth inhibition or tumor regression of cell line-based or patient-derived xenograft models of human gastric or breast cancer. Similar to FPA144, FGFR2 amplification or mRNA overexpression predicted high response to BAY 1179470 treatment [95].

As some FGF members, such as FGF18, are abundant in gastric carcinogenesis, using FGF ligand traps is another strategy to neutralize FGF and quench malignancies [85]. An FGF "ligand trap" is comprised of a fusion protein of an immunoglobulin Fc fragment and a soluble FGFR extracellular domain that competitively binds with FGF1, 2, 3, 7, and 10 to suppress ligand-dependent FGFR signaling [93]. For example, the FGF traps FP-1039 (GSK3052230) and sFGFR3 are soluble proteins that contain the extracellular regions of FGFR1 and FGFR3, respectively [96], thus they can successfully neutralize the oncogenic role of FGFs. Another good example is NSC12, acting as an extracellular FGF trap. It can be employed in anti-angiogenic and anti-vascular endothelial growth factor therapy as an FGF antagonist [97].

\subsection{Small Molecules: Non-Selective and Selective FGFR Inhibitors}

Apart from the antibodies or traps, small molecules can also generally and effectively inhibit tyrosine kinase receptor-related signaling (non-selective FGFR inhibitors). SOMCL-085 is a novel 
FGFR-dominant multi-target kinase inhibitor. This compound can simultaneously inhibit the angiogenesis kinases such as vascular endothelial growth factor receptor (VEGFR) and platelet-derived growth factor receptor (PDGFR). SOMCL-085 potently inhibits FGFR1, FGFR2, and FGFR3 kinase activity, with $\mathrm{IC}_{50}$ values of $1.8,1.9$, and $6.9 \mathrm{nmol} / \mathrm{L}$, respectively [99]. In the FGFR1-amplified lung cancer cell line H1581-xenograft mice and FGFR2-amplified GC cell line SNU16-xenograft mice, oral administration of SOMCL-085 for 21 days substantially inhibited tumor growth without loss of body weight. Nintedanib, a triple-angiokinase inhibitor, is a potent and selective inhibitor for tumor angiogenesis through the blocking of the tyrosine kinase activities of VEGFR1-3, PDGFR-alpha and -beta, together with FGFR1-3 [100]. In combination with docetaxel, nintedanib has been approved for the second-line treatment of adenocarcinoma non-small cell lung cancer (NSCLC). In human GC cell lines driven by an FGFR2 amplification, such as KatoIII, nintedanib is also confirmed to be highly effective. Regorafenib has also demonstrated survival benefits in patients with metastatic colorectal and gastrointestinal stromal tumors. More importantly, FGFR2 amplification was the only genetic alteration associated with in vitro sensitivity to regorafenib. Regorafenib induces G1 phase cell cycle arrest in SNU16 and KATOIII GC cells and suppresses their xenograft formation abilities [101]. S49076 is a novel and potent inhibitor of MET, AXL/MER, and FGFR1/2/3. S49076 potentially blocks cellular phosphorylation of MET, AXL, and FGFRs and inhibits downstream signaling pathways in vitro and in vivo. S49076 alone can cause tumor growth arrest in bevacizumab-resistant cancer cells. Based on the favorable and novel pharmacologic profile of S49076, a phase I study is currently being conducted in patients with advanced solid tumors [102]. Ponatinib (AP24534), an oral multitargeted tyrosine kinase inhibitor, has been explored in a pivotal phase II trial in patients with chronic myelogenous leukemia due to its potent ability against BCR-ABL. It has also been shown to inhibit the in vitro kinase activity of all four FGFRs. In a panel of 14 cell lines representing multiple tumor types (endometrial, bladder, gastric, breast, lung, and colon) and containing FGFRs dysregulated by amplification, overexpression, or mutation, ponatinib inhibited FGFR-mediated signaling with $\mathrm{IC}_{50}$ values below $40 \mathrm{nmol} / \mathrm{L}$, supporting it as a potent pan-FGFR inhibitor in patients with FGFR-driven cancers [103].

To avoid the off-target effects of non-selective inhibitors, novel selective FGFR inhibitors were generated and employed for specifically blocking the FGF-FGFR cascade in GC. Among all the selective FGFR inhibitors, AZD4547 is the most famous [17]. It is a selective FGFR1, 2, 3 tyrosine kinase inhibitor with potent preclinical activity in FGFR2-amplified gastric adenocarcinoma SNU16 and SGC083 xenograft animal models, together with the patient-derived cells (PDCs) [104]. The randomized phase II SHINE study (NCT01457846) investigated whether AZD4547 improved clinical outcome versus paclitaxel as a second-line treatment in patients with advanced gastric adenocarcinoma displaying FGFR2 polysomy or gene amplification detected by fluorescence in situ hybridization (FISH). However, the final results indicated AZD4547 failed to significantly improve progression-free survival compared with paclitaxel in GC patients with FGFR2 amplification or polysomy [78]. The related molecular mechanism needs to be further addressed. LY2874455, a potent oral selective pan-FGFR inhibitor, was investigated for its efficacy in a phase I clinical trial. LY2874455 was gradually absorbed and generally showed linear pharmacokinetics. The effective half-life span was approximately $12 \mathrm{~h}$. In 15 GC patients, one patient had a partial response, while 12 patients had stable disease. Thus, LY2874455 has a recommended phase II dosing of $16 \mathrm{mg}$ BID in solid-organ cancer patients [105]. However, in FGFR2-amplified GC patients, some will eventually develop an acquired LY2874455 resistance due to a novel FGFR2-ACSL5 fusion protein that is formed [106]. Based on the structure, medicinal chemistry optimization, and unique ADME assays of a covalent drug discovery program, a novel compound, namely PRN1371, was discovered to serve as a highly selective and potent FGFR1-4 inhibitor [107]. In combination with the de novo synthesis program 'SYNOPSIS' to generate high scoring and synthetically accessible compounds, alofanib (RPT835) was found to be an effective inhibitor of the FGF/FGFR2 pathway. RPT835 potently inhibited growth of KATOIII GC cells with a GI 50 value of $10 \mathrm{nmol} / \mathrm{L}$ [108]. ARQ 087 is a novel, ATP competitive, small molecule, multi-kinase inhibitor with potent in vitro and in vivo activity against FGFR-addicted cell lines and tumors. It exhibited $\mathrm{IC}_{50}$ 
values of $1.8 \mathrm{nM}$ for FGFR2, and $4.5 \mathrm{nM}$ for FGFR1 and 3. ARQ 087 has anti-proliferative activity in cell lines driven by FGFR dysregulation, including amplifications, fusions, and mutations, such as the SNU16 cell line. It is currently being investigated in a phase I/II clinical trial [109]. BGJ398, a pan-FGFR inhibitor, was also investigated in a GC model. In vitro, FGFR inhibition was most effective in KKLS cells (high FGFR1, FGFR2IIIc, no FGFR2IIIb expression) with inhibition of growth and motility. BGJ398 also showed partial activity in MKN45 cells (intermediate FGFR1, high FGFR2IIIb, low FGFR2IIIc expression), while TMK-1 cells (low FGFR1, no FGFR2IIIb and FGFR2IIIc expression) showed a negative response to this drug [110]. Some of the non-selective and selective FGFR inhibitors that have been investigated in gastric adenocarcinoma are listed in Table 2.

Table 2. The list of non-selective and selective FGFR tyrosine kinase inhibitors reported in GC.

\begin{tabular}{ccc}
\hline Non-Selective FGFR Inhibitors & Main Targets & References \\
\hline SOMCL-085 & FGFR, VEGFR, and PDGFR & {$[99]$} \\
Nintedanib & FGFR, VEGFR, and PDGFR & {$[100]$} \\
Regorafenib & FGFR2, VEGFR1-3, PDGFR, c-Kit, and RET & {$[101]$} \\
S49076 & MET, AXL/MER, and FGFR1-3 & {$[102]$} \\
Ponatinib & BCR-ABL, VEGFR2-3, and FGFR1-4 & {$[103]$} \\
\hline Selective FGFR inhibitors & & \\
\hline AZD4547 & FGFR1, FGFR2 and FGFR3 & {$[17,78]$} \\
LY2874455 & FGFR1, FGFR2, FGFR3 and FGFR4 & {$[105]$} \\
PRN1371 & FGFR1, FGFR2, FGFR3 and FGFR4 & {$[107]$} \\
RPT835 & FGFR2 & {$[108]$} \\
ARQ 087 & FGFR1, FGFR2 and FGFR3 & {$[109]$} \\
BGJ398 & FGFR1, FGFR2 and FGFR3 & {$[110]$} \\
\hline
\end{tabular}

\section{Conclusions and Future Directions}

Although we have made great progress in understanding the molecular mechanisms and crosstalk of FGF-FGFR in gastric carcinogenesis, and are even trying to employ small molecules or specific antibodies to block the oncogenic-driven role of FGF-FGFR signaling, several important issues need to be addressed urgently in future studies. First of all, GC can be subgrouped into intestinal and diffuse type from the histological classification, and it can also be stratified as four molecular subtypes according to TCGA molecular classification, Epstein-Barr virus (EBV)-positive tumors, microsatellite instable (MSI) tumors, genomically stable (GS) tumors, and tumors with chromosomal instability (CIN) [13]. Each subtype has its distinct molecular features and the etiology together with pathological processes are quite different among the subtypes. Thus, we need to re-evaluate the genetic and epigenetic changes and clinical correlations in a large cohort of FGF-FGFR for each subgroup to confirm the impact of different genetic backgrounds on FGF-FGFR activation. For example, in a small size cohort study, high FGFR4 expression correlated with tumor progression and survival in both diffuse and intestinal GC, whereas high expression of FGFR1 and 2 correlated with tumor progression and survival only in diffuse type GC [111]. Secondly, as FGF-FGFR crosstalks with multiple signaling pathways, such as the RAS-MAPK pathway, PI3K-Akt-mTOR pathway [112], and PKC-GSK3 $\beta$ pathway, we need to stratify our primary samples again according to different crosstalks by the immunohistochemistry method combined with FISH analysis. We will re-evaluate the clinical significance and perform co-administration of multiple anti-cancer drugs to achieve synergistic effects. The successful development of highly specific anti-FGFR personalized strategies will rely on our deeper knowledge of the key alterations that drive oncogenesis in GC [113]. Based on the identification of novel key downstream effectors of the FGF-FGFR cascade in gastric carcinogenesis, we aim to effectively and accurately target FGFR-related signaling in this precision medicine era. 
Author Contributions: W.K. and K.F.T. provided direction and instruction in preparing this manuscript. J.Z., Y.Z. and W.K. reviewed the literature and drafted this manuscript. P.M.K.T., A.S.L.C. and J.Y. reviewed the manuscript and made significant revisions on the drafts.

Funding: The current manuscript is supported by Research Grants Council of the Hong Kong Special Administrative Region, China [Project No.: CUHK 14110016 and 14118518 (for GRF projects)], Innovation and Technology Fund of Hong Kong (Project No.: (ITS/068/18, PiH/009/19, PiH/010/19, InP/008/19, InP/009/19), and CUHK Direct Grant for Research (2017.001 and 2018.002) from The Chinese University of Hong Kong.

Acknowledgments: We acknowledge the support from the Core Utilities of Cancer Genomics and Pathobiology of Department of Anatomical and Cellular Pathology, The Chinese University of Hong Kong.

Conflicts of Interest: The authors declare no conflict of interest.

\section{References}

1. Bray, F.; Ferlay, J.; Soerjomataram, I.; Siegel, R.L.; Torre, L.A.; Jemal, A. Global cancer statistics 2018: GLOBOCAN estimates of incidence and mortality worldwide for 36 cancers in 185 countries. CA Cancer J. Clin. 2018, 68, 394-424. [CrossRef] [PubMed]

2. Correa, P. Human gastric carcinogenesis: A multistep and multifactorial process-First American Cancer Society Award Lecture on Cancer Epidemiology and Prevention. Cancer Res. 1992, 52, 6735-6740. [PubMed]

3. Uemura, N.; Okamoto, S.; Yamamoto, S.; Matsumura, N.; Yamaguchi, S.; Yamakido, M.; Taniyama, K.; Sasaki, N.; Schlemper, R.J. Helicobacter pylori infection and the development of gastric cancer. N. Engl. J. Med. 2001, 345, 784-789. [CrossRef] [PubMed]

4. Pharoah, P.D.; Guilford, P.; Caldas, C. International Gastric Cancer Linkage C: Incidence of gastric cancer and breast cancer in CDH1 (E-cadherin) mutation carriers from hereditary diffuse gastric cancer families. Gastroenterology 2001, 121, 1348-1353. [CrossRef] [PubMed]

5. Hansford, S.; Kaurah, P.; Li-Chang, H.; Woo, M.; Senz, J.; Pinheiro, H.; Schrader, K.A.; Schaeffer, D.F.; Shumansky, K.; Zogopoulos, G.; et al. Hereditary Diffuse Gastric Cancer Syndrome: CDH1 Mutations and Beyond. JAMA Oncol. 2015, 1, 23-32. [CrossRef] [PubMed]

6. Yanai, K.; Nakamura, M.; Akiyoshi, T.; Nagai, S.; Wada, J.; Koga, K.; Noshiro, H.; Nagai, E.; Tsuneyoshi, M.; Tanaka, M.; et al. Crosstalk of hedgehog and Wnt pathways in gastric cancer. Cancer Lett. 2008, 263, 145-156. [CrossRef]

7. Corso, S.; Ghiso, E.; Cepero, V.; Sierra, J.R.; Migliore, C.; Bertotti, A.; Trusolino, L.; Comoglio, P.M.; Giordano, S. Activation of HER family members in gastric carcinoma cells mediates resistance to MET inhibition. Mol. Cancer 2010, 9, 121. [CrossRef]

8. Fu, Y.F.; Gui, R.; Liu, J. HER-2-induced PI3K signaling pathway was involved in the pathogenesis of gastric cancer. Cancer Gene Ther. 2015, 22, 145-153. [CrossRef]

9. Riquelme, I.; Saavedra, K.; Espinoza, J.A.; Weber, H.; García, P.; Nervi, B.; Garrido, M.; Corvalán, A.H.; Roa, J.C.; Bizama, C. Molecular classification of gastric cancer: Towards a pathway-driven targeted therapy. Oncotarget 2015, 6, 24750-24779. [CrossRef]

10. Cui, Z.-L.; Han, F.-F.; Peng, X.-H.; Chen, X.; Luan, C.-Y.; Han, R.-C.; Xu, W.-G.; Guo, X.-J. Yes-Associated Protein 1 Promotes Adenocarcinoma Growth and Metastasis through Activation of the Receptor Tyrosine Kinase Axl. Int. J. Immunopathol. Pharmacol. 2012, 25, 989-1001. [CrossRef]

11. Kang, W.; Cheng, A.S.; Yu, J.; To, K.F. Emerging role of Hippo pathway in gastric and other gastrointestinal cancers. World J. Gastroenterol. 2016, 22, 1279-1288. [CrossRef] [PubMed]

12. Zhou, Y.; Huang, T.; Zhang, J.; Wong, C.C.; Zhang, B.; Dong, Y.; Wu, F.; Tong, J.H.M.; Wu, W.K.K.; Cheng, A.S.L.; et al. TEAD1/4 exerts oncogenic role and is negatively regulated by miR-4269 in gastric tumorigenesis. Oncogene 2017, 36, 6518-6530. [CrossRef] [PubMed]

13. The Cancer Genome Atlas Research Network. Comprehensive molecular characterization of gastric adenocarcinoma. Nature 2014, 513, 202-209. [CrossRef] [PubMed]

14. Friesel, R.; Neilson, K.M. Ligand-independent Activation of Fibroblast Growth Factor Receptors by Point Mutations in the Extracellular, Transmembrane, and Kinase Domains. J. Boil. Chem. 1996, 271, 25049-25057.

15. Thisse, B.; Thisse, C. Functions and regulations of fibroblast growth factor signaling during embryonic development. Dev. Biol. 2005, 287, 390-402. [CrossRef] [PubMed] 
16. Dieci, M.V.; Arnedos, M.; Andre, F.; Soria, J.C. Fibroblast Growth Factor Receptor Inhibitors as a Cancer Treatment: From a Biologic Rationale to Medical Perspectives. Cancer Discov. 2013, 3, 264-279. [CrossRef] [PubMed]

17. Xie, L.; Su, X.; Zhang, L.; Yin, X.; Tang, L.; Zhang, X.; Xu, Y.; Gao, Z.; Liu, K.; Zhou, M.; et al. FGFR2 gene amplification in gastric cancer predicts sensitivity to the selective FGFR inhibitor AZD4547. Clin. Cancer Res. 2013, 19, 2572-2583. [CrossRef]

18. Su, X.; Zhan, P.; Gavine, P.R.; Morgan, S.; Womack, C.; Ni, X.; Shen, D.; Bang, Y.-J.; Im, S.-A.; Kim, W.H.; et al. FGFR2 amplification has prognostic significance in gastric cancer: Results from a large international multicentre study. Br. J. Cancer 2014, 110, 967-975. [CrossRef]

19. Ahn, S.; Lee, J.; Hong, M.; Kim, S.T.; Park, S.H.; Choi, M.G.; Lee, J.-H.; Sohn, T.S.; Bae, J.M.; Kim, S.; et al. FGFR2 in gastric cancer: Protein overexpression predicts gene amplification and high $\mathrm{H}$-index predicts poor survival. Mod. Pathol. 2016, 29, 1095-1103. [CrossRef]

20. Katoh, M.; Nakagama, H. FGF receptors: Cancer biology and therapeutics. Med. Res. Rev. 2014, 34, $280-300$. [CrossRef]

21. Itoh, N.; Ornitz, D.M. Fibroblast growth factors: From molecular evolution to roles in development, metabolism and disease. J. Biochem. 2011, 149, 121-130. [CrossRef]

22. Ghedini, G.C.; Ronca, R.; Presta, M.; Giacomini, A. Future applications of FGF/FGFR inhibitors in cancer. Expert Rev. Anticancer. Ther. 2018, 18, 861-872. [CrossRef] [PubMed]

23. Yu, P.; Wilhelm, K.; Dubrac, A.; Tung, J.K.; Alves, T.C.; Fang, J.S.; Xie, Y.; Zhu, J.; Chen, Z.; De Smet, F.; et al. FGF-dependent metabolic control of vascular development. Nature 2017, 545, 224-228. [CrossRef] [PubMed]

24. Maddaluno, L.; Urwyler, C.; Werner, S. Fibroblast growth factors: Key players in regeneration and tissue repair. Development 2017, 144, 4047-4060. [CrossRef]

25. Hsu, P.I.; Chow, N.H.; Lai, K.H.; Yang, H.B.; Chan, S.H.; Lin, X.Z.; Cheng, J.S.; Huang, J.S.; Ger, L.P.; Huang, S.M.; et al. Implications of serum basic fibroblast growth factor levels in chronic liver diseases and hepatocellular carcinoma. Anticancer. Res. 1997, 17, 2803-2809. [PubMed]

26. Poon, R.T.-P.; Ng, I.O.-L.; Lau, C.; Yu, W.-C.; Fan, S.-T.; Wong, J. Correlation of serum basic fibroblast growth factor levels with clinicopathologic features and postoperative recurrence in hepatocellular carcinoma. Am. J. Surg. 2001, 182, 298-304. [CrossRef]

27. Jibiki, N.; Saito, N.; Kameoka, S.; Kobayashi, M. Clinical Significance of Fibroblast Growth Factor (FGF) Expression in Colorectal Cancer. Int. Surg. 2014, 99, 493-499. [CrossRef]

28. Helsten, T.; Schwaederle, M.; Kurzrock, R. Fibroblast growth factor receptor signaling in hereditary and neoplastic disease: Biologic and clinical implications. Cancer Metastasis Rev. 2015, 34, 479-496. [CrossRef]

29. Korc, M.; Friesel, R. The Role of Fibroblast Growth Factors in Tumor Growth. Curr. Cancer Drug Targets 2009, 9, 639-651. [CrossRef]

30. Lee, P.; Johnson, D.; Cousens, L.; Fried, V.; Williams, L. Purification and complementary DNA cloning of a receptor for basic fibroblast growth factor. Sci. 1989, 245, 57-60. [CrossRef]

31. Johnson, D.E.; Williams, L.T. Structural and functional diversity in the FGF receptor multigene family. Adv. Cancer Res. 1993, 60, 1-41.

32. Haugsten, E.M.; Wiedlocha, A.; Olsnes, S.; Wesche, J. Roles of Fibroblast Growth Factor Receptors in Carcinogenesis. Mol. Cancer Res. 2010, 8, 1439-1452. [CrossRef]

33. Holzmann, K.; Grunt, T.; Heinzle, C.; Sampl, S.; Steinhoff, H.; Reichmann, N.; Kleiter, M.; Hauck, M.; Marian, B. Alternative Splicing of Fibroblast Growth Factor Receptor IgIII Loops in Cancer. J. Nucleic Acids 2012, 2012, 950508. [CrossRef]

34. Yeh, B.K.; Igarashi, M.; Eliseenkova, A.V.; Plotnikov, A.N.; Sher, I.; Ron, D.; Aaronson, S.A.; Mohammadi, M. Structural basis by which alternative splicing confers specificity in fibroblast growth factor receptors. Proc. Natl. Acad. Sci. USA 2003, 100, 2266-2271. [CrossRef]

35. Babina, I.S.; Turner, N.C. Advances and challenges in targeting FGFR signalling in cancer. Nat. Rev. Cancer 2017, 17, 318-332. [CrossRef]

36. Trueb, B. Biology of FGFRL1, the fifth fibroblast growth factor receptor. Cell. Mol. Life Sci. 2011, 68, 951-964. [CrossRef]

37. Helsten, T.; Elkin, S.; Arthur, E.; Tomson, B.N.; Carter, J.; Kurzrock, R. The FGFR Landscape in Cancer: Analysis of 4,853 Tumors by Next-Generation Sequencing. Clin. Cancer Res. 2016, 22, 259-267. [CrossRef] 
38. Latko, M.; Czyrek, A.; Porebska, N.; Kucinska, M.; Otlewski, J.; Zakrzewska, M.; Opalinski, L. Cross-Talk between Fibroblast Growth Factor Receptors and Other Cell Surface Proteins. Cells 2019, 8, 455. [CrossRef]

39. Mulloy, B.; Linhardt, R.J. Order out of complexity - protein structures that interact with heparin. Curr. Opin. Struct. Boil. 2001, 11, 623-628. [CrossRef]

40. Beenken, A.; Mohammadi, M. The FGF family: Biology, pathophysiology and therapy. Nat. Rev. Drug Discov. 2009, 8, 235-253. [CrossRef]

41. Venero Galanternik, M.; Kramer, K.L.; Piotrowski, T. Heparan Sulfate Proteoglycans Regulate Fgf Signaling and Cell Polarity during Collective Cell Migration. Cell Rep. 2015, 10, 414-428. [CrossRef]

42. Goetz, R.; Mohammadi, M. Exploring mechanisms of FGF signalling through the lens of structural biology. Nat. Rev. Mol. Cell Biol. 2013, 14, 166-180. [CrossRef]

43. Xu, H.; Lee, K.W.; Goldfarb, M. Novel Recognition Motif on Fibroblast Growth Factor Receptor Mediates Direct Association and Activation of SNT Adapter Proteins. J. Boil. Chem. 1998, 273, 17987-17990. [CrossRef]

44. Hoch, R.V.; Soriano, P. Context-specific requirements for Fgfr1 signaling through Frs2 and Frs3 during mouse development. Development 2006, 133, 663-673. [CrossRef]

45. Gotoh, N.; Laks, S.; Nakashima, M.; Lax, I.; Schlessinger, J. FRS2 family docking proteins with overlapping roles in activation of MAP kinase have distinct spatial-temporal patterns of expression of their transcripts. FEBS Lett. 2004, 564, 14-18. [CrossRef]

46. Mohammadi, M.; Olsen, S.K.; Ibrahimi, O.A. Structural basis for fibroblast growth factor receptor activation. Cytokine Growth Factor Rev. 2005, 16, 107-137. [CrossRef]

47. Zhang, Y.; McKeehan, K.; Lin, Y.; Zhang, J.; Wang, F. Fibroblast growth factor receptor 1 (FGFR1) tyrosine phosphorylation regulates binding of FGFR substrate 2alpha (FRS2alpha) but not FRS2 to the receptor. Mol. Endocrinol. 2008, 22, 167-175. [CrossRef]

48. Fürthauer, M.; Lin, W.; Ang, S.-L.; Thisse, B.; Thisse, C. Sef is a feedback-induced antagonist of Ras/MAPK-mediated FGF signalling. Nat. Cell Biol. 2002, 4, 170-174. [CrossRef]

49. Tsang, M.; Friesel, R.; Kudoh, T.; Dawid, I.B. Identification of Sef, a novel modulator of FGF signalling. Nat. Cell Biol. 2002, 4, 165-169. [CrossRef]

50. Tsang, M.; Dawid, I.B. Promotion and attenuation of FGF signaling through the Ras-MAPK pathway. Sci. Stke 2004, 2004, pe17. [CrossRef]

51. Dey, J.H.; Bianchi, F.; Voshol, J.; Bonenfant, D.; Oakeley, E.J.; Hynes, N.E. Targeting Fibroblast Growth Factor Receptors Blocks PI3K/AKT Signaling, Induces Apoptosis, and Impairs Mammary Tumor Outgrowth and Metastasis. Cancer Res. 2010, 70, 4151-4162. [CrossRef]

52. Eswarakumar, V.; Lax, I.; Schlessinger, J.; Eswarakumar, J. Cellular signaling by fibroblast growth factor receptors. Cytokine Growth Factor Rev. 2005, 16, 139-149. [CrossRef]

53. Turner, N.; Grose, R. Fibroblast growth factor signalling: From development to cancer. Nat. Rev. Cancer 2010, 10, 116-129. [CrossRef]

54. Kamata, T. Keratinocyte growth factor regulates proliferation and differentiation of hematopoietic cells expressing the receptor gene K-sam. Exp. Hematol. 2002, 30, 297-305. [CrossRef]

55. Cailliau, K.; Browaeys-Poly, E.; Vilain, J.P. Fibroblast growth factors 1 and 2 differently activate MAP kinase in Xenopus oocytes expressing fibroblast growth factor receptors 1 and 4. Biochim. Biophys. Acta 2001, 1538, 228-233. [CrossRef]

56. Peters, K.G.; Marie, J.; Wilson, E.; Ives, H.E.; Escobedo, J.; Del Rosario, M.; Mirda, D.; Williams, L.T. Point mutation of an FGF receptor abolishes phosphatidylinositol turnover and $\mathrm{Ca}^{2+}$ flux but not mitogenesis. Nature 1992, 358, 678-681. [CrossRef]

57. Dudka, A.A.; Sweet, S.M.; Heath, J.K. Signal transducers and activators of transcription-3 binding to the fibroblast growth factor receptor is activated by receptor amplification. Cancer Res. 2010, 70, 3391-3401. [CrossRef]

58. Candi, E.; Rufini, A.; Terrinoni, A.; Giamboi-Miraglia, A.; Lena, A.M.; Mantovani, R.; Knight, R.; Melino, G. DeltaNp63 regulates thymic development through enhanced expression of FgfR2 and Jag2. Proc. Natl. Acad. Sci. USA 2007, 104, 11999-12004. [CrossRef]

59. Katoh, M.; Katoh, M. Cross-talk of WNT and FGF signaling pathways at GSK3beta to regulate beta-catenin and SNAIL signaling cascades. Cancer Biol. Ther. 2006, 5, 1059-1064. [CrossRef] 
60. Fogarty, M.P.; Emmenegger, B.A.; Grasfeder, L.L.; Oliver, T.G.; Wechsler-Reya, R.J. Fibroblast growth factor blocks Sonic hedgehog signaling in neuronal precursors and tumor cells. Proc. Natl. Acad. Sci. USA 2007, 104, 2973-2978. [CrossRef]

61. Dudley, A.T.; Godin, R.E.; Robertson, E.J. Interaction between FGF and BMP signaling pathways regulates development of metanephric mesenchyme. Genes Dev. 1999, 13, 1601-1613. [CrossRef]

62. Peters, K.G.; Werner, S.; Chen, G.; Williams, L.T. Two FGF receptor genes are differentially expressed in epithelial and mesenchymal tissues during limb formation and organogenesis in the mouse. Development 1992, 114, 233-243.

63. Orr-Urtreger, A.; Bedford, M.T.; Burakova, T.; Arman, E.; Zimmer, Y.; Yayon, A.; Givol, D.; Lonai, P. Developmental Localization of the Splicing Alternatives of Fibroblast Growth Factor Receptor-2 (FGFR2). Dev. Boil. 1993, 158, 475-486. [CrossRef]

64. Eswarakumar, V.P.; Monsonego-Ornan, E.; Pines, M.; Antonopoulou, I.; Morriss-Kay, G.M.; Lonai, P. The IIIc alternative of Fgfr2 is a positive regulator of bone formation. Development 2002, 129, 3783-3793.

65. Zhang, X.; Ibrahimi, O.A.; Olsen, S.K.; Umemori, H.; Mohammadi, M.; Ornitz, D.M. Receptor specificity of the fibroblast growth factor family. The complete mammalian FGF family. J. Boil. Chem. 2006, 281, 15694-15700. [CrossRef]

66. Ornitz, D.M.; Xu, J.; Colvin, J.S.; McEwen, D.G.; MacArthur, C.A.; Coulier, F.; Gao, G.; Goldfarb, M. Receptor specificity of the fibroblast growth factor family. J. Biol. Chem. 1996, 271, 15292-15297. [CrossRef]

67. Nyeng, P.; Norgaard, G.A.; Kobberup, S.; Jensen, J. FGF10 signaling controls stomach morphogenesis. Dev.Boil. 2007, 303, 295-310. [CrossRef]

68. Hattori, Y.; Odagiri, H.; Nakatani, H.; Miyagawa, K.; Naito, K.; Sakamoto, H.; Katoh, O.; Yoshida, T.; Sugimura, T.; Terada, M. K-sam, an amplified gene in stomach cancer, is a member of the heparin-binding growth factor receptor genes. Proc. Natl. Acad. Sci. USA 1990, 87, 5983-5987. [CrossRef]

69. Nakatani, H.; Sakamoto, H.; Yoshida, T.; Yokota, J.; Tahara, E.; Sugimura, T.; Terada, M. Isolation of an Amplified DNA Sequence in Stomach Cancer. Jpn. J. Cancer Res. 1990, 81, 707-710. [CrossRef]

70. Cristescu, R.; Lee, J.; Nebozhyn, M.; Kim, K.-M.; Ting, J.C.; Wong, S.S.; Liu, J.; Yue, Y.G.; Wang, J.; Yu, K.; et al. Molecular analysis of gastric cancer identifies subtypes associated with distinct clinical outcomes. Nat. Med. 2015, 21, 449-456. [CrossRef]

71. Deng, N.; Goh, L.K.; Wang, H.; Das, K.; Tao, J.; Tan, I.B.; Zhang, S.; Lee, M.; Wu, J.; Lim, K.H.; et al. A comprehensive survey of genomic alterations in gastric cancer reveals systematic patterns of molecular exclusivity and co-occurrence among distinct therapeutic targets. Gut 2012, 61, 673-684. [CrossRef]

72. Jung, E.-J.; Jung, E.-J.; Min, S.Y.; Kim, M.A.; Kim, W.H. Fibroblast growth factor receptor 2 gene amplification status and its clinicopathologic significance in gastric carcinoma. Hum. Pathol. 2012, 43, 1559-1566. [CrossRef]

73. Matsumoto, K.; Arao, T.; Hamaguchi, T.; Shimada, Y.; Kato, K.; Oda, I.; Taniguchi, H.; Koizumi, F.; Yanagihara, K.; Sasaki, H.; et al. FGFR2 gene amplification and clinicopathological features in gastric cancer. Br. J. Cancer 2012, 106, 727-732. [CrossRef]

74. Park, Y.S.; Na, Y.-S.; Ryu, M.-H.; Lee, C.-W.; Park, H.J.; Lee, J.-K.; Park, S.R.; Ryoo, B.-Y.; Kang, Y.-K. FGFR2 Assessment in Gastric Cancer Using Quantitative Real-Time Polymerase Chain Reaction, Fluorescent In Situ Hybridization, and Immunohistochemistry. Am. J. Clin. Pathol. 2015, 143, 865-872. [CrossRef]

75. Das, K.; Gunasegaran, B.; Tan, I.B.; Deng, N.; Lim, K.H.; Tan, P. Mutually exclusive FGFR2, HER2, and KRAS gene amplifications in gastric cancer revealed by multicolour FISH. Cancer Lett. 2014, 353, 167-175. [CrossRef]

76. Kuboki, Y.; Schatz, C.A.; Koechert, K.; Schubert, S.; Feng, J.; Wittemer-Rump, S.; Ziegelbauer, K.; Krahn, T.; Nagatsuma, A.K.; Ochiai, A. In situ analysis of FGFR2 mRNA and comparison with FGFR2 gene copy number by dual-color in situ hybridization in a large cohort of gastric cancer patients. Gastric Cancer 2018, 21, 401-412. [CrossRef]

77. Hosoda, K.; Yamashita, K.; Ushiku, H.; Ema, A.; Moriya, H.; Mieno, H.; Washio, M.; Watanabe, M. Prognostic relevance of FGFR2 expression in stage II/III gastric cancer with curative resection and S-1 chemotherapy. Oncol. Lett. 2018, 15, 1853-1860.

78. Van Cutsem, E.; Bang, Y.J.; Mansoor, W.; Petty, R.D.; Chao, Y.; Cunningham, D.; Ferry, D.R.; Smith, N.R.; Frewer, P.; Ratnayake, J.; et al. A randomized, open-label study of the efficacy and safety of AZD4547 monotherapy versus paclitaxel for the treatment of advanced gastric adenocarcinoma with FGFR2 polysomy or gene amplification. Ann. Oncol. 2017, 28, 1316-1324. [CrossRef] 
79. Nakazawa, K.; Yashiro, M.; Hirakawa, K. Keratinocyte growth factor produced by gastric fibroblasts specifically stimulates proliferation of cancer cells from scirrhous gastric carcinoma. Cancer Res. 2003, 63, 8848-8852.

80. Huang, T.; Wang, L.; Liu, D.; Li, P.; Xiong, H.; Zhuang, L.; Sun, L.; Yuan, X.; Qiu, H. FGF7/FGFR2 signal promotes invasion and migration in human gastric cancer through upregulation of thrombospondin-1. Int. J. Oncol. 2017, 50, 1501-1512. [CrossRef]

81. Ren, C.; Chen, H.; Han, C.; Fu, D.; Wang, F.; Wang, D.; Ma, L.; Zhou, L.; Han, D. The anti-apoptotic and prognostic value of fibroblast growth factor 9 in gastric cancer. Oncotarget 2016, 7, 36655-36665. [CrossRef]

82. Ooi, A.; Oyama, T.; Nakamura, R.; Tajiri, R.; Ikeda, H.; Fushida, S.; Nakamura, H.; Dobashi, Y. Semi-comprehensive analysis of gene amplification in gastric cancers using multiplex ligation-dependent probe amplification and fluorescence in situ hybridization. Mod. Pathol. 2015, 28, 861-871. [CrossRef]

83. Cerami, E.; Gao, J.; Dogrusoz, U.; Gross, B.E.; Sumer, S.O.; Aksoy, B.A.; Jacobsen, A.; Byrne, C.J.; Heuer, M.L.; Larsson, E.; et al. The cBio cancer genomics portal: An open platform for exploring multidimensional cancer genomics data. Cancer Discov. 2012, 2, 401-404. [CrossRef]

84. Sun, Q.; Lin, P.; Zhang, J.; Li, X.; Yang, L.; Huang, J.; Zhou, Z.; Liu, P.; Liu, N. Expression of Fibroblast Growth Factor 10 Is Correlated with Poor Prognosis in Gastric Adenocarcinoma. Tohoku J. Exp. Med. 2015, 236, 311-318. [CrossRef]

85. Zhang, J.; Zhou, Y.; Huang, T.; Wu, F.; Pan, Y.; Dong, Y.; Wang, Y.; Chan, A.K.Y.; Liu, L.; Kwan, J.S.H.; et al. FGF18, a prominent player in FGF signaling, promotes gastric tumorigenesis through autocrine manner and is negatively regulated by miR-590-5p. Oncogene 2019, 38, 33-46. [CrossRef]

86. Chang, J.; Wang, S.; Zhang, Z.; Liu, X.; Wu, Z.; Geng, R.; Ge, X.; Dai, C.; Liu, R.; Zhang, Q.; et al. Multiple receptor tyrosine kinase activation attenuates therapeutic efficacy of the fibroblast growth factor receptor 2 inhibitor AZD4547 in FGFR2 amplified gastric cancer. Oncotarget 2015, 6, 2009-2022. [CrossRef]

87. Liu, Y.J.; Shen, D.; Yin, X.; Gavine, P.; Zhang, T.; Su, X.; Zhan, P.; Xu, Y.; Lv, J.; Qian, J.; et al. HER2, MET and FGFR2 oncogenic driver alterations define distinct molecular segments for targeted therapies in gastric carcinoma. Br. J. Cancer 2014, 110, 1169-1178. [CrossRef]

88. Lau, W.M.; Teng, E.; Huang, K.K.; Tan, J.W.; Das, K.; Zang, Z.; Chia, T.; The, M.; Kono, K.; Yong, W.P.; et al. Acquired Resistance to FGFR Inhibitor in Diffuse-Type Gastric Cancer through an AKT-Independent PKC-Mediated Phosphorylation of GSK3beta. Mol. Cancer Ther. 2018, 17, 232-242. [CrossRef]

89. Huang, T.; Liu, D.; Wang, Y.; Li, P.; Sun, L.; Xiong, H.; Dai, Y.; Zou, M.; Yuan, X.; Qiu, H. FGFR2 Promotes Gastric Cancer Progression by Inhibiting the Expression of Thrombospondin4 via PI3K-Akt-Mtor Pathway. Cell. Physiol. Biochem. 2018, 50, 1332-1345. [CrossRef]

90. Zhu, D.-Y.; Guo, Q.-S.; Li, Y.-L.; Cui, B.; Guo, J.; Liu, J.-X.; Li, P. Twist1 correlates with poor differentiation and progression in gastric adenocarcinoma via elevation of FGFR2 expression. World J. Gastroenterol. 2014, 20, 18306-18315. [CrossRef]

91. Grygielewicz, P.; Dymek, B.; Bujak, A.; Gunerka, P.; Stanczak, A.; Lamparska-Przybysz, M.; Wieczorek, M.; Dzwonek, K.; Zdzalik, D. Epithelial-mesenchymal transition confers resistance to selective FGFR inhibitors in SNU-16 gastric cancer cells. Gastric Cancer 2016, 19, 53-62. [CrossRef]

92. Hallinan, N.; Finn, S.; Cuffe, S.; Rafee, S.; O’Byrne, K.; Gately, K. Targeting the fibroblast growth factor receptor family in cancer. Cancer Treat. Rev. 2016, 46, 51-62. [CrossRef]

93. Hui, Q.; Jin, Z.; Li, X.; Liu, C.; Wang, X. FGF Family: From Drug Development to Clinical Application. Int. J. Mol. Sci. 2018, 19, 1875. [CrossRef]

94. Zhao, W.-M.; Wang, L.; Park, H.; Chhim, S.; Tanphanich, M.; Yashiro, M.; Kim, K.J. Monoclonal Antibodies to Fibroblast Growth Factor Receptor 2 Effectively Inhibit Growth of Gastric Tumor Xenografts. Clin. Cancer Res. 2010, 16, 5750-5758. [CrossRef]

95. Sommer, A.; Kopitz, C.; Schatz, C.A.; Nising, C.F.; Mahlert, C.; Lerchen, H.G.; Stelte-Ludwig, B.; Hammer, S.; Greven, S.; Schuhmacher, J.; et al. Preclinical Efficacy of the Auristatin-Based Antibody-Drug Conjugate BAY 1187982 for the Treatment of FGFR2-Positive Solid Tumors. Cancer Res. 2016, 76, 6331-6339. [CrossRef]

96. Katoh, M. Therapeutics Targeting FGF Signaling Network in Human Diseases. Trends Pharmacol. Sci. 2016, 37, 1081-1096. [CrossRef]

97. Ronca, R.; Giacomini, A.; Di Salle, E.; Coltrini, D.; Pagano, K.; Ragona, L.; Matarazzo, S.; Rezzola, S.; Maiolo, D.; Torella, R.; et al. Long-Pentraxin 3 Derivative as a Small-Molecule FGF Trap for Cancer Therapy. Cancer Cell 2015, 28, 225-239. [CrossRef] 
98. Pierce, K.L.; Deshpande, A.M.; Stohr, B.A.; Gemo, A.T.; Patil, N.S.; Brennan, T.J.; Bellovin, D.I.; Palencia, S.; Giese, T.; Huang, C.; et al. FPA144, a humanized monoclonal antibody for both FGFR2-amplified and nonamplified, FGFR2b-overexpressing gastric cancer patients. J. Clin. Oncol. 2014, 32, e15074. [CrossRef]

99. Jiang, X.F.; Dai, Y.; Peng, X.; Shen, Y.Y.; Su, Y.; Wei, M.M.; Liu, W.R.; Ding, Z.B.; Zhang, A.; Shi, Y.H.; et al. SOMCL-085, a novel multi-targeted FGFR inhibitor, displays potent anticancer activity in FGFR-addicted human cancer models. Acta Pharmacol. Sin. 2018, 39, 243-250. [CrossRef]

100. Hilberg, F.; Tontsch-Grunt, U.; Baum, A.; Le, A.T.; Doebele, R.C.; Lieb, S.; Gianni, D.; Voss, T.; Garin-Chesa, P.; Haslinger, C.; et al. Triple Angiokinase Inhibitor Nintedanib Directly Inhibits Tumor Cell Growth and Induces Tumor Shrinkage via Blocking Oncogenic Receptor Tyrosine Kinases. J. Pharmacol. Exp. Ther. 2018, 364, 494-503. [CrossRef]

101. Cha, Y.; Kim, H.-P.; Lim, Y.; Han, S.-W.; Song, S.-H.; Kim, T.-Y. FGFR2 amplification is predictive of sensitivity to regorafenib in gastric and colorectal cancers in vitro. Mol. Oncol. 2018, 12, 993-1003. [CrossRef]

102. Burbridge, M.F.; Bossard, C.J.; Saunier, C.; Fejes, I.; Bruno, A.; Leonce, S.; Ferry, G.; Da Violante, G.; Bouzom, F.; Cattan, V.; et al. S49076 is a novel kinase inhibitor of MET, AXL, and FGFR with strong preclinical activity alone and in association with bevacizumab. Mol. Cancer Ther. 2013, 12, 1749-1762. [CrossRef]

103. Gozgit, J.M.; Wong, M.J.; Moran, L.; Wardwell, S.; Mohemmad, Q.K.; Narasimhan, N.I.; Shakespeare, W.C.; Wang, F.; Clackson, T.; Rivera, V.M. Ponatinib (AP24534), a multitargeted pan-FGFR inhibitor with activity in multiple FGFR-amplified or mutated cancer models. Mol. Cancer Ther. 2012, 11, 690-699. [CrossRef]

104. Jang, J.; Kim, H.K.; Bang, H.; Kim, S.T.; Kim, S.Y.; Park, S.H.; Lim, H.Y.; Kang, W.K.; Lee, J.; Kim, K.-M. Antitumor Effect of AZD4547 in a Fibroblast Growth Factor Receptor 2-Amplified Gastric Cancer Patient-Derived Cell Model1. Transl. Oncol. 2017, 10, 469-475. [CrossRef]

105. Michael, M.; Bang, Y.J.; Park, Y.S.; Kang, Y.K.; Kim, T.M.; Hamid, O.; Thornton, D.; Tate, S.C.; Raddad, E.; Tie, J. A Phase 1 Study of LY2874455, an Oral Selective pan-FGFR Inhibitor, in Patients with Advanced Cancer. Target. Oncol. 2017, 12, 463-474. [CrossRef]

106. Kim, S.Y.; Ahn, T.; Bang, H.; Ham, J.S.; Kim, J.; Kim, S.T.; Jang, J.; Shim, M.; Kang, S.Y.; Park, S.H.; et al. Acquired resistance to LY2874455 in FGFR2-amplified gastric cancer through an emergence of novel FGFR2-ACSL5 fusion. Oncotarget 2017, 8, 15014-15022. [CrossRef]

107. Brameld, K.A.; Owens, T.D.; Verner, E.; Venetsanakos, E.; Bradshaw, J.M.; Phan, V.T.; Tam, D.; Leung, K.; Shu, J.; LaStant, J.; et al. Discovery of the Irreversible Covalent FGFR Inhibitor 8-(3-(4-Acryloylpiperazin-1-yl) propyl)-6-(2,6-dichloro-3,5-dimethoxyphenyl)-2-(me thylamino) pyrido [2,3-d] pyrimidin-7 (8 H)-one (PRN1371) for the Treatment of Solid Tumors. J. Med. Chem. 2017, 60, 6516-6527. [CrossRef]

108. Tsimafeyeu, I.; Daeyaert, F.; Joos, J.B.; Aken, K.V.; Ludes-Meyers, J.; Byakhov, M.; Tjulandin, S. Molecular Modeling, de novo Design and Synthesis of a Novel, Extracellular Binding Fibroblast Growth Factor Receptor 2 Inhibitor Alofanib (RPT835). Med. Chem. 2016, 12, 303-317. [CrossRef]

109. Hall, T.G.; Yu, Y.; Eathiraj, S.; Wang, Y.; Savage, R.E.; Lapierre, J.M.; Schwartz, B.; Abbadessa, G. Preclinical Activity of ARQ 087, a Novel Inhibitor Targeting FGFR Dysregulation. PLoS ONE 2016, 11, e0162594. [CrossRef]

110. Schmidt, K.; Moser, C.; Hellerbrand, C.; Zieker, D.; Wagner, C.; Redekopf, J.; Schlitt, H.J.; Geissler, E.K.; Lang, S.A. Targeting Fibroblast Growth Factor Receptor (FGFR) with BGJ398 in a Gastric Cancer Model. Anticancer Res. 2015, 35, 6655-6665.

111. Inokuchi, M.; Murase, H.; Otsuki, S.; Kawano, T.; Kojima, K. Different clinical significance of FGFR1-4 expression between diffuse-type and intestinal-type gastric cancer. World J. Surg. Oncol. 2017, 15, 2. [CrossRef]

112. Pearson, A.; Smyth, E.; Babina, I.S.; Herrera-Abreu, M.T.; Tarazona, N.; Peckitt, C.; Kilgour, E.; Smith, N.R.; Geh, C.; Rooney, C.; et al. High-Level Clonal FGFR Amplification and Response to FGFR Inhibition in a Translational Clinical Trial. Cancer Discov. 2016, 6, 838-851. [CrossRef]

113. Hierro, C.; Alsina, M.; Sanchez, M.; Serra, V.; Rodon, J.; Tabernero, J. Targeting the fibroblast growth factor receptor 2 in gastric cancer: Promise or pitfall? Ann. Oncol. 2017, 28, 1207-1216. [CrossRef]

(C) 2019 by the authors. Licensee MDPI, Basel, Switzerland. This article is an open access article distributed under the terms and conditions of the Creative Commons Attribution (CC BY) license (http://creativecommons.org/licenses/by/4.0/). 


\title{
Fibroblast Growth Factor Receptors (FGFRs): Structures and Small Molecule Inhibitors
}

\author{
Shuyan Dai ${ }^{1}$, Zhan Zhou ${ }^{1}$, Zhuchu Chen ${ }^{1}$, Guangyu $\mathrm{Xu}^{2, *}$ and Yongheng Chen ${ }^{1, *}$ \\ 1 NHC Key Laboratory of Cancer Proteomics \& Laboratory of Structural Biology, Xiangya Hospital, Central \\ South University, Changsha 410008, Hunan, China; syandai@hotmail.com (S.D.); \\ zhouzhan285@163.com (Z.Z.); chenzhuchu@126.com (Z.C.) \\ 2 Key Laboratory of Chemical Biology and Traditional Chinese Medicine Research (Ministry of Education), \\ College of Chemistry and Chemical Engineering, Hunan Normal University, \\ Changsha 410081, Hunan, China \\ * Correspondence: gyxu@hunnu.edu.cn (G.X.); yonghenc@163.com (Y.C.); \\ Tel.: +731-88872530 (G.X.); +86-731-84327542 (Y.C.)
}

Received: 15 May 2019; Accepted: 12 June 2019; Published: 18 June 2019

\begin{abstract}
Fibroblast growth factor receptors (FGFRs) are a family of receptor tyrosine kinases expressed on the cell membrane that play crucial roles in both developmental and adult cells. Dysregulation of FGFRs has been implicated in a wide variety of cancers, such as urothelial carcinoma, hepatocellular carcinoma, ovarian cancer and lung adenocarcinoma. Due to their functional importance, FGFRs have been considered as promising drug targets for the therapy of various cancers. Multiple small molecule inhibitors targeting this family of kinases have been developed, and some of them are in clinical trials. Furthermore, the pan-FGFR inhibitor erdafitinib (JNJ-42756493) has recently been approved by the U.S. Food and Drug Administration (FDA) for the treatment of metastatic or unresectable urothelial carcinoma (mUC). This review summarizes the structure of FGFR, especially its kinase domain, and the development of small molecule FGFR inhibitors.
\end{abstract}

Keywords: fibroblast growth factor receptors; structure; kinase inhibitor; targeted therapy

\section{Introduction}

The human fibroblast growth factor receptor (FGFR) family consists of four members: FGFR1 to FGFR4. Despite being encoded by separate genes, the four members share high homology, with their sequence identity varying from $56 \%$ to $71 \%$ [1]. Similar to other receptor tyrosine kinases (RTKs), FGFRs are expressed on the cell membrane and can be stimulated and activated by extracellular signals. The native ligand of FGFRs is fibroblast growth factors (FGFs) [2-4]. The binding of FGFs drives the dimerization of FGFRs; subsequently, a transautophosphorylation event of the intracellular kinase domain is induced, followed by the activation of downstream transduction pathways [5,6]. Through triggering downstream signaling pathways, FGFRs participate in various vital physiological processes, such as proliferation, differentiation, cell migration and survival [7-9].

Aberrant expression of FGFRs has been shown in various kinds of solid tumors, and moreover, the aberrancy is considered an oncogenic signaling pathway [10-12]. It is believed that small molecules that competitively bind to the adenosine triphosphate (ATP) pocket of aberrant FGFRs while exhibiting little or no toxicity provide limitless prospects for the treatment of relevant tumors. The structure of FGFRs, especially the kinase domain, and the design of small molecular inhibitors have attracted intensive study in the past two decades. Multiple small molecule inhibitors have been developed, and some of them are currently being used in clinical trials, such as FGF401, which targets FGFR4 for the treatment of hepatocarcinoma (HCC) [13]; AZD4547, which targets FGFR1-3 for the treatment of a variety of tumors [14]. Moreover, erdafitinib (JNJ-42756493) [15] has been approved recently by U.S. 
Food and Drug Administration (FDA) for the treatment of mUC. More than 20 FGFR kinase/inhibitor complex structures have been determined to-date, and these structures have yielded extensive insights into the understanding of inactivation of FGFRs for related disease therapy.

\section{Organization of FGFR}

FGFRs share a canonical RTK architecture. From the N- to the C-terminus, all four FGFR members contain a large extracellular ligand-binding domain that comprises three immunoglobulin (Ig)-like subunits (D1, D2 and D3) followed by a single transmembrane helix and an intracellular tyrosine kinase domain [1,16] (Figure 1A). The linker region between D1 and D2 contains a highly conserved motif that is rich in aspartate acids, called the acid box [17]. The detailed function of those structural units will be further introduced below.

A

trans-membrane helix

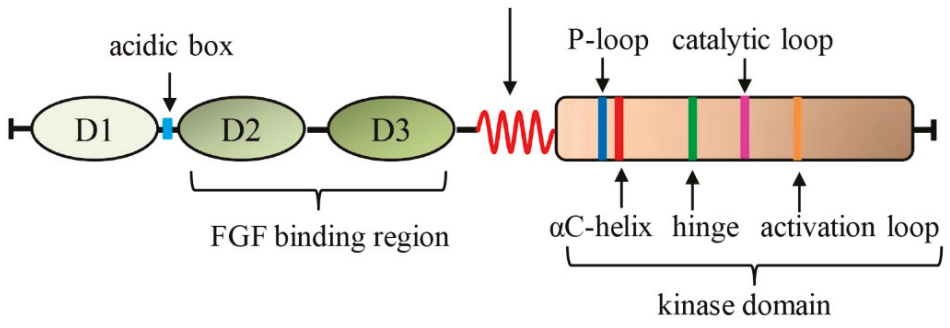

B
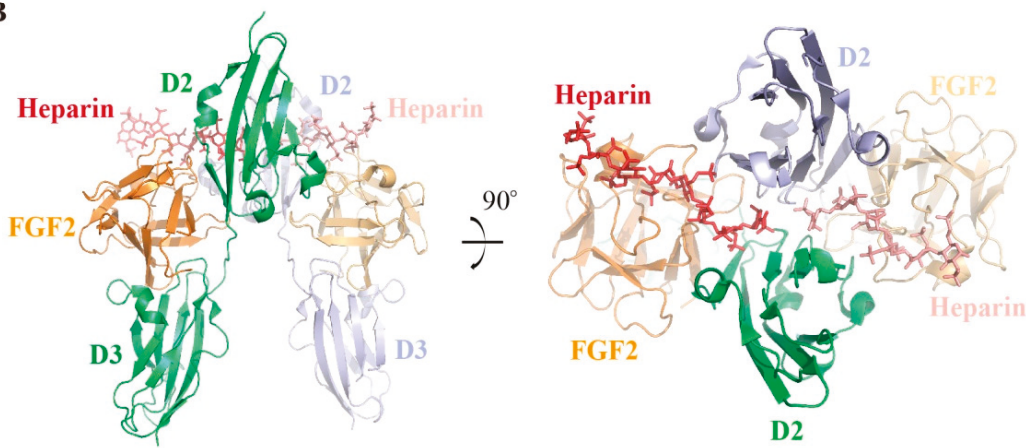

Figure 1. Schematic diagram of FGFRs and the structure of the FGFR extracellular domain. (A) Organization of FGFRs. Important functional elements are highlighted. (B) Crystal structure of the FGF2:FGFR1:heparin ternary complex (PDB ID 1FQ9). The two copies of FGFR1 molecules are colored in green and light blue respectively. Heparin molecules are shown in red stick representation; FGF2 (colored in orange) and FGFR1 are shown in cartoon representation.

FGFs are the native ligand for this family of kinases. Through its extracellular domain, FGFR recognizes and is stimulated by specific FGFs. The FGF binding pocket is formed by the D2 and D3 subregions [18]. There have been contradicting views regarding the stoichiometry of the FGF/FGFR complex. Schlessinger, J. et al. solved the ternary complex structure of FGF2/FGFR1/heparin [19]. With the help of heparin, FGFR1 was dimerized after the binding of FGF2 to form the complex at a symmetric 2:2:2 stoichiometry ratio; both the FGF2 and heparin molecules simultaneously contacted the two FGFR1 monomers (Figure 1B). In the FGF1/FGFR2/heparin crystal structure solved by Pellegrini et al., the complex was assembled by asymmetric 2:2:1 stoichiometry [20]. By utilizing nuclear magnetic resonance, Saxena et al. studied the interactions of FGF1(FGF2)/FGFR4/HM (HM: heparin mimetics) 
complex, and their results supported the formation of the symmetric mode of FGF/FGFR dimerization in solution [21]. Interestingly, although all FGFs have a heparin sulfate binding site on their surface [22,23], endocrine FGFs such as FGF21 and FGF23 show a lower binding affinity to heparin sulfate [23] and require Klotho coreceptors instead to act as cofactors for FGFR activation [24-26].

In addition to acting as the ligand sensor, the extracellular domain also undertakes an autoinhibitory role, which relies on regulation by D1 and the acid box $[27,28]$. Several studies have proposed that the acid box could competitively bind to the heparin binding site of D2 to suppress heparin binding, while D1 forms intramolecular contacts with D2-D3, thus blocking the binding of FGFs [28-30]. Nevertheless, the mechanisms of autoinhibition need to be further clarified.

\section{Structure of FGFR Kinase Domain}

The intracellular tyrosine kinase domain is the most well studied region of the FGFR protein. This domain exhibits the canonical bilobed architecture of protein kinases [31-35]. The fold of the N-terminal small lobe (N-lobe, 100 amino acid residues) consists of a five-stranded antiparallel $\beta$-sheet ( $\beta 1-\beta 5)$ and the $\alpha \mathrm{C}$-helix, an important regulatory element. The C-terminal large lobe (C-lobe, $\sim 200$ amino acid residues) predominately comprises seven a helices $(\alpha \mathrm{D}, \alpha \mathrm{E}, \alpha \mathrm{EF}, \alpha \mathrm{F}-\alpha \mathrm{I})$ (Figure 2A). The active site, which is responsible for ATP and substrate protein binding, is located in a clef between the two lobes (Figure 2B).

A

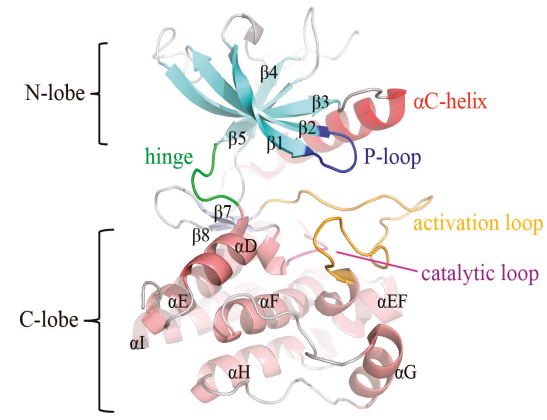

C

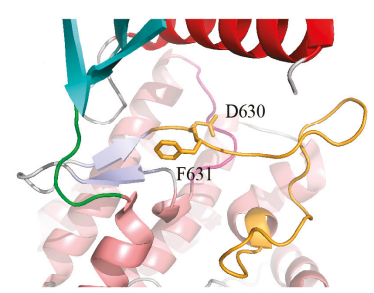

B

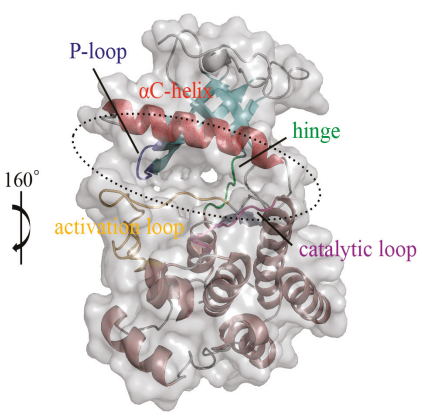

D

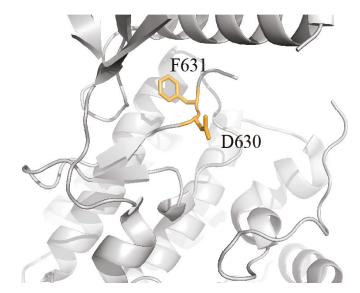

Figure 2. Structure of the FGFR kinase domain. (A) Overall crystal structure of FGFR4 in cartoon representation. The five $\beta$-sheets of the $\mathrm{N}$-lobe are labeled in cyan, and the helixes of the $\mathrm{C}$-lobe are colored in salmon. The $\alpha \mathrm{C}$ helix (red), P-loop (blue), catalytic loop (magenta), activation loop (bright orange) and hinge (green) are highlighted. (B) Surface presentation of FGFR4. The ATP binding pocket located between the N- and C-lobe is indicated by the dashed circle. (C) DFG-out conformation of the FGFR4 activation loop. The side chains of D630 and F631 are shown in stick representation. (D) DFG-in status of the FGFR4 activation loop. (A-C) were prepared from PDB ID 4UXQ; (D) was prepared from PDB ID 5JKG. 
The C-lobe folds tightly with the $\alpha$ F-helix to form a hydrophobic core, around which the other secondary segments are packed. In addition to the primary helixes, the C-lobe contains a short helix located between the activation loop (A-loop) and the $\alpha$ F-helix named the $\alpha$ EF-helix, which is conserved among all FGFR members as well as other protein kinases [36]. Two short $\beta$-strands ( $\beta 7$ and $\beta 8$ ) (Figure 2A) between the catalytic loop and activation loop (introduced below) interact with each other and are believed to participate in the regulation of FGFR activation [37]. In contrast to the C-lobe, the N-lobe folds in a more flexible manner, which benefits the binding and release of ATP/ADP and substrates.

There are several functionally important loops in both lobes. The loop between $\beta 8$ and the $\alpha E F-h e l i x$ is an activation loop (A-loop), which is essential for kinase activation [38-40]. The conformation of the highly conserved Asp-Phe-Gly motif (DFG-motif) in the A-loop is an indicator of kinase activity status [39,41]. Generally, the DFG-motif exists in two states: the active DFG-in and inactive DFG-out conformations [42,43] (Figure 2C,D). In the DFG-in state, the aspartate residue of the DFG-motif plays an essential role in ATP binding through the coordination of all three phosphate groups of ATP, either directly or via magnesium ions, while these interactions are sterically impossible when the motif is flipped into the DFG-out conformation. Phosphorylation is catalyzed by the conserved aspartate of the His-Arg-Asp (HRD) motif in the catalytic ( $\alpha \mathrm{E}-\beta 7$ ) loop [44]. The glycine rich P-loop (also called the nucleotide binding loop), located between the $\beta 1$ - and $\beta 2$-strands, folds over to enclose ATP for phosphotransfer [45]. The so-called molecular brake located at the hinge region that connects the $\mathrm{N}$ and C-lobes plays a critical role in the regulation of autoinhibition and activation [46].

The catalytic activity of the kinase domain is precisely controlled. There are two general conformations for all protein kinases, including those of the FGFR family. Activation typically involves changes in the orientation of the $\alpha \mathrm{C}$-helix in the small lobe and the activation loop in the C-lobe. During the catalytic cycle, the active kinase toggles between open and closed conformations. In the open form, the kinase binds MgATP and the protein substrate, while during catalysis, the kinase adopts the closed form. Once catalysis is completed, the MgADP and phosphorylated substrate are released, and the enzyme recovers to the open conformation, preparing for the next catalytic cycle $[16,47]$.

\section{Characteristics of FGFR/Inhibitor Interaction}

As noted above, aberrantly expressed FGFRs have been implicated in various tumors. Therefore, extensive work has been performed on the development of FGFR inhibitors. The inhibitors that are in clinical trials or approved by the FDA for clinical use are summarized in Table 1, and the chemical structures of those inhibitors are shown in Figure 3.

Table 1. FGFR inhibitors that are in clinical trials or approved by the FDA.

\begin{tabular}{|c|c|c|c|c|c|}
\hline $\begin{array}{l}\text { Inhibitor } \\
\text { Name }\end{array}$ & $\begin{array}{l}\text { Binding } \\
\text { Features }\end{array}$ & IC50 (nM) & PDB ID & $\begin{array}{l}\text { Clinical Trial } \\
\text { Phase/Number }\end{array}$ & Reference \\
\hline $\begin{array}{c}\text { JNJ-42756493 } \\
\text { (Erdafitinib) }\end{array}$ & $\begin{array}{c}\text { Pan-FGFR } \\
\text { Reversible } \\
\text { Type I }\end{array}$ & $\begin{array}{l}\text { FGFR1: } 1.2 \\
\text { FGFR2: } 2.5 \\
\text { FGFR3: } 3.0 \\
\text { FGFR4: } 5.7\end{array}$ & $\mathrm{n} / \mathrm{a}$ & FDA approved & [15] \\
\hline AZD4547 & $\begin{array}{c}\text { Pan-FGFR } \\
\text { Reversible } \\
\text { Type I }\end{array}$ & $\begin{array}{l}\text { FGFR1: } 0.2 \\
\text { FGFR2: } 2.5 \\
\text { FGFR3: } 1.8 \\
\text { FGFR4: } 165\end{array}$ & 4V05 & $\begin{array}{c}\text { Phase I/II } \\
\text { NCT02824133 }\end{array}$ & {$[14,34]$} \\
\hline Ly2874455 & $\begin{array}{c}\text { Pan-FGFR } \\
\text { Reversible } \\
\text { Type I }\end{array}$ & $\begin{array}{c}\text { FGFR1: } 2.8 \\
\text { FGFR2: } 2.6 \\
\text { FGFR3: } 6.4 \\
\text { FGFR4: } 6\end{array}$ & $5 \mathrm{JKG}$ & $\begin{array}{c}\text { Phase I } \\
\text { NCT01212107 }\end{array}$ & {$[33,48]$} \\
\hline
\end{tabular}


Table 1. Cont.

\begin{tabular}{|c|c|c|c|c|c|}
\hline $\begin{array}{l}\text { Inhibitor } \\
\text { Name }\end{array}$ & $\begin{array}{l}\text { Binding } \\
\text { Features }\end{array}$ & IC50 (nM) & PDB ID & $\begin{array}{l}\text { Clinical Trial } \\
\text { Phase/Number }\end{array}$ & Reference \\
\hline CH5183284 & $\begin{array}{c}\text { Pan-FGFR } \\
\text { Reversible } \\
\text { Type I }\end{array}$ & $\begin{array}{l}\text { FGFR1: } 9.3 \\
\text { FGFR2: } 7.6 \\
\text { FGFR3: } 22 \\
\text { FGFR4: } 290\end{array}$ & $5 \mathrm{~N} 7 \mathrm{~V}$ & $\begin{array}{c}\text { Phase II/III } \\
\text { NCT03344536 }\end{array}$ & [49] \\
\hline NVP-BGJ398 & $\begin{array}{c}\text { Pan-FGFR } \\
\text { Reversible } \\
\text { Type I }\end{array}$ & $\begin{array}{c}\text { FGFR1: } 0.9 \\
\text { FGFR2: } 1.4 \\
\text { FGFR3: } 1 \\
\text { FGFR4: } 60\end{array}$ & 3ТT0 & $\begin{array}{c}\text { Phase II } \\
\text { NCT02706691 }\end{array}$ & [50] \\
\hline INCB054828 & $\begin{array}{c}\text { Pan-FGFR } \\
\text { Reversible } \\
\text { Type I }\end{array}$ & $\begin{array}{l}\text { FGFR1: } 0.4 \\
\text { FGFR2: } 0.5 \\
\text { FGFR3: } 1.2 \\
\text { FGFR4: } 30\end{array}$ & $\mathrm{n} / \mathrm{a}$ & $\begin{array}{c}\text { Phase II } \\
\text { NCT03011372 }\end{array}$ & [51] \\
\hline Rogaratinib & $\begin{array}{c}\text { Pan-FGFR } \\
\text { Reversible } \\
\text { Type I }\end{array}$ & $\begin{array}{c}\text { FGFR1: } \\
\text { 12-15 } \\
\text { FGFR2: <1 } \\
\text { FGFR3: } 19 \\
\text { FGFR4: } 33\end{array}$ & $\mathrm{n} / \mathrm{a}$ & $\begin{array}{c}\text { Phase II/III } \\
\text { NCT03410693 }\end{array}$ & [52] \\
\hline PRN1371 & $\begin{array}{c}\text { Pan-FGFR } \\
\text { Irreversible } \\
\text { Type I }\end{array}$ & $\begin{array}{l}\text { FGFR1: } 0.6 \\
\text { FGFR2: } 1.3 \\
\text { FGFR3: } 4.1 \\
\text { FGFR4: } 19.3\end{array}$ & $\mathrm{n} / \mathrm{a}$ & $\begin{array}{c}\text { Phase I } \\
\text { NCT02608125 }\end{array}$ & [53] \\
\hline TAS-120 & $\begin{array}{c}\text { Pan-FGFR } \\
\text { Irreversible } \\
\text { Type I }\end{array}$ & $\begin{array}{l}\text { FGFR1: } 3.9 \\
\text { FGFR2: } 1.3 \\
\text { FGFR3: } 1.6 \\
\text { FGFR4: } 8.3\end{array}$ & $6 \mathrm{M} 2 \mathrm{Q}$ & $\begin{array}{c}\text { Phase I/II } \\
\text { NCT02052778 }\end{array}$ & [54] \\
\hline BLU-554 & $\begin{array}{c}\text { FGFR4 } \\
\text { selective } \\
\text { Irreversible, } \\
\text { Type I }\end{array}$ & $\begin{array}{c}\text { FGFR1: } 624 \\
\text { FGFR2: } \\
1202 \\
\text { FGFR3: } \\
\text { 2203 } \\
\text { FGFR4: } 5\end{array}$ & $\mathrm{n} / \mathrm{a}$ & $\begin{array}{c}\text { Phase I } \\
\text { NCT02508467 }\end{array}$ & [55] \\
\hline Н3В-6527 & $\begin{array}{c}\text { FGFR4 } \\
\text { selective } \\
\text { Irreversible, } \\
\text { Type I }\end{array}$ & $\begin{array}{c}\text { FGFR1: } 320 \\
\text { FGFR2: } \\
1290 \\
\text { FGFR3: } \\
1060 \\
\text { FGFR4: } \\
<1.2\end{array}$ & $5 \mathrm{VND}$ & $\begin{array}{c}\text { Phase I } \\
\text { NCT02834780 }\end{array}$ & [56] \\
\hline FGF401 & $\begin{array}{c}\text { FGFR4 } \\
\text { selective } \\
\text { Reversible } \\
\text { Covalent, } \\
\text { Type I }\end{array}$ & $\begin{array}{l}\text { FGFR1-3: } \\
\text { >10,000 } \\
\text { FGFR4: } 1.1\end{array}$ & $6 \mathrm{JPJ}$ & $\begin{array}{c}\text { Phase I/II } \\
\text { NCT02325739 }\end{array}$ & {$[13,57]$} \\
\hline
\end{tabular}

FGFR inhibitors can generally be divided into two groups according to their binding behaviors, namely, type I and type II inhibitors [58,59]. Type I inhibitors bind FGFRs in the DFG-in enzymatic active conformation in an ATP-competitive manner, while the binding of type II requires the DFG-motif to be flipped to the DFG-out state [60,61]. The X-ray crystallographic structures of AZD4547 (PDB ID 4V05) [34] and PD173074 (PDB ID 2FGI) [62] bound to FGFR1 demonstrate that these two inhibitors are type I inhibitors. Taking the FGFR1/AZD4547 structure as an example, the AZD4547 occupying the 
ATP pocket of FGFR1 forms a hydrogen bond with the backbone nitrogen atom of the DFG aspartate (Asp641) and forms three hydrogen bonds with the hinge residues [34] (Figure 4A). In contrast, both the DFG-motifs of FGFR4 and FGFR1 are flipped out into an inactive conformation in the FGFR4/ponatinib (PDB ID 4UXQ) and FGFR1/ponatinib (PDB ID 4V04) structures [34]. In addition to the basal interactions, a hydrogen bond formed between ponatinib and the side chain of the strictly conserved glutamate from the $\alpha$ C-helix (Glu520 in FGFR4 and Glu531 in FGFR1) was also observed, which is characteristic of a type II inhibitor [61] (Figure 4B). Thus, these structures reveal ponatinib to be a type II inhibitor for FGFRs. As a consequence, the flip of the phenylalanine side chain breaks the regulatory spine and creates an additional induced-fit hydrophobic pocket that allows deeper binding of the inhibitor and provides better selectivity [34,43] as well as slower dissociation kinetics [34,63].

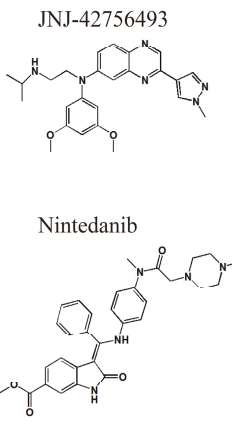

CH5183284
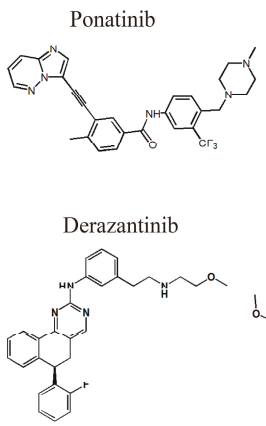

BGJ398

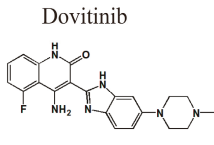

AZD4547

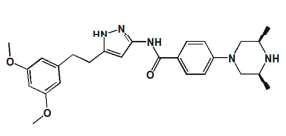

Lucitanib

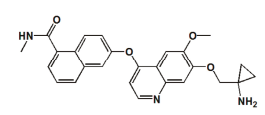

Ly2874455

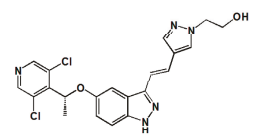

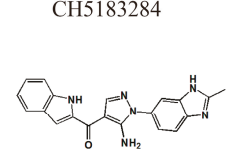
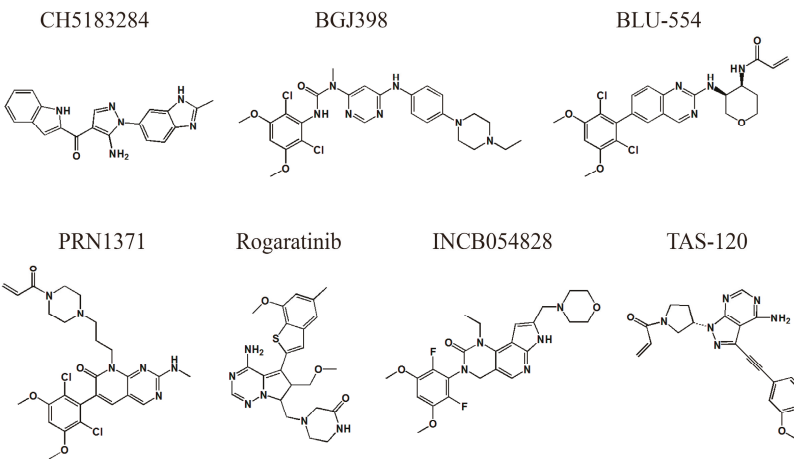

Rogaratinib

INCB054828

TAS-120
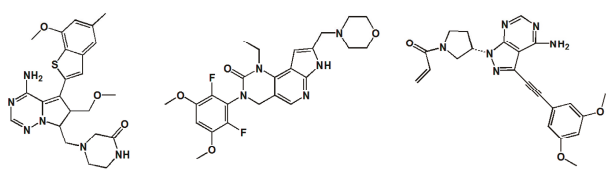

FGF401

H3B-6527

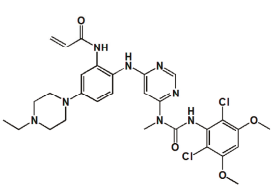

Figure 3. Chemical structure of FGFR small molecule inhibitors.

The interaction between a small molecule inhibitor and protein kinase can be covalent (irreversible) or noncovalent (reversible) [64,65]. Typically, covalent inhibitors have a functional group known as the warhead, which can improve binding affinity and selectivity through covalent interaction with a certain residue of the target kinase $[65,66]$. Moreover, a well-designed warhead could provide better performance against drug resistance than reversible inhibitors $[67,68]$. The reported covalent reactive residues in protein kinases include cysteine [69], aspartic acid [70], lysine [71] and others [72]. For FGFRs, the conserved cysteine of the P-loop (C488 in FGFR1, C491 in FGFR2, C482 in FGFR3 and C477 in FGFR4) and the unique C552 in FGFR4 from the hinge region are the covalent binding sites. The FGFR4/FIIN-2 complex structure (PDB accession number 4QQC) is the first solved irreversible structure, where FIIN-2 formed a covalent bond through its reactive acrylamide group with the hydrosulfonyl side chain of FGFR4 C488 [73] (Figure 4C). 
A

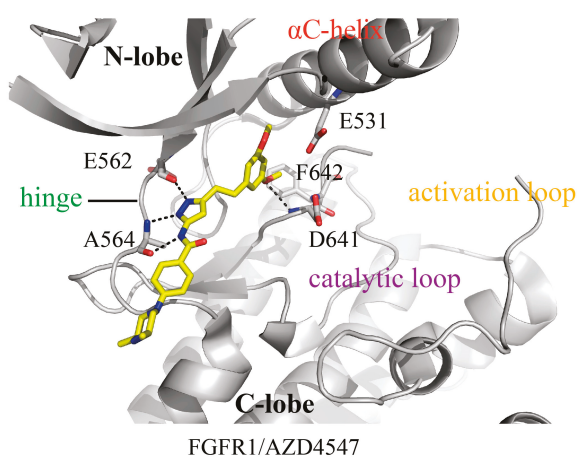

$\mathrm{C}$

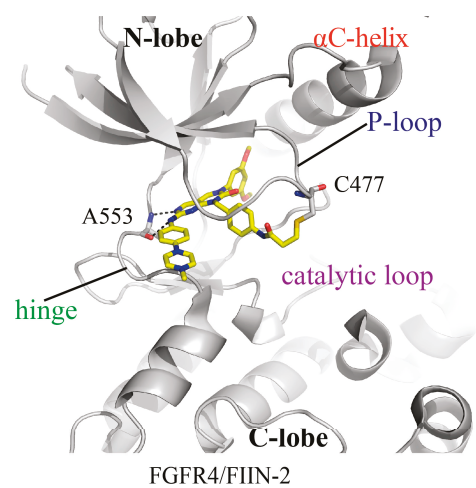

B

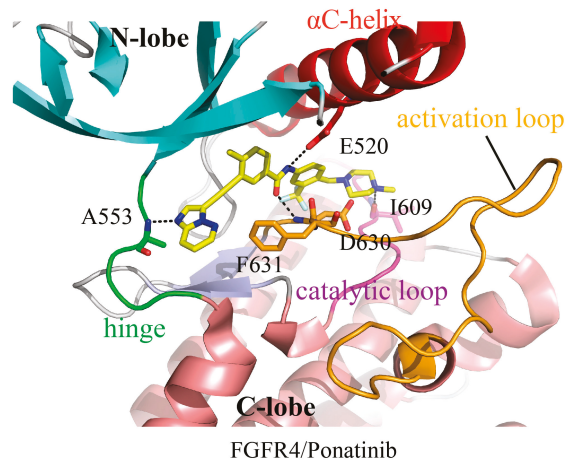

$\mathrm{D}$

$\begin{array}{lc} & 541 \\ \text { FGFR4 } & \text { TQEGPLYVIVECAAKGNLREFLR } \\ \text { FGFR1 } & \text { TQDGPLYVIVEYASKGNLREYLQ } \\ \text { FGFR2 } & \text { TQDGPLYVIVEYASKGNLREYLR } \\ \text { FGFR3 } & \text { TQGGPLYVIVEYAAKGNLREFLR }\end{array}$

$\mathrm{E}$

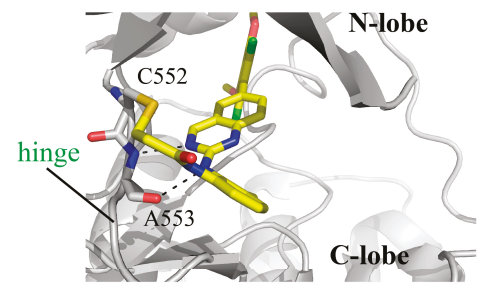

FGFR4/BLU-9931

Figure 4. FGFRs/inhibitor interaction features. All inhibitors are presented in yellow stick representation. (A) Structure of AZD4547 bound to FGFR1. The side chains of A564, E562 and D641, which directly form hydrogen bonds with the inhibitor and F642 of the DFG-motif, are shown. The hydrogen bonds are indicated by dashed lines. AZD4547 binds FGFR1 into DFG-in status, the side chain of F642 points out from the ATP pocket. (B) The structure of FGFR4 in a complex with ponatinib. The DFG-motif of FGFR4 flipped to an out conformation with F631 benzene ring flipped into the ATP-pocket and D630 point out from the pocket. (C) Covalent interaction of FIIN-2 and FGFR4. The side chains of A553 and C477, which interact with ponatinib, are shown in stick representation. Covalent bond formed between C477 and the acrylamide group of FIIN-2. (D) Sequence alignment of the FGFR hinge region. The C552 in FGR4 is replaced by a tyrosine in the other 3 members. The gatekeeper residues which locate at the kinases hinge region and play an essential role in determining pocket accessibility for inhibitors are highlighted in green. (E) Structure of BLU-9931 in complex with FGFR4. Unlike the pan-FGFR covalent inhibitors, BLU-9931 targets the unique C552 of FGFR4 to form covalent interactions.

\section{Current Status of Small Molecule FGFR Inhibitor Development}

Designing specific small molecule inhibitors targeting protein kinases is challenging because the ATP binding pockets of the human kinome are similar [74,75]. Inhibitor research for FGFRs has gone through several stages. Initially, nonselective multiple-kinase inhibitors were developed to treat FGFR aberrations. Those nonselective inhibitors, including ponatinib [76], dovitinib [77] lucitanib [78] and nintedanib [79] (see Table 1 for details), were originally designed for other kinases and then proved to have potent inhibition activity toward FGFRs. For instance, the type II inhibitor ponatinib was 
originally developed to overcome the BCR-ABL T315I gatekeeper mutant and showed single-digit nanomolar binding strength to FGFR1-4 in later researches [76]. Although nonselective inhibitors might be clinically beneficial and achieve therapeutic success to some extent, the development of those inhibitors has been restricted due to the undesirable off-target toxicities [80,81].

To overcome the off-target effects of nonselective inhibitors, efforts have been made to develop FGFR-selective inhibitors (pan-FGFR inhibitors). In the earlier stage, multiple noncovalent pan-FGFR inhibitors were developed, including the well-known AZD4547 [14] and LY2874455 [48] (see Table 1 for details). Those pan-FGFR inhibitors are typically type I inhibitors. For example, AZD4547 is capable of potently inhibiting FGFR1-3 but shows negligible binding affinity to FGFR4. AZD4547 is currently in phase II clinical trials. However, preclinical data show that AZD4547 is not able to overcome the gatekeeper mutation V555M in FGFR3 [82]. Unlike AZD4547, the inhibitor LY2874455 shows inhibition efficacy against all 4 FGFRs, and the crystal structure as well as in vitro and vivo experiments confirmed that this inhibitor maintained equal inhibitory ability against the gatekeeper mutant V550M/V550L of FGFR4 [32].

Given that covalent inhibitors confer better binding kinetics and selectivity than noncovalent ones, developing irreversible inhibitors of FGFRs has attracted intensive pharmaceutical and academic attention in recent years. Since the first covalent inhibitor FIIN-1 [83], this field has achieved much progress. A number of FGFR covalent inhibitors have been developed, and some of those agents are already in clinical trials (see Table 1 for details). Moreover, several irreversible inhibitor/FGFR structures have been revealed by crystal structures, including FGFR4/BLU9931 (PDB ID: 4XCU) [84], FGFR4/FIIN-3 (PDB ID: 4R6V) [73], FGFR4/FIIN-2 (PDB ID: 4QQ5) [85], FGFR4/CGA159527 (PDB ID: 5NUD) [86], FGFR1(Y563C)/H3B-6527 (PDB ID: 5VND) [86], and FGFR1/TAS-120 (PDB ID: 6MZW) [54].

The kinase domains of FGFRs are highly homologous, with sequence identity varying from $74 \%$ to $77 \%$ [34]. Unexpectedly, most of the reported pan-FGFR reversible inhibitors tend to bind FGFR1-3 but exhibit greatly reduced potency toward FGFR4 [87]. The underlying mechanism is not quite clear. Tucker et al. proposed that the innate flexibility of the FGFR4 kinase domain might be responsible for the decrease in binding ability [34]. This feature, together with the unique C552 of FGFR4, which replaces a tyrosine in FGFR1-3, confers the opportunity to develop FGFR4-selective inhibitors $[88,89]$ (Figure 4D). Indeed, H3B-6527 [56], BLU-9931 [84], BLU-554 [84] and FGF401 [90] were developed as FGFR4-selective covalent inhibitors that target C552 for irreversible binding (Figure 4E). Among these four molecules, FGF401 is the most interesting because the covalent bond it forms is reversible, which reduces the off-target effect and prolongs the residence time [91,92]. The crystal structure of FGF401/FGFR4 was recently reported by our laboratory (PDB ID 6JPJ) [57], and its potential utility is currently under intensive research.

In addition to the kinase domain, the ectodomains of FGFRs have also attracted intensive interests for drug discovery. Unlike the highly conserved kinase domain, the ectodomains of FGFRs are less conserved; targeting this domain may offer better isoform selectivity. The dominant strategy to target FGFR ectodomains is using monoclonal antibody/antibody-drug conjugate [93]. Several anti-FGFR monoclonal antibodies have been developed, and some of them are in clinical trials [94-98]. In addition, efforts have been made in the development of small molecule inhibitor targeting FGFR ectodomains. An inhibitor, SSR128129E, which allosterically binds to the ectodomain of FGFR in a non-FGF competitive manner, has been reported to inhibit FGF-induced signaling $[99,100]$.

\section{FGFR Gatekeeper Mutation and Drug Resistance}

The long-term efficacy of kinase inhibitors in cancer treatment is often disturbed by acquired resistance. One common mechanism of resistance is generated by mutating the so-called gatekeeper residue of the kinase domain [101]. The gatekeeper mutation has been reported in various kinases, such as Bcr-Abl (T315I) [102], EGFR (T790M) [68], PDGFR (T674I) [103], FGFR1 (V561M) [104] and FGFR2 (V565I) [105]. The gatekeeper residue lies at the beginning of the hinge region and controls the accessibility of the hydrophobic pocket. Most protein kinases harbor a threonine that plays a major 
role in the interaction with the inhibitor by forming a critical hydrogen bond via its side chain hydroxyl oxygen. The mutation of this residue to a bulky hydrophobic amino acid, either Met or Ile, breaks the hydrogen interaction and introduces steric hindrance for inhibitor binding [76,106].

The drug resistance of FGFR gatekeeper mutations has been extensively verified, both in vitro and in vivo. For instance, the V564M mutation of FGFR2 confers the ability to resist dovitinib and BGJ398 [107]. Furthermore, an array of FGFR gatekeeper mutations have been identified in clinical samples. For example, the FGFR4 V550M mutation was detected in 13\% of neuroendocrine breast carcinomas [108]. In FGFRs, the gatekeeper residue is a valine (Figure 4D); as a consequence, its side chain cannot form hydrogen interactions with inhibitors (see above context), and the resistance thus arises mainly through the introduction of steric hindrance. Several FGFR inhibitors have been shown to have the ability to overcome FGFR gatekeeper mutations. For example, Ly2874455 has almost equal binding affinity to wild-type FGFR4, FGFR4 (V550M), FGFR4 (V550L) FGFR1 (V561M), FGFR2 (V564F) and FGFR3 (V555M) [32]; FGF401 has similar affinity to wild-type FGFR4, FGFR4 (V550M) and FGFR4 (V550L) [57]; FIIN-2 shows a binding affinity loss of $\sim 10$-fold for FGFR4 (V550L) compared with the wild-type kinase [85].

\section{Conclusions}

Increasing evidence indicates that aberrant Fibroblast growth factor receptors (FGFR)signaling plays a crucial role in tumorigenesis and progression. Now, small molecule inhibitors targeting FGFRs offer a novel and effective strategy for the therapy of cancers caused by FGFR aberrations. Efforts and progress have been made in the field of FGFR inhibitor development. Some small molecules show promising antitumor activity and are evaluated in clinical trials. Recently, the pan-FGFR inhibitor erdafitinib has been approved by the Food and Drug Administration for the treatment of mUC, making it the first approved FGFR-targeted drug. However, there are still challenges in the field of FGFR inhibitor development, such as the need for more potent and selective FGFR inhibitors, and inhibitors with the ability to overcome gatekeeper mutations. Most FGFR inhibitors currently under evaluation are typical type I inhibitors that occupy only the ATP binding pocket. Development of type II FGFR inhibitors, which could be inserted deeper into the pocket, could confer better potency and selectivity. In addition, the development of covalent irreversible or covalent reversible FGFR inhibitors might be another strategy to improve safety and efficacy for cancer treatment.

Author Contributions: All authors were involved in the design of the review, and in writing or revising the manuscript. All authors approved the submitted version.

Funding: This research was funded by National Natural Science Foundation of China (81372904 and 81570537 to Y.C.).

Conflicts of Interest: The authors declare no conflict of interest.

\section{References}

1. Itoh, N.; Ornitz, D.M. Evolution of the Fgf and Fgfr gene families. Trends Genet. 2004, 20, 563-569. [CrossRef] [PubMed]

2. Schlessinger, J. Cell signaling by receptor tyrosine kinases. Cell 2000, 103, 211-225. [CrossRef]

3. Weiner, H.L.; Zagzag, D. Growth factor receptor tyrosine kinases: Cell adhesion kinase family suggests a novel signaling mechanism in cancer. Cancer Investig. 2000, 18, 544-554. [CrossRef]

4. Lemmon, M.A.; Schlessinger, J. Cell signaling by receptor tyrosine kinases. Cell 2010, 141, 1117-1134. [CrossRef] [PubMed]

5. Schlessinger, J. Cell signaling by receptor tyrosine kinases: From basic concepts to clinical applications. Eur. J. Cancer Suppl. 2006, 4, 3-26. [CrossRef]

6. Schlessinger, J. Receptor Tyrosine Kinases: Legacy of the First Two Decades. Cold Spring Harb. Perspect. Biol. 2014. [CrossRef] [PubMed]

7. Ornitz, D.M.; Itoh, N. The Fibroblast Growth Factor signaling pathway. Wiley Interdiscip. Rev. Dev. Biol. 2015, 4, 215-266. [CrossRef] [PubMed] 
8. Andrew, B.; Moosa, M. The FGF family: Biology, pathophysiology and therapy. Nat. Rev. Drug Discov. 2009, 8, 235-253.

9. Karel, D.; Enrique, A. FGF signalling: Diverse roles during early vertebrate embryogenesis. Development 2010, 137, 3731-3742.

10. Gowardhan, B.; Douglas, D.A.; Mathers, M.E.; McKie, A.B.; McCracken, S.R.C.; Robson, C.N.; Leung, H.Y. Evaluation of the fibroblast growth factor system as a potential target for therapy in human prostate cancer. Br. J. Cancer 2005, 92, 320-327. [CrossRef]

11. Brooks, A.N.; Kilgour, E.; Smith, P.D. Molecular pathways: Fibroblast growth factor signaling: A new therapeutic opportunity in cancer. Clin. Cancer Res. 2012, 18, 1855-1862. [CrossRef] [PubMed]

12. Turner, N.; Grose, R. Fibroblast growth factor signalling: From development to cancer. Nat. Rev. Cancer 2010, 10, 116-129. [CrossRef] [PubMed]

13. Porta, D.G.; Weiss, A.; Fairhurst, R.A.; Wartmann, M.; Stamm, C.; Reimann, F.; Buhles, A.; Kinyamu-Akunda, J.; Sterker, D.; Murakami, M. Abstract 2098: NVP-FGF401, a first-in-class highly selective and potent FGFR4 inhibitor for the treatment of HCC. Cancer Res. 2017. [CrossRef]

14. Gavine, P.R.; Mooney, L.; Kilgour, E.; Thomas, A.P.; Al-Kadhimi, K.; Beck, S.; Rooney, C.; Coleman, T.; Baker, D.; Mellor, M.J.; et al. AZD4547: An orally bioavailable, potent, and selective inhibitor of the fibroblast growth factor receptor tyrosine kinase family. Cancer Res. 2012, 72, 2045-2056. [CrossRef] [PubMed]

15. Perera, T.P.S.; Jovcheva, E.; Mevellec, L.; Vialard, J.; De Lange, D.; Verhulst, T.; Paulussen, C.; Van De Ven, K.; King, P.; Freyne, E.; et al. Discovery and Pharmacological Characterization of JNJ-42756493 (Erdafitinib), a Functionally Selective Small-Molecule FGFR Family Inhibitor. Mol. Cancer Ther. 2017, 16, 1010-1020. [CrossRef]

16. Farrell, B.; Breeze, A.L. Structure, activation and dysregulation of fibroblast growth factor receptor kinases: Perspectives for clinical targeting. Biochem. Soc. Trans. 2018, 46, 1753-1770. [CrossRef] [PubMed]

17. Sanchez-Heras, E.; Howell, F.V.; Williams, G.; Doherty, P. The fibroblast growth factor receptor acid box is essential for interactions with $\mathrm{N}$-cadherin and all of the major isoforms of neural cell adhesion molecule. J. Biol. Chem. 2006, 281, 35208-35216. [CrossRef]

18. Wang, F.; Kan, M.; Xu, J.; Yan, G.; McKeehan, W.L. Ligand-specific structural domains in the fibroblast growth factor receptor. J. Biol. Chem. 1995, 270, 10222-10230. [CrossRef]

19. Schlessinger, J.; Plotnikov, A.N.; Ibrahimi, O.A.; Eliseenkova, A.V.; Yeh, B.K.; Yayon, A.; Linhardt, R.J.; Mohammadi, M. Crystal structure of a ternary FGF-FGFR-heparin complex reveals a dual role for heparin in FGFR binding and dimerization. Mol. Cell 2000, 6, 743-750. [CrossRef]

20. Pellegrini, L.; Burke, D.F.; von Delft, F.; Mulloy, B.; Blundell, T.L. Crystal structure of fibroblast growth factor receptor ectodomain bound to ligand and heparin. Nature 2000, 407, 1029-1034. [CrossRef]

21. Saxena, K.; Schieborr, U.; Anderka, O.; Duchardt-Ferner, E.; Elshorst, B.; Gande, S.L.; Janzon, J.; Kudlinzki, D.; Sreeramulu, S.; Dreyer, M.K.; et al. Influence of heparin mimetics on assembly of the FGF.FGFR4 signaling complex. J. Biol. Chem. 2010, 285, 26628-26640. [CrossRef] [PubMed]

22. Eriksson, A.E.; Cousens, L.S.; Weaver, L.H.; Matthews, B.W. Three-dimensional structure of human basic fibroblast growth factor. Proc. Natl. Acad. Sci. USA 1991, 88, 3441-3445. [CrossRef] [PubMed]

23. Goetz, R.; Mohammadi, M. Exploring mechanisms of FGF signalling through the lens of structural biology. Nat. Rev. Mol. Cell Biol. 2013, 14, 166-180. [CrossRef] [PubMed]

24. Razzaque, M.S. The FGF23-Klotho axis: Endocrine regulation of phosphate homeostasis. Nat. Rev. Endocrinol. 2009, 5, 611-619. [CrossRef] [PubMed]

25. Chen, G.; Liu, Y.; Goetz, R.; Fu, L.; Jayaraman, S.; Hu, M.C.; Moe, O.W.; Liang, G.; Li, X.; Mohammadi, M. alpha-Klotho is a non-enzymatic molecular scaffold for FGF23 hormone signalling. Nature 2018, 553, 461-466. [CrossRef] [PubMed]

26. Lee, S.; Choi, J.; Mohanty, J.; Sousa, L.P.; Tome, F.; Pardon, E.; Steyaert, J.; Lemmon, M.A.; Lax, I.; Schlessinger, J. Structures of beta-klotho reveal a 'zip code'-like mechanism for endocrine FGF signalling. Nature 2018, 553, 501-505. [CrossRef] [PubMed]

27. Mohammadi, M.; Olsen, S.K.; Ibrahimi, O.A. Structural basis for fibroblast growth factor receptor activation. Cytokine Growth Factor Rev. 2005, 16, 107-137. [CrossRef]

28. Kalinina, J.; Dutta, K.; Ilghari, D.; Beenken, A.; Goetz, R.; Eliseenkova, A.V.; Cowburn, D.; Mohammadi, M. The alternatively spliced acid box region plays a key role in FGF receptor autoinhibition. Structure 2012, 20, 77-88. [CrossRef] 
29. Olsen, S.K.; Ibrahimi, O.A.; Raucci, A.; Zhang, F.; Eliseenkova, A.V.; Yayon, A.; Basilico, C.; Linhardt, R.J.; Schlessinger, J.; Mohammadi, M. Insights into the molecular basis for fibroblast growth factor receptor autoinhibition and ligand-binding promiscuity. Proc. Natl. Acad. Sci. USA 2004, 101, 935-940. [CrossRef]

30. Wang, F.; Kan, M.; Yan, G.; Xu, J.; McKeehan, W.L. Alternately spliced NH2-terminal immunoglobulin-like Loop I in the ectodomain of the fibroblast growth factor (FGF) receptor 1 lowers affinity for both heparin and FGF-1. J. Biol. Chem. 1995, 270, 10231-10235. [CrossRef]

31. Mohammadi, M.; Schlessinger, J.; Hubbard, S.R. Structure of the FGF receptor tyrosine kinase domain reveals a novel autoinhibitory mechanism. Cell 1996, 86, 577-587. [CrossRef]

32. Wu, D.; Guo, M.; Min, X.; Dai, S.; Li, M.; Tan, S.; Li, G.; Chen, X.; Ma, Y.; Li, J.; et al. LY2874455 potently inhibits FGFR gatekeeper mutants and overcomes mutation-based resistance. Chem. Commun. (Camb.) 2018, 54, 12089-12092. [CrossRef] [PubMed]

33. Wu, D.; Guo, M.; Philips, M.A.; Qu, L.; Jiang, L.; Li, J.; Chen, X.; Chen, Z.; Chen, L.; Chen, Y. Crystal Structure of the FGFR4/LY2874455 Complex Reveals Insights into the Pan-FGFR Selectivity of LY2874455. PLoS ONE 2016, 11, e0162491. [CrossRef] [PubMed]

34. Tucker, J.A.; Klein, T.; Breed, J.; Breeze, A.L.; Overman, R.; Phillips, C.; Norman, R.A. Structural insights into FGFR kinase isoform selectivity: Diverse binding modes of AZD4547 and ponatinib in complex with FGFR1 and FGFR4. Structure 2014, 22, 1764-1774. [CrossRef] [PubMed]

35. Ni, F.; Kung, A.; Duan, Y.; Shah, V.; Amador, C.D.; Guo, M.; Fan, X.; Chen, L.; Chen, Y.; McKenna, C.E.; et al. Remarkably Stereospecific Utilization of ATP alpha,beta-Halomethylene Analogues by Protein Kinases. J. Am. Chem. Soc. 2017, 139, 7701-7704. [CrossRef] [PubMed]

36. Knighton, D.R.; Zheng, J.H.; Ten Eyck, L.F.; Ashford, V.A.; Xuong, N.H.; Taylor, S.S.; Sowadski, J.M. Crystal structure of the catalytic subunit of cyclic adenosine monophosphate-dependent protein kinase. Science 1991, 253, 407-414. [CrossRef] [PubMed]

37. Yang, Y.; Ye, Q.; Jia, Z.; Cote, G.P. Characterization of the Catalytic and Nucleotide Binding Properties of the alpha-Kinase Domain of Dictyostelium Myosin-II Heavy Chain Kinase A. J. Biol. Chem. 2015, 290, 23935-23946. [CrossRef]

38. Furdui, C.M.; Lew, E.D.; Schlessinger, J.; Anderson, K.S. Autophosphorylation of FGFR1 kinase is mediated by a sequential and precisely ordered reaction. Mol. Cell 2006, 21, 711-717. [CrossRef]

39. Kornev, A.P.; Taylor, S.S.; Ten Eyck, L.F. A helix scaffold for the assembly of active protein kinases. Proc. Natl. Acad. Sci. USA 2008, 105, 14377-14382. [CrossRef]

40. Duan, Y.; Chen, L.; Chen, Y.; Fan, X.G. c-Src binds to the cancer drug Ruxolitinib with an active conformation. PLOS ONE 2014, 9, e106225. [CrossRef]

41. Hu, J.; Ahuja, L.G.; Meharena, H.S.; Kannan, N.; Kornev, A.P.; Taylor, S.S.; Shaw, A.S. Kinase regulation by hydrophobic spine assembly in cancer. Mol. Cell. Biol. 2015, 35, 264-276. [CrossRef] [PubMed]

42. Hari, S.B.; Merritt, E.A.; Maly, D.J. Sequence determinants of a specific inactive protein kinase conformation. Chem. Biol. 2013, 20, 806-815. [CrossRef] [PubMed]

43. Vijayan, R.S.; He, P.; Modi, V.; Duong-Ly, K.C.; Ma, H.; Peterson, J.R.; Dunbrack, R.L., Jr.; Levy, R.M. Conformational analysis of the DFG-out kinase motif and biochemical profiling of structurally validated type II inhibitors. J. Med. Chem. 2015, 58, 466-479. [CrossRef] [PubMed]

44. Klein, T.; Vajpai, N.; Phillips, J.J.; Davies, G.; Holdgate, G.A.; Phillips, C.; Tucker, J.A.; Norman, R.A.; Scott, A.D.; Higazi, D.R.; et al. Structural and dynamic insights into the energetics of activation loop rearrangement in FGFR1 kinase. Nat. Commun. 2015. [CrossRef]

45. Guimaraes, C.R.; Rai, B.K.; Munchhof, M.J.; Liu, S.; Wang, J.; Bhattacharya, S.K.; Buckbinder, L. Understanding the impact of the P-loop conformation on kinase selectivity. J. Chem. Inf. Model. 2011,51,1199-1204. [CrossRef] [PubMed]

46. Chen, H.; Ma, J.; Li, W.; Eliseenkova, A.V.; Xu, C.; Neubert, T.A.; Miller, W.T.; Mohammadi, M. A molecular brake in the kinase hinge region regulates the activity of receptor tyrosine kinases. Mol. Cell 2007, 27,717-730. [CrossRef] [PubMed]

47. Roskoski, R., Jr. Src protein-tyrosine kinase structure, mechanism, and small molecule inhibitors. Pharm. Res. 2015, 94, 9-25. [CrossRef] [PubMed]

48. Zhao, G.; Li, W.Y.; Chen, D.; Henry, J.R.; Li, H.Y.; Chen, Z.; Zia-Ebrahimi, M.; Bloem, L.; Zhai, Y.; Huss, K.; et al. A novel, selective inhibitor of fibroblast growth factor receptors that shows a potent broad spectrum of antitumor activity in several tumor xenograft models. Mol. Cancer. Ther. 2011, 10, 2200-2210. [CrossRef] 
49. Nakanishi, Y.; Akiyama, N.; Tsukaguchi, T.; Fujii, T.; Sakata, K.; Sase, H.; Isobe, T.; Morikami, K.; Shindoh, H.; Mio, T; et al. The fibroblast growth factor receptor genetic status as a potential predictor of the sensitivity to CH5183284/Debio 1347, a novel selective FGFR inhibitor. Mol. Cancer. Ther. 2014, 13, 2547-2558. [CrossRef]

50. Guagnano, V.; Furet, P.; Spanka, C.; Bordas, V.; Le Douget, M.; Stamm, C.; Brueggen, J.; Jensen, M.R.; Schnell, C.; Schmid, H.; et al. Discovery of 3-(2,6-dichloro-3,5-dimethoxy-phenyl)-1-\{6-[4 -(4-ethyl-piperazin-1-yl)-phenylamin o]-pyrimidin-4-yl\}-1-methyl-urea (NVP-BGJ398), a potent and selective inhibitor of the fibroblast growth factor receptor family of receptor tyrosine kinase. J. Med. Chem. 2011, 54, 7066-7083. [CrossRef]

51. Karkera, J.D.; Cardona, G.M.; Bell, K.; Gaffney, D.; Portale, J.C.; Santiago-Walker, A.; Moy, C.H.; King, P.; Sharp, M.; Bahleda, R.; et al. Oncogenic Characterization and Pharmacologic Sensitivity of Activating Fibroblast Growth Factor Receptor (FGFR) Genetic Alterations to the Selective FGFR Inhibitor Erdafitinib. Mol. Cancer. Ther. 2017, 16, 1717-1726. [CrossRef] [PubMed]

52. Collin, M.P.; Lobell, M.; Hubsch, W.; Brohm, D.; Schirok, H.; Jautelat, R.; Lustig, K.; Bomer, U.; Vohringer, V.; Heroult, M.; et al. Discovery of Rogaratinib (BAY 1163877): A pan-FGFR Inhibitor. Chem. Med. Chem. 2018, 13, 437-445. [CrossRef] [PubMed]

53. Brameld, K.A.; Owens, T.D.; Verner, E.; Venetsanakos, E.; Bradshaw, J.M.; Phan, V.T.; Tam, D.; Leung, K.; Shu, J.; LaStant, J.; et al. Discovery of the Irreversible Covalent FGFR Inhibitor 8-(3-(4-Acryloylpiperazin-1-yl) propyl)-6-(2,6-dichloro-3,5-dimethoxyphenyl)-2-(me thylamino)pyrido[2,3-d]pyrimidin-7(8H)-one (PRN1371) for the Treatment of Solid Tumors. J. Med. Chem. 2017, 60, 6516-6527. [CrossRef] [PubMed]

54. Kalyukina, M.; Yosaatmadja, Y.; Middleditch, M.J.; Patterson, A.V.; Smaill, J.B.; Squire, C.J. TAS-120 Cancer Target Binding: Defining Reactivity and Revealing the First Fibroblast Growth Factor Receptor 1 (FGFR1) Irreversible Structure. ChemMedChem 2019, 14, 494-500. [CrossRef] [PubMed]

55. Kim, R.; Sharma, S.; Meyer, T.; Sarker, D.; Macarulla, T.; Sung, M.; Choo, S.P.; Shi, H.; Schmidt-Kittler, O.; Clifford, C.; et al. First-in-human study of BLU-554, a potent, highly-selective FGFR4 inhibitor designed for hepatocellular carcinoma (HCC) with FGFR4 pathway activation. Eur. J. Cancer 2016, 69, S41. [CrossRef]

56. Joshi, J.J.; Coffey, H.; Corcoran, E.; Tsai, J.; Huang, C.L.; Ichikawa, K.; Prajapati, S.; Hao, M.H.; Bailey, S.; Wu, J.; et al. H3B-6527 Is a Potent and Selective Inhibitor of FGFR4 in FGF19-Driven Hepatocellular Carcinoma. Cancer Res. 2017, 77, 6999-7013. [CrossRef]

57. Zhou, Z.; Chen, X.; Fu, Y.; Zhang, Y.; Dai, S.; Li, J.; Chen, L.; Xu, G.; Chen, Z.; Chen, Y. Characterization of FGF401 as a reversible covalent inhibitor of fibroblast growth factor receptor 4. Chem. Commun. (Camb.) 2019. [CrossRef]

58. Roskoski, R., Jr. ERK1/2 MAP kinases: Structure, function, and regulation. Pharm. Res. 2012, 66, $105-143$.

59. Dar, A.C.; Shokat, K.M. The evolution of protein kinase inhibitors from antagonists to agonists of cellular signaling. Annu. Rev. Biochem. 2011, 80, 769-795. [CrossRef]

60. Norman, R.A.; Schott, A.K.; Andrews, D.M.; Breed, J.; Foote, K.M.; Garner, A.P.; Ogg, D.; Orme, J.P.; Pink, J.H.; Roberts, K.; et al. Protein-ligand crystal structures can guide the design of selective inhibitors of the FGFR tyrosine kinase. J. Med. Chem. 2012, 55, 5003-5012. [CrossRef]

61. Liu, Y.; Gray, N.S. Rational design of inhibitors that bind to inactive kinase conformations. Nat. Chem. Biol. 2006, 2, 358-364. [CrossRef] [PubMed]

62. Mohammadi, M.; Froum, S.; Hamby, J.M.; Schroeder, M.C.; Panek, R.L.; Lu, G.H.; Eliseenkova, A.V.; Green, D.; Schlessinger, J.; Hubbard, S.R. Crystal structure of an angiogenesis inhibitor bound to the FGF receptor tyrosine kinase domain. EMBO J. 1998, 17, 5896-5904. [CrossRef] [PubMed]

63. Davis, M.I.; Hunt, J.P.; Herrgard, S.; Ciceri, P.; Wodicka, L.M.; Pallares, G.; Hocker, M.; Treiber, D.K.; Zarrinkar, P.P. Comprehensive analysis of kinase inhibitor selectivity. Nat. Biotechnol. 2011, 29, 1046-1051. [CrossRef]

64. Baillie, T.A. Targeted Covalent Inhibitors for Drug Design. Angew. Chem. Int. Ed. 2016, 55, 13408-13421. [CrossRef] [PubMed]

65. Awoonor-Williams, E.; Walsh, A.G.; Rowley, C.N. Modeling covalent-modifier drugs. Biochim. Biophys. Acta 2017, 1865, 1664-1675. [CrossRef]

66. Liu, Q.; Sabnis, Y.; Zhao, Z.; Zhang, T.; Buhrlage, S.J.; Jones, L.H.; Gray, N.S. Developing irreversible inhibitors of the protein kinase cysteinome. Chem. Biol. 2013, 20, 146-159. [CrossRef] [PubMed]

67. Serafimova, I.M.; Pufall, M.A.; Krishnan, S.; Duda, K.; Cohen, M.S.; Maglathlin, R.L.; McFarland, J.M.; Miller, R.M.; Frodin, M.; Taunton, J. Reversible targeting of noncatalytic cysteines with chemically tuned electrophiles. Nat. Chem. Biol. 2012, 8, 471-476. [CrossRef] 
68. Zhou, W.; Ercan, D.; Chen, L.; Yun, C.H.; Li, D.; Capelletti, M.; Cortot, A.B.; Chirieac, L.; Iacob, R.E.; Padera, R.; et al. Novel mutant-selective EGFR kinase inhibitors against EGFR T790M. Nature 2009, 462, 1070-1074. [CrossRef]

69. Zhang, J.; Yang, P.L.; Gray, N.S. Targeting cancer with small molecule kinase inhibitors. Nat. Rev. Cancer 2009, 9, 28-39. [CrossRef]

70. Powis, G.; Bonjouklian, R.; Berggren, M.M.; Gallegos, A.; Abraham, R.; Ashendel, C.; Zalkow, L.; Matter, W.F.; Dodge, J.; Grindey, G.; et al. Wortmannin, a potent and selective inhibitor of phosphatidylinositol-3-kinase. Cancer Res. 1994, 54, 2419-2423.

71. Fox, T.; Fitzgibbon, M.J.; Fleming, M.A.; Hsiao, H.M.; Brummel, C.L.; Su, M.S. Kinetic mechanism and ATP-binding site reactivity of p38gamma MAP kinase. FEBS Lett. 1999, 461, 323-328. [CrossRef]

72. Shannon, D.A.; Weerapana, E. Covalent protein modification: The current landscape of residue-specific electrophiles. Curr. Opin. Chem. Biol. 2015, 24, 18-26. [CrossRef] [PubMed]

73. Tan, L.; Wang, J.; Tanizaki, J.; Huang, Z.; Aref, A.R.; Rusan, M.; Zhu, S.J.; Zhang, Y.; Ercan, D.; Liao, R.G.; et al. Development of covalent inhibitors that can overcome resistance to first-generation FGFR kinase inhibitors. Proc. Natl. Acad. Sci. USA 2014, 111, E4869-E4877. [CrossRef] [PubMed]

74. Paul, S.M.; Mytelka, D.S.; Dunwiddie, C.T.; Persinger, C.C.; Munos, B.H.; Lindborg, S.R.; Schacht, A.L. How to improve R\&D productivity: The pharmaceutical industry's grand challenge. Nat. Rev. Drug Discov. 2010, 9, 203-214. [CrossRef] [PubMed]

75. Fedorov, O.; Muller, S.; Knapp, S. The (un)targeted cancer kinome. Nat. Chem. Biol. 2010, 6, 166-169. [CrossRef] [PubMed]

76. O'Hare, T.; Shakespeare, W.C.; Zhu, X.; Eide, C.A.; Rivera, V.M.; Wang, F.; Adrian, L.T.; Zhou, T.; Huang, W.S.; Xu, Q.; et al. AP24534, a pan-BCR-ABL inhibitor for chronic myeloid leukemia, potently inhibits the T315I mutant and overcomes mutation-based resistance. Cancer Cell 2009, 16, 401-412. [CrossRef] [PubMed]

77. Trudel, S.; Li, Z.H.; Wei, E.; Wiesmann, M.; Chang, H.; Chen, C.; Reece, D.; Heise, C.; Stewart, A.K. CHIR-258, a novel, multitargeted tyrosine kinase inhibitor for the potential treatment of $t(4 ; 14)$ multiple myeloma. Blood 2005, 105, 2941-2948. [CrossRef]

78. Bello, E.; Colella, G.; Scarlato, V.; Oliva, P.; Berndt, A.; Valbusa, G.; Serra, S.C.; D’Incalci, M.; Cavalletti, E.; Giavazzi, R.; et al. E-3810 is a potent dual inhibitor of VEGFR and FGFR that exerts antitumor activity in multiple preclinical models. Cancer Res. 2011, 71, 1396-1405. [CrossRef]

79. Hilberg, F.; Roth, G.J.; Krssak, M.; Kautschitsch, S.; Sommergruber, W.; Tontsch-Grunt, U.; Garin-Chesa, P.; Bader, G.; Zoephel, A.; Quant, J.; et al. BIBF 1120: Triple angiokinase inhibitor with sustained receptor blockade and good antitumor efficacy. Cancer Res. 2008, 68, 4774-4782. [CrossRef]

80. Nogova, L.; Sequist, L.V.; Perez Garcia, J.M.; Andre, F.; Delord, J.P.; Hidalgo, M.; Schellens, J.H.; Cassier, P.A.; Camidge, D.R.; Schuler, M.; et al. Evaluation of BGJ398, a Fibroblast Growth Factor Receptor 1-3 Kinase Inhibitor, in Patients with Advanced Solid Tumors Harboring Genetic Alterations in Fibroblast Growth Factor Receptors: Results of a Global Phase I, Dose-Escalation and Dose-Expansion Study. J. Clin. Oncol. 2017, 35, 157-165. [CrossRef]

81. Degirolamo, C.; Sabba, C.; Moschetta, A. Therapeutic potential of the endocrine fibroblast growth factors FGF19, FGF21 and FGF23. Nat. Rev. Drug Discov. 2016, 15, 51-69. [CrossRef]

82. Chell, V.; Balmanno, K.; Little, A.S.; Wilson, M.; Andrews, S.; Blockley, L.; Hampson, M.; Gavine, P.R.; Cook, S.J. Tumour cell responses to new fibroblast growth factor receptor tyrosine kinase inhibitors and identification of a gatekeeper mutation in FGFR3 as a mechanism of acquired resistance. Oncogene 2013, 32, 3059-3070. [CrossRef] [PubMed]

83. Zhou, W.; Hur, W.; McDermott, U.; Dutt, A.; Xian, W.; Ficarro, S.B.; Zhang, J.; Sharma, S.V.; Brugge, J.; Meyerson, M.; et al. A structure-guided approach to creating covalent FGFR inhibitors. Chem. Biol. 2010, 17, 285-295. [CrossRef] [PubMed]

84. Hagel, M.; Miduturu, C.; Sheets, M.; Rubin, N.; Weng, W.; Stransky, N.; Bifulco, N.; Kim, J.L.; Hodous, B.; Brooijmans, N.; et al. First Selective Small Molecule Inhibitor of FGFR4 for the Treatment of Hepatocellular Carcinomas with an Activated FGFR4 Signaling Pathway. Cancer Discov. 2015, 5, 424-437. [CrossRef] [PubMed]

85. Huang, Z.; Tan, L.; Wang, H.; Liu, Y.; Blais, S.; Deng, J.; Neubert, T.A.; Gray, N.S.; Li, X.; Mohammadi, M. DFG-out mode of inhibition by an irreversible type-1 inhibitor capable of overcoming gate-keeper mutations in FGF receptors. ACS Chem. Biol. 2015, 10, 299-309. [CrossRef] [PubMed] 
86. Fairhurst, R.A.; Knoepfel, T.; Leblanc, C.; Buschmann, N.; Gaul, C.; Blank, J.; Galuba, I.; Trappe, J.; Zou, C.; Voshol, J.; et al. Approaches to selective fibroblast growth factor receptor 4 inhibition through targeting the ATP-pocket middle-hinge region. MedChem Comm 2017, 8, 1604-1613. [CrossRef]

87. Ho, H.K.; Yeo, A.H.; Kang, T.S.; Chua, B.T. Current strategies for inhibiting FGFR activities in clinical applications: Opportunities, challenges and toxicological considerations. Drug Discov. Today 2014, 19, 51-62. [CrossRef]

88. Katoh, M. FGFR inhibitors: Effects on cancer cells, tumor microenvironment and whole-body homeostasis (Review). Int. J. Mol. Med. 2016, 38, 3-15. [CrossRef]

89. Lu, X.; Chen, H.; Patterson, A.V.; Smaill, J.B.; Ding, K. Fibroblast Growth Factor Receptor 4 (FGFR4) Selective Inhibitors as Hepatocellular Carcinoma Therapy: Advances and Prospects. J. Med. Chem. 2019, 62, 2905-2915. [CrossRef]

90. Hierro, C.; Rodon, J.; Tabernero, J. Fibroblast Growth Factor (FGF) Receptor/FGF Inhibitors: Novel Targets and Strategies for Optimization of Response of Solid Tumors. Semin. Oncol. 2015, 42, 801-819. [CrossRef]

91. Bradshaw, J.M.; McFarland, J.M.; Paavilainen, V.O.; Bisconte, A.; Tam, D.; Phan, V.T.; Romanov, S.; Finkle, D.; Shu, J.; Patel, V.; et al. Prolonged and tunable residence time using reversible covalent kinase inhibitors. Nat. Chem. Biol. 2015, 11, 525-531. [CrossRef] [PubMed]

92. Knoepfel, T.; Furet, P.; Mah, R.; Buschmann, N.; Leblanc, C.; Ripoche, S.; Graus-Porta, D.; Wartmann, M.; Galuba, I.; Fairhurst, R.A. 2-Formylpyridyl Ureas as Highly Selective Reversible-Covalent Inhibitors of Fibroblast Growth Factor Receptor 4. ACS Med. Chem. Lett. 2018, 9, 215-220. [CrossRef] [PubMed]

93. Shabani, M.; Hojjat-Farsangi, M. Targeting Receptor Tyrosine Kinases Using Monoclonal Antibodies: The Most Specific Tools for Targeted-Based Cancer Therapy. Curr. Drug Targets 2016, 17, 1687-1703. [CrossRef] [PubMed]

94. Pierce, K.L.; Deshpande, A.M.; Stohr, B.A.; Gemo, A.T.; Patil, N.S.; Brennan, T.J.; Bellovin, D.I.; Palencia, S.; Giese, T.; Huang, C.; et al. FPA144, a humanized monoclonal antibody for both FGFR2-amplified and nonamplified, FGFR2b-overexpressing gastric cancer patients. J. Clin. Oncol. 2014. [CrossRef]

95. Sommer, A.; Kopitz, C.; Schatz, C.A.; Nising, C.F.; Mahlert, C.; Lerchen, H.G.; Stelte-Ludwig, B.; Hammer, S.; Greven, S.; Schuhmacher, J.; et al. Preclinical Efficacy of the Auristatin-Based Antibody-Drug Conjugate BAY 1187982 for the Treatment of FGFR2-Positive Solid Tumors. Cancer Res. 2016, 76, 6331-6339. [CrossRef] [PubMed]

96. Schatz, C.A.; Kopitz, C.; Wittemer-Rump, S.; Sommer, A.; Lindbom, L.; Osada, M.; Yamanouchi, H.; Huynh, H.; Krahn, T.; Asadullah, K. Abstract 4766: Pharmacodynamic and stratification biomarker for the anti-FGFR2 antibody (BAY1179470) and the FGFR2-ADC. Cancer Res. 2014. [CrossRef]

97. Trudel, S.; Bergsagel, P.L.; Singhal, S.; Niesvizky, R.; Comenzo, R.L.; Bensinger, W.I.; Lebovic, D.; Choi, Y.; Lu, D.; French, D.; et al. A Phase I Study of the Safety and Pharmacokinetics of Escalating Doses of MFGR1877S, a Fibroblast Growth Factor Receptor 3 (FGFR3) Antibody, in Patients with Relapsed or Refractory t(4;14)-Positive Multiple Myeloma. Blood 2012, 120, 4029.

98. Blackwell, C.; Sherk, C.; Fricko, M.; Ganji, G.; Barnette, M.; Hoang, B.; Tunstead, J.; Skedzielewski, T.; Alsaid, H.; Jucker, B.M.; et al. Inhibition of FGF/FGFR autocrine signaling in mesothelioma with the FGF ligand trap, FP-1039/GSK3052230. Oncotarget 2016, 7, 39861-39871. [CrossRef]

99. Bono, F.; De Smet, F.; Herbert, C.; De Bock, K.; Georgiadou, M.; Fons, P.; Tjwa, M.; Alcouffe, C.; Ny, A.; Bianciotto, M.; et al. Inhibition of tumor angiogenesis and growth by a small-molecule multi-FGF receptor blocker with allosteric properties. Cancer Cell 2013, 23, 477-488. [CrossRef]

100. Herbert, C.; Schieborr, U.; Saxena, K.; Juraszek, J.; De Smet, F.; Alcouffe, C.; Bianciotto, M.; Saladino, G.; Sibrac, D.; Kudlinzki, D.; et al. Molecular mechanism of SSR128129E, an extracellularly acting, small-molecule, allosteric inhibitor of FGF receptor signaling. Cancer Cell 2013, 23, 489-501. [CrossRef]

101. Babina, I.S.; Turner, N.C. Advances and challenges in targeting FGFR signalling in cancer. Nat. Rev. Cancer 2017, 17, 318-332. [CrossRef] [PubMed]

102. Cheetham, G.M.; Charlton, P.A.; Golec, J.M.; Pollard, J.R. Structural basis for potent inhibition of the Aurora kinases and a T315I multi-drug resistant mutant form of Abl kinase by VX-680. Cancer Lett. 2007, 251, 323-329. [CrossRef] [PubMed]

103. Weisberg, E.; Choi, H.G.; Ray, A.; Barrett, R.; Zhang, J.; Sim, T.; Zhou, W.; Seeliger, M.; Cameron, M.; Azam, M.; et al. Discovery of a small-molecule type II inhibitor of wild-type and gatekeeper mutants of BCR-ABL, 
PDGFRalpha, Kit, and Src kinases: Novel type II inhibitor of gatekeeper mutants. Blood 2010, 115, 4206-4216. [CrossRef] [PubMed]

104. Ryan, M.R.; Sohl, C.D.; Luo, B.; Anderson, K.S. The FGFR1 V561M Gatekeeper Mutation Drives AZD4547 Resistance through STAT3 Activation and EMT. Mol. Cancer Res. 2019, 17, 532-543. [CrossRef] [PubMed]

105. Byron, S.A.; Chen, H.; Wortmann, A.; Loch, D.; Gartside, M.G.; Dehkhoda, F.; Blais, S.P.; Neubert, T.A.; Mohammadi, M.; Pollock, P.M. The N550K/H mutations in FGFR2 confer differential resistance to PD173074, dovitinib, and ponatinib ATP-competitive inhibitors. Neoplasia 2013, 15, 975-988. [CrossRef] [PubMed]

106. Yoza, K.; Himeno, R.; Amano, S.; Kobashigawa, Y.; Amemiya, S.; Fukuda, N.; Kumeta, H.; Morioka, H.; Inagaki, F. Biophysical characterization of drug-resistant mutants of fibroblast growth factor receptor 1. Genes Cells 2016, 21, 1049-1058. [CrossRef]

107. Goyal, L.; Saha, S.K.; Liu, L.Y.; Siravegna, G.; Leshchiner, I.; Ahronian, L.G.; Lennerz, J.K.; Vu, P.; Deshpande, V.; Kambadakone, A.; et al. Polyclonal Secondary FGFR2 Mutations Drive Acquired Resistance to FGFR Inhibition in Patients with FGFR2 Fusion-Positive Cholangiocarcinoma. Cancer Discov. 2017, 7, 252-263. [CrossRef]

108. Ang, D.; Ballard, M.; Beadling, C.; Warrick, A.; Schilling, A.; O'Gara, R.; Pukay, M.; Neff, T.L.; West, R.B.; Corless, C.L.; et al. Novel mutations in neuroendocrine carcinoma of the breast: Possible therapeutic targets. Appl. Immunohistochem. Mol. Morphol. 2015, 23, 97-103. [CrossRef]

(C) 2019 by the authors. Licensee MDPI, Basel, Switzerland. This article is an open access article distributed under the terms and conditions of the Creative Commons Attribution (CC BY) license (http://creativecommons.org/licenses/by/4.0/). 



\title{
Fibroblast Growth Factor Receptor Signaling in Skin Cancers
}

\author{
Malgorzata Czyz \\ Department of Molecular Biology of Cancer, Medical University of Lodz, 6/8 Mazowiecka Street, 92-215 Lodz, \\ Poland; malgorzata.czyz@umed.lodz.pl; Tel.: +48-42-272-57-02
}

Academic Editor: Klaus Holzmann

Received: 15 May 2019; Accepted: 3 June 2019; Published: 4 June 2019

\begin{abstract}
Fibroblast growth factor (FGF)/Fibroblast growth factor receptor (FGFR) signaling regulates various cellular processes during the embryonic development and in the adult organism. In the skin, fibroblasts and keratinocytes control proliferation and survival of melanocytes in a paracrine manner via several signaling molecules, including FGFs. FGF/FGFR signaling contributes to the skin surface expansion in childhood or during wound healing, and skin protection from UV light damage. Aberrant FGF/FGFR signaling has been implicated in many disorders, including cancer. In melanoma cells, the FGFR expression is low, probably because of the strong endogenous mutation-driven constitutive activation of the downstream mitogen-activated protein kinase-extracellular signal-regulated kinase (MAPK-ERK) signaling pathway. FGFR1 is exceptional as it is expressed in the majority of melanomas at a high level. Melanoma cells that acquired the capacity to synthesize FGFs can influence the neighboring cells in the tumor niche, such as endothelial cells, fibroblasts, or other melanoma cells. In this way, FGF/FGFR signaling contributes to intratumoral angiogenesis, melanoma cell survival, and development of resistance to therapeutics. Therefore, inhibitors of aberrant FGF/FGFR signaling are considered as drugs in combination treatment. The ongoing LOGIC-2 phase II clinical trial aims to find out whether targeting the FGF/FGFR signaling pathway with BGJ398 may be a good therapeutic strategy in melanoma patients who develop resistance to $\mathrm{v}$-Raf murine sarcoma viral oncogene homolog B (BRAF)/MEK inhibitors.
\end{abstract}

Keywords: FGF; fibroblast growth factor; FGFR; autocrine signaling; skin; melanoma; squamous and basal cell carcinoma; seborrheic keratosis; targeted therapy; resistance

\section{Introduction}

Receptor tyrosine kinases (RTKs) are cell membrane proteins comprising about 20 families with nearly 60 members. The Fibroblast Growth Factor Receptor (FGFR) family of human RTKs consists of four highly conserved transmembrane receptors (FGFR1-4) and one FGF receptor without an intracellular domain (FGFR5). FGFRs are mainly localized at the cell surface; however, they are also present inside of the cells, in the nucleus and mitochondria [1]. The common structure of cell membrane-localized FGFRs consists of a large ligand-binding extracellular region with three immunoglobulin-like (Ig-like) domains, a transmembrane helical region, and a cytoplasmic region with a catalytically active tyrosine kinase domain. The alternative splicing of the third Ig-like domain results in a variety of FGFR isoforms with different ligand specificities [2,3]. FGFR activity is modulated in diverse ways, including posttranslational modifications and formation of complexes with selected ligands and other cell membrane proteins [4]. Over 20 distinct fibroblast growth factors (FGFs) have been identified as the ligands of FGFRs. Binding of FGFs to FGFRs is assisted by cofactors, heparin sulfate proteoglycans (HSPGs) in paracrine FGF signaling, or Klotho coreceptor in endocrine signaling [5]. It triggers the dimerization of receptor monomers in the membrane and cross-autophosphorylation 
of tyrosine residues in the cytoplasmic kinase domain, which is followed by binding of various downstream effector molecules, including phospholipase C gamma (PLC $\gamma$ ), fibroblast growth factor receptor substrate 2 (FRS2), son of sevenless (SOS), and growth factor receptor-bound 2 (GRB2). FRS2 is an adaptor/scaffold protein, which acts downstream of a limited number of RTKs, including FGFR [6]. FRS2 was annotated as amplified in skin-derived tumors in the Cancer Genome Project dataset $[7,8]$ and emerged as a potential therapeutic target in melanoma [9]. Binding of adaptor proteins induces the activation of several signaling pathways, such as protein kinase $\mathrm{C}$ (PKC), mitogen-activated protein kinase-extracellular signal-regulated kinase (MAPK-ERK), phosphoinositide 3-kinase/protein kinase $\mathrm{B}$ (PI3K/AKT), and signal transducer and activator of transcription 3/5 (STAT3/5) signaling pathway (Figure 1). 


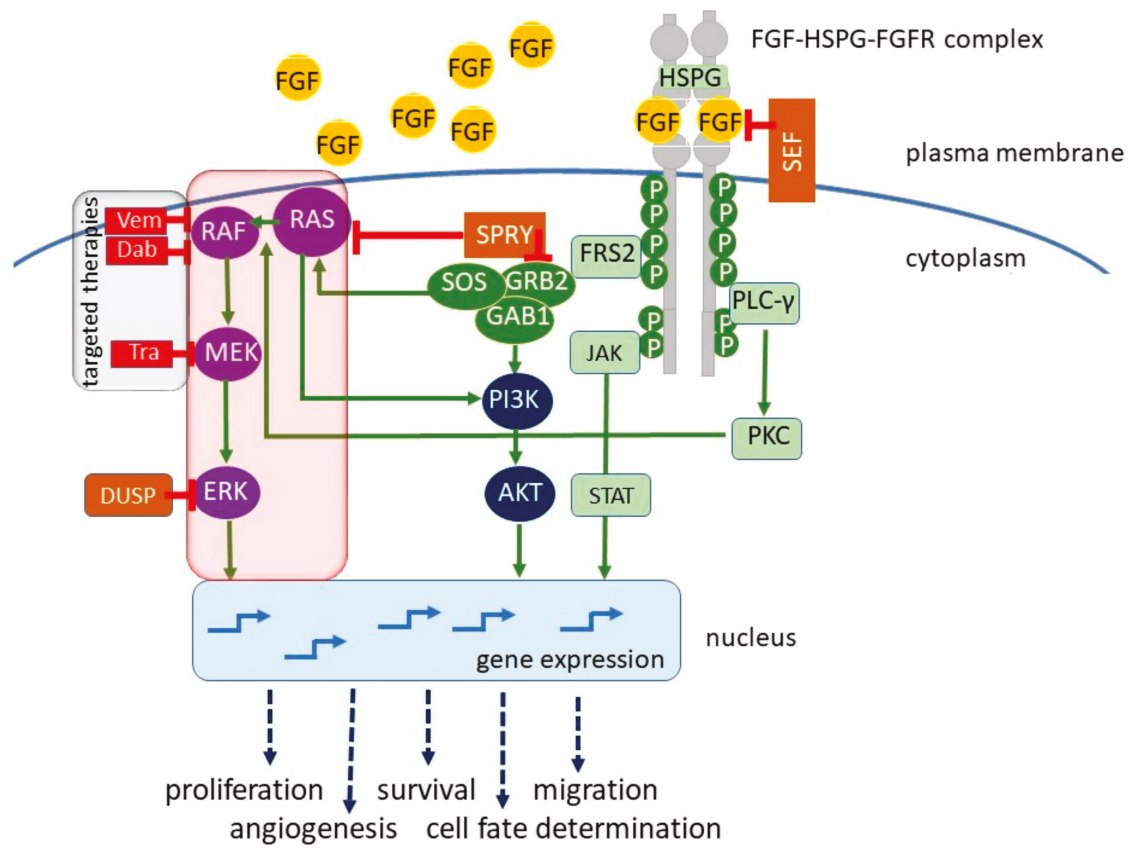

Figure 1. Fibroblast growth factor receptors (FGFRs) are highly conserved transmembrane receptors consisting of three extracellular immunoglobulin-like (Ig-like) domains, a transmembrane helical region, and a cytoplasmic region with kinase activity. The fibroblast growth factor (FGF) ligand and its cofactor heparin sulfate proteoglycan (HSPG) bind to FGFR monomers, leading to dimerization and tyrosine cross-autophosphorylation of the cytoplasmic domain. This induces various signaling pathways, resulting in cellular proliferation, survival, migration, angiogenesis, and cell fate determination in embryogenesis and in response to microenvironmental signals, including therapeutics. FGF/FGFR signaling can be stimulated in a paracrine manner, mainly in physiological settings, or in an autocrine manner as demonstrated in various cancers. In melanoma, FGF/FGFR signaling is largely suppressed by mutation-driven enhanced activity of the RAS (Rat sarcoma oncogene)/BRAF (v-Raf murine sarcoma viral oncogene homolog B)/MEK (mitogen-activated protein kinase)/ERK (extracellular signal-regulated kinase) pathway (red framed). Melanoma cells that acquire the ability to secrete FGFs and stimulate FGFR in a paracrine or autocrine manner can contribute to angiogenesis and cell-fate decisions involving transitions between different phenotypes, including phenotypes resistant to targeted therapies (grey framed). Dab, dabrafenib; DUSP, dual-specificity phosphatase; FRS2, FGFR substrate 2; GAB1, GRB2-associated binding protein 1; GRB2, growth factor receptor protein 2; JAK, Janus kinase; PKC, protein kinase C; PLC- $\gamma$, phospholipase C gamma; SOS, son of sevenless; SEF, similar expression to FGF; SPRY, Sprouty; STAT, signal transducer and activator of transcription; Tra, trametinib; Vem, vemurafenib.

FGFR triggered signaling pathways play crucial roles in morphogenesis during embryonic development [10]. Signaling from FGFR is also important for controlling the nervous system, angiogenesis, metabolism, endocrine function, wound healing, and tissue repair in the adult organism. FGF signaling regulates expression of genes by modulating microRNA abundance [11,12]. Besides being involved in normal development, abnormal activities of FGRFs has been documented in hereditary diseases and a wide range of cancers [13-17]. In the recently performed large-scale high-throughput study, the dysregulation of FGFRs was found in $7 \%$ of cancers [18].

Melanoma belongs to a group of highly lethal cancers. Several signaling pathways are constitutively activated in melanoma [19]. Recently developed technologies, including next-generation sequencing (NGS), led to a new genetic-based classification of melanoma [20,21]. The activity of the MAPK 
signaling pathway, comprising the cascade of RAS (Rat sarcoma oncogene)/RAF (v-Raf murine sarcoma viral oncogene homolog B)/MEK (mitogen-activated protein kinase kinase)/ERK, is very frequently altered in melanoma by somatic mutations [22]. About $50 \%$ of melanoma patients harbor activating mutations in $B R A F$ with $\mathrm{BRAF}^{\mathrm{V} 600 \mathrm{E}}$ as the main protein product, whereas NRAS is mutated in about $15-20 \%$ of cases $[23,24]$. The constitutive activity of the MAPK signaling pathway results in elevated proliferation rate and enhanced survival potential of melanomas. Therefore, in addition to the development of immunotherapies, the main effort is focused on targeted therapies with $\mathrm{BRAF}^{\mathrm{V} 600}$ and MEK inhibitors [25]. Several drugs have been accepted by the FDA (Food and Drug Administration) and EMA (European Medicines Agency) for the treatment of melanoma patients in unresectable stages of tumor development [25-29]. Although targeted therapies are very promising, they are challenged by intrinsic resistance and the development of acquired resistance in approximately one-half of the melanoma patients within a few months [30-37].

Depending on the tumor type and its major driving oncogenes, the FGF/FGFR signaling can be differently utilized by tumor cells to maintain their malignancy and can be variably affected by therapeutics, especially those targeting tumor-specific oncoproteins $[16,38]$. This review article summarizes the current knowledge on the fibroblast growth factor receptor signaling in skin cancers with a focus on melanoma.

\section{Fibroblast Growth Factor Receptor Signaling Pathway in Normal Skin}

The skin is a complex organ. The epidermal layer is composed of keratinocytes, melanocytes, and inflammatory cells. The dermal layer is made up of fibroblasts, hair follicles, blood vessels, sweat glands, nerve endings, monocytes/macrophages, and T cells. Melanocytes are neural crest-derived cells, and mature melanocytes are anchored to the basement membrane, which holds together epidermis and dermis. Under physiological conditions, receptor signaling pathways are tightly controlled to provide skin homeostasis. Melanocytes produce pigment-containing melanosomes and transport them through dendrites to keratinocytes [39]. Melanocytes are under control of keratinocytes, and each melanocyte is connected with more than 30 keratinocytes to form the melanin unit protecting the skin from UV light. Several secreted factors, such as FGF2 (also known as bFGF, basic fibroblast growth factor), stem cell factor (SCF), hepatocyte growth factor (HGF), melanocyte stimulating hormone (MESH), and endothelin (ET), have been detected in the normal skin [40]. When secreted in response to ultraviolet (UV) light, they contribute to the stimulation of pigmentation and melanocyte proliferation via their receptors on melanocytes, e.g., FGF2 is secreted by UVB-exposed keratinocytes [41]. In general, melanocytes rarely undergo mitosis. Melanocytes mainly proliferate during the expansion of the skin surface in childhood or wound healing, and at a low rate upon stimulation by sunlight exposure. To undergo mitosis, they detach from keratinocyte and the basement membrane, and their dendrites are drawn out. After division, they migrate along the basement membrane to form a new melanin unit with keratinocytes. In normal skin, FGF2 is secreted by fibroblasts in the dermal layer and keratinocytes in the epidermis. FGF2 is highly mitogenic for melanocytes in vitro [41-43]. FGF2 could induce a transformed phenotype in melanocytes [44]; however, in another study, overexpression of FGF2 in skin xenografts induced hyperpigmentation and proliferation of melanocytes but no malignant transformation [45]. Melanoma-like lesions appeared when FGF2 overexpression was combined with UVB but not UVA [45,46]. FGF2 was also shown to be a melanocyte survival factor [47]. It was demonstrated that dermal nevus cells were able to survive in 3D type 1 collagen culture, whereas normal melanocytes underwent apoptosis unless the collagen culture was supplemented with FGF2 [47]. It was suggested that the higher level of FGF2 in the microenvironment of dermal nevus-derived melanocytes allowed melanocytes to adapt to grow in the dermis, which might be important for the development of melanoma. 


\section{Fibroblast Growth Factor Receptor Signaling in Melanoma and Other Skin Cancers}

The development and progression of melanoma is a complex process that usually consists of six steps: 1 . common acquired melanocytic nevi; 2 . melanocytic nevi with hyperplasia; 3 . melanocytic nevi with dysplasia; 4 . the radial growth phase (RGP) of primary melanoma; 5 . the vertical growth phase (VGP) of primary melanoma; 6 . metastatic melanoma [48]. In addition to being produced by neighboring fibroblasts and keratinocytes, FGF2 can be synthesized by cells in nevi and melanoma cells. In dysplastic nevi, moderate to high FGF2 levels were detected, whereas in common acquired nevi, contradicting data were obtained $[49,50]$. Melanoma progression was shown to be accompanied by increased FGF2 expression [51]. It was demonstrated that the switch to growth factor independence could distinguish RGP lesions from tumorigenic VGP primary melanoma [52]. A comparison of FGFR expression revealed an absent or very weak expression in nevi and diverse expression levels in primary and metastatic melanomas [53]. In a more recent study, FGF2 was detected in $72 \%$ of non-dysplastic nevi and only in $18 \%$ of dysplastic nevi [54]. Co-expression of FGF2 and FGFR1 was found in $60 \%$ of non-dysplastic nevi and in 18\% of dysplastic ones. FGFR1 was detected in $86 \%$, whereas FGF2 in $45 \%$ of primary melanomas [54]. In 2/3 of melanomas, FGF2 was also expressed by keratinocytes in the epidermis, and FGFR1 was commonly detected in the epidermis [54]. As suggested by the authors of the latter study, the discrepancies between the results obtained by different laboratories may be due to different primary antibodies used to detect FGF2 and FGFR1.

It has been demonstrated that melanoma cells stimulate their FGFRs in an autocrine manner [49,50,55-60]. An in vitro study showing melanoma cell independence on exogenously added growth factors suggested that the growth factors received by melanoma cells in a paracrine manner play a minor role [61]. Patient-derived $\mathrm{BRAF}^{\mathrm{V} 600 \mathrm{E}}$ melanoma cells could grow without serum and exogenous growth factors, FGF2, EGF (epidermal growth factor), and HGF (hepatocyte growth factor), for at least 4 months without substantial changes in viability and cell phenotype. Neither the cell cycle nor the activity of pathways important for melanoma maintenance, such as MAPK-ERK, WNT (wingless/integrated)/ $\beta$-catenin, and NF-KB (nuclear factor kappa B), were affected by the absence or presence of FGF2, HGF, and EGF used alone or in combination. More interestingly, lack of exogenous growth factors did not influence acute cell response to vemurafenib and trametinib, targeting $\mathrm{BRAF}^{\mathrm{V} 600 \mathrm{E}}$ and MEK1/2, respectively [61]. Although the expression of growth factors in melanoma cells was not blocked in this study and endogenous and exogenous level was only measured for HGF, another growth factor considered as important for melanoma [62], it is possible to conclude that endogenous growth factors released by melanoma cells and/or mutation-driven activation of the MAPK-ERK signaling pathway seem to be sufficient to maintain the major functions in a subset of melanoma cell lines. Mutation status might be also important for the sensitivity of melanoma cells to growth factors. It was demonstrated that wild-type melanoma cell lines were more responsive to FGF2 than BRAF mutant or NRAS mutant cell lines [63].

Co-expression of the pair FGF2 and FGFR1 in melanoma was extensively characterized in the 1980s and 1990s [64,65]. Early studies indicated that FGF2/FGFR1 might be of importance for autocrine growth control and melanoma progression $[42,44,53,66,67]$. Suppression of the FGFR1 activity inhibited cell proliferation and survival and induced cell differentiation [68,69]. Down-regulation of FGF2 in melanoma cells inhibited proliferation and colony formation $[42,66]$. Melanoma cells could not survive in vitro and in vivo if FGFR1 or FG2F were targeted [65,66,70-72]. Inhibition of FGF2 and FGFR1 in melanoma cells in vivo induced apoptosis and blocked intratumoral angiogenesis and tumor growth [65,71]. More recent studies have shown that the majority of melanoma cell lines concomitantly overexpressed FGF2, FGF5, and FGF18 and diverse isoforms of FGFRs [73]. For instance, FGF2 transcript levels were more than 100-fold higher in half of melanoma cell lines than in normal melanocytes [73], and FGF5 in 1/3 of melanoma cell lines [74]. FGF5 protein has been recently shown to be endogenously overexpressed in a subset of melanoma cell lines and in more than $60 \%$ of benign nevi and melanoma specimens [74]. These results were supported by data from the Cancer Genome Atlas [74]. Blockade of FGF/FGFR signaling by genetic constructs or kinase inhibitors inhibited 
melanoma growth, and synergistic anti-melanoma effects were obtained in vitro and in vivo if BRAF inhibitors were combined with FGFR inhibition [73].

Another interesting study suggesting that inhibition of the FGF/FGFR signaling might improve the response to targeted therapies has been published most recently [75]. In metastatic uveal melanoma (UM) that developed resistance to inhibitors of bromodomain and extra-terminal domain (BET) proteins, elevated levels of stromal FGF2, but not other factors, were detected. In addition, BET inhibitors could enhance FGFR expression in UM cell lines and patient tumor samples, while FGFR inhibitors (AZD4547 and BLU9931) reversed the effects of stromal FGF2. These results strongly suggest that co-inhibition of the FGF2/FGFR signaling pathway is necessary to prevent the development of resistance and improve the efficacy of BET inhibitors [75]. It was shown in a mouse model that FGF2 was secreted by liver cells at the site of metastasis. This may indicate that paracrine secretion of growth factors by organ-specific cells in the tumor microenvironment and elevated expression of FGFRs in melanoma cells are resistance mechanisms reducing treatment efficacy.

It has been shown that the FGF2/FGFR1 signaling is also crucial for melanoma angiogenesis as FGF2 secretion by melanoma cells could induce the mitogenic effects on endothelial cells and fibroblasts [65]. In VGP primary melanomas, co-expression of FGF2 and FGFR1 is significantly associated with increased density of microvessels [76]. FGF2 is known to stimulate the expression of vascular endothelial growth factor (VEGF) in vascular endothelial cells [77]. In a mouse model, when FGF2 was injected at the melanoma inoculation site during the initial phase of tumor growth, both, VEGF-A-dependent neovascularization of the host stroma and melanoma metastasis were enhanced [78]. Cancer cell metabolism substantially contributes to angiogenesis, metastasis, and suppression of the immune system. One of the important factors is lactate, which is secreted by cancer cells to the tumor microenvironment (TME), and it is taken up by endothelial cells. This, in turn, results in up-regulation of FGF2 and vascular endothelial growth factor receptor 2 (VEGFR2) signaling and induction of angiogenesis [79].

Besides melanocyte-originated melanoma, skin cancers include keratinocyte-originated seborrheic keratosis (SK), squamous cell carcinoma (SCC), and basal cell carcinoma (BCC), among others. Abnormal FGF/FGFR3 signaling has been observed in SK [80-84]. FGFR3 activation is, however, insufficient to drive skin tumors, such as SCC, and additional genetic and/or microenvironmental factors are required for epidermis to progress malignancy [85]. Autocrine FGF10/FGFR2 signaling may promote cutaneous SCC, representing about $25 \%$ of non-melanoma skin cancers [86]. It was shown in a mouse model of SCC that the enhanced mTOR (a mammalian target of rapamycin) signaling obtained in keratinocytes by deletion of Pten (phosphatase and tensin homolog deleted on chromosome 10) strongly enhanced the level of Fgf10 protein, and Pten deletion-induced skin cancers were inhibited by epidermal Fgfr2 deletion [86]. Moreover, in clinical samples, almost all SCC specimens showed a PTEN loss and an increase in FGF10 when compared to normal skin from a patient undergoing abdominoplasty. A previously published report suggested a tumor-suppressive function of the FGFR2 signaling in the skin [87]. These two opposing functions of FGF10 signaling-tumor suppressive and oncogenic-might be explained by different magnitudes of signaling [86]. In addition, the oncogenic function of FGF10/FGFR2 signaling might be especially potent in PTEN-deficient epidermis. It has been shown that the activity of PI3K in PTEN-deficient skin lesions is enhanced by the FGF-activated RAS-MAPK signaling [88].

It has been reported that FGF2 is overexpressed in another nonmelanocytic skin cancer, BCC [89]. FGF2 could induce angiogenesis and survival in BCC via STAT3 and PI3K/AKT pathways [90]. Dobesilate, the inhibitor of FGF, reduced the level of phosphorylated STAT3 in BCC, which was accompanied by the promotion of apoptosis, and inhibition of proliferation and angiogenesis [91]. In addition, dobesilate showed high efficacy in the topical treatment of two BCC patients [91]. 


\section{Mutations of Genes Encoding FGFRs in Melanoma and Other Skin Cancers}

The primary mechanism for abnormal signaling is connected with point mutations and chromosomal translocations that often result in constitutive dimerization and kinase activation of growth factor receptors, including FGFRs [92,93]. FGFR overexpression can be also achieved by repression of miRNAs targeting FGFR transcripts or up-regulation of long-non-coding RNAs (lncRNAs) sequestering FGFR-targeting miRNAs [94]. Elements of FGF/FGFR signaling are very frequently mutated kinases in cancers $[18,95]$, and DNA sequencing of cancer specimens has revealed a plethora of mutations in genes encoding FGFRs and FGFs.

According to an analysis based on data from cbioportal at http://www.cbioportal.org/public-portal (accessed November 2014) by Helsten et al. [17], the frequency of aberrations in FGFR reached 20\% in melanoma, and it is lower than in bladder urothelial carcinoma (35\%) or lung, squamous cell carcinoma (about $27 \%$ ). The frequency of FGF alterations in melanoma is about $38 \%$, and the highest frequency is observed in head and neck squamous cell carcinoma (nearly 54\%). Based on a large-scale high-throughput study, the same group showed that melanoma exerts one of the lowest frequencies of FGFR aberrations among all tested cancers [18].

Amplification of genes encoding FGFR can be found in many cancers, including head and neck squamous cell carcinoma, non-small-cell lung, breast and gastric cancers [96-100], but not in melanoma.

Chromosomal translocations can lead to fusion proteins with oncogenic potential. The most common fusion partner for FGFR, especially FGFR2 and FGFR3, is TACC3 encoding transforming acidic coiled-coil containing protein 3 [18]. This fusion was originally identified in glioblastoma [101] and was recently shown to cluster within transcriptional subgroups that have metabolic functions [102]. FGFR3-TACC3 fusion leads to a loss of the miR-99a binding site, which results in an up-regulation of fusion protein translation [103]. The first case of melanoma harboring an FGFR3-TACC3 fusion has been reported recently [104].

Non-synonymous somatic point mutations occur most commonly in FGFR3. Several somatic activating mutations have been found in FGFR3 in seborrheic keratoses, one of the most frequent benign epidermal tumors in older patients [81,105]. It has been demonstrated that one of these mutations in the extracellular domain of FGFR3, leading to the Ser249Cys substitution, can induce benign skin tumors in mouse epidermis [81]. The mutation leading to Arg248Cys has been also detected in adenoid seborrheic keratoses, very common benign skin tumors [106]. Mutations localized in the positions 248 and 249, with cysteine substitution, are thought to increase the stability of the FGFR3 dimer, which stimulates the receptor in a ligand-independent manner [107]. Stimulated receptor induces the transcription factor forkhead box N1 (FOXN1), which triggers the expression of FGFR3, and this feedback loop may antagonize RAS activity by promoting differentiation [84]. More recently, it has been reported that activating mutations in FGFR3 leading to Arg248Cys, Ser249Cys, and Gly697Cys can cause mild hyperplasia in the skin but are insufficient to induce benign or malignant skin cancers, including SK and SCC [85]. The Lys650Met FGFR3 mutation has been detected in acanthosis nigricans (AC), a benign skin tumor $[108,109]$. A mutation leading to the Gly380Arg substitution has been found in FGFR3 of cutaneous SCC [110]. The Gly380Arg FGFR3 mutation increases the ligand-independent phosphorylation of FGFR3, which causes constitutive activation of the downstream signaling pathways [111]. Interestingly, activating FGFR3 mutations, mainly Arg248Cys, can be also found in epidermal nevi, a common congenital skin lesion with an incidence of 1 in 1000 people [112]. It has been suggested that a large part of epidermal nevi results from mosaicism of activating FGFR3 mutations in the epidermis or postzygotic mutations in early embryonic development [112].

Pro252Arg/Ser/Thr somatic mutations in the fragment FGFR1 encoding its extracellular domain were identified among genetic drivers in human melanoma cell lines [113]. These gain of function mutations might increase receptor-ligand binding affinity by reducing the dissociation rate of the FGF ligand [114,115].

Similarly, as for genotypes of FGFR4 in breast, colorectal, and lung cancers [116,117], an SNP (rs351855) that results in Gly388Arg substitution can be found in melanomas. Fifty-five percent of 
melanoma patients had a homozygous Gly388 allele, while 45\% harbored at least one Arg388 allele [118]. Most recently, this mutation was found in three out of eight patient-derived melanoma cell lines [119]. Arg388 allele correlated with tumor thickness and was earlier associated with increased cell motility and invasiveness [116]. It has been shown that the Gly388Arg substitution induces conformational changes in receptor structure, which results in the enhanced association of FGFR4 with STAT3 [120]. The analysis of the literature has shown the lack of unambiguous evidence of the pro-tumorigenic and pro-metastatic impact of FGFR4 Arg388 allele in the majority of cancers, including melanoma [121].

Although loss-of-function mutations are not common in cancer, these type of aberrations (more than 20 different point mutations) have been found in FGFR2 in some melanoma cell lines and uncultured primary and metastatic tumors [122]. Therefore, FGFR2 was classified as melanoma suppressor [123]. Loss of receptor activity might be achieved through diverse mechanisms, such as loss of ligand binding affinity, retention in the endoplasmic reticulum, impaired receptor dimerization, and loss of tyrosinase activity [122]. It has been suggested that the inhibition of FGFR2 activity contributes to melanoma; however, the mechanism(s) is not clear, and reintroduction of FGFR2 failed to suppress melanoma cell proliferation [122]. As FGFR2 could induce melanoma growth arrest through interaction with stroma, its inhibition might promote melanoma invasion [123].

FGFR mutations reported in skin cancer patients and cell lines, and their relative location on the proteins are summarized in Table 1.

Table 1. Mutations of genes encoding fibroblast growth factor receptors (FGFRs) in melanoma and other skin cancers.

\begin{tabular}{|c|c|c|c|}
\hline Cancer & Gene & Encoded Substitution/Fusion & Refs \\
\hline acanthosis nigricans & FGFR3 & K650M & {$[108,109]$} \\
\hline adenoid seborrheic keratoses & FGFR3 & R248C & [106] \\
\hline squamous cell carcinoma & FGFR3 & G380R & [110] \\
\hline \multirow{5}{*}{ melanoma } & FGFR1 & $\mathrm{P} 252 \mathrm{R} / \mathrm{S} / \mathrm{T}$ & [113] \\
\hline & FGFR2 & $\begin{array}{l}\text { S24F, V77M, E160A, H213Y, E219K, } \\
\text { G227E, V248D, R251Q, G271E, } \\
\text { G305R, T370R, W474X, E475K, } \\
\text { D530N, E574K, E636K, M640I, I642V, } \\
\text { A648T, S688F, G702S, P708S, R759X, } \\
\text { R759Q, L770V }\end{array}$ & [122] \\
\hline & FGFR3 & FGFR3-TACC3 fusion & [104] \\
\hline & FGFR3 & P451S & [119] \\
\hline & FGFR4 & G388R & {$[118,119]$} \\
\hline
\end{tabular}

\section{FGF/FGFR Signaling in Resistance to Targeted Therapies}

The mechanisms of intrinsic and acquired resistance of melanoma to targeted therapies are diverse [36,124-128]. Reduced drug sensitivity of melanomas has been associated with increased expression/activities of multi-RTKs, including epidermal growth factor receptor (EGFR), cellular mesenchymal-epithelial transition factor (c-MET, hepatocyte growth factor receptor), platelet-derived growth factor receptor beta (PDGFRB), insulin growth factor 1 receptor (IGF1R), and AXL receptor tyrosinase kinase (AXL) [127,129-140]. FGF was among growth factors that were capable of attenuating the antiproliferative effects of vemurafenib, which was accompanied by the reduced ability of vemurafenib to inhibit pMEK [140]. This effect was reversed by FGFR inhibitor PD173074 [140]. FGF1 reactivated pERK in most melanoma cell lines treated with $\mathrm{BRAF}^{\mathrm{V} 600 \mathrm{E}}$ inhibitor in another study [132].

In drug-naïve $\mathrm{BRAF}^{\mathrm{V} 600}$ melanoma cells, the RTK expression is low, probably because the strong endogenous BRAF/NRAS mutation-driven activation of the MAPK-ERK signaling pathway selects, 
against cells with active RTK signaling, to prevent senescence [125]. FGFR1 and to some extent FGFR4 are exceptional in contrast to FGFR2-3, and these receptors are expressed in the majority of melanomas $[54,73,141]$. FGFR1 has been listed among resistance-associated genes expressed transiently in melanoma cells [142]. As suggested by Grimm et al., elevated FGFR1 expression allows melanoma cells to react immediately on ligands that are induced by drugs [143]. A therapy-induced senescence-like state is one of the options for melanoma cells to survive the treatment (Figure 2).

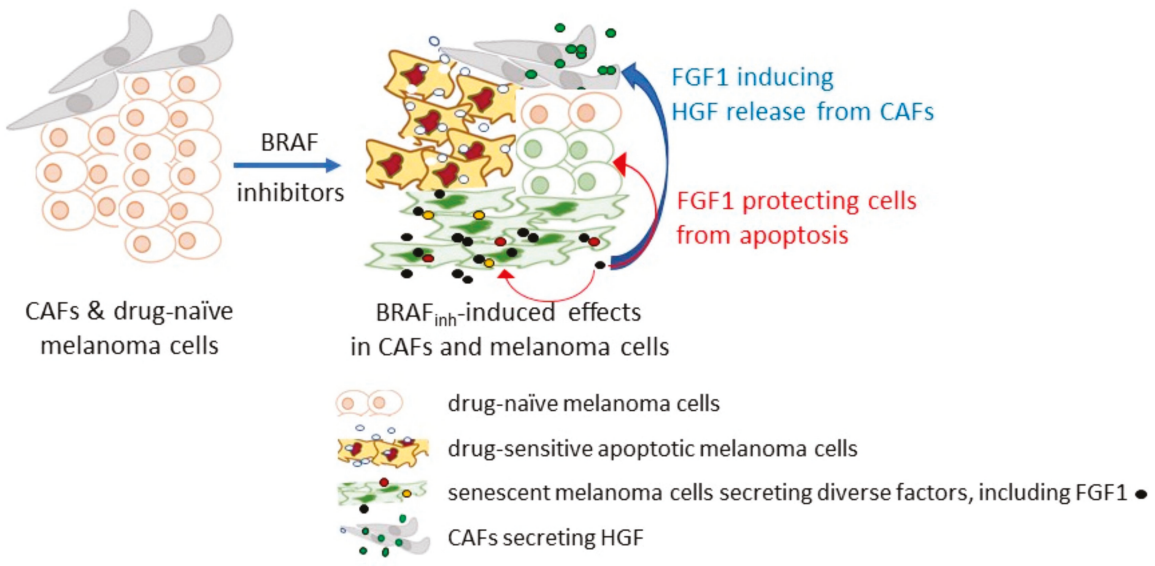

Figure 2. A simplified schematic illustration of fibroblast growth factor 1 (FGF1)-induced resistance to BRAF ( $v$-Raf murine sarcoma viral oncogene homolog B) inhibitors in melanoma cells. BRAF inhibitors, e.g., vemurafenib and mitogen-activated protein kinase (MEK) inhibitors, e.g., trametinib can induce diverse changes in drug-naïve melanoma cells, including apoptosis and premature senescence, while some melanoma cells remain unaffected. Thus, targeted therapy enhances the phenotypic heterogeneity of the neoplastic lesion in the tumor niche. Melanoma cells exerting the senescence-associated secretory phenotype (SASP) secrete several cytokines and growth factors, including FGF1. FGF1 released by senescent melanoma cells can stimulate melanoma cells and neighboring cells. Melanoma cells become protected from apoptosis, whereas cancer-associated fibroblasts (CAFs) are stimulated to secrete hepatocyte growth factor (HGF). Although these mechanisms are not universal and are not observed in all tested melanoma cell lines, they can contribute to drug resistance in a subset of melanomas. The scheme was prepared based on the study of Grimm et al. [143].

It has been demonstrated that the inhibition of $\mathrm{BRAF}^{\mathrm{V} 600}$ can induce a secretome with stimulating effects on both adjacent drug naïve melanoma cells and fibroblasts [143]. FGFs were found among senescence-associated factors expressed and released by melanoma cells in response to BRAF and MEK inhibition. It has been shown that FGF1 could limit the pro-apoptotic activities of drugs and activate fibroblasts to secrete HGF [143]. This contributes to "minimal residual disease", while continued BRAF ${ }^{\mathrm{V} 600}$ inhibition may induce secondary resistance mechanisms [143]. When inhibitors of FGFR have been applied in parallel to BRAF inhibitors, the resistance of melanoma cells was diminished. As the regulation of FGF1 was jointly mediated by FRA1 repression and activation of the PI3K/AKT signaling pathway, which is considered as the compensatory pathway activated by targeted therapy against $\mathrm{BRAF}^{\mathrm{V} 600 / \mathrm{MEK}}[144,145]$, obtained results suggest that targeting FGF/FGFR signaling pathway might be considered as the opportunity to block the compensatory pathway.

Considering cross-talk between the FGF/FGFR signaling and other oncogenic signaling pathways, the role of FGF/FGFR signaling in the development of acquired resistance to therapies targeting oncoproteins is conceivable. FGFR3 activation has been proposed as a mechanism of acquired resistance to vemurafenib in $\mathrm{BRAF}^{\mathrm{V} 600 \mathrm{E}}$ melanomas [146] and to cetuximab in KRAS wild type 
SCC [147]. Inhibition of the FGFR3/RAS axis could restore the sensitivity of resistant melanoma cells to vemurafenib [146].

A new variant of FGFR2 with a mutation leading to Gly542Glu was reported in $\mathrm{BRAF}^{\mathrm{V} 600 \mathrm{E}}$ melanoma patient during ongoing treatment with dabrafenib, a BRAF ${ }^{\mathrm{V} 600 \mathrm{E}}$ inhibitor, as an example of intratumor clonal evolution resulting in the development of resistance [148].

\section{FGF and FGFR as Molecular Prognostic Markers}

Several factors, such as dose of sun exposure, age, gender, fair skin phenotype, and previous keratinocyte skin cancers, are considered as causal factors of initiation and progression of melanoma, with UV-induced damage of DNA as the primary cause of melanoma development [149-151]. However, the incidence of melanoma in childhood and at sites that are minimally exposed to the sun are also registered $[152,153]$. There is hope that genome-wide studies will help to identify the heritable and environmentally-triggered contributors to melanoma risk [153-157]. Another important issue is to correctly prognose the melanoma progression. The 10-year survival among patients diagnosed with melanoma localized to the skin (stage I-II) is high if surgical treatment is applied. But still, it is unclear how to predict the recurrence in melanoma patients. Therefore, the development of prognostic tools applied at the time of diagnosis and surgery, which could select stage I-II melanoma patients of high recurrence risk for adjuvant therapy, might further reduce the burden of untreated melanomas. As melanoma is a highly heterogeneous tumor [32], currently used clinicopathologic prognostic markers do not account for all registered variability connected with melanoma progression. Also, none of the key mutations that emerged as the result of genome-wide genetic analysis could be unambiguously linked to melanoma progression. Therefore, molecular classifiers predictive of melanoma outcomes are in focus of several studies, and several models, including MelaPRO and GenoMELPREDICT, have been developed, and genetics consortium GenoMEL (www.genomel.org) has been established.

Loss-of-function mutations in FGFR2 and activating mutations in FGFR4 have been connected with melanoma. In a large nested case-control study of Caucasian women, however, neither mutations in FGFR2 nor in FGFR4 has been found as contributing to an inherited predisposition to three skin cancer types, including melanoma, SCC, and BCC [158].

Therefore, it has been suggested that rather than serving as predisposition indicators, genetic aberrations in FGFR2 and FGFR4 might be used as potential biomarkers of melanoma progression. FGFR4 has been detected at the protein level in $45 \%$ of 137 melanoma specimens [118]. It has been demonstrated that its expression correlated with pTNM melanoma stage $(p=0.046)$, vascularity $(p=0.001)$, microulceration $(p=0.009)$, metastases $(p=0.025)$, overall survival $(p=0.047)$, and disease-free survival $(p=0.046)$. Moreover, Arg 388 polymorphism was present in $45 \%$ of melanoma patients. The FGFR4Arg388 allele has been associated with intensified melanoma motility and invasiveness [116]. Therefore, both, expression of FGFR4 and Arg388 genotype was suggested as potential biomarkers for the progression of melanoma, although no further study has been published to extend these findings.

Analysis of 418 manuscripts revealed several proteins that could potentially stratify melanoma, and FGFR1 was listed among 67 proteins categorized as contributing to self-sufficiency in growth signals [159]; although in an earlier study, there was no correlation between expression of FGFR1 and the usual clinicopathological features of melanoma [141].

Up to date, none of the elements of the FGF/FGFR signaling pathway is included as the biomarker in the diagnosis of melanoma.

\section{Targeting Aberrant FGF/FGFR Signaling in the Clinic}

The FGFR inhibitors are still under development, and there are currently no FGFR-targeted therapies, which are approved for the treatment of cancer patients [160-162]. Results of early-phase clinical trials from different cancers suggest that selective FGFR inhibitors could be useful in treating 
patients with FGFR fusions and selected patients with FGFR2 amplification [160]. It is thought that inhibitors of FGFRs evoke effects on cancer cells but also indirectly through paracrine signaling on angiogenesis and immune evasion [94].

While in the preclinical melanoma models, a small molecule FGFR inhibitor SU5402 has demonstrated activity [73], it was not tested in clinical trials. In a phase I study, lenvatinib, an oral tyrosine kinase inhibitor, was evaluated in melanoma patients, where tumor shrinkage was observed, but many adverse effects were also reported [163].

In the ongoing LOGIC-2 phase II clinical trial (https://clinicaltrials.gov/ct2/show/NCT02159066) for advanced BRAF melanomas, one of the FGFR1-3 selective inhibitors, BGJ398 (Infigratinib), was included as the third agent used in combination with LGX818 (Encorafenib), a potent BRAF inhibitor, and MEK162 (Binimetinib), a selective inhibitor of MEK. The study design allows applying BGJ398 after progression from therapy against BRAF/MEK. The estimated LOGIC-2 study completion date for advanced melanoma patients is 2022. BGJ398 was/is investigated in several clinical trials (phase I and II), and, so far, the analysis of the results indicated a substantial variability in response rates. In a phase I study, it demonstrated partial response in four patients with FGFR1-amplified non-small-cell carcinoma (NSCLC) and stable disease in 14 patients [164]. Only one breast cancer patient with FGFR1-amplified showed tumor regression, and partial responses were reported in FGFR3-mutated bladder cancer, while in one patient with hepatocellular carcinoma (HCC) and in one patient with cholangiocarcinoma (CCA), both with FGFR2-BICC1 (bicaudal C homolog 1) fusion gene, tumor shrinkage was observed [155]. In the ongoing phase II clinical studies, BGJ398 is investigated in advanced-stage gastrointestinal stromal tumors (https://clinicaltrials.gov/ct2/show/NCT02257541) and advanced CCA (https://clinicaltrials.gov/ct2/show/NCT02150967) and other hematological and solid cancers with FGFR genetic alterations (https://clinicaltrials.gov/ct2/show/NCT02160041). Three patients with CCA, who progressed on BGJ398, acquired recurrent polyclonal point mutations in the kinase domain of fusion FGFR2, which led to the development of drug resistance [165].

Several challenges are recognized in clinical trials applying FGFR inhibitors, including dosing limitations, diversity of the mechanisms activating FGF/FGFR signaling, and difficulties in prospective selection of patients with specific aberrations in FGFRs.

\section{Conclusions}

The FGF/FGFR signaling does not markedly contribute to the development of melanoma as it is suppressed by the overactivated RAS/BRAF/MEK/ERK (MAPK) signaling pathway. When BRAF/MEK inhibitors are applied, and the pathway is blocked, the FGF/FGFR signaling might be considered as one of the mechanisms that lead to the development of resistance limiting the success of targeted therapies against BRAF and MEK. First attempts are undertaken in the clinics (LOGIC-2) to find out whether targeting the FGF/FGFR signaling pathway with BGJ398 (Infigratinib) may be a good therapeutic strategy in combination with BRAF/MEK inhibitors.

Funding: This work was supported by the Grant 2014/15/B/NZ7/00947 from National Science Center (Poland).

Acknowledgments: I would like to thank Markus Düchler for helpful comments and Julita Talaj for her assistance in the preparation of the reference list.

Conflicts of Interest: The author declares no conflict of interest.

\section{References}

1. Porębska, N.; Latko, M.; Kucińska, M.; Zakrzewska, M.; Otlewski, J.; Opaliński, Ł. Targeting Cellular Trafficking of Fibroblast Growth Factor Receptors as a Strategy for Selective Cancer Treatment. J. Clin. Med. 2018, 8, E7. [CrossRef] [PubMed]

2. Eswarakumar, V.P.; Lax, I.; Schlessinger, J. Cellular signaling by fibroblast growth factor receptors. Cytokine Growth Factor Rev. 2005, 16, 139-149. [CrossRef] [PubMed] 
3. Holzmann, K.; Grunt, T.; Heinzle, C.; Sampl, S.; Steinhoff, H.; Reichmann, N.; Kleiter, M.; Hauck, M.; Marian, B. Alternative Splicing of Fibroblast Growth Factor Receptor IgIII Loops in Cancer. J. Nucleic Acids 2012, 2012, 950508. [CrossRef] [PubMed]

4. Latko, M.; Czyrek, A.; Porębska, N.; Kucińska, M.; Otlewski, J.; Zakrzewska, M.; Opaliński, Ł. Cross-Talk between Fibroblast Growth Factor Receptors and Other Cell Surface Proteins. Cells 2019, 8, E455. [CrossRef] [PubMed]

5. Belov, A.A.; Mohammadi, M. Molecular mechanisms of fibroblast growth factor signaling in physiology and pathology. Cold Spring Harb. Perspect. Biol. 2013, 5, a015958. [CrossRef] [PubMed]

6. Gotoh, N. Regulation of growth factor signaling by FRS2 family docking/scaffold adaptor proteins. Cancer Sci. 2008, 99, 1319-1325. [CrossRef]

7. Bignell, G.R.; Greenman, C.D.; Davies, H.; Butler, A.P.; Edkins, S.; Andrews, J.M.; Buck, G.; Chen, L.; Beare, D.; Latimer, C.; et al. Signatures of mutation and selection in the cancer genome. Nature 2010, 463, 893-898. [CrossRef] [PubMed]

8. Bignell, G.R.; Santarius, T.; Pole, J.C.; Butler, A.P.; Perry, J.; Pleasance, E.; Greenman, C.; Menzies, A.; Taylor, S.; Edkins, S.; et al. Architectures of somatic genomic rearrangement in human cancer amplicons at sequence-level resolution. Genome Res. 2007, 17, 1296-1303. [CrossRef]

9. Valsesia, A.; Rimoldi, D.; Martinet, D.; Ibberson, M.; Benaglio, P.; Quadroni, M.; Waridel, P.; Gaillard, M.; Pidoux, M.; Rapin, B.; et al. Network-guided analysis of genes with altered somatic copy number and gene expression reveals pathways commonly perturbed in metastatic melanoma. PLoS ONE 2011, 6, e18369. [CrossRef]

10. Dorey, K.; Amaya, E. FGF signalling: Diverse roles during early vertebrate embryogenesis. Development 2010, 137, 3731-3742. [CrossRef]

11. Bobbs, A.S.; Saarela, A.V.; Yatskievych, T.A.; Antin, P.B. Fibroblast growth factor (FGF) signaling during gastrulation negatively modulates the abundance of microRNAs that regulate proteins required for cell migration and embryo patterning. J. Biol. Chem. 2012, 287, 38505-38514. [CrossRef]

12. Schelch, K.; Wagner, C.; Hager, S.; Pirker, C.; Siess, K.; Lang, E.; Lin, R.; Kirschner, M.B.; Mohr, T.; Brcic, L.; et al. FGF2 and EGF induce epithelial-mesenchymal transition in malignant pleural mesothelioma cells via a MAPKinase/MMP1 signal. Carcinogenesis 2018, 39, 534-545. [CrossRef] [PubMed]

13. Dienstmann, R.; Rodon, J.; Prat, A.; Perez-Garcia, J.; Adamo, B.; Felip, E.; Cortes, J.; Iafrate, A.J.; Nuciforo, P.; Tabernero, J. Genomic aberrations in the FGFR pathway: Opportunities for targeted therapies in solid tumors. Ann. Oncol. 2014, 25, 552-563. [CrossRef] [PubMed]

14. Turner, N.; Grose, R. Fibroblast growth factor signalling: From development to cancer. Nat. Rev. Cancer 2010, 10, 116-129. [CrossRef] [PubMed]

15. Touat, M.; Ileana, E.; Postel-Vinay, S.; André, F.; Soria, J.C. Targeting FGFR Signaling in Cancer. Clin. Cancer Res. 2015, 21, 2684-2694. [CrossRef] [PubMed]

16. Prieto-Dominguez, N.; Shull, A.Y.; Teng, Y. Making way for suppressing the FGF19/FGFR4 axis in cancer. Future Med. Chem. 2018, 10, 2457-2470. [CrossRef] [PubMed]

17. Helsten, T.; Schwaederle, M.; Kurzrock, R. Fibroblast growth factor receptor signaling in hereditary and neoplastic disease: Biologic and clinical implications. Cancer Metastasis Rev. 2015, 34, 479-496. [CrossRef] [PubMed]

18. Helsten, T.; Elkin, S.; Arthur, E.; Tomson, B.N.; Carter, J.; Kurzrock, R. The FGFR Landscape in Cancer: Analysis of 4,853 Tumors by Next-Generation Sequencing. Clin. Cancer Res. 2016, 22, 259-267. [CrossRef] [PubMed]

19. Adler, N.R.; Haydon, A.; McLean, C.A.; Kelly, J.W.; Mar, V.J. Metastatic pathways in patients with cutaneous melanoma. Pigment Cell Melanoma Res. 2017, 30, 13-27. [CrossRef] [PubMed]

20. Hayward, N.K.; Wilmott, J.S.; Waddell, N.; Johansson, P.A.; Field, M.A.; Nones, K.; Patch, A.M.; Kakavand, H.; Alexandrov, L.B.; Burke, H.; et al. Whole-genome landscapes of major melanoma subtypes. Nature 2017, 545, 175-180. [CrossRef] [PubMed]

21. The Cancer Genome Atlas Network. Genomic classification of cutaneous melanoma. Cell 2015, 161, 1681-1696. [CrossRef] [PubMed]

22. Hodis, E.; Watson, I.R.; Kryukov, G.V.; Arold, S.T.; Imielinski, M.; Theurillat, J.P.; Nickerson, E.; Auclair, D.; Li, L.; Place, C.; et al. A landscape of driver mutations in melanoma. Cell 2012, 150, 251-263. [CrossRef] [PubMed] 
23. Ascierto, P.A.; Kirkwood, J.M.; Grob, J.J.; Simeone, E.; Grimaldi, A.M.; Maio, M.; Palmieri, G.; Testori, A.; Marincola, F.M.; Mozzillo, N. The role of BRAF V600 mutation in melanoma. J. Transl. Med. 2012, 10, 85. [CrossRef] [PubMed]

24. Muñoz-Couselo, E.; Adelantado, E.Z.; Ortiz, C.; García, J.S.; Perez-Garcia, J. NRAS-mutant melanoma: Current challenges and future prospect. Onco Targets Ther. 2017, 10, 3941-3947. [CrossRef] [PubMed]

25. Luke, J.J.; Flaherty, K.T.; Ribas, A.; Long, G.V. Targeted agents and immunotherapies: Optimizing outcomes in melanoma. Nat. Rev. Clin. Oncol. 2017, 14, 463-482. [CrossRef]

26. Volpe, V.O.; Klufas, D.M.; Hegde, U.; Grant-Kels, J.M. The new paradigm of systemic therapies for metastatic melanoma. J. Am. Acad. Dermatol. 2017, 77, 356-368. [CrossRef]

27. Ugurel, S.; Röhmel, J.; Ascierto, P.A.; Flaherty, K.T.; Grob, J.J.; Hauschild, A.; Larkin, J.; Long, G.V.; Lorigan, P.; McArthur, G.A.; et al. Survival of patients with advanced metastatic melanoma: The impact of novel therapies-update 2017. Eur. J. Cancer 2017, 83, 247-257. [CrossRef]

28. Kaufman, H.L.; Margolin, K.; Sullivan, R. Management of Metastatic Melanoma in 2018. JAMA Oncol. 2018, 4, 857-858. [CrossRef]

29. Gatzka, M.V. Targeted Tumor Therapy Remixed-An Update on the Use of Small-Molecule Drugs in Combination Therapies. Cancers (Basel) 2018, 10, E155. [CrossRef]

30. Wang, L.; Leite de Oliveira, R.; Huijberts, S.; Bosdriesz, E.; Pencheva, N.; Brunen, D.; Bosma, A.; Song, J.Y.; Zevenhoven, J.; Los-de Vries, G.T.; et al. An Acquired Vulnerability of Drug-Resistant Melanoma with Therapeutic Potential. Cell 2018, 173, 1413-1425. [CrossRef]

31. Teh, J.L.F.; Aplin, A.E. Playing the Melanoma Endgame. Clin. Cancer Res. 2018, 24, 4629-4630. [CrossRef]

32. Ahmed, F.; Haass, N.K. Microenvironment-Driven Dynamic Heterogeneity and Phenotypic Plasticity as a Mechanism of Melanoma Therapy Resistance. Front. Oncol. 2018, 8, 173. [CrossRef] [PubMed]

33. Welsh, S.J.; Rizos, H.; Scolyer, R.A.; Long, G.V. Resistance to combination BRAF and MEK inhibition in metastatic melanoma: Where to next? Eur. J. Cancer 2016, 62, 76-85. [CrossRef] [PubMed]

34. Rizos, H.; Menzies, A.M.; Pupo, G.M.; Carlino, M.S.; Fung, C.; Hyman, J.; Haydu, L.E.; Mijatov, B.; Becker, T.M.; Boyd, S.C.; et al. BRAF inhibitor resistance mechanisms in metastatic melanoma: Spectrum and clinical impact. Clin. Cancer Res. 2014, 20, 1965-1977. [CrossRef]

35. Van Allen, E.M.; Wagle, N.; Sucker, A.; Treacy, D.J.; Johannessen, C.M.; Goetz, E.M.; Place, C.S.; Taylor-Weiner, A.; Whittaker, S.; Kryukov, G.V. The genetic landscape of clinical resistance to RAF inhibition in metastatic melanoma. Cancer Discov. 2014, 4, 94-109. [CrossRef]

36. Luebker, S.A.; Koepsell, S.A. Diverse Mechanisms of BRAF Inhibitor Resistance in Melanoma Identified in Clinical and Preclinical Studies. Front. Oncol. 2019, 9, 268. [CrossRef]

37. Eggermont, A.; Suciu, S.; Kicinski, M. Estimation of Distant Metastasis-free Survival in Trials of Adjuvant Therapy for Melanoma. N. Engl. J. Med. 2019, 380, 1374-1376. [CrossRef]

38. Heinzle, C.; Sutterlüty, H.; Grusch, M.; Grasl-Kraupp, B.; Berger, W.; Marian, B. Targeting fibroblast-growth-factor-receptor-dependent signaling for cancer therapy. Expert Opin. Ther. Targets 2011, 15, 829-846. [CrossRef]

39. Jimbow, K. Current update and trends in melanin pigmentation and melanin biology. Keio J. Med. 1995, 44, 9-18. [CrossRef]

40. Haass, N.K.; Herlyn, M. Normal human melanocyte homeostasis as a paradigm for understanding melanoma. J. Investig. Dermatol. Symp. Proc. 2005, 10, 153-163. [CrossRef]

41. Halaban, R.; Langdon, R.; Birchall, N.; Cuono, C.; Baird, A.; Scott, G.; Moellmann, G.; McGuire, J. Basic fibroblast growth factor from human keratinocytes is a natural mitogen for melanocytes. J. Cell. Biol. 1988, 107, 1611-1619. [CrossRef] [PubMed]

42. Halaban, R.; Kwon, B.S.; Ghosh, S.; Delli Bovi, P.; Baird, A. bFGF as an autocrine growth factor for human melanomas. Oncogene Res. 1988, 3, 177-186. [PubMed]

43. Root, L.L.; Shipley, G.D. Human dermal fibroblasts express multiple bFGF and aFGF proteins. In Vitro Cell. Dev. Biol. 1991, 27A, 815-822. [CrossRef] [PubMed]

44. Nesbit, M.; Nesbit, H.K.; Bennett, J.; Andl, T.; Hsu, M.Y.; Dejesus, E.; McBrian, M.; Gupta, A.R.; Eck, S.L.; Herlyn, M. Basic fibroblast growth factor induces a transformed phenotype in normal human melanocytes. Oncogene 1999, 18, 6469-6476. [CrossRef] [PubMed]

45. Berking, C.; Takemoto, R.; Satyamoorthy, K.; Elenitsas, R.; Herlyn, M. Basic fibroblast growth factor and ultraviolet B transform melanocytes in human skin. Am. J. Pathol. 2001, 158, 943-953. [CrossRef] 
46. Berking, C.; Takemoto, R.; Satyamoorthy, K.; Shirakawa, T.; Eskandarpour, M.; Hansson, J.; VanBelle, P.A.; Elder, D.E.; Herlyn, M. Induction of melanoma phenotypes in human skin by growth factors and ultraviolet B. Cancer Res. 2004, 64, 807-811. [CrossRef]

47. Alanko, T.; Rosenberg, M.; Saksela, O. FGF expression allows nevus cells to survive in three-dimensional collagen gel under conditions that induce apoptosis in normal human melanocytes. J. Invest. Dermatol. 1999, 113, 111-116. [CrossRef]

48. Clark, W.H., Jr.; Elder, D.E.; Guerry, D., 4th; Epstein, M.N.; Greene, M.H.; Van Horn, M. A study of tumor progression: The precursor lesions of superficial spreading and nodular melanoma. Hum. Pathol. 1984, 15, 1147-1165. [CrossRef]

49. Scott, G.; Stoler, M.; Sarkar, S.; Halaban, R. Localization of basic fibroblast growth factor mRNA in melanocytic lesions by in situ hybridization. J. Invest. Dermatol. 1991, 96, 318-322. [CrossRef]

50. Reed, J.A.; McNutt, N.S.; Albino, A.P. Differential expression of basic fibroblast growth factor (bFGF) in melanocytic lesions demonstrated by in situ hybridisation. Implications for tumor progression. Am. J. Pathol. 1994, 144, 329-336.

51. Birck, A.; Kirkin, A.F.; Zeuthen, J.; Hou-Jensen, K. Expression of basic fibroblast growth factor and vascular endothelial factor in primary and metastatic melanoma from the same patients. Melanoma Res. 1999, 9, 375-381. [CrossRef]

52. Kath, R.; Jambrosic, J.A.; Holland, L.; Rodeck, U.; Herlyn, M. Development of invasive and growth factor-independent cell variants from primary human melanomas. Cancer Res. 1991, 51, 2205-2211.

53. Ahmed, N.U.; Ueda, M.; Ito, A.; Ohashi, A.; Funasaka, Y.; Ichihashi, M. Expression of fibroblast growth factor receptors in naevus-cell naevus and malignant melanoma. Melanoma Res. 1997, 7, 299-305. [CrossRef]

54. Giehl, K.A.; Nägele, U.; Volkenandt, M.; Berking, C. Protein expression of melanocyte growth factors (bFGF, SCF) and their receptors (FGFR-1, c-kit) in nevi and melanoma. J. Cutan. Pathol. 2007, 34, 7-14. [CrossRef]

55. Halaban, R.; Ghosh, S.; Baird, A. bFGF is the putative natural growth factor for human melanocytes. In Vitro Cell. Dev. Biol. 1987, 23, 47-52. [CrossRef]

56. Halaban, R.; Langdon, R.; Birchall, N.; Cuono, C.; Baird, A.; Scott, G.; Moellmann, G.; McGuire, J. Paracrine stimulation of melanocytes by keratinocytes through basic fibroblast growth factor. Ann. NY Acad. Sci. 1988, 548, 180-190. [CrossRef]

57. Albino, A.P.; Davis, B.M.; Nanus, D.M. Induction of growth factor RNA expression in human malignant melanoma: Markers of transformation. Cancer Res. 1991, 51, 4815-4820.

58. Al-Alousi, S.; Barnhill, R.; Blessing, K.; Barksdale, S. The prognostic significance of basic fibroblast growth factor in cutaneous malignant melanoma. J. Cutan. Pathol. 1996, 23, 506-510. [CrossRef]

59. Al-Alousi, S.; Carlson, J.A.; Blessing, K.; Cook, M.; Karaoli, T.; Barnhill, R.L. Expression of basic fibroblast growth factor in desmoplastic melanoma. J. Cutan. Pathol. 1996, 23, 118-125. [CrossRef]

60. Meier, F.; Caroli, U.; Satyamoorthy, K.; Schittek, B.; Bauer, J.; Berking, C.; Moller, H.; Maczey, E.; Rassner, G.; Herlyn, M.; et al. Fibroblast growth factor-2 but not Mel-CAM and/or beta3 integrin promotes progression of melanocytes to melanoma. Exp. Dermatol. 2003, 12, 296-306. [CrossRef]

61. Zalesna, I.; Osrodek, M.; Hartman, M.L.; Rozanski, M.; Sztiller-Sikorska, M.; Niewinna, K.; Nejc, D.; Czyz, M. Exogenous growth factors bFGF, EGF and HGF do not influence viability and phenotype of V600EBRAF melanoma cells and their response to vemurafenib and trametinib in vitro. PLoS ONE 2017, 12, e0183498. [CrossRef]

62. Czyz, M. HGF/c-MET Signaling in Melanocytes and Melanoma. Int. J. Mol. Sci. 2018, 19, E3844. [CrossRef]

63. Garay, T.; Molnár, E.; Juhász, É.; László, V.; Barbai, T.; Dobos, J.; Schelch, K.; Pirker, C.; Grusch, M.; Berger, W.; et al. Sensitivity of Melanoma Cells to EGFR and FGFR Activation but Not Inhibition is Influenced by Oncogenic BRAF and NRAS Mutations. Pathol. Oncol. Res. 2015, 21, 957-968. [CrossRef]

64. Kaipainen, A.; Vlaykova, T.; Hatva, E.; Bohling, T.; Jekunen, A.; Pyrhonen, S.; Alitalo, K. Enhanced expression of the tie receptor tyrosine kinase mesenger RNA in the vascular endothelium of metastatic melanomas. Cancer Res. 1994, 54, 6571-6577.

65. Wang, Y.; Becker, D. Antisense targeting of basic fibroblast growth factor and fibroblast growth factor receptor-1 in human melanomas blocks intratumoral angiogenesis and tumor growth. Nat. Med. 1997, 3, 887-893. [CrossRef]

66. Becker, D.; Meier, C.B.; Herlyn, M. Proliferation of human malignant melanomas is inhibited by antisense oligodeoxynucleotides targeted against basic fibroblast growth factor. EMBO J. 1989, 8, 3685-3691. [CrossRef] 
67. Meier, F.; Nesbit, M.; Hsu, M.Y.; Martin, B.; Van Belle, P.; Elder, D.E.; Schaumburg-Lever, G.; Garbe, C.; Walz, T.M.; Donatien, P.; et al. Human melanoma progression in skin reconstructs: Biological significance of bFGF. Am. J. Pathol. 2000, 156, 193-200. [CrossRef]

68. Yayon, A.; Ma, Y.S.; Safran, M.; Klagsbrun, M.; Halaban, R. Suppression of autocrine cell proliferation and tumorigenesis of human melanoma cells and fibroblast growth factor transformed fibroblasts by a kinase deficient FGF receptor 1: Evidence for the involvement of Src-family kinases. Oncogene 1997, 14, 2999-3009. [CrossRef]

69. Becker, D.; Lee, P.L.; Rodeck, U.; Herlyn, M. Inhibition of the fibroblast growth factor receptor 1 (FGFR-1) gene in human melanocytes and malignant melanomas leads to inhibition of proliferation and signs indicative of differentiation. Oncogene 1992, 7, 2303-2313.

70. Graeven, U.; Rodeck, U.; Karpinski, S.; Jost, M.; Philippou, S.; Schmiegel, W. Modulation of angiogenesis and tumorigenicity of human melanocytic cells by vascular endothelial growth factor and basic fibroblast growth factor. Cancer Res. 2001, 61, 7282-7290.

71. Valesky, M.; Spang, A.J.; Fisher, G.W.; Farkas, D.L.; Becker, D. Noninvasive dynamic fluorescence imaging of human melanomas reveals that targeted inhibition of bFGF or FGFR-1 in melanoma cells blocks tumor growth by apoptosis. Mol. Med. 2002, 8, 103-112. [CrossRef]

72. Ozen, M.; Medrano, E.E.; Ittmann, M. Inhibition of proliferation and survival of melanoma cells by adenoviral-mediated expression of dominant negative fibroblast growth factor receptor. Melanoma Res. 2004, 14, 13-21. [CrossRef]

73. Metzner, T.; Bedeir, A.; Held, G.; Peter-Vörösmarty, B.; Ghassemi, S.; Heinzle, C.; Spiegl-Kreinecker, S.; Marian, B.; Holzmann, K.; Grasl-Kraupp, B.; et al. Fibroblast growth factor receptors as therapeutic targets in human melanoma: Synergism with BRAF inhibition. J. Invest. Dermatol. 2011, 131, 2087-2095. [CrossRef]

74. Ghassemi, S.; Vejdovszky, K.; Sahin, E.; Ratzinger, L.; Schelch, K.; Mohr, T.; Peter-Vörösmarty, B.; Brankovic, J.; Lackner, A.; Leopoldi, A.; et al. FGF5 is expressed in melanoma and enhances malignancy in vitro and in vivo. Oncotarget 2017, 8, 87750-87762. [CrossRef]

75. Chua, V.; Orloff, M.; The, J.L.; Sugase, T.; Liao, C.; Purwin, T.J.; Lam, B.Q.; Terai, M.; Ambrosini, G.; Carvajal, R.D.; et al. Stromal fibroblast growth factor 2 reduces the efficacy of bromodomain inhibitors in uveal melanoma. EMBO Mol. Med. 2019, 11, e9081. [CrossRef]

76. Straume, O.; Akslen, L.A. Importance of vascular phenotype by basic fibroblast growth factor, and influence of the angiogenic factors basic fibroblast growth factor/fibroblast growth factor receptor-1 and ephrin-A1/EphA2 on melanoma progression. Am. J. Pathol. 2002, 160, 1009-1019. [CrossRef]

77. Seghezzi, G.; Patel, S.; Ren, C.J.; Gualandris, A.; Pintucci, G.; Robbins, E.S.; Shapiro, R.L.; Galloway, A.C.; Rifkin, D.B.; Mignatti, P. Fibroblast growth factor-2 (FGF-2) induces vascular endothelial growth factor (VEGF) expression in the endothelial cells of forming capillaries: An autocrine mechanism contributing to angiogenesis. J. Cell. Biol. 1998, 141, 1659-1673. [CrossRef]

78. Tsunoda, S.; Nakamura, T.; Sakurai, H.; Saiki, I. Fibroblast growth factor-2-induced host stroma reaction during initial tumor growth promotes progression of mouse melanoma via vascular endothelial growth factor A-dependent neovascularization. Cancer Sci. 2007, 98, 541-548. [CrossRef]

79. Sonveaux, P.; Copetti, T.; De Saedeleer, C.J.; Végran, F.; Verrax, J.; Kennedy, K.M.; Moon, E.J.; Dhup, S.; Danhier, P.; Frérart, F.; et al. Targeting the lactate transporter MCT1 in endothelial cells inhibits lactate-induced HIF-1 activation and tumor angiogenesis. PLoS ONE 2012, 7, e33418. [CrossRef]

80. Kubo, Y.; Matsudate, Y.; Fukui, N.; Nakasuka, A.; Sogawa, M.; Oshima, M.; Mizutani, T.; Otsu, M.; Murao, K.; Hashimoto, I. Molecular tumorigenesis of the skin. J. Med. Invest. 2014, 61, 7-14. [CrossRef]

81. Logié, A.; Dunois-Lardé, C.; Rosty, C.; Levrel, O.; Blanche, M.; Ribeiro, A.; Gasc, J.M.; Jorcano, J.; Werner, S.; Sastre-Garau, X.; et al. Activating mutations of the tyrosine kinase receptor FGFR3 are associated with benign skin tumors in mice and humans. Hum. Mol. Genet. 2005, 14, 1153-1160. [CrossRef]

82. Hafner, C.; Hartmann, A.; van Oers, J.M.; Stoehr, R.; Zwarthoff, E.C.; Hofstaedter, F.; Landthaler, M.; Vogt, T. FGFR3 mutations in seborrheic keratoses are already present in flat lesions and associated with age and localization. Mod. Pathol. 2007, 20, 895-903. [CrossRef]

83. Hafner, C.; Toll, A.; Fernández-Casado, A.; Earl, J.; Marqués, M.; Acquadro, F.; Méndez-Pertuz, M.; Urioste, M.; Malats, N.; Burns, J.E.; et al. Multiple oncogenic mutations and clonal relationship in spatially distinct benign human epidermal tumors. Proc. Natl. Acad. Sci. USA 2010, 107, 20780-20785. [CrossRef] 
84. Mandinova, A.; Kolev, V.; Neel, V.; Hu, B.; Stonely, W.; Lieb, J.; Wu, X.; Colli, C.; Han, R.; Pazin, M.J.; et al. A positive FGFR3/FOXN1 feedback loop underlies benign skin keratosis versus squamous cell carcinoma formation in humans. J. Clin. Invest. 2009, 119, 3127-3137. [CrossRef]

85. Duperret, E.K.; Oh, S.J.; McNeal, A.; Prouty, S.M.; Ridky, T.W. Activating FGFR3 mutations cause mild hyperplasia in human skin, but are insufficient to drive benign or malignant skin tumors. Cell Cycle 2014, 13, 1551-1559. [CrossRef]

86. Hertzler-Schaefer, K.; Mathew, G.; Somani, A.K.; Tholpady, S.; Kadakia, M.P.; Chen, Y.; Spandau, D.F.; Zhang, X. Pten loss induces autocrine FGF signaling to promote skin tumorigenesis. Cell Rep. 2014, 6, 818-826. [CrossRef]

87. Grose, R.; Fantl, V.; Werner, S.; Chioni, A.M.; Jarosz, M.; Rudling, R.; Cross, B.; Hart, I.R.; Dickson, C. The role of fibroblast growth factor receptor $2 \mathrm{~b}$ in skin homeostasis and cancer development. EMBO J. 2007, 26, 1268-1278. [CrossRef]

88. Mathew, G.; Hannan, A.; Hertzler-Schaefer, K.; Wang, F.; Feng, G.S.; Zhong, J.; Zhao, J.J.; Downward, J.; Zhang, X. Targeting of Ras-mediated FGF signaling suppresses Pten-deficient skin tumor. Proc. Natl. Acad. Sci. USA 2016, 113, 13156-13161. [CrossRef]

89. Arbiser, J.L.; Byers, H.R.; Cohen, C.; Arbeit, J. Altered basic fibroblast growth factor expression in common epidermal neoplasms: Examination with in situ hybridization and immunohistochemistry. J. Am. Acad. Dermatol. 2000, 42, 973-977. [CrossRef]

90. Jee, S.H.; Chu, C.Y.; Chiu, H.C.; Huang, Y.L.; Tsai, W.L.; Liao, Y.H.; Kuo, M.L. Interleukin-6 induced basic fibroblast growth factor-dependent angiogenesis in basal cell carcinoma cell line via JAK/STAT3 and PI3-kinase/Akt pathways. J. Invest. Dermatol. 2004, 123, 1169-1175. [CrossRef]

91. Cuevas, P.; Calvo, M.; Angulo, J.; Cuevas-Bourdier, A.N.; Giménez-Gallego, G. Efficacy of the fibroblast growth factor inhibitor 2,5-dihydroxyphenylsulfonate in basal cell carcinoma: A histopathological and inmunohistochemical study. J. Dermatolog. Treat. 2011, 22, 348-352. [CrossRef]

92. Gallo, L.H.; Nelson, K.N.; Meyer, A.N.; Donoghue, D.J. Functions of Fibroblast Growth Factor Receptors in cancer defined by novel translocations and mutations. Cytokine Growth Factor Rev. 2015, 26, 425-449. [CrossRef]

93. Wu, Y.M.; Su, F.; Kalyana-Sundaram, S.; Khazanov, N.; Ateeq, B.; Cao, X.; Lonigro, R.J.; Vats, P.; Wang, R.; Lin, S.F.; et al. Identification of targetable FGFR gene fusions in diverse cancers. Cancer Discov. 2013, 3, 636-647. [CrossRef]

94. Katoh, M. FGFR inhibitors: Effects on cancer cells, tumor microenvironment and whole-body homeostasis (Review). Int. J. Mol. Med. 2016, 38, 3-15. [CrossRef]

95. Greenman, C.; Stephens, P.; Smith, R.; Dalgliesh, G.L.; Hunter, C.; Bignell, G.; Davies, H.; Teague, J.; Butler, A.; Stevens, C.; et al. Patterns of somatic mutation in human cancer genomes. Nature 2007, 446, 153-158. [CrossRef]

96. Dubot, C.; Bernard, V.; Sablin, M.P.; Vacher, S.; Chemlali, W.; Schnitzler, A.; Pierron, G.; Ait Rais, K.; Bessoltane, N.; Jeannot, E.; et al. Comprehensive genomic profiling of head and neck squamous cell carcinoma reveals FGFR1 amplifications and tumour genomic alterations burden as prognostic biomarkers of survival. Eur. J. Cancer 2018, 91, 47-55. [CrossRef]

97. Cihoric, N.; Savic, S.; Schneider, S.; Ackermann, I.; Bichsel-Naef, M.; Schmid, R.A.; Lardinois, D.; Gugger, M.; Bubendorf, L.; Zlobec, I.; et al. Prognostic role of FGFR1 amplification in early-stage non-small cell lung cancer. Br. J. Cancer 2014, 110, 2914-2922. [CrossRef]

98. Yang, W.; Yao, Y.W.; Zeng, J.L.; Liang, W.J.; Wang, L.; Bai, C.Q.; Liu, C.H.; Song, Y. Prognostic value of FGFR1 gene copy number in patients with non-small cell lung cancer: A meta-analysis. J. Thorac. Dis. 2014, 6, 803-809. [CrossRef]

99. Lee, H.J.; Seo, A.N.; Park, S.Y.; Kim, J.Y.; Park, J.Y.; Yu, J.H.; Ahn, J.H.; Gong, G. Low prognostic implication of fibroblast growth factor family activation in triple-negative breast cancer subsets. Ann. Surg. Oncol. 2014, 21, 1561-1568. [CrossRef]

100. Matsumoto, K.; Arao, T.; Hamaguchi, T.; Shimada, Y.; Kato, K.; Oda, I.; Taniguchi, H.; Koizumi, F.; Yanagihara, K.; Sasaki, H.; et al. FGFR2 gene amplification and clinicopathological features in gastric cancer. Br. J. Cancer 2012, 106, 727-732. [CrossRef] 
101. Singh, D.; Chan, J.M.; Zoppoli, P.; Niola, F.; Sullivan, R.; Castano, A.; Liu, E.M.; Reichel, J.; Porrati, P.; Pellegatta, S.; et al. Transforming fusions of FGFR and TACC genes in human glioblastoma. Science 2012, 337, 1231-1235. [CrossRef]

102. Frattini, V.; Pagnotta, S.M.; Talla; Fan, J.J.; Russo, M.V.; Lee, S.B.; Garofano, L.; Zhang, J.; Shi, P.; Lewis, G.; et al. A metabolic function of FGFR3-TACC3 gene fusions in cancer. Nature 2018, 553, 222-227. [CrossRef]

103. Parker, B.C.; Annala, M.J.; Cogdell, D.E.; Granberg, K.J.; Sun, Y.; Ji, P.; Li, X.; Gumin, J.; Zheng, H.; Hu, L.; et al. The tumorigenic FGFR3-TACC3 gene fusion escapes miR-99a regulation in glioblastoma. J. Clin. Invest. 2013, 123, 855-865. [CrossRef]

104. Lee, J.; Lee, J.; Hong, S.D.; Jang, K-T.; Lee, S.J. FGFR3-TACC3: A novel gene fusion in malignant melanoma. Precis. Future Med. 2018, 2, 71-75. [CrossRef]

105. Heidenreich, B.; Denisova, E.; Rachakonda, S.; Sanmartin, O.; Dereani, T.; Hosen, I.; Nagore, E.; Kumar, R. Genetic alterations in seborrheic keratoses. Oncotarget 2017, 8, 36639-36649. [CrossRef]

106. Hafner, C.; van Oers, J.M.; Hartmann, A.; Landthaler, M.; Stoehr, R.; Blaszyk, H.; Hofstaedter, F.; Zwarthoff, E.C.; Vogt, T. High frequency of FGFR3 mutations in adenoid seborrheic keratoses. J. Invest. Dermatol. 2006, 126, 2404-2407. [CrossRef]

107. Hafner, C.; Vogt, T.; Hartmann, A. FGFR3 mutations in benign skin tumors. Cell Cycle 2006, 5, 2723-2728. [CrossRef]

108. Bellus, G.A.; Bamshad, M.J.; Przylepa, K.A.; Dorst, J.; Lee, R.R.; Hurko, O.; Jabs, E.W.; Curry, C.J.; Wilcox, W.R.; Lachman, R.S.; et al. Severe achondroplasia with developmental delay and acanthosis nigricans (SADDAN): Phenotypic analysis of a new skeletal dysplasia caused by a Lys650Met mutation in fibroblast growth factor receptor 3. Am. J. Med. Genet. 1999, 85, 53-65. [CrossRef]

109. Muguet Guenot, L.; Aubert, H.; Isidor, B.; Toutain, A.; Mazereeuw-Hautier, J.; Collet, C.; Bourrat, E.; Denis Musquer, M.; Barbarot, S.; Groupe de Recherche de la Société Française de Dermatologie Pédiatrique. Acanthosis nigricans, hypochondroplasia, and FGFR3 mutations: Findings with five new patients, and a review of the literature. Pediatr. Dermatol. 2019, 36, 242-246. [CrossRef]

110. Capalbo, C.; Belardinilli, F.; Filetti, M.; Parisi, C.; Petroni, M.; Colicchia, V.; Tessitore, A.; Santoni, M.; Coppa, A.; Giannini, G.; et al. Effective treatment of a platinum-resistant cutaneous squamous cell carcinoma case by EGFR pathway inhibition. Mol. Clin. Oncol. 2018, 9, 30-34. [CrossRef]

111. L'Hôte, C.G.; Knowles, M.A. Cell responses to FGFR3 signalling: Growth, differentiation and apoptosis. Exp. Cell Res. 2005, 304, 417-431. [CrossRef]

112. Hafner, C.; van Oers, J.M.; Vogt, T.; Landthaler, M.; Stoehr, R.; Blaszyk, H.; Hofstaedter, F.; Zwarthoff, E.C.; Hartmann, A. Mosaicism of activating FGFR3 mutations in human skin causes epidermal nevi. J. Clin. Invest. 2006, 116, 2201-2207. [CrossRef]

113. Dahl, C.; Christensen, C.; Jönsson, G.; Lorentzen, A.; Skjødt, M.L.; Borg, Å.; Pawelec, G.; Guldberg, P. Mutual exclusivity analysis of genetic and epigenetic drivers in melanoma identifies a link between p14 ARF and RAR $\beta$ signaling. Mol. Cancer Res. 2013, 11, 1166-1178. [CrossRef]

114. Ibrahimi, O.A.; Eliseenkova, A.V.; Plotnikov, A.N.; Yu, K.; Ornitz, D.M.; Mohammadi, M. Structural basis for fibroblast growth factor receptor 2 activation in Apert syndrome. Proc. Natl. Acad. Sci. USA 2001, 98, 7182-7187. [CrossRef]

115. Ibrahimi, O.A.; Zhang, F.; Eliseenkova, A.V.; Itoh, N.; Linhardt, R.J.; Mohammadi, M. Biochemical analysis of pathogenic ligand-dependent FGFR2 mutations suggests distinct pathophysiological mechanisms for craniofacial and limb abnormalities. Hum. Mol. Genet. 2004, 13, 2313-2324. [CrossRef]

116. Bange, J.; Prechtl, D.; Cheburkin, Y.; Specht, K.; Harbeck, N.; Schmitt, M.; Knyazeva, T.; Müller, S.; Gärtner, S.; Sures, I.; et al. Cancer progression and tumor cell motility are associated with the FGFR4 Arg388 allele. Cancer Res. 2002, 62, 840-847.

117. Templeton, A.J.; Diez-Gonzalez, L.; Ace, O.; Vera-Badillo, F.; Seruga, B.; Jordán, J.; Amir, E.; Pandiella, A.; Ocaña, A. Prognostic relevance of receptor tyrosine kinase expression in breast cancer: A meta-analysis. Cancer Treat. Rev. 2014, 40, 1048-1055. [CrossRef]

118. Streit, S.; Mestel, D.S.; Schmidt, M.; Ullrich, A.; Berking, C. FGFR4 Arg388 allele correlates with tumour thickness and FGFR4 protein expression with survival of melanoma patients. Br. J. Cancer 2006, 94, 1879-1886. [CrossRef]

119. Hartman, M.L.; Sztiller-Sikorska, M.; Czyz, M. Whole-exome sequencing reveals novel genetic variants associated with diverse phenotypes of melanoma cells. Mol. Carcinog. 2019, 58, 588-602. [CrossRef] 
120. Ulaganathan, V.K.; Sperl, B.; Rapp, U.R.; Ullrich, A. Germline variant FGFR4 p.G388R exposes a membrane-proximal STAT3 binding site. Nature 2015, 528, 570-574. [CrossRef]

121. Heinzle, C.; Erdem, Z.; Paur, J.; Grasl-Kraupp, B.; Holzmann, K.; Grusch, M.; Berger, W.; Marian, B. Is fibroblast growth factor receptor 4 a suitable target of cancer therapy? Curr. Pharm. Des. 2014, 20, 2881-2898. [CrossRef]

122. Gartside, M.G.; Chen, H.; Ibrahimi, O.A.; Byron, S.A.; Curtis, A.V.; Wellens, C.L.; Bengston, A.; Yudt, L.M.; Eliseenkova, A.V.; Ma, J.; et al. Loss-of-function fibroblast growth factor receptor-2 mutations in melanoma. Mol. Cancer Res. 2009, 7, 41-54. [CrossRef]

123. Rother, J.; Jones, D. Molecular markers of tumor progression in melanoma. Curr. Genomics 2009, 10, $231-239$. [CrossRef]

124. Wagle, N.; Emery, C.; Berger, M.F.; Davis, M.J.; Sawyer, A.; Pochanard, P.; Kehoe, S.M.; Johannessen, C.M.; Macconaill, L.E.; Hahn, W.C.; et al. Dissecting therapeutic resistance to RAF inhibition in melanoma by tumor genomic profiling. J. Clin. Oncol. 2011, 29, 3085-3096. [CrossRef]

125. Sun, C.; Wang, L.; Huang, S.; Heynen, G.J.; Prahallad, A.; Robert, C.; Haanen, J.; Blank, C.; Wesseling, J.; Willems, S.M. Reversible and adaptive resistance to BRAF(V600E) inhibition in melanoma. Nature 2014, 508, 118-122. [CrossRef]

126. Zaleśna, I.; Hartman, M.L.; Czyż, M. BRAF mutation in progression and therapy of melanoma, papillary thyroid carcinoma and colorectal adenocarcinoma. Postepy Hig. Med. Dosw. 2016, 70, 471-488. [CrossRef]

127. Song, C.; Piva, M.; Sun, L.; Hong, A.; Moriceau, G.; Kong, X.; Zhang, H.; Lomeli, S.; Qian, J.; Yu, C.C.; et al. Recurrent Tumor Cell-Intrinsic and -Extrinsic Alterations during MAPKi-Induced Melanoma Regression and Early Adaptation. Cancer Discov. 2017, 7, 1248-1265. [CrossRef]

128. Lu, H.; Liu, S.; Zhang, G.; Wu, B.; Zhu, Y.; Frederick, D.T.; Hu, Y.; Zhong, W.; Randell, S.; Sadek, N.; et al. PAK signalling drives acquired drug resistance to MAPK inhibitors in BRAF-mutant melanomas. Nature 2017, 550, 133-136. [CrossRef]

129. Nazarian, R.; Shi, H.; Wang, Q.; Kong, X.; Koya, R.C.; Lee, H.; Chen, Z.; Lee, M.K.; Attar, N.; Sazegar, H.; et al. Melanomas acquire resistance to B-RAF(V600E) inhibition by RTK or N-RAS upregulation. Nature 2010, 468, 973-977. [CrossRef]

130. Müller, J.; Krijgsman, O.; Tsoi, J.; Robert, L.; Hugo, W.; Song, C.; Kong, X.; Possik, P.A.; Cornelissen-Steijger, P.D.; Geukes Foppen, M.H.; et al. Low MITF/AXL ratio predicts early resistance to multiple targeted drugs in melanoma. Nat. Commun. 2014, 5, 5712. [CrossRef]

131. Turajlic, S.; Furney, S.J.; Stamp, G.; Rana, S.; Ricken, G.; Oduko, Y.; Saturno, G.; Springer, C.; Hayes, A.; Gore, M.; et al. Whole-genome sequencing reveals complex mechanisms of intrinsic resistance to BRAF inhibition. Ann. Oncol. 2014, 25, 959-967. [CrossRef]

132. Straussman, R.; Morikawa, T.; Shee, K.; Barzily-Rokni, M.; Qian, Z.R.; Du, J.; Davis, A.; Mongare, M.M.; Gould, J.; Frederick, D.T.; et al. Tumour micro-environment elicits innate resistance to RAF inhibitors through HGF secretion. Nature 2012, 487, 500-504. [CrossRef]

133. Zuo, Q.; Liu, J.; Huang, L.; Qin, Y.; Hawley, T.; Seo, C.; Merlino, G.; Yu, Y. AXL/AKT axis mediated-resistance to BRAF inhibitor depends on PTEN status in melanoma. Oncogene 2018, 37, 3275-3289. [CrossRef]

134. Chan, X.Y.; Singh, A.; Osman, N.; Piva, T.J. Role Played by Signalling Pathways in Overcoming BRAF Inhibitor Resistance in Melanoma. Int. J. Mol. Sci. 2017, 18, E1527. [CrossRef]

135. Kwong, L.N.; Davies, M.A. Targeted therapy for melanoma: Rational combinatorial approaches. Oncogene 2014, 33, 1-9. [CrossRef]

136. Villanueva, J.; Vultur, A.; Lee, J.T.; Somasundaram, R.; Fukunaga-Kalabis, M.; Cipolla, A.K.; Wubbenhorst, B.; Xu, X.; Gimotty, P.A.; Kee, D.; et al. Acquired resistance to BRAF inhibitors mediated by a RAF kinase switch in melanoma can be overcome by cotargeting MEK and IGF-1R/PI3K. Cancer Cell 2010, 18, 683-695. [CrossRef]

137. Girotti, M.R.; Pedersen, M.; Sanchez-Laorden, B.; Viros, A.; Turajlic, S.; Niculescu-Duvaz, D.; Zambon, A.; Sinclair, J.; Hayes, A.; Gore, M.; et al. Inhibiting EGF receptor or SRC family kinase signaling overcomes BRAF inhibitor resistance in melanoma. Cancer Discov. 2013, 3, 158-167. [CrossRef]

138. Wilson, T.R.; Fridlyand, J.; Yan, Y.; Penuel, E.; Burton, L.; Chan, E.; Peng, J.; Lin, E.; Wang, Y.; Sosman, J.; et al. Widespread potential for growth-factor-driven resistance to anticancer kinase inhibitors. Nature 2012, 487 , 505-509. [CrossRef] 
139. Vergani, E.; Vallacchi, V.; Frigerio, S.; Deho, P.; Mondellini, P.; Perego, P.; Cassinelli, G.; Lanzi, C.; Testi, M.A.; Rivoltini, L.; et al. Identification of MET and SRC activation in melanoma cell lines showing primary resistance to PLX4032. Neoplasia 2011, 13, 1132-1142. [CrossRef]

140. Lito, P.; Pratilas, C.A.; Joseph, E.W.; Tadi, M.; Halilovic, E.; Zubrowski, M.; Huang, A.; Wong, W.L.; Callahan, M.K.; Merghoub, T.; et al. Relief of profound feedback inhibition of mitogenic signaling by RAF inhibitors attenuates their activity in BRAFV600E melanomas. Cancer Cell 2012, 22, 668-682. [CrossRef]

141. Xerri, L.; Battyani, Z.; Grob, J.J.; Parc, P.; Hassoun, J.; Bonerandi, J.J.; Birnbaum, D. Expression of FGF1 and FGFR1 in human melanoma tissues. Melanoma Res. 1996, 6, 223-230. [CrossRef]

142. Shaffer, S.M.; Dunagin, M.C.; Torborg, S.R.; Torre, E.A.; Emert, B.; Krepler, C.; Beqiri, M.; Sproesser, K.; Brafford, P.A.; Xiao, M.; et al. Rare cell variability and drug-induced reprogramming as a mode of cancer drug resistance. Nature 2017, 546, 431-435. [CrossRef]

143. Grimm, J.; Hufnagel, A.; Wobser, M.; Borst, A.; Haferkamp, S.; Houben, R.; Meierjohann, S. BRAF inhibition causes resilience of melanoma cell lines by inducing the secretion of FGF1. Oncogenesis 2018, 7, 71. [CrossRef]

144. Deng, W.; Gopal, Y.N.; Scott, A.; Chen, G.; Woodman, S.E.; Davies, M.A. Role and therapeutic potential of PI3K-mTOR signaling in de novo resistance to BRAF inhibition. Pigment. Cell Melanoma Res. 2012, 25, 248-258. [CrossRef]

145. Shi, H.; Kong, X.; Ribas, A.; Lo, R.S. Combinatorial treatments that overcome PDGFR $\beta$-driven resistance of melanoma cells to V600EB-RAF inhibition. Cancer Res. 2011, 71, 5067-5074. [CrossRef]

146. Yadav, V.; Zhang, X.; Liu, J.; Estrem, S.; Li, S.; Gong, X.Q.; Buchanan, S.; Henry, J.R.; Starling, J.J.; Peng, S.B. Reactivation of mitogen-activated protein kinase (MAPK) pathway by FGF receptor 3 (FGFR3)/Ras mediates resistance to vemurafenib in human B-RAF V600E mutant melanoma. J. Biol. Chem. 2012, 287, 28087-28098. [CrossRef]

147. Oliveras-Ferraros, C.; Cufí, S.; Queralt, B.; Vazquez-Martin, A.; Martin-Castillo, B.; de Llorens, R.; Bosch-Barrera, J.; Brunet, J.; Menendez, J.A. Cross-suppression of EGFR ligands amphiregulin and epiregulin and de-repression of FGFR3 signalling contribute to cetuximab resistance in wild-type KRAS tumour cells. Br. J. Cancer 2012, 106, 1406-1414. [CrossRef]

148. Mak, G.; Arkenau, H.T.; Chin, M. Resistance surveillance in a BRAF mutant melanoma patient on long-term BRAF-inhibitor treatment. Melanoma Res. 2014, 24, 408-412. [CrossRef]

149. Emri, G.; Paragh, G.; Tósaki, Á.; Janka, E.; Kollár, S.; Hegedûs, C.; Gellén, E.; Horkay, I.; Koncz, G.; Remenyik, É. Ultraviolet radiation-mediated development of cutaneous melanoma: An update. J. Photochem. Photobiol. B. 2018, 185, 169-175. [CrossRef]

150. Markovic, S.N.; Erickson, L.A.; Rao, R.D.; Weenig, R.H.; Pockaj, B.A.; Bardia, A.; Vachon, C.M.; Schild, S.E.; McWilliams, R.R.; Hand, J.L.; et al. Malignant melanoma in the 21st century, part 1: Epidemiology, risk factors, screening, prevention, and diagnosis. Mayo Clin. Proc. 2007, 82, 364-380. [CrossRef]

151. Whiteman, D.C.; Bray, C.A.; Siskind, V.; Hole, D.; MacKie, R.M.; Green, A.C. A comparison of the anatomic distribution of cutaneous melanoma in two populations with different levels of sunlight: The west of Scotland and Queensland, Australia 1982-2001. Cancer Causes Control. 2007, 18, 485-491. [CrossRef]

152. Lu, C.; Zhang, J.; Nagahawatte, P.; Easton, J.; Lee, S.; Liu, Z.; Ding, L.; Wyczalkowski, M.A.; Valentine, M.; Navid, F.; et al. The genomic landscape of childhood and adolescent melanoma. J. Invest. Dermatol. 2015, 135, 816-823. [CrossRef]

153. Pellegrini, C.; Botta, F.; Massi, D.; Martorelli, C.; Facchetti, F.; Gandini, S.; Maisonneuve, P.; Avril, M.F.; Demenais, F.; Bressac-de Paillerets, B.; et al. MC1R variants in childhood and adolescent melanoma: A retrospective pooled analysis of a multicentre cohort. Lancet Child. Adolesc. Health 2019, 3, 332-342. [CrossRef]

154. Kypreou, K.P.; Stefanaki, I.; Antonopoulou, K.; Karagianni, F.; Ntritsos, G.; Zaras, A.; Nikolaou, V.; Kalfa, I.; Chasapi, V.; Polydorou, D.; et al. Prediction of Melanoma Risk in a Southern European Population Based on a Weighted Genetic Risk Score. J. Invest. Dermatol. 2016, 136, 690-695. [CrossRef]

155. Cust, A.E.; Drummond, M.; Kanetsky, P.A. Assessing the Incremental Contribution of Common Genomic Variants to Melanoma Risk Prediction in Two Population-Based Studies. J. Invest. Dermatol. 2018, 138, 2617-2624. [CrossRef]

156. Taylor, N.J.; Mitra, N.; Qian, L.; Avril, M.F.; Bishop, D.T.; Paillerets, B.B.; Bruno, W.; Calista, D.; Cuellar, F.; Cust, A.E.; et al. Estimating CDKN2A mutation carrier probability among global familial melanoma cases using GenoMELPREDICT. J. Am. Acad. Dermatol. 2019. [CrossRef] 
157. Duffy, D.L.; Zhu, G.; Li, X.; Sanna, M.; Iles, M.M.; Jacobs, L.C.; Evans, D.M.; Yazar, S.; Beesley, J.; Law, M.H.; et al. Novel pleiotropic risk loci for melanoma and nevus density implicate multiple biological pathways. Nat. Commun. 2019, 10, 299. [CrossRef]

158. Nan, H.; Qureshi, A.A.; Hunter, D.J.; Han, J. Genetic variants in FGFR2 and FGFR4 genes and skin cancer risk in the Nurses' Health Study. BMC Cancer 2009, 9, 172. [CrossRef]

159. Gould Rothberg, B.E.; Rimm, D.L. Biomarkers: The useful and the not so useful-An assessment of molecular prognostic markers for cutaneous melanoma. J. Invest. Dermatol. 2010, 130, 1971-1987. [CrossRef]

160. Babina, I.S.; Turner, N.C. Advances and challenges in targeting FGFR signalling in cancer. Nat. Rev. Cancer 2017, 17, 318-332. [CrossRef]

161. Farrell, B.; Breeze, A.L. Structure, activation and dysregulation of fibroblast growth factor receptor kinases: Perspectives for clinical targeting. Biochem. Soc. Trans. 2018, 46, 1753-1770. [CrossRef]

162. Lang, L.; Teng, Y. Fibroblast Growth Factor Receptor 4 Targeting in Cancer: New Insights into Mechanisms and Therapeutic Strategies. Cells 2019, 8, E31. [CrossRef]

163. Hong, D.S.; Kurzrock, R.; Wheler, J.J.; Naing, A.; Falchook, G.S.; Fu, S.; Kim, K.B.; Davies, M.A.; Nguyen, L.M.; George, G.C.; et al. Phase I Dose-Escalation Study of the Multikinase Inhibitor Lenvatinib in Patients with Advanced Solid Tumors and in an Expanded Cohort of Patients with Melanoma. Clin. Cancer Res. 2015, 21, 4801-4810. [CrossRef]

164. Nogova, L.; Sequist, L.V.; Perez Garcia, J.M.; Andre, F.; Delord, J.P.; Hidalgo, M.; Schellens, J.H.; Cassier, P.A.; Camidge, D.R.; Schuler, M.; et al. Evaluation of BGJ398, a Fibroblast Growth Factor Receptor 1-3 Kinase Inhibitor, in Patients With Advanced Solid Tumors Harboring Genetic Alterations in Fibroblast Growth Factor Receptors: Results of a Global Phase I, Dose-Escalation and Dose-Expansion Study. J. Clin. Oncol. 2017, 35, 157-165.

165. Goyal, L.; Saha, S.K.; Liu, L.Y.; Siravegna, G.; Leshchiner, I.; Ahronian, L.G.; Lennerz, J.K.; Vu, P.; Deshpande, V.; Kambadakone, A.; et al. Polyclonal Secondary FGFR2 Mutations Drive Acquired Resistance to FGFR Inhibition in Patients with FGFR2 Fusion-Positive Cholangiocarcinoma. Cancer Discov. 2017, 7, 252-263. [CrossRef]

(C) 2019 by the author. Licensee MDPI, Basel, Switzerland. This article is an open access article distributed under the terms and conditions of the Creative Commons Attribution (CC BY) license (http://creativecommons.org/licenses/by/4.0/). 


\title{
FGF19-FGFR4 Signaling in Hepatocellular Carcinoma
}

\author{
Aroosha Raja ${ }^{1}$, Inkeun Park ${ }^{2}$, Farhan Haq ${ }^{1, *}$ and Sung-Min Ahn ${ }^{2,3, *}$ \\ 1 Department of Biosciences, Comsats University, Islamabad 45550, Pakistan; aroosha.raja@gmail.com \\ 2 Division of Medical Oncology, Department of Internal Medicine, Gachon University Gil Medical Center, \\ Incheon 21565, Korea; ingni79@hanmail.net \\ 3 Department of Genome Medicine and Science, College of Medicine, Gachon University, Incheon 21565, Korea \\ * Correspondence: farhan.haq@comsats.edu.pk (F.H.); smahn@gachon.ac.kr or \\ ahnsungmin@gmail.com (S.-M.A.); Tel.: +92-334-954-0954 (F.H.); +92-032-460-2177 or 010-3648-7437 (S.-M.A.)
}

Received: 10 May 2019; Accepted: 29 May 2019; Published: 4 June 2019

\begin{abstract}
Hepatocellular carcinoma (HCC) is the sixth most common type of cancer, with an increasing mortality rate. Aberrant expression of fibroblast growth factor 19-fibroblast growth factor receptor 4 (FGF19-FGFR4) is reported to be an oncogenic-driver pathway for HCC patients. Thus, the FGF19-FGFR4 signaling pathway is a promising target for the treatment of HCC. Several pan-FGFR (1-4) and FGFR4-specific inhibitors are in different phases of clinical trials. In this review, we summarize the information, recent developments, binding modes, selectivity, and clinical trial phases of different available FGFR4/pan-FGF inhibitors. We also discuss future perspectives and highlight the points that should be addressed to improve the efficacy of these inhibitors.
\end{abstract}

Keywords: prognosis; FGF19; FGFR4; HCC; inhibitors

\section{Introduction}

Hepatocellular carcinoma (HCC) is the sixth most common type of cancer, with the fourth highest mortality rate [1]. Despite advancements in therapeutic strategies, the response rate and overall survival rate are still low [2]. The most common cause of HCC is liver cirrhosis from any etiology including hepatitis $\mathrm{B}$ and hepatitis $\mathrm{C}$ infection, excessive alcohol consumption, diabetes mellitus, and non-alcoholic fatty liver disease [3]. Moreover, various molecular pathways are involved in the initiation and progression of HCC [4]. With respect to these pathways, there is evidence demonstrating the role of fibroblast growth factor pathway genes in HCC prognosis [5].

The fibroblast growth factors ( $F G F s$ ) family comprises a large family of growth factors that are found in different multicellular organisms [6]. The FGFs signal through four transmembrane tyrosine kinase fibroblast growth factor receptors (FGFRs) namely FGFR1, FGFR2, FGFR3, and FGFR4 [7]. FGFs-FGFRs are involved in regulation of many biological processes such as embryonic development, cell proliferation, differentiation, and tissue repair [8]. FGF-FGFR dysregulation is also widely reported in different types of diseases, disorders, and cancers [9]. Notably, aberrant expression of FGF19/FGFR4 contributes to HCC progression [10].

Since sorafenib marked a new era in molecularly targeted therapy in advanced HCC [11], various drugs such as lenvatinib, regorafenib, cabozantinib, nivolumab, and ramucirumab have subsequently demonstrated overall survival benefits for patients [12-16]. However, the treatment outcome of metastatic HCC is still unsatisfactory, with a median overall survival below 15 months [12]. Thus, more effective treatment options for advanced HCC are needed. This can be achieved by a better understanding of the underlying genetic mechanisms involved in HCC. This review aims to provide comprehensive landscape of current information available on the FGF19-FGFR4 pathway. It also discusses recent advancements on FGF19-FGFR4 inhibitors in HCC. The data is obtained by systematic analysis of the literature and by using different text-mining approaches. 


\section{Overview of FGFR4 and FGF19}

\subsection{Structure and Function of FGFR4}

Fibroblast growth factor receptor 4 (FGFR4) is a protein coding gene and is a member of tyrosine kinase receptors family. The human FGFR4 gene is located on chromosome 5 and measures 11.41 bp in length [17]. The FGFR4 protein coded by two full transcripts of FGFR4 gene consists of $\sim 800$ amino acids, with molecular weight of around 95-110 kDa [18]. The structure of FGFR4 proteins contains three immunoglobin-like domains (D1-D3), a transmembrane domain, and the kinase domain [19]. (Figure 1) Among these immunoglobin-like domains, first two have role in receptor auto-inhibition, while the third domain is involved in specific binding of ligands [20]. The kinase domain (intracellular) is important in activation of downstream pathways [21]. Further, the kinase domain comprises the N-terminal (smaller) and C-terminal (larger) canonical domains [22]. FGF receptors differ from each other in tissue specificity and ligand-binding affinity. However, good identity scores are found between the kinase domains of FGFR4 and other FGF receptors [22]. The expression of FGFR4 is highly tissue-specific due to its unique ligand binding affinity [23]. At a functional level, FGFR4 is predominantly involved in regeneration of muscles, regulation of lipid metabolism, bile acid biosynthesis, cell proliferation, differentiation, glucose uptake, and myogenesis [24]. Of note, it is reported that FGFR4 is mostly expressed in liver tissue [25].

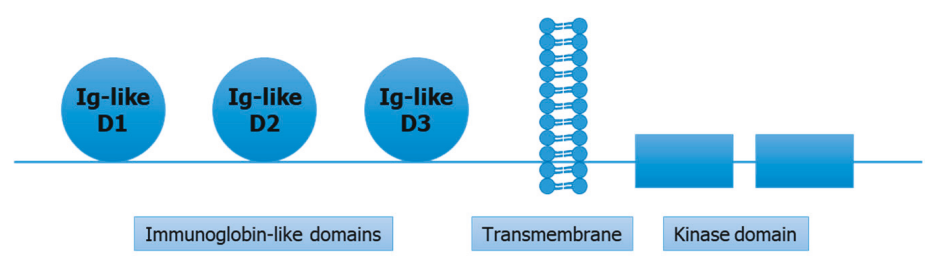

Figure 1. Structural overview of fibroblast growth factor receptor 4 (FGFR4) protein.

\subsection{FGFR4 in Cancer}

FGFR4 exerts a combination of biological effects that contribute to different hallmarks of cancer (Figure 2) [26]. Functional analysis demonstrated induction of both increased local growth and enhanced metastasis by mutated FGFR4 [27]. Xu et al. described germline mutations in FGFR4 i.e., glycine to arginine transition at position 388 in the transmembrane domain of FGFR4 receptor, which results in the formation of FGFR4 arg388 allele, leading to higher cancer risk [28]. Due to broad ligand binding spectrum of FGFR4, it is reportedly involved in multiple tumor types including HCC, breast cancer, colorectal cancer, rhabdomyosarcoma, and lung cancer [29-33]. 


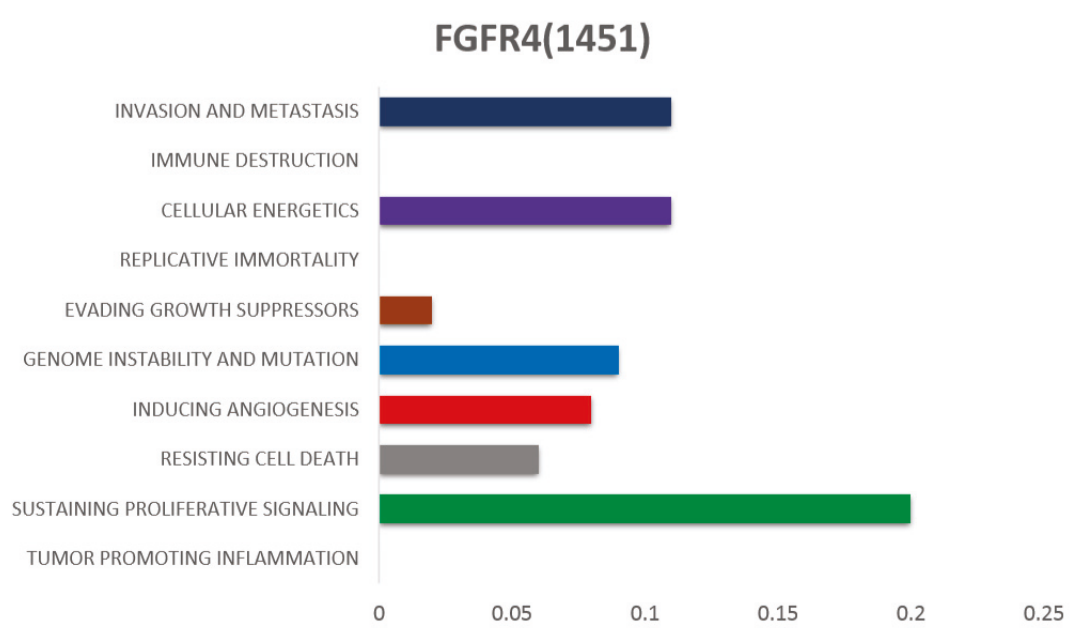

Figure 2. The association of FGFR 4 with different hallmarks of cancer, as reported in the literature. (Scales of bars from left to right represent the lowest to highest number of associations reported)

\subsection{Structure and Function of FGF19}

Out of three endogenous fibroblast growth factors (FGF19, FGF21, and FGF23), FGF19 binds to FGFR4 with highest affinity [34]. The human FGF19 gene is located on chromosome 11q13. In mice, the FGF15 gene is an orthologue of the human FGF19 gene [6]. The farnesoid X receptor (FXR) is activated by the secretion of bile acid from the gall bladder to the small intestine, which ultimately stimulates FGF19 secretion from the ileum $[35,36]$. The primary roles of FGF19 are found in bile acid synthesis, gallbladder filling, glycogen synthesis, gluconeogenesis, and protein synthesis [37]. FGF19 contributes to several hallmarks of cancer (Figure 3). Interestingly, FGF19 and FGF21 (endogenous fibroblast growth factors) are also most commonly involved in regulation of different functions occurring in liver [38]. Nicholes et al. demonstrated in transgenic mice that overexpression of FGF19 is involved in liver dysplasia [39]. In our recent study, amplification of FGF19 was found to be significantly associated with cirrhosis and also increased the risk of HCC [40]. Similarly, in our other study we used the fluorescence in situ hybridization technique and found the similar oncogenic patterns of FGF19 in HCC [41]. Copy number amplification of FGF19 is also highly reported in The Cancer Genome Atlas (TCGA) data [42]. Notably, the role of FGF19 at expression level is also frequently reported in HCC prognosis [43,44]. 


\section{FGFR19(940)}

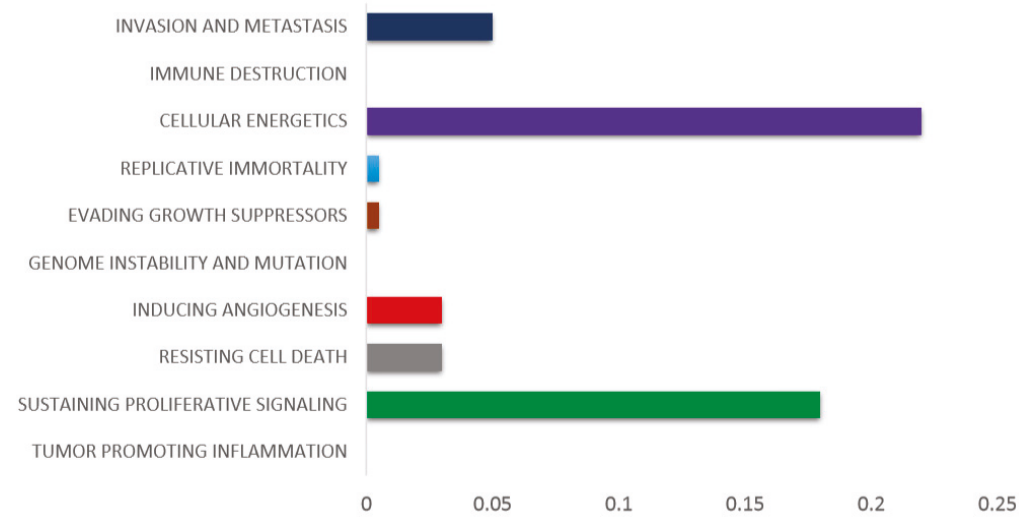

Figure 3. The association of FGF19 with different hallmarks of cancer, as reported in the literature. (Scales of bars from left to right represent the lowest to highest number of associations reported)

\subsection{Mechanism of FGFR4 Activation}

Specific ligand receptor binding spectrum in FGFs lead to autophosphorylation and formation of multiple complex [45]. FGFR4 is regulated using its co-receptor klotho-beta (KLB) (a transmembrane protein) [46]. The involvement of $K L B$ co-receptor is reported in hepatocytes and adipose and pancreatic tissues [47]. FGFR4 and KLB are found to be overexpressed in mature hepatocytes [48]. In addition, $K L B$ is required for FGF19-FGFR4 complex activation [49] (Figure 4). 


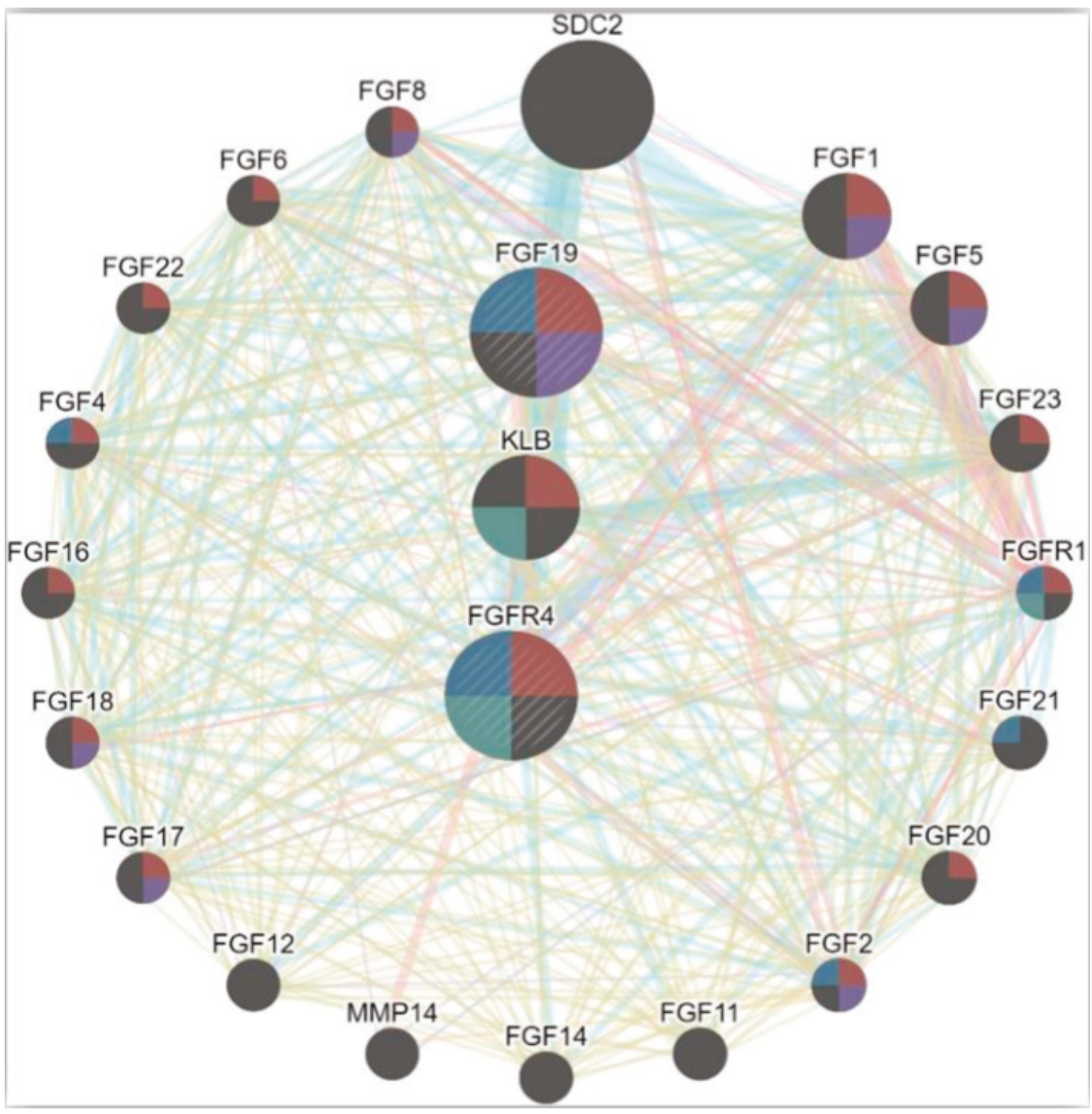

Figure 4. Interaction network of FGFR4 with different genes with high potency and functional similarity. The interaction network is based on various parameters including co-expression, genetic interactivity, shared protein domains, co-localization and physical interactions.

FGFR4 related pathways have predominant involvement in proliferation, differentiation, survival, and migration of cells. (Figure 4) Multiple signaling cascades such as GSK3 $\beta / \beta$-catenin, PI3K/AKT, PLC $\gamma /$ DAG/PKC, and RAS/RAF/MAPK are modulated by FGFR4 activation $[10,50,51]$ (Figure 5).

FGFR4 selectively binds FGF19 ligand [49,52]. FGF19 is also reported as a functional partner of FGFR4, with the highest score in analysis through the STRING (https://string-db.org/) database. 


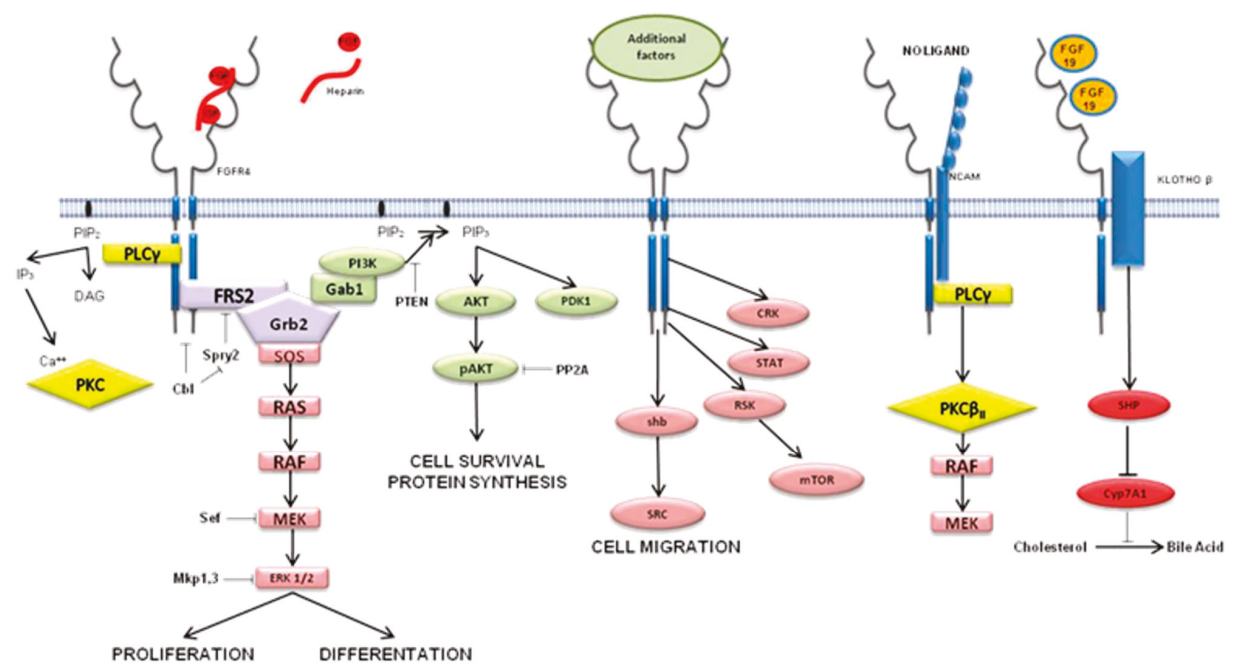

Figure 5. Involvement of FGFR4-related signaling pathways. Involvement in cell proliferation is depicted on the far left; next to it the cell survival signaling pathway is shown, and on the right side the cell migration pathway is explained (adapted from Atlas of Genetics and Cytogenetics in Oncology and Haematology).

\subsection{FGF19-FGFR4 Pathway in HCC}

FGF19/FGFR4 activation leads to the formation of FGF receptor substrate 2 (FRS2) and growth factor receptor-bound protein 2 (GRB2) complex, ultimately activating Ras-Raf-ERK1/2MAPK and $P I 3 K-A k t$ pathways. (Figure 6) These pathways are predominantly involved in tumor proliferation and anti-apoptosis. (Figure 6).

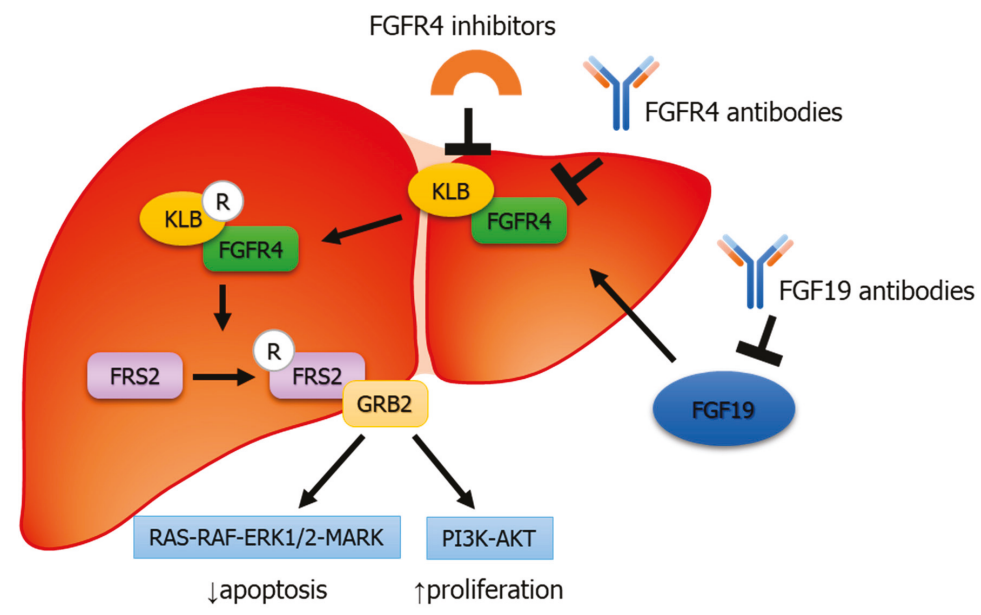

Figure 6. Binding mechanism of FGF19 to FGFR4 leads to FRS2 along with recruitment of growth factor receptor-bound protein 2 (GRB2), ultimately leading to activation of the Ras-Raf-ERK1/2 MAPK and PI3K-Akt pathways.

As discussed, frequent studies reported the anomalous expression of FGF19-FGFR4 complex enhances the progression of HCC [31,44]. In a study conducted on mice model, Cui et al. suggested 
FGF19 as a potential therapeutic target for the treatment of HCC [53]. FGFR4 dysregulation and its correlation with TGF- $\beta 1$ also suggested FGFR4 as potential therapeutic target of HCC patients with invasiveness and metastasis [43,54].

\section{Targeting FGF19-FGFR4 in HCC}

FGF19/FGFR4 inhibition is thought to lead to anti-tumor activities [55]. Thus, several FGFR (1-4) inhibitors are under trial for different types of malignancies including HCC [56] (Figure 7).

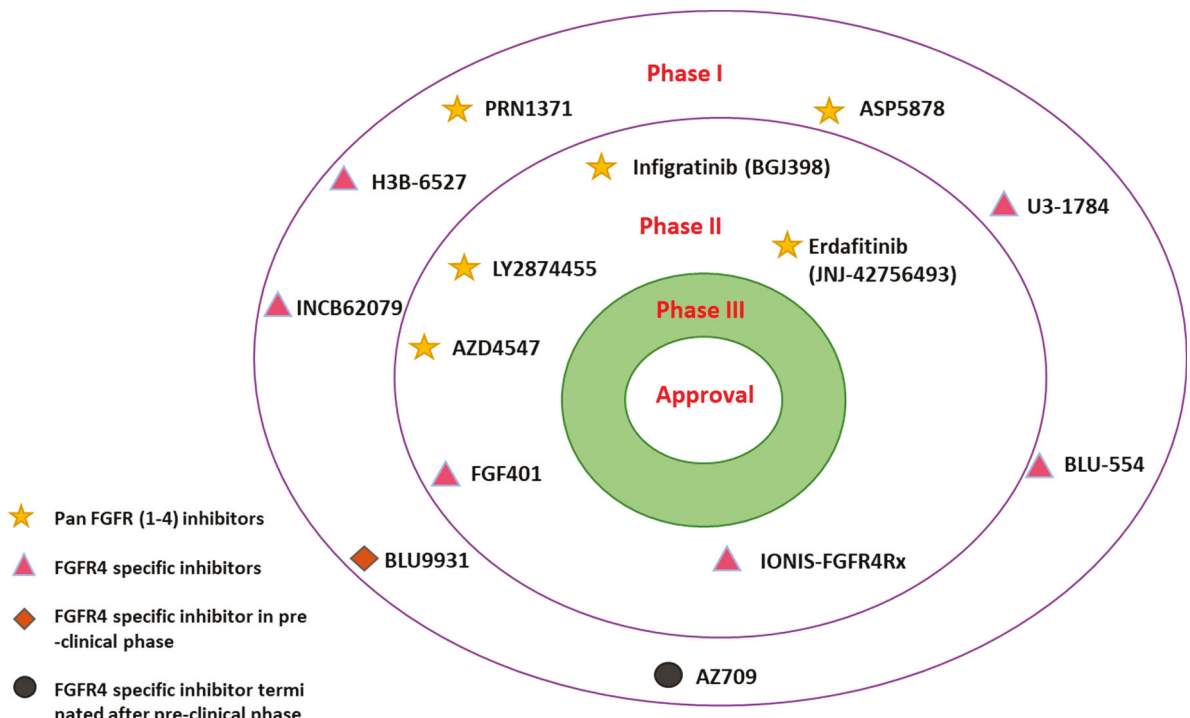

nated after pre-clinical phase

Figure 7. Selected overview of pan-FGFRs and FGFR4-specific inhibitors in different stages of clinical trials for hepatocellular carcinoma (HCC).

\subsection{Pan-FGFR (1-4) Inhibitors}

Multiple pan-FGFR (1-4) inhibitors are under-development in different phases of clinical trials (Figure 7). LY2874455 (NCT01212107), AZD4547 (NCT02038673), infigratinib (NCT02160041), and erdafitinib (NCT02365597) drugs are designed to target pan-FGFRs and are in phase II of development and clinical trials (Table 1).

LY2874455 is a small molecule inhibitor developed by Eli Lilly [53] (Figure 8). It has shown promising effects against advanced and metastatic cancers such as myelomas, lung, bladder, and gastric cancer [57]. Its highly effective inhibitory action suggests that it can be effective potential drug for $\mathrm{HCC}$ in the near future. 
Table 1. Pan-FGFR inhibitors in different phases of clinical trials.

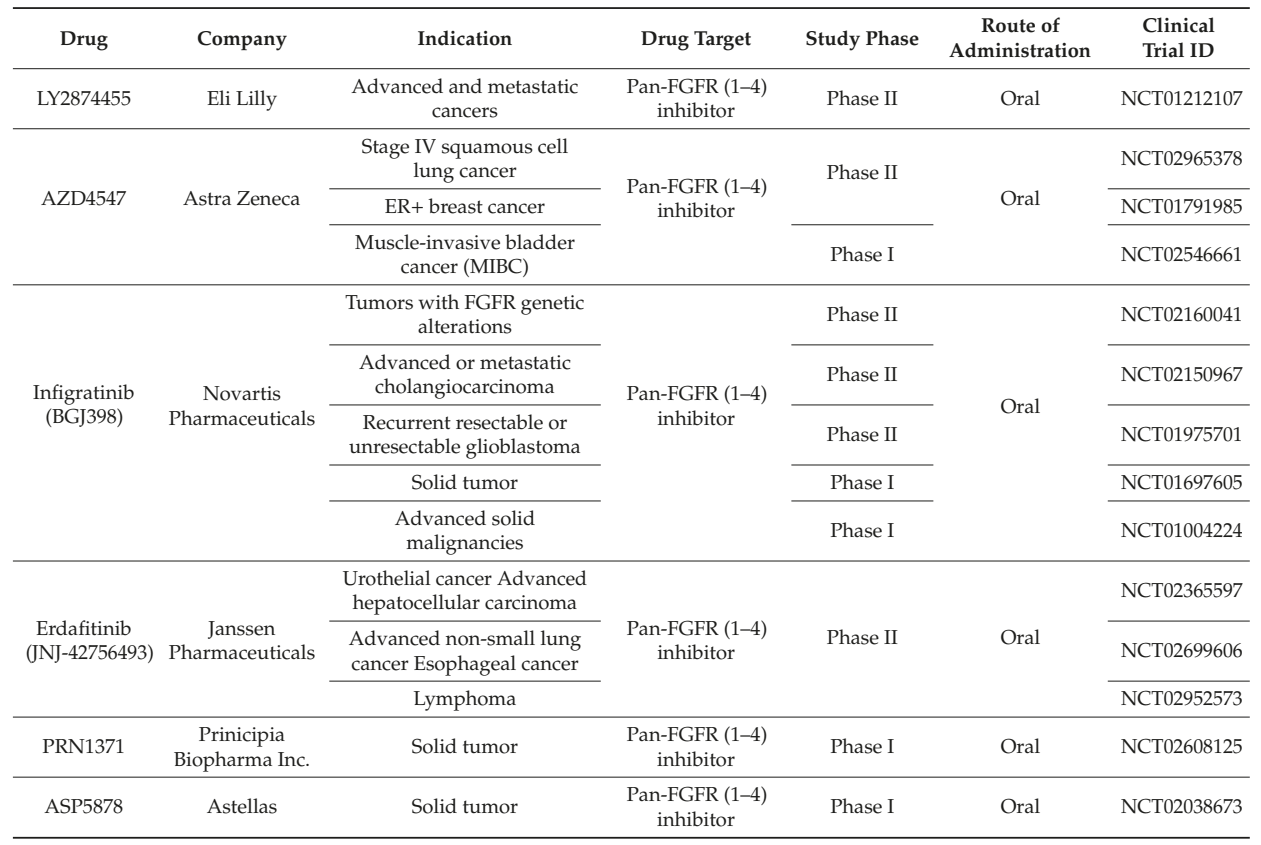

ER+ breast cancer: estrogen-receptor-positive breast cancer.

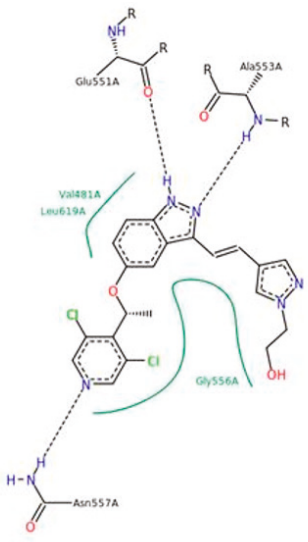

$\mathbf{a}$

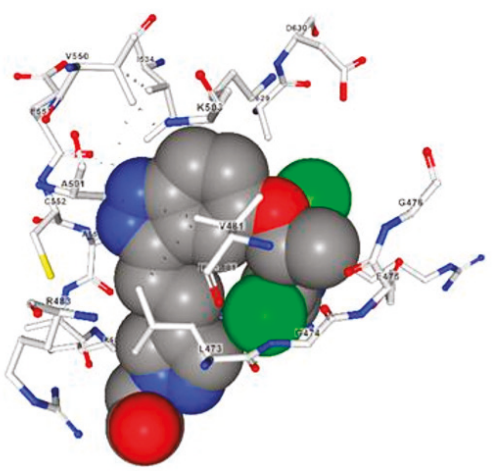

b

Figure 8. (a) Structure of LY2874455, and (b) binding mode of LY2874455 with the FGFR4 kinase domain (PDB code 5JKG).

AZD4547 was developed to specifically target pan-FGFR (1-4) in solid tumors. However, AZD4547 showed good efficacy against FGFR (1-3) but weaker activity against FGFR4 [58], suggesting low efficacy when specifically targeting FGFR4.

Infigratinib (BGJ398), which targets FGFR (1-3) with high affinity and FGFR4 with less affinity, was developed by Novartis Pharmaceuticals. It is currently in phase II for tumors with alteration of FGFR and for glioblastomas, solid tumors, hematologic malignancies, and advanced cholangiocarcinoma. 
Infigratinib showed an effective response against FGFR signaling pathways in HCC [59]. However, FDA-approved clinical trials are yet to be conducted for infigratinib in HCC [59].

Janssen Pharmaceuticals reported erdafitinib (JNJ-42756493), a pan-FGFR (1-4) inhibitor (Figure 9), which is currently under phase II of clinical trials for advanced HCC. It significantly inhibited FGFR-overexpressing tumor cells in HCC [60].

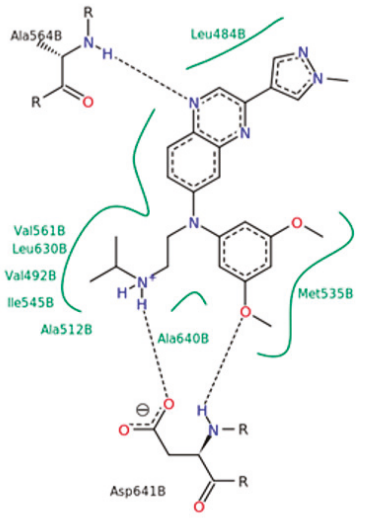

a

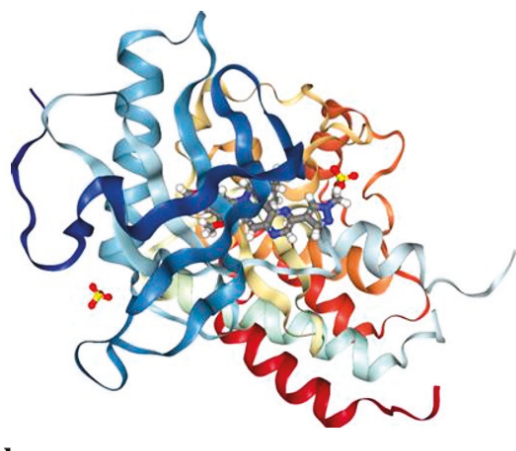

b

Figure 9. (a) Structure of JNJ-42756493 (b) Interaction of JNJ-42756493 with FGFR1 (PDB code 5EW8).

PRN1371 (NCT02608125) and ASP5878 (NCT02038673) are drugs designed to target pan-FGFRs and are in phase I of development and clinical trials. PRN1371 was developed by Principia Biopharma Inc. for solid tumors. It is an irreversible inhibitor that specifically targets FGFRs. The inhibitory action of this drug has been reported in many tumor types like HCC, gastric, and lung cancer [61]. Astellas developed ASP5878 to target pan-FGFRs (1-4) in solid tumors. Importantly, ASP5878 also inhibited HCC cell lines exhibiting overexpression of FGF19 in the pre-clinical phase. In addition, this small inhibitor molecule improved the efficacy of sorafenib [62].

\subsection{FGFR4-Specific Inhibitors}

As discussed, the overexpression of FGFR4 is most frequently reported receptor compared to FGFR (1-3) in HCC initiation and progression. However, selectivity of pan-FGFR inhibitors is comparatively lower for FGFR4. Thus, Prieto-Dominguez et al. outlined different targeted therapeutics available for the FGF19-FGFR4 complex [29]. A number of drugs are under different phases of clinical trials which specifically target FGF19/FGFR4. Two potential drug candidates in the phase II stage of clinical trials, namely IONIS-FGFR4Rx (NCT02476019) and FGF-401 (NCT02325739), are reported (Table 2). 
Table 2. FGFR4-specific inhibitors under different phases of clinical trials.

\begin{tabular}{|c|c|c|c|c|c|c|}
\hline Drug & Company & Indication & Drug Target & Study Phase & $\begin{array}{c}\text { Route of } \\
\text { Administration }\end{array}$ & $\begin{array}{l}\text { Clinical } \\
\text { Trial ID }\end{array}$ \\
\hline IONIS-FGFR4Rx & $\begin{array}{c}\text { Ionis } \\
\text { Pharmaceuticals }\end{array}$ & $\begin{array}{l}\text { Obesity and insulin } \\
\text { sensitivity }\end{array}$ & FGFR4-specific & Phase II & Subcutaneous & NCT02476019 \\
\hline FGF401 & Novartis AG & $\begin{array}{l}\text { Hepatocellular } \\
\text { carcinoma Solid } \\
\text { malignancies }\end{array}$ & FGFR4-specific & $\begin{array}{c}\text { Phase II } \\
\text { (recruiting } \\
\text { status) }\end{array}$ & Oral & NCT02325739 \\
\hline Н3В-6527 & $\begin{array}{l}\text { H3 Biomedicine } \\
\text { Inc. }\end{array}$ & $\begin{array}{l}\text { Hepatocellular } \\
\text { carcinoma }\end{array}$ & FGFR4-specific & Phase I & Oral & NCT02834780 \\
\hline U3-1784 & $\begin{array}{l}\text { Daiichi Sankyo } \\
\text { Inc. }\end{array}$ & $\begin{array}{l}\text { Advanced solid } \\
\text { tumor } \\
\text { Hepatocellular } \\
\text { carcinoma }\end{array}$ & FGFR4-specific & $\begin{array}{c}\text { Phase I } \\
\text { (Terminated) }\end{array}$ & Intravenous & NCT02690350 \\
\hline BLU-554 & $\begin{array}{c}\text { Blueprint } \\
\text { Medicines Corp. }\end{array}$ & $\begin{array}{c}\text { Hepatocellular } \\
\text { carcinoma (orphan } \\
\text { drug designation for } \\
\text { HCC by the U.S. } \\
\text { FDA) }\end{array}$ & FGFR4-specific & Phase I & Oral & NCT02508467 \\
\hline AZ709 & AstraZeneca & $\begin{array}{l}\text { Hepatocellular } \\
\text { carcinoma }\end{array}$ & FGFR4-specific & $\begin{array}{c}\text { Inactive } \\
\text { (Pre-clinical) }\end{array}$ & Unspecified & \\
\hline
\end{tabular}

U.S. FDA: U.S. Food and Drug Administration.

IONIS-FGFR4Rx, previously known as ISIS-FGFR4Rx, exhibited antisense inhibitor activity against FGFR4 [59]. IONIS-FGFR4Rx has undergone a phase II clinical trial for obesity, specifically targeting FGFR4 in liver and fat tissues. It is not only effective in reducing obesity but also improves insulin sensitivity [63]. Thus, we suggest that conducting trials with IONIS-FGFR4Rx in HCC patients may give significant results.

FGF401 was developed by Novartis and specifically targets FGFR4 in HCC patients. According to the most recent update, FGF401 is in phase II of clinical trials for HCC, expected to be completed by the year 2020. FGF401, with an $\mathrm{IC}_{50}$, exhibited at least 1000-fold potency for inhibiting FGFR4 kinase activity compared to other FGFRs (1-3) [64].

H3B-6527 (NCT02834780), U3-1784 (NCT02690350), and BLU-554 (NCT02508467) are reported to be in phase I clinical trials to specifically target FGFR4.

H3B-6527 is a small inhibitor molecule developed by $\mathrm{H} 3$ Biomedicine Inc for targeting FGFR4-overexpression in advanced HCC and cholangiocarcinoma (IHCC) patients. In preclinical trials, H3B-6527 proved to be effective in terms of repressing tumor growth in a xenograft model of HCC which exhibited activated aberrant FGF19-FGFR4 signaling [65].

The human monoclonal drug U3-1784 is under-development by Daiichi Sankyo Inc for HCC and other solid tumors. This antibody specifically binds to FGFR4 and is most effective (approximately 90\%) in FGF19-expressing models, suggesting it as a potential drug for HCC with an activated FGF19-FGFR4 pathway. However, according to a recent update, the clinical trials for this drug have been terminated [66].

BLU-554, a FGFR4-specific inhibitor, is under recruiting phase by Blueprint Medicines Corp. for HCC and cholangiocarcinoma patients. In addition, it was also granted an orphan drug designation in 2015 by the U.S. FDA for HCC [67].

Lastly, AZ709 showed good selective inhibition of FGFR4 in HCC, as recently reported by AstraZeneca, and is in the preclinical stage of development. However, no progress has been reported on this drug to date (reported at the 2013 NCRI Cancer Conference, Liverpool, UK).

\subsection{Irreversible FGFR4 Inhibitors}

Two irreversible FGFR4 inhibitors have also been recently reported, including INCB62079 (ClinicalTrials.gov Identifier: NCT03144661) and BLU9931 [68] (Figure 7, Table 3). INCB62079, developed by the Incyte Corporation, showed effective dose-dependent and compound-selective activity against cancer cells exhibiting active FGF19-FGFR4. Additionally, it showed good efficacy in 
Hep3b hepatocellular cancer xenograft model in pre-clinical trial phase. INCB62079 is currently in phase I clinical trials (ClinicalTrials.gov Identifier: NCT03144661) for HCC.

Table 3. FGFR4-specific irreversible inhibitors under different phases of clinical trials.

\begin{tabular}{ccccccc}
\hline Drug & Company & Indication & Drug Target & Study Phase & $\begin{array}{c}\text { Route of } \\
\text { Administration }\end{array}$ & $\begin{array}{c}\text { Clinical } \\
\text { Trial ID }\end{array}$ \\
\hline INCB62079 & $\begin{array}{c}\text { Incyte } \\
\text { Corporation }\end{array}$ & Liver cancer & $\begin{array}{c}\text { FGFR4-specific } \\
\text { (irreversible) }\end{array}$ & Phase I & Unspecified & NCT03144661 \\
\hline BLU9931 & $\begin{array}{c}\text { Blueprint } \\
\text { Medicines Corp. }\end{array}$ & $\begin{array}{c}\text { Hepatocellular } \\
\text { carcinoma }\end{array}$ & $\begin{array}{c}\text { FGFR4-specific } \\
\text { (irreversible) }\end{array}$ & Pre-clinical & Oral & \\
\hline
\end{tabular}

Blueprint Medicines Corp reported the remarkable drug BLU9931, a small irreversible inhibitor of FGFR4. It is currently in the pre-clinical stage of development for HCC and has not been approved by the U.S. FDA. In the preclinical trial phase, BLU9931 exhibited potent antitumor activity in mice with an HCC tumor xenograft with amplified FGF19 and high expression of FGF19 at the mRNA level. Recently, it has been reported that FGF19 shows resistance to sorafenib, but BLU9931 is involved in improving sorafenib efficacy by inactivating FGFR4 signaling [68].

Apart from the drugs reported in different clinical trials, different studies are underway to find new potent inhibitors against FGF19/FGFR. For instance, Cheuk et al. developed a chimeric antibody 3A11ScFvFc (mice antibody Fv + Human IgG1Fc) to specifically target FGFR4 in HCC [69]. Chen et al. found $A B S K-011$ to be involved in suppressing high FGFR4 expression, which ultimately results in HCC tumor suppression. ABSK-011, acting as irreversible inhibitor, selectively modifies cys552, which is the residue present within the active site of FGFR4. Of note, safety studies have also been conducted for this inhibitor [70]. Lee et al. examined the effect of the HM81422 inhibitor on the FGFR4-FGFR19 pathway. They successfully demonstrated that HM81422 can potentially target FGFR4 activated pathways. However, further elucidation is still required to understand the role of this inhibitor in HCC [71]. Furthermore, different pharmacological approaches suggested significant involvement of the drug sorafenib in inhibiting tyrosine kinase pathways. Initially, Gao et al. reported sorafenib as potential tyrosine kinase inhibitor which improves overall survival rate in HCC patients [68]. Later, Matsuki et al. revealed that sorafenib has no particular effect on the oncogenic FGF signaling pathway. However, the involvement of the drug lenvatinib was also recently reported [68]. Lenvatinib reportedly inhibits FGF pathways in HCC cell lines. Of note, studies suggested that it can be used as a pan-FGFR (1-4) inhibitor [68]. However, the specificity of lenvatinib against the FGF19-FGFR4 signaling pathway still remains unclear [72].

\section{Discussion and Conclusions}

Compelling evidence supports the involvement of the FGF19-FGFR4 signaling pathway in HCC [43]. Therefore, this pathway is considered to be a promising therapeutic target for the treatment of HCC. Interestingly, a number of different inhibitors and drugs have been reported to target FGF and FGFR signaling pathways. Despite promising advancements, it is still challenging to completely address all the underlying perspectives of this pathway. These perspectives, if clearly addressed, can improve the efficacy and potency of drugs available for HCC. The detailed analysis of available data revealed that FGFR4 is structurally distinct from other FGF receptors (1-3) and also exhibits variable inhibition potency towards different available FGFR drugs [73]. Perhaps, this distinct characteristic of FGFR4 should be exploited in depth to develop FGFR4-specific inhibitors to improve drug efficacy for HCC. Importantly, the evidence derived from primates suggests that anti-FGF19 antibody treatment is mostly accompanied with dose-related liver toxicity [74]. Therefore, the likelihood of adverse effects of FGF/FGFR drugs should be properly envisaged to assure best possible and safe outcomes along with reduced dose-dependent side effects. 
In addition, the correlation of FGF19 gene amplification and HCC is reported to be highly significant, and it is consequently thought to act as potential biomarker for HCC [75]. Therefore, copy number gain of FGF19 and FGFR4 should be taken into consideration when designing potential inhibitors of these genes and their pathways.

Conceptually, it is shown that the patients having elevated bile acid concentrations and diabetes have a higher risk of developing HCC [44,53]. Therefore, these complications should be taken into account along with the inhibition of FGF19-FGFR4 pathways to avoid potential adverse impacts and minimize safety risks in HCC patients.

Overall, the degree of FGF-FGFR inhibition in HCC is not satisfactory. This perhaps gives an indication towards elucidating other factors that are simultaneously involved in the FGF-FGFR signaling pathway. For instance, KLB (the co-receptor of FGFR4) is reportedly considered as a novel drug candidate as it is mostly found involved in inducing FGFR4 overexpression and is also found in an elevated state in HCC [46,76]. Thus, in the future klotho-specific inhibitors can be considered to potentially maximize antitumor and therapeutic benefits in HCC by terminating FGF19-binding to FGFR4. Lastly, developing drugs that act on key SNPs of FGFR4 i.e., Gly388 to Arg388, may also be clinically relevant.

In conclusion, most of the FGFR4-specific inhibitors are in pre-clinical phases. Progression of these potential inhibitors to advance clinical trial phases coupled with comprehensive research and improvements can revolutionize the available therapeutic options for HCC.

Author Contributions: S.-M.A. and F.H. contributed to the conception and design of the study. A.R. performed the literature review. A.R. and F.H. wrote the manuscript. A.R., I.P., and S.-M.A. contributed in revising the manuscript.

Funding: This research was supported by a grant from the Korea Health Technology R\&D Project through the Korea Health Industry Development Institute, funded by the Ministry of Health and Welfare (HI16C1985) and by a grant from Gachon University Gil Medical Center (FRD2016-20). The research was also funded by Higher Education Commission of Pakistan through National Research Program For Universities (NRPU \# 7036).

Conflicts of Interest: The authors declare no conflict of interest.

\section{References}

1. Bray, F.; Ferlay, J.; Soerjomataram, I.; Siegel, R.L.; Torre, L.A.; Jemal, A. Global cancer statistics 2018: GLOBOCAN estimates of incidence and mortality worldwide for 36 cancers in 185 countries. CA Cancer J. Clin. 2018, 68, 394-424. [CrossRef] [PubMed]

2. Huang, S.; He, X. The role of microRNAs in liver cancer progression. Br. J. Cancer 2011, 104, $235-240$. [CrossRef] [PubMed]

3. Kulik, L.; El-Serag, H.B. Epidemiology and Management of Hepatocellular Carcinoma. Gastroenterology 2019, 156, 477-491. [CrossRef] [PubMed]

4. Moeini, A.; Cornellà, H.; Villanueva, A. Emerging Signaling Pathways in Hepatocellular Carcinoma. LIC 2012, 1, 83-93. [CrossRef] [PubMed]

5. Zheng, N.; Wei, W.; Wang, Z. Emerging roles of FGF signaling in hepatocellular carcinoma. Transl. Cancer Res. 2016, 5, 1-6. [PubMed]

6. Ornitz, D.M.; Itoh, N. Fibroblast growth factors. Genome Biol. 2001, 2, reviews3005.1-reviews3005.12. [CrossRef] [PubMed]

7. Itoh, N.; Ornitz, D.M. Fibroblast growth factors: From molecular evolution to roles in development, metabolism and disease. J. Biochem. 2011, 149, 121-130. [CrossRef] [PubMed]

8. Wilkie, A.; Morriss-Kay, G.M.; Yvonne Jones, E.; Heath, J.K. Functions of fibroblast growth factors and their receptors. Curr. Biol. 1995, 5, 500-507. [CrossRef]

9. Turner, N.; Grose, R. Fibroblast growth factor signalling: From development to cancer. Nat. Rev. Cancer 2010, 10, 116-129. [CrossRef]

10. Zhao, H.; Lv, F.; Liang, G.; Huang, X.; Wu, G.; Zhang, W.; Yu, L.; Shi, L.; Teng, Y. FGF19 promotes epithelial-mesenchymal transition in hepatocellular carcinoma cells by modulating the GSK3 $\beta / \beta$ - catenin signaling cascade via FGFR4 activation. Oncotarget 2015, 7, 13575-13586. 
11. Llovet, J.M.; Hilgard, P.; de Oliveira, A.C.; Forner, A.; Zeuzem, S.; Galle, P.R.; Häussinger, D.; Moscovici, M. Sorafenib in Advanced Hepatocellular Carcinoma. N. Engl. J. Med. 2008, 13. [CrossRef] [PubMed]

12. Kudo, M.; Finn, R.S.; Qin, S.; Han, K.-H.; Ikeda, K.; Piscaglia, F.; Baron, A.; Park, J.-W.; Han, G.; Jassem, J.; et al. Lenvatinib versus sorafenib in first-line treatment of patients with unresectable hepatocellular carcinoma: A randomised phase 3 non-inferiority trial. Lancet 2018, 391, 1163-1173. [CrossRef]

13. Bruix, J.; Qin, S.; Merle, P.; Granito, A.; Huang, Y.-H.; Bodoky, G.; Pracht, M.; Yokosuka, O.; Rosmorduc, O.; Breder, V.; et al. Regorafenib for patients with hepatocellular carcinoma who progressed on sorafenib treatment (RESORCE): A randomised, double-blind, placebo-controlled, phase 3 trial. Lancet 2017, 389, 56-66. [CrossRef]

14. Abou-Alfa, G.K.; Meyer, T.; Cheng, A.-L.; El-Khoueiry, A.B.; Rimassa, L.; Ryoo, B.-Y.; Cicin, I.; Merle, P.; Chen, Y.; Park, J.-W.; et al. Cabozantinib in Patients with Advanced and Progressing Hepatocellular Carcinoma. N. Engl. J. Med. 2018, 379, 54-63. [CrossRef] [PubMed]

15. Zhu, A.X.; Kang, Y.-K.; Yen, C.-J.; Finn, R.S.; Galle, P.R.; Llovet, J.M.; Assenat, E.; Brandi, G.; Pracht, M.; Lim, H.Y.; et al. Ramucirumab after sorafenib in patients with advanced hepatocellular carcinoma and increased $\alpha$-fetoprotein concentrations (REACH-2): A randomised, double-blind, placebo-controlled, phase 3 trial. Lancet Oncol. 2019, 20, 282-296. [CrossRef]

16. El-Khoueiry, A.B.; Sangro, B.; Yau, T.; Crocenzi, T.S.; Kudo, M.; Hsu, C.; Kim, T.-Y.; Choo, S.-P.; Trojan, J.; Welling, T.H.; et al. Nivolumab in patients with advanced hepatocellular carcinoma (CheckMate 040): An open-label, non-comparative, phase 1/2 dose escalation and expansion trial. Lancet 2017, 389, 2492-2502. [CrossRef]

17. Transcript: FGFR4-201 (ENST00000292408.8)—Protein summary-Homo sapiens_Ensembl genome browser 95. Available online: https://asia.ensembl.org/Homo_sapiens/Transcript/ProteinSummary?g= ENSG00000160867;r=5:177086886-177098144;t=ENST00000292408 (accessed on 19 January 2019).

18. Partanen, J.; Mäkelä, T.P.; Eerola, E.; Korhonen, J.; Hirvonen, H.; Claesson-Welsh, L.; Alitalo, K. FGFR-4, a novel acidic fibroblast growth factor receptor with a distinct expression pattern. EMBO J. 1991, 10, 1347-1354. [CrossRef]

19. Mohammadi, M.; Olsen, S.K.; Ibrahimi, O.A. Structural basis for fibroblast growth factor receptor activation. Cytok. Growth Factor Rev. 2005, 16, 107-137. [CrossRef]

20. Wang, F.; Kan, M.; Yan, G.; Xu, J.; McKeehan, W.L. Alternately Spliced NH2-terminal Immunoglobulin-like Loop I in the Ectodomain of the Fibroblast Growth Factor (FGF) Receptor 1 Lowers Affinity for both Heparin and FGF-1. J. Biol. Chem. 1995, 270, 10231-10235. [CrossRef]

21. Ornitz, D.M.; Itoh, N. The Fibroblast Growth Factor signaling pathway. Wiley Interdiscip. Rev. Dev. Biol. 2015, 4, 215-266. [CrossRef]

22. Tucker, J.A.; Klein, T.; Breed, J.; Breeze, A.L.; Overman, R.; Phillips, C.; Norman, R.A. Structural Insights into FGFR Kinase Isoform Selectivity: Diverse Binding Modes of AZD4547 and Ponatinib in Complex with FGFR1 and FGFR4. Structure 2014, 22, 1764-1774. [CrossRef] [PubMed]

23. Horlick, R.A.; Stack, S.L.; Cooke, G.M. Cloning, expression and tissue distribution of the gene encoding rat fibroblast growth factor receptor subtype 4. Gene 1992, 120, 291-295. [CrossRef]

24. Reference, G.H. FGFR4 Gene. Available online: https://ghr.nlm.nih.gov/gene/FGFR4 (accessed on 19 January 2019).

25. Hughes, S.E. Differential Expression of the Fibroblast Growth Factor Receptor (FGFR) Multigene Family in Normal Human Adult Tissues. J. Histochem. Cytochem. 1997, 45, 1005-1019. [CrossRef] [PubMed]

26. Gross, S.; Rahal, R.; Stransky, N.; Lengauer, C.; Hoeflich, K.P. Targeting cancer with kinase inhibitors. J. Clin. Investig. 2015, 125, 1780-1789. [CrossRef]

27. Greenman, C.; Stephens, P.; Smith, R.; Dalgliesh, G.L.; Hunter, C.; Bignell, G.; Davies, H.; Teague, J.; Butler, A.; Stevens, C.; et al. Patterns of somatic mutation in human cancer genomes. Nature 2007, 446, 153-158. [CrossRef] [PubMed]

28. Xu, W.; Li, Y.; Wang, X.; Chen, B.; Wang, Y.; Liu, S.; Xu, J.; Zhao, W.; Wu, J. FGFR4 transmembrane domain polymorphism and cancer risk: A meta-analysis including 8555 subjects. Eur. J. Cancer 2010, 46, 3332-3338. [CrossRef] [PubMed]

29. Ye, Y.-W.; Zhang, X.; Zhou, Y.; Wu, J.; Zhao, C.; Yuan, L.; Wang, G.; Du, C.; Wang, C.; Shi, Y. The correlations between the expression of FGFR4 protein and clinicopathological parameters as well as prognosis of gastric cancer patients. J. Surg. Oncol. 2012, 106, 872-879. [CrossRef] 
30. Spinola, M.; Leoni, V.P.; Tanuma, J.; Pettinicchio, A.; Frattini, M.; Signoroni, S.; Agresti, R.; Giovanazzi, R.; Pilotti, S.; Bertario, L.; et al. FGFR4 Gly388Arg polymorphism and prognosis of breast and colorectal cancer. Oncol. Rep. 2005, 14, 415-419. [CrossRef]

31. Matakidou, A.; el Galta, R.; Rudd, M.F.; Webb, E.L.; Bridle, H.; Eisen, T.; Houlston, R.S. Further observations on the relationship between the FGFR4 Gly388Arg polymorphism and lung cancer prognosis. Br. J. Cancer 2007, 96, 1904-1907. [CrossRef]

32. Vi, J.G.T.; Cheuk, A.T.; Tsang, P.S.; Chung, J.-Y.; Song, Y.K.; Desai, K.; Yu, Y.; Chen, Q.-R.; Shah, K.; Youngblood, V.; et al. Identification of FGFR4-activating mutations in human rhabdomyosarcomas that promote metastasis in xenotransplanted models. J. Clin. Investig. 2009, 119, 3395-3407. [CrossRef]

33. Sheu, M.-J.; Hsieh, M.-J.; Chiang, W.-L.; Yang, S.-F.; Lee, H.-L.; Lee, L.-M.; Yeh, C.-B. Fibroblast Growth Factor Receptor 4 Polymorphism Is Associated with Liver Cirrhosis in Hepatocarcinoma. PLoS ONE 2015, 10, e0122961. [CrossRef] [PubMed]

34. Lee, K.J.; Jang, Y.O.; Cha, S.-K.; Kim, M.Y.; Park, K.-S.; Eom, Y.W.; Baik, S.K. Expression of Fibroblast Growth Factor 21 and $\beta$-Klotho Regulates Hepatic Fibrosis through the Nuclear Factor- $\mathrm{kB}$ and c-Jun N-Terminal Kinase Pathways. Gut Liver 2018, 12, 449-456. [CrossRef] [PubMed]

35. Liu, W.-Y.; Xie, D.-M.; Zhu, G.-Q.; Huang, G.-Q.; Lin, Y.-Q.; Wang, L.-R.; Shi, K.-Q.; Hu, B.; Braddock, M.; Chen, Y.-P.; et al. Targeting fibroblast growth factor 19 in liver disease: A potential biomarker and therapeutic target. Expert Opin. Ther. Targets 2015, 19, 675-685. [CrossRef] [PubMed]

36. Kurosu, H.; Choi, M.; Ogawa, Y.; Dickson, A.S.; Goetz, R.; Eliseenkova, A.V.; Mohammadi, M.; Rosenblatt, K.P.; Kliewer, S.A.; Kuro-o, M. Tissue-specific Expression of $\beta$ Klotho and Fibroblast Growth Factor (FGF) Receptor Isoforms Determines Metabolic Activity of FGF19 and FGF21. J. Biol. Chem. 2007, 282, 26687-26695. [CrossRef] [PubMed]

37. Kir, S.; Kliewer, S.A.; Mangelsdorf, D.J. Roles of FGF19 in Liver Metabolism. Cold Spring Harb. Symp. Quant. Biol. 2011, 76, 139-144. [CrossRef] [PubMed]

38. Fukumoto, S. Actions and Mode of Actions of FGF19 Subfamily Members. Endocr. J. 2008, 55, 23-31. [CrossRef] [PubMed]

39. Nicholes, K.; Guillet, S.; Tomlinson, E.; Hillan, K.; Wright, B.; Frantz, G.D.; Pham, T.A.; Dillard-Telm, L.; Tsai, S.P.; Stephan, J.-P.; et al. A Mouse Model of Hepatocellular Carcinoma: Ectopic Expression of Fibroblast Growth Factor 19 in Skeletal Muscle of Transgenic Mice. Am. J. Pathol. 2002, 160, 2295-2307. [CrossRef]

40. Ahn, S.-M.; Jang, S.J.; Shim, J.H.; Kim, D.; Hong, S.-M.; Sung, C.O.; Baek, D.; Haq, F.; Ansari, A.A.; Lee, S.Y.; et al. Genomic portrait of resectable hepatocellular carcinomas: Implications of RB1 and FGF19 aberrations for patient stratification. Hepatology 2014, 60, 1972-1982. [CrossRef]

41. Kang, H.J.; Haq, F.; Sung, C.O.; Choi, J.; Hong, S.-M.; Eo, S.-H.; Jeong, H.J.; Shin, J.; Shim, J.H.; Lee, H.C.; et al. Characterization of Hepatocellular Carcinoma Patients with FGF19 Amplification Assessed by Fluorescence in situ Hybridization: A Large Cohort Study. LIC 2019, 8, 12-23. [CrossRef]

42. Ally, A.; Balasundaram, M.; Carlsen, R.; Chuah, E.; Clarke, A.; Dhalla, N.; Holt, R.A.; Jones, S.J.M.; Lee, D.; $\mathrm{Ma}, \mathrm{Y}$; , et al. Comprehensive and Integrative Genomic Characterization of Hepatocellular Carcinoma. Cell 2017, 169, 1327-1341. [CrossRef]

43. Miura, S.; Mitsuhashi, N.; Shimizu, H.; Kimura, F.; Yoshidome, H.; Otsuka, M.; Kato, A.; Shida, T.; Okamura, D.; Miyazaki, M. Fibroblast growth factor 19 expression correlates with tumor progression and poorer prognosis of hepatocellular carcinoma. BMC Cancer 2012, 12, 56. [CrossRef] [PubMed]

44. Wu, X.; Ge, H.; Lemon, B.; Vonderfecht, S.; Weiszmann, J.; Hecht, R.; Gupte, J.; Hager, T.; Wang, Z.; Lindberg, R.; et al. FGF19-induced Hepatocyte Proliferation Is Mediated through FGFR4 Activation. J. Biol. Chem. 2010, 285, 5165-5170. [CrossRef] [PubMed]

45. Powers, C.J.; McLeskey, S.W.; Wellstein, A. Fibroblast growth factors, their receptors and signaling. Endocr. Relat. Cancer 2000, 7, 165-197. [CrossRef] [PubMed]

46. Poh, W.; Wong, W.; Ong, H.; Aung, M.O.; Lim, S.G.; Chua, B.T.; Ho, H.K. Klotho-beta overexpression as a novel target for suppressing proliferation and fibroblast growth factor receptor-4 signaling in hepatocellular carcinoma. Mol. Cancer 2012, 11, 14. [CrossRef] [PubMed]

47. Ito, S.; Kinoshita, S.; Shiraishi, N.; Nakagawa, S.; Sekine, S.; Fujimori, T.; Nabeshima, Y. Molecular cloning and expression analyses of mouse $\beta$ klotho, which encodes a novel Klotho family protein. Mech. Dev. 2000, 98, 115-119. [CrossRef] 
48. Li, Y.; Zhang, W.; Doughtie, A.; Cui, G.; Li, X.; Pandit, H.; Yang, Y.; Li, S.; Martin, R. Up-regulation of fibroblast growth factor 19 and its receptor associates with progression from fatty liver to hepatocellular carcinoma. Oncotarget 2016, 7, 52329-52339. [CrossRef]

49. Lin, B.C.; Wang, M.; Blackmore, C.; Desnoyers, L.R. Liver-specific Activities of FGF19 Require Klotho beta. J. Biol. Chem. 2007, 282, 27277-27284. [CrossRef]

50. Pratsinis, H.; Armatast, A.A.; Kletsas, D. Response of Fetal and Adult Cells to Growth Factors. In Human Fetal Tissue Transplantation; Bhattacharya, N., Stubblefield, P., Eds.; Springer: London, UK, 2013; pp. 65-77. ISBN 978-1-4471-4171-6.

51. Tiong, K.H.; Tan, B.S.; Choo, H.L.; Chung, F.F.-L.; Hii, L.-W.; Tan, S.H.; Khor, N.T.W.; Wong, S.F.; See, S.-J.; Tan, Y.-F.; et al. Fibroblast growth factor receptor 4 (FGFR4) and fibroblast growth factor 19 (FGF19) autocrine enhance breast cancer cells survival. Oncotarget 2016, 7, 57633-57650. [CrossRef] [PubMed]

52. Xie, M.-H.; Holcomb, I.; Deuel, B.; Dowd, P.; Huang, A.; Vagts, A.; Foster, J.; Liang, J.; Brush, J.; Gu, Q.; et al. FGF-19, a Novel Fibroblast Growth Factor with Unique Specificity for FGFR4. Cytokine 1999, 11, 729-735. [CrossRef]

53. Cui, G.; Martin, R.C.; Jin, H.; Liu, X.; Pandit, H.; Zhao, H.; Cai, L.; Zhang, P.; Li, W.; Li, Y. Up-regulation of FGF15/19 signaling promotes hepatocellular carcinoma in the background of fatty liver. J. Exp. Clin. Cancer Res. 2018, 37, 136. [CrossRef] [PubMed]

54. Ho, H.K.; Pok, S.; Streit, S.; Ruhe, J.E.; Hart, S.; Lim, K.S.; Loo, H.L.; Aung, M.O.; Lim, S.G.; Ullrich, A. Fibroblast growth factor receptor 4 regulates proliferation, anti-apoptosis and alpha-fetoprotein secretion during hepatocellular carcinoma progression and represents a potential target for therapeutic intervention. J. Hepatol. 2009, 50, 118-127. [CrossRef] [PubMed]

55. Lin, B.C.; Desnoyers, L.R. FGF19 and Cancer. In Endocrine FGFs and Klothos; Kuro-o, M., Ed.; Springer: New York, NY, USA, 2012; pp. 183-194. ISBN 978-1-4614-0887-1.

56. Touat, M.; Ileana, E.; Postel-Vinay, S.; André, F.; Soria, J.-C. Targeting FGFR Signaling in Cancer. Clin. Cancer Res. 2015, 21, 2684-2694. [CrossRef] [PubMed]

57. Michael, M.; Bang, Y.-J.; Park, Y.S.; Kang, Y.-K.; Kim, T.M.; Hamid, O.; Thornton, D.; Tate, S.C.; Raddad, E.; Tie, J. A Phase 1 Study of LY2874455, an Oral Selective pan-FGFR Inhibitor, in Patients with Advanced Cancer. Target. Oncol. 2017, 12, 463-474. [CrossRef] [PubMed]

58. Saka, H.; Kitagawa, C.; Kogure, Y.; Takahashi, Y.; Fujikawa, K.; Sagawa, T.; Iwasa, S.; Takahashi, N.; Fukao, T.; Tchinou, C.; et al. Safety, tolerability and pharmacokinetics of the fibroblast growth factor receptor inhibitor AZD4547 in Japanese patients with advanced solid tumours: A Phase I study. Investig. New Drugs 2017, 35, 451-462. [CrossRef] [PubMed]

59. Huynh, H.; Lee, L.Y.; Goh, K.Y.; Ong, R.; Hao, H.-X.; Huang, A.; Wang, Y.; Porta, D.G.; Chow, P.; Chung, A. Infigratinib mediates vascular normalization, impairs metastasis and improves chemotherapy in hepatocellular carcinoma. Hepatology 2018. [CrossRef] [PubMed]

60. Nishina, T.; Takahashi, S.; Iwasawa, R.; Noguchi, H.; Aoki, M.; Doi, T. Safety, pharmacokinetic, and pharmacodynamics of erdafitinib, a pan-fibroblast growth factor receptor (FGFR) tyrosine kinase inhibitor, in patients with advanced or refractory solid tumors. Investig. New Drugs 2018, 36, 424-434. [CrossRef]

61. Brameld, K.A. Abstract SY30-01: Discovery of the highly selective covalent FGFR1-4 inhibitor PRN1371, currently in development for the treatment of solid tumors. Cancer Res. 2016, 76, SY30-01.

62. Futami, T.; Okada, H.; Kihara, R.; Kawase, T.; Nakayama, A.; Suzuki, T.; Kameda, M.; Shindoh, N.; Terasaka, T.; Hirano, M.; et al. ASP5878, a Novel Inhibitor of FGFR1, 2, 3, and 4, Inhibits the Growth of FGF19-Expressing Hepatocellular Carcinoma. Mol. Cancer Ther. 2017, 16, 68-75. [CrossRef]

63. Martinussen, C.; Bojsen-Moller, K.N.; Svane, M.S.; Dejgaard, T.F.; Madsbad, S. Emerging drugs for the treatment of obesity. Expert Opin. Emerg. Drugs 2017, 22, 87-99. [CrossRef]

64. Weiss, A.; Porta, D.G.; Reimann, F.; Buhles, A.; Stamm, C.; Fairhurst, R.A.; Kinyamu-Akunda, J.; Sterker, D.; Murakami, M.; Wartmann, M.; et al. Abstract 2103: NVP-FGF401: Cellular and in vivo profile of a novel highly potent and selective FGFR4 inhibitor for the treatment of FGF19/FGFR4/KLB+ tumors. Cancer Res. 2017, 77, 2103.

65. Selvaraj, A.; Corcoran, E.; Coffey, H.; Prajapati, S.; Hao, M.-H.; Larsen, N.; Tsai, J.; Satoh, T.; Ichikawa, K.; Joshi, J.J.; et al. Abstract 3126: H3B6527, a selective and potent FGFR4 inhibitor for FGF19-driven hepatocellular carcinoma. Cancer Res. 2017, 77, 3126. 
66. Bartz, R.; Fukuchi, K.; Lange, T.; Gruner, K.; Ohtsuka, T.; Watanabe, I.; Hayashi, S.; Redondo-Müller, M.; Takahashi, M.; Agatsuma, T.; et al. Abstract 3852: U3-1784, a human anti-FGFR4 antibody for the treatment of cancer. Cancer Res. 2016, 76, 3852.

67. Kim, R.; Sharma, S.; Meyer, T.; Sarker, D.; Macarulla, T.; Sung, M.; Choo, S.P.; Shi, H.; Schmidt-Kittler, O.; Clifford, C.; et al. First-in-human study of BLU-554, a potent, highly-selective FGFR4 inhibitor designed for hepatocellular carcinoma (HCC) with FGFR4 pathway activation. Eur. J. Cancer 2016, 69, S41. [CrossRef]

68. Gao, L.; Shay, C.; Lv, F.; Wang, X.; Teng, Y. Implications of FGF19 on sorafenib-mediated nitric oxide production in hepatocellular carcinoma cells-A short report. Cell Oncol. 2018, 41, 85-91. [CrossRef] [PubMed]

69. Cheuk, A.; Shivaprasad, N.; Skarzynski, M.; Baskar, S.; Azorsa, P.; Khan, J. Abstract 5618: Anti-FGFR4 antibody drug conjugate for immune therapy of rhabdomyosarcoma and hepatocellular carcinoma. Cancer Res. 2018, 78,5618 .

70. Chen, Z. Abstract LB-272: Discovery and characterization of a novel FGFR4 Inhibitor for the treatment of hepatocellular carcinoma. Cancer Res. 2018, 78, LB-272.

71. Lee, J.; Kang, H.; Koo, K.; Ha, Y.; Lim, S.Y.; Byun, J.-Y.; Yu, H.; Song, T.; Lee, M.; Jung, S.H.; et al. Abstract 4780: A novel, potent and selective FGFR4 inhibitor, HM81422 in hepatocellular carcinoma with FGFR4-driven pathway activation. Cancer Res. 2018, 78, 4780.

72. Matsuki, M.; Hoshi, T.; Yamamoto, Y.; Ikemori-Kawada, M.; Minoshima, Y.; Funahashi, Y.; Matsui, J. Lenvatinib inhibits angiogenesis and tumor fibroblast growth factor signaling pathways in human hepatocellular carcinoma models. Cancer Med. 2018, 7, 2641-2653. [CrossRef]

73. Ho, H.K.; Yeo, A.H.L.; Kang, T.S.; Chua, B.T. Current strategies for inhibiting FGFR activities in clinical applications: Opportunities, challenges and toxicological considerations. Drug Discov. Today 2014, 19, 51-62. [CrossRef]

74. Pai, R.; French, D.; Ma, N.; Hotzel, K.; Plise, E.; Salphati, L.; Setchell, K.D.R.; Ware, J.; Lauriault, V.; Schutt, L.; et al. Antibody-Mediated Inhibition of Fibroblast Growth Factor 19 Results in Increased Bile Acids Synthesis and Ileal Malabsorption of Bile Acids in Cynomolgus Monkeys. Toxicol. Sci. 2012, 126, 446-456. [CrossRef]

75. Kaibori, M.; Sakai, K.; Ishizaki, M.; Matsushima, H.; De Velasco, M.A.; Matsui, K.; Iida, H.; Kitade, H.; Kwon, A.-H.; Nagano, H.; et al. Increased FGF19 copy number is frequently detected in hepatocellular carcinoma with a complete response after sorafenib treatment. Oncotarget 2016, 7, 49091-49098. [CrossRef] [PubMed]

76. Tang, X.; Wang, Y.; Fan, Z.; Ji, G.; Wang, M.; Lin, J.; Huang, S.; Meltzer, S.J. Klotho: A tumor suppressor and modulator of the Wnt/ $\beta$-catenin pathway in human hepatocellular carcinoma. Lab. Investig. 2016, 96, 197-205. [CrossRef] [PubMed]

(C) 2019 by the authors. Licensee MDPI, Basel, Switzerland. This article is an open access article distributed under the terms and conditions of the Creative Commons Attribution (CC BY) license (http://creativecommons.org/licenses/by/4.0/). 


\title{
Review \\ Cross-Talk between Fibroblast Growth Factor Receptors and Other Cell Surface Proteins
}

\author{
Marta Latko, Aleksandra Czyrek, Natalia Porębska, Marika Kucińska, Jacek Otlewski, \\ Małgorzata Zakrzewska and Łukasz Opaliński *

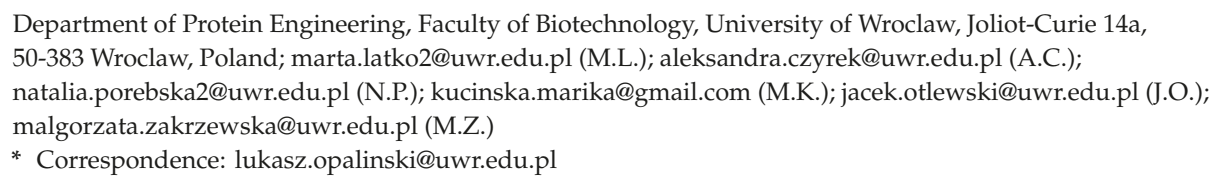

Received: 25 April 2019; Accepted: 13 May 2019; Published: 14 May 2019

\begin{abstract}
Fibroblast growth factors (FGFs) and their receptors (FGFRs) constitute signaling circuits that transmit signals across the plasma membrane, regulating pivotal cellular processes like differentiation, migration, proliferation, and apoptosis. The malfunction of FGFs/FGFRs signaling axis is observed in numerous developmental and metabolic disorders, and in various tumors. The large diversity of FGFs/FGFRs functions is attributed to a great complexity in the regulation of FGFs/FGFRs-dependent signaling cascades. The function of FGFRs is modulated at several levels, including gene expression, alternative splicing, posttranslational modifications, and protein trafficking. One of the emerging ways to adjust FGFRs activity is through formation of complexes with other integral proteins of the cell membrane. These proteins may act as coreceptors, modulating binding of FGFs to FGFRs and defining specificity of elicited cellular response. FGFRs may interact with other cell surface receptors, like G-protein-coupled receptors (GPCRs) or receptor tyrosine kinases (RTKs). The cross-talk between various receptors modulates the strength and specificity of intracellular signaling and cell fate. At the cell surface FGFRs can assemble into large complexes involving various cell adhesion molecules (CAMs). The interplay between FGFRs and CAMs affects cell-cell interaction and motility and is especially important for development of the central nervous system. This review summarizes current stage of knowledge about the regulation of FGFRs by the plasma membrane-embedded partner proteins and highlights the importance of FGFRs-containing membrane complexes in pathological conditions, including cancer.
\end{abstract}

Keywords: fibroblast growth factor receptors; signaling; receptor cross-talk; coreceptor; membrane proteins

\section{Introduction}

Fibroblast growth factor receptors 1-4 (FGFR1-4) form a group of receptor tyrosine kinases (RTKs) that are present on the surface of various cell types. FGFRs govern plethora of key cellular processes, including proliferation, migration, differentiation, and apoptosis, and their proper functioning is critical for development of the human body and homeostasis [1]. Alterations in FGFR1-4 are frequently detected in variety of developmental diseases and cancers, like prostate, breast, lung, and ovarian cancers [2,3]. The overall structure of FGFRs is typical for RTKs with an N-terminal region including three immunoglobulin-like domains D1-D3 exposed to the extracellular space, a single transmembrane span and a cytosolic tyrosine kinase domain (Figure 1a) [1,4]. The extracellular part of FGFRs constitutes binding sites for their natural ligands, FGFs, heparan cofactors, and a number of partner proteins $[5,6]$. Additionally, the ectodomain of FGFRs includes several motifs that prevent receptor autoactivation in the absence of growth factors [7-10]. The transmembrane helix of FGFRs anchors the receptors in the 
membrane and facilitates dimerization [11]. In the cytosol, the juxtamembrane (JM) region of FGFRs is involved in receptor dimerization and moderates transmission of signals [12-14]. The initiation of intracellular signaling circuits requires activation of FGFRs split kinase domain [1,5]. FGFR1-3 are subjected to alternative splicing in their extracellular region, yielding $\mathrm{b}$ and $\mathrm{c}$ isoforms of the receptors that differ in expression pattern and ligand specificity [15-17]. The FGFR family includes also fifth member-FGFRL1 (FGFR5)—which is homologous to FGFRs in the extracellular region, but lacks the cytosolic tyrosine kinase domain $[18,19]$.

Classically, the transmission of signals through the plasma membrane via FGFRs requires binding of appropriate growth factors and subsequent receptor activation. The canonical FGFs (FGF1-FGF10, FGF16, FGF17, FGF18, FGF20, and FGF22) are effective ligands in FGFRs binding and activation. In an inactive state monomeric FGFRs bind canonical FGFs, which triggers conformational changes in the receptor, resulting in dimerization and transactivation of cytosolic tyrosine kinases $[1,20]$. Sequential phosphorylation of tyrosine residues within the cytosolic tail of FGFRs creates docking sites for downstream signaling proteins [1,21]. The signals are further propagated through several pathways: Ras/Raf-mitogen-activated protein kinase/extracellular signal regulated kinase kinase (MEK)-extracellular signal regulated kinase (ERK), phosphoinositide 3-kinase (PI3K)/protein kinase B $(\mathrm{AKT}) /$ mammalian target of rapamycin (mTOR), phospholipase $\mathrm{C} \gamma(\mathrm{PLC} \gamma)$, and signal transducer and activator of transcription (STAT) [1,20].

FGFR-dependent signaling can be adjusted in several ways, including the diversified tissue distribution, different expression level of signaling components and their alternative splicing, which influences tissue development and disease progression [1]. Transmission of signals can be further modulated by ligand type, as FGFR complexes with different FGFs may vary in the strength and duration of propagated signals, which in turn decides cell fate [20,22]. FGFRs signaling can be modified as well by spontaneous receptor dimerization in the absence of ligands [23]. The posttranslational modifications, like glycosylation, ubiquitination, and phosphorylation, influence ligand binding and constitute negative feedback mechanisms for inhibition of FGFRs signaling [24-28]. Additionally, the cellular trafficking of FGFRs may regulate signals specificity, intensity, and timing [29-31].

One of the emerging means to modulate FGFRs activity is via formation of complexes with other plasma membrane proteins. Assembly of such complexes can be critical for transmission of signals, which is the case for endocrine FGFs (FGF19, FGF21, and FGF23) [32]. Partner proteins may deliver cofactors that facilitate formation of productive signaling modules or regulate the cellular transport of FGFRs [1]. Distinct types of cell surface receptors interact with FGFRs, leading to integration of different signaling routes or modulation of signal transmission. Several high throughput studies led to the discovery of numerous potential interaction partners of FGFRs within the plasma membrane [33-35]. However, the biological significance for most of them still needs to be elucidated.

In the next chapters we focus on the interplay between FGFRs and their binding partners in the regulation of signaling and cell behavior.

\section{Cross-Talk between FGFRs and G-Protein-Coupled Receptors in Regulation of the Central Nervous System}

G-protein-coupled receptors (GPCRs) constitute one of the largest groups of receptors responsible for signal transmission [36-38]. GPCRs are composed of an N-terminal extracellular domain, seven transmembrane helices, and a C-terminal region directed to the cytosol. Stimulation of GPCRs by extracellular ligands induces conformational changes within GPCRs, triggering intracellular signaling pathways modulated by heterotrimeric $G$ proteins $[39,40]$. Due to their wide diversity GPCRs modulate numerous processes, including, among others, nervous system transmission, visual, gustatory and smell sensing, inflammation, and recognition of cell density [41]. 
a

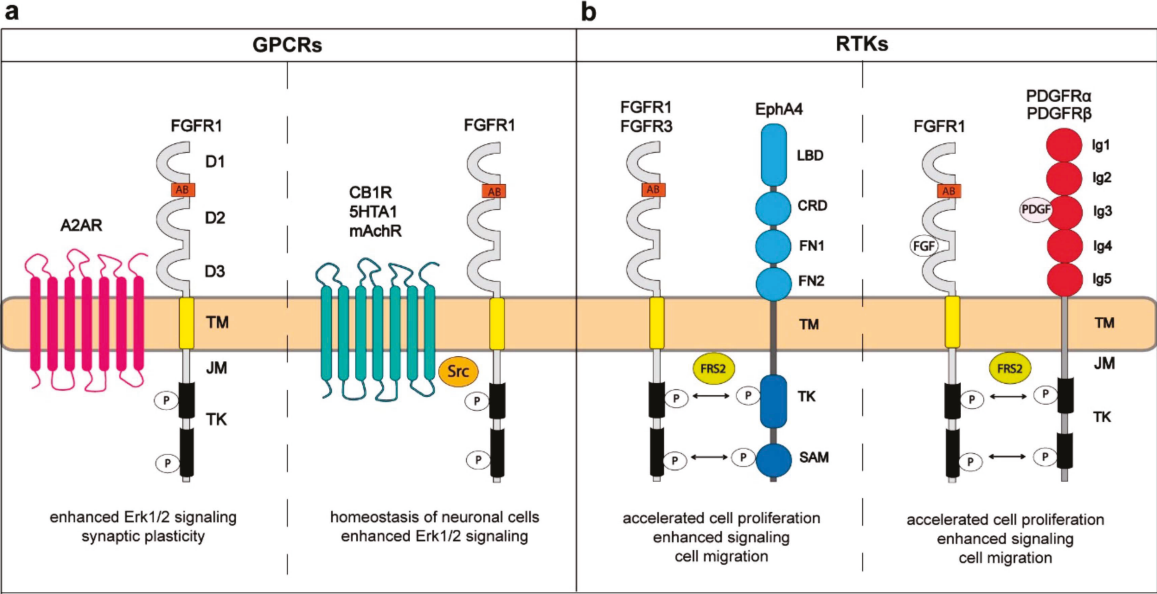

Figure 1. (a) Interplay between fibroblast growth factor receptors (FGFRs) and G-protein-coupled receptors (GPCRs) (a) and other receptor tyrosine kinases (RTKs) (b) in the regulation of downstream signaling. The extracellular region of FGFRs is composed of immunoglobulin like domains D1-D3 (gray) and the acidic box (AB; red). FGFRs are anchored in the plasma membrane by a single transmembrane helix (yellow). The cytosolic part of FGFRs consists of the juxtamembrane domain (JM) and the split tyrosine kinase domain (TK; black). GPCR-FGFR complexes may involve Src as a mediator between receptors or form functional heterocomplexes without involvement of Src. (b) FGFRs interact with other RTK members in the plasma membrane and can be directly activated by intracellular tyrosine kinase domains of partner proteins like Eph receptors or PDGFRs. EphA4 receptor contains the N-terminal ligand binding domain (LBD) followed by the cysteine rich domain (CDR) and two fibronectin type III domains (FN1-2). EphA4 is embedded in the membrane by a single transmembrane domain (TM). The cytosol-oriented region of EphA4 is composed of the tyrosine kinase domain (TK) and the sterile alpha motif (SAM). The TK domain of EphA4 interacts with JM region of FGFRs. PDGFRs contain five immunoglobulin-like domains (Ig1-Ig5) in their extracellular region, a single transmembrane span (TM), and intracellular juxtamembrane (JM) and tyrosine kinase (TK) domains. TK of PDGFRs directly phosphorylates FGFRs.

Various members of GPCRs and RTKs form heterocomplexes, which trigger intracellular signaling and cellular response different from that induced by RTKs or GPCRs alone [42]. The alterations in transmitted signals by GPCRs-RTKs heterocomplexes is achieved by the transactivation of RTKs by GPCRs which may occur via two distinct mechanisms: one relying on GPCRs activation and signaling that results in release of RTKs ligands and subsequent RTKs activation and second mechanism that involves a direct interaction and subsequent activation of RTKs by GPCRs [42]. The transactivation of RTKs by GPCRs was already demonstrated for a large number of RTKs, including epidermal growth factor receptors (EGFRs), platelet-derived growth factor receptors (PDGFRs), and insulin-like growth factor receptors (IGFRs) [42].

In the central nervous system (CNS), GPCR-dependent signaling controls proliferation, migration, survival, and differentiation of neurons [43]. FGFRs are expressed in different areas of brain. While FGFR1 is widely found in the hippocampus and in various parts of the cortex, FGFR2 and FGFR3 proteins are scattered throughout the CNS, and their expression profile changes with the brain development. FGFR4 is less abundant than other FGFRs and is mainly localized to the medial habenular nucleus [44-48]. The FGFRs are involved in the development, function and maintenance of the CNS [49]. Yeast two-hybrid (Y2H) screens revealed FGFR1 as a binding partner of G-protein-coupled receptor (GPCR)-adenosine receptor A2AR. The FGFR1-A2AR interaction was further confirmed by pull-down and coimmunoprecipitation [50]. The simultaneous stimulation of PC12 cells with A2AR agonist 
and FGF2 results in enhanced activation of downstream signaling pathways in comparison to single treatments, pointing on the synergistic effect of both receptors on cellular signaling. The enhanced activation of extracellular regulated kinases 1/2 (ERK1/2) requires assembly of the FGFR1-A2AR complex, pointing on the functional relevance of this interaction. The modulation of signaling by FGFR1-A2AR heterocomplexes was found to be important for regulation of the synaptic plasticity (Figure 1a) [50].

Cannabinoid receptor 1 (CB1R) is GPCR-ubiquitous in neurons, mediates the biological action of endogenous and synthetic cannabinoids, and regulates homeostasis of neuronal cells [51]. CB1R-FGFR1 interaction in neurons was demonstrated by means of coimmunoprecipitation. CB1R induces the transactivation of FGFR1 via protein kinase C (PKC) that in turn activates Fyn and Src. The latter proteins trigger activation of FGFR1 by phosphorylating key tyrosine residues of the receptor kinase domain [52]. The formation of CB1R-FGFR1 complexes occurs in lipid rafts of the plasma membrane, leads to activation of ERK1/2, and is important for neuronal differentiation (Figure 1a).

Using the proximity ligation assay (PLA) the interaction of FGFR1 with muscarinic acetylocholine receptor $(\mathrm{mAChR})$ subtype M1R was visualized [53]. Upon stimulation of hippocampal neurons with M1R agonist oxotremorine-M the activation of FGFR1 was observed. The exact mechanism of FGFR1 transactivation is not clear, however it involves Src tyrosine kinase that phosphorylates FGFR1 [53]. The cross-talk between mAChR and FGFR1 enhances neurite growth (Figure 1a) [53].

Binding between FGFR1 and 5-hydroxytriptamine receptor 1A (5-HT1A) was also demonstrated with PLA, but it was further confirmed by coimmunoprecipitation and bioluminescence resonance energy transfer (BRET) in a wide variety of cell types [54-56]. The number of FGFR1-5-HT1A complexes increases upon stimulation of cells with the FGF2 and 5-HT1A agonist 7-(Dipropylamino)-5,6,7,8-tetrahydronaphthalen-1-ol (8-OH-DPAT), confirming the functional interplay between these receptors [55]. Activation of 5-HTA1 with 8-OH-DPAT causes subsequent FGFR1 phosphorylation mediated by Src [55]. The simultaneous activation of FGFR1 and 5-HTA1 results in synergistically enhanced signaling that induces growth and controls homeostasis of neuronal cells (Figure 1a) [55]. Interestingly, the FGFR1-5-HT1A heterocomplexes display anti-depressive effects and thus may constitute targets for treatment of mood disorders [55,57-59].

Mu-opoid receptor (MOR) binds with high affinity to enkephalins and endorphins that modulate neuronal excitability. In rat glioma C6 cells MOR induces rapid activation of ERK1/2 via the transactivation of FGFR1. Again, the exact mechanism of this transactivation is unknown. Also the direct interaction between MOR and FGFR1 has not been yet demonstrated [60].

Summarizing, various members of GPCRs affect activity of FGFRs through the transactivation, which usually requires formation of the direct interaction between these receptors and involves Src as a bridging factor. The cross-talk between GPCRs and FGFRs is especially relevant for the development and functioning of neurons. GPCRs constitute large group of receptors, however only few members of the GPCRs family were demonstrated to bind FGFR1. The function of one type of receptors can be modulated by binding to other group of receptors. Since GPCRs play diverse pivotal functions in cells, the involvement of FGFRs in the regulation of GPCRs needs to be elucidated.

\section{Interplay between FGFRs and Other RTKs}

Diversification of signals transmitted by FGFRs can be also achieved by the interplay with other members of RTK family. The cross-talk between RTKs can occur via formation of receptor heterocomplexes and subsequent tyrosine phosphorylation of one receptor by tyrosine kinase of the other one. Alternatively, the transphosphorylation of RTKs in the complex can be mediated by the cytosolic kinase, like Src [61].

Eph receptors are activated by ephrin ligands and constitute the largest family of RTKs [62,63]. Based on sequence similarity and preference for ephrins A or B, Eph receptors are divided into EphA (EphA1-EphA10) and EphB (EphB1-EphB6) receptors [64]. The Eph receptors contain structural features characteristic for RTKs: an extracellular ligand binding region, a transmembrane domain, 
and an intracellular tyrosine kinase module [65]. The N-terminal extracellular part of Eph receptors is composed of ephrin binding domain followed by the cysteine rich EGF-like motif and two fibronectin type III repeats (FN3) FN1 and FN2. The cytosolic region of Eph receptors includes the juxtamembrane domain, the tyrosine kinase and the sterile alpha motif (SAM) (Figure 1b) [66]. Remarkably, activation of Eph receptors by ephrins requires the assembly of cell to cell contacts, as ephrins are embedded in the plasma membrane by the glycosylphosphatidylinositol (GPI) anchor (ephrins A) or the transmembrane helix (ephrins B) [64]. Binding of Eph receptor to ephrin present on the surface of aligned cell is followed by the juxtaposition of cytoplasmic kinase domain that evokes the transphosphorylation of receptor tyrosine residues initiating downstream signaling cascades [67]. The Eph receptor-ephrin complexes can be further arranged into high order assemblies that modulate cellular signaling $[68,69]$. The Eph receptor-ephrin complexes adjust cell adhesion, organization of cytoskeleton, angiogenesis, neural development, and plasticity [70].

EphA4 receptor emerged as binding partner of FGFR3 in $\mathrm{Y} 2 \mathrm{H}$ screens [71]. Further experiments, including coimmunoprecipitation revealed that the tyrosine kinase domain of Eph4 directly interacts with the JM domain of FGFR1-4 [71]. The formation of EphA4-FGFR complexes requires phosphorylation of tyrosine residues within JM domain of Eph4. Kinase domains of EphA4 and FGFRs can transphosphorylate each other. Furthermore, EphA4 ligand ephrin-A1 enhances FGFRs signaling, indicating significance of the FGFRs transactivation by EphA4 for the modulation of intracellular signal propagation [72]. Signals transmitted via FGF2/FGFR1/EphA4 complexes are enhanced in relation to FGF2/FGFR1, resulting in accelerated cell proliferation and migration [67]. In addition, the interaction between EphA4 and the fibroblast growth factor receptor substrate 2 alpha (FRS2 $\alpha$ ), a protein required for FGFRs signaling [73] was demonstrated with $\mathrm{Y} 2 \mathrm{H}$ and pull down experiments. Noteworthy, the ternary complex, involving FGFR1, EphA4, and FRS2 $\alpha$ was detected. Thus, FRS2 $\alpha$ acts as a tethering molecule that integrates signals from both receptors and regulates self-renewal, differentiation, and proliferation of neural stem/progenitor cells [74,75]. The cross-talk between Eph and FGFRs and Eph receptors was further confirmed by the observation that FGFRs phosphorylate EphA receptor target molecule, ephexin-1 [76]. Furthermore, Dlg-1, a scaffolding protein directly interacting with EphA receptors, can modulate FGFRs signaling (Figure 1b) [77,78].

Platelet-derived growth factor receptors alpha and beta (PDGFR $\alpha$ and PDGFR $\alpha$ ) are RTKs that are activated by five different platelet-derived growth factors (PDGF): PDGF-AA, PDGF-BB, PDGF-AB, PDGF-CC, and PDGF-DD [79,80]. Through regulation of cellular signaling PDGFRs influence cell motility, proliferation, and angiogenesis and aberrant PDGFRs are implicated in cancer [79]. PDGFRs are composed of the extracellular region divided into five Ig-like domains, from which Ig2 and Ig3 form the PDGF binding site, a single transmembrane span, and the intracellular tyrosine kinase domain (Figure 1b) [81,82]. In vitro and in vivo experiments using solid-phase assay (SPA), coimmunoprecipitation, and Förster Resonance Energy Transfer (FRET) revealed that PDGFR $\alpha$ interacts with high affinity with FGFR1 [83]. The formation of PDGFR $\alpha$-FGFR1 complexes is facilitated by the presence of ligands for both receptors [83]. The interaction between PDGFR $\beta$ and FGFR1 was demonstrated by means of coimmunoprecipitation [84]. In this receptor heterocomplex PDGFR $\beta$ directly phosphorylates FGFR1 on tyrosine residues [84]. Interestingly, FRS2 functions as a bridging molecule between PDGFR $\beta$ and FGFR1 (Figure 1b) [84]. The interplay is not only observed between the receptors but also at the level of their ligands. PDGF-BB and FGF2 interact with each other and activity of individual ligands in PDGF-BB-FGF2 complex is altered [85-87]. Remarkably, PDGFRs and FGFRs are often dysregulated in cancer and are targets of numerous therapeutic approaches [88].

Summarizing, FGFRs assemble into large multiprotein complexes with other RTK members and accessory proteins. The tyrosine kinase domains of different RTKs are able to transphosphorylate each other, initiating signals and adjusting their strength and specificity. Importantly, the interplay between RTKs is often coordinated at the level of FRS2. The fact that different members of RTKs can transactivate each other suggests the presence of an additional level of complexity in RTKs signaling. The family of RTKs is composed of 58 members; however, to date only few RTKs have been implicated 
in the FGFRs transactivation. Further studies on the interplay of FGFRs with other RTKs may uncover novel cellular regulatory mechanisms. Numerous FGFR-targeted anticancer therapies aim on the inhibition of FGFs interaction with FGFRs. Since FGFRs can be activated by other receptors in the absence of ligands, the detailed knowledge about FGFRs interplay with other RTKs may help in the development of novel therapeutics downregulating FGFRs signaling.

\section{Modulation of FGFRs Activity by Cell-Surface Proteins Involved in Adhesion}

Establishing cell-cell contacts requires an extensive remodeling of cellular components. Communication between cells involves interactions that are mediated by various cell adhesion molecules (CAMs). At the cell-cell interface extensive signaling is triggered, which coordinates remodeling of cellular structures. Noteworthy, FGFRs emerged as CAMs binding partners that participate in the signaling initiated by CAMs at cell-cell contacts (Figure 2).

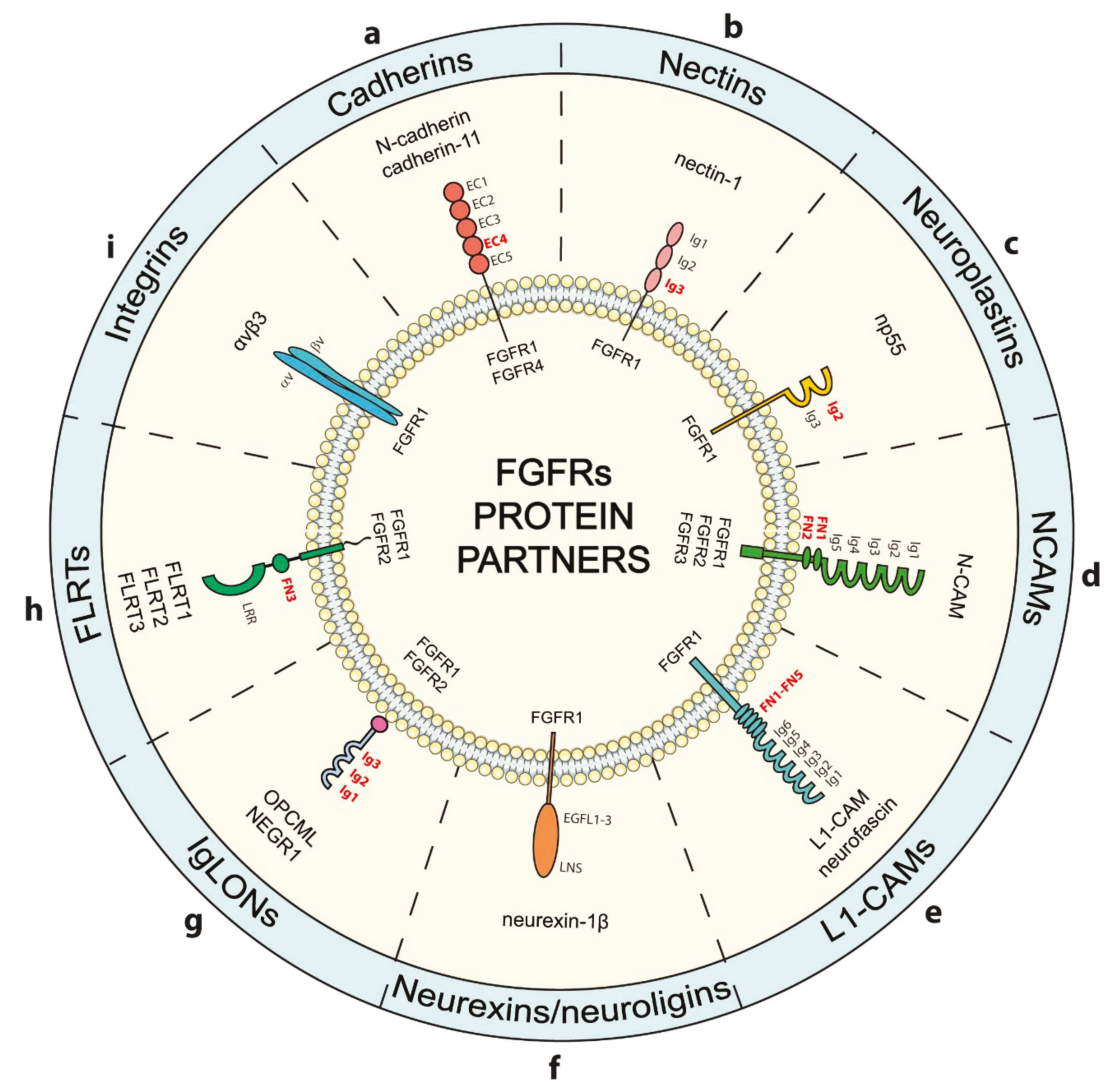

Figure 2. Cross-talk between FGFRs and various cell adhesion molecules. The interaction of particular FGFR with members of CAMs subgroup is indicated. The domain architecture of FGFR partner proteins is shown. Domains (where identified) responsible for the interaction between the partner protein and FGFR are indicated in red. (a) Cadherins reported to interact with FGFR1 and FGFR4 contain five EC domains in their extracellular region, a single transmembrane helix, and a cytosoilc tail interacting with several signaling proteins. (b) Nectins are composed of three immunoglobulin-like domains Ig1-Ig3, a single transmembrane domain, and a cytosolic region. Nectins bind FGFR1 using the Ig3 domain (c) Neuroplastin (Np55) contains two immunoglobulin-like Ig1-Ig2 domains in their extracellular region 
and are embedded in the membrane by a single transmembrane helix, exposing short tail into the cytosol. Np55-FGFR1 interaction involves the Ig2 domain of Np55 (d) NCAMs expose on the surface of the cells five immunoglobulin-like domains Ig1-Ig5 and two fibronectin type III domains FN1 and FN2. The cytosolic tail of NCAMs varies in length. NCAMs bind FGFR1-FGFR3 using FN1-FN2 domains (e) L1-CAM is a single spanning plasma membrane protein with six Ig-like domains (Ig1-Ig6) and five fibronectin type III domains (FN1-FN5) in its extracellular region. FGFR1 binding requires the FN1-FN5 region of L1-CAM (f) Neurexins contain different numbers of the laminin-neurexin-sex hormone binding globulin domains (LNS) and three EGF-like domains (EGFL1-3), a single transmembrane span and the cytosolic tail interacting with cytoskeletal and signaling proteins. The extracellular region of neurexin $1-\beta$ interacts with FGFR1. (g) Ig-LON family members: OPCML and NEGR1 interact with FGFR1 and FGFR2. Ig-LON proteins contain three immunoglobulin-like domains Ig1-Ig3 that are implicated in FGFR binding. (h) FLRTs are single spanning transmembrane proteins containing the leucine-rich repeat domain (LRR) and the FN3 domain in their extracellular region. FLRTs employ the FN3 domain for FGFR1 and FGFR2 binding. (i) Integrins are composed of different $\alpha$ and $\beta$ subunits. Integrin $\alpha v \beta 3$ forms complexes with FGFR1.

\subsection{Cadherins}

Cadherins are integral membrane proteins that are involved in the formation of specific cell-cell contacts, the adherens junctions (AJs) [89]. AJs are regulated by the alternative splicing of cadherins and are important for tissue development, homeostasis of epithelium and are implicated in different types of cancer [90-92]. Cadherins on opposing cells interact with each other via extracellular regions composed of five domains (EC1-EC5) in a calcium-dependent manner (Figure 2a) [92]. The cytosolic tail of cadherins binds catenins and other intracellular factors that link cadherin complexes to the cytoskeleton and forms signaling platforms at the cell-cell interface [90].

Neuronal cadherin (N-cadherin, cadherin-2) is expressed in various cell types, but its highest level is detected in neuronal and mesenchymal cells, where it coordinates cell migration and proliferation [93]. The functional interaction between N-cadherin and FGFRs was demonstrated in numerous cells, where $\mathrm{N}$-cadherin was shown to activate FGFRs and receptor-downstream signaling (Figure 2a) [94-96]. The interaction between N-cadherin and FGFR1 was demonstrated by means of coimmunoprecipitation in different cell lines $[97,98]$. The binding studies with truncated variants of FGFR1 revealed that the acidic box of the receptor extracellular region is required for the interaction with $\mathrm{N}$-cadherin $[97,98]$. Fluorescence microscopy analyses revealed that in transfected NIH3T3 cells N-cadherin and FGFR1 colocalize at the plasma membrane, however the N-cadherin-FGFR1 complexes are less abundant at the cell-cell contact sites where N-cadherin is enriched, suggesting dynamic nature of this interaction [97]. Formation of N-cadherin complexes with FGFR1 in breast cancer cells causes decreased internalization and lysosomal degradation of FGFR1, and sustained receptor signaling via MAPKs. Thus, N-cadherin may promote invasiveness of cancer cells not only by regulating cell-cell interactions, but also by affecting FGFR1 levels and activity [98-100]. Silencing of N-cadherin results in the accelerated FGFR1 degradation, whereas overproduction of N-cadherin is accompanied by increased levels of FGFR1. Thus, N-cadherin stabilizes FGFR1 and simultaneously enhances FGF2-induced proliferation and differentiation of epiblast stem cells [101]. Using coimmunoprecipitation, the interaction of N-cadherin with FGFR4 was demonstrated in pancreatic tumor cells and was dependent on neural cell adhesion molecule (N-CAM) [102]. Moreover, FGFR4-388Arg mutant frequently observed in various cancers induces signaling cascades that lead to enhanced $\mathrm{N}$-cadherin expression and modulates epithelial to mesenchymal transition (EMT) [103].

Cadherin-11 is widely expressed in mesenchymal cells like osteoblasts and neurons, and is important for tissue development during embryogenesis [104,105]. It is implicated in migration of cancer cells and in epithelial to mesenchymal transition [106-109]. The formation of complexes between FGFR1 and the cadherin- $11 / \beta$-catenin adhesion complexes was demonstrated by coimmunoprecipitation (Figure 2a) [110]. Pull-down experiments revealed that the cadherin-11-FGFR1 interaction occurs through their extracellular domains. Cadherin-11 initiates intracellular signaling pathways via FGFR1 
and recruits FGFR1 into areas of cell-cell contacts [49]. The cadherin-11-induced FGFR1 signaling stimulates neurite outgrowth [49].

\subsection{Nectins}

Nectins comprise a group of four plasma membrane proteins (Nectin-1-4) involved in formation of cell-cell contacts that are relevant in the neural development and disorders, and cancer [111]. Nectins contain an extracellular region composed of three immunolglobulin-like (Ig) domains, a single transmembrane helix, and a cytosolic domain (Figure 2b). Nectins from one cell can oligomerize in trans orientation with nectins present on the opposing cell, which results in cell adhesion. Depending on the involvement of accessory proteins nectins can be involved in establishing several types of adhesion complexes [111,112]. Using surface plasmon resonance (SPR) a direct interaction between Ig2-Ig3 domains of FGFRs and Ig3 of nectin-1 was demonstrated (Figure 2b). Binding of Ig3 of nectin-1 to FGFR1 results in receptor activation. Nectin-1 induces neurite outgrowth in hippocampal neurons in FGFR1-dependent manner, indicating that nectin-1 co-clusters with FGFR1 at the cell-cell contacts to stimulate differentiation and development of neurons [113].

\subsection{Neuroplastins}

Neuroplastins are cell adhesion molecules from immunoglobulin superfamily [114]. Neuroplastin Np55 is expressed in numerous cell types and tissues [115]. Np55 contains two Ig-like domains-Ig2 and Ig3-oriented towards the extracellular space, a single transmembrane span, and a short cytoplasmic tail (Figure 2c) [116]. SPR analysis revealed that Np55 directly interacts with the $\operatorname{Ig} 2-\operatorname{Ig} 3$ region of FGFR1 (Figure 2c). Binding of Np55 to FGFR1 present on the cell surface leads to receptor activation and initiation of downstream signaling. Although FGF2 and Np55 bind to the same region of FGFR1, these proteins elicit different effects on the receptor. Np55-FGFR1 complexes stimulate neurite outgrowth in primary hippocampal neurons, while FGF2-FGFR1 does not, which suggests different mode of intracellular signaling activation by these two FGFR1 ligands. Peptide based on Np55 extracellular domain was able to activate FGFR1 and downstream signaling and displayed antidepressant effects [117].

\subsection{N-CAMs}

Neural cell adhesion molecules (N-CAMs) are cell surface glycoproteins involved in axonal growth, cell migration, synaptic plasticity, and cell differentiation, and are implicated in various diseases including cancer $[118,119]$. N-CAMs contain five Ig-like domains and two FN3 domains in their extracellular region. NCAM-140 and NCAM-180 are embedded in the plasma membrane via transmembrane helices and display cytoplasmic tails of different length (Figure $2 \mathrm{~d}$ ). In contrast NCAM-120 utilizes the glycosylphosphatidylinositol (GPI) moiety for attachment to the cell surface [120].

The functional interplay between FGFRs and N-CAMs in neurite outgrowth was initially demonstrated by Williams et al. [94]. Subsequent studies confirmed a direct interaction of N-CAMs and FGFRs in different types of cells, including cancer cells [97,102,121-123]. The FN3 domains are responsible for the N-CAMs interaction with the Ig2-Ig3 region of FGFRs (Figure 2d) [124-126]. N-CAMs bind to FGFR1-FGFR3, but not to FGFR4, and these interactions depend on the receptor splice variants [127]. Binding of N-CAMs to FGFRs results in activation of the receptor and initiation of signaling cascades. The N-CAMs-FGFRs interplay is important for neuronal tissue development, but is also implicated in cancer. The N-CAMs/FGFRs complexes are observed in epithelial ovarian carcinoma, where they stimulate cancer cell migration and invasion $[128,129]$. The N-CAMs/FGFRs signaling may also modulate EMT [130]. Interestingly, N-CAMs can affect the cellular trafficking of FGFRs. Activation of FGFR1 by FGFs triggers receptor internalization and lysosomal degradation. In contrast, N-CAM-FGFR1 complexes are internalized, but the majority of the receptor is recycled 
from endosomes to the cell surface [121]. This differential FGFR1 cellular transport determines distinct cell fate depending on stimulation with FGF or N-CAM proteins [73].

\subsection{L1-CAMs}

L1-CAM is a cell surface glycoprotein that contains six Ig-like domains and five FN3 motifs in its extracellular region, a single TM span, and an intracellular tail that binds several signaling proteins (Figure 2e) [118]. The functional link between FGFR1 and L1-CAM was established by the observation that extracellular region of L1-CAM activates FGFR1, stimulating neurite outgrowth [94]. SPR experiments demonstrated a direct interaction between L1-CAM FN3 domains 1-5 and FGFR1 Ig2-Ig3 domains that was dependent on ATP [131]. Noteworthy, the cross-talk between FGFR1 and L1-CAM plays a role in proliferation and motility of glioma cells. The soluble, extracellular region of L1-CAM is often released by the cells due to the limited proteolysis involving ADAM-10 protease [132]. By binding to FGFR1 the extracellular region of L1-CAM leads to receptor activation, resulting in stimulation of glioma cell proliferation and motility [133]. The multiprotein complex of L1-CAM, FGFR1, and secreted glycoprotein Anosmin-1, which is involved in cell adhesion, motility, and differentiation, were also implicated in neurite branching [134-139].

Neurofascins are L1-CAM group members that control neurite outgrowth and synaptic organization [140]. The interaction between neurofascin (isoform NF166) and FGFR1 was demonstrated by coimmunoprecipitation [141]. Experiments with truncated versions of neurofascin revealed presence of two binding sites for FGFR1: an extracellular and an intracellular. Nevertheless, only the intracellular region of neurofascin is critical for FGFR1-dependent neurite outgrowth [141,142].

\subsection{Neurexins}

Neurexins and neuroligins are neuronal CAMs that regulate synaptic organization and function $[143,144]$. Presynaptic neurexins consist of the extracellular region containing from one to six laminin- neurexin-sex hormone binding globulin domains (LNS) and three epidermal growth factor like (EGF-like) domains, O-glycosylation sites, a single transmembrane span, and the cytosolic region recruiting various intracellular cytoskeletal and signaling proteins (Figure 2f) [144]. Postsynaptic neuroligins are composed of the extracellular acetylcholinesterase-like domain, a region enriched in glycosylation sites, a single transmembrane helix and the C-terminal intracellular PDZ domain recognition motif. Neurexins and neuroligins form trans-synaptic tethers that organize structure and function of synapses [144]. SPR experiments revealed a direct interaction between extracellular domain of FGFR1 and ectodomain of neurexin-1 $\beta$ (Figure 2f) [145]. Neurexin-1 $\beta$ binding leads to the activation of FGFR1 and receptor-downstream signaling cascades in a dose-dependent manner [145].

\subsection{IgLONs}

IgLONs are CAMs from immunoglobulin superfamily composed of three Ig-like domains that are attached to the cell membrane via GPI anchor (Figure 2g) [146]. Neuronal growth regulator 1 (NEGR1) is IgLON member that regulates neuronal maturation [147]. The functional interplay between NEGR1 and FGFRs in neuronal development and disease was initially suggested by Pischedda et al. and Casey et al. $[148,149]$. This was further confirmed by detection of the interaction between extracellular regions of NEGR1 and FGFR2 (Figure 2g). NEGR1 influences FGFR2 intracellular trafficking, favoring receptor recycling. The prolonged intracellular trafficking of FGFR2 in endosome compartments results in enhanced receptor-dependent signaling. Importantly, it was demonstrated that the coordinated cortical development requires the functional interplay between FGFR2 and NEGR1 [150].

Opioid binding protein cell adhesion molecule (OPCML) is another IgLON member linked with FGFRs. OPCML is a tumor suppressor implicated in various cancers [151-155]. Coimmunoprecipitation revealed that OPCML interacts with FGFR1. Furthermore, pull down experiments with recombinant OPCML and FGFR1 truncations showed that the Ig1-Ig3 region of OPCML directly interacts with the extracellular domain of FGFR1 (Figure 2g). Binding of OPCML to FGFR1 and a few other RTK 
members results in their downregulation, which is likely a result of their altered intracellular trafficking and decreased recycling [156].

\subsection{FLRTS}

Fibronectin leucine-rich transmembrane (FLRTs) proteins comprise a group of three cell surface glycoproteins involved in cell adhesion during vascularization and synapse development [157-161]. FLRTs contain the N-terminal extracellular region composed of the leucine-rich repeat domain (LRR) and the FN3 domain. FLTRs are embedded in the cell membrane via a single transmembrane helix and contain a short cytoplasmic tail (Figure 2h) [162]. FLRTs mediate cell-cell contacts mainly through the interaction of LRR domains of FLRTs on neighboring cells or with latrophilin [157,162]. Coimmunoprecipitation, pull-down and BRET experiments revealed that the FN3 domain of FLRT2 and FLRT3 interacts with FGFR2 and FGFR1, respectively (Figure 2h) [162,163]. Assembly of the FLRT-FGFR complexes is mediated by the interaction between intracellular regions of these proteins $[164,165]$. FGFR1-dependent signaling leads to the tyrosine phosphorylation of the intracellular tail of FLRT1. In addition, formation of the FLRT1-FGFR1 complexes enhances receptor signaling upon stimulation with FGF ligand, which accelerates neurite outgrowth in MAPK-dependent manner [166].

\subsection{Integrins}

Integrins are adhesion molecules that recognize ligands present in the extracellular matrix and on the cell surface, playing a key role in establishing cell contacts and regulating intracellular signaling [167]. Subunits $\alpha$ (18 isoforms) and $\beta$ ( 8 isoforms) assemble into 24 functional integrins that vary in terms of ligand specificity and cellular function (Figure 2i) [168]. Integrin-dependent signaling modulates survival, migration, and differentiation of cells [169]. Dysregulation of integrin adhesion complexes is widely implicated in various cancer types [170]. Coimmunoprecipitation experiments confirmed assembly of the ternary complex containing FGF1, FGFR1 and integrin $\alpha \mathrm{v} \beta 3$, with FGF1 acting as a bridging factor (Figure 2i). These multiprotein complexes are important for sustained activation of FGFR1-dependent kinases ERK1/2 [171]. Interestingly, the integrin binding-deficient mutant of FGF-1 (R50E) is capable of binding and activating FGFR1, however it fails to induce cell proliferation and migration, pointing on the functional relevance of integrin $\alpha \mathrm{v} \beta 3$ in FGF1 action [172,173]. The integrin binding site within FGF2 was identified as well; however the involvement of FGF2 in bridging FGFR1 and integrin $\alpha \mathrm{v} \beta 3$ has still to be determined [174].

Cell-cell contacts are complex signaling platforms that regulate behavior of neighboring cells and thus are strongly implicated in cancer. FGFRs are modulated by a number of different CAMs at the cell-cell interface. The FGFR-CAM interaction involves extracellular domains of these proteins, suggesting formation of complexes in cis and trans orientation. The FGFRs-CAMs interplay may adjust the strength of cell-cell attachment, which is relevant for migration of cancer cells and thus may constitute the target for future anticancer therapies.

\section{Novel Activities Acquired by FGFRs upon Binding to Specific Coreceptors}

Coreceptors are cell surface molecules that modulate the interaction of primary receptors with ligands. Usually, specific ligands require assembly of the ternary complexes involving ligand, receptor and coreceptor to initiate signal propagation. The perfect examples of FGFRs coreceptors are Klotho proteins that are necessary for endocrine FGFs (FGF19, FGF21, and FGF23) to trigger signaling. Functional FGFR signaling modules involve also specific polysaccharides, heparan sulfate (HS) chains, which stabilize receptor-ligand complexes. In this chapter we focus on coreceptors of FGFRs and their role in modulating FGFRs specificity and activity.

\subsection{Heparan Sulfate Proteoglycans}

The formation of FGF-FGFR complexes requires presence of HS [175,176]. HS directly binds FGFs and FGFRs stabilizing the ternary complex and facilitating FGFR autophosphorylation [177]. 
HS chains are covalently attached to the serine residues of a subset of cell surface proteins, forming heparan sulfate proteoglycans (HSPGs). HSPGs are secreted into the extracellular space or are attached to the plasma membrane either via GPI anchor or transmembrane helix [178]. HSPGs participate in FGF signaling by regulating availability of FGFs to FGFRs and by adjusting the FGF-FGFR complex dynamics (Figure 3a) [179].

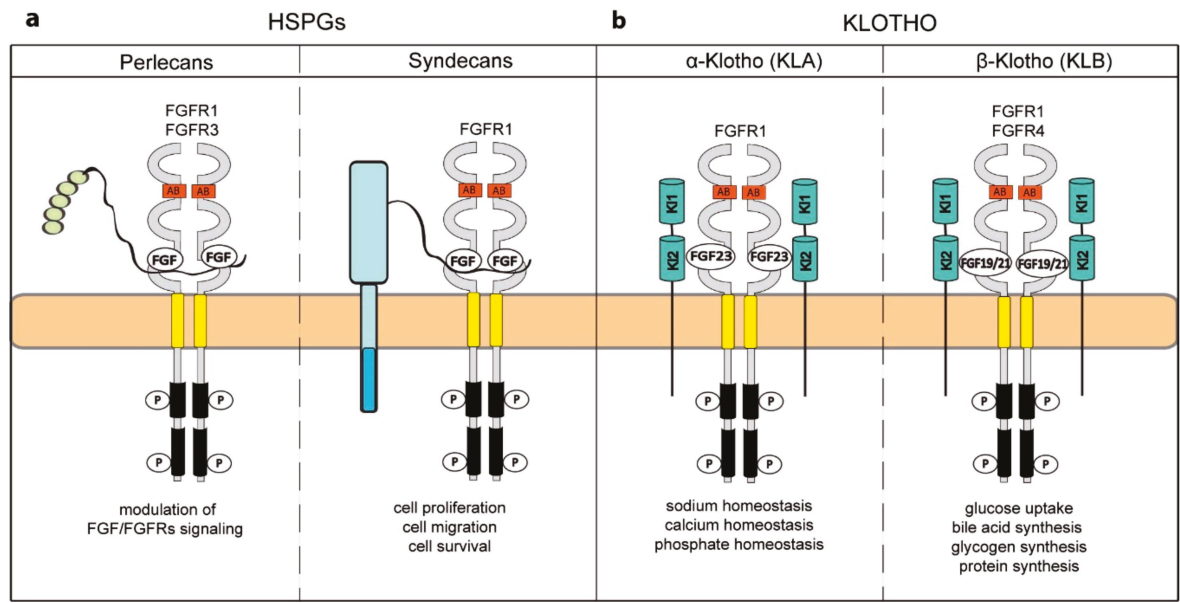

Figure 3. Involvement of coreceptors in the FGFRs signaling. (a) Heparan sulfate proteoglycans (HSPGs) provide polysaccharide chains that stabilize FGF-FGFR complexes and regulate availability of ligands. HSPGs are either integral membrane proteins (syndecans) or secreted glycoproteins (perlecans), which form ternary complexes with FGF-FGFR. (b) Klotho proteins $\alpha$ (KLA) and $\beta$ (KLB) are necessary for FGF23 and FGF19/FGF21 signaling, respectively.

Perlecan is high molecular weight, multidomain HSPG ubiquitous in the extracellular space. The HS chains are attached to the N-terminal domain of perlecan [180]. Perlecan interacts with several FGFs, providing their storage in the extracellular matrix, thus adjusting their accessibility to FGFRs [181-184]. In the absence of FGF perlecan is able to bind FGFR3, but perlecan-FGFR1 interaction requires presence of the growth factor [182]. The ternary complexes involving FGFs (FGF20 or FGF18), perlecan, and FGFRs affect FGFRs signaling and resulting cellular response (Figure 3a) [181,185]. Interestingly, perlecan isolated from diverse tissues differentially modulates FGF/FGFRs signaling, highlighting the importance of HS structure for FGFRs [183].

Syndecans are composed of an N-terminal extracellular domain with attached several sugar chains, including HS, a single transmembrane helix and a C-terminal cytosolic tail [186]. The N-terminal domain of syndecans interacts with several proteins, including growth factors, extracellular matrix proteins and chemokines, the transmembrane helix facilitates oligomerization of syndecans, while the intracellular region interacts with numerous signaling and cytoskeletal proteins $[187,188]$. Syndecans via HS chains interact with FGFs and FGFRs with relatively low affinity, but still facilitating formation of ternary signaling complexes [189-192]. Syndecan-dependent modulation of FGF/FGFR complexes is relevant for cell proliferation, migration and survival (Figure 3a) [193-196]. The cellular trafficking of FGFRs is tightly regulated and constitutes a mechanism for adjustment of signaling pathways and cellular fate [29]. In endothelial cells syndecan-4 initiates the internalization of syndecan-4/FGF2/FGFR1 complexes via micropinocytosis that is independent of clathrin and dynamin, and involves RhoG and Rab4. The altered trafficking of FGFR1 changes kinetics of MAPK signaling important for survival of endothelial cells [197]. 
Another group of HSPGs that adjust cellular signaling pathways triggered by growth factors are GPI-anchored glypicans [198]. Glypican-1 interacts with FGFs, modulating their activity and accessibility for FGFRs [199-201]. However, in brain endothelial cells and in glioma cells the overexpression of glypican-1 facilitates mitogenic response triggered by FGF2 [202,203].

\subsection{Klotho Coreceptors}

The FGF family includes a subgroup of endocrine FGFs-FGF19, FGF21, and FGF23-which largely differ from canonical FGFs in their structure and mode of action. Endocrine FGFs circulate throughout the human body regulating numerous metabolic processes [204,205]. In contrast to canonical FGFs, endocrine FGFs display low affinity to FGFRs and cell surface heparans [206-208]. To form functional signaling complexes with FGFRs endocrine FGFs require obligatory coreceptors from Klotho family: $\alpha$-Klotho (KLA) and $\beta$-Klotho (KLB) [209-213]. Klotho proteins are plasma membrane proteins containing two tandem KL1 and KL2 repeats with similarity to family 1 glucosidases in their extracellular region, a single transmembrane helix, and a short cytoplasmic tail [214,215].

KLA was discovered as a protein involved in aging process and is necessary for FGF23 signaling [211,214]. The obligate involvement of KLA in the formation of productive FGF23-FGFR1 signaling complex was enlightened by recent structural studies [216]. KLA interacts directly with FGFR1 and forms a high-affinity binding site for FGF23. FGF23 binds FGFR1 with its $\mathrm{N}$-terminus, while the C-terminal region of FGF23 directly interacts with KLA, forming the KLA-FGF23-FGFR1 signaling complex (Figure 3b) [216]. Interestingly, dimerization of such complexes and receptor activation remain dependent on the binding of heparan sulfate [216]. This ternary complex acts mainly in kidneys, regulating sodium, calcium and phosphate homeostasis and its imbalance leads to various metabolic diseases, like acute and chronic uremia and premature aging [217-225].

KLB is a homologue of KLA that facilitates formation of signaling complexes containing FGFR-FGF19, mainly in hepatocytes, and FGFRs-FGF21 in adipocytes (Figure 3b) [226-229]. The molecular bases of FGFR-FGF19/FGF21-KLB signaling complex assembly largely resemble FGFR1-FGF23-KLA. KLB utilizes both KL1 and KL2 of the extracellular domain for direct binding to FGF19/FGF21 C-terminal domains [230,231]. The KLB-FGF19 complex binds FGFR1 and FGFR4, while KLB-FGF21 can form the ternary complex only with FGFR1 [227]. The dimerized KLB-FGF21-FGFR1 complexes in adipocytes induce catabolic processes, stimulate glucose uptake, and improve insulin sensitivity [232]. Noteworthy, acting as a fasting hormone, FGF21 significantly extends lifespan $[233,234]$. In hepatocytes the KLB-FGF19-FGFR4 complexes are formed in response to feeding and downregulate synthesis of bile acid $[235,236]$. Additionally, these complexes contribute to the regulation of blood glucose level by stimulating synthesis of glycogen $[237,238]$. The dysregulation of FGF19/FGF21 is implicated in metabolic diseases, aging, and cancer [217,239-241].

\section{Modulation of FGFRs by Other Cell Surface Proteins}

There are plasma membrane proteins that interact with FGFRs but cannot be assigned to the above described categories. One of them is transforming growth factor $\beta$ receptor III (TGFBRIII), which is also known as betaglycan. It is a coreceptor of TGFBRI and TBFBRII that lacks an intracellular kinase activity [242]. The interaction between TGFBRIII and FGFR1 was demonstrated by coimmunoprecipitation in neuroblastoma. The TGFBRIII-FGFR1 interaction is stimulated by FGF2 and the assembly of ternary complexes enhances FGF2 signaling and promotes neuronal differentiation [243]. In addition, FGF2 binds to the glysocaminoglycan chains (GAG) present on the extracellular region of TGFBRIII, which may regulate availability of the FGF2 to FGFRs on the cell surface [244].

Another FGFRs' interactor is Sef (similar expression to fgf genes), a receptor-like protein composed of an extracellular region containing the FN3 domain, a single transmembrane helix and an intracellular domain with similarity to the interleukin 17 receptor [245]. Besides membrane bound Sef, secreted and cytosolic isoforms of Sef are generated [246]. The expression of Sef is induced by FGF signaling in various cell types [245,247-249]. The interaction of various Sef isoforms with intracellular region of FGFRs 
was demonstrated with coimmunoprecipitation [246,249-253]. Sef is an inhibitor of FGFR-dependent signaling acting either directly at the level of the receptor and/or on downstream intracellular kinases [254]. FGFR-dependent activation of ERK/MAPK and Akt is blocked by Sef, resulting in inhibition of cell proliferation [250,255]. Sef can also induce apoptosis and affect FGF-induced differentiation in various cell types [255]. Notably, the FGFRs-Sef interplay was implicated in prostate cancer $[256,257]$.

\section{Conclusions}

The cellular fate is very rarely determined by isolated signaling units. Instead, it is rather a result of extensive cross-communication between numerous diverse ligand/receptor systems. Secreted FGFs and their receptors are well studied signaling molecules. However, a number of recent reports largely changed the view about FGFs/FGFRs as separate signaling modules. FGFs/FGFRs are integrated into the complex cellular signaling at many levels and are subjected to diverse regulatory mechanisms. The cross-talk between FGFRs and other cell surface receptors, adhesion molecules, and coreceptors effectively modulates cellular processes such as proliferation, motility, differentiation, and death. The list of FGFRs binding partners within the plasma membrane is expanding; however it is still far from complete. As FGFRs expose large domains towards the extracellular space and the cytosol, the activity of these receptors might be further modulated by currently unknown secreted and/or intracellular proteins, respectively. Certainly, further studies aiming on the identification of novel FGFRs binding proteins and deciphering the relevance of FGFRs' complexes are required. Moreover, the application of complementary in vitro and in vivo experimental approaches is required for the validation and in-depth characterization of identified interactions. Structural data revealed the molecular mechanism of FGFR tyrosine kinase activation facilitating the design of diverse FGFR small molecule inhibitors that are currently tested as anticancer drugs [258]. Similarly, understanding how FGFRs cooperate with other cell surface receptors may lead to the development of novel inhibitors targeting FGFR-dependent processes.

As FGFRs are embedded in the plasma membrane, the activity and distribution of these receptors can be additionally affected by properties of the cell membrane (membrane composition, organization, curvature, etc.). Additionally, the alternative splicing of FGFRs and partner proteins may constitute another regulatory mechanism of the assembly of multiprotein signaling complexes. Further studies in this direction are unquestionably required. The spatiotemporal regulation of FGFRs constitutes another way to adjust cellular signaling. Some binding partners affect cellular trafficking of FGFRs, influencing selected transport mechanism and subcellular destination of the receptors. This in turn affects the kinetics and specificity of signaling and modulates cellular response. As FGFRs and number of partner proteins are implicated in various diseases including cancer, the deeper understanding of the interplay between FGFRs and other components of the cell membrane may facilitate treatment of life-threatening diseases.

Author Contributions: Ł.O., M.Z. and J.O. designed the manuscript. M.L., A.C., N.P. and M.K. performed literature studies and collected data. N.P. and M.K. prepared the figures. E.O. and M.Z. wrote the manuscript. All authors provided conceptual input, edited the text, and approved the final version of the manuscript.

Funding: This work was carried out within the First TEAM programme (POIR.04.04.00-00-43B2/17-00) of the Foundation for Polish Science cofinanced by the European Union under the European Regional Development Fund, awarded to Ł.O. MZ work was supported by the National Science Centre, Poland (grant Sonata Bis 2015/18/E/NZ3/00501).

Conflicts of Interest: The authors declare no conflicts of interest 


\section{Abbreviations}

5-HT1A 5-hydroksytriptamine receptor 1A

8-OH-DPAT 7(Dipropylamino)-5,6,7,8-tetrahydronaphthalen-1-ol

A2AR adenosine receptor

AJ

adherens junction

AKT

ATP

protein kinase B

BRET

adenosine triphosphate

CAMs

bioluminescence resonance energy transfer

CB1R

cell adhesion molecules

CNS

cannabinoid receptor 1

Dlg-1

EGFs

EGFRs

EMT

Eph

ERK1/2

FGFs

central nervous system

disks large homolog 1

epidermal growth factors

epidermal growth factor receptors

FGFRs

FGFRL1

epithelial to mesenchymal transition

FLRTs

FN3

ephrin

extracellular regulated kinases 1/2

fibroblast growth factors

fibroblast growth factor receptors

fibroblast growth factor receptor like 1

FRET

fibronectin leucine-rich transmembranes

FRS2

fibronectin type III

Förster Resonance Energy Transfer

GAG

fibroblast growth factor receptor substrate 2

GPCRs

GPI

glysocaminoglycan

G-protein-coupled receptors

HS

glycosylphosphatidylinositol

HSPGs heparan sulfate proteoglycans

IGFR

insulin-like growth factor receptor

JM juxtamembrane

KLA

$\alpha$-klotho

KLB $\quad \beta$-klotho

L1-CAM L1 cell adhesion molecule

LNS laminin, neurexin, sex hormone binding globulin

LPR leucine-rich repeat domain

mAChR muscarinic acetylcholine receptor

MAPK mitogen-activated protein kinase

MOR mu-opioid receptor

mTOR mammalian target of rapamycin

N-CAMs neural cell adhesion molecules

NEGR1 neuronal growth regulator 1

NFs neurofascins

Np55 neuroplastin 55

OPCML opioid binding protein cell adhesion molecule

PDGFs platelet-derived growth factors

PDGFRs platelet-derived growth factor receptors

PI13K phosphoinositide 3-kinase

PKC

protein kinase $\mathrm{C}$

PLA

PLCX

proximity ligation assay

RTKs

phospholipase CX

SAM

receptor tyrosine kinases

sterile alpha motif 
Sef

SPA

SPR

STAT

TGFs

TGFBRs

$\mathrm{Y} 2 \mathrm{H}$ similar expression to fgf genes

solid-phase assay

surface plasmon resonance

signal transducer and activator of transcription

transforming growth factors

transforming growth factor receptors

yeast two-hybrid

\section{References}

1. Ornitz, D.M.; Itoh, N. The Fibroblast Growth Factor signaling pathway. Wiley Interdiscip. Rev. Dev. Biol. 2015, 4, 215-266. [CrossRef] [PubMed]

2. Helsten, T.; Elkin, S.; Arthur, E.; Tomson, B.N.; Carter, J.; Kurzrock, R. The FGFR Landscape in Cancer: Analysis of 4,853 Tumors by Next-Generation Sequencing. Clin. Cancer Res. 2016, 22, 259-267. [CrossRef] [PubMed]

3. Hallinan, N.; Finn, S.; Cuffe, S.; Rafee, S.; O’Byrne, K.; Gately, K. Targeting the fibroblast growth factor receptor family in cancer. Cancer Treat. Rev. 2016, 46, 51-62. [CrossRef] [PubMed]

4. Ornitz, D.M.; Marie, P.J. Fibroblast growth factor signaling in skeletal development and disease. Genes Dev. 2015, 29, 1463-1486. [CrossRef] [PubMed]

5. Mohammadi, M.; Olsen, S.K.; Ibrahimi, O.A. Structural basis for fibroblast growth factor receptor activation. Cytokine Growth Factor Rev. 2005, 16, 107-137. [CrossRef] [PubMed]

6. Plotnikov, A.N.; Hubbard, S.R.; Schlessinger, J.; Mohammadi, M. Crystal structures of two FGF-FGFR complexes reveal the determinants of ligand-receptor specificity. Cell 2000, 101, 413-424. [CrossRef]

7. Olsen, S.K.; Ibrahimi, O.A.; Raucci, A.; Zhang, F.; Eliseenkova, A.V.; Yayon, A.; Basilico, C.; Linhardt, R.J.; Schlessinger, J.; Mohammadi, M. Insights into the molecular basis for fibroblast growth factor receptor autoinhibition and ligand-binding promiscuity. Proc. Natl. Acad. Sci. USA 2004, 101, 935-940. [CrossRef]

8. Kalinina, J.; Dutta, K.; Ilghari, D.; Beenken, A.; Goetz, R.; Eliseenkova, A.V.; Cowburn, D.; Mohammadi, M. The alternatively spliced acid box region plays a key role in FGF receptor autoinhibition. Structure 2012, 20, 77-88. [CrossRef]

9. Kiselyov, V.V.; Kochoyan, A.; Poulsen, F.M.; Bock, E.; Berezin, V. Elucidation of the mechanism of the regulatory function of the Ig1 module of the fibroblast growth factor receptor 1. Protein Sci. 2006, 15, 2318-2322. [CrossRef] [PubMed]

10. Opalinski, L.; Szczepara, M.; Sokolowska-Wedzina, A.; Zakrzewska, M.; Otlewski, J. The autoinhibitory function of D1 domain of FGFR1 goes beyond the inhibition of ligand binding. Int. J. Biochem. Cell Biol. 2017, 89, 193-198. [CrossRef] [PubMed]

11. Peng, W.C.; Lin, X.; Torres, J. The strong dimerization of the transmembrane domain of the fibroblast growth factor receptor (FGFR) is modulated by C-terminal juxtamembrane residues. Protein Sci. 2009, 18, 450-459. [CrossRef] [PubMed]

12. Lin, H.Y.; Xu, J.; Ischenko, I.; Ornitz, D.M.; Halegoua, S.; Hayman, M.J. Identification of the cytoplasmic regions of fibroblast growth factor (FGF) receptor 1 which play important roles in induction of neurite outgrowth in PC12 cells by FGF-1. Mol. Cell Biol. 1998, 18, 3762-3770. [CrossRef]

13. Burgar, H.R.; Burns, H.D.; Elsden, J.L.; Lalioti, M.D.; Heath, J.K. Association of the signaling adaptor FRS2 with fibroblast growth factor receptor 1 (Fgfr1) is mediated by alternative splicing of the juxtamembrane domain. J. Biol. Chem. 2002, 277, 4018-4023. [CrossRef] [PubMed]

14. Sarabipour, S.; Hristova, K. FGFR3 unliganded dimer stabilization by the juxtamembrane domain. J. Mol. Biol. 2015, 427, 1705-1714. [CrossRef]

15. Miki, T.; Bottaro, D.P.; Fleming, T.P.; Smith, C.L.; Burgess, W.H.; Chan, A.M.; Aaronson, S.A. Determination of ligand-binding specificity by alternative splicing: Two distinct growth factor receptors encoded by a single gene. Proc. Natl. Acad. Sci. USA 1992, 89, 246-250. [CrossRef] [PubMed]

16. Chellaiah, A.T.; McEwen, D.G.; Werner, S.; Xu, J.; Ornitz, D.M. Fibroblast growth factor receptor (FGFR) 3. Alternative splicing in immunoglobulin-like domain III creates a receptor highly specific for acidic FGF/FGF-1. J. Biol. Chem. 1994, 269, 11620-11627. [PubMed] 
17. Gong, S.G. Isoforms of receptors of fibroblast growth factors. J. Cell. Physiol. 2014, 229, 1887-1895. [CrossRef] [PubMed]

18. Wiedemann, M.; Trueb, B. Characterization of a novel protein (FGFRL1) from human cartilage related to FGF receptors. Genomics 2000, 69, 275-279. [CrossRef] [PubMed]

19. Trueb, B.; Zhuang, L.; Taeschler, S.; Wiedemann, M. Characterization of FGFRL1, a novel fibroblast growth factor (FGF) receptor preferentially expressed in skeletal tissues. J. Biol. Chem. 2003, 278, 33857-33865. [CrossRef]

20. Goetz, R.; Mohammadi, M. Exploring mechanisms of FGF signalling through the lens of structural biology. Nat. Rev. Mol. Cell Biol. 2013, 14, 166-180. [CrossRef]

21. Furdui, C.M.; Lew, E.D.; Schlessinger, J.; Anderson, K.S. Autophosphorylation of FGFR1 kinase is mediated by a sequential and precisely ordered reaction. Mol. Cell 2006, 21, 711-717. [CrossRef]

22. Zinkle, A.; Mohammadi, M. A threshold model for receptor tyrosine kinase signaling specificity and cell fate determination. F1000Researcharch 2018, 7. [CrossRef]

23. Sarabipour, S.; Hristova, K. Mechanism of FGF receptor dimerization and activation. Nat. Commun. 2016, 7, 10262. [CrossRef]

24. Duchesne, L.; Tissot, B.; Rudd, T.R.; Dell, A.; Fernig, D.G. N-glycosylation of fibroblast growth factor receptor 1 regulates ligand and heparan sulfate co-receptor binding. J. Biol. Chem. 2006, 281, 27178-27189. [CrossRef] [PubMed]

25. Polanska, U.M.; Duchesne, L.; Harries, J.C.; Fernig, D.G.; Kinnunen, T.K. N-Glycosylation regulates fibroblast growth factor receptor/EGL-15 activity in Caenorhabditis elegans in vivo. J. Biol. Chem. 2009, 284, 33030-33039. [CrossRef] [PubMed]

26. Brooks, A.N.; Kilgour, E.; Smith, P.D. Molecular pathways: Fibroblast growth factor signaling: A new therapeutic opportunity in cancer. Clin. Cancer Res. 2012, 18, 1855-1862. [CrossRef] [PubMed]

27. Haugsten, E.M.; Malecki, J.; Bjorklund, S.M.; Olsnes, S.; Wesche, J. Ubiquitination of fibroblast growth factor receptor 1 is required for its intracellular sorting but not for its endocytosis. Mol. Biol. Cell 2008, 19, 3390-3403. [CrossRef] [PubMed]

28. Zakrzewska, M.; Haugsten, E.M.; Nadratowska-Wesolowska, B.; Oppelt, A.; Hausott, B.; Jin, Y.; Otlewski, J.; Wesche, J.; Wiedlocha, A. ERK-mediated phosphorylation of fibroblast growth factor receptor 1 on Ser777 inhibits signaling. Sci. Signal. 2013, 6, ra11. [CrossRef]

29. Porebska, N.; Latko, M.; Kucinska, M.; Zakrzewska, M.; Otlewski, J.; Opalinski, L. Targeting Cellular Trafficking of Fibroblast Growth Factor Receptors as a Strategy for Selective Cancer Treatment. J. Clin. Med. 2018, 8, 7. [CrossRef] [PubMed]

30. Hitosugi, T.; Fan, J.; Chung, T.W.; Lythgoe, K.; Wang, X.; Xie, J.; Ge, Q.; Gu, T.L.; Polakiewicz, R.D.; Roesel, J.L.; et al. Tyrosine phosphorylation of mitochondrial pyruvate dehydrogenase kinase 1 is important for cancer metabolism. Mol. Cell 2011, 44, 864-877. [CrossRef] [PubMed]

31. Stachowiak, M.K.; Maher, P.A.; Stachowiak, E.K. Integrative nuclear signaling in cell development-a role for FGF receptor-1. DNA Cell Biol. 2007, 26, 811-826. [CrossRef] [PubMed]

32. Itoh, N.; Ohta, H.; Konishi, M. Endocrine FGFs: Evolution, Physiology, Pathophysiology, and Pharmacotherapy. Front. Endocrinol. (Lausanne) 2015, 6, 154. [CrossRef] [PubMed]

33. Vecchione, A.; Cooper, H.J.; Trim, K.J.; Akbarzadeh, S.; Heath, J.K.; Wheldon, L.M. Protein partners in the life history of activated fibroblast growth factor receptors. Proteomics 2007, 7, 4565-4578. [CrossRef] [PubMed]

34. Balek, L.; Nemec, P.; Konik, P.; Kunova Bosakova, M.; Varecha, M.; Gudernova, I.; Medalova, J.; Krakow, D.; Krejci, P. Proteomic analyses of signalling complexes associated with receptor tyrosine kinase identify novel members of fibroblast growth factor receptor 3 interactome. Cell Signal. 2018, 42, 144-154. [CrossRef] [PubMed]

35. Kostas, M.; Haugsten, E.M.; Zhen, Y.; Sorensen, V.; Szybowska, P.; Fiorito, E.; Lorenz, S.; Jones, N.; de Souza, G.A.; Wiedlocha, A.; et al. Protein Tyrosine Phosphatase Receptor Type G (PTPRG) Controls Fibroblast Growth Factor Receptor (FGFR) 1 Activity and Influences Sensitivity to FGFR Kinase Inhibitors. Mol. Cell Proteom. 2018, 17, 850-870. [CrossRef] [PubMed]

36. Calebiro, D.; Koszegi, Z. The subcellular dynamics of GPCR signaling. Mol. Cell Endocrinol. 2019. [CrossRef] [PubMed]

37. Calebiro, D.; Jobin, M.L. Hot spots for GPCR signaling: Lessons from single-molecule microscopy. Curr. Opin. Cell Biol. 2018, 57,57-63. [CrossRef] 
38. Milligan, G.; Ward, R.J.; Marsango, S. GPCR homo-oligomerization. Curr. Opin. Cell. Biol. 2018, 57, 40-47. [CrossRef] [PubMed]

39. Thal, D.M.; Glukhova, A.; Sexton, P.M.; Christopoulos, A. Structural insights into G-protein-coupled receptor allostery. Nature 2018, 559, 45-53. [CrossRef] [PubMed]

40. Mahoney, J.P.; Sunahara, R.K. Mechanistic insights into GPCR-G protein interactions. Curr. Opin. Struct. Biol. 2016, 41, 247-254. [CrossRef] [PubMed]

41. Husted, A.S.; Trauelsen, M.; Rudenko, O.; Hjorth, S.A.; Schwartz, T.W. GPCR-Mediated Signaling of Metabolites. Cell Metab. 2017, 25, 777-796. [CrossRef] [PubMed]

42. Di Liberto, V.; Mudo, G.; Belluardo, N. Crosstalk between receptor tyrosine kinases (RTKs) and G protein-coupled receptors (GPCR) in the brain: Focus on heteroreceptor complexes and related functional neurotrophic effects. Neuropharmacology 2018. [CrossRef] [PubMed]

43. Leung, C.C.Y.; Wong, Y.H. Role of G Protein-Coupled Receptors in the Regulation of Structural Plasticity and Cognitive Function. Molecules 2017, 22, 1239. [CrossRef] [PubMed]

44. Belluardo, N.; Wu, G.; Mudo, G.; Hansson, A.C.; Pettersson, R.; Fuxe, K. Comparative localization of fibroblast growth factor receptor-1, -2 , and -3 mRNAs in the rat brain: In situ hybridization analysis. J. Comp. Neurol. 1997, 379, 226-246. [CrossRef]

45. Gonzalez, A.M.; Berry, M.; Maher, P.A.; Logan, A.; Baird, A. A comprehensive analysis of the distribution of FGF-2 and FGFR1 in the rat brain. Brain Res. 1995, 701, 201-226. [CrossRef]

46. Ford-Perriss, M.; Abud, H.; Murphy, M. Fibroblast growth factors in the developing central nervous system. Clin. Exp. Pharm. Physiol. 2001, 28, 493-503. [CrossRef]

47. Choubey, L.; Collette, J.C.; Smith, K.M. Quantitative assessment of fibroblast growth factor receptor 1 expression in neurons and glia. Peer]. 2017, 5, e3173. [CrossRef] [PubMed]

48. Itoh, N.; Yazaki, N.; Tagashira, S.; Miyake, A.; Ozaki, K.; Minami, M.; Satoh, M.; Ohta, M.; Kawasaki, T. Rat FGF receptor-4 mRNA in the brain is expressed preferentially in the medial habenular nucleus. Brain Res. Mol. Brain Res. 1994, 21, 344-348. [CrossRef]

49. Turner, C.A.; Akil, H.; Watson, S.J.; Evans, S.J. The fibroblast growth factor system and mood disorders. Biol. Psychiatry 2006, 59, 1128-1135. [CrossRef]

50. Flajolet, M.; Wang, Z.; Futter, M.; Shen, W.; Nuangchamnong, N.; Bendor, J.; Wallach, I.; Nairn, A.C.; Surmeier, D.J.; Greengard, P. FGF acts as a co-transmitter through adenosine A(2A) receptor to regulate synaptic plasticity. Nat. Neurosci. 2008, 11, 1402-1409. [CrossRef]

51. Howlett, A.C.; Barth, F.; Bonner, T.I.; Cabral, G.; Casellas, P.; Devane, W.A.; Felder, C.C.; Herkenham, M.; Mackie, K.; Martin, B.R.; et al. International Union of Pharmacology. XXVII. Classification of cannabinoid receptors. Pharm. Rev. 2002, 54, 161-202. [CrossRef] [PubMed]

52. Asimaki, O.; Leondaritis, G.; Lois, G.; Sakellaridis, N.; Mangoura, D. Cannabinoid 1 receptor-dependent transactivation of fibroblast growth factor receptor 1 emanates from lipid rafts and amplifies extracellular signal-regulated kinase 1/2 activation in embryonic cortical neurons. J. Neurochem. 2011, 116, 866-873. [CrossRef] [PubMed]

53. Di Liberto, V.; Borroto-Escuela, D.O.; Frinchi, M.; Verdi, V.; Fuxe, K.; Belluardo, N.; Mudo, G. Existence of muscarinic acetylcholine receptor $(\mathrm{mAChR})$ and fibroblast growth factor receptor (FGFR) heteroreceptor complexes and their enhancement of neurite outgrowth in neural hippocampal cultures. Biochim. Biophys. Acta Gen. Subj. 2017, 1861, 235-245. [CrossRef]

54. Borroto-Escuela, D.O.; Narvaez, M.; Perez-Alea, M.; Tarakanov, A.O.; Jimenez-Beristain, A.; Mudo, G.; Agnati, L.F.; Ciruela, F.; Belluardo, N.; Fuxe, K. Evidence for the existence of FGFR1-5-HT1A heteroreceptor complexes in the midbrain raphe 5-HT system. Biochem. Biophys. Res. Commun. 2015, 456, 489-493. [CrossRef] [PubMed]

55. Borroto-Escuela, D.O.; Romero-Fernandez, W.; Mudo, G.; Perez-Alea, M.; Ciruela, F.; Tarakanov, A.O.; Narvaez, M.; Di Liberto, V.; Agnati, L.F.; Belluardo, N.; et al. Fibroblast growth factor receptor 15-hydroxytryptamine 1A heteroreceptor complexes and their enhancement of hippocampal plasticity. Biol. Psychiatry 2012, 71, 84-91. [CrossRef]

56. Borroto-Escuela, D.O.; Perez-Alea, M.; Narvaez, M.; Tarakanov, A.O.; Mudo, G.; Jimenez-Beristain, A.; Agnati, L.F.; Ciruela, F.; Belluardo, N.; Fuxe, K. Enhancement of the FGFR1 signaling in the FGFR1-5-HT1A heteroreceptor complex in midbrain raphe 5-HT neuron systems. Relevance for neuroplasticity and depression. Biochem. Biophys. Res. Commun. 2015, 463, 180-186. [CrossRef] [PubMed] 
57. Borroto-Escuela, D.O.; DuPont, C.M.; Li, X.; Savelli, D.; Lattanzi, D.; Srivastava, I.; Narvaez, M.; Di Palma, M.; Barbieri, E.; Andrade-Talavera, Y.; et al. Disturbances in the FGFR1-5-HT1A Heteroreceptor Complexes in the Raphe-Hippocampal 5-HT System Develop in a Genetic Rat Model of Depression. Front. Cell Neurosci. 2017, 11, 309. [CrossRef] [PubMed]

58. Borroto-Escuela, D.O.; Tarakanov, A.O.; Fuxe, K. FGFR1-5-HT1A Heteroreceptor Complexes: Implications for Understanding and Treating Major Depression. Trends Neurosci. 2016, 39, 5-15. [CrossRef] [PubMed]

59. Borroto-Escuela, D.O.; Carlsson, J.; Ambrogini, P.; Narvaez, M.; Wydra, K.; Tarakanov, A.O.; Li, X.; Millon, C.; Ferraro, L.; Cuppini, R.; et al. Understanding the Role of GPCR Heteroreceptor Complexes in Modulating the Brain Networks in Health and Disease. Front. Cell Neurosci. 2017, 11, 37. [CrossRef] [PubMed]

60. Belcheva, M.M.; Haas, P.D.; Tan, Y.; Heaton, V.M.; Coscia, C.J. The fibroblast growth factor receptor is at the site of convergence between mu-opioid receptor and growth factor signaling pathways in rat C6 glioma cells. J. Pharm. Exp. 2002, 303, 909-918. [CrossRef] [PubMed]

61. Volinsky, N.; Kholodenko, B.N. Complexity of receptor tyrosine kinase signal processing. Cold Spring Harb. Perspect. Biol. 2013, 5, a009043. [CrossRef] [PubMed]

62. Saha, N.; Robev, D.; Mason, E.O.; Himanen, J.P.; Nikolov, D.B. Therapeutic potential of targeting the Eph/ephrin signaling complex. Int. J. Biochem. Cell Biol. 2018, 105, 123-133. [CrossRef]

63. Pasquale, E.B. Eph receptor signalling casts a wide net on cell behaviour. Nat. Rev. Mol. Cell Biol. 2005, 6, 462-475. [CrossRef]

64. Lisabeth, E.M.; Falivelli, G.; Pasquale, E.B. Eph receptor signaling and ephrins. Cold Spring Harb. Perspect. Biol. 2013, 5. [CrossRef] [PubMed]

65. Shiuan, E.; Chen, J. Eph Receptor Tyrosine Kinases in Tumor Immunity. Cancer Res. 2016, 76, 6452-6457. [CrossRef] [PubMed]

66. Himanen, J.P.; Rajashankar, K.R.; Lackmann, M.; Cowan, C.A.; Henkemeyer, M.; Nikolov, D.B. Crystal structure of an Eph receptor-ephrin complex. Nature 2001, 414, 933-938. [CrossRef] [PubMed]

67. Kalo, M.S.; Pasquale, E.B. Signal transfer by Eph receptors. Cell Tissue Res. 1999, 298, 1-9. [CrossRef]

68. Janes, P.W.; Nievergall, E.; Lackmann, M. Concepts and consequences of Eph receptor clustering. Semin. Cell Dev. Biol. 2012, 23, 43-50. [CrossRef]

69. Himanen, J.P.; Yermekbayeva, L.; Janes, P.W.; Walker, J.R.; Xu, K.; Atapattu, L.; Rajashankar, K.R.; Mensinga, A.; Lackmann, M.; Nikolov, D.B.; et al. Architecture of Eph receptor clusters. Proc. Natl. Acad. Sci. USA 2010, 107, 10860-10865. [CrossRef] [PubMed]

70. Schmucker, D.; Zipursky, S.L. Signaling downstream of Eph receptors and ephrin ligands. Cell 2001, 105, 701-704. [CrossRef]

71. Yokote, H.; Fujita, K.; Jing, X.; Sawada, T.; Liang, S.; Yao, L.; Yan, X.; Zhang, Y.; Schlessinger, J.; Sakaguchi, K. Trans-activation of EphA4 and FGF receptors mediated by direct interactions between their cytoplasmic domains. Proc. Natl. Acad. Sci. USA 2005, 102, 18866-18871. [CrossRef] [PubMed]

72. Fukai, J.; Yokote, H.; Yamanaka, R.; Arao, T.; Nishio, K.; Itakura, T. EphA4 promotes cell proliferation and migration through a novel EphA4-FGFR1 signaling pathway in the human glioma U251 cell line. Mol. Cancer 2008, 7, 2768-2778. [CrossRef] [PubMed]

73. Gotoh, N. Regulation of growth factor signaling by FRS2 family docking/scaffold adaptor proteins. Cancer Sci. 2008, 99, 1319-1325. [CrossRef] [PubMed]

74. Sawada, T.; Jing, X.; Zhang, Y.; Shimada, E.; Yokote, H.; Miyajima, M.; Sakaguchi, K. Ternary complex formation of EphA4, FGFR and FRS2alpha plays an important role in the proliferation of embryonic neural stem/progenitor cells. Genes Cells 2010, 15, 297-311. [CrossRef] [PubMed]

75. Sawada, T.; Arai, D.; Jing, X.; Furushima, K.; Chen, Q.; Kawakami, K.; Yokote, H.; Miyajima, M.; Sakaguchi, K. Trans-Activation between EphA and FGFR Regulates Self-Renewal and Differentiation of Mouse Embryonic Neural Stem/Progenitor Cells via Differential Activation of FRS2alpha. PLoS ONE 2015, 10, e0128826. [CrossRef]

76. Zhang, Y.; Sawada, T.; Jing, X.; Yokote, H.; Yan, X.; Sakaguchi, K. Regulation of ephexin1, a guanine nucleotide exchange factor of Rho family GTPases, by fibroblast growth factor receptor-mediated tyrosine phosphorylation. J. Biol. Chem. 2007, 282, 31103-31112. [CrossRef] [PubMed]

77. Lee, S.; Shatadal, S.; Griep, A.E. Dlg-1 Interacts With and Regulates the Activities of Fibroblast Growth Factor Receptors and EphA2 in the Mouse Lens. Invest. Ophthalmol. Vis. Sci. 2016, 57, 707-718. [CrossRef] [PubMed] 
78. Lee, S.; Griep, A.E. Loss of Dlg-1 in the mouse lens impairs fibroblast growth factor receptor signaling. PLoS ONE 2014, 9, e97470. [CrossRef] [PubMed]

79. Cao, Y. Multifarious functions of PDGFs and PDGFRs in tumor growth and metastasis. Trends Mol. Med. 2013, 19, 460-473. [CrossRef]

80. Papadopoulos, N.; Lennartsson, J. The PDGF/PDGFR pathway as a drug target. Mol. Asp. Med. 2018, 62, 75-88. [CrossRef] [PubMed]

81. Shim, A.H.; Liu, H.; Focia, P.J.; Chen, X.; Lin, P.C.; He, X. Structures of a platelet-derived growth factor/propeptide complex and a platelet-derived growth factor/receptor complex. Proc. Natl. Acad. Sci. USA 2010, 107, 11307-11312. [CrossRef] [PubMed]

82. Miyazawa, K.; Backstrom, G.; Leppanen, O.; Persson, C.; Wernstedt, C.; Hellman, U.; Heldin, C.H.; Ostman, A. Role of immunoglobulin-like domains 2-4 of the platelet-derived growth factor alpha-receptor in ligand-receptor complex assembly. J. Biol. Chem. 1998, 273, 25495-25502. [CrossRef]

83. Faraone, D.; Aguzzi, M.S.; Ragone, G.; Russo, K.; Capogrossi, M.C.; Facchiano, A. Heterodimerization of FGF-receptor 1 and PDGF-receptor-alpha: A novel mechanism underlying the inhibitory effect of PDGF-BB on FGF-2 in human cells. Blood 2006, 107, 1896-1902. [CrossRef] [PubMed]

84. Chen, P.Y.; Simons, M.; Friesel, R. FRS2 via fibroblast growth factor receptor 1 is required for platelet-derived growth factor receptor beta-mediated regulation of vascular smooth muscle marker gene expression. J. Biol. Chem. 2009, 284, 15980-15992. [CrossRef] [PubMed]

85. Russo, K.; Ragone, R.; Facchiano, A.M.; Capogrossi, M.C.; Facchiano, A. Platelet-derived growth factor-BB and basic fibroblast growth factor directly interact in vitro with high affinity. J. Biol. Chem. 2002, 277, 1284-1291. [CrossRef] [PubMed]

86. De Marchis, F.; Ribatti, D.; Giampietri, C.; Lentini, A.; Faraone, D.; Scoccianti, M.; Capogrossi, M.C.; Facchiano, A. Platelet-derived growth factor inhibits basic fibroblast growth factor angiogenic properties in vitro and in vivo through its alpha receptor. Blood 2002, 99, 2045-2053. [CrossRef]

87. Facchiano, A.; De Marchis, F.; Turchetti, E.; Facchiano, F.; Guglielmi, M.; Denaro, A.; Palumbo, R.; Scoccianti, M.; Capogrossi, M.C. The chemotactic and mitogenic effects of platelet-derived growth factor-BB on rat aorta smooth muscle cells are inhibited by basic fibroblast growth factor. J. Cell Sci. 2000, 113 Pt 16, 2855-2863.

88. Kono, S.A.; Heasley, L.E.; Doebele, R.C.; Camidge, D.R. Adding to the mix: Fibroblast growth factor and platelet-derived growth factor receptor pathways as targets in non-small cell lung cancer. Curr. Cancer Drug Targets 2012, 12, 107-123. [CrossRef] [PubMed]

89. Takeichi, M. Morphogenetic roles of classic cadherins. Curr. Opin. Cell Biol. 1995, 7, 619-627. [CrossRef]

90. Kourtidis, A.; Lu, R.; Pence, L.J.; Anastasiadis, P.Z. A central role for cadherin signaling in cancer. Exp. Cell Res. 2017, 358, 78-85. [CrossRef]

91. Gloushankova, N.A.; Rubtsova, S.N.; Zhitnyak, I.Y. Cadherin-mediated cell-cell interactions in normal and cancer cells. Tissue Barriers 2017, 5, e1356900. [CrossRef] [PubMed]

92. Fontenete, S.; Pena-Jimenez, D.; Perez-Moreno, M. Heterocellular cadherin connections: Coordinating adhesive cues in homeostasis and cancer. F1000Research 2017, 6, 1010. [CrossRef]

93. Nguyen, T.; Mege, R.M. N-Cadherin and Fibroblast Growth Factor Receptors crosstalk in the control of developmental and cancer cell migrations. Eur. J. Cell Biol. 2016, 95, 415-426. [CrossRef]

94. Williams, E.J.; Furness, J.; Walsh, F.S.; Doherty, P. Activation of the FGF receptor underlies neurite outgrowth stimulated by L1, N-CAM, and N-cadherin. Neuron 1994, 13, 583-594. [CrossRef]

95. Saffell, J.L.; Williams, E.J.; Mason, I.J.; Walsh, F.S.; Doherty, P. Expression of a dominant negative FGF receptor inhibits axonal growth and FGF receptor phosphorylation stimulated by CAMs. Neuron 1997, 18, 231-242. [CrossRef]

96. Ronn, L.C.; Doherty, P.; Holm, A.; Berezin, V.; Bock, E. Neurite outgrowth induced by a synthetic peptide ligand of neural cell adhesion molecule requires fibroblast growth factor receptor activation. J. Neurochem. 2000, 75, 665-671. [CrossRef] [PubMed]

97. Sanchez-Heras, E.; Howell, F.V.; Williams, G.; Doherty, P. The fibroblast growth factor receptor acid box is essential for interactions with N-cadherin and all of the major isoforms of neural cell adhesion molecule. J. Biol. Chem. 2006, 281, 35208-35216. [CrossRef]

98. Suyama, K.; Shapiro, I.; Guttman, M.; Hazan, R.B. A signaling pathway leading to metastasis is controlled by N-cadherin and the FGF receptor. Cancer Cell 2002, 2, 301-314. [CrossRef] 
99. Hulit, J.; Suyama, K.; Chung, S.; Keren, R.; Agiostratidou, G.; Shan, W.; Dong, X.; Williams, T.M.; Lisanti, M.P.; Knudsen, K.; et al. N-cadherin signaling potentiates mammary tumor metastasis via enhanced extracellular signal-regulated kinase activation. Cancer Res. 2007, 67, 3106-3116. [CrossRef]

100. Qian, X.; Anzovino, A.; Kim, S.; Suyama, K.; Yao, J.; Hulit, J.; Agiostratidou, G.; Chandiramani, N.; McDaid, H.M.; Nagi, C.; et al. N-cadherin/FGFR promotes metastasis through epithelial-to-mesenchymal transition and stem/progenitor cell-like properties. Oncogene 2014, 33, 3411-3421. [CrossRef]

101. Takehara, T.; Teramura, T.; Onodera, Y.; Frampton, J.; Fukuda, K. Cdh2 stabilizes FGFR1 and contributes to primed-state pluripotency in mouse epiblast stem cells. Sci. Rep. 2015, 5, 14722. [CrossRef] [PubMed]

102. Cavallaro, U.; Niedermeyer, J.; Fuxa, M.; Christofori, G. N-CAM modulates tumour-cell adhesion to matrix by inducing FGF-receptor signalling. Nat. Cell Biol. 2001, 3, 650-657. [CrossRef]

103. Quintanal-Villalonga, A.; Ojeda-Marquez, L.; Marrugal, A.; Yague, P.; Ponce-Aix, S.; Salinas, A.; Carnero, A.; Ferrer, I.; Molina-Pinelo, S.; Paz-Ares, L. The FGFR4-388arg Variant Promotes Lung Cancer Progression by N-Cadherin Induction. Sci. Rep. 2018, 8, 2394. [CrossRef]

104. Kimura, Y.; Matsunami, H.; Inoue, T.; Shimamura, K.; Uchida, N.; Ueno, T.; Miyazaki, T.; Takeichi, M. Cadherin-11 expressed in association with mesenchymal morphogenesis in the head, somite, and limb bud of early mouse embryos. Dev. Biol. 1995, 169, 347-358. [CrossRef]

105. Sfikakis, P.P.; Vlachogiannis, N.I.; Christopoulos, P.F. Cadherin-11 as a therapeutic target in chronic, inflammatory rheumatic diseases. Clin. Immunol. 2017, 176, 107-113. [CrossRef]

106. Birtolo, C.; Pham, H.; Morvaridi, S.; Chheda, C.; Go, V.L.; Ptasznik, A.; Edderkaoui, M.; Weisman, M.H.; Noss, E.; Brenner, M.B.; et al. Cadherin-11 Is a Cell Surface Marker Up-Regulated in Activated Pancreatic Stellate Cells and Is Involved in Pancreatic Cancer Cell Migration. Am. J. Pathol. 2017, 187, 146-155. [CrossRef]

107. Ortiz, A.; Lee, Y.C.; Yu, G.; Liu, H.C.; Lin, S.C.; Bilen, M.A.; Cho, H.; Yu-Lee, L.Y.; Lin, S.H. Angiomotin is a novel component of cadherin-11/beta-catenin/p120 complex and is critical for cadherin-11-mediated cell migration. FASEB J. 2015, 29, 1080-1091. [CrossRef] [PubMed]

108. Kim, N.H.; Choi, S.H.; Lee, T.R.; Lee, C.H.; Lee, A.Y. Cadherin 11, a miR-675 target, induces N-cadherin expression and epithelial-mesenchymal transition in melasma. J. Invest. Derm. 2014, 134, 2967-2976. [CrossRef] [PubMed]

109. Chu, K.; Cheng, C.J.; Ye, X.; Lee, Y.C.; Zurita, A.J.; Chen, D.T.; Yu-Lee, L.Y.; Zhang, S.; Yeh, E.T.; Hu, M.C.; et al. Cadherin-11 promotes the metastasis of prostate cancer cells to bone. Mol. Cancer Res. 2008, 6, 1259-1267. [CrossRef] [PubMed]

110. Boscher, C.; Mege, R.M. Cadherin-11 interacts with the FGF receptor and induces neurite outgrowth through associated downstream signalling. Cell Signal. 2008, 20, 1061-1072. [CrossRef] [PubMed]

111. Mizutani, K.; Takai, Y. Nectin spot: A novel type of nectin-mediated cell adhesion apparatus. Biochem. J. 2016, 473, 2691-2715. [CrossRef] [PubMed]

112. Huang, K.; Lui, W.Y. Nectins and nectin-like molecules (Necls): Recent findings and their role and regulation in spermatogenesis. Semin. Cell Dev. Biol. 2016, 59, 54-61. [CrossRef]

113. Bojesen, K.B.; Clausen, O.; Rohde, K.; Christensen, C.; Zhang, L.; Li, S.; Kohler, L.; Nielbo, S.; Nielsen, J.; Gjorlund, M.D.; et al. Nectin-1 binds and signals through the fibroblast growth factor receptor. J. Biol. Chem. 2012, 287, 37420-37433. [CrossRef]

114. Owczarek, S.; Berezin, V. Neuroplastin: Cell adhesion molecule and signaling receptor. Int. J. Biochem. Cell Biol. 2012, 44, 1-5. [CrossRef]

115. Langnaese, K.; Mummery, R.; Gundelfinger, E.D.; Beesley, P.W. Immunoglobulin superfamily members gp65 and gp55: Tissue distribution of glycoforms. FEBS Lett. 1998, 429, 284-288. [CrossRef]

116. Langnaese, K.; Beesley, P.W.; Gundelfinger, E.D. Synaptic membrane glycoproteins gp65 and gp55 are new members of the immunoglobulin superfamily. J. Biol. Chem. 1997, 272, 821-827. [CrossRef] [PubMed]

117. Owczarek, S.; Kiryushko, D.; Larsen, M.H.; Kastrup, J.S.; Gajhede, M.; Sandi, C.; Berezin, V.; Bock, E.; Soroka, V. Neuroplastin-55 binds to and signals through the fibroblast growth factor receptor. FASEB J. 2010, 24, 1139-1150. [CrossRef] [PubMed]

118. Colombo, F.; Meldolesi, J. L1-CAM and N-CAM: From Adhesion Proteins to Pharmacological Targets. Trends Pharm. Sci. 2015, 36, 769-781. [CrossRef] 
119. Sytnyk, V.; Leshchyns'ka, I.; Schachner, M. Neural Cell Adhesion Molecules of the Immunoglobulin Superfamily Regulate Synapse Formation, Maintenance, and Function. Trends Neurosci. 2017, 40, 295-308. [CrossRef] [PubMed]

120. Aonurm-Helm, A.; Jaako, K.; Jurgenson, M.; Zharkovsky, A. Pharmacological approach for targeting dysfunctional brain plasticity: Focus on neural cell adhesion molecule (NCAM). Pharm. Res. 2016, 113, 731-738. [CrossRef] [PubMed]

121. Francavilla, C.; Cattaneo, P.; Berezin, V.; Bock, E.; Ami, D.; de Marco, A.; Christofori, G.; Cavallaro, U. The binding of NCAM to FGFR1 induces a specific cellular response mediated by receptor trafficking. J. Cell Biol. 2009, 187, 1101-1116. [CrossRef]

122. Christensen, C.; Lauridsen, J.B.; Berezin, V.; Bock, E.; Kiselyov, V.V. The neural cell adhesion molecule binds to fibroblast growth factor receptor 2. FEBS Lett. 2006, 580, 3386-3390. [CrossRef] [PubMed]

123. Francavilla, C.; Loeffler, S.; Piccini, D.; Kren, A.; Christofori, G.; Cavallaro, U. Neural cell adhesion molecule regulates the cellular response to fibroblast growth factor. J. Cell Sci. 2007, 120, 4388-4394. [CrossRef] [PubMed]

124. Hansen, S.M.; Li, S.; Bock, E.; Berezin, V. Synthetic NCAM-derived ligands of the fibroblast growth factor receptor. Adv. Exp. Med. Biol. 2010, 663, 355-372. [CrossRef] [PubMed]

125. Abe, K.; Ohuchi, H.; Tanabe, H.; Imanaka, K.; Asano, H.; Kato, M.; Yokote, Y.; Kyo, S. Aortic root remodeling and coronary artery bypass grafting for acute type A aortic dissection involving the left main coronary artery; report of a case. Kyobu Geka 2005, 58, 897-901.

126. Kiselyov, V.V.; Soroka, V.; Berezin, V.; Bock, E. Structural biology of NCAM homophilic binding and activation of FGFR. J. Neurochem. 2005, 94, 1169-1179. [CrossRef]

127. Christensen, C.; Berezin, V.; Bock, E. Neural cell adhesion molecule differentially interacts with isoforms of the fibroblast growth factor receptor. Neuroreport 2011, 22, 727-732. [CrossRef]

128. Zecchini, S.; Bombardelli, L.; Decio, A.; Bianchi, M.; Mazzarol, G.; Sanguineti, F.; Aletti, G.; Maddaluno, L.; Berezin, V.; Bock, E.; et al. The adhesion molecule NCAM promotes ovarian cancer progression via FGFR signalling. EMBO Mol. Med. 2011, 3, 480-494. [CrossRef]

129. Colombo, N.; Cavallaro, U. The interplay between NCAM and FGFR signalling underlies ovarian cancer progression. Ecancermedicalscience 2011, 5, 226. [CrossRef]

130. Zivotic, M.; Tampe, B.; Muller, G.; Muller, C.; Lipkovski, A.; Xu, X.; Nyamsuren, G.; Zeisberg, M.; Markovic-Lipkovski, J. Modulation of NCAM/FGFR1 signaling suppresses EMT program in human proximal tubular epithelial cells. PLoS ONE 2018, 13, e0206786. [CrossRef] [PubMed]

131. Kulahin, N.; Li, S.; Hinsby, A.; Kiselyov, V.; Berezin, V.; Bock, E. Fibronectin type III (FN3) modules of the neuronal cell adhesion molecule L1 interact directly with the fibroblast growth factor (FGF) receptor. Mol. Cell Neurosci. 2008, 37, 528-536. [CrossRef]

132. Riedle, S.; Kiefel, H.; Gast, D.; Bondong, S.; Wolterink, S.; Gutwein, P.; Altevogt, P. Nuclear translocation and signalling of L1-CAM in human carcinoma cells requires ADAM10 and presenilin/gamma-secretase activity. Biochem. J. 2009, 420, 391-402. [CrossRef] [PubMed]

133. Mohanan, V.; Temburni, M.K.; Kappes, J.C.; Galileo, D.S. L1CAM stimulates glioma cell motility and proliferation through the fibroblast growth factor receptor. Clin. Exp. Metastasis 2013, 30, 507-520. [CrossRef] [PubMed]

134. Gonzalez-Martinez, D.; Kim, S.H.; Hu, Y.; Guimond, S.; Schofield, J.; Winyard, P.; Vannelli, G.B.; Turnbull, J.; Bouloux, P.M. Anosmin-1 modulates fibroblast growth factor receptor 1 signaling in human gonadotropin-releasing hormone olfactory neuroblasts through a heparan sulfate-dependent mechanism. J. Neurosci. 2004, 24, 10384-10392. [CrossRef]

135. Bribian, A.; Barallobre, M.J.; Soussi-Yanicostas, N.; de Castro, F. Anosmin-1 modulates the FGF-2-dependent migration of oligodendrocyte precursors in the developing optic nerve. Mol. Cell Neurosci. 2006, 33, 2-14. [CrossRef] [PubMed]

136. Garcia-Gonzalez, D.; Clemente, D.; Coelho, M.; Esteban, P.F.; Soussi-Yanicostas, N.; de Castro, F. Dynamic roles of FGF-2 and Anosmin-1 in the migration of neuronal precursors from the subventricular zone during pre- and postnatal development. Exp. Neurol. 2010, 222, 285-295. [CrossRef] [PubMed]

137. Murcia-Belmonte, V.; Esteban, P.F.; Garcia-Gonzalez, D.; De Castro, F. Biochemical dissection of Anosmin-1 interaction with FGFR1 and components of the extracellular matrix. J. Neurochem. 2010, 115, 1256-1265. [CrossRef] 
138. Hu, Y.; Guimond, S.E.; Travers, P.; Cadman, S.; Hohenester, E.; Turnbull, J.E.; Kim, S.H.; Bouloux, P.M. Novel mechanisms of fibroblast growth factor receptor 1 regulation by extracellular matrix protein anosmin-1. J. Biol. Chem. 2009, 284, 29905-29920. [CrossRef] [PubMed]

139. Diaz-Balzac, C.A.; Lazaro-Pena, M.I.; Ramos-Ortiz, G.A.; Bulow, H.E. The Adhesion Molecule KAL-1/anosmin-1 Regulates Neurite Branching through a SAX-7/L1CAM-EGL-15/FGFR Receptor Complex. Cell Rep. 2015, 11, 1377-1384. [CrossRef] [PubMed]

140. Kriebel, M.; Wuchter, J.; Trinks, S.; Volkmer, H. Neurofascin: A switch between neuronal plasticity and stability. Int. J. Biochem. Cell Biol. 2012, 44, 694-697. [CrossRef]

141. Kirschbaum, K.; Kriebel, M.; Kranz, E.U.; Potz, O.; Volkmer, H. Analysis of non-canonical fibroblast growth factor receptor 1 (FGFR1) interaction reveals regulatory and activating domains of neurofascin. J. Biol. Chem. 2009, 284, 28533-28542. [CrossRef] [PubMed]

142. Pruss, T.; Kranz, E.U.; Niere, M.; Volkmer, H. A regulated switch of chick neurofascin isoforms modulates ligand recognition and neurite extension. Mol. Cell Neurosci. 2006, 31, 354-365. [CrossRef] [PubMed]

143. Sudhof, T.C. Neuroligins and neurexins link synaptic function to cognitive disease. Nature 2008, 455, $903-911$. [CrossRef] [PubMed]

144. Rudenko, G. Neurexins - versatile molecular platforms in the synaptic cleft. Curr. Opin. Struct. Biol. 2019, 54, 112-121. [CrossRef] [PubMed]

145. Gjorlund, M.D.; Nielsen, J.; Pankratova, S.; Li, S.; Korshunova, I.; Bock, E.; Berezin, V. Neuroligin-1 induces neurite outgrowth through interaction with neurexin-1beta and activation of fibroblast growth factor receptor-1. FASEB J. 2012, 26, 4174-4186. [CrossRef]

146. Kubick, N.; Brosamle, D.; Mickael, M.E. Molecular Evolution and Functional Divergence of the IgLON Family. Evol. Bioinform. Online 2018, 14, 1176934318775081. [CrossRef] [PubMed]

147. Funatsu, N.; Miyata, S.; Kumanogoh, H.; Shigeta, M.; Hamada, K.; Endo, Y.; Sokawa, Y.; Maekawa, S. Characterization of a novel rat brain glycosylphosphatidylinositol-anchored protein (Kilon), a member of the IgLON cell adhesion molecule family. J. Biol. Chem. 1999, 274, 8224-8230. [CrossRef] [PubMed]

148. Pischedda, F.; Piccoli, G. The IgLON Family Member Negr1 Promotes Neuronal Arborization Acting as Soluble Factor via FGFR2. Front. Mol. Neurosci. 2015, 8, 89. [CrossRef]

149. Casey, J.P.; Magalhaes, T.; Conroy, J.M.; Regan, R.; Shah, N.; Anney, R.; Shields, D.C.; Abrahams, B.S.; Almeida, J.; Bacchelli, E.; et al. A novel approach of homozygous haplotype sharing identifies candidate genes in autism spectrum disorder. Hum. Genet. 2012, 131, 565-579. [CrossRef] [PubMed]

150. Szczurkowska, J.; Pischedda, F.; Pinto, B.; Manago, F.; Haas, C.A.; Summa, M.; Bertorelli, R.; Papaleo, F.; Schafer, M.K.; Piccoli, G.; et al. NEGR1 and FGFR2 cooperatively regulate cortical development and core behaviours related to autism disorders in mice. Brain 2018, 141, 2772-2794. [CrossRef]

151. Sellar, G.C.; Watt, K.P.; Rabiasz, G.J.; Stronach, E.A.; Li, L.; Miller, E.P.; Massie, C.E.; Miller, J.; Contreras-Moreira, B.; Scott, D.; et al. OPCML at 11q25 is epigenetically inactivated and has tumor-suppressor function in epithelial ovarian cancer. Nat. Genet. 2003, 34, 337-343. [CrossRef] [PubMed]

152. Chen, H.; Ye, F.; Zhang, J.; Lu, W.; Cheng, Q.; Xie, X. Loss of OPCML expression and the correlation with CpG island methylation and LOH in ovarian serous carcinoma. Eur. J. Gynaecol. Oncol. 2007, 28, 464-467.

153. Reed, J.E.; Dunn, J.R.; du Plessis, D.G.; Shaw, E.J.; Reeves, P.; Gee, A.L.; Warnke, P.C.; Sellar, G.C.; Moss, D.J.; Walker, C. Expression of cellular adhesion molecule 'OPCML' is down-regulated in gliomas and other brain tumours. Neuropathol. Appl. Neurobiol. 2007, 33, 77-85. [CrossRef]

154. Cui, Y.; Ying, Y.; van Hasselt, A.; Ng, K.M.; Yu, J.; Zhang, Q.; Jin, J.; Liu, D.; Rhim, J.S.; Rha, S.Y.; et al. OPCML is a broad tumor suppressor for multiple carcinomas and lymphomas with frequently epigenetic inactivation. PLoS ONE 2008, 3, e2990. [CrossRef]

155. Zhang, N.; Xu, J.; Wang, Y.; Heng, X.; Yang, L.; Xing, X. Loss of opioid binding protein/cell adhesion molecule-like gene expression in gastric cancer. Oncol. Lett. 2018, 15, 9973-9977. [CrossRef] [PubMed]

156. McKie, A.B.; Vaughan, S.; Zanini, E.; Okon, I.S.; Louis, L.; de Sousa, C.; Greene, M.I.; Wang, Q.; Agarwal, R.; Shaposhnikov, D.; et al. The OPCML tumor suppressor functions as a cell surface repressor-adaptor, negatively regulating receptor tyrosine kinases in epithelial ovarian cancer. Cancer Discov. 2012, 2, 156-171. [CrossRef]

157. O'Sullivan, M.L.; de Wit, J.; Savas, J.N.; Comoletti, D.; Otto-Hitt, S.; Yates, J.R., 3rd; Ghosh, A. FLRT proteins are endogenous latrophilin ligands and regulate excitatory synapse development. Neuron 2012, 73, 903-910. [CrossRef] [PubMed] 
158. Sando, R.; Jiang, X.; Sudhof, T.C. Latrophilin GPCRs direct synapse specificity by coincident binding of FLRTs and teneurins. Science 2019, 363. [CrossRef] [PubMed]

159. Del Toro, D.; Ruff, T.; Cederfjall, E.; Villalba, A.; Seyit-Bremer, G.; Borrell, V.; Klein, R. Regulation of Cerebral Cortex Folding by Controlling Neuronal Migration via FLRT Adhesion Molecules. Cell 2017, 169, 621-635.e616. [CrossRef] [PubMed]

160. Jackson, V.A.; Mehmood, S.; Chavent, M.; Roversi, P.; Carrasquero, M.; Del Toro, D.; Seyit-Bremer, G.; Ranaivoson, F.M.; Comoletti, D.; Sansom, M.S.; et al. Super-complexes of adhesion GPCRs and neural guidance receptors. Nat. Commun. 2016, 7, 11184. [CrossRef] [PubMed]

161. Lacy, S.E.; Bonnemann, C.G.; Buzney, E.A.; Kunkel, L.M. Identification of FLRT1, FLRT2, and FLRT3: A novel family of transmembrane leucine-rich repeat proteins. Genomics 1999, 62, 417-426. [CrossRef] [PubMed]

162. Karaulanov, E.E.; Bottcher, R.T.; Niehrs, C. A role for fibronectin-leucine-rich transmembrane cell-surface proteins in homotypic cell adhesion. EMBO Rep. 2006, 7, 283-290. [CrossRef] [PubMed]

163. Bottcher, R.T.; Pollet, N.; Delius, H.; Niehrs, C. The transmembrane protein XFLRT3 forms a complex with FGF receptors and promotes FGF signalling. Nat. Cell Biol. 2004, 6, 38-44. [CrossRef] [PubMed]

164. Wei, K.; Xu, Y.; Tse, H.; Manolson, M.F.; Gong, S.G. Mouse FLRT2 interacts with the extracellular and intracellular regions of FGFR2. J. Dent. Res. 2011, 90, 1234-1239. [CrossRef] [PubMed]

165. Haines, B.P.; Wheldon, L.M.; Summerbell, D.; Heath, J.K.; Rigby, P.W. Regulated expression of FLRT genes implies a functional role in the regulation of FGF signalling during mouse development. Dev. Biol. 2006, 297, 14-25. [CrossRef] [PubMed]

166. Wheldon, L.M.; Haines, B.P.; Rajappa, R.; Mason, I.; Rigby, P.W.; Heath, J.K. Critical role of FLRT1 phosphorylation in the interdependent regulation of FLRT1 function and FGF receptor signalling. PLoS ONE 2010, 5, e10264. [CrossRef] [PubMed]

167. Humphries, J.D.; Chastney, M.R.; Askari, J.A.; Humphries, M.J. Signal transduction via integrin adhesion complexes. Curr. Opin. Cell Biol. 2019, 56, 14-21. [CrossRef]

168. Barczyk, M.; Carracedo, S.; Gullberg, D. Integrins. Cell Tissue Res. 2010, 339, 269-280. [CrossRef] [PubMed]

169. Harburger, D.S.; Calderwood, D.A. Integrin signalling at a glance. J. Cell Sci. 2009, 122, 159-163. [CrossRef]

170. Hamidi, H.; Ivaska, J. Every step of the way: Integrins in cancer progression and metastasis. Nat. Rev. Cancer 2018, 18, 533-548. [CrossRef]

171. Yamaji, S.; Saegusa, J.; Ieguchi, K.; Fujita, M.; Mori, S.; Takada, Y.K.; Takada, Y. A novel fibroblast growth factor-1 (FGF1) mutant that acts as an FGF antagonist. PLoS ONE 2010, 5, e10273. [CrossRef] [PubMed]

172. Mori, S.; Wu, C.Y.; Yamaji, S.; Saegusa, J.; Shi, B.; Ma, Z.; Kuwabara, Y.; Lam, K.S.; Isseroff, R.R.; Takada, Y.K.; et al. Direct binding of integrin alphavbeta3 to FGF1 plays a role in FGF1 signaling. J. Biol. Chem. 2008, 283, 18066-18075. [CrossRef]

173. Mori, S.; Tran, V.; Nishikawa, K.; Kaneda, T.; Hamada, Y.; Kawaguchi, N.; Fujita, M.; Saegusa, J.; Takada, Y.K.; Matsuura, N.; et al. A dominant-negative FGF1 mutant (the R50E mutant) suppresses tumorigenesis and angiogenesis. PLoS ONE 2013, 8, e57927. [CrossRef]

174. Mori, S.; Hatori, N.; Kawaguchi, N.; Hamada, Y.; Shih, T.C.; Wu, C.Y.; Lam, K.S.; Matsuura, N.; Yamamoto, H.; Takada, Y.K.; et al. The integrin-binding defective FGF2 mutants potently suppress FGF2 signalling and angiogenesis. Biosci. Rep. 2017, 37. [CrossRef] [PubMed]

175. Yayon, A.; Klagsbrun, M.; Esko, J.D.; Leder, P.; Ornitz, D.M. Cell surface, heparin-like molecules are required for binding of basic fibroblast growth factor to its high affinity receptor. Cell 1991, 64, 841-848. [CrossRef]

176. Rapraeger, A.C.; Krufka, A.; Olwin, B.B. Requirement of heparan sulfate for bFGF-mediated fibroblast growth and myoblast differentiation. Science 1991, 252, 1705-1708. [CrossRef]

177. Pellegrini, L.; Burke, D.F.; von Delft, F.; Mulloy, B.; Blundell, T.L. Crystal structure of fibroblast growth factor receptor ectodomain bound to ligand and heparin. Nature 2000, 407, 1029-1034. [CrossRef]

178. Bishop, J.R.; Schuksz, M.; Esko, J.D. Heparan sulphate proteoglycans fine-tune mammalian physiology. Nature 2007, 446, 1030-1037. [CrossRef] [PubMed]

179. Matsuo, I.; Kimura-Yoshida, C. Extracellular modulation of Fibroblast Growth Factor signaling through heparan sulfate proteoglycans in mammalian development. Curr. Opin. Genet. Dev. 2013, 23, 399-407. [CrossRef] [PubMed]

180. Lord, M.S.; Tang, F.; Rnjak-Kovacina, J.; Smith, J.G.W.; Melrose, J.; Whitelock, J.M. The multifaceted roles of perlecan in fibrosis. Matrix Biol. 2018, 68-69, 150-166. [CrossRef] 
181. Chuang, C.Y.; Lord, M.S.; Melrose, J.; Rees, M.D.; Knox, S.M.; Freeman, C.; Iozzo, R.V.; Whitelock, J.M. Heparan sulfate-dependent signaling of fibroblast growth factor 18 by chondrocyte-derived perlecan. Biochemistry 2010, 49, 5524-5532. [CrossRef] [PubMed]

182. Knox, S.; Merry, C.; Stringer, S.; Melrose, J.; Whitelock, J. Not all perlecans are created equal: Interactions with fibroblast growth factor (FGF) 2 and FGF receptors. J. Biol. Chem. 2002, 277, 14657-14665. [CrossRef] [PubMed]

183. Smith, S.M.; West, L.A.; Govindraj, P.; Zhang, X.; Ornitz, D.M.; Hassell, J.R. Heparan and chondroitin sulfate on growth plate perlecan mediate binding and delivery of FGF-2 to FGF receptors. Matrix Biol. 2007, 26, 175-184. [CrossRef] [PubMed]

184. Smith, S.M.; West, L.A.; Hassell, J.R. The core protein of growth plate perlecan binds FGF-18 and alters its mitogenic effect on chondrocytes. Arch. Biochem. Biophys. 2007, 468, 244-251. [CrossRef] [PubMed]

185. Aviezer, D.; Hecht, D.; Safran, M.; Eisinger, M.; David, G.; Yayon, A. Perlecan, basal lamina proteoglycan, promotes basic fibroblast growth factor-receptor binding, mitogenesis, and angiogenesis. Cell 1994, 79, 1005-1013. [CrossRef]

186. Theocharis, A.D.; Karamanos, N.K. Proteoglycans remodeling in cancer: Underlying molecular mechanisms. Matrix Biol. 2019, 75-76, 220-259. [CrossRef]

187. Afratis, N.A.; Nikitovic, D.; Multhaupt, H.A.; Theocharis, A.D.; Couchman, J.R.; Karamanos, N.K. Syndecans-Key regulators of cell signaling and biological functions. FEBS J. 2017, 284, 27-41. [CrossRef] [PubMed]

188. Chung, H.; Multhaupt, H.A.; Oh, E.S.; Couchman, J.R. Minireview: Syndecans and their crucial roles during tissue regeneration. FEBS Lett. 2016, 590, 2408-2417. [CrossRef]

189. Bernfield, M.; Sanderson, R.D. Syndecan, a developmentally regulated cell surface proteoglycan that binds extracellular matrix and growth factors. Philos. Trans. R. Soc. Lond. B Biol. Sci. 1990, 327, 171-186. [CrossRef] [PubMed]

190. Olwin, B.B.; Rapraeger, A. Repression of myogenic differentiation by aFGF, bFGF, and K-FGF is dependent on cellular heparan sulfate. J. Cell Biol. 1992, 118, 631-639. [CrossRef]

191. Clasper, S.; Vekemans, S.; Fiore, M.; Plebanski, M.; Wordsworth, P.; David, G.; Jackson, D.G. Inducible expression of the cell surface heparan sulfate proteoglycan syndecan-2 (fibroglycan) on human activated macrophages can regulate fibroblast growth factor action. J. Biol. Chem. 1999, 274, 24113-24123. [CrossRef] [PubMed]

192. Wu, X.; Kan, M.; Wang, F.; Jin, C.; Yu, C.; McKeehan, W.L. A rare premalignant prostate tumor epithelial cell syndecan-1 forms a fibroblast growth factor-binding complex with progression-promoting ectopic fibroblast growth factor receptor 1. Cancer Res. 2001, 61, 5295-5302. [PubMed]

193. Iwabuchi, T.; Goetinck, P.F. Syndecan-4 dependent FGF stimulation of mouse vibrissae growth. Mech. Dev. 2006, 123, 831-841. [CrossRef] [PubMed]

194. Filla, M.S.; Dam, P.; Rapraeger, A.C. The cell surface proteoglycan syndecan-1 mediates fibroblast growth factor-2 binding and activity. J. Cell Physiol. 1998, 174, 310-321. [CrossRef]

195. Jang, E.; Albadawi, H.; Watkins, M.T.; Edelman, E.R.; Baker, A.B. Syndecan-4 proteoliposomes enhance fibroblast growth factor-2 (FGF-2)-induced proliferation, migration, and neovascularization of ischemic muscle. Proc. Natl. Acad. Sci. USA 2012, 109, 1679-1684. [CrossRef] [PubMed]

196. Murakami, M.; Nguyen, L.T.; Zhuang, Z.W.; Moodie, K.L.; Carmeliet, P.; Stan, R.V.; Simons, M. The FGF system has a key role in regulating vascular integrity. J. Clin. Invest. 2008, 118, 3355-3366. [CrossRef] [PubMed]

197. Elfenbein, A.; Lanahan, A.; Zhou, T.X.; Yamasaki, A.; Tkachenko, E.; Matsuda, M.; Simons, M. Syndecan 4 regulates FGFR1 signaling in endothelial cells by directing macropinocytosis. Sci. Signal. 2012, 5, ra36. [CrossRef] [PubMed]

198. Fico, A.; Maina, F.; Dono, R. Fine-tuning of cell signaling by glypicans. Cell Mol. Life Sci. 2011, 68, $923-929$. [CrossRef]

199. Galli, A.; Roure, A.; Zeller, R.; Dono, R. Glypican 4 modulates FGF signalling and regulates dorsoventral forebrain patterning in Xenopus embryos. Development 2003, 130, 4919-4929. [CrossRef]

200. Gutierrez, J.; Brandan, E. A novel mechanism of sequestering fibroblast growth factor 2 by glypican in lipid rafts, allowing skeletal muscle differentiation. Mol. Cell Biol. 2010, 30, 1634-1649. [CrossRef] [PubMed] 
201. Berman, B.; Ostrovsky, O.; Shlissel, M.; Lang, T.; Regan, D.; Vlodavsky, I.; Ishai-Michaeli, R.; Ron, D. Similarities and differences between the effects of heparin and glypican-1 on the bioactivity of acidic fibroblast growth factor and the keratinocyte growth factor. J. Biol. Chem. 1999, 274, 36132-36138. [CrossRef] [PubMed]

202. Qiao, D.; Meyer, K.; Mundhenke, C.; Drew, S.A.; Friedl, A. Heparan sulfate proteoglycans as regulators of fibroblast growth factor-2 signaling in brain endothelial cells. Specific role for glypican-1 in glioma angiogenesis. J. Biol. Chem. 2003, 278, 16045-16053. [CrossRef] [PubMed]

203. Su, G.; Meyer, K.; Nandini, C.D.; Qiao, D.; Salamat, S.; Friedl, A. Glypican-1 is frequently overexpressed in human gliomas and enhances FGF-2 signaling in glioma cells. Am. J. Pathol. 2006, 168, 2014-2026. [CrossRef]

204. Itoh, N.; Nakayama, Y.; Konishi, M. Roles of FGFs As Paracrine or Endocrine Signals in Liver Development, Health, and Disease. Front. Cell Dev. Biol. 2016, 4, 30. [CrossRef] [PubMed]

205. Itoh, N. Hormone-like (endocrine) Fgfs: Their evolutionary history and roles in development, metabolism, and disease. Cell Tissue Res. 2010, 342, 1-11. [CrossRef] [PubMed]

206. Yu, X.; Ibrahimi, O.A.; Goetz, R.; Zhang, F.; Davis, S.I.; Garringer, H.J.; Linhardt, R.J.; Ornitz, D.M.; Mohammadi, M.; White, K.E. Analysis of the biochemical mechanisms for the endocrine actions of fibroblast growth factor-23. Endocrinology 2005, 146, 4647-4656. [CrossRef]

207. Yie, J.; Wang, W.; Deng, L.; Tam, L.T.; Stevens, J.; Chen, M.M.; Li, Y.; Xu, J.; Lindberg, R.; Hecht, R.; et al. Understanding the physical interactions in the FGF21/FGFR/beta-Klotho complex: Structural requirements and implications in FGF21 signaling. Chem. Biol. Drug Des. 2012, 79, 398-410. [CrossRef] [PubMed]

208. Goetz, R.; Ohnishi, M.; Kir, S.; Kurosu, H.; Wang, L.; Pastor, J.; Ma, J.; Gai, W.; Kuro-o, M.; Razzaque, M.S.; et al. Conversion of a paracrine fibroblast growth factor into an endocrine fibroblast growth factor. J. Biol. Chem. 2012, 287, 29134-29146. [CrossRef] [PubMed]

209. Adams, A.C.; Cheng, C.C.; Coskun, T.; Kharitonenkov, A. FGF21 requires betaklotho to act in vivo. PLoS ONE 2012, 7, e49977. [CrossRef] [PubMed]

210. Ding, X.; Boney-Montoya, J.; Owen, B.M.; Bookout, A.L.; Coate, K.C.; Mangelsdorf, D.J.; Kliewer, S.A. betaKlotho is required for fibroblast growth factor 21 effects on growth and metabolism. Cell Metab. 2012, 16, 387-393. [CrossRef] [PubMed]

211. Kurosu, H.; Ogawa, Y.; Miyoshi, M.; Yamamoto, M.; Nandi, A.; Rosenblatt, K.P.; Baum, M.G.; Schiavi, S.; Hu, M.C.; Moe, O.W.; et al. Regulation of fibroblast growth factor-23 signaling by klotho. J. Biol. Chem. 2006, 281, 6120-6123. [CrossRef]

212. Urakawa, I.; Yamazaki, Y.; Shimada, T.; Iijima, K.; Hasegawa, H.; Okawa, K.; Fujita, T.; Fukumoto, S.; Yamashita, T. Klotho converts canonical FGF receptor into a specific receptor for FGF23. Nature 2006, 444, 770-774. [CrossRef] [PubMed]

213. Kharitonenkov, A.; Dunbar, J.D.; Bina, H.A.; Bright, S.; Moyers, J.S.; Zhang, C.; Ding, L.; Micanovic, R.; Mehrbod, S.F.; Knierman, M.D.; et al. FGF-21/FGF-21 receptor interaction and activation is determined by betaKlotho. J. Cell Physiol. 2008, 215, 1-7. [CrossRef] [PubMed]

214. Kuro-o, M.; Matsumura, Y.; Aizawa, H.; Kawaguchi, H.; Suga, T.; Utsugi, T.; Ohyama, Y.; Kurabayashi, M.; Kaname, T.; Kume, E.; et al. Mutation of the mouse klotho gene leads to a syndrome resembling ageing. Nature 1997, 390, 45-51. [CrossRef] [PubMed]

215. Matsumura, Y.; Aizawa, H.; Shiraki-Iida, T.; Nagai, R.; Kuro-o, M.; Nabeshima, Y. Identification of the human klotho gene and its two transcripts encoding membrane and secreted klotho protein. Biochem. Biophys. Res. Commun. 1998, 242, 626-630. [CrossRef]

216. Chen, G.; Liu, Y.; Goetz, R.; Fu, L.; Jayaraman, S.; Hu, M.C.; Moe, O.W.; Liang, G.; Li, X.; Mohammadi, M. alpha-Klotho is a non-enzymatic molecular scaffold for FGF23 hormone signalling. Nature 2018, 553, 461-466. [CrossRef] [PubMed]

217. Luo, Y.; Ye, S.; Li, X.; Lu, W. Emerging Structure-Function Paradigm of Endocrine FGFs in Metabolic Diseases. Trends Pharm. Sci. 2019, 40, 142-153. [CrossRef] [PubMed]

218. Takashi, Y.; Fukumoto, S. FGF23 beyond Phosphotropic Hormone. Trends Endocrinol. Metab. 2018, 29, 755-767. [CrossRef] [PubMed]

219. Farrow, E.G.; Davis, S.I.; Summers, L.J.; White, K.E. Initial FGF23-mediated signaling occurs in the distal convoluted tubule. J. Am. Soc. Nephrol. 2009, 20, 955-960. [CrossRef] 
220. Andrukhova, O.; Smorodchenko, A.; Egerbacher, M.; Streicher, C.; Zeitz, U.; Goetz, R.; Shalhoub, V.; Mohammadi, M.; Pohl, E.E.; Lanske, B.; et al. FGF23 promotes renal calcium reabsorption through the TRPV5 channel. EMBO J. 2014, 33, 229-246. [CrossRef] [PubMed]

221. Goetz, R.; Nakada, Y.; Hu, M.C.; Kurosu, H.; Wang, L.; Nakatani, T.; Shi, M.; Eliseenkova, A.V.; Razzaque, M.S.; Moe, O.W.; et al. Isolated C-terminal tail of FGF23 alleviates hypophosphatemia by inhibiting FGF23-FGFR-Klotho complex formation. Proc. Natl. Acad. Sci. USA 2010, 107, 407-412. [CrossRef] [PubMed]

222. Andrukhova, O.; Zeitz, U.; Goetz, R.; Mohammadi, M.; Lanske, B.; Erben, R.G. FGF23 acts directly on renal proximal tubules to induce phosphaturia through activation of the ERK1/2-SGK1 signaling pathway. Bone 2012, 51, 621-628. [CrossRef] [PubMed]

223. Martin, A.; David, V.; Quarles, L.D. Regulation and function of the FGF23/Klotho endocrine pathways. Physiol. Rev. 2012, 92, 131-155. [CrossRef] [PubMed]

224. Villanueva, L.S.; Gonzalez, S.G.; Tomero, J.A.S.; Aguilera, A.; Junco, E.O. Bone mineral disorder in chronic kidney disease: Klotho and FGF23; cardiovascular implications. Nefrologia 2016, 36, 333-464. [CrossRef]

225. Lu, X.; Hu, M.C. Klotho/FGF23 Axis in Chronic Kidney Disease and Cardiovascular Disease. Kidney Dis. 2017, 3, 15-23. [CrossRef]

226. Shiohama, A.; Sasaki, T.; Noda, S.; Minoshima, S.; Shimizu, N. Molecular cloning and expression analysis of a novel gene DGCR8 located in the DiGeorge syndrome chromosomal region. Biochem. Biophys. Res. Commun. 2003, 304, 184-190. [CrossRef]

227. Kurosu, H.; Choi, M.; Ogawa, Y.; Dickson, A.S.; Goetz, R.; Eliseenkova, A.V.; Mohammadi, M.; Rosenblatt, K.P.; Kliewer, S.A.; Kuro-o, M. Tissue-specific expression of betaKlotho and fibroblast growth factor (FGF) receptor isoforms determines metabolic activity of FGF19 and FGF21. J. Biol. Chem. 2007, 282, 26687-26695. [CrossRef]

228. Ogawa, Y.; Kurosu, H.; Yamamoto, M.; Nandi, A.; Rosenblatt, K.P.; Goetz, R.; Eliseenkova, A.V.; Mohammadi, M.; Kuro-o, M. BetaKlotho is required for metabolic activity of fibroblast growth factor 21. Proc. Natl. Acad. Sci. USA 2007, 104, 7432-7437. [CrossRef]

229. Adams, A.C.; Yang, C.; Coskun, T.; Cheng, C.C.; Gimeno, R.E.; Luo, Y.; Kharitonenkov, A. The breadth of FGF21's metabolic actions are governed by FGFR1 in adipose tissue. Mol. Metab. 2012, 2, 31-37. [CrossRef] [PubMed]

230. Lee, S.; Choi, J.; Mohanty, J.; Sousa, L.P.; Tome, F.; Pardon, E.; Steyaert, J.; Lemmon, M.A.; Lax, I.; Schlessinger, J. Structures of beta-klotho reveal a 'zip code'-like mechanism for endocrine FGF signalling. Nature 2018, 553, 501-505. [CrossRef] [PubMed]

231. Shi, S.Y.; Lu, Y.W.; Richardson, J.; Min, X.; Weiszmann, J.; Richards, W.G.; Wang, Z.; Zhang, Z.; Zhang, J.; Li, Y. A systematic dissection of sequence elements determining beta-Klotho and FGF interaction and signaling. Sci. Rep. 2018, 8, 11045. [CrossRef] [PubMed]

232. Kharitonenkov, A.; Shiyanova, T.L.; Koester, A.; Ford, A.M.; Micanovic, R.; Galbreath, E.J.; Sandusky, G.E.; Hammond, L.J.; Moyers, J.S.; Owens, R.A.; et al. FGF-21 as a novel metabolic regulator. J. Clin. Investig. 2005, 115, 1627-1635. [CrossRef] [PubMed]

233. Zhang, Y.; Xie, Y.; Berglund, E.D.; Coate, K.C.; He, T.T.; Katafuchi, T.; Xiao, G.; Potthoff, M.J.; Wei, W.; Wan, Y.; et al. The starvation hormone, fibroblast growth factor-21, extends lifespan in mice. eLife 2012, 1, e00065. [CrossRef] [PubMed]

234. Salminen, A.; Kaarniranta, K.; Kauppinen, A. Regulation of longevity by FGF21: Interaction between energy metabolism and stress responses. Ageing Res. Rev. 2017, 37, 79-93. [CrossRef] [PubMed]

235. Inagaki, T.; Choi, M.; Moschetta, A.; Peng, L.; Cummins, C.L.; McDonald, J.G.; Luo, G.; Jones, S.A.; Goodwin, B.; Richardson, J.A.; et al. Fibroblast growth factor 15 functions as an enterohepatic signal to regulate bile acid homeostasis. Cell Metab. 2005, 2, 217-225. [CrossRef] [PubMed]

236. Yu, C.; Wang, F.; Kan, M.; Jin, C.; Jones, R.B.; Weinstein, M.; Deng, C.X.; McKeehan, W.L. Elevated cholesterol metabolism and bile acid synthesis in mice lacking membrane tyrosine kinase receptor FGFR4. J. Biol. Chem. 2000, 275, 15482-15489. [CrossRef] [PubMed]

237. Fu, L.; John, L.M.; Adams, S.H.; Yu, X.X.; Tomlinson, E.; Renz, M.; Williams, P.M.; Soriano, R.; Corpuz, R.; Moffat, B.; et al. Fibroblast growth factor 19 increases metabolic rate and reverses dietary and leptin-deficient diabetes. Endocrinology 2004, 145, 2594-2603. [CrossRef] [PubMed] 
238. Kir, S.; Beddow, S.A.; Samuel, V.T.; Miller, P.; Previs, S.F.; Suino-Powell, K.; Xu, H.E.; Shulman, G.I.; Kliewer, S.A.; Mangelsdorf, D.J. FGF19 as a postprandial, insulin-independent activator of hepatic protein and glycogen synthesis. Science 2011, 331, 1621-1624. [CrossRef] [PubMed]

239. Kuro, O.M. The Klotho proteins in health and disease. Nat. Rev. Nephrol. 2019, 15, 27-44. [CrossRef]

240. Babaknejad, N.; Nayeri, H.; Hemmati, R.; Bahrami, S.; Esmaillzadeh, A. An Overview of FGF19 and FGF21: The Therapeutic Role in the Treatment of the Metabolic Disorders and Obesity. Horm. Metab. Res. 2018, 50, 441-452. [CrossRef] [PubMed]

241. Alvarez-Sola, G.; Uriarte, I.; Latasa, M.U.; Urtasun, R.; Barcena-Varela, M.; Elizalde, M.; Jimenez, M.; Rodriguez-Ortigosa, C.M.; Corrales, F.J.; Fernandez-Barrena, M.G.; et al. Fibroblast Growth Factor 15/19 in Hepatocarcinogenesis. Dig. Dis. 2017, 35, 158-165. [CrossRef] [PubMed]

242. Lopez-Casillas, F.; Cheifetz, S.; Doody, J.; Andres, J.L.; Lane, W.S.; Massague, J. Structure and expression of the membrane proteoglycan betaglycan, a component of the TGF-beta receptor system. Cell 1991, 67, 785-795. [CrossRef]

243. Knelson, E.H.; Gaviglio, A.L.; Tewari, A.K.; Armstrong, M.B.; Mythreye, K.; Blobe, G.C. Type III TGF-beta receptor promotes FGF2-mediated neuronal differentiation in neuroblastoma. J. Clin. Investig. 2013, 123, 4786-4798. [CrossRef] [PubMed]

244. Andres, J.L.; DeFalcis, D.; Noda, M.; Massague, J. Binding of two growth factor families to separate domains of the proteoglycan betaglycan. J. Biol. Chem. 1992, 267, 5927-5930. [PubMed]

245. Furthauer, M.; Lin, W.; Ang, S.L.; Thisse, B.; Thisse, C. Sef is a feedback-induced antagonist of Ras/MAPK-mediated FGF signalling. Nat. Cell Biol. 2002, 4, 170-174. [CrossRef] [PubMed]

246. Preger, E.; Ziv, I.; Shabtay, A.; Sher, I.; Tsang, M.; Dawid, I.B.; Altuvia, Y.; Ron, D. Alternative splicing generates an isoform of the human Sef gene with altered subcellular localization and specificity. Proc. Natl. Acad. Sci. USA 2004, 101, 1229-1234. [CrossRef] [PubMed]

247. Harduf, H.; Halperin, E.; Reshef, R.; Ron, D. Sef is synexpressed with FGFs during chick embryogenesis and its expression is differentially regulated by FGFs in the developing limb. Dev. Dyn. 2005, 233, 301-312. [CrossRef]

248. Lin, W.; Furthauer, M.; Thisse, B.; Thisse, C.; Jing, N.; Ang, S.L. Cloning of the mouse Sef gene and comparative analysis of its expression with Fgf8 and Spry2 during embryogenesis. Mech. Dev. 2002, 113, 163-168. [CrossRef]

249. Yang, R.B.; Ng, C.K.; Wasserman, S.M.; Komuves, L.G.; Gerritsen, M.E.; Topper, J.N. A novel interleukin-17 receptor-like protein identified in human umbilical vein endothelial cells antagonizes basic fibroblast growth factor-induced signaling. J. Biol. Chem. 2003, 278, 33232-33238. [CrossRef] [PubMed]

250. Kovalenko, D.; Yang, X.; Nadeau, R.J.; Harkins, L.K.; Friesel, R. Sef inhibits fibroblast growth factor signaling by inhibiting FGFR1 tyrosine phosphorylation and subsequent ERK activation. J. Biol. Chem. 2003, 278, 14087-14091. [CrossRef]

251. Xiong, S.; Zhao, Q.; Rong, Z.; Huang, G.; Huang, Y.; Chen, P.; Zhang, S.; Liu, L.; Chang, Z. hSef inhibits PC-12 cell differentiation by interfering with Ras-mitogen-activated protein kinase MAPK signaling. J. Biol. Chem. 2003, 278, 50273-50282. [CrossRef] [PubMed]

252. Rong, Z.; Ren, Y.; Cheng, L.; Li, Z.; Li, Y.; Sun, Y.; Li, H.; Xiong, S.; Chang, Z. Sef-S, an alternative splice isoform of sef gene, inhibits NIH3T3 cell proliferation via a mitogen-activated protein kinases p42 and p44 (ERK1/2)-independent mechanism. Cell Signal. 2007, 19, 93-102. [CrossRef] [PubMed]

253. Tsang, M.; Friesel, R.; Kudoh, T.; Dawid, I.B. Identification of Sef, a novel modulator of FGF signalling. Nat. Cell Biol. 2002, 4, 165-169. [CrossRef] [PubMed]

254. Korsensky, L.; Ron, D. Regulation of FGF signaling: Recent insights from studying positive and negative modulators. Semin. Cell Dev. Biol. 2016, 53, 101-114. [CrossRef] [PubMed]

255. Ziv, I.; Fuchs, Y.; Preger, E.; Shabtay, A.; Harduf, H.; Zilpa, T.; Dym, N.; Ron, D. The human sef-a isoform utilizes different mechanisms to regulate receptor tyrosine kinase signaling pathways and subsequent cell fate. J. Biol. Chem. 2006, 281, 39225-39235. [CrossRef] [PubMed]

256. Murphy, T.; Darby, S.; Mathers, M.E.; Gnanapragasam, V.J. Evidence for distinct alterations in the FGF axis in prostate cancer progression to an aggressive clinical phenotype. J. Pathol. 2010, 220, 452-460. [CrossRef] [PubMed] 
257. Hori, S.; Wadhwa, K.; Pisupati, V.; Zecchini, V.; Ramos-Montoya, A.; Warren, A.Y.; Neal, D.E.; Gnanapragasam, V.J. Loss of hSef promotes metastasis through upregulation of EMT in prostate cancer. Int. J. Cancer 2017, 140, 1881-1887. [CrossRef] [PubMed]

258. Katoh, M. Fibroblast growth factor receptors as treatment targets in clinical oncology. Nat. Rev. Clin. Oncol. 2019, 16, 105-122. [CrossRef] [PubMed]

(C) 2019 by the authors. Licensee MDPI, Basel, Switzerland. This article is an open access article distributed under the terms and conditions of the Creative Commons Attribution (CC BY) license (http://creativecommons.org/licenses/by/4.0/). 


\title{
Fibroblast Growth Factor Receptor 4 Targeting in Cancer: New Insights into Mechanisms and Therapeutic Strategies ${ }^{\dagger}$
}

\author{
Liwei Lang ${ }^{1}$ and Yong Teng ${ }^{1,2,3, *}$ \\ 1 Department of Oral Biology and Diagnostic Sciences, Dental College of Georgia, Augusta University, \\ Augusta, GA 30912, USA; llang@augusta.edu \\ 2 Georgia Cancer Center, Department of Biochemistry and Molecular Biology, Medical College of Georgia, \\ Augusta University, Augusta, GA 30912, USA \\ 3 Department of Medical Laboratory, Imaging and Radiologic Sciences, College of Allied Health, \\ Augusta University, Augusta, GA 30912, USA \\ * Correspondence: yteng@augusta.edu; Tel: +1-706-446-5611; Fax: +1-706-721-9415 \\ + Running Title: Targeting FGFR4 for cancer therapy.
}

Received: 30 November 2018; Accepted: 8 January 2019; Published: 9 January 2019

\begin{abstract}
Fibroblast growth factor receptor 4 (FGFR4), a tyrosine kinase receptor for FGFs, is involved in diverse cellular processes, including the regulation of cell proliferation, differentiation, migration, metabolism, and bile acid biosynthesis. High activation of FGFR4 is strongly associated with the amplification of its specific ligand FGF19 in many types of solid tumors and hematologic malignancies, where it acts as an oncogene driving the cancer development and progression. Currently, the development and therapeutic evaluation of FGFR4-specific inhibitors, such as BLU9931 and H3B-6527, in animal models and cancer patients, are paving the way to suppress hyperactive FGFR4 signaling in cancer. This comprehensive review not only covers the recent discoveries in understanding FGFR4 regulation and function in cancer, but also reveals the therapeutic implications and applications regarding emerging anti-FGFR4 agents. Our aim is to pinpoint the potential of FGFR4 as a therapeutic target and identify new avenues for advancing future research in the field.
\end{abstract}

Keywords: FGFR4; FGF19; gene regulation; cancer signaling; anticancer

\section{Introduction}

Fibroblast growth factor receptors (FGFRs) have been found to play a vital role in tumorigenesis and cancer progression through increased cell proliferation, metastasis, and survival [1,2]. Compared with the other three FGFR family members, the signaling pathways and mechanisms of FGFR4 involved in cancer development are less characterized. The expression of FGFR4 is strictly regulated in human adult organs and tissues after fetal development, suggesting it perhaps has a particular relevance to tissue functions. Recently, elevated FGFR4 has been tightly correlated with cancer development and progression, making it an attractive target to develop novel and effective anticancer therapeutics. More efforts have been focused on developing selective inhibitors to target FGFR4, which show particular promise as an anticancer monotherapy or an adjunct treatment.

\section{Molecular Characters of FGFR4 and Its Ligands}

\subsection{The Molecular Structure of FGFR4}

FGFR4 is one of four family members harboring tyrosine kinase (TK) domains. The human FGFR4 gene is located on the long arm of chromosome 5 (5q 35.1). The FGFR4 gene consists of 18 exons 
and has five transcript variants with three of them encoding the FGFR4 isoform 1 (Figure 1A) [3]. The 802 amino acid (aa) core region in the FGFR4 protein contains four parts, signal peptide (1-21 aa), extracellular region (22-369 aa), transmembrane region (70-390 aa), and the intracellular region (391-802 aa) (Figure 1B). Similar to the other three FGFR members, the extracellular region of FGFR4 consists of three immunoglobulin-like domains (IgI, IgII, and IgIII), which are essential for specific ligand-binding. IgI is located in 50-107 aa with a length of 97 aa. IgII and IgIII are located in order in 157-241 aa and 264-351 aa. Compared with the other three family members, FGFR4 does not have a splice variant on the IgIII [4]. Several ligand binding sites have been identified, such as 273, 278-280, 309-310, 316, and 337 aa. The TK domains locate in the C terminal from 454-767 aa with several tyrosine (Y) for autocatalysis, such as Y642, Y643, and Y764 (Figure 2).

A

A

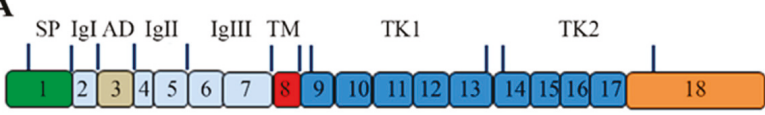

B

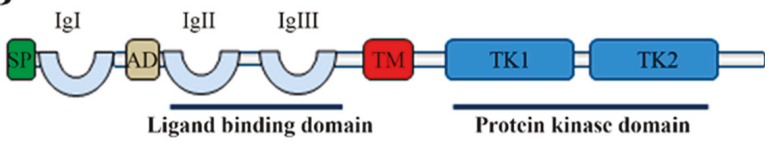

$\square$ Signal peptide $\square$ Acid box $\square$ IgG like domain

Transmembrane domain $\square$ Tyrosine kinase domain

Figure 1. The molecular structure of FGFR4. (A) The illustration of FGFR4 with mRNA structure. The transcript variant 1 of FGFR4 contains 18 exons and encodes isoform 1 of FGFR4 protein with the main function domains. (B) The main domains of FGFR4 with the corresponding function.

\subsection{The Ligands of FGFR4}

FGFs are a family of 22 different proteins in vertebrates and are classified into seven subfamilies including FGF1, FGF4, FGF7, FGF8, FGF9, FGF19 ligand subfamily, and FGF11 subfamily [5]. The members of FGF11 subfamily are not ligands of FGFRs and are known as FGF homologous factors [5], while all other six subfamilies work as ligands to bind with FGFR4 (Figure 2) [6]. In other words, ten canonical FGF subfamily members (FGF1, FGF2, FGF4, FGF6, FGF7, FGF8, FGF9, FGF16, FGF17, and FGF18) and three FGF19 subfamily members (FGF19, FGF21, and FGF23) have the potential to bind FGFR4 (Figure 2). Canonical FGFs bind to and activate FGFR4 with heparin/heparin sulfate (HS) [7], while FGF19 subfamily members need $\beta$-klotho (KLB) as a co-receptor to bind with FGFR4. FGF1, FGF4, and FGF8, have a higher affinity to bind FGFR4 than other canonical FGFs. Most importantly, FGF19, as an endocrine ligand, has a more specific selective affinity to FGFR4 than other FGFR members [8,9]. 


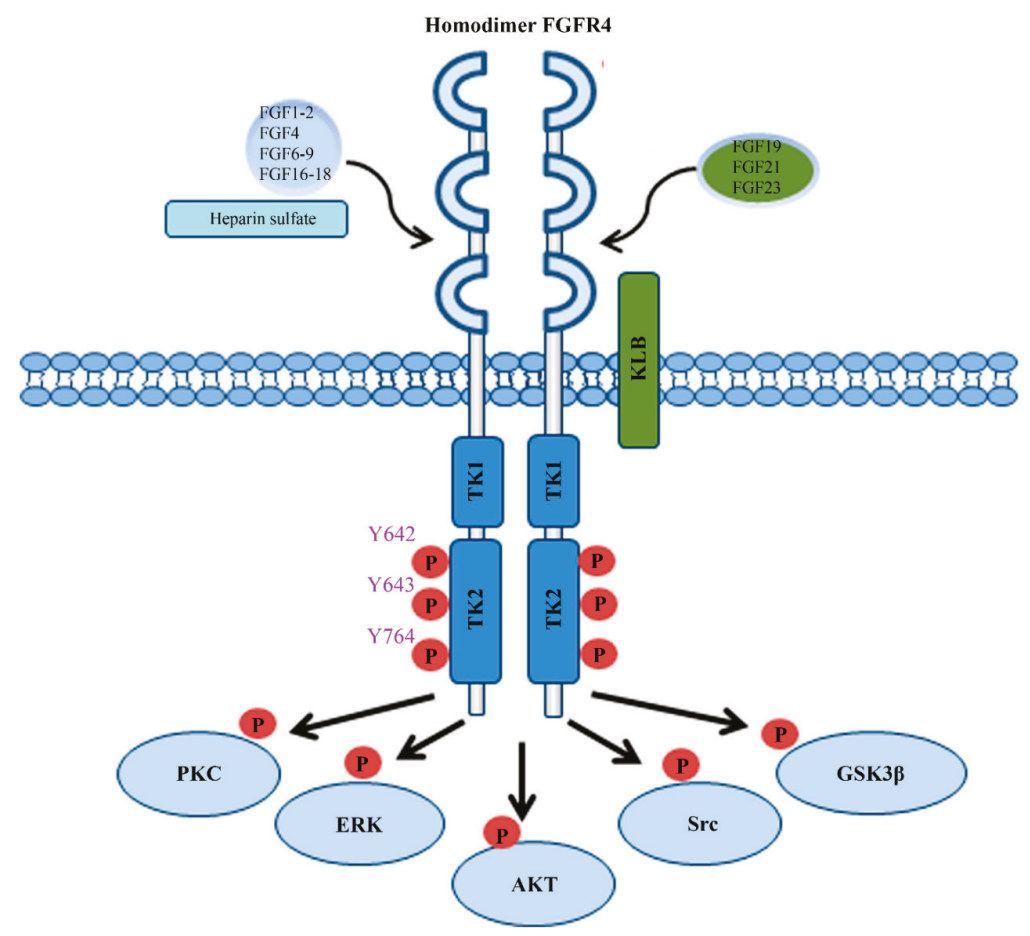

Figure 2. The FGF/FGFR4 signal axis. The signal transduction mediated by the FGF/FGFR4 axis is extremely complex, which includes PKC, ERK1/2, AKT, Src, and GSK3 $\beta$ signaling cascades. The homodimer of FGFR4 forms when binding to either canonical FGF subfamily members (FGF1, FGF2, FGF4, FGF6, FGF7, FGF8, FGF9, FGF16, FGF17, and FGF18) or FGF19 subfamily members (FGF19, FGF21, and FGF23). Heparin or heparin sulfate is required for the binding of canonical FGF subfamily members to FGFR4, whereas KLB acts as a co-receptor of FGFR4 to facilitate FGFR4 interacting with FGF19 subfamily members. When FGFR4 forms protein complexes with FGFs, it can be phosphorylated on three main tyrosine residues: Y642, Y643, and Y764.

\subsection{The Physiologic Functions of FGFR4}

As an important mediator of homeostasis in the liver, FGFR4 function is required for the maintenance of both lipid and glucose metabolism under normal dietary conditions, in addition to its established role in cholesterol [10]. Particularly, FGFR4 activated by endocrine FGF19 represses the gluconeogenesis and stimulates of glycogen and protein synthesis in hepatocytes [11]. The liver-protective effect of FGFR4 becomes even clearer in the model of carbon tetrachloride-induced liver damage, where more significant liver fibrosis was observed in FGFR4 knock-out compared with wild-type (wt) mice [12]. The importance of FGFR4 in controlling bile acids was also established. It has been reported that bile acids secretion and cholesterol metabolism are regulated by FGF19 through binding to FGFR4 in physical activity [13]. It is worth mentioning that the FGF6/FGFR4 pathway plays important role in myoblast differentiation and myotube regeneration [14,15].

\section{The Genetic Alterations of FGFR4 Gene in Cancer}

The high expression levels of FGFR4 can be detected during fetal human and mouse embryonic development. However, deletion of FGFR4 does not lead to developmental abnormalities in adult mice only with changed cholesterol metabolism and elevated bile acids [16,17]. The expression of FGFR4 is dramatically decreased although it still consistently expresses in several organs, especially 
in the liver. Gene alterations of FGFRs, including amplification, translocation, and mutation of gain of function, have been linked to tumorigenesis and cancer progression in solid and hematological malignancies. Recently, one study was conducted to evaluate the alterations of FGFR genes in a variety of cancer types [18], which showed that gene alterations of FGFRs occurred in $7.1 \%$ of 4853 solid tumors, with the majority being gene amplification ( $66 \%$ of the aberrations), followed by mutations $(26 \%)$ and translocations ( $8 \%$ ). Amplification was the predominant type of alteration for the FGFR4 gene, accounting for $78 \%$ of all FGFR4 gene alterations. Interestingly, the amplified FGFR4 gene was identified in $10 \%$ of breast cancer, which more frequently harbors estrogen- and progesterone-receptor with lymph-node metastases [11]. Unlike FGFR1, the translocation of FGFR4 is very rare in human cancers [18]. Two point mutations in the TK domains of the FGFR4 gene, K535 and E550, have been identified in rhabdomyosarcoma [19]. Another activating point mutation in FGFR4 gene (Y367C) inducing constitutive FGFR4 dimerization, has been found in MDA-MB-453 breast cancer cells [20]. Although the gene alteration is relatively low, FGFR4 overexpression has been reported in many types of cancer. Increased FGFR4 mRNA expression has been detected in one-third of hepatocellular carcinoma (HCC) [21]. In another study, elevated FGFR4 mRNA levels were detected in $32 \%$ of breast cancer samples [22]. FGFR4 overexpression is also observed in 64\% (153/238) of oropharyngeal squamous cell carcinoma and $41 \%(87 / 212)$ of oral squamous cell carcinoma [23]. Overexpressed FGFR4 has also been found in pancreatic carcinomas and derived cell lines, which are mediated by an intronic enhancer activated by hepatic nuclear factor 1 alpha [24]. Additionally, highly FGFR4 expression was detected in rhabdomyosarcoma [19].

As a specific ligand of FGFR4, FGF19 can bind and active FGFR4 with the co-receptor KLB. FGFR4 consistently activated by amplified FGF19 has been identified in several types of cancer. The FGF19 gene is located on chromosome 11q13.3, a region commonly amplified in human cancer. The amplification of the FGF19 gene was found in liver cancer, breast cancer, lung cancer, bladder cancer, head and neck squamous cell carcinoma (HNSCC), and esophageal cancer [25-29]. For example, the frequency of the amplified FGF19 gene is as high as 15\% in HCC [26]. Moreover, compared with adjacent normal liver tissues, HCC tissues have significantly elevated mRNA levels of FGF19 [30], suggesting the increased mRNA expression is tightly associated with its amplification. A similar tendency was also identified in HNSCC where FGF19 amplification corresponds with an increased dependency upon FGF19-FGFR4 autocrine signaling [31].

\section{Mechanisms and Functions of FGFR4 in Cancer Development and Treatment}

Accumulating observations indicate that the FGFR4 plays vital roles for cancer development, especially for those harboring FGF19 amplification. Unlike other family members, the mechanisms and functions of FGFR4 are still poorly characterized at the molecular level in cancer development and progression. Here, the novel observations of mechanisms and functions about oncogenic FGFR4 signaling in cancer development and progression have been summarized and discussed.

\subsection{FGFR4-Mediated AKT and ERK Signaling Cascades Promote Cancer Development}

The MAPK-ERK and PI3K-AKT signaling are two main pathways regulated by the FGF/FGFR protein complexes. After binding FGFs with HS or KLB, FGFR4 will be activated through autophosphorylation and forms a homodimer (Figure 2). FGFR4 also has the potential to form a heterodimer receptor with other family members, especially with FGFR3 [32,33]. Mechanistic studies showed that phosphorylated FGFR4 recruits and phosphorylates two important intracellular targets, phospholipase $\gamma$ (PLC $\gamma$ ) and FGFR substrate 2 (FRS2) [4]. MAPK then can be stimulated by activated protein kinase C (PKC) through PLC $\gamma$. Meanwhile, the MAPK and PI3K-AKT pathway can be triggered by activated FRS2 through recruitment of growth factor receptor bound 2 (GRB2) (Figure 3) [4]. Upregulated activity of AKT and ERK1/2 leads to enhanced cell proliferation and survival in HCC upon the activation of FGF19/FGFR4 signaling (Figure 3) [34-36]. 


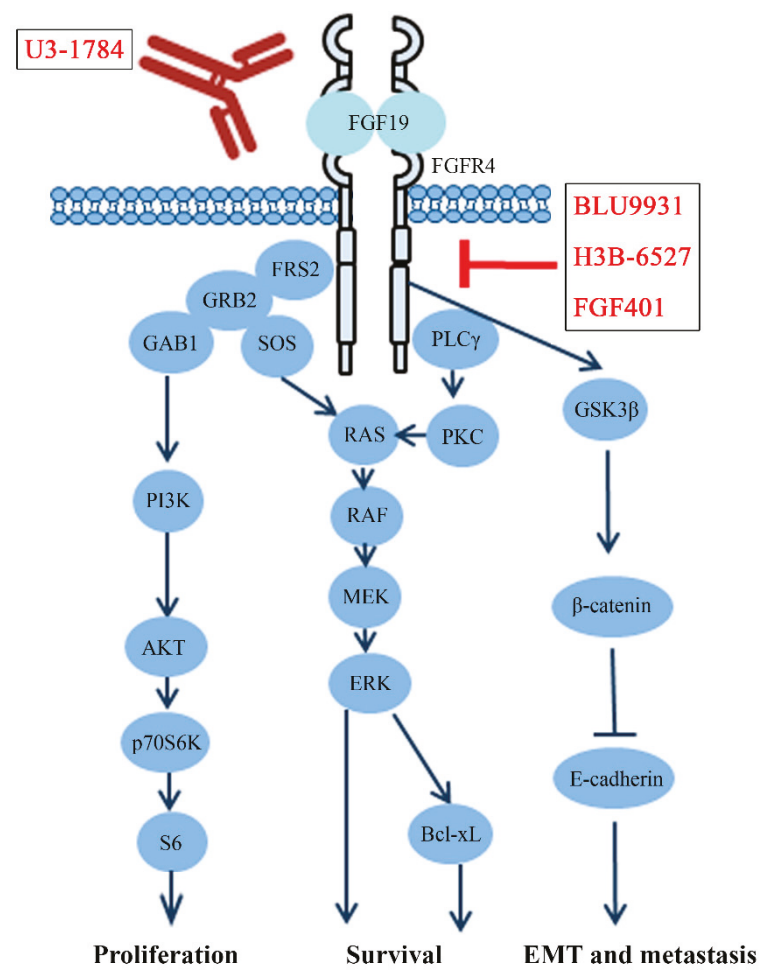

Figure 3. The signal transduction cascades of FGF19/FGFR4 in cancer development and progression. In cancer cells, once FGFR4 receives the extracellular signal from FGF19, it activates many downstream pathways, including PI3K-AKT, MEK-ERK, and GSK3 $\beta$ - $\beta$-catenin, leading to increased tumor-promoting activities. FGFR4 activation can be blocked by two non-genetic strategies, using either monoclonal antibodies (e.g., U3-1798) or selective small-molecule inhibitors (e.g., BLU9931, H3B-6527 and FGF401).

FGF19 is more highly expressed in the breast cancer tissue than the adjacent normal tissue [37], and co-expression of FGFR4 and FGF19 accounts over 28\% primary breast cancer [38]. AKT phosphorylation is strongly associated with co-expression of FGF19 and FGFR4, which can be blocked with FGF19 antibody (1A6) or siRNA-mediated silencing of FGF19 in breast cancer cells [27]. Our recent findings reveal that FGFR4-mediated hyperactivation of AKT increases breast cancer cells proliferation, but not metastasis [37]. Inactivation of FGFR4 by its inhibitor BLU9931 significantly attenuates FGF19-induced tumor-promoting activity, suggesting interruption of FGFR4 function is sufficient to affect FGF19-driven breast cancer [37].

Our recent study demonstrates that FGF19 amplification and overexpression are associated with a poorer overall survival rate for HNSCC patients, provoking FGFR4-dependent ERK/AKT-p70S6K-S6 signaling activation to increase HNSCC cell proliferation [31]. Blocking activation of FGFR4 by small hairpin RNAs (shRNAs) or BLU9931, not only attenuates FGF19-induced ERK1/2 and AKT activation, but also abrogates its ability to induce cell proliferation [31]. FGFR4-induced activation of ERK1/2 and AKT pathways was also correlated with increased cell proliferation and survival in colorectal cancer (CRC) [39]. 


\subsection{The FGF19-FGFR4 Axis Promotes Epithelial-Mesenchymal Transition (EMT) to Accelerate Metastasis}

The FGF19-FGFR4 axis has been linked to metastasis and poor survival [26]. FGFR4 is predominantly expressed in the liver and responsive for FGF19 stimulation to regulate cholesterol metabolism. There is no doubt that elevated FGF19-FGFR4 signaling is associated with HCC progression, especially for metastasis [26,40]. Our research team has demonstrated that the FGF19/FGFR4 axis facilitates HCC cell EMT through upregulating GSK3 $\beta$ - $\beta$-catenin signaling and consequently increases HCC metastasis (Figure 3) [30]. Recently, a vital role of FGFR4 was found in CRC metastasis. Activated FGFR4 phosphorylates AKT, ERK1/2, and Src, leading to increased CRC cell invasion. Silencing FGFR4 reduces adhesion, migration, and invasion of CRC cells [39]. Further study shows that depletion FGFR4 by CRISPR-Cas9 results in the morphological changes and reduced metastasis ability, accompanied by upregulation of E-cadherin and downregulation of Snail and other EMT mediators [39]. Moreover, FGFR4-GSK3 $\beta$ - $\beta$-catenin is also elucidated in CRC metastasis. Elevated expression of Forkhead box C1 (FOXC1) is tightly correlated with metastasis of CRC, and FGFR4 is the main target of this gene [41]. BLU9931, the specific inhibitor of FGFR4, can inhibit the activation of GSK3 $\beta$ / $\beta$-catenin induced by FOXC1 overexpression in vitro and metastatic colonization of CRC in vivo [41].

\subsection{FGFR4-Associated Chemotherapy Resistance in Cancer}

Cancer cells may develop a mechanism that inactivates the drug which represents the main obstacle for cancer treatment. It can be achieved by cancer cells through different mechanisms, such as drug inactivation, drug target alteration, drug efflux, DNA damage repair, cell death inhibition, and EMT [42]. The FGF19-FGFR4 axis has participated in chemotherapy resistance in several types of cancers. The expression levels of FGFR4 are significantly increased in doxorubicin-resistant breast cancer clones [43]. Moreover, FGFR4 overexpression has been detected in those insensitive breast cancer cell lines to doxorubicin [43]. Silencing FGFR4 with small interfering RNA (siRNA) in chemo-resistant clones increases their sensitivity to doxorubicin. Furthermore, inhibition of FGFR4 with an antagonistic antibody also enhances the sensitivity of endogenously FGFR4-expressing cell lines to doxorubicin. Inhibition of apoptosis by FGFR4 is the main mechanism of doxorubicin resistance in breast cancer [43]. Bcl-xL, an anti-apoptotic protein, is upregulated by FGFR4 via MAPK cascade and responsive for the increased resistance to doxorubicin [43]. Other studies indicate that upregulation of FGF19-FGFR4 signaling increases drug resistance to doxorubicin in basal-like breast cancer [27]. Inactivation of FGFR4 signaling by an anti-FGF19 antibody or siRNA-mediated FGF19 gene silencing, can sensitize FGFR4 ${ }^{+} / \mathrm{FGF}^{+}{ }^{+}$breast cancer cells to doxorubicin treatment [27]. Increased sensitivity to 5 -fluorouracil (5-FU) or oxaliplatin treatment has also been observed after FGFR4 silencing in CRC cells [44].

As a multiple TKI, sorafenib is an efficient target therapy agent to treat HCC. However, sorafenib is always restricted to continuous administration by occurring drug resistance with unknown mechanisms $[45,46]$. Recently, our study shows that activation of the FGF19-FGFR4 axis is one of the main mechanisms for sorafenib resistance in the treatment of HCC [47]. The outbalanced oxidative stress induced by reactive oxygen species (ROS) plays a pivotal role in apoptosis [48]. Mechanistically, sorafenib induces ROS-associated apoptosis, but this can be suppressed by FGF19 overexpression in HCC cells. The FGF19-FGFR4 axis has the potential to assist HCC cancer cells to escape apoptosis in sorafenib treatment through suppression of ROS. FGFR4 knockout increases the sensitivity to sorafenib treatment in HCC cells, accompanied by enhanced cell apoptosis [47]. Silencing the FGF19 gene or inactivating FGFR4 with the FGFR-pan inhibitor ponatinib, significantly increases the sensitivity to sorafenib in sorafenib-resistant HCC cells, with induced apoptosis and accumulated ROS generation [47]. Additionally, a similar phenomenon is also observed in young adult mouse colonic epithelial cells. Upregulating FGF19-FGFR4 signaling significantly reduces ROS-mediated apoptosis caused by $\mathrm{H}_{2} \mathrm{O}_{2}$ through blocking the caspase-3 pathway [49], which also prevents prostate cancer cells from apoptosis in TNF $\alpha$ treatment [50]. 


\section{Develop Specific FGFR4 Inhibitors Targeting Cancer Harboring Elevated FGF19/FGFR4 Signaling}

As a promising target, FGFR4 attracts intensive pharmaceutical and academic attention to develop novel target therapy against cancers driven by FGFR4. Three strategies have been developed to target FGFR4, including neutral antibodies, antisense oligonucleotides, and small molecule inhibitors. Two monoclonal neutralizing antibodies of FGFR4, LD-1, and U3-1784, have been developed to competitively targeting extracellular Ig domains of FGFR4. The therapeutic efficacy of U3-1784 is currently being evaluated in Phase I clinical trials for the treatment of HCC and other advanced solid tumors [51] (Table 1 and Figure 3). As an antisense oligonucleotide targeting FGFR4 mRNA, ISIS-FGFR4RX has entered a Phase I clinical trial for obesity (NCT02476019). However, the potential anticancer activity of ISIS-FGFR4RX has not been reported.

Comparing two strategies above, targeting FGFR4 using small-molecule inhibitors is more feasible and can be developed through structure-guided drug design. Not surprisingly, multi-targeted tyrosine kinase inhibitors (mTKIs) can be used to inactivate FGFR4 by disrupting ATP binding in its TK domains. The anticancer activity of many mTKIs, including lenvatinib and ponatinib, have been tested on FGFR-driven solid tumors in animals or in clinical trials (Table 1) [52-54]. However, the limited selective activity of mTKIs on FGFRs induces less efficiency and increases side effects in these treatments. Therefore, pan-FGFR inhibitors are developed and are being evaluated in clinical trials to treat cancers driven by abnormal FGFR pathways. Most of these inhibitors target ATP binding pocket in the TK domains of FGFRs through reversible or covalent bonds. For example, ponatinib can impede the autophosphorylation activity of FGFRs by binding to the hinge region of FGFRs and block the ATP-binding cassette motif [5]. As such, ponatinib has the great potential to inhibit the enzyme activity of FGFRs which are always hyperactive in cancer cells. Other inhibitors in this category include ATP-competitive inhibitors NVP-BGJ398 [55] and AZD4547 [56], ATP-binding pocket inhibitor LY2874455 [57], and FGFRs-FIIN-3 which generates a covalent bond with a conserved cysteine located in the ATP binding site (Table 1) [58]. However, the low specificity of these pan-FGFR inhibitors to FGFR4 cannot sufficiently suppress the oncogenic FGFR4 signaling. For example, the $\mathrm{IC}_{50}$ of AZD4547 on FGFR4 is over 100-fold higher than other FGFR members [59]. Moreover, inevitable on-target toxicities and off-target activity resulting from the use of nonspecific FGFR inhibitors lead to several adverse effects such as soft-tissue mineralization and hyperphosphatemia [60]. Such disadvantages eventually limit their usage in cancer patients.

Compared with other FGFR family members, FGFR4 is more specifically expressed in the liver and several other organs for bile acid secretion and cholesterol metabolism. Therefore, the generation of more specific inhibitors which only abolish FGFR4 can improve FGFR4 sensitivity and overcome the drawbacks of pan-FGFR inhibitors. BLU9931 is the first selective FGFR4 inhibitor for the treatment of HCC with an activated FGFR4 signaling pathway [61,62]. As a novel irreversible kinase inhibitor, BLU9931 creates a covalent bond with Cysteine 552 near the ATP-binding site that is only present in FGFR4 among FGFRs [62]. BLU9931 can effectively inhibit HCC tumor harboring elevated FGF19-FGFR4 axis in vivo. Moreover, BLU9931 also displays the potent anticancer ability in breast cancer, CRC, and HNSCC with upregulated FGF19-FGFR4 signaling [37,41]. BLU554 was derived from BLU9931 with improved pharmaceutical properties, which is now in Phase I clinical trial to treat HCC with elevated FGF19-FGFR4 axis (NCT02508467) (Table 1) [63]. H3B-6527 is another selective FGFR4 inhibitor currently in clinical trials for HCC treatment (Table 1). H3B-6527 also targets Cysteine 552 through forming a covalent bond near the ATP binding site of FGFR4, and it exhibits an inhibitory effect on FGFR4 activation in FGF19-driven HCC in vitro and in vivo [64]. By studying a panel of 40 HCC cell lines and 30 HCC patient-derived xenograft models, the expression levels of FGF19 are implicated as a predictive biomarker for H3B-6527 response [64]. Moreover, the combination of H3B-6527 with the CDK4/6 inhibitor palbociclib has a superior effect on the repression of tumors in a xenograft model of HCC [64]. FGF401 is another selective FGFR4 inhibitor, which is under investigation in a phase I/II study to treat HCC with FGFR4 and KLB expression (NCT02325739) [65] 
(Table 1). FGF401 is evaluated to treat HCC as the single use or combined with a humanized anti-PD1 IgG4 antibody PDR001 [65]. These novel FGFR4-targeting therapies provide a novel and promising approach which could potentially be developed into a therapeutic strategy to combat cancer.

Table 1. Clinical trials of FGFR4 inhibitors for cancer treatment.

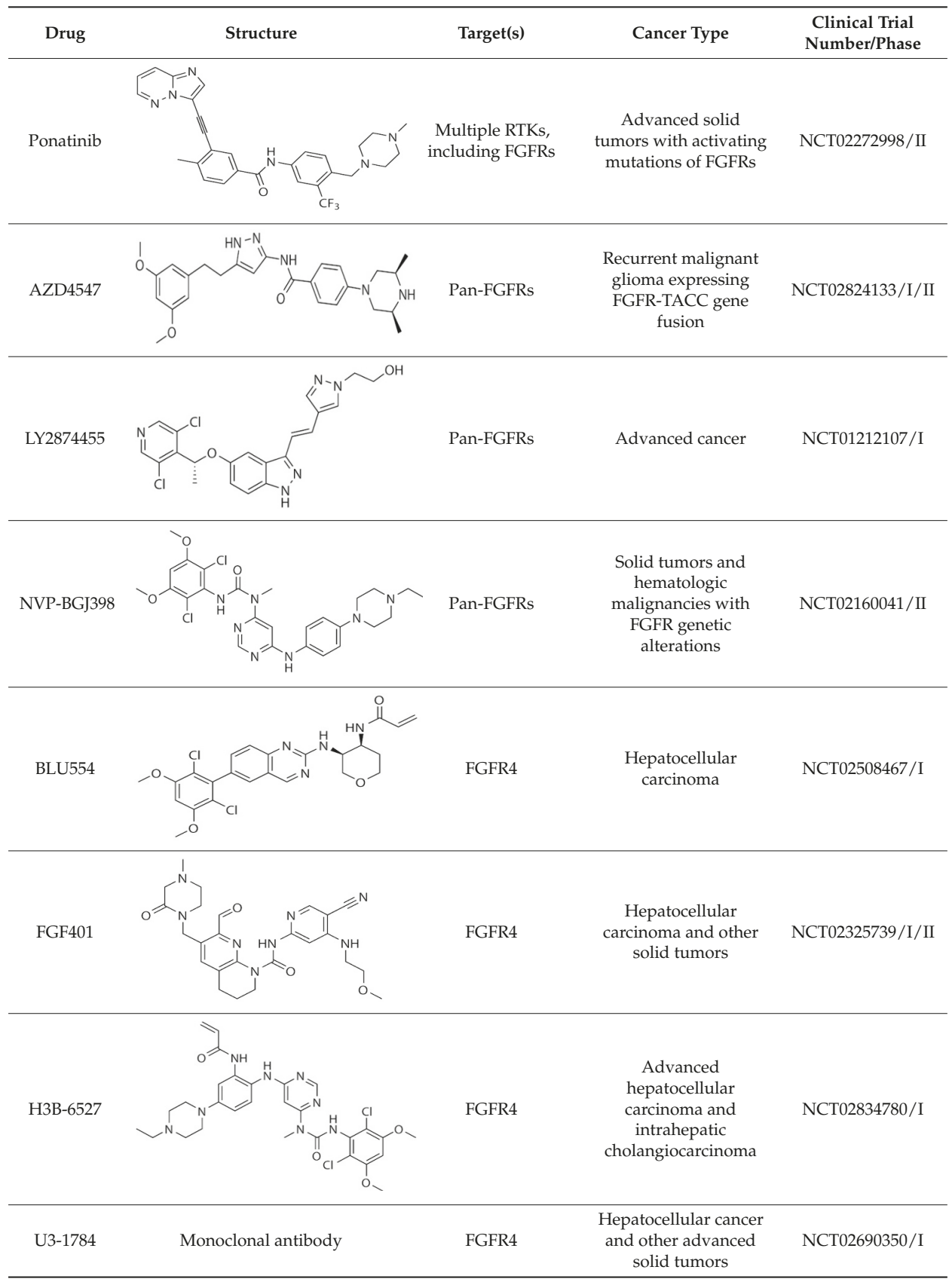




\section{Conclusions}

Increasing evidence indicates that upregulation of FGF19-FGFR4 signaling plays an essential role in tumorigenesis and cancer progression. FGFR4 has been proven as an attracted target to develop a novel therapy for the subgroup of cancers associated with the FGF19-FGFR4 pathway. Given that overexpression of FGFR4 significantly correlates with EpCAM, a marker of hepatic cancer stem cells, within the fatty liver-steatosis-cirrhosis-HCC sequence [66], FGFR4 may have the ability to regulate cancer stem cells and lead to chemoresistance in HCC or other cancers. Gaining these insights will improve our comprehensive understanding of the role of FGFR4 in cancer development and treatment. Recently, more specific inhibitors targeting FGFR4 have been developed and evaluated, which are demonstrating promise as a single agent therapy or in combination with other anticancer agents. Thus, there is no doubt that FGFR4-targeting inhibitors offer the most immediate prospects of reducing cancer mortality rate. Perhaps, we can design stapled peptides [67] to incorporate the hydrophobic staple at the interface of FGF19-FGFR4 binding sites, which would increase the specificity of signal targeting compared to the FGFR4 inhibitors that are commercially available. Although the critical role of FGFR4 in metastasis has been demonstrated in animal models of several cancers, prospective studies are warranted to provide evidence regarding the therapeutic efficacy of FGFR4 inhibitors in clinical metastatic cancers.

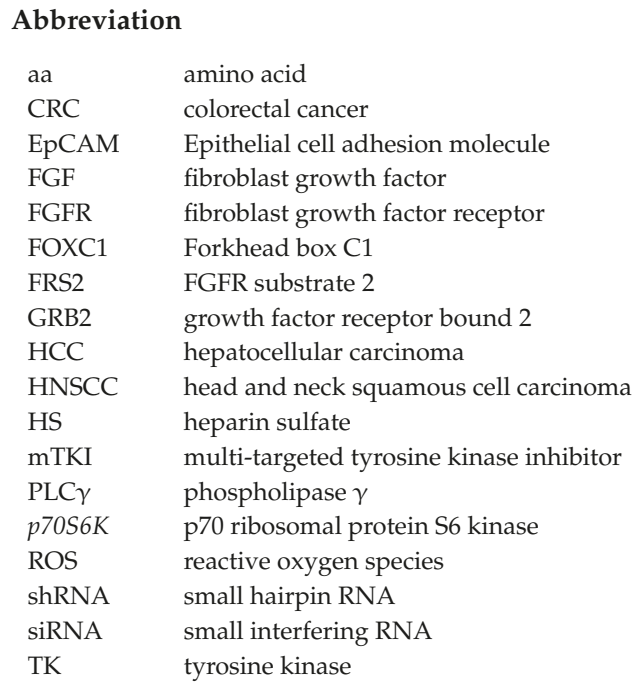

Author Contributions: Writing-L.L., Y.T.; Review and editing-Y.T.

Funding: This research received no external funding.

Conflicts of Interest: The authors declare no conflict of interest.

\section{References}

1. Babina, I.S.; Turner, N.C. Advances and challenges in targeting FGFR signalling in cancer. Nat. Rev. Cancer 2017, 17, 318-332. [CrossRef]

2. Porta, R.; Borea, R.; Coelho, A.; Khan, S.; Araújo, A.; Reclusa, P.; Franchina, T.; Van Der Steen, N.; Van Dam, P.; Ferri, J. FGFR a promising druggable target in cancer: Molecular biology and new drugs. Crit. Rev. Oncol./Hematol. 2017, 113, 256-267. [CrossRef]

3. Heinzle, C.; Erdem, Z.; Paur, J.; Grasl-Kraupp, B.; Holzmann, K.; Grusch, M.; Berger, W.; Marian, B. Is fibroblast growth factor receptor 4 a suitable target of cancer therapy? Curr. Pharm. Des. 2014, 20, 2881-2898. [CrossRef] 
4. Touat, M.; Ileana, E.; Postel-Vinay, S.; André, F.; Soria, J.C. Targeting FGFR signaling in cancer. Clin. Cancer Res. 2015, 21, 2684-2694. [CrossRef]

5. Prieto-Dominguez, N.; Shull, A.Y.; Teng, Y. Making way for suppressing the FGF19/FGFR4 axis in cancer. Future Med. Chem. 2018, 10, 2457-2469. [CrossRef]

6. Helsten, T.; Schwaederle, M.; Kurzrock, R. Fibroblast growth factor receptor signaling in hereditary and neoplastic disease: Biologic and clinical implications. Cancer Metastasis Rev. 2015, 34, 479-496. [CrossRef]

7. Lin, B.C.; Wang, M.; Blackmore, C.; Desnoyers, L.R. Liver-specific activities of FGF19 require Klotho beta. J. Biol. Chem. 2007, 282, 27277-27284. [CrossRef]

8. Ornitz, D.M.; Xu, J.; Colvin, J.S.; McEwen, D.G.; MacArthur, C.A.; Coulier, F.; Gao, G.; Goldfarb, M. Receptor specificity of the fibroblast growth factor family. J. Biol. Chem. 1996, 271, 15292-15297. [CrossRef]

9. Zhang, X.; Ibrahimi, O.A.; Olsen, S.K.; Umemori, H.; Mohammadi, M.; Ornitz, D.M. Receptor specificity of the fibroblast growth factor family, part II. J. Biol. Chem. 2006, 281, 15694-15700. [CrossRef]

10. Huang, X.; Yang, C.; Luo, Y.; Jin, C.; Wang, F.; McKeehan, W.L. FGFR4 Prevents Hyperlipidemia and Insulin Resistance but Underlies High Fat Diet-Induced Fatty Liver. Diabetes 2007, 56, 2501-2510. [CrossRef]

11. Kir, S.; Beddow, S.A.; Samuel, V.T.; Miller, P.; Previs, S.F.; Suino-Powell, K.; Xu, H.E.; Shulman, G.I.; Kliewer, S.A.; Mangelsdorf, D.J. FGF19 as a postprandial, insulin-independent activator of hepatic protein and glycogen synthesis. Science 2011, 331, 1621-1624. [CrossRef] [PubMed]

12. Yu, C.; Wang, F.; Jin, C.; Wu, X.; Chan, W.-K.; McKeehan, W.L. Increased carbon tetrachloride-induced liver injury and fibrosis in FGFR4-deficient mice. Am. J. Pathol. 2002, 161, 2003-2010. [CrossRef]

13. Wu, A.L.; Coulter, S.; Liddle, C.; Wong, A.; Eastham-Anderson, J.; French, D.M.; Peterson, A.S.; Sonoda, J. FGF19 regulates cell proliferation, glucose and bile acid metabolism via FGFR4-dependent and independent pathways. PLoS ONE 2011, 6, e17868. [CrossRef] [PubMed]

14. Floss, T.; Arnold, H.-H.; Braun, T. A role for FGF-6 in skeletal muscle regeneration. Genes Dev. 1997, 11, 2040-2051. [CrossRef] [PubMed]

15. Zhao, P.; Hoffman, E.P. Embryonic myogenesis pathways in muscle regeneration. Dev. Dyn. 2004, 229, 380-392. [CrossRef] [PubMed]

16. Partanen, J.; Mäkelä, T.; Eerola, E.; Korhonen, J.; Hirvonen, H.; Claesson-Welsh, L.; Alitalo, K. FGFR-4, a novel acidic fibroblast growth factor receptor with a distinct expression pattern. EMBO J. 1991, 10, 1347-1354. [CrossRef] [PubMed]

17. Weinstein, M.; Xu, X.; Ohyama, K.; Deng, C.-X. FGFR-3 and FGFR-4 function cooperatively to direct alveogenesis in the murine lung. Development 1998, 125, 3615-3623. [PubMed]

18. Helsten, T.; Elkin, S.; Arthur, E.; Tomson, B.N.; Carter, J.; Kurzrock, R. The FGFR landscape in cancer: Analysis of 4,853 tumors by next-generation sequencing. Clin. Cancer Res. 2016, 22, 259-267. [CrossRef] [PubMed]

19. Taylor, J.G.; Cheuk, A.T.; Tsang, P.S.; Chung, J.Y.; Song, Y.K.; Desai, K.; Yu, Y.; Chen, Q.R.; Shah, K.; Youngblood, V. Identification of FGFR4-activating mutations in human rhabdomyosarcomas that promote metastasis in xenotransplanted models. J. Clin. Investig. 2009, 119, 3395-3407.

20. Roidl, A.; Foo, P.; Wong, W.; Mann, C.; Bechtold, S.; Berger, H.; Streit, S.; Ruhe, J.; Hart, S.; Ullrich, A. The FGFR4 Y367C mutant is a dominant oncogene in MDA-MB453 breast cancer cells. Oncogene 2010, 29, 1543-1552. [CrossRef]

21. Ho, H.K.; Pok, S.; Streit, S.; Ruhe, J.E.; Hart, S.; Lim, K.S.; Loo, H.L.; Aung, M.O.; Lim, S.G.; Ullrich, A. Fibroblast growth factor receptor 4 regulates proliferation, anti-apoptosis and alpha-fetoprotein secretion during hepatocellular carcinoma progression and represents a potential target for therapeutic intervention. J. Hepatol. 2009, 50, 118-127. [CrossRef] [PubMed]

22. Penault-Llorca, F.; Bertucci, F.; Adélaïde, J.; Parc, P.; Coulier, F.; Jacquemier, J.; Birnbaum, D.; Delapeyrière, O. Expression of FGF and FGF receptor genes in human breast cancer. Int. J. Cancer 1995, 61, 170-176. [CrossRef] [PubMed]

23. Koole, K.; Van Kempen, P.M.; Van Bockel, L.W.; Smets, T.; Van Der Klooster, Z.; Dutman, A.C.; Peeters, T.; Koole, R.; Van Diest, P.; Van Es, R.J. FGFR4 is a potential predictive biomarker in oral and oropharyngeal squamous cell carcinoma. Pathobiology 2015, 82, 280-289. [CrossRef] [PubMed]

24. Shah, R.N.; Ibbitt, J.C.; Alitalo, K.; Hurst, H.C. FGFR4 overexpression in pancreatic cancer is mediated by an intronic enhancer activated by HNF1 $\alpha$. Oncogene 2002, 21, 8251-8261. [CrossRef] [PubMed] 
25. Huang, X.; Gollin, S.M.; Raja, S.; Godfrey, T.E. High-resolution mapping of the 11q13 amplicon and identification of a gene, TAOS1, that is amplified and overexpressed in oral cancer cells. Proc. Natl. Acad. Sci. USA 2002, 99, 11369-11374. [CrossRef] [PubMed]

26. Sawey, E.T.; Chanrion, M.; Cai, C.; Wu, G.; Zhang, J.; Zender, L.; Zhao, A.; Busuttil, R.W.; Yee, H.; Stein, L. Identification of a therapeutic strategy targeting amplified FGF19 in liver cancer by Oncogenomic screening. Cancer Cell 2011, 19, 347-358. [CrossRef] [PubMed]

27. Tiong, K.H.; Tan, B.S.; Choo, H.L.; Chung, F.F.; Hii, L.W.; Tan, S.H.; Khor, N.T.; Wong, S.F.; See, S.J.; Tan, Y.F.; et al. Fibroblast growth factor receptor 4 (FGFR4) and fibroblast growth factor 19 (FGF19) autocrine enhance breast cancer cells survival. Oncotarget 2016, 7, 57633. [CrossRef]

28. Zhang, X.; Kong, M.; Zhang, Z.; Xu, S.; Yan, F.; Wei, L.; Zhou, J. FGF 19 genetic amplification as a potential therapeutic target in lung squamous cell carcinomas. Thorac. Cancer 2017, 8, 655-665. [CrossRef]

29. Hoover, H.; Li, J.; Marchese, J.; Rothwell, C.; Borawoski, J.; Jeffery, D.A.; Gaither, L.A.; Finkel, N. Quantitative proteomic verification of membrane proteins as potential therapeutic targets located in the 11q13 amplicon in cancers. J. Proteome Res. 2015, 14, 3670-3679. [CrossRef]

30. Zhao, H.; Lv, F.; Liang, G.; Huang, X.; Wu, G.; Zhang, W.; Yu, L.; Shi, L.; Teng, Y. FGF19 promotes epithelial-mesenchymal transition in hepatocellular carcinoma cells by modulating the GSK3beta/betacatenin signaling cascade via FGFR4 activation. Oncotarget 2016, 7, 13575-13586. [CrossRef]

31. Gao, L.; Lang, L.; Zhao, X.; Shay, C.; Shull, A.Y.; Teng, Y. FGF19 Amplification Reveals an Oncogenic Dependency upon Autocrine FGF19/FGFR4 Signaling in Head and Neck Squamous Cell Carcinoma. Oncogene 2018, in press. [CrossRef] [PubMed]

32. Paur, J.; Nika, L.; Maier, C.; Moscu-Gregor, A.; Kostka, J.; Huber, D.; Mohr, T.; Heffeter, P.; Schrottmaier, W.C.; Kappel, S. Fibroblast growth factor receptor 3 isoforms: Novel therapeutic targets for hepatocellular carcinoma? Hepatology 2015, 62, 1767-1778. [CrossRef] [PubMed]

33. Del Piccolo, N.; Sarabipour, S.; Hristova, K. A new method to study heterodimerization of membrane proteins and its application to fibroblast growth factor receptors. J. Biol. Chem. 2017, 292, 1288-1301. [CrossRef] [PubMed]

34. Manning, B.D.; Toker, A. AKT/PKB signaling: Navigating the network. Cell 2017, 169, 381-405. [CrossRef] [PubMed]

35. Degirolamo, C.; Sabba, C.; Moschetta, A. Therapeutic potential of the endocrine fibroblast growth factors FGF19, FGF21 and FGF23. Nat. Rev. Drug Discov. 2016, 15, 51-69. [CrossRef] [PubMed]

36. Panera, N.; Ceccarelli, S.; Nobili, V.; Alisi, A. Targeting FGF19 binding to its receptor system: A novel therapeutic approach for hepatocellular carcinoma. Hepatology 2015, 62, 1324. [CrossRef] [PubMed]

37. Zhao, X.; Xu, F.; Dominguez, N.P.; Xiong, Y.; Xiong, Z.; Peng, H.; Shay, C.; Teng, Y. FGFR4 provides the conduit to facilitate FGF19 signaling in breast cancer progression. Mol. Carcinog. 2018, 57, 1616-1625. [CrossRef] [PubMed]

38. Dallol, A.; Buhmeida, A.; Merdad, A.; Al-Maghrabi, J.; Gari, M.A.; Abu-Elmagd, M.M.; Elaimi, A.; Assidi, M.; Chaudhary, A.G.; Abuzenadah, A.M. Frequent methylation of the KLOTHO gene and overexpression of the FGFR4 receptor in invasive ductal carcinoma of the breast. Tumor Biol. 2015, 36, 9677-9683. [CrossRef]

39. Peláez-García, A.; Barderas, R.; Torres, S.; Hernández-Varas, P.; Teixidó, J.; Bonilla, F.; de Herreros, A.G.; Casal, J.I. FGFR4 role in epithelial-mesenchymal transition and its therapeutic value in colorectal cancer. PLoS ONE 2013, 8, e63695. [CrossRef]

40. Miura, S.; Mitsuhashi, N.; Shimizu, H.; Kimura, F.; Yoshidome, H.; Otsuka, M.; Kato, A.; Shida, T.; Okamura, D.; Miyazaki, M. Fibroblast growth factor 19 expression correlates with tumor progression and poorer prognosis of hepatocellular carcinoma. BMC Cancer 2012, 12, 56. [CrossRef]

41. Liu, J.; Zhang, Z.; Li, X.; Chen, J.; Wang, G.; Tian, Z.; Qian, M.; Chen, Z.; Guo, H.; Tang, G. Forkhead box C1 promotes colorectal cancer metastasis through transactivating ITGA7 and FGFR4 expression. Oncogene 2018, 37, 5477-5491. [CrossRef] [PubMed]

42. Housman, G.; Byler, S.; Heerboth, S.; Lapinska, K.; Longacre, M.; Snyder, N.; Sarkar, S. Drug resistance in cancer: An overview. Cancers 2014, 6, 1769-1792. [CrossRef] [PubMed]

43. Roidl, A.; Berger, H.-J.; Kumar, S.; Bange, J.; Knyazev, P.; Ullrich, A. Resistance to chemotherapy is associated with fibroblast growth factor receptor 4 up-regulation. Clin. Cancer Res. 2009, 15, 2058-2066. [CrossRef] [PubMed] 
44. Turkington, R.; Longley, D.; Allen, W.; Stevenson, L.; McLaughlin, K.; Dunne, P.; Blayney, J.; Salto-Tellez, M.; Van Schaeybroeck, S.; Johnston, P. Fibroblast growth factor receptor 4 (FGFR4): A targetable regulator of drug resistance in colorectal cancer. Cell. Death Disease 2014, 5, e1046. [CrossRef] [PubMed]

45. Van Malenstein, H.; Dekervel, J.; Verslype, C.; Van Cutsem, E.; Windmolders, P.; Nevens, F.; van Pelt, J. Long-term exposure to sorafenib of liver cancer cells induces resistance with epithelial-to-mesenchymal transition, increased invasion and risk of rebound growth. Cancer Lett. 2013, 329, 74-83. [CrossRef] [PubMed]

46. Villanueva, A.; Llovet, J.M. Second-line therapies in hepatocellular carcinoma: Emergence of resistance to sorafenib. Clin. Cancer Res. 2012, 18, 1824-1826. [CrossRef] [PubMed]

47. Gao, L.; Wang, X.; Tang, Y.; Huang, S.; Hu, C.-A.A.; Teng, Y. FGF19/FGFR4 signaling contributes to the resistance of hepatocellular carcinoma to sorafenib. J. Exp. Clin. Cancer Res. 2017, 36, 1-10. [CrossRef]

48. Octavia, Y.; Brunner-La Rocca, H.P.; Moens, A.L. NADPH oxidase-dependent oxidative stress in the failing heart: From pathogenic roles to therapeutic approach. Free Radic. Biol. Med. 2012, 52, 291-297. [CrossRef]

49. Valastyan, S.; Weinberg, R.A. Tumor metastasis: Molecular insights and evolving paradigms. Cell 2011, 147, 275-292. [CrossRef]

50. Hu, L.; Cong, L. Fibroblast growth factor 19 is correlated with an unfavorable prognosis and promotes progression by activating fibroblast growth factor receptor 4 in advanced-stage serous ovarian cancer. Oncol. Rep. 2015, 34, 2683-2691. [CrossRef]

51. Bartz, R.; Fukuchi, K.; Lange, T.; Gruner, K.; Ohtsuka, T.; Watanabe, I.; Hayashi, S.; Redondo-Müller, M.; Takahashi, M.; Agatsuma, T. U3-1784, a human anti-FGFR4 antibody for the treatment of cancer. Cancer Res. 2016, 76, 3852. [CrossRef]

52. Dienstmann, R.; Rodon, J.; Prat, A.; Perez-Garcia, J.; Adamo, B.; Felip, E.; Cortes, J.; Iafrate, A.; Nuciforo, P.; Tabernero, J. Genomic aberrations in the FGFR pathway: Opportunities for targeted therapies in solid tumors. Ann. Oncol. 2014, 25, 552-563. [CrossRef] [PubMed]

53. Li, S.Q.; Cheuk, A.T.; Shern, J.F.; Song, Y.K.; Hurd, L.; Liao, H.; Wei, J.S.; Khan, J. Targeting wild-type and mutationally activated FGFR4 in rhabdomyosarcoma with the inhibitor ponatinib (AP24534). PLoS ONE 2013, 8, e76551. [CrossRef]

54. Cabanillas, M.E.; Schlumberger, M.; Jarzab, B.; Martins, R.G.; Pacini, F.; Robinson, B.; McCaffrey, J.C.; Shah, M.H.; Bodenner, D.L.; Topliss, D. A phase 2 trial of lenvatinib (E7080) in advanced, progressive, radioiodine-refractory, differentiated thyroid cancer: A clinical outcomes and biomarker assessment. Cancer 2015, 121, 2749-2756. [CrossRef] [PubMed]

55. Wolf, J.; LoRusso, P.M.; Camidge, R.D.; Perez, J.M.; Tabernero, J.; Hidalgo, M.; Schuler, M.; Tian, G.G.; Soria, J.C.; Delord, J.P. Abstract LB-122: A phase I dose escalation study of NVP-BGJ398, a selective pan FGFR inhibitor in genetically preselected advanced solid tumors. Cancer Res. 2012, 72, LB-122. [CrossRef]

56. Gavine, P.R.; Mooney, L.; Kilgour, E.; Thomas, A.P.; Al-Kadhimi, K.; Beck, S.; Rooney, C.; Coleman, T.; Baker, D.; Mellor, M.J. AZD4547: An orally bioavailable, potent, and selective inhibitor of the fibroblast growth factor receptor tyrosine kinase family. Cancer Res. 2012, 72, 2045-2056. [CrossRef]

57. Tie, J.; Bang, Y.-J.; Park, Y.S.; Kang, Y.-K.; Monteith, D.; Hartsock, K.; Thornton, D.E.; Michael, M. Abstract CT215: A phase I trial of LY2874455, a fibroblast growth factor receptor inhibitor, in patients with advanced cance. Cancer Res. 2014, 74, CT215. [CrossRef]

58. Tan, L.; Wang, J.; Tanizaki, J.; Huang, Z.; Aref, A.R.; Rusan, M.; Zhu, S.-J.; Zhang, Y.; Ercan, D.; Liao, R.G. Development of covalent inhibitors that can overcome resistance to first-generation FGFR kinase inhibitors. Proc. Natl. Acad. Sci. USA 2014, 111, e4869-e4877. [CrossRef]

59. Repana, D.; Ross, P. Targeting FGF19/FGFR4 Pathway: A Novel Therapeutic Strategy for Hepatocellular Carcinoma. Diseases 2015, 3, 294-305. [CrossRef]

60. Dieci, M.V.; Arnedos, M.; Andre, F.; Soria, J.C. Fibroblast growth factor receptor inhibitors as a cancer treatment: From a biologic rationale to medical perspectives. Cancer Discov. 2013, 3, 264-279. [CrossRef]

61. Gao, L.; Shay, C.; Lv, F.; Wang, X.; Teng, Y. Implications of FGF19 on sorafenib-mediated nitric oxide production in hepatocellular carcinoma cells-a short report. Cell. Oncol. 2018, 41, 85-91. [CrossRef] [PubMed]

62. Hagel, M.; Miduturu, C.; Sheets, M.; Rubin, N.; Weng, W.; Stransky, N.; Bifulco, N.; Kim, J.L.; Hodous, B.; Brooijmans, N. First selective small molecule inhibitor of FGFR4 for the treatment of hepatocellular carcinomas with an activated FGFR4 signaling pathway. Cancer Discov. 2015, 5, 1-14. [CrossRef] [PubMed] 
63. Kim, R.; Sharma, S.; Meyer, T.; Sarker, D.; Macarulla, T.; Sung, M.; Choo, S.; Shi, H.; Schmidt-Kittler, O.; Clifford, C. First-in-human study of BLU-554, a potent, highly-selective FGFR4 inhibitor designed for hepatocellular carcinoma (HCC) with FGFR4 pathway activation. Eur. J. Cancer 2016, 69, S41. [CrossRef]

64. Joshi, J.J.; Coffey, H.; Corcoran, E.; Tsai, J.; Huang, C.-L.; Ichikawa, K.; Prajapati, S.; Hao, M.-H.; Bailey, S.; Wu, J. H3B-6527 Is a Potent and Selective Inhibitor of FGFR4 in FGF19-Driven Hepatocellular Carcinoma. Cancer Res. 2017, 77, 6999-7013. [CrossRef] [PubMed]

65. Chan, S.L.; Yen, C.-J.; Schuler, M.; Lin, C.-C.; Choo, S.P.; Weiss, K.-H.; Geier, A.; Okusaka, T.; Lim, H.Y.; Macarulla, T. Abstract CT106: Ph I/II study of FGF401 in adult pts with HCC or solid tumors characterized by FGFR4/KLB expression. Cancer Res. 2017, 77, CT106. [CrossRef]

66. Li, Y.; Zhang, W.; Doughtie, A.; Cui, G.; Li, X.; Pandit, H.; Yang, Y.; Li, S.; Martin, R. Up-regulation of fibroblast growth factor 19 and its receptor associates with progression from fatty liver to hepatocellular carcinoma. Oncotarget 2016, 7, 52329-52339. [CrossRef] [PubMed]

67. Xie, X.; Gao, L.; Shull, A.Y.; Teng, Y. Stapled peptides: Providing the best of both worlds in drug development. Future Med. Chem. 2016, 8, 1969-1980. [CrossRef] [PubMed]

(C) 2019 by the authors. Licensee MDPI, Basel, Switzerland. This article is an open access article distributed under the terms and conditions of the Creative Commons Attribution (CC BY) license (http:/ / creativecommons.org/licenses/by/4.0/). 

MDPI

St. Alban-Anlage 66

4052 Basel

Switzerland

Tel. +41 616837734

Fax +41 613028918

www.mdpi.com

Cells Editorial Office

E-mail: cells@mdpi.com www.mdpi.com/journal/cells

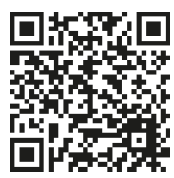



MDPI

St. Alban-Anlage 66

4052 Basel

Switzerland

Tel: +41 616837734

Fax: +41 613028918

www.mdpi.com 